Wesley Góis

\title{
ELEMENTOS FINITOS HÍBRIDOS E HÍBRIDO-MISTOS DE TENSÃO COM ENRIQUECIMENTO NODAL
}

Tese apresentada à Escola de Engenharia de São Carlos, Universidade de São Paulo, como parte dos requisitos para a obtenção do título de Doutor em Engenharia de Estruturas.

Orientador: Prof. Tit. Sergio Persival Baroncini Proença

São Carlos

2009 

Candidato: Engenheiro WESLEY GÓIS

Tese defendida e julgada em 14/05/2009 perante a Comissão Julgadora:

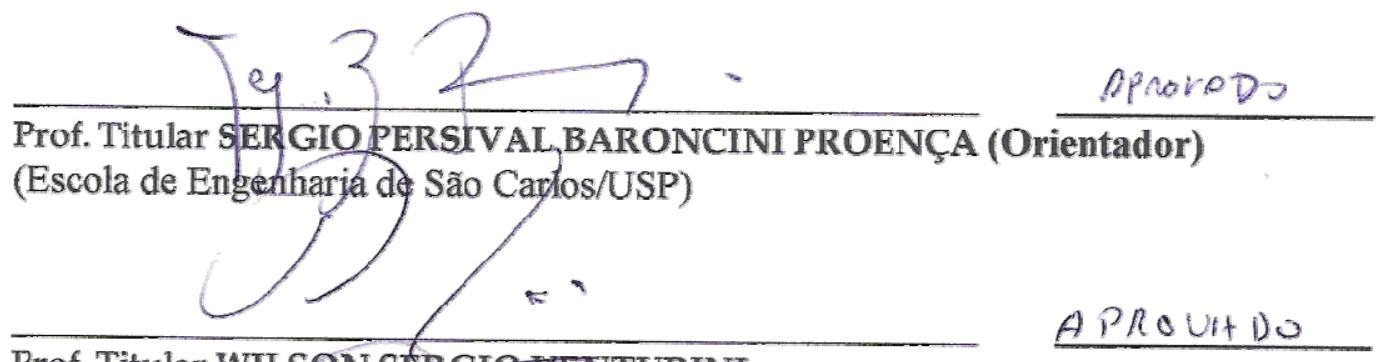

Prof. Titular WILSON SERGIO VENTURINI

(Escola de Engenharia de São Carlos/USP)

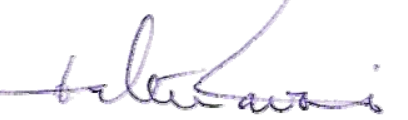

APROVADO

Prof. Titular WALTER SAVASSI

(Escola de Engenharia de São Carlos/USP)

$$
\text { Alaisio \& Arran }
$$

Prof. Titular ALOÍSIO ERNESTO ASSAN

Aprovodo

(Universidade Estadual de Campinas/UNICAMP)

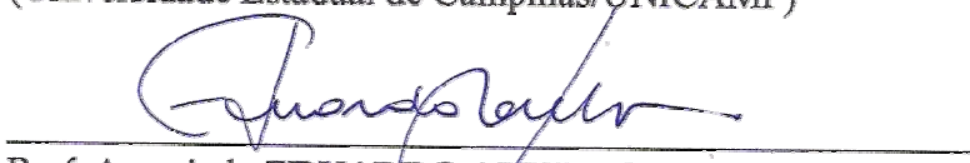

ApRovado

Prof. Associado EDUARDO ALBERTO FANCELLO

(Universidade Federal de Santa Catarina/UFSC)

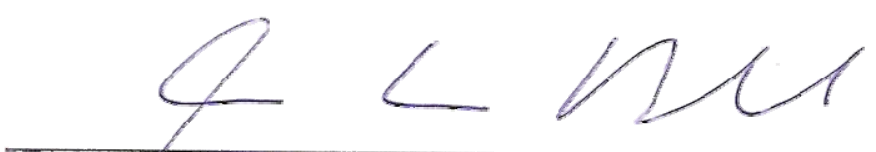

Prof. Associado MARCIO ANTONIO RAMALHO

Coordenador do Programa de Pós-Graduação em

Engenharia Civil (Engenharia de Estruturas)

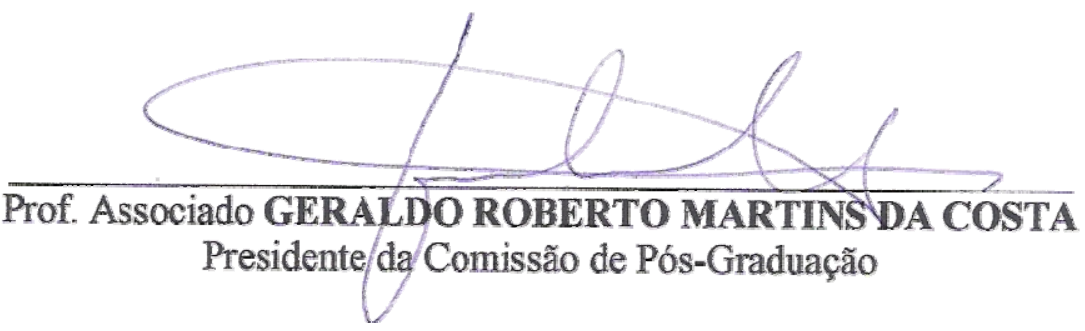


Dedico este trabalho aos meus pais Bomfim e Creuza 



\section{AGRADECIMENTOS}

Ao Prof. Tit. Sergio Persival Baroncini Proença, pela amizade, pela orientação competente e oportunidades concedidas durante estes sete anos de convivência. Prof. Sérgio! Também agradeço por todos aqueles sábados que você se dispôs a passar comigo no Departamento de Engenharia de Estruturas, quando das minhas idas e vindas, São Carlos-São José dos Campos.

Aos professores da Universidade Federal de Sergipe, em especial, Ângela Tereza Costa Sales e Josafá de Oliveira Filho pela amizade e confiança.

Aos funcionários e professores do Departamento de Engenharia de Estruturas da Escola de Engenharia de São Carlos, pela disposição em ajudar.

À minha amiga Denise Conceição, pelos anos de convivência em São Carlos.

Aos meus amigos Ava Brandão e Daniel Barbosa, pela amizade, pelo acolhimento e paciência que tiveram comigo durante as idas e vindas, São Carlos-São José dos Campos.

Aos eternos amigos-irmãos do Vôlei CAASO, pelas lutas, angústias, brigas, choros, derrotas e conquistas. Em especial ao João Guilherme (JoGui), Paulino, Rafel, Rodrigo (Slow), Marcelinho, William, Ricardo, Julio (Maromba), Vitão, Ricardo (Xaxá), Marcinha (Japa), Malu, Dudão e todos os outros atletas e técnicos que passaram por essa grande família.

Aos muitos amigos da USP/São Carlos, pelo companheirismo. Em especial ao Francisco Adriano, Ivan Torres, Fabiana, Larissa Kirchhof, Luciana Pizzo, Oscar Begambre, Walter Luiz, Claudius, Sandrinha, Leandro, Marcelo, Leonardo, Madalena, Kelly, Luciana, Ana Rosa, Eddy e Dani.

Ao amigo Ricardo de Sá Teles, pela amizade, pela honestidade, pela acolhida e pelos agradáveis momentos compartilhados em São Carlos.

Aos amigos da minha cidade natal, Ribeirópolis-SE, pela torcida.

À CAPES, pela bolsa de estudo concedida.

À minha irmã Maria Cleide e ao meu cunhado Antônio Mendonça, pela atenção e apoio, sobretudo, nos momentos difíceis que passei em São Paulo. Aos meus queridos sobrinhos Helder, Heider e Hedlla pelo carinho e acolhida em Osasco.

Aos meus irmãos e sobrinhos, pelas orações e amor que sempre me deram.

Aos meus pais Bomfim e Creuza, por tudo que me ensinaram a minha eterna gratidão.

A Deus, ao Sagrado Coração de Jesus e à Bem-Aventurada Virgem Maria do Monte Carmelo, pela minha vida repleta de graças e fontes inesgotáveis de amor e fé. 



\section{RESUMO}

GÓIS, W. (2009). Elementos finitos híbridos e híbrido-mistos de tensão com enriquecimento nodal. Tese (Doutorado) - Escola de Engenharia de São Carlos, Universidade de São Paulo, São Carlos.

Neste trabalho, a técnica de enriquecimento da partição da unidade é estendida e adaptada para duas formulações não-convencionais para a elasticidade plana: a formulação híbrida de tensão (FHT) e a formulação híbrido-mista de tensão (FHMT). Estas formulações são ditas não-convencionais, pois não recorrem a princípios variacionais clássicos. Elementos finitos triangulares e quadrilaterais com enriquecimento nodal são desenvolvidos para avaliação da forma discreta das duas formulações estudadas. Na FHMT, três campos são aproximados de forma independente: tensões e deslocamentos no domínio e deslocamentos no contorno. O conceito de partição da unidade é então utilizado para garantir continuidade de cada um dos campos envolvidos na FHMT e realizar o procedimento de enriquecimento nodal. Funções polinomiais são utilizadas para enriquecer cada uma das aproximações dos campos da FHMT. A sensibilidade das respostas em relação a redes distorcidas é avaliada. Além disso, abordam-se aspectos relativos à convergência e estabilidade da solução numérica. Especificamente para a FHT, dois campos são independentemente aproximados: tensões no domínio e deslocamentos na fronteira estática. As aproximações das tensões, que por definição não estão atreladas a nós, devem primeiramente satisfazer a condição de equilíbrio no domínio. O conceito de partição da unidade é empregado, neste caso, para dar continuidade aos deslocamentos entre as fronteiras dos elementos. O enriquecimento polinomial da partição de unidade é então aplicado às aproximações dos deslocamentos no contorno. Para o campo de tensões no domínio, desenvolve-se uma técnica específica de enriquecimento nodal. Mais uma vez, aspectos relativos à sensibilidade à distorção de redes e convergência são estudados e avaliados. Finalmente, alguns exemplos numéricos são apresentados para ilustrar o desempenho de ambas as abordagens, especialmente quando a técnica de enriquecimento é aplicada.

Palavras-chave: Método dos Elementos Finitos; Método dos Elementos Finitos Generalizados; Formulação Híbrida e Híbrido-Mista de Tensão; Estabilidade do Método dos Elementos Finitos. 



\section{ABSTRACT}

GÓIS, W. (2009). Stress hybrid and hybrid-mixed finite elements with nodal enrichment. PhD Thesis - São Carlos School of Engineering, University of São Paulo, São Carlos.

In the present work, the partition of unity enrichment concept is basically applied to non-conventional stress hybrid-mixed and hybrid formulations in plane elasticity. These formulations are referred to as non-conventional because no Variational Principles are explored. From these, triangular and quadrilateral finite elements with selective nodal enrichment are then derived. In the stress hybrid-mixed approach, three independent fields are approximated: stress and displacement fields in the domain and displacement fields on the static boundary. The partition of unity concept is then used to provide continuity to all the fields involved. Afterwards, the nodal enrichment feature is explored. Polynomial functions are employed to enrich each one of the approximation fields. Besides, some aspects concerning convergence and stability of the numerical solutions obtained are addressed. On the other hand, in the hybrid approach, two independent fields are approximated: stress fields in the domain and displacement fields on the static boundary. However, the approximation of the stress field must first satisfy the equilibrium condition in the domain without involving nodal values in its definition. Hence, the partition of unity concept is used to provide continuity of displacements between the boundaries of the elements. The partition of unity based nodal enrichment is then applied to the boundary displacement fields. Nevertheless, enrichment of the stress field can also be carried out with exploring a specific and original technique that permits applied the partition of unity concept but in such way as to preserve satisfaction of the equilibrium condition in the domain. Again, convergence and stability aspects of the hybrid approach are briefly addressed. Finally, some numerical examples are presented to illustrate the performance of both approaches derived, especially when combined possibilities of enrichment are explored.

Keywords: Finite Element Method; Generalized Finite Element Method; Stress Hybrid and Hybrid-Mixed Formulations; Stability of Finite Element Method. 



\section{Lista de Figuras}

Figura 2.1 - Corpo elástico submetido a forças de volume e de superfície e a representação das partes complementares do contorno.

Figura 2.2 - Elemento de volume $\boldsymbol{\Omega}$ e contornos $\boldsymbol{\Gamma}_{t}, \boldsymbol{\Gamma}_{u}$ e $\boldsymbol{\Gamma}_{\boldsymbol{i}} \ldots \ldots \ldots \ldots \ldots \ldots . . . \ldots . . . . .16$

Figura 2.3 - Dois elementos de volume $\boldsymbol{\Omega}$ e contornos $\boldsymbol{\Gamma}_{t}, \boldsymbol{\Gamma}_{u}$ e $\boldsymbol{\Gamma}_{i} \ldots \ldots \ldots \ldots . . . . .$.

Figura 2.4 - Dois elementos de volume $\boldsymbol{\Omega}$ e contornos $\boldsymbol{\Gamma}_{t}, \boldsymbol{\Gamma}_{u}$ e $\boldsymbol{\Gamma}_{\boldsymbol{i}}$ continuidade e reciprocidade em forma fraca.

Figura 3.1 - Enriquecimento da Partição da Unidade num domínio global $\boldsymbol{x y}$..

Figura 3.2 - FHMT e FHT - nuvens de influência para as redes de cobertura: domínio (bidimensional) e contorno (unidimensional)

Figura 4.1 - Elemento quadrilateral de quatro nós.

Figura 4.2 - Elemento finito linear com sistema local de referência com origem no seu centro.

Figura 4.3 - Elemento triangular de três nós.

Figura 4.4 - Elemento triangular de três nós no espaço $\xi_{1} \xi_{2}$

Figura 4.5 - Elemento finito linear com sistema de referência $\boldsymbol{\xi}$

59

Figura 7.1 - Chapa com 100 unidades de momento concentrado. 108

Figura 7.2 - Conjunto de Redes Quadrilaterais e Triangulares: Exemplo 1.

Figura 7.3 - Tensão plana $-\boldsymbol{\sigma}_{\boldsymbol{x}}$ - elemento quadrilateral e triangular sem enriquecimento $-(\boldsymbol{\%}=\boldsymbol{0})-$ FHMT.

Figura 7.4 - Tensão plana $-\sigma_{x}$ - elemento quadrilateral e triangular sem enriquecimento - $(\boldsymbol{\%} \boldsymbol{\mu}=\boldsymbol{0})$ - Aproximação Quadrática das Tensões - FHT... 
Figura 7.5 - Nós enriquecidos no Exemplo 1- FHT e FHMT.

Figura 7.6 - Sensibilidade do deslocamento à distorção da rede - Elemento Quadrilateral - FHT, FHMT e MEF Clássico

Figura 7.7 - Sensibilidade do deslocamento à distorção da rede - Elemento Triangular - FHT, FHMT e MEF Clássico.

Figura 7.8 - Sensibilidade do deslocamento à distorção da rede - Elemento Quadrilateral - FHT e MEF Clássico.....

Figura 7.9 - Sensibilidade do deslocamento à distorção da rede - Elemento Triangular - FHT e MEF clássico

Figura 7.10 - Sensibilidade do deslocamento à distorção da rede - Elemento Quadrilateral - FHT e Punch e Atluri (1984).

Figura 7.11 - Sensibilidade do deslocamento à distorção da rede - Elemento Quadrilateral - FHMT e MEF clássico.

Figura 7.12 - Sensibilidade do deslocamento à distorção da rede - Elemento Triangular - FHMT e MEF clássico

Figura 7.13 - Sensibilidade do deslocamento à distorção da rede - Elemento Quadrilateral - FHMT e Punch and Atluri (1984).

Figura 7.14 - Tensão plana $-\sigma_{x}$ - elemento quadrilateral e triangular sem enriquecimento $-(\boldsymbol{\%} \boldsymbol{\mu}=\boldsymbol{8 0})-$ FHMT

Figura 7.15 - Tensão plana - $\boldsymbol{\sigma}_{\boldsymbol{x}}$ - elemento quadrilateral e triangular sem enriquecimento - $(\boldsymbol{\%} \boldsymbol{\mu}=\boldsymbol{8 0})$ - Aproximação Quadrática das Tensões - FHT. Figura 7.16 - Tensão plana $-\boldsymbol{\sigma}_{\boldsymbol{x}}$ - elemento quadrilateral com enriquecimento
seletivo dos Deslocamentos no Contorno $(\boldsymbol{x})-(\boldsymbol{\%} \boldsymbol{\mu}=\boldsymbol{8 0})-$ Aproximação Quadrática das Tensões - FHT.

Tensão plana - $\boldsymbol{\sigma}_{\boldsymbol{x}}$ - elemento triangular com enriquecimento

Figura 7.17 - seletivo dos Deslocamentos no Contorno $(\boldsymbol{x})-(\boldsymbol{\%} \boldsymbol{\mu}=\boldsymbol{8 0})-$ Aproximação Quadrática das Tensões - FHT. 
Tensão plana - $\boldsymbol{\sigma}_{\boldsymbol{x}}$ - elemento quadrilateral com enriquecimento

Figura 7.18 - das Tensões e Deslocamentos no Domínio $\left(y^{2}\right)$ e Deslocamentos no Contorno $(\boldsymbol{x})-(\boldsymbol{\%} \boldsymbol{\mu}=\boldsymbol{8 0})-$ FHMT...............

Figura 7.19 - Tensão plana - $\sigma_{x}$ - elemento triangular com enriquecimento das Tensões e Deslocamentos no Domínio $\left(y^{2}\right)$ e Deslocamentos no Contorno $(\boldsymbol{x})-(\boldsymbol{\%} \boldsymbol{\mu} \boldsymbol{8 0})-$ FHMT

Figura 7.20 - Sensibilidade da energia de deformação à distorção da rede Elemento Quadrilateral - FHT, FHMT e MEF clássico.

Figura 7.21 - Sensibilidade da energia de deformação à distorção da rede Elemento Triangular - FHT, FHMT e MEF clássico.

Figura 7.22 - Sensibilidade da energia de deformação à distorção da rede Elemento Quadrilateral - FHT e MEF clássico.

Figura 7.23 - Sensibilidade da energia de deformação à distorção da rede Elemento Triangular- FHT e MEF clássico.

Figura 7.24 - Sensibilidade da energia de deformação à distorção da rede Elemento Quadrilateral - FHMT e MEF clássico.

Figura 7.25 - Sensibilidade da energia de deformação à distorção da rede Elemento Triangular- FHMT e MEF clássico.

Figura 7.26 - Exemplo 2 - Chapa com 100 unidades de força por unidade de comprimento.

Figura 7.27 - Exemplo 2 - Deslocamento $\boldsymbol{u}_{\boldsymbol{y}}$ para a discretização adotada.......... 127

Figura 7.28 - Exemplo 2 - Tensões Planas para a discretização adotada. 128

Figura 7.29 - Tensões planas - elemento quadrilateral sem enriquecimento $(\% \boldsymbol{\mu}=\boldsymbol{0})-$ FHMT. 130

Figura 7.30 - Tensões planas - elemento quadrilateral sem enriquecimento $(\boldsymbol{\%}=\boldsymbol{0})$ - Aproximação Quadrática das Tensões - FHT. 
Figura 7.31 - Tensões planas - elemento triangular sem enriquecimento $(\boldsymbol{\%}=\boldsymbol{0})$ - Aproximação Quadrática das Tensões - FHT.

Figura 7.32 - Tensões planas - elemento quadrilateral - $(\boldsymbol{\%} \boldsymbol{\mu}=\boldsymbol{0})$ - MEF.........

Figura 7.33 - Sensibilidade do deslocamento à distorção de rede - Elemento Quadrilateral - FHT, FHMT e MEF Clássico

Figura 7.34 - Sensibilidade do deslocamento à distorção da rede - Elemento Triangular - FHT, FHMT e MEF Clássico.

Figura 7.35 - Sensibilidade do deslocamento à distorção da rede - Elemento Quadrilateral - FHT e MEF Clássico.

Figura 7.36 - Sensibilidade do deslocamento à distorção da rede - Elemento Triangular - FHT e MEF clássico

Figura 7.37 - Sensibilidade do deslocamento à distorção da rede - Elemento Quadrilateral - FHT e Punch and Atluri (1984).

Figura 7.38 - Sensibilidade do deslocamento à distorção da rede - Elemento Quadrilateral - FHMT e MEF clássico.

Sensibilidade do deslocamento à distorção da rede - Elemento

Figura 7.39 - Triangular - FHMT e MEF clássico.

Figura 7.40 - Sensibilidade do deslocamento à distorção da rede - Elemento Quadrilateral - FHMT e Punch and Altluri (1984).

Tensão plana - $\boldsymbol{\sigma}_{\boldsymbol{x}}$ - elemento quadrilateral com enriquecimento

Figura 7.41 - seletivo dos Deslocamentos no Contorno $(\boldsymbol{x})-(\boldsymbol{\%}=\boldsymbol{0})$ e $(\boldsymbol{\%}=\boldsymbol{8 0})$ - Aproximação Quadrática das Tensões - FHT.

Figura 7.42 - Tensão plana $-\sigma_{x}$ - elemento triangular com enriquecimento seletivo dos Deslocamentos no Contorno $(\boldsymbol{x})-(\boldsymbol{\%} \boldsymbol{\mu}=\boldsymbol{8 0})-$ Aproximação Quadrática das Tensões - FHT.

Figura 7.43 - Tensão plana $-\boldsymbol{\sigma}_{\boldsymbol{x}}$ - elemento quadrilateral com enriquecimento das Tensões e Deslocamentos no Domínio $\left(y^{2}\right)$ e Deslocamentos no Contorno $(\boldsymbol{x})-(\boldsymbol{\%} \boldsymbol{\mu}=\boldsymbol{8 0})$ - FHMT. 
Figura 7.44 - Tensão plana - $\sigma_{x}$ - elemento triangular com enriquecimento das Tensões e Deslocamentos no Domínio $\left(\boldsymbol{y}+\boldsymbol{y}^{2}\right)$ e Deslocamentos no Contorno $(\boldsymbol{x})-(\boldsymbol{\%} \boldsymbol{\mu}=\boldsymbol{8 0})$ - FHMT.

Figura 7.45 - Sensibilidade da energia de deformação à distorção da rede Elemento Quadrilateral - FHT, FHMT e MEF clássico.

Figura 7.46 -

Sensibilidade da energia de deformação à distorção da rede Elemento Triangular - FHT, FHMT e MEF clássico.

Figura 7.47 - Sensibilidade da energia de deformação à distorção da rede Elemento Quadrilateral - FHT e MEF clássico.

Figura 7.48 - Sensibilidade da energia de deformação à distorção da rede Elemento Triangular- FHT e MEF clássico.

Figura 7.49 - Sensibilidade da energia de deformação à distorção da rede Elemento Quadrilateral - FHMT e MEF clássico.

Figura 7.50 - Sensibilidade da energia de deformação à distorção da rede Elemento Triangular - FHMT e MEF clássico...... 146

Figura 7.51 - Exemplo 3 - Painel de Cook. 147

Figura 7.52 - Conjunto de redes distorcidas: Exemplo 3

Figura 7.53 - Convergência da energia de deformação $U_{\text {exemplo } 3}$ - Elemento Quadrilateral - FHT/FHMT e MEF clássico.

Figura 7.54 - Convergência da energia de deformação $\boldsymbol{U}_{\text {exemplo } 3}$ - Elemento Triangular - FHT/FHMT e MEF clássico

Figura 7.55 - Convergência da energia de deformação $U_{\text {exemplo } 3}$ - Elemento Quadrilateral - FHMT e MEF clássico.

Figura 7.56 - Convergência da energia de deformação $U_{\text {exemplo } 3}$ - Elemento Triangular - FHMT e MEF clássico.

Figura 7.57 - Convergência da energia de deformação $\boldsymbol{U}_{\text {exemplo } 3}$ - Elemento Quadrilateral - FHT e MEF clássico...... 
Figura 7.58 - Convergência da energia de deformação $U_{\text {exemplo } 3}$ - Elemento Triangular - FHT e MEF clássico.

Figura 7.59 - Chapa tracionada.

Figura 7.60 - Chapa tracionada com fenda central.

Figura 7.61 - Simetria da chapa tracionada com fenda central.

Figura 7.62 - Redes quadrilaterais regulares - chapa tracionada.

Figura 7.63 - Redes triangulares regulares - chapa tracionada

Figura 7.64 - Convergência da energia de deformação $\boldsymbol{U}_{\text {chapa tracionada }}$ - Elemento Quadrilateral - FHT, FHMT e MEF clássico

Figura 7.65 - Convergência da energia de deformação $\boldsymbol{U}_{\text {chapa tracionada }}$ - Elemento Quadrilateral - FHMT e MEF clássico....

Figura 7.66 - Convergência da energia de deformação $\boldsymbol{U}_{\text {chapa tracionada }}$ - Elemento Quadrilateral - FHT e MEF clássico.

Figura 7.67 - Convergência da energia de deformação $\boldsymbol{U}_{\text {chapa tracionada }}$ - Elemento Triangular - FHT, FHMT e MEF clássico

Figura 7.68 - Convergência da energia de deformação $\boldsymbol{U}_{\text {chapa tracionada }}$ - Elemento Triangular - FHMT e MEF clássico.

Figura 7.69 - Convergência da energia de deformação $\boldsymbol{U}_{\text {chapa tracionada }}$ - Elemento Triangular - FHT e MEF clássico.

Figura 7.70 - Tensões planas para rede regular $\mathbf{1 6 \times 1 6}$ - aproximação quadrática das tensões - Elemento Quadrilateral - FHT - sem enriquecimento.

Figura 7.71 - Tensões planas para rede regular $16 \times 16$ - aproximação quadrática das tensões - Elemento Quadrilateral - FHT enriquecimento dos Deslocamentos no Contorno $(\boldsymbol{x})$. 
Figura 7.72 - Tensões planas para rede regular $\mathbf{1 6} \times \mathbf{1 6}$ com- Elemento Triangular - FHMT - sem enriquecimento.

Figura 7.73 - Tensões planas para rede regular $\mathbf{1 6} \times \mathbf{1 6}$ com - Elemento Triangular - FHMT - enriquecimento das Tensões e Deslocamentos no Domínio $\left(\boldsymbol{y}^{2}+\boldsymbol{y}\right)$ dos Deslocamentos no Contorno $(x)$

Aproximação típica dos deslocamentos nas direções $\boldsymbol{x}\left(\boldsymbol{u}_{\boldsymbol{x}}\right)$

Figura 7.74 - e $\boldsymbol{y}\left(\boldsymbol{u}_{\boldsymbol{y}}\right)$ para rede regular $\mathbf{1 6} \times \mathbf{1 6}$ - aproximação quadrática das tensões - Elemento Quadrilateral - FHT - enriquecimento dos Deslocamentos no Contorno $(\boldsymbol{x})$.

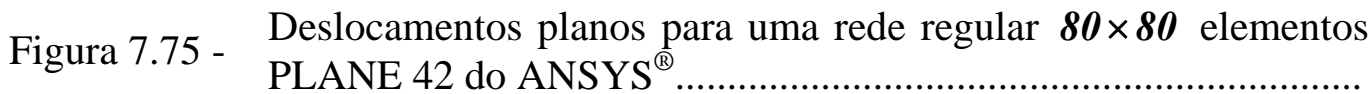

Figura 7.76 - Representação do campo de tensões para uma rede regular $\mathbf{8 0} \times \mathbf{8 0}$ elementos PLANE 42 do ANSYS ${ }^{\circledR}$.

Figura 7.77 - Redes quadrilaterais regulares - chapa tracionada com fenda central.

Figura 7.78 - Redes triangulares regulares - chapa tracionada com fenda central.

Figura 7.79 - Enriquecimento seletivo - chapa tracionado com fenda - rede quadrilateral regular $6 \times 6$.

Figura 7.80 - Convergência da energia de deformação $\boldsymbol{U}_{\text {chapa tracionada com fenda }}{ }^{-}$ Elemento Quadrilateral - FHT, FHMT e MEF clássico..

Figura 7.81 - Convergência da energia de deformação $\boldsymbol{U}_{\text {chapa tracionada com fenda }}{ }^{-}$ Elemento Quadrilateral - FHMT e MEF clássico

Figura 7.82 - Convergência da energia de deformação $\boldsymbol{U}_{\text {chapa tracionada com fenda }}{ }^{-}$ Elemento Triangular - FHT, FHMT e MEF clássico.

Figura 7.83 - Convergência da energia de deformação $U_{\text {chapa tracionada com fenda }}{ }^{-}$ Elemento Triangular - FHMT e MEF clássico.. 
Figura 7.84 - Convergência da energia de deformação $\boldsymbol{U}_{\text {chapa tracionada com fenda }}{ }^{-}$ Elemento Quadrilateral - FHT e MEF clássico.

Figura 7.85 - Convergência da energia de deformação $\boldsymbol{U}_{\text {chapa tracionada com fenda }}{ }^{-}$ Elemento Triangular - FHT e MEF clássico....................................

Figura 7.86 - Tensões planas para rede regular $24 \times 24$ - Elemento Quadrilateral - FHMT - sem enriquecimento.

Figura 7.87 - Tensões planas para rede regular $24 \times 24$ - Elemento Quadrilateral - FHMT - com enriquecimento das Tensões e Deslocamentos no Domínio $\left(\boldsymbol{y}^{2}+\boldsymbol{x}^{2}+\boldsymbol{x}+\boldsymbol{y}+\boldsymbol{x y}\right)$ e dos Deslocamentos no Contorno $(x)$

Figura 7.88 - Tensões planas para rede regular $24 \times 24-$ Elemento Triangular - FHMT - sem enriquecimento

Figura 7.89 - Tensões planas para rede regular $24 \times 24-$ Elemento Triangular - FHMT - com enriquecimento das Tensões e Deslocamentos no Domínio $\left(\boldsymbol{y}^{2}+\boldsymbol{x}^{2}+\boldsymbol{x}+\boldsymbol{y}+\boldsymbol{x y}\right)$ e dos Deslocamentos no Contorno $(x)$

Figura 7.90 - Tensões planas para rede regular $24 \times 24$ - aproximação constante das tensões - Elemento Quadrilateral - FHT - sem enriquecimento.

Figura 7.91 - Aproximação típica dos deslocamentos nas direções $\boldsymbol{x}\left(\boldsymbol{u}_{\boldsymbol{x}}\right)$ e $\boldsymbol{y}\left(\boldsymbol{u}_{\boldsymbol{y}}\right)$ para rede regular $24 \times 24$ - aproximação constante das tensões - Elemento Quadrilateral - FHT - sem enriquecimento.

Figura 7.92 -

Tensões planas para rede regular $24 \times 24$ - aproximação constante das tensões - Elemento Quadrilateral - FHT enriquecimento das Tensões no Domínio $\left(y^{2}\right)$ e dos Deslocamentos no Contorno $(x)$

Figura 7.93 - Rede irregular com 28 elementos quadrilaterais. 
Tensões planas para rede irregular - aproximação quadrática das Figura 7.95 - tensões - Elemento Quadrilateral - FHT - sem

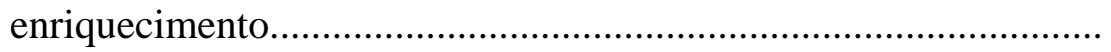

Aproximação típica dos deslocamentos nas direções $\boldsymbol{x}\left(\boldsymbol{u}_{\boldsymbol{x}}\right)$

Figura 7.96 - e $\boldsymbol{y}\left(\boldsymbol{u}_{\boldsymbol{y}}\right)$ para rede irregular - aproximação quadrática das tensões - Elemento Quadrilateral - FHT - sem enriquecimento....

Figura 7.97 - Representação do campo de tensões para uma rede regular $240 \times 240$ elementos PLANE 42 do ANSYS ${ }^{\circledR}$.

Figura 7.98 - Deslocamentos planos para uma rede regular $240 \times \mathbf{2 4 0}$ elementos PLANE 42 do ANSYS ${ }^{\circledR}$.....

Figura 7.99 - Resultados do teste “inf-sup” - Elemento Quadrilateral FHT/FHMT sem enriquecimento

Figura 7.100 - Resultados do teste “inf-sup” - Elemento Quadrilateral - FHMT com enriquecimento.

Figura 7.101 - Resultados do teste “inf-sup” - Elemento Quadrilateral - FHT com enriquecimento.

Figura 7.102 - Resultados do teste “inf-sup” - Elemento Quadrilateral - FHT com enriquecimento....

Figura 7.103 - Resultados do teste “inf-sup” - Elemento Triangular FHT/FHMT sem enriquecimento

Figura 7.104 - Resultados do teste “inf-sup” - Elemento Triangular - FHMT com enriquecimento.

Figura 7.105 - Resultados do teste "inf-sup" - Elemento Triangular - FHT com enriquecimento.

Figura 7.106 - Resultados do teste "inf-sup” - Elemento Triangular - FHT com enriquecimento.

Figura 7.107 - Resultados do teste “inf-sup” - Elemento Quadrilateral - FHT sem enriquecimento. 
Figura 7.108 - Resultados do teste “inf-sup” - Elemento Quadrilateral - FHMT sem enriquecimento

Figura 7.109 - Resultados do teste “inf-sup” - Elemento Quadrilateral - FHT com enriquecimento.

Figura 7.110 - Resultados do teste “inf-sup” - Elemento Quadrilateral - FHMT com enriquecimento

Figura 7.111 - Resultados do teste "inf-sup” - Elemento Triangular - FHT sem enriquecimento.

Figura 7.112 - Resultados do teste “inf-sup” - Elemento Triangular - FHMT sem enriquecimento.

Figura 7.113 - Resultados do teste “inf-sup” - Elemento Triangular - FHT sem enriquecimento.

Figura 7.114 - Resultados do teste “inf-sup” - Elemento Triangular - FHMT com enriquecimento.

Figura 7.115 - Resultados do teste “inf-sup” - Elemento Quadrilateral - FHT sem enriquecimento.

Figura 7.116 - Resultados do teste “inf-sup” - Elemento Quadrilateral - FHMT sem enriquecimento

Figura 7.117 - Resultados do teste “inf-sup” - Elemento Triangular - FHT sem enriquecimento.

Figura 7.118 - Resultados do teste "inf-sup" - Elemento Triangular - FHMT sem enriquecimento.

Figura 7.119 - Resultados do teste “inf-sup” - Elemento Triangular - FHMT com enriquecimento.

Figura 7.120 - Resultados do teste “inf-sup” - Elemento Quadrilateral - FHMT com enriquecimento.

Figura 7.121 - Resultados do teste “inf-sup” - Elemento Quadrilateral - FHT com enriquecimento.

Figura 7.122 - Resultados do teste "inf-sup" - Elemento Triangular - FHT com enriquecimento. 


\title{
Lista de Símbolos
}

\author{
No Cap.1- item 1.1, deslocamento de um corpo elástico \\ $\boldsymbol{u}-$ \\ No Cap.1- item 1.1, tensões num corpo elástico \\ $\varepsilon-$ \\ No Cap.1- item 1.1, deformações num corpo elástico \\ $\boldsymbol{\Omega}-$ \\ Domínio de um corpo elástico \\ $\boldsymbol{\Gamma}$ - $\quad$ Contorno regular que limita o domínio de um corpo elástico \\ $\boldsymbol{L}$ - $\quad$ Operador diferencial divergente \\ $\boldsymbol{b}$ - $\quad$ Vetor de forças volúmicas \\ $\boldsymbol{\sigma}$ - $\quad$ No Cap.2- item 2.2, vetor coluna formado com as componentes do \\ tensor de tensões \\ $\sigma_{x}, \sigma_{y}$ e $\tau_{x y}-$ Componentes planas das tensões \\ $\boldsymbol{\varepsilon}$ - $\quad$ No Cap.2- item 2.2, vetor coluna formado com as componentes do \\ tensor de deformações \\ $\boldsymbol{\varepsilon}_{x}, \boldsymbol{\varepsilon}_{y}$ e $\boldsymbol{\gamma}_{x y}-$ Componentes planas das deformações \\ No Cap.2- item 2.2, vetor coluna formado com as componentes planas \\ $\boldsymbol{u}$ - dos deslocamentos: $\boldsymbol{u}_{\boldsymbol{x}}$ e $\boldsymbol{u}_{\boldsymbol{y}}$ \\ $f$ - $\quad$ Matriz de flexibilidade para materiais elásticos, lineares e isótropos \\ $v$ - $\quad$ Coeficiente de Poisson \\ $\overline{\boldsymbol{u}}-\quad$ Vetor de deslocamentos impostos \\ $\overline{\boldsymbol{t}}$ - $\quad$ Vetor de forças de superfície \\ $\quad N$ - $\quad$ Matriz construída com as componentes do vetor normal ao contorno \\ $\Gamma_{\boldsymbol{t}}$ - $\quad$ Parte do contorno regular onde são impostas as forças de superfície \\ $\Gamma_{u}-\quad$ Parte do contorno regular onde são impostos os deslocamentos \\ $\boldsymbol{M}_{\Omega}$ - $\quad$ Função de ponderação da lei constitutiva \\ $\boldsymbol{P}_{\boldsymbol{\Omega}}$ - $\quad$ Função de ponderação da equação de equilíbrio \\ $\boldsymbol{R}_{\boldsymbol{I}_{t}}$ - $\quad$ Função de ponderação da equação de equilíbrio no contorno
}




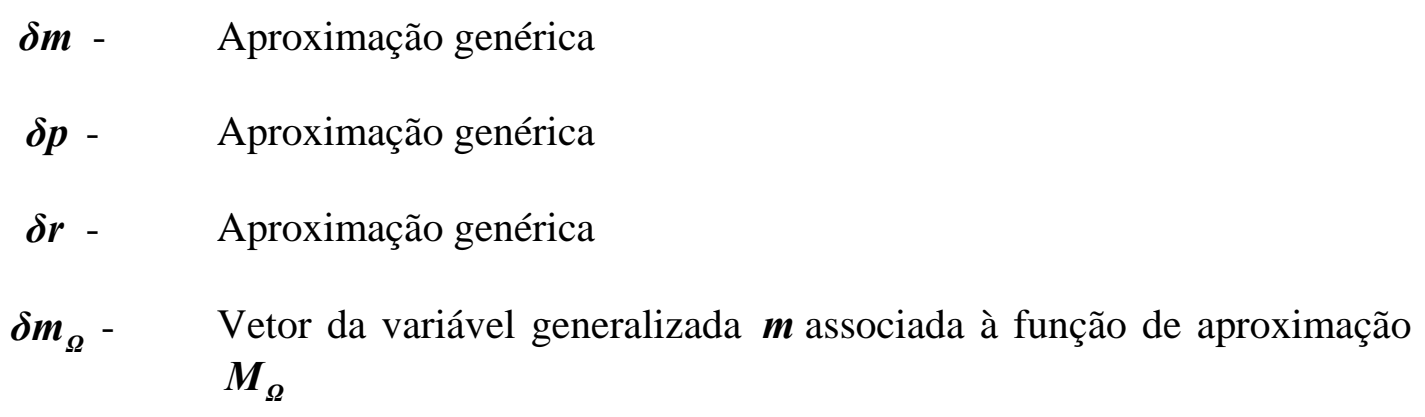

$\boldsymbol{\delta} \boldsymbol{p}_{\boldsymbol{\Omega}}$ - $\quad$ Vetor da variável generalizada $\boldsymbol{p}$ associada à função de aproximação $P_{\Omega}$

$\boldsymbol{\delta}_{\boldsymbol{\Gamma}_{t}}$ - Vetor da variável generalizada $\boldsymbol{r}$ associada à função de aproximação $\boldsymbol{R}_{\Gamma_{t}}$

$\boldsymbol{u}_{\boldsymbol{\Gamma}}$ - $\quad$ Vetor deslocamento na parte do contorno regular onde são impostas as forças de superfície

$S_{\Omega}$ - Matriz que guarda as funções de aproximação dos campos de tensão no domínio $\boldsymbol{\Omega}$

$\boldsymbol{U}_{\boldsymbol{\Omega}}$ - Matriz que guarda as funções de aproximação do campo de deslocamento no domínio $\boldsymbol{\Omega}$

$\boldsymbol{U}_{\boldsymbol{\Gamma}_{t}}$ - Matriz que coleta as funções de aproximação do campo de deslocamento no contorno $\boldsymbol{\Gamma}_{\boldsymbol{t}}$

$\boldsymbol{s}_{\boldsymbol{\Omega}}$ - $\quad$ Vetor das tensões generalizadas associadas às funções de aproximação dos campos de tensão em $\boldsymbol{S}_{\boldsymbol{\Omega}}$

$\boldsymbol{q}_{\boldsymbol{\Omega}}$ - Vetor dos deslocamentos generalizados associadas às funções de aproximação dos campos de deslocamento em $\boldsymbol{U}_{\boldsymbol{\Omega}}$

$\boldsymbol{q}_{\boldsymbol{\Gamma}_{\boldsymbol{t}}}$ - Vetor que contem os pesos que podem ser interpretados como deslocamentos generalizados no contorno $\Gamma_{t}$

$\boldsymbol{e}_{\boldsymbol{\Gamma}_{u}}-\quad$ Vetor cujos termos são integrações no contorno $\boldsymbol{\Gamma}_{\boldsymbol{u}}$ sobre as aproximações do campo de tensões no domínio e do vetor de deslocamentos impostos

$\boldsymbol{Q}_{\boldsymbol{\Omega}}$ - $\quad$ Vetor cujos termos são integrações no domínio $\boldsymbol{\Omega}$ sobre as aproximações do campo de deslocamentos no domínio e do vetor de forças volúmicas

$\boldsymbol{Q}_{\boldsymbol{\Gamma}_{\boldsymbol{t}}}$ - Vetor cujos termos são integrações no contorno $\boldsymbol{\Gamma}_{\boldsymbol{t}}$ sobre as aproximações do campo de deslocamento no contorno e do vetor de forças de superfície 
$\boldsymbol{F}$ - $\quad$ Matriz quadrada cujos termos são integrações no domínio $\boldsymbol{\Omega}$ sobre as aproximações do campo de tensões no domínio e dos termos da matriz de flexibilidade

$\boldsymbol{A}_{\boldsymbol{\Omega}}$ - $\quad$ Matriz cujos termos são integrações no domínio $\boldsymbol{\Omega}$ sobre as aproximações do campo de tensões e deslocamentos no domínio

$\boldsymbol{A}_{\boldsymbol{\Gamma}_{t}}$ - Matriz cujos termos são integrações no contorno $\boldsymbol{\Gamma}_{\boldsymbol{t}}$ sobre as aproximações do campo de tensões no domínio e deslocamento no contorno

$\boldsymbol{\Gamma}_{\boldsymbol{i}}-\quad$ Contorno entre as partes discretas do domínio $\boldsymbol{\Omega}$

$\boldsymbol{u}_{\boldsymbol{\Gamma}_{\boldsymbol{t}}}$ - Vetor deslocamento na parte do contorno regular onde são impostas as forças de superfície

$\boldsymbol{t}_{\boldsymbol{\Gamma}_{u}}$ - Vetor de forças na parte do contorno regular onde são impostas os deslocamentos

$\boldsymbol{W}_{\boldsymbol{T}_{u}}$ - $\quad$ Matriz que coleta as funções de aproximação das forças no contorno $\Gamma_{u}$

$\boldsymbol{o}_{\Gamma_{u}}-\quad$ Vetor que contem os pesos que podem ser interpretados como forças generalizadas no contorno $\boldsymbol{\Gamma}_{u}$

$\boldsymbol{u}_{\boldsymbol{\Gamma}_{i}}$ - Vetor deslocamento no contorno entre as partes discretas do domínio $\boldsymbol{\Omega}$ (fronteira entre elementos)

$\boldsymbol{U}_{\boldsymbol{\Gamma}_{i}}$ - Matriz que coleta as funções de aproximação do campo de deslocamento no contorno $\boldsymbol{\Gamma}_{\boldsymbol{i}}$

$\boldsymbol{q}_{\boldsymbol{\Gamma}_{i}}$ - Vetor que contem os pesos que podem ser interpretados como deslocamentos generalizados no contorno $\boldsymbol{\Gamma}_{\boldsymbol{i}}$

$\boldsymbol{t}_{\boldsymbol{\Gamma}_{\boldsymbol{i}}}-\quad$ Vetor de forças no contorno entre as partes discretas do domínio $\boldsymbol{\Omega}$ (fronteira entre elementos)

$\boldsymbol{W}_{\boldsymbol{\Gamma}_{i}}$ - Matriz que coleta as funções de aproximação das forças no contorno $\Gamma_{i}$

$\boldsymbol{o}_{\boldsymbol{\Gamma}_{\boldsymbol{i}}}-\quad$ Vetor que contem os pesos que podem ser interpretados como forças generalizadas no contorno $\boldsymbol{\Gamma}_{\boldsymbol{i}}$

$\boldsymbol{Q}_{\boldsymbol{\Gamma}_{\boldsymbol{i}}}$ - $\quad$ Vetor cujos termos são integrações no contorno $\boldsymbol{\Gamma}_{\boldsymbol{i}}$ sobre as aproximações do campo de deslocamento no contorno $\boldsymbol{\Gamma}_{\boldsymbol{i}}$ e do vetor de forças de superfície $\boldsymbol{t}_{\boldsymbol{\Gamma}_{i}}$ 
$\boldsymbol{A}_{\boldsymbol{\Gamma}_{\boldsymbol{i}}}$ - Matriz cujos termos são integrações no contorno $\boldsymbol{\Gamma}_{\boldsymbol{i}}$ sobre as aproximações do campo de tensões no domínio e deslocamento no contorno $\boldsymbol{\Gamma}_{\boldsymbol{i}}$

$\boldsymbol{C}_{\boldsymbol{\Gamma}_{\boldsymbol{i}}}$ - Matriz cujos termos são integrações no contorno $\boldsymbol{\Gamma}_{\boldsymbol{i}}$ sobre as aproximações das forças e deslocamento no contorno $\boldsymbol{\Gamma}_{\boldsymbol{i}}$

$\boldsymbol{\delta}_{\boldsymbol{t}}-\quad$ Forças virtuais no contorno $\boldsymbol{\Gamma}_{\boldsymbol{i}}$

$\boldsymbol{\delta}_{\boldsymbol{\Gamma}_{\boldsymbol{i}}}-\quad$ Vetor que contem os pesos que podem ser interpretados como forças virtuais generalizadas no contorno $\boldsymbol{\Gamma}_{i}$

$\boldsymbol{x}$ - $\quad$ No Cap.3- item 3.1, um ponto qualquer

$\boldsymbol{\varphi}_{j}(\boldsymbol{x})$ - Conjunto de funções de forma definidas no ponto $\boldsymbol{x}$

$\omega_{j}-\quad$ Região de influência ou nuvem

$C_{0}^{\infty}\left(\omega_{j}\right)$ - $\quad \begin{aligned} & \text { Conjunto de funções cujas derivadas até a ordem infinita apresentam } \\ & \text { suporte compacto }\end{aligned}$

$\boldsymbol{x}_{\boldsymbol{j}}-\quad$ Nó qualquer do domínio $\boldsymbol{\Omega}$

$\boldsymbol{u}(\boldsymbol{x}) \boldsymbol{=} \boldsymbol{k}$ - Função aproximada

$\boldsymbol{j}$ - $\quad$ Nó de índice $\boldsymbol{j}$

$\tilde{\boldsymbol{u}}_{j}=\boldsymbol{k}-\quad$ Conjunto de dados definido em cada ponto $\boldsymbol{x}_{\boldsymbol{j}}, \boldsymbol{j}=\mathbf{1}, \ldots, \boldsymbol{N}$

$\hat{\boldsymbol{u}}(\boldsymbol{x})$ - $\quad$ Função aproximadora global

$\boldsymbol{x y}$ - Domínio global plano

$\boldsymbol{\xi}$ - $\quad$ Função paramétrica

$\boldsymbol{x}$ - $\quad$ No Cap.3- item 3.1, função num domínio global

$\boldsymbol{P}$ - $\quad$ No Cap.3- item 3.1, refinamento adaptativo

$\boldsymbol{\Omega}_{e}-\quad$ Domínio de um determinado elemento finito

g - $\quad$ Função de suporte compacto

$\boldsymbol{h}$ - $\quad$ Função enriquecedora

$\boldsymbol{x}_{\boldsymbol{j}_{\boldsymbol{T}}}-\quad$ Nó qualquer do contorno $\boldsymbol{\Gamma}_{\boldsymbol{i}}$ ou $\boldsymbol{\Gamma}_{\boldsymbol{t}}$ 
$\boldsymbol{\omega}_{j_{\Gamma}}-\quad$ Região de influência ou nuvem no contorno $\boldsymbol{\Gamma}_{\boldsymbol{i}}$ ou $\boldsymbol{\Gamma}_{\boldsymbol{t}}$

$\mathfrak{R}_{N_{T}}^{p}$ - A família de funções para o campo de deslocamentos no contorno $\Gamma_{i}$ ou $\Gamma_{t}$

$N_{\Gamma}-\quad$ É o número total de nós no contorno $\Gamma\left(\Gamma_{i}+\Gamma_{t}+\Gamma_{u}\right)$

$\boldsymbol{p}$ - $\quad$ No Cap.3- item 3.2, é o grau máximo da aproximação Lagrangiana resultante

$U_{\Gamma_{j}}$ - É a função de forma (PU) para o campo de deslocamento referente aos nós do contorno $\Gamma\left(\Gamma_{i}+\Gamma_{t}+\Gamma_{u}\right)\left(j=1, \ldots, N_{\Gamma}\right)$

$\boldsymbol{O}_{\boldsymbol{j n}_{\boldsymbol{n}_{\boldsymbol{e}}}}$ - São as $\boldsymbol{n}_{\boldsymbol{e}_{r}}$ funções que multiplicam (enriquecem) a função de forma de contorno definidas em cada nó de índice $\boldsymbol{j}$

$I(j)$ - É o contador para o número de funções adicionadas a cada nó de índice $j$

$\mathfrak{J}_{e}^{p}$ - $\quad$ A família de funções para o campo de tensões no domínio $\boldsymbol{\Omega}$

$\boldsymbol{S}_{\Omega_{e}}$ - É a função aproximativa para o campo de tensão, referentes aos elementos $\boldsymbol{e}=1, \ldots, n_{\Omega}$ do domínio $\boldsymbol{\Omega}$

$\boldsymbol{g}_{j}$ - $\quad$ São funções com suporte compacto sobre nuvens definidas em cada nó de índice $\boldsymbol{j}$ com auxílio da rede de elementos finitos de cobertura

$\boldsymbol{N}$ - $\quad$ No Cap.3- item 3.2, é o número total de nós no domínio $\boldsymbol{\Omega}$

$\boldsymbol{L}_{\boldsymbol{j}}$ - $\quad$ São funções que multiplicam ou enriquecem as funções $\boldsymbol{g}_{\boldsymbol{j}}$

$\mathfrak{J}_{N}^{p}-\quad$ Família de funções para o campo de tensões no domínio $\boldsymbol{\Omega}$

$S_{\Omega_{j}}$ - É a função de forma (PU) para os campos de tensão referentes aos nós $\boldsymbol{j}=\mathbf{1}, \ldots, \boldsymbol{N}$ do domínio $\boldsymbol{\Omega}$

$\boldsymbol{\Theta}_{N}^{p}$ - $\quad$ A família de funções para o campo de deslocamentos no domínio $\boldsymbol{\Omega}$

$\boldsymbol{U}_{\boldsymbol{\Omega}_{j}}$ - $\quad$ É a função de forma (PU) para os campos de deslocamentos referentes aos nós $\boldsymbol{j}=\mathbf{1}, \ldots, \boldsymbol{N}$ do domínio $\boldsymbol{\Omega}$

$\boldsymbol{\Xi}_{N_{r}}^{p}$ - A família de funções para o campo de deslocamentos no contorno $\boldsymbol{\Gamma}_{\boldsymbol{i}}$ ou $\Gamma_{t}$ 
$\boldsymbol{U}_{\Gamma_{j}}$ - É a função de forma (Partição da Unidade) para o campo de deslocamento referente dos nós do contorno $\Gamma\left(\boldsymbol{\Gamma}_{i}+\boldsymbol{\Gamma}_{t}+\boldsymbol{\Gamma}_{u}\right)$ $\left(j=1, \ldots, N_{\Gamma}\right)$

$\boldsymbol{L}_{\boldsymbol{j} \boldsymbol{n}_{e}}, \boldsymbol{M}_{j \boldsymbol{n}_{e}}-$ São as $\boldsymbol{n}_{e}$ funções que multiplicam ou enriquecem a função de forma de domínio definida em cada nó de índice $\boldsymbol{j}$

$\boldsymbol{O}_{\boldsymbol{j n}_{\boldsymbol{n}} \boldsymbol{n}}$ - São as $\boldsymbol{n}_{\boldsymbol{e}_{r}}$ funções que multiplicam (enriquecem) a função de forma de contorno definida em cada nó de índice $\boldsymbol{j}$

$\boldsymbol{n}_{\Omega}$ - Rede de elementos finitos ditos de domínio

$\boldsymbol{n}_{\Gamma_{t}}-\quad$ Elementos no contorno de Neumann $\boldsymbol{\Gamma}_{t_{e_{t}}}$

$\boldsymbol{n}_{\boldsymbol{\Gamma}_{i}}-\quad$ Elementos do contorno entre os elementos de domínio $\boldsymbol{\Gamma}_{\boldsymbol{i}_{e_{i}}}$

$\boldsymbol{s}_{\boldsymbol{\Omega}_{e}}-\quad$ Vetor que guarda os parâmetros generalizados correspondentes às aproximações locais para as tensões no domínio

$\boldsymbol{q}_{\boldsymbol{\Omega}_{e}}$ - Vetor que guarda os parâmetros generalizados correspondentes às aproximações locais para os deslocamentos no domínio

$\boldsymbol{q}_{\Gamma_{e_{\tau_{i}}}}$ - Vetor que guarda os parâmetros generalizados correspondentes às aproximações locais para os deslocamentos no contorno de Neumann $\Gamma_{t_{e_{t}}}$

$\boldsymbol{q}_{\Gamma_{e_{T_{i}}}}$ - Vetor que guarda os parâmetros generalizados correspondentes às aproximações locais para os deslocamentos no contorno entre os elementos de domínio $\boldsymbol{\Gamma}_{i_{e_{i}}}$

$\boldsymbol{e}-\quad$ Elemento no domínio $\boldsymbol{\Omega}$

$\boldsymbol{e}_{\Gamma_{t}}-\quad$ Elemento no contorno de Neumann $\boldsymbol{\Gamma}_{\boldsymbol{t}_{e_{\tau_{t}}}}$

$\boldsymbol{e}_{\Gamma_{i}}-\quad$ Elemento no contorno entre os elementos de domínio $\boldsymbol{\Gamma}_{i_{e_{i}}}$

$\boldsymbol{F}_{\boldsymbol{e}}$ e $\boldsymbol{A}_{\boldsymbol{e}}$ - Matrizes Booleanas que extraem os graus de liberdade do elemento finito de domínio $\boldsymbol{\Omega}_{e}$

$\boldsymbol{A}_{\boldsymbol{r}_{\boldsymbol{e}_{\boldsymbol{T}_{i}}}}$ e $\boldsymbol{A}_{\boldsymbol{r}_{\boldsymbol{e}_{\boldsymbol{r}_{i}}}}$ - São as matrizes Booleanas que extraem, respectivamente, os graus de liberdade dos elementos no contorno $\boldsymbol{\Gamma}_{\boldsymbol{t}_{e_{\tau_{i}}}}$ e $\boldsymbol{\Gamma}_{\boldsymbol{i}_{e_{r_{i}}}}$

$\tilde{\boldsymbol{\sigma}}_{e}-\quad$ Aproximações locais dos campos de tensões no domínio $\boldsymbol{\Omega}_{e}$ 
$\tilde{\boldsymbol{u}}_{e}$ - $\quad$ Aproximações locais dos campos de deslocamento no domínio $\boldsymbol{\Omega}_{\boldsymbol{e}}$

$\tilde{\boldsymbol{u}}_{\boldsymbol{T}_{t}}$ - $\quad$ Aproximações locais dos campos de deslocamento no contorno de Neumann $\boldsymbol{\Gamma}_{\boldsymbol{t}_{\boldsymbol{T}_{t}}}$

$\boldsymbol{u}_{\boldsymbol{T}_{i}}$ - $\quad$ Aproximações locais dos campos de deslocamento no contorno entre os elementos de domínio $\boldsymbol{\Gamma}_{i_{e_{i}}}$

$\boldsymbol{n e}_{\Gamma_{u}}-\quad$ Elementos com deslocamentos prescritos

$\boldsymbol{F}_{\boldsymbol{e}}$ - Matriz quadrada cujos termos são integrações no domínio $\boldsymbol{\Omega}_{\boldsymbol{e}}$ sobre as aproximações do campo de tensões no domínio e dos termos da matriz de flexibilidade

$\boldsymbol{A}_{\boldsymbol{\Omega}_{e}}$ - Matriz cujos termos são integrações no domínio $\boldsymbol{\Omega}_{\boldsymbol{e}}$ sobre as aproximações do campo de tensões e deslocamentos no domínio

$\boldsymbol{A}_{\boldsymbol{\Gamma}_{t_{e_{\tau_{i}}}}}-\quad$ Matriz cujos termos são integrações no contorno $\boldsymbol{\Gamma}_{\boldsymbol{t}_{e_{\tau_{t}}}}$ sobre as aproximações do campo de tensões no domínio e deslocamento no contorno

$\boldsymbol{A}_{\boldsymbol{\Gamma}_{i_{e_{i}}}}$ - Matriz cujos termos são integrações no contorno $\boldsymbol{\Gamma}_{i_{e_{r_{i}}}}$ sobre as aproximações do campo de tensões no domínio e deslocamento no contorno

$\boldsymbol{e}_{\Gamma_{u_{\text {ue }} e_{u}}}$ - Vetor cujos termos são integrações no contorno $\boldsymbol{\Gamma}_{u_{\text {ne }_{u}}}$ sobre as aproximações do campo de tensões no domínio e do vetor de deslocamentos impostos

$\boldsymbol{Q}_{\Omega_{e}}$ - Vetor cujos termos são integrações no domínio $\boldsymbol{\Omega}_{e}$ sobre as aproximações do campo de deslocamentos no domínio e do vetor de forças volúmicas

$\boldsymbol{Q}_{\Gamma_{e_{\tau_{t}}}}$ - Vetor cujos termos são integrações no contorno $\boldsymbol{\Gamma}_{\boldsymbol{t}_{\boldsymbol{e}_{\tau_{t}}}}$ sobre as aproximações do campo de deslocamento no contorno e do vetor de forças de superfície

$\xi \boldsymbol{\eta}-\quad$ Coordenadas normalizadas ortogonais

$\boldsymbol{x y}$ - Coordenadas cartesianas

$\varphi_{j}$ - Funções bilineares Lagrangianas para elementos finitos quadrilaterais, $j=1,2,3,4$

$\xi$ e $\boldsymbol{\eta}$ - $\quad$ Coordenadas adimensionais variando entre -1 e 1 

$\boldsymbol{M}$ - $\quad$ Matriz composta pelas funções de interpolação $\boldsymbol{\varphi}_{j}$
$c$ - Vetor $\boldsymbol{c}$ que guarda as coordenadas nodais do elemento finito quadrilateral

$\boldsymbol{x}_{\boldsymbol{i}}$ e $\boldsymbol{y}_{\boldsymbol{i}}$ - Coordenadas nodais do elemento finito quadrilateral, $\boldsymbol{i}=\mathbf{1 , 4}$

$\boldsymbol{J}$ - $\quad$ Matriz Jacobiana

$\boldsymbol{\psi}_{\boldsymbol{i}}$ - $\quad$ Funções lineares Lagrangianas clássicas, $\boldsymbol{i}=\mathbf{1 , 2}$

$\boldsymbol{M}_{\boldsymbol{\Gamma}}$ - Matriz composta pelas funções de interpolação $\boldsymbol{\psi}_{\boldsymbol{i}}$

$\boldsymbol{x}_{\boldsymbol{i}}$ e $\boldsymbol{y}_{\boldsymbol{i}}-\quad$ Coordenadas nodais do elemento finito linear, $\boldsymbol{i}=\mathbf{1 , 2}$

$c_{\Gamma}-\quad$ Vetor que guarda as coordenadas nodais no domínio físico do elemento finito no contorno

$\boldsymbol{d} \boldsymbol{s}$ - Diferencial de comprimento no domínio global $\boldsymbol{x y}$

$\boldsymbol{d} \boldsymbol{\xi}$ - Diferencial de comprimento no domínio paramétrico

$\boldsymbol{h}_{\boldsymbol{j} \boldsymbol{n}_{\boldsymbol{e}}}$ - São as $\boldsymbol{n}_{\boldsymbol{e}}$ funções polinomiais que multiplicam ou enriquecem as tensões e deslocamentos de domínio definida em cada nó de índice $\boldsymbol{j}$

$\hat{\sigma}$ - $\quad$ Aproximações das tensões no domínio

$\boldsymbol{s}_{\boldsymbol{\Omega}_{j}}$ - $\quad$ Os graus de liberdade de tensões associadas às funções de forma originais

$\boldsymbol{b}_{j i}$ - Os novos parâmetros nodais correspondentes a cada uma das parcelas de enriquecimento das tensões no domínio

$\hat{\boldsymbol{u}}$ - $\quad$ Aproximação dos deslocamentos no domínio

$\boldsymbol{u}_{\boldsymbol{\Omega}_{j}}$ - Os graus de liberdade em deslocamento associados às funções de forma originais

$\boldsymbol{c}_{j i}$ - $\quad$ Os novos parâmetros nodais correspondentes a cada uma das parcelas de enriquecimento dos deslocamentos no domínio

$\hat{\boldsymbol{u}}_{\boldsymbol{\Gamma}}-\quad$ Aproximação dos deslocamentos no contorno

$\boldsymbol{u}_{\boldsymbol{\Gamma}_{j}}$ - Os graus de liberdade em deslocamento associados às funções de forma originais

$\boldsymbol{d}_{j i}$ - Os novos parâmetros nodais correspondentes a cada uma das parcelas de enriquecimento dos deslocamentos no contorno 
$\boldsymbol{h}_{\boldsymbol{j} \boldsymbol{n}_{\boldsymbol{e}_{\boldsymbol{T}}}}$ - São as $\boldsymbol{n}_{\boldsymbol{e}_{\boldsymbol{T}}}$ funções polinomiais que multiplicam ou enriquecem os deslocamentos no contorno definida em cada nó de índice $\boldsymbol{j}$

$\boldsymbol{A}_{\boldsymbol{j}}, \boldsymbol{j}=\mathbf{1}, \ldots, 4$ - Matriz de enriquecimento polinomial no domínio do elemento quadrilateral

$\boldsymbol{\Delta}_{\boldsymbol{\Gamma}_{j}}, \boldsymbol{j}=\mathbf{1 , 2}-$ Matriz de enriquecimento polinomial no contorno do elemento

$\boldsymbol{I}_{3}$ e $\boldsymbol{I}_{2}$ - $\quad$ Matrizes identidades de terceira e segunda ordem respectivamente

$\boldsymbol{p}(\boldsymbol{\xi}, \boldsymbol{\eta})$ - $\quad$ Função escrita num domínio paramétrico $\xi \boldsymbol{\eta}$

$|\boldsymbol{J}|$ - $\quad$ Determinante da matriz jacobiana

$\boldsymbol{n}$ e $\boldsymbol{m}$ - $\quad$ No Cap.4- item 4.3.1.4, são os números de pontos de Gauss utilizados em cada direção $\boldsymbol{\xi}$ e $\boldsymbol{\eta}$

$\boldsymbol{w}_{\boldsymbol{i}}$ e $\boldsymbol{w}_{j}$ - $\quad$ No Cap.4- item 4.3.1.4, são os pesos associados aos pontos de Gauss $\boldsymbol{i}$ e $\boldsymbol{j}$

$\boldsymbol{s}(\boldsymbol{\xi}, \boldsymbol{\eta})$ - Função escrita num domínio paramétrico $\xi \boldsymbol{\xi}$

$\boldsymbol{q}(\boldsymbol{\xi}, \boldsymbol{\eta})$ - $\quad$ Função escrita num domínio paramétrico $\xi \boldsymbol{\eta}$

$\boldsymbol{r}\left(\boldsymbol{\xi}_{\text {constante }}, \boldsymbol{\eta}\right) \quad$ Função escrita num contorno paramétrico $\boldsymbol{\xi}_{\text {constante }}$ ou $\boldsymbol{\eta}_{\text {constante }}$

ou

$r\left(\xi, \eta_{\text {constante }}\right)$

$\boldsymbol{P}$ - $\quad$ Ponto interno ao elemento de domínio triangular $\boldsymbol{\Omega}_{\boldsymbol{e}}$

$A_{e}$ - Área do elemento triangular

$A_{1}$ - Área do triângulo $P 12$

$A_{2}$ - Área do triângulo $P 23$

$A_{3}$ - $\quad$ Área do triângulo $P 31$

$\xi_{i}-\quad$ Coordenadas triangulares normalizadas, $i=1,3$

$\boldsymbol{x}_{\boldsymbol{i}}$ e $\boldsymbol{y}_{\boldsymbol{i}}$ - Coordenadas nodais do elemento finito triangular, $\boldsymbol{i}=\mathbf{1 , 3}$

$\boldsymbol{M}_{T}-\quad$ Matriz composta pelas coordenadas triangulares normalizadas

$\boldsymbol{c}_{T}$ - Vetor que guarda as coordenadas nodais do elemento finito triangular

$\boldsymbol{M}_{\boldsymbol{I}_{T}}$ - Matriz composta pelas funções de interpolação $\psi_{i}$ 
$\boldsymbol{c}_{\Gamma_{T}}$ - Vetor $\boldsymbol{c}_{\Gamma_{T}}$ que guarda as coordenadas nodais no domínio físico do elemento finito no contorno

$\boldsymbol{A}_{j}, \boldsymbol{j}=\mathbf{1}, \ldots, \mathbf{3}$ - Matriz de enriquecimento polinomial no domínio do elemento triangular

$p\left(\xi_{1}, \xi_{2}\right)-\quad$ Função escrita num domínio paramétrico $\xi_{1} \xi_{2}$

$s\left(\xi_{1}, \xi_{2}\right)-\quad$ Função escrita num domínio paramétrico $\xi_{1} \xi_{2}$

$q\left(\xi_{1}, \xi_{2}\right)$ - Função escrita num domínio paramétrico $\xi_{1} \xi_{2}$

$\boldsymbol{w}_{\boldsymbol{i}}$ - $\quad$ No Cap.4- item 4.3.2.4, é o peso associado a cada ponto de Hammer $\boldsymbol{i}$

$r(\xi)$ - $\quad$ Função escrita num contorno paramétrico $\xi$

$\boldsymbol{A}$ - $\quad$ No Cap.4- item 4.3.2.4, matriz de composição

b - $\quad$ No Cap.4- item 4.3.2.4, vetor de composição

$\boldsymbol{B}$ - $\quad$ No Cap.4- item 4.3.2.4, matriz diagonal

$\varepsilon$ - $\quad$ No Cap.4- item 4.3.2.4, um número muito pequeno

$\boldsymbol{a}_{\boldsymbol{j}}$ - $\quad$ No Cap.4- item 4.3.2.4, é o erro na iteração $\boldsymbol{j}$

$\bar{r}_{\boldsymbol{j}}$ - $\quad$ No Cap.4- item 4.3.2.4, é o resíduo na iteração $\boldsymbol{j}$

$\boldsymbol{A}(\boldsymbol{x}, \boldsymbol{y})$ - $\quad$ No Cap.5- item 5.3.1.2, função de Airy

$\boldsymbol{a}, \boldsymbol{b}, \boldsymbol{c}, \boldsymbol{d}$ e $\boldsymbol{e}$ - No Cap.5- item 5.3.1.2, são parâmetros generalizados das tensões no domínio

$\boldsymbol{b}_{\boldsymbol{j}}$ - Novos parâmetros das tensões correspondentes ao enriquecimento

$\boldsymbol{A}$ - $\quad$ No Cap.5- item 5.3.1.3, função de Airy

$S_{\Omega_{e}}^{*} \quad \quad \quad$ Matriz de interpolação das tensões enriquecidas

$\Sigma_{j} \quad$ - Matriz de enriquecimento das tensões no domínio que reúne as

$(\boldsymbol{j = 1 , \ldots , 4 )} \quad$ interpolações de suporte compacto com $\boldsymbol{x}, \boldsymbol{y}$ centrados nos nós $\boldsymbol{j}$ da rede de elementos finitos

$\boldsymbol{q}_{\Gamma_{j}}-\quad$ São graus de liberdade em deslocamento no contorno associados às funções de forma originais

$\boldsymbol{d}_{\boldsymbol{j} \boldsymbol{l}}$ - São os novos parâmetros nodais correspondentes a cada uma das parcelas de enriquecimento dos deslocamentos no contorno 
$\xi_{j}$ - Coordenada adimensional para o elemento da rede de cobertura de contorno

$\boldsymbol{l}, \boldsymbol{k}$ - $\quad$ No Cap.5- item 5.3.1.3, são os nós enriquecidos do elemento quadrilateral

$\boldsymbol{n}$ - $\quad$ No Cap.5- item 5.3.1.3, é o número de nós enriquecidos no mesmo elemento

$\boldsymbol{J}_{11}^{-1}, \boldsymbol{J}_{12}^{-1}, \boldsymbol{J}_{21}^{-1}$ Elementos da inversa da matriz Jacobiana

e $J_{22}^{-1}-$

$\boldsymbol{n}_{\boldsymbol{x}} \quad$ No Cap.6- item 6.2.2, número de componentes de um vetor $\boldsymbol{x}$ qualquer

$\boldsymbol{n}_{\boldsymbol{y}} \quad$ No Cap.6- item 6.2.2, número de componentes de um vetor $\boldsymbol{y}$ qualquer

$\boldsymbol{A}$ - $\quad$ No Cap.6- item 6.2.2, matriz com dimensão $\left(\boldsymbol{n}_{\boldsymbol{x}} \times \boldsymbol{n}_{\boldsymbol{x}}\right)$

$\boldsymbol{B}$ - $\quad$ No Cap.6- item 6.2.2, matriz com dimensão $\left(\boldsymbol{n}_{\boldsymbol{x}} \times \boldsymbol{n}_{\boldsymbol{y}}\right)$

$\boldsymbol{x}$ - $\quad$ No Cap.6- item 6.2.2, vetor com dimensão $\left(\boldsymbol{n}_{\boldsymbol{x}}\right)$

$\boldsymbol{y}$ - $\quad$ No Cap.6- item 6.2.2, vetor com dimensão $\left(\boldsymbol{n}_{\boldsymbol{y}}\right)$

$\boldsymbol{f}_{\boldsymbol{1}}$ e $\boldsymbol{f}_{2}$ - No Cap.6- item 6.2.2, são termos independentes do sistema de equações lineares da eq.(6.1)

$\boldsymbol{C}$ - $\quad$ No Cap.6- item 6.2.2, matriz com dimensão $\left(\boldsymbol{n}_{\boldsymbol{y}} \times \boldsymbol{n}_{\boldsymbol{x}}\right)$

$\boldsymbol{D}$ - $\quad$ No Cap.6- item 6.2.2, matriz com dimensão $\left(\boldsymbol{n}_{\boldsymbol{y}} \times \boldsymbol{n}_{\boldsymbol{y}}\right)$

$\varphi \quad$ No Cap.6- item 6.3.2, função admissível ou solução

$\psi \quad$ No Cap.6- item 6.3.2, função peso ou teste

$\boldsymbol{B}(\varphi, \psi)-\quad$ Forma bilinear

$\boldsymbol{W}$ - $\quad$ Espaço de Hilbert

$\boldsymbol{L}^{2}(\boldsymbol{\Omega})$ - Espaço das funções quadrado integráveis no domínio $\boldsymbol{\Omega}$

$\boldsymbol{F}(\psi)$ - Funcional linear contínuo

$\boldsymbol{W}_{\boldsymbol{n}} \quad$ Subespaço de dimensão finita

$\boldsymbol{Q}_{n}\left(\boldsymbol{\Omega}^{(m)}\right)$ - Aproximação polinomial de grau “ $\boldsymbol{n}$ ”no elemento $\boldsymbol{m}$ 
$\boldsymbol{\varphi}_{n}$ - $\quad$ No Cap.6- item 6.3.2, solução aproximada por elementos finitos

$\boldsymbol{\psi}_{n}$ - $\quad$ No Cap.6- item 6.3.2, função peso ou teste no subespaço de dimensão finita

$\|\cdot\|_{S}$ - $\quad$ Norma no espaço das funções admissíveis

$\|\cdot\|_{T}$ - $\quad$ Norma no espaço das funções testes

inf - Valor ínfimo

$\boldsymbol{\eta}_{\boldsymbol{n}}$ - $\quad$ No Cap.6- item 6.3.2, qualquer aproximação do espaço discretizado $W_{n}$

$\boldsymbol{k}_{\boldsymbol{m}}$ - $\quad$ No Cap.6- item 6.3.2, constante da condição de continuidade da forma bilinear

$\lambda$ - $\quad$ No Cap.6- item 6.3.2, constante de estabilidade

$\lambda_{n}$ - No Cap.6- item 6.3.2, constante de estabilidade determinado numericamente

sup - Valor supremo

$\Phi^{n}(\varphi)$ - Aproximabilidade

B - $\quad$ No Cap.6- item 6.3.3, matriz simétrica com dimensão $\boldsymbol{n} \times \boldsymbol{n}$

S e T - $\quad$ No Cap.6- item 6.3.3, são matrizes simétricas positivo-definidas obtidas das normas $\|\cdot\|_{S}^{2}$ e $\|\cdot\|_{T}^{2}$

$\boldsymbol{\eta}$ e $\boldsymbol{\psi}$ - $\quad$ No Cap.6- item 6.3.3, são vetores que reúnem os valores nodais de $\boldsymbol{\eta}_{\boldsymbol{n}}$ e $\psi_{n}$, respectivamente

$\mathbf{R}(\boldsymbol{\eta}, \boldsymbol{\psi})$ - $\quad$ No Cap.6- item 6.3.3, forma bilinear

$\xi$ - $\quad$ No Cap.6- item 6.3.3, vetor de composição

$\mathbf{x}$ - $\quad$ No Cap.6- item 6.3.3, autovetores da eq.(6.34)

$\boldsymbol{\mu}$ - $\quad$ No Cap.6- item 6.3.3, autovalores da eq.(6.34) 


\section{Lista de Tabelas}

Tabela 7.1 - Exemplo $1-$ FHMT sem enriquecimento nodal- $\boldsymbol{\mu}=\boldsymbol{0}$ - resultados para deslocamento e energia de deformação.

Tabela 7.2 - Exemplo 1 - FHT sem enriquecimento nodal $\boldsymbol{-} \boldsymbol{\mu}=\boldsymbol{0}$ - resultados para deslocamento e energia de deformação

Tabela 7.3 - Exemplo 1 - MEF Clássico $-\boldsymbol{\mu}=\boldsymbol{0}$ - resultados para deslocamento e energia de deformação.

Tabela 7.4 - Exemplo 2 - FHMT sem enriquecimento $\boldsymbol{\mu} \boldsymbol{\mu}=\boldsymbol{0}$ - resultados para deslocamento e energia de deformação.

Tabela 7.5 - Exemplo 2 - FHT sem enriquecimento- $\boldsymbol{\mu}=\boldsymbol{0}$ - resultados para deslocamento e energia de deformação

Tabela 7.6 - Exemplo 1 - MEF Clássico $-\boldsymbol{\mu}=\boldsymbol{0}$ - resultados para deslocamento e energia de deformação

Tabela 7.7 - Exemplo 3 - FHMT sem enriquecimento - resultados para o deslocamento no ponto $\mathrm{A}$

Tabela 7.8 - Exemplo 3 - FHT sem enriquecimento- resultados para o deslocamento no ponto A

Tabela 7.9 - Exemplo 3 - FHMT com enriquecimento das tensões no domínio $\left(\boldsymbol{y}^{2}\right)$ e deslocamentos no contorno $(\boldsymbol{x})$ - resultados para o deslocamento no ponto A

Tabela 7.10 - Exemplo 3 - FHT com enriquecimento dos deslocamentos no contorno $(\boldsymbol{x})$ - resultados para o deslocamento no ponto A............

Tabela 7.11 - Graus de liberdade para a chapa tracionada - elemento quadrilateral - FHMT

Tabela 7.12 - Graus de liberdade para a chapa tracionada - elemento triangular - FHMT.

Tabela 7.13 - Graus de liberdade para a chapa tracionada - elemento quadrilateral com aproximação constante para o campo de tensão - FHT 
Tabela 7.14 - Graus de liberdade para a chapa tracionada - elemento triangular com aproximação constante para o campo de tensão - FHT.

Tabela 7.15 - Graus de liberdade para a chapa tracionada - elemento quadrilateral com aproximação linear para o campo de tensão FHT..

Tabela 7.16 - Graus de liberdade para a chapa tracionada - elemento triangular com aproximação linear para o campo de tensão - FHT.

Tabela 7.17 - Graus de liberdade para a chapa tracionada - elemento quadrilateral com aproximação quadrática para o campo de tensão -FHT.

Tabela 7.18 - Graus de liberdade para a chapa tracionada - elemento triangular com aproximação quadrática para o campo de tensão - FHT.

Tabela 7.19 - Autovalores para a chapa tracionada - elemento quadrilateral sem enriquecimento - FHMT.

Tabela 7.20 - Autovalores para a chapa tracionada - elemento triangular - sem enriquecimento - FHMT.

Tabela 7.21 - Autovalores para a chapa tracionada - sem enriquecimento FHT.

Tabela 7.22 - Graus de liberdade para a chapa com fenda central - elemento quadrilateral - FHMT.

Tabela 7.23 - Graus de liberdade para a chapa com fenda central - elemento triangular - FHMT.

Tabela 7.24 - Graus de liberdade para a chapa tracionada com fenda central elemento quadrilateral com aproximação constante para o campo de tensão - FHT.

Tabela 7.25 - Graus de liberdade para a chapa tracionada com fenda central elemento triangular com aproximação constante para o campo de tensão - FHT.

Tabela 7.26 - Graus de liberdade para a chapa tracionada com fenda central elemento quadrilateral com aproximação linear para o campo de tensão - FHT.

Tabela 7.27 - Graus de liberdade para a chapa tracionada com fenda central elemento triangular com aproximação linear para o campo de tensão - FHT.

Tabela 7.28 - Graus de liberdade para a chapa tracionada com fenda central elemento quadrilateral com aproximação quadrática para o campo de tensão - FHT. 
Tabela 7.29 - Graus de liberdade para a chapa tracionada com fenda central elemento triangular com aproximação quadrática para o campo de tensão - FHT..........................................................................

Tabela 7.30 - Autovalores para a chapa com fenda central - elemento quadrilateral - sem enriquecimento - FHMT.

Tabela 7.31 - Autovalores para a chapa com fenda central - elemento triangular - sem enriquecimento - FHMT.......................................................

Tabela 7.32 - Autovalores para a chapa tracionada com fenda central - sem enriquecimento - FHT. 


\section{ABREVIATURAS}

PVC - Problema de Valor de Contorno

MEF - $\quad$ Método dos Elementos Finitos

MMQM - Método dos Mínimos Quadrados Móveis

PU - $\quad$ Partição da Unidade

MEFG - Método dos Elementos Finitos Generalizados

FHT - $\quad$ Formulação Híbrida de Tensão

FHMT - Formulação Híbrido-Mista de Tensão

EPT - $\quad$ Estado Plano de Tensão

hp - $\quad$ Processos h-adaptativo e p-adaptativo do Método dos Elementos Finitos 


\section{SUMÁRIO}

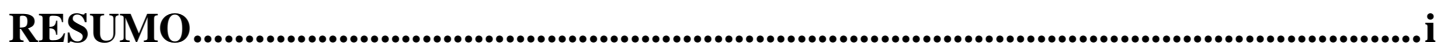

ABSTRACT ….............................................................................................................iii

LISTA DE FIGURAS.................................................................................................

LISTA DE SÍMBOLOS .....................................................................................................

LISTA DE TABELAS ...............................................................................................xxvii

ABREVIATURAS ..................................................................................................

1. INTRODUÇÃO ........................................................................................................1

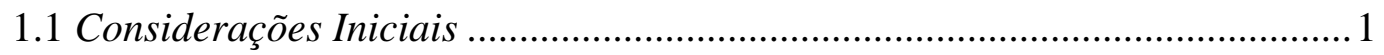

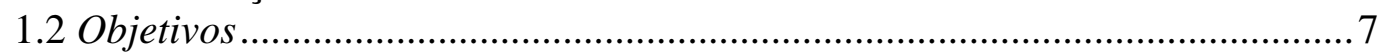

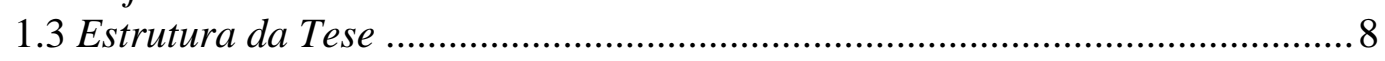

2. FORMULAÇÃO HÍBRIDA E HÍBRIDO-MISTA DE TENSÃO PARA

ELASTICIDA PLANA .................................................................................................9

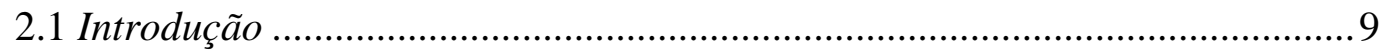

2.2 Formulações Híbrido-Mistas para a Elasticidade Plana .................................. 9

2.3 Formulações Híbridas para a Elasticidade Plana ........................................... 25

3. FHT E FHMT COM ENRIQUECIMENTO NODAL - MÉTODO DOS ELEMENTOS FINITOS GENERALIZADOS........................................................27

3.1 O Método dos Elementos Finitos Generalizados (MEFG) ………………..... 27

3.2 FHMT e FHT com Enriquecimento Nodal ....................................................... 31

3.2.1 FHMT com Enriquecimento Nodal ............................................................31

3.2.2 FHT com Enriquecimento Nodal................................................................ 33

4. MODELO DISCRETO DA FHMT COM ENRIQUECIMENTO NODAL... 35

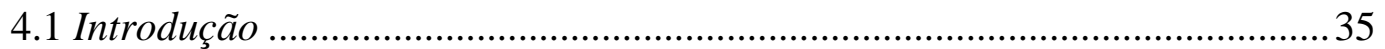

4.2 Modelo Discreto da FHMT com Enriquecimento Nodal ................................35

4.3 Elementos Finitos Híbrido-Mistos de Tensão com Enriquecimento Nodal

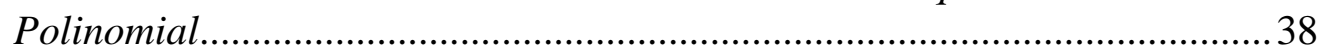

4.3.1 Elemento Quadrilateral de Quatro Nós ....................................................38

4.3.1.1 Características Geométricas e Transformação de Coordenadas...............38

4.3.1.2 Funções Aproximativas do Elemento Quadrilateral de Quatro Nós da FHMT

4.3.1.3 Enriquecimento das Funções Aproximativas do Elemento Quadrilateral

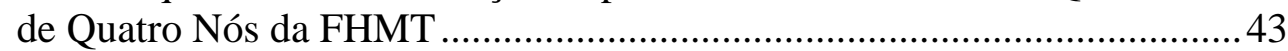

4.3.1.4 Integrais Intervenientes........................................................................ 51

4.3.2 Elemento Triangular de Três Nós ..............................................................5 
4.3.2.1 Características Geométricas e Transformação de Coordenadas ........... 55

4.3.2.2 Funções Aproximativas do Elemento Triangular de Três Nós da FHMT

4.3.2.3 Enriquecimento das Funções Aproximativas do Elemento Triangular

de Três Nós da FHMT .

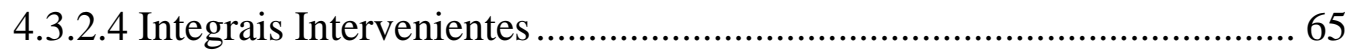

4.3.2.5 Solução do Sistema de Equações da FHMT com Enriquecimento Nodal - Método de Babuška.

5. MODELO DISCRETO DA FHT COM ENRIQUECIMENTO NODAL ...... 71

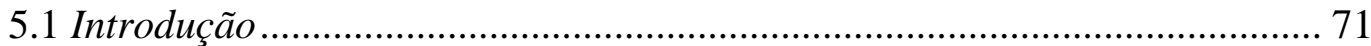

5.2 Modelo Discreto da FHT com Enriquecimento Nodal ................................ 71

5.3 Elementos Finitos Híbridos de Tensão com Enriquecimento Nodal

Polinomial 72

5.3.1 Elemento Quadrilateral de Quatro Nós................................................ 72

5.3.1.1 Características Geométricas e Transformação de Coordenadas ............ 72

5.3.1.2 Funções Aproximativas do Elemento Quadrilateral de Quatro Nós da FHT

5.3.1.3 Enriquecimento das Funções Aproximativas do Elemento Quadrilateral

de Quatro Nós da FHT ............................................................................. 75

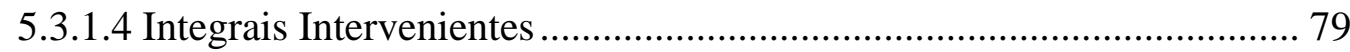

5.3.2 Elemento Triangular de Três Nós......................................................... 84

5.3.2.1 Características Geométricas e Transformação de Coordenadas ............ 84

5.3.2.2 Funções Aproximativas do Elemento Triangular de Três Nós da

FHT ... 84

5.3.2.3 Enriquecimento das Funções Aproximativas do Elemento Triangular de Três Nós da FHT.

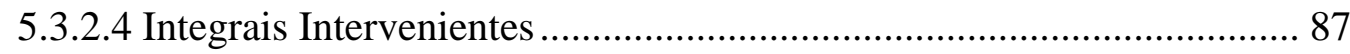

\section{ESTUDO DAS CONDIÇÕES DE CONVERGÊNCIA DA FHT E FHMT COM ENRIQUECIMENTO NODAL ...............................................................89}

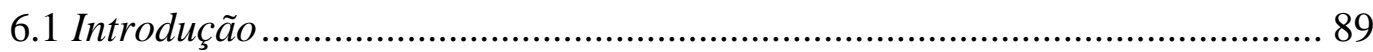

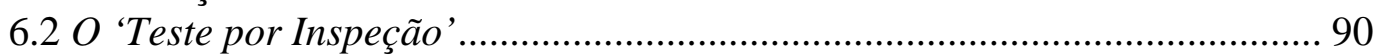

6.2.1 Considerações Iniciais............................................................................ 90

6.2.2 O 'Teste por Inspeção'.......................................................................... 90

6.3 Estudo da Condição de Babuška-Brezzi (inf-sup) Aplicada à FHT e

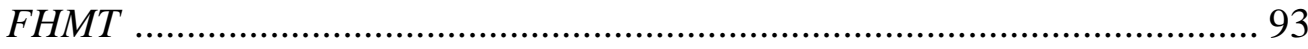

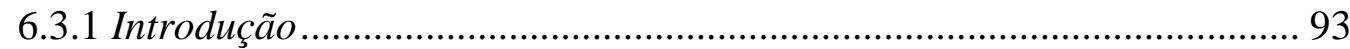

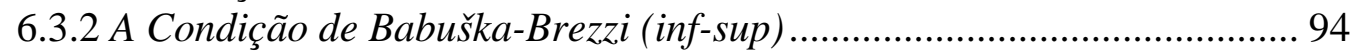

6.3.3 Determinação Numérica de $\lambda$............................................................... 97

6.3.4 O Teste Numérico da Condição de Babuška-Brezzi (inf-sup) Aplicada à

FHMT com Enriquecimento Nodal............................................................ 99

6.3.5 O Teste Numérico da Condição de Babuška-Brezzi (inf-sup) Aplicada à

FHT com Enriquecimento Nodal ........................................................... 103 


\section{ANÁLISE NUMÉRICA DA FHT E FHTMT COM ENRIQUECIMENTO}

NODAL

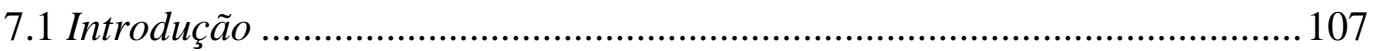

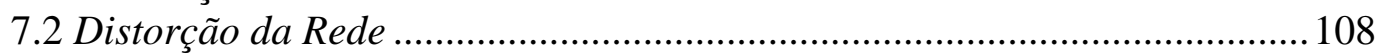

7.2.1 Exemplo 1 - Viga Engastada - Momento Concentrado ..........................108

7.2.2 Exemplo 2 - Viga Engastada - Cisalhamento .........................................126

7.2.3 Exemplo 3 - Painel de Cook................................................................... 146

$7.3 \mathrm{O}$ 'Teste por Inspeção' Aplicado à FHT/FHMT com Enriquecimento

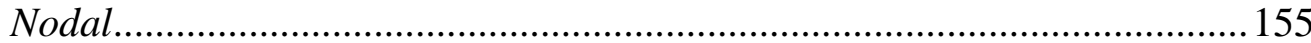

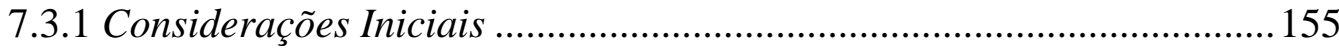

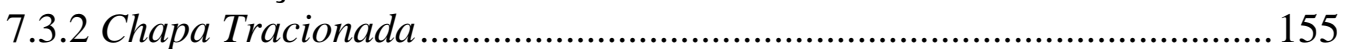

7.3.3Chapa Tracionada com Fenda Central....................................................174

7.4 O Teste "inf-sup" Aplicado à FHT/FHMT com Enriquecimento Nodal:

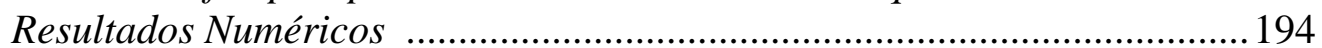

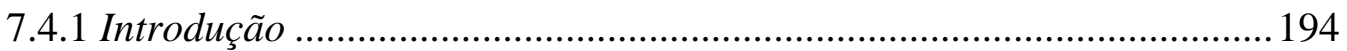

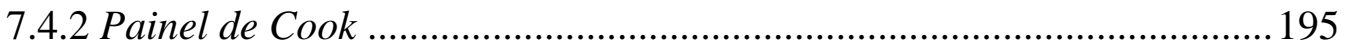

7.4.3 Chapa Tracionada ........................................................................... 199

7.4.4 Chapa Tracionada com Fenda Central.................................................204

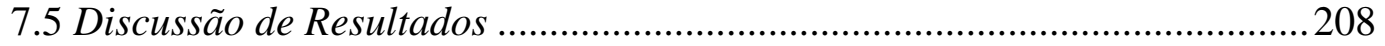

8. CONSIDERAÇÕES FINAIS E CONCLUSÕES ................................................211

REFERÊNCIAS BIBLIOGRÁFICAS E BIBLIOGRAFIA BÁSICA..................215

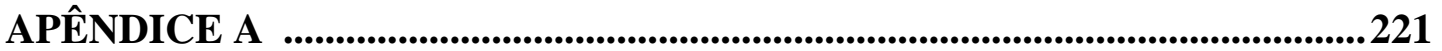





\section{Introdução}

\subsection{Considerações Iniciais}

Entender o comportamento das estruturas e dos materiais que as constituem é problema intrínseco a qualquer ramo da engenharia. No desenvolvimento de produtos (edifícios, carros, pontes, aviões, navios, próteses que substituem parte da estrutura óssea humana, etc.), exige-se, especificamente da engenharia de estruturas, a formulação de modelos mecânicos-estruturais que simulem o mais próximo quanto possível, a realidade dos fenômenos envolvidos.

Basicamente, segundo a abordagem pela mecânica do contínuo, a descrição matemática dos fenômenos de interesse envolve a interação de três grandezas-chave: os deslocamentos $(\boldsymbol{u})$, as tensões $(\boldsymbol{\sigma})$ e as deformações $(\boldsymbol{\varepsilon})$. Assim, representar e quantificar realisticamente cada uma dessas grandezas é fundamental para sucesso de qualquer projeto na engenharia de estruturas.

Dessa forma, um modelo matemático consistente envolve restrições sobre os deslocamentos, as tensões e as deformações no tocante ao equilíbrio, compatibilidade, leis constitutivas e condições de contorno específicas de cada fenômeno estudado. Classicamente, na mecânica das estruturas e dos materiais, o modelo matemático expresso pelo conjunto de relações associadas às restrições, e que permite a determinação das grandezas-chave, constitui o denominado Problema de Valor no Contorno (PVC). Os operadores matemáticos envolvidos nos modelos caracterizam diferentes classes de problemas.

Soluções analíticas de uma classe de PVC, na maioria das vezes obtidas com hipóteses simplificadoras, restringem-se a um pequeno número de problemas. O emprego de métodos numéricos permite ampliar as possibilidades de análise e solução de toda uma classe de PVC. 
Os métodos numéricos substituem a resolução analítica de um PVC pela introdução de formas aproximadas, ou discretizadas, do modelo matemático, gerando os correspondentes modelos discretos. Em tais modelos, se objetiva determinar os deslocamentos $(\boldsymbol{u})$, as tensões $(\sigma)$ e as deformações $(\varepsilon)$ em um número finito de pontos.

Dentre os métodos numéricos, a forma convencional “em deslocamentos” do Método dos Elementos Finitos (MEF) é uma das ferramentas mais difundidas e aplicadas na resolução aproximada de um PVC. Essa consolidação deve-se, em parte, à sua simplicidade conceitual, mas também à facilidade de implementação computacional.

Entretanto, quando funções polinomiais de baixo grau, aproximativas dos campos de deslocamentos, são utilizadas no MEF clássico, pode-se obter resultados insatisfatórios em termos da representação de grandezas que dependam de ordens superiores de derivadas desses campos. É o caso, principalmente, de problemas que envolvam regiões com singularidade (que se caracterizam por elevados gradientes de tensão), Szabó e Babuška (1991). Esse fato ocorre porque as derivações implicam em redução da ordem da aproximação.

Mas há outras situações em que o MEF clássico também sofre limitações. Por exemplo, na análise de problemas no âmbito da elasticidade quase incompressível, mesmo com uma discretização razoável, utilizando elementos finitos clássicos, pode-se obter soluções grosseiras, Bathe (1996). Além disso, a solução obtida via MEF clássico também fica comprometida, quando se empregam redes de elementos finitos excessivamente distorcidos.

Por outro lado, na utilização do MEF convencional em problemas que envolvam grandes deformações ou propagação de trincas pode ser necessária a mudança contínua da rede de elementos finitos. Ocorre que a mudança da discretização do problema é normalmente bastante onerosa computacionalmente.

Por isso, durante as últimas décadas, muitos esforços têm sido direcionados na tentativa de desenvolvimento de metodologias numéricas não-convencionais que combinem boa capacidade de aproximação com baixo custo computacional.

Boa parte das metodologias propostas fundamenta-se em princípios variacionais mistos. Uma boa revisão sobre os princípios variacionais clássicos encontra-se em Desai e Abel (1972), Góis (2004) e Zienkiewicz (2000).

Formas variacionais conhecidas na literatura por funcionais de Hellinger-Reissner e Hu-Washizu, conduziram ao desenvolvimento da Formulação Mista (e suas variações) do MEF, Góis (2004) e Zienkiewicz (2000). As formas mistas do MEF são assim denominadas, 
por envolverem funcionais dependentes de mais de um campo definido no domínio estudado $(\boldsymbol{\Omega})$, como: tensão $(\boldsymbol{\sigma})$ e deslocamento $(\boldsymbol{u})$ no funcional de Hellinger-Reissner e tensão $(\boldsymbol{\sigma})$, deslocamento $(\boldsymbol{u})$ e deformação $(\boldsymbol{\varepsilon})$ no funcional de Hu-Washizu.

As formulações mistas são comumente utilizadas para análise de cascas, placas e de problemas no âmbito da elasticidade quase incompressível, pois contornam os entraves apresentados nos estudos desses casos com o MEF clássico, Bathe (1996).

Para além das formulações mistas, o trabalho pioneiro de Pian (1964) apresentou um funcional que envolvia tensão $(\boldsymbol{\sigma})$ no domínio $(\boldsymbol{\Omega})$ e deslocamento $(\boldsymbol{u})$ nas interfaces de subdomínios de $(\boldsymbol{\Omega})$. Definiu-se este funcional como funcional híbrido e dele surgiu a Formulação Híbrida de Tensão do MEF. Pian (1964) comenta que nesta formulação é possível obter boas soluções dos campos de tensão $(\boldsymbol{\sigma})$ e deslocamento $(\boldsymbol{u})$ e ainda explorar a vantagem de "relaxar” o requerimento de continuidade dos campos aproximados.

Vale salientar, entretanto, que a dependência em relação a um princípio variacional associado não é condição necessária para o desenvolvimento de uma formulação do MEF. Nesse contexto, destacam-se as formas não-convencionais do MEF apresentadas em Freitas, Almeida e Pereira (1996), que dispensam o emprego de princípios variacionais e se constituem essencialmente em formulações em resíduos ponderados.

No âmbito dessas formulações, em Freitas, Almeida e Pereira (1996), o problema clássico da elasticidade linear é estudado por meio de formas não-convencionais onde campos de tensão $(\boldsymbol{\sigma})$ e de deslocamento $(\boldsymbol{u})$ podem ser simultaneamente aproximados. São seis as formas não-convencionais apresentadas: As Formulações Híbrido-Mistas de Tensão e de Deslocamento, as formulações Híbridas de Tensão e de Deslocamento e as formulações Híbrido-Trefftz de Tensão e de Deslocamento. Na obtenção de cada uma delas, não se utilizam princípios variacionais, mas ponderações de Galerkin das equações de equilíbrio, compatibilidade e constitutiva envolvidas no problema da elasticidade linear.

As formas híbrido-mistas são primeiramente ditas mistas porque se fundamentam na aproximação direta de dois campos incompatíveis no domínio: tensão $(\boldsymbol{\sigma})$ e deslocamento $(\boldsymbol{u})$. Como os campos de deslocamento ou de forças podem independentemente ser aproximados no contorno $(\boldsymbol{\Gamma})$, a formulação é também caracterizada como Híbrida.

Ocorre que ao se adotar uma abordagem híbrido-mista ou híbrida, os campos de contorno precisam ser compatibilizados, nos sentidos: cinemático e estático, com os campos de domínio. Como proposta geral, um ou outra dessas compatibilizações é imposta em forma 
forte, enquanto a restante é inserida em forma fraca. Por exemplo, o modelo resultante diz-se híbrido-misto de tensão quando o equilíbrio de forças no contorno entre elementos é imposto de forma ponderada. Já o modelo é denominado híbrido-misto de deslocamento quando a compatibilidade de deslocamentos é imposta de forma ponderada nas fronteiras comuns a elementos.

As abordagens híbridas podem ser obtidas das formas Híbrido-Mistas pela simples escolha de funções interpoladoras dos campos de tensão ou deformação satisfazendo previamente as condições de equilíbrio ou compatibilidade no volume, respectivamente.

Já nas Formulações Híbrido-Trefftz, no modelo de tensão as aproximações das tensões no domínio são derivadas de potenciais de tensão ditos: 'bi-harmônicos', pois satisfazem à equação de Beltrami. Na variante de deslocamento, as aproximações de deslocamento no domínio são obtidas de potenciais de deslocamentos harmônicos que satisfazem à equação de Navier.

A implementação numérica das formulações não-convencionais proposta neste trabalho tem por base uma combinação de recursos dos métodos sem malha e do MEF.

Os métodos sem malha, Duarte (1995), são definidos como métodos numéricos para solução de um PVC, onde as equações que governam o modelo discreto independem totalmente ou em parte, de uma rede de elementos finitos.

Dentre os métodos sem malha, destaca-se o Método das Nuvens "hp” (“hp” Clouds), apresentado nos trabalhos de Duarte e Oden $(1995,1996)$, onde o modelo discreto é construído a partir de pontos nodais dispersos no domínio, sem nenhuma conectividade. Igualmente à maioria dos métodos sem malha, as funções aproximativas do Método das Nuvens “hp” são determinadas pelo Método dos Mínimos Quadrados Móveis (MMQM), Lancaster e Salkauskas $(1981,1990)$. Tais funções de forma constituem uma Partição da Unidade (PU) (essencialmente um conjunto de funções cuja soma, num ponto, é igual à unidade).

Mas a principal característica desse método empregada neste trabalho é a possibilidade de enriquecimento nodal das funções de aproximação que constituem uma partição da unidade. O enriquecimento nodal pode ser definido como a ampliação das bases aproximativas envolvidas, sem a necessidade de introduzir novos pontos nodais ao domínio.

A combinação de características favoráveis dos métodos sem malha e do MEF adotada neste trabalho é o Método dos Elementos Finitos Generalizados (MEFG), Babuška, Caloz e Osborn (1994), Babuška e Melenk (1997), Duarte (1996), Duarte e Oden (1995, 1996), Melenk e Babuška (1996) e Oden, Duarte e Zienkiewicz (1998). Essencialmente, uma rede de cobertura é empregada para definir nuvens suporte dentro das quais as funções de forma do 
MEF clássico são usadas como PU. Estas funções de forma podem então ser enriquecidas dentro de cada nuvem, mediante a multiplicação da PU por funções de interesse; o enriquecimento resultante tem caráter nodal.

Nos trabalhos de Pimenta, Proença e Freitas (2002) e Góis (2004), os elementos Híbrido-Mistos de Tensão foram pioneiramente utilizados no MEFG como rede de cobertura (definição de nuvens associadas aos pontos nodais). Já as funções aproximativas dos campos de tensão e deslocamento no domínio e deslocamento no contorno foram tomadas como PU. Além disso, a técnica de enriquecimento nodal foi aplicada aos campos envolvidos na Formulação Híbrido-Mista de Tensão, possibilitando assim, uma nova apresentação ao MEFG.

Mas um questionamento pode ser levantado no tocante à aplicação dessas formas nãoconvencionais do MEF: Elas sempre possibilitarão soluções convergentes?

A formalização matemática das condições necessárias e suficientes para convergência de soluções aproximadas obtidas com formas não-convencionais do MEF, como por exemplo, as adotadas neste trabalho e apresentadas originalmente em Freitas, Almeida e Pereira (1996), quase inexistem.

Para a formulação mista clássica do MEF, o estudo das condições necessárias e suficientes para convergência está bem consolidado, como mostram os trabalhos de Babuška (1996), Bathe (1996, 2001), Brezzi e Bathe (1990), Chapelle e Bathe (1993), Iosilevich, Bathe e Brezzi (1997), Zienkiewicz et al. (1986) e Zienkiewicz e Lefebvre (1987).

A convergência de soluções aproximativas obtidas com o MEF para qualquer formulação variacional fica garantida se basicamente duas condições são atendidas: consistência e estabilidade.

A consistência é composta pelos aspectos de representatividade e compatibilidade (ou conformidade) das aproximações, essencialmente ratificadas pelo emprego da PU. Já a estabilidade depende de boas propriedades, como a densidade, da forma bilinear atrelada à formulação adotada. As condições ditas de elipsidade (ligada à solvibilidade) e (inf-sup) de Babuška-Brezzi (ligada à densidade do operador), proposta de modo independente por Babuška (1971, 1973) e Brezzi (1974), determinam as boas propriedades da forma bilinear e, de resto, do PVC.

No âmbito dos estudos envolvendo as formulações clássicas do MEF, em Chapelle e Bathe (1993), Bathe, Iosilevich e Chapelle (2000) e Bathe (2001), é ressaltado que para certa discretização as condições de consistência e elipsidade são relativamente fáceis de serem verificadas. Ainda nestes trabalhos, os autores comentam da dificuldade de verificar 
analiticamente se a condição Babuška-Brezzi (inf-sup) é satisfeita para uma discretização em elementos finitos.

Em Chapelle e Bathe (1993) foi apresentada uma alternativa numérica, portanto envolvendo o operador discretizado, denominada de "teste inf-sup”, para análise da condição de Babuška-Brezzi (inf-sup). Em Bathe (1996, 2001), Bathe, Iosileviche e Chapelle (2000), Chapelle e Bathe (1993) e Iosilevich, Bathe e Brezzi (1997), o "teste inf-sup” foi utilizado em diversos problemas (cascas, placas, incompressibilidade) analisados com elementos finitos mistos, para averiguar se as soluções geradas atendiam à condição de Babuška-Brezzi (infsup). Como já mencionado, na literatura técnica poucos são os trabalhos que utilizam da condição de Babuška-Brezzi (inf-sup) para consolidar aspectos de convergência de formulações Híbrido-Mistas e Híbridas.

No âmbito das formulações não-convencionais, em Góis (2004), analisou-se a condição de elipsidade da Formulação Híbrido-Mista de Tensão com enriquecimento nodal por meio de testes numéricos simples baseados nos trabalhos de Zienkiewicz et al. (1986) e Zienkiewicz e Lefebvre (1987). De forma incipiente, em Góis (2004), a condição de BabuškaBrezzi (inf-sup) foi aplicada à Formulação Híbrido-Mista de Tensão, estendendo a metodologia proposta em Chapelle e Bathe (1993).

Góis e Proença (2005, 2006a, 2007b, 2007c) avançaram no tema da aplicação da condição de Babuška-Brezzi (inf-sup) na Formulação Híbrido-Mista de Tensão com enriquecimento nodal.

Em Góis e Proença (2006b, 2007a, 2007c) a Formulação Híbrida de Tensão com enriquecimento nodal foi apresentada, focando-se principalmente aspectos relacionados às condições necessárias e suficientes para convergência de soluções obtidas com esta formulação.

Os estudos realizados por um lado confirmaram alguns entraves dessas formulações, principalmente no tocante à geração de modos espúrios cinemáticos, que podem ocorrer quando da adoção de um enriquecimento livre dos campos de deslocamentos, sem que se tome cuidado de compensá-lo pelo enriquecimento do campo de tensões. Por outro lado, uma vez que se aplique o recurso do enriquecimento com um critério adequado, ressaltaram-se as potencialidades apresentadas pelas formulações não-convencionais combinadas com a técnica de enriquecimento nodal, justificando a continuidade de pesquisas nesse tema. 


\subsection{Objetivos}

O trabalho tem por objetivo principal oferecer uma contribuição ao estudo de formulações não-convencionais do PVC, tendo-se como base a Formulação Híbrida de Tensão (FHT) e a Formulação Híbrido-Mista de Tensão (FHMT), combinada com uma técnica de enriquecimento nodal de aproximações definidas por uma rede de elementos finitos.

O método dos elementos finitos generalizados é essencialmente aplicado na FHMT. A extensão do método para a FHT não é, entretanto, imediata. Uma contribuição inovadora, nesse sentido, é o desenvolvimento de uma metodologia que possibilita o enriquecimento nodal das aproximações dos campos de tensão $(\boldsymbol{\sigma})$ que originalmente não estão atreladas a nós, no caso da FHT.

Um estudo numérico sobre as condições necessárias e suficientes para convergência das soluções obtidas com as FHT e FHMT com enriquecimento nodal, também é outro ponto de abordagem original do trabalho.

Como primeira etapa da pesquisa, tem-se a implementação computacional da proposta inicial da aplicação do MEFG na FHT e na FHMT. Nesta fase, são desenvolvidos elementos triangulares e quadrilaterais enriquecidos nodalmente por funções polinomiais. Procura-se, por meio de exemplos clássicos da elasticidade linear, verificar também a sensibilidade das aproximações resultantes geradas a partir de redes distorcidas. Ainda, resultados obtidos da FHT/FHMT com enriquecimento nodal são confrontados com resultados obtidos de um ‘software’ comercial fundamentado na formulação clássica do MEF.

Na segunda parte do trabalho, analisam-se os aspectos matemáticos necessários e suficientes para convergência das soluções obtidas da FHT e FHMT com enriquecimento nodal pela verificação numérica da condição de Babuška-Brezzi, Babuška $(1971,1973)$ e Brezzi (1974). 


\subsection{Estrutura da Tese}

O conteúdo do trabalho está organizado como se segue:

- No capítulo 1, inicialmente, apresentam-se as considerações iniciais do trabalho, a revisão bibliográfica que fundamenta a pesquisa, os trabalhos já desenvolvidos e publicados pelo autor no tema desta tese. Posteriormente, destacam-se os objetivos do trabalho e a estruturação desta tese.

- No capítulo 2, após uma breve revisão dos conceitos básicos da teoria da elasticidade utilizados no trabalho, formulam-se matematicamente a FHT e a FHMT com base em resíduos ponderados.

- No capítulo 3, foca-se o conceito da partição da unidade (PU) e o enriquecimento desta partição na formulação em deslocamentos do MEF, ou seja, como o Método dos Elementos Finitos Generalizados (MEFG) foi apresentado originalmente. O capítulo é finalizado com a aplicação do MEFG aos campos de aproximação envolvidos na FHT e FHMT.

- O capítulo 4 trata da construção dos elementos finitos isoparamétricos triangular e quadrilateral da FHMT com enriquecimento nodal.

- O capítulo 5 apresenta os elementos finitos triangular e quadrilateral da FHT com enriquecimento nodal. Aqui, destaca-se a metodologia inovadora que possibilitou a aplicação do MEFG à FHT.

- No capítulo 6, estudam-se as condições necessárias e suficientes para estabilidade das soluções numéricas obtidas com a FHT/FHMT enriquecidas. Nesta parte, salientam-se os testes numéricos desenvolvidos para verificar as condições gerais de garantia de estabilidade: o ‘Teste por Inspeção’ e o teste “inf-sup”.

- $\quad$ No capítulo 7 são apresentados os exemplos numéricos e os resultados são discutidos.

- $\quad$ No capítulo 8 são apresentadas as considerações finais e conclusões. 


\section{Formulação Híbrida e Híbrido-Mista de Tensão para Elasticidade}

\subsection{Introdução}

Neste item apresentam-se as formulações híbrido-mistas para a consideração de problemas lineares em Mecânica dos Sólidos e Estruturas. A forma apresentada é dita nãoconvencional, uma vez que não se baseia em nenhum princípio variacional como usualmente apresentado nos textos sobre o tema, mas sim no método dos resíduos ponderados. A formalização aqui descrita segue o trabalho de Proença (2008).

\subsection{Formulações Híbrido-Mistas para a Elasticidade Plana}

As relações do problema elástico de valor de contorno são aqui resumidas, para que as formulações Híbrida e Híbrido-Mista de Tensão sejam posteriormente mais facilmente entendidas. Não existe, portanto, o objetivo de entrar em detalhes da teoria da elasticidade, os quais podem ser encontrados em obras como Xu (1992), Timoshenko e Goodier (1980) e Valliappan (1981).

No caso geral, o PVC em elasticidade linear, para o estado plano de tensão (EPT), é regido em forma forte pelo seguinte conjunto de relações:

- Equações de Equilíbrio:

$\boldsymbol{L} \boldsymbol{\sigma}+\boldsymbol{b}=\boldsymbol{0}$, em $\boldsymbol{\Omega}$

onde: 


$$
L=\left[\begin{array}{ccc}
\frac{\partial}{\partial x} & 0 & \frac{\partial}{\partial y} \\
0 & \frac{\partial}{\partial y} & \frac{\partial}{\partial x}
\end{array}\right]
$$

é o operador diferencial divergente, $\sigma$ é representado pelo vetor coluna formado com as componentes do tensor de tensão em um sistema cartesiano de coordenadas $x$ e $y$ e $\boldsymbol{b}$ é o vetor de forças volúmicas.

- Equação de Compatibilidade:

$$
\boldsymbol{\varepsilon}-\boldsymbol{L}^{T} \boldsymbol{u}=\boldsymbol{0}, \text { em } \boldsymbol{\Omega},
$$

onde:

$$
u=\left\{\begin{array}{l}
u_{x} \\
u_{y}
\end{array}\right\}
$$

é o vetor de deslocamentos e $\boldsymbol{\varepsilon}$ é representado pelo vetor coluna formado com as componentes dos tensores de deformação em um sistema cartesiano de coordenadas $x$ e $y$.

- Lei Constitutiva:

$$
\varepsilon=f \sigma
$$

onde:

$$
\sigma=\left\{\begin{array}{l}
\sigma_{x} \\
\sigma_{y} \\
\tau_{x y}
\end{array}\right\} ; \varepsilon=\left\{\begin{array}{c}
1 / 2 \varepsilon_{x} \\
1 / 2 \varepsilon_{y} \\
1 / 2 \gamma_{x y}
\end{array}\right\} ; f=\frac{1}{E}\left[\begin{array}{ccc}
1 & -v & 0 \\
-v & 1 & 0 \\
0 & 0 & 2(1+v)
\end{array}\right],
$$

onde $\boldsymbol{E}$ é o módulo de elasticidade e $\boldsymbol{v}$ é o coeficiente de Poisson. Nessa notação, $\boldsymbol{f}$ é a matriz flexibilidade para materiais elásticos lineares isótropos.

- As condições de contorno da elasticidade plana são:

$$
\overline{\boldsymbol{u}}=\boldsymbol{u}, \text { em } \boldsymbol{\Gamma}_{u}
$$




$$
\bar{t}=N \sigma, \text { em } \Gamma_{t}
$$

onde:

$$
N=\left[\begin{array}{ccc}
n_{x} & 0 & n_{y} \\
0 & n_{y} & n_{x}
\end{array}\right]
$$

é a matriz construída com as componentes do vetor $\boldsymbol{n}$ normal ao contorno, $\overline{\boldsymbol{u}}$ é o vetor dos deslocamentos impostos em $\boldsymbol{\Gamma}_{\boldsymbol{u}}$ e $\overline{\boldsymbol{t}}=\left[\begin{array}{l}\overline{\boldsymbol{t}}_{\boldsymbol{x}} \\ \overline{\boldsymbol{t}_{\boldsymbol{y}}}\end{array}\right]$ é o vetor das forças superficiais aplicadas em $\boldsymbol{\Gamma}_{\boldsymbol{t}}$.

Ainda nas eq.(2.1) a eq.(2.5) $\boldsymbol{\Omega}$ é um domínio limitado por um contorno regular $\Gamma=\Gamma_{u} \cup \Gamma_{t}$, ver figura 2.1 .

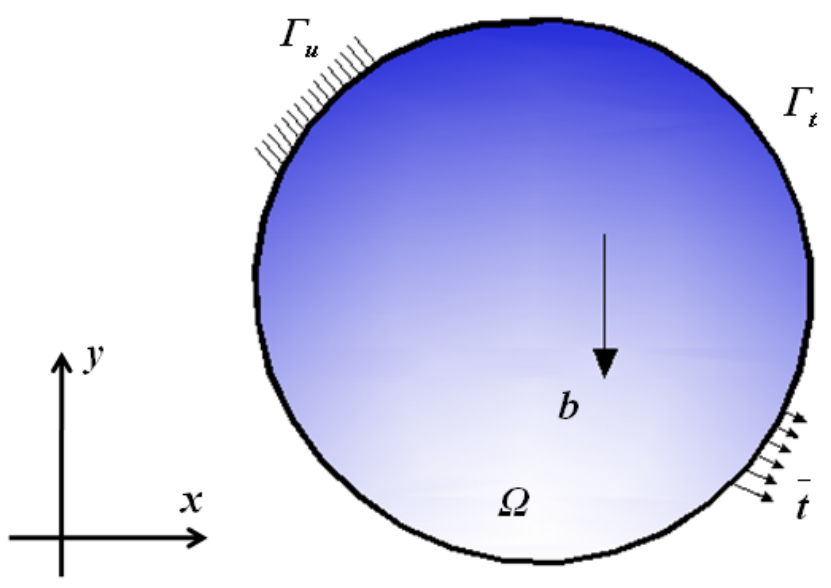

Figura 2.1 - Corpo elástico submetido a forças de volume e de superfície e a representação das partes complementares do contorno.

Vale salientar que as equações aqui apresentadas referem-se a problemas regidos pela Teoria da Elasticidade Linear, considerando-se apenas a isotropia e dentro dos limites das deformações infinitesimais e pequenos deslocamentos.

Agora, admitindo-se neste desenvolvimento que as condições no contorno (de Dirichlet) sejam atendidas em forma forte, todas as outras condições podem ser impostas em forma fraca mediante ponderações, como indicam as relações abaixo:

$$
\int_{\Omega} M_{\Omega}^{T}\left(L^{T} u-f \sigma\right) d \Omega=0
$$




$$
\begin{aligned}
& \int_{\Omega} P_{\Omega}^{T}(L \sigma+b) d \Omega=0 \\
& \int_{\Gamma_{t}} R_{\Gamma_{t}}^{T}(\bar{t}-N \sigma) d \Gamma=0
\end{aligned}
$$

Os operadores de ponderação que aparecem nas relações anteriores resultaram das seguintes definições adotadas para as funções ponderadoras:

$$
\begin{gathered}
\delta m=M_{\Omega} \delta m_{\Omega} \\
\delta p=P_{\Omega} \delta p_{\Omega} \\
\delta r=R_{\Gamma_{t}} \delta r_{\Gamma_{t}}
\end{gathered}
$$

O sistema formado pelas eq.(2.6) a (2.8) é primeiramente alterado pela integração por partes da eq.(2.6), obtendo-se:

$$
\int_{\Omega}\left(L M_{\Omega}\right)^{T} u d \Omega+\int_{\Omega} M_{\Omega}^{T} f \sigma d \Omega-\int_{\Gamma_{t}}\left(N M_{\Omega}\right)^{T} u_{\Gamma} d \Gamma-\int_{\Gamma_{u}}\left(N M_{\Omega}\right)^{T} \bar{u} d \Gamma=0
$$

Na relação anterior colocam-se em destaque os campos considerados independentes e que justificam a denominação formulação híbrido-mista: deslocamentos e tensões no domínio $\boldsymbol{\Omega}$ e deslocamentos na parte $\boldsymbol{\Gamma}_{\boldsymbol{t}}$ do contorno. Admite-se, portanto, que:

- As aproximações para deslocamentos no domínio e no contorno são diferentes. Ambos os campos são compatibilizados em forma fraca mediante a relação anterior.

- As tensões e deslocamentos no domínio são incompatíveis.

Em termos mais gerais, nos contornos as grandezas desconhecidas podem ser aproximadas de forma independente das aproximações adotadas no domínio.

Como nos contornos $\boldsymbol{\Gamma}_{\boldsymbol{u}}$ e $\boldsymbol{\Gamma}_{\boldsymbol{t}}$ do domínio $\boldsymbol{\Omega}$ são conhecidos $\overline{\boldsymbol{u}}$ e $\overline{\boldsymbol{t}}$, respectivamente, neles são aproximadas, também respectivamente, as grandezas $\boldsymbol{t}$ e $\boldsymbol{u}$. 
As aproximações para os campos incógnitos escrevem-se:

$$
\begin{gathered}
\boldsymbol{\sigma}=\boldsymbol{S}_{\boldsymbol{\Omega}} \boldsymbol{s}_{\boldsymbol{\Omega}} \text { em } \boldsymbol{\Omega} \\
\boldsymbol{u}=\boldsymbol{U}_{\boldsymbol{\Omega}} \boldsymbol{q}_{\boldsymbol{\Omega}} \text { em } \boldsymbol{\Omega} \\
\boldsymbol{u}_{\boldsymbol{\Gamma}}=\boldsymbol{U}_{\boldsymbol{\Gamma}_{\boldsymbol{t}}} \boldsymbol{q}_{\Gamma_{t}} \text { em } \boldsymbol{\Gamma}_{\boldsymbol{t}}
\end{gathered}
$$

onde vetor $\boldsymbol{s}_{\boldsymbol{\Omega}}$ pode ser interpretado como o vetor das tensões generalizadas associadas às funções de aproximação dos campos de tensão em $\boldsymbol{S}_{\Omega}$.

Já $\boldsymbol{U}_{\Omega}$ é a matriz que guarda as funções de aproximação do campo de deslocamento no domínio $\boldsymbol{\Omega}$ e $\boldsymbol{q}_{\boldsymbol{\Omega}}$ é o vetor que agrupa os graus de liberdade generalizados associados. Finalmente, $\boldsymbol{U}_{\boldsymbol{\Gamma}_{t}}$ é a matriz que coleta as funções de aproximação do campo de deslocamento no contorno $\boldsymbol{\Gamma}_{\boldsymbol{t}}$ e $\boldsymbol{q}_{\boldsymbol{\Gamma}_{t}}$ é o vetor que contem os pesos que podem ser interpretados como deslocamentos generalizados no contorno $\boldsymbol{\Gamma}_{t}$.

Substituindo-se essas aproximações no conjunto de relações ponderadas (eq.(2.12), eq.(2.7) e eq.(2.8)), obtém-se o seguinte sistema:

$$
\left[\begin{array}{ccc}
\int_{\Omega} M_{\Omega}^{T} f S_{\Omega} d \Omega & \int_{\Omega}\left(L M_{\Omega}\right)^{T} U_{\Omega} d \Omega & -\int_{\Gamma_{t}}\left(N M_{\Omega}\right)^{T} U_{\Gamma_{t}} d \Gamma \\
\int_{\Omega} P_{\Omega}^{T}\left(L M_{\Omega}\right) d \Omega & 0 & 0 \\
-\int_{\Gamma_{t}} R_{\Gamma_{\sigma}}^{T}\left(N M_{\Omega}\right) d \Gamma & 0 & 0
\end{array}\right]\left\{\begin{array}{l}
s_{\Omega} \\
q_{\Omega} \\
q_{\Gamma_{t}}
\end{array}\right\}=\left\{\begin{array}{c}
e_{\Gamma_{u}} \\
-Q_{\Omega} \\
-Q_{\Gamma_{t}}
\end{array}\right\}
$$

onde:

$$
\begin{aligned}
& e_{\Gamma_{u}}=\int_{\Gamma_{u}}\left(N M_{\Omega}\right)^{T} \bar{u} d \Gamma \\
& Q_{\Omega}=\int_{\Omega} P_{\Omega}^{T} \bar{b} d \Omega
\end{aligned}
$$




$$
Q_{\Gamma_{t}}=\int_{\Gamma_{t}} R_{\Gamma_{t}}^{T} \bar{t} d \Gamma
$$

Como se pode observar, o sistema anterior (eq.(2.16)) não apresenta simetria. Tal condição pode ser recuperada impondo-se as seguintes restrições ('Galerkin') sobre o conjunto de funções de aproximação:

$$
\begin{gathered}
M_{\Omega}=S_{\Omega} \\
P_{\Omega}=U_{\Omega} \\
R_{\Gamma_{t}}=U_{\Gamma_{t}}
\end{gathered}
$$

O sistema simétrico apresenta-se com a seguinte notação compacta:

$$
\left[\begin{array}{ccc}
F & A_{\Omega} & -A_{\Gamma_{t}} \\
A_{\Omega}^{T} & 0 & 0 \\
-A_{\Gamma_{t}}^{T} & 0 & 0
\end{array}\right]\left\{\begin{array}{l}
s_{\Omega} \\
q_{\Omega} \\
q_{\Gamma_{t}}
\end{array}\right\}=\left\{\begin{array}{c}
e_{\Gamma_{u}} \\
-Q_{\Omega} \\
-Q_{\Gamma_{t}}
\end{array}\right\}
$$

onde:

$$
\begin{aligned}
& F=\int_{\Omega} S_{\Omega}^{T} f S_{\Omega} d \Omega \\
& A_{\Omega}=\int_{\Omega}\left(L S_{\Omega}\right)^{T} U_{\Omega} d \Omega \\
& A_{\Gamma_{t}}=\int_{\Gamma_{t}}\left(N S_{\Omega}\right)^{T} U_{\Gamma_{t}} d \Gamma \\
& e_{\Gamma_{u}}=\int_{\Gamma_{u}}\left(N S_{\Omega}\right)^{T} \bar{u} d \Gamma \\
& Q_{\Omega}=\int_{\Omega} U_{\Omega}^{T} \bar{b} d \Omega
\end{aligned}
$$




$$
Q_{\Gamma_{t}}=\int_{\Gamma_{t}} U_{\Gamma_{t}}^{T} \bar{t} d \Gamma
$$

Ao se introduzir a discretização do volume $\boldsymbol{\Omega}$ em elementos, dentro de cada um deles definem-se aproximações para os campos independentes de tensão e deslocamento. Entretanto, aparece uma nova parte do contorno que não foi caracterizada na formalização anterior: trata-se do contorno $\boldsymbol{\Gamma}_{i}$ (entre elementos), e seus campos de deslocamentos e forças precisam ser 'compatibilizados’ com os campos internos dos elementos adjacentes. Assim sendo, individualizam-se novas incógnitas que podem ser aproximadas como campos independentes em $\boldsymbol{\Gamma}_{\boldsymbol{i}}$.

Em geral, as variáveis 'discretizáveis' para o elemento de volume $\boldsymbol{\Omega}$ e contornos $\boldsymbol{\Gamma}_{\boldsymbol{t}}$, $\Gamma_{u}$ e $\boldsymbol{\Gamma}_{i}$, indicado na figura 2.2, são:

$$
\begin{aligned}
& \boldsymbol{S}=\boldsymbol{S}_{\Omega} \boldsymbol{s}_{\Omega} \text { em } \boldsymbol{\Omega} \\
& \boldsymbol{u}=\boldsymbol{U}_{\boldsymbol{\Omega}} \boldsymbol{q}_{\boldsymbol{\Omega}} \text { em } \boldsymbol{\Omega} \\
& \boldsymbol{u}_{\Gamma_{t}}=\boldsymbol{U}_{\boldsymbol{\Gamma}_{t}} \boldsymbol{q}_{\Gamma_{t}} \text { em } \boldsymbol{\Gamma}_{\boldsymbol{t}} \\
& \boldsymbol{t}_{\boldsymbol{\Gamma}_{u}}=\boldsymbol{W}_{\Gamma_{u}} \boldsymbol{o}_{\Gamma_{u}} \text { em } \boldsymbol{\Gamma}_{\boldsymbol{u}} \\
& \boldsymbol{u}_{\boldsymbol{\Gamma}_{i}}=\boldsymbol{U}_{\boldsymbol{\Gamma}_{i}} \boldsymbol{q}_{\Gamma_{i}} \text { em } \boldsymbol{\Gamma}_{\boldsymbol{i}} \\
& \boldsymbol{t}_{\boldsymbol{\Gamma}_{i}}=\boldsymbol{W}_{\boldsymbol{\Gamma}_{i}} \boldsymbol{o}_{\Gamma_{i}} \text { em } \boldsymbol{\Gamma}_{\boldsymbol{i}}
\end{aligned}
$$

onde $\Gamma_{i}$ é a fronteira entre elementos. 


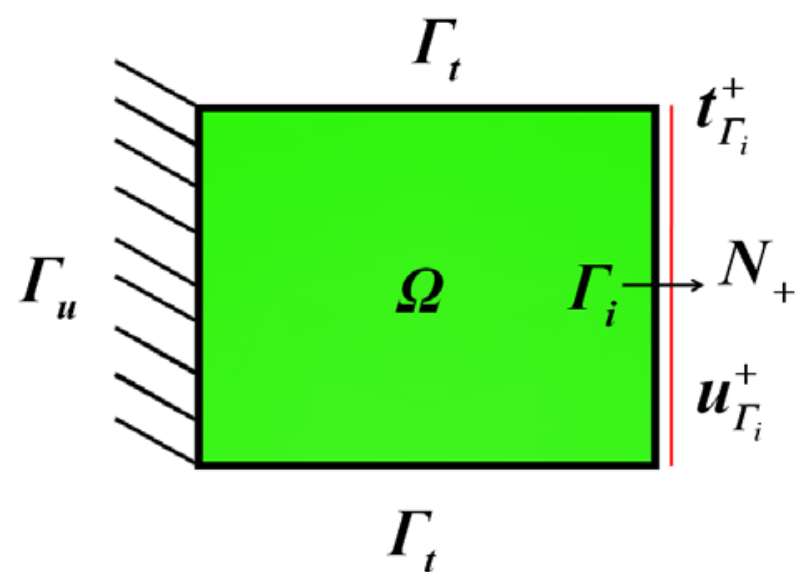

Figura 2.2 - Elemento de volume $\boldsymbol{\Omega}$ e contornos $\boldsymbol{\Gamma}_{t}, \boldsymbol{\Gamma}_{u}$ e $\boldsymbol{\Gamma}_{\boldsymbol{i}}$.

Em razão da existência de $\boldsymbol{\Gamma}_{\boldsymbol{i}}$ no elemento, as condições de compatibilidade e equilíbrio são então estendidas de modo a incluir os novos campos e condições sobre esse contorno. Resumidamente, a relação (2.12) passa a incluir uma nova parcela integral no contorno $\boldsymbol{\Gamma}_{\boldsymbol{i}}$ e uma nova equação é introduzida, exprimindo a forma fraca do equilíbrio entre forças nesse contorno e tensões no domínio do elemento (adiante, retomam-se essas considerações mais detalhadamente). O sistema resolvente para um elemento fica, então, dado por:

$$
\left[\begin{array}{cccc}
F & A_{\Omega} & -A_{\Gamma_{t}} & -A_{\Gamma_{i}} \\
A_{\Omega}^{T} & 0 & 0 & 0 \\
-A_{\Gamma_{t}}^{T} & 0 & 0 & 0 \\
-A_{\Gamma_{i}}^{T} & 0 & 0 & 0
\end{array}\right]\left\{\begin{array}{c}
s_{\Omega} \\
q_{\Omega} \\
q_{\Gamma_{t}} \\
q_{\Gamma_{i}}^{+}
\end{array}\right\}=\left\{\begin{array}{c}
e_{\Gamma_{u}} \\
-Q_{\Omega} \\
-Q_{\Gamma_{t}} \\
-Q_{\Gamma_{i}}^{+}
\end{array}\right\}
$$

Valendo as definições das eq.(2.24) a eq.(2.29) e ainda

$$
\begin{aligned}
& A_{\Gamma_{i}}=\int_{\Gamma_{i}}\left(N S_{\Omega}\right)^{T} U_{\Gamma_{i}} d \Gamma \\
& Q_{\Gamma_{i}}^{+}=\int_{\Gamma_{i}} U_{\Gamma_{i}}^{T} t_{\Gamma_{i}}^{+} d \Gamma
\end{aligned}
$$

Nota-se na equação de equilíbrio em $\boldsymbol{\Gamma}_{\boldsymbol{i}}$ que $\boldsymbol{t}_{\boldsymbol{\Gamma}_{\boldsymbol{i}}}^{+}$também é desconhecida. Naturalmente o sistema descrito pode se apresentar com características particulares. Por exemplo, a parte de contorno $\boldsymbol{\Gamma}_{\boldsymbol{i}}$ simplesmente não existe numa discretização com um único 
elemento. Por outro lado, em se tratando de elemento interior, as partes $\boldsymbol{\Gamma}_{\boldsymbol{t}}$ e $\boldsymbol{\Gamma}_{\boldsymbol{u}}$ não existem. Nesses casos, os sistemas dos elementos correspondentes apresentam matrizes com diferentes ordens e diferente número de variáveis.

Ao considerar um mosaico de elementos, a sua união deve contemplar as condições de continuidade de deslocamentos e de equilíbrio de forças nos contornos internos entre elementos. Entretanto, com o objetivo de reduzir o número de incógnitas associado ao mosaico de elementos, é usual a imposição em forma forte de uma dessas condições, impondo-se a outra em forma fraca.

A condição sobre os deslocamentos é dita de continuidade, sendo expressa em forma forte em $\boldsymbol{\Gamma}_{\boldsymbol{i}}$ comum por: $\boldsymbol{u}_{\boldsymbol{\Gamma}_{i}}^{+}=\boldsymbol{u}_{\boldsymbol{\Gamma}_{i}}^{-}$. A condição sobre as forças é dita de reciprocidade, sendo expressa no contorno comum por: $\boldsymbol{t}_{\boldsymbol{\Gamma}_{i}}^{+}=-\boldsymbol{t}_{\boldsymbol{\Gamma}_{i}}^{-}$. De acordo com a hipótese que venha a ser admitida em $\boldsymbol{\Gamma}_{\boldsymbol{i}}$, a outra deverá ser imposta em forma fraca, passando esta fronteira a ser entendida como um contorno de Neumann ou de Dirichlet, isto é, estático ou cinemático, respectivamente.

Para entender melhor as hipóteses de compatibilização a serem adotadas entre elementos, é importante analisar, inicialmente, a composição e o significado de cada uma das equações do sistema apresentado para um único elemento, ver eq.(2.36).

Em primeiro lugar, vale lembrar que as relações indicadas decorrem de ponderações sobre as equações de compatibilidade e equilíbrio no domínio, e equilíbrio nas partes $\boldsymbol{\Gamma}_{\boldsymbol{t}}$ e $\boldsymbol{\Gamma}_{\boldsymbol{i}}$ do contorno. A condição de contorno em deslocamentos dos pontos pertencentes à $\boldsymbol{\Gamma}_{\boldsymbol{u}}$ é admitida como satisfeita em forma forte e, portanto, não há ponderação a ela associada.

A primeira equação representada na eq.(2.36) tem origem na integração por partes da condição de compatibilidade entre deslocamentos e deformações do elemento, o que acaba por inserir também a compatibilização com os campos de deslocamentos nas partes $\boldsymbol{\Gamma}_{\boldsymbol{t}}$ e $\boldsymbol{\Gamma}_{\boldsymbol{i}}$ do contorno. Levando-se em conta, por um lado, que os campos de deslocamentos no domínio e no contorno são representados por aproximações independentes e, por outro, que o contorno se divide em regiões distintas, tal integração fornece:

$$
\int_{\Omega} S_{\Omega}^{T} f \sigma d \Omega=-\int_{\Omega}\left(L S_{\Omega}\right)^{T} u d \Omega+\int_{\Gamma_{t}}\left(N S_{\Omega}\right)^{T} u_{\Gamma_{t}} d \Gamma+\int_{\Gamma_{i}}\left(N_{+} S_{\Omega}\right)^{T} u_{\Gamma_{i}} d \Gamma+\int_{\Gamma_{u}}\left(N S_{\Omega}\right)^{T} \bar{u} d \Gamma
$$


Dessa condição, introduzindo-se as aproximações para as tensões (eq.(2.30)) e deslocamentos (eq.(2.30)) no domínio e deslocamentos nos contornos $\boldsymbol{\Gamma}_{\boldsymbol{t}}$ (eq.(2.32)) e $\boldsymbol{\Gamma}_{\boldsymbol{i}}$ (eq.(2.34)), resulta a primeira equação do sistema da eq.(2.36):

$$
F s_{\Omega}+A_{\Omega} q_{\Omega}-A_{\Gamma_{t}} q_{\Gamma_{t}}-A_{\Gamma_{i}} q_{\Gamma_{i}}^{+}=e_{\Gamma_{u}}
$$

com as definições pontuadas nas eq.(2.24) a eq.(2.27) e eq.(2.37).

Outra relação que aparece na eq.(2.36) é a que exprime o equilíbrio entre forças na fronteira $\boldsymbol{\Gamma}_{\boldsymbol{i}}$ e tensões no domínio do elemento:

$$
\int_{\Gamma}\left(U_{\Gamma_{i}}\right)^{T}\left(N_{+} \sigma-t_{\Gamma_{i}}^{+}\right) d \Gamma=0
$$

Substituindo-se nessa condição a aproximação para o campo de tensões no domínio (eq.(2.30)), resulta a quarta equação do sistema do elemento representada na eq.(2.36):

$$
A_{\Gamma_{i}}^{T} s_{\Omega}-Q_{\Gamma_{i}}^{+}=0
$$

As outras equações (segunda e terceira da eq.(2.36)) referem-se ao equilíbrio no volume e à condição de Neumann no contorno $\boldsymbol{\Gamma}_{t}$, sendo de interpretação mais simples.

É importante observar, neste ponto, que o sistema da eq.(2.36) indicado para um elemento apresenta 4 equações e 5 incógnitas (a 5 incógnita é a $\boldsymbol{t}_{\boldsymbol{\Gamma}_{i}}^{+}$).

Considere-se, agora, o exemplo de dois elementos, indicado na figura 2.3. Se nenhuma hipótese de equilíbrio ('reciprocidade') ou de continuidade na fronteira comum é adotada 'a priori', as seguintes aproximações são válidas no contorno $\boldsymbol{\Gamma}_{\boldsymbol{i}}$ de cada elemento: $\boldsymbol{u}_{\boldsymbol{\Gamma}_{i}}^{+}=\boldsymbol{U}_{\boldsymbol{\Gamma}_{i}}^{+} \boldsymbol{q}_{\boldsymbol{\Gamma}_{\boldsymbol{i}}}^{+}$, $\boldsymbol{t}_{\boldsymbol{\Gamma}_{i}}^{+}=\boldsymbol{W}_{\boldsymbol{\Gamma}_{i}}^{+} \boldsymbol{o}_{\Gamma_{i}}^{+}, \boldsymbol{u}_{\boldsymbol{\Gamma}_{i}}^{-}=\boldsymbol{U}_{\boldsymbol{\Gamma}_{i}}^{-} \boldsymbol{q}_{\Gamma_{i}}^{-}$e $\boldsymbol{t}_{\boldsymbol{\Gamma}_{i}}^{-}=\boldsymbol{W}_{\boldsymbol{\Gamma}_{i}}^{-} \boldsymbol{o}_{\boldsymbol{\Gamma}_{i}}^{-}$. Assim sendo, nesta situação geral, $\boldsymbol{q}_{\boldsymbol{\Gamma}_{i}}^{+}, \boldsymbol{q}_{\boldsymbol{\Gamma}_{i}}^{-}$, $\boldsymbol{o}_{\boldsymbol{\Gamma}_{i}}^{+}$e $\boldsymbol{o}_{\boldsymbol{\Gamma}_{i}}^{-}$ são incógnitas adicionais a determinar. Dada à independência adotada para os campos envolvidos resulta, ao considerar o sistema de equações dos dois elementos, um total de 8 equações e 10 incógnitas envolvidas. As duas equações adicionais para tornar o sistema determinado são, justamente, de continuidade e reciprocidade na fronteira comum. 


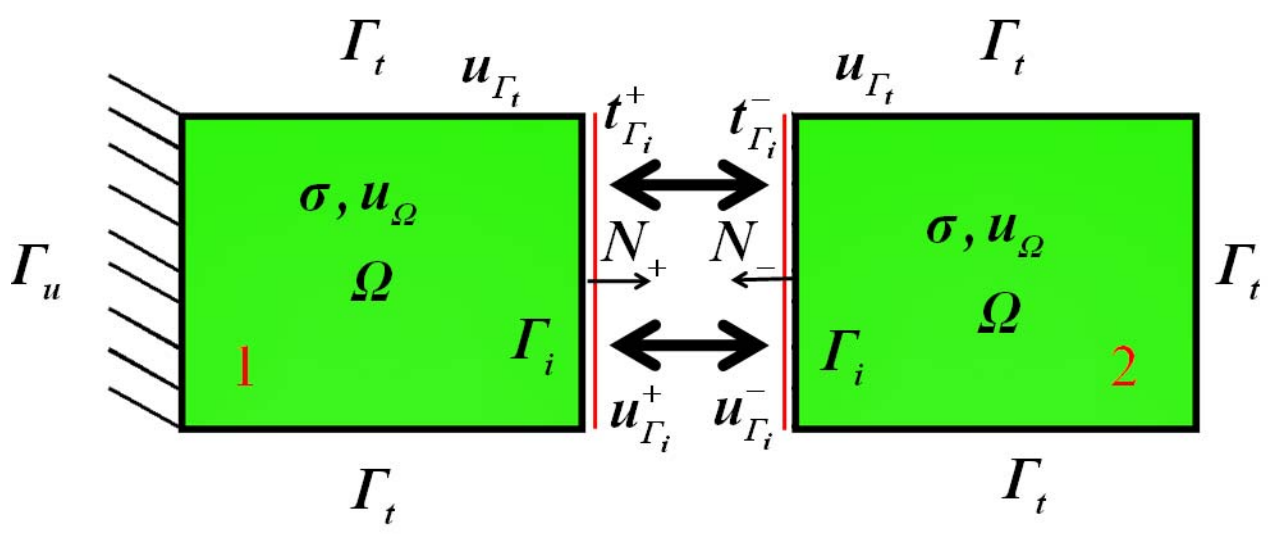

Figura 2.3 - Dois elementos de volume $\boldsymbol{\Omega}$ e contornos $\boldsymbol{\Gamma}_{t}, \boldsymbol{\Gamma}_{u}$ e $\boldsymbol{\Gamma}_{i}$.

A imposição em forma forte de uma das condições, de continuidade ou de equilíbrio, permite substituir na fronteira $\boldsymbol{\Gamma}_{\boldsymbol{i}}$ as duas incógnitas, em deslocamentos ou forças, respectivamente, por uma incógnita comum. O sistema resolvente passa a apresentar, então, 9 incógnitas e 9 equações, sendo montado primeiramente com as condições sobre cada elemento referentes à compatibilidade e equilíbrio no volume e equilíbrio em $\boldsymbol{\Gamma}_{\boldsymbol{t}}$ e $\boldsymbol{\Gamma}_{\boldsymbol{i}}$, dispostas em seqüência, sem sobreposição. Há, ainda, uma equação adicional relativa à forma fraca da outra condição, de reciprocidade ou de continuidade, envolvendo as incógnitas em forças ou deslocamentos, respectivamente, no contorno comum $\boldsymbol{\Gamma}_{\boldsymbol{i}}$.

Seja, então, uma primeira hipótese que consiste em impor que na fronteira comum $\boldsymbol{\Gamma}_{\boldsymbol{i}}$ haja 'a priori' reciprocidade de forças incógnitas entre elementos, isto é: $\boldsymbol{t}_{\boldsymbol{\Gamma}_{i}}^{+}=-\boldsymbol{t}_{\boldsymbol{\Gamma}_{i}}^{-}=\boldsymbol{t}=\boldsymbol{W}_{\boldsymbol{\Gamma}_{i}} \boldsymbol{o}_{\Gamma_{i}}$. Segue que a outra condição, de continuidade nos deslocamentos, passa a ser imposta em forma ponderada. Nesta situação $\boldsymbol{\Gamma}_{\boldsymbol{i}}$ pode ser interpretado como um contorno cinemático ou de Dirichlet. O número de incógnitas em $\boldsymbol{\Gamma}_{\boldsymbol{i}}$ se reduz, portanto, a: $\boldsymbol{q}_{\Gamma_{i}}^{+}, \boldsymbol{q}_{\Gamma_{i}}^{-}$e $\boldsymbol{o}_{\Gamma_{i}}$

No que segue apresenta-se a configuração do sistema de acordo com as condições implícitas na hipótese adotada. Inicialmente, a condição de reciprocidade das forças na fronteira $\boldsymbol{\Gamma}_{\boldsymbol{i}}$ introduz alguma modificação nas relações referentes ao equilíbrio na fronteira comum, abaixo reproduzidas:

$$
A_{\Gamma_{i}}^{+T} s_{\Omega}^{1}=Q_{\Gamma_{i}}^{+}
$$




$$
A_{\Gamma_{i}}^{-T} s_{\Omega}^{2}=Q_{\Gamma_{i}}^{-}
$$

Pela reciprocidade admitida, cada termo correspondente à forma linear, isto é: $\boldsymbol{Q}_{\boldsymbol{\Gamma}_{i}}^{+} \mathrm{e}$ $\boldsymbol{Q}_{\boldsymbol{\Gamma}_{i}}^{-}$, passa a ser escrito como:

$$
\begin{aligned}
& Q_{\Gamma_{i}}^{+}=\int_{\Gamma_{i}^{+}} U_{\Gamma_{i}}^{+^{T}} t d \Gamma \\
& Q_{\Gamma_{i}}^{-}=-\int_{\Gamma_{i}^{-}} U_{\Gamma_{i}}^{-T} t d \Gamma
\end{aligned}
$$

Levando-se em conta a aproximação adotada para a força $\boldsymbol{t}$, essas formas lineares passam a ter novas redações. Portanto, no caso do elemento 1 (figura 2.3), obtém-se:

$$
\begin{aligned}
& Q_{\Gamma_{i}}^{+}=\int_{\Gamma_{i}^{+}} U_{\Gamma_{i}}^{+^{T}} t d \Gamma=\int_{\Gamma_{i}^{+}} U_{\Gamma_{i}}^{+^{T}} W_{\Gamma_{i}} \boldsymbol{o}_{\Gamma_{i}} d \Gamma \\
& Q_{\Gamma_{i}}^{+}=\underbrace{\left[\int_{\Gamma_{i}^{+}} U_{\Gamma_{i}}^{+^{T}} \boldsymbol{W}_{\Gamma_{i}} d \Gamma\right]}_{{C_{\Gamma_{i}}^{T}}^{T}} \boldsymbol{o}_{\Gamma_{i}}=C_{\Gamma_{i}}^{+^{T}} \boldsymbol{o}_{\Gamma_{i}}
\end{aligned}
$$

No caso do elemento 2 (figura 2.3), analogamente, resulta:

$$
\begin{gathered}
Q_{\Gamma_{i}}^{-}=-\int_{\Gamma_{i}^{-}} U_{\Gamma_{i}}^{-T} t d \Gamma=-\int_{\Gamma_{i}^{-}} U_{\Gamma_{i}}^{-T} W_{\Gamma_{i}} o_{\Gamma_{i}} d \Gamma \\
Q_{\Gamma_{i}}^{-}=\underbrace{\left[\int_{\Gamma_{i}^{-}} U_{\Gamma_{i}}^{-T} W_{\Gamma_{i}} d \Gamma\right]}_{C_{\Gamma_{i}}^{T}} o_{\Gamma_{i}}=C_{\Gamma_{i}}^{-^{T}} o_{\Gamma_{i}}
\end{gathered}
$$

Assim sendo, as relações de equilíbrio na fronteira comum $\boldsymbol{\Gamma}_{\boldsymbol{i}}$ passam a ser escritas para o elemento 1 como:

$$
A_{\Gamma_{i}}^{+^{T}} s_{\Omega}^{1}=Q_{\Gamma_{i}}^{T} \rightarrow A_{\Gamma_{i}}^{+^{T}} s_{\Omega}^{1}=C_{\Gamma_{i}}^{+^{T}} o_{\Gamma_{i}}
$$


ou

$$
A_{\Gamma_{i}}^{+T} s_{\Omega}^{1}-C_{\Gamma_{i}}^{+^{T}} \boldsymbol{o}_{\Gamma_{i}}=0
$$

E para o elemento 2:

$$
A_{\Gamma_{i}}^{-T} s_{\Omega}^{1}+C_{\Gamma_{i}}^{-T} o_{\Gamma_{i}}=0
$$

Em relação à representação da continuidade dos deslocamentos em $\boldsymbol{\Gamma}_{\boldsymbol{i}}$, em forma fraca, tal condição pode ser imposta tomando-se forças virtuais como ponderadoras, sendo, então, expressa como:

$$
\int_{\Gamma_{i}} \delta t^{T}\left(u_{\Gamma_{i}}^{+}-u_{\Gamma_{i}}^{-}\right) d \Gamma=0
$$

Considerando-se que as forças virtuais possam ser escritas como: $\delta \boldsymbol{t}=\boldsymbol{W}_{\boldsymbol{\Gamma}_{i}} \boldsymbol{\delta} \boldsymbol{o}_{\Gamma_{i}}$, a condição anterior passa a:

$$
\begin{aligned}
& \delta o_{\Gamma_{i}}^{T} \int_{\Gamma_{i}} W_{\Gamma_{i}}^{T}\left(u_{\Gamma_{i}}^{+}-u_{\Gamma_{i}}^{-}\right) d \Gamma=0 \\
& \int_{\Gamma_{i}} W_{\Gamma_{i}}^{T} u_{\Gamma_{i}}^{+} d \Gamma-\int_{\Gamma_{i}} W_{\Gamma_{i}}^{T} u_{\Gamma_{i}}^{-} d \Gamma=0 \\
& {\left[\int_{\Gamma_{i}} W_{\Gamma_{i}}^{T} U_{\Gamma_{i}}^{+} d \Gamma\right] q_{\Gamma_{i}}^{+}-\left[\int_{\Gamma_{i}} W_{\Gamma_{i}}^{T} U_{\Gamma_{i}}^{-} d \Gamma\right] q_{\Gamma_{i}}^{-}=0} \\
& C_{\Gamma_{i}}^{+} q_{\Gamma_{i}}^{+}-C_{\Gamma_{i}}^{-} q_{\Gamma_{i}}^{-}=0
\end{aligned}
$$

Portanto, de acordo com essa hipótese de compatibilização (em deslocamentos), o sistema resolvente para dois elementos fica composto pelas contribuições das relações de compatibilidade e equilíbrio no volume e equilíbrios nos contornos $\boldsymbol{\Gamma}_{t}$ e $\boldsymbol{\Gamma}_{\boldsymbol{i}}$, para cada elemento, mais a relação de continuidade dos deslocamentos na fronteira comum $\boldsymbol{\Gamma}_{\boldsymbol{i}}$.

Tal sistema apresenta-se na forma: 


$$
\left[\begin{array}{ccccccccc}
\boldsymbol{F}^{1} & A_{\Omega}^{1} & -A_{\Gamma_{t}}^{1} & -A_{\Gamma_{t}}^{+} & 0 & 0 & 0 & 0 & 0 \\
A_{\Omega}^{I^{T}} & 0 & 0 & 0 & 0 & 0 & 0 & 0 & 0 \\
-A_{\Gamma_{t}}^{1^{T}} & 0 & 0 & 0 & 0 & 0 & 0 & 0 & 0 \\
-A_{\Gamma_{t}}^{+^{T}} & 0 & 0 & 0 & 0 & 0 & 0 & 0 & C_{\Gamma_{i}}^{+^{T}} \\
0 & 0 & 0 & 0 & F^{2} & A_{\Omega}^{2} & -A_{\Gamma_{t}}^{2} & -A_{\Gamma_{t}}^{-} & 0 \\
0 & 0 & 0 & 0 & A_{\Omega}^{2^{T}} & 0 & 0 & 0 & 0 \\
0 & 0 & 0 & 0 & -A_{\Gamma_{t}}^{2^{T}} & 0 & 0 & 0 & 0 \\
0 & 0 & 0 & 0 & -A_{\Gamma_{t}}^{-T} & 0 & 0 & 0 & C_{\Gamma_{i}}^{-T} \\
0 & 0 & 0 & C_{\Gamma_{i}}^{+} & 0 & 0 & 0 & C_{\Gamma_{i}}^{-} & 0
\end{array}\right]\left\{\begin{array}{c}
s_{\Omega}^{1} \\
\boldsymbol{q}_{\Omega}^{1} \\
\boldsymbol{q}_{\Gamma_{t}}^{1} \\
\boldsymbol{q}_{\Gamma_{i}}^{+} \\
\boldsymbol{s}_{\Omega}^{2} \\
\boldsymbol{q}_{\Omega}^{2} \\
\boldsymbol{q}_{\Gamma_{t}}^{2} \\
\boldsymbol{q}_{\Gamma_{i}} \\
\boldsymbol{o}_{\Gamma_{i}}
\end{array}\right\}=\left\{\begin{array}{c}
\boldsymbol{e}_{\Gamma_{u}}^{1} \\
-Q_{\Omega}^{1} \\
-Q_{\Gamma_{t}}^{1} \\
0 \\
\boldsymbol{c}_{\Gamma_{u}}^{2} \\
-Q_{\Omega}^{2} \\
-Q_{\Gamma_{t}}^{2} \\
0 \\
0
\end{array}\right\}
$$

onde $\boldsymbol{e}_{\Gamma_{u}}^{1}$ e $\boldsymbol{e}_{\Gamma_{u}}^{2}$ existem somente se os respectivos elementos possuírem partes de seus contornos contidas no contorno global de Dirichlet e com restrições não-nulas nos deslocamentos.

A segunda hipótese consiste em impor 'a priori' a continuidade de deslocamentos na fronteira comum, valendo: $\boldsymbol{u}_{\boldsymbol{\Gamma}_{i}}^{+}=\boldsymbol{u}_{\boldsymbol{\Gamma}_{i}}^{-}=\boldsymbol{u}_{\boldsymbol{\Gamma}_{i}}=\boldsymbol{U}_{\boldsymbol{\Gamma}_{\boldsymbol{i}}} \boldsymbol{q}_{\boldsymbol{\Gamma}_{i}}$. A reciprocidade de forças passa a ser imposta em forma ponderada. Portanto, nessa hipótese $\boldsymbol{\Gamma}_{\boldsymbol{i}}$ pode ser entendida como uma fronteira de Neumann.

Em razão da continuidade de deslocamentos, a primeira equação de cada elemento (figura 2.3) tem seu termo associado à fronteira $\boldsymbol{\Gamma}_{\boldsymbol{i}}$ sofrendo uma pequena modificação, uma vez que as variáveis da aproximação para o campo de deslocamentos na fronteira comum entre elementos não mais se distinguem.

Assim sendo, resulta:

$$
\begin{gathered}
\int_{\Gamma_{i}^{+}}\left(N_{+} S_{\Omega}^{l}\right)^{T} u_{\Gamma_{i}^{+}}^{+} d \Gamma=\underbrace{\left[\int_{\Gamma_{i}^{+}}\left(N_{+} S_{\Omega}^{l}\right)^{T} U_{\Gamma_{i}} d \Gamma\right]}_{A_{\Gamma_{i}}^{+}} q_{\Gamma_{i}}=A_{\Gamma_{i}}^{+} q_{\Gamma_{i}} \\
\int_{\Gamma_{i}^{-}}\left(N_{-} S_{\Omega}^{2}\right)^{T} u_{\Gamma_{i}^{-}}^{-} d \Gamma=-\underbrace{\left[\int_{\Gamma_{i}^{-}}\left(N_{-} S_{\Omega}^{2}\right)^{T} U_{\Gamma_{i}} d \Gamma\right]}_{A_{\Gamma_{i}}^{-}} q_{\Gamma_{i}}=-A_{\Gamma_{i}}^{-} q_{\Gamma_{i}}
\end{gathered}
$$


A equação adicional, de reciprocidade de forças na fronteira $\boldsymbol{\Gamma}_{\boldsymbol{i}}$, é imposta em forma ponderada. Tal condição escreve-se:

$$
\int_{\Gamma_{i}} \delta u_{\Gamma_{i}}^{T}\left(t_{\Gamma_{i}}^{+}+t_{\Gamma_{i}}^{-}\right) d \Gamma=0
$$

Substituindo-se $\delta \boldsymbol{u}_{\Gamma_{i}}^{T}=\delta \boldsymbol{q}_{\Gamma_{i}}^{T} \boldsymbol{U}_{\Gamma_{i}}^{T}$ resultam, sucessivamente:

$$
\begin{gathered}
\delta q_{\Gamma_{i}}^{T} \int_{\Gamma_{i}} U_{\Gamma_{i}}^{T}\left(t_{\Gamma_{i}}^{+}+t_{\Gamma_{i}}^{-}\right) d \Gamma=0 \\
\int_{\Gamma_{i}} U_{\Gamma_{i}}^{T} t_{\Gamma_{i}}^{+} d \Gamma+\int_{\Gamma_{i}} U_{\Gamma_{i}}^{T} t_{\Gamma_{i}}^{-} d \Gamma=0
\end{gathered}
$$

Uma vantagem desta alternativa é a utilização da condição de equilíbrio no contorno, considerando-se o regime de tensões no interior de cada elemento, como forma de redução do número de incógnitas. Assim, introduzindo-se a condição de equilíbrio no contorno $\boldsymbol{\Gamma}_{\boldsymbol{i}}$ comum a cada um dos elementos, segue que:

$$
\begin{aligned}
& \int_{\Gamma_{i}} U_{\Gamma_{i}}^{T} N_{+} \sigma_{\Omega}^{1} d \Gamma+\int_{\Gamma_{i}} U_{\Gamma_{i}}^{T} N_{-} \sigma_{\Omega}^{2} d \Gamma=0 \\
& \underbrace{\left[\int_{\Gamma_{i}} U_{\Gamma_{i}}^{T} N_{+} S_{\Omega}^{1} d \Gamma\right]}_{A_{\Gamma_{i}}^{T}} s_{\Omega}^{1}+\underbrace{\left[\int_{\Gamma_{i}} U_{\Gamma_{i}}^{T} N_{-} S_{\Omega}^{2} d \Gamma\right]}_{A_{\Gamma_{i}}^{T}} s_{\Omega}^{2}=0 \\
& A_{\Gamma_{i}^{+}}^{+^{T}} s_{\Omega}^{1}+A_{\Gamma_{i}}^{\sigma^{T}} s_{\Omega}^{2}=0
\end{aligned}
$$

Portanto, de acordo com essa hipótese, o sistema resolvente fica composto pelas contribuições das relações de compatibilidade e equilíbrio no volume e equilíbrio no contorno $\boldsymbol{\Gamma}_{t}$, de cada elemento, mais as relações de reciprocidade de forças na fronteira comum $\boldsymbol{\Gamma}_{\boldsymbol{i}}$. Tal sistema apresenta-se na forma: 


$$
\left[\begin{array}{ccccccc}
\boldsymbol{F}^{1} & A_{\Omega}^{1} & -A_{\Gamma_{t}}^{1} & 0 & 0 & 0 & -A_{\Gamma_{i}}^{+} \\
A_{\Omega}^{I^{T}} & 0 & 0 & 0 & 0 & 0 & 0 \\
-A_{\Gamma_{t}}^{I^{T}} & 0 & 0 & 0 & 0 & 0 & 0 \\
0 & 0 & 0 & F^{2} & A_{\Omega}^{2} & -A_{\Gamma_{t}}^{2} & -A_{\Gamma_{i}}^{-} \\
0 & 0 & 0 & A_{\Omega}^{2^{T}} & 0 & 0 & 0 \\
0 & 0 & 0 & -A_{\Gamma_{t}}^{2^{T}} & 0 & 0 & 0 \\
-A_{\Gamma_{i}}^{+T} & 0 & 0 & -A_{\Gamma_{i}}^{-T} & 0 & 0 & 0
\end{array}\right]\left\{\begin{array}{c}
s_{\Omega}^{1} \\
q_{\Omega}^{1} \\
\boldsymbol{q}_{\Gamma_{t}}^{1} \\
s_{\Omega}^{2} \\
\boldsymbol{q}_{\Omega}^{2} \\
\boldsymbol{q}_{\Gamma_{t}}^{2} \\
q_{\Gamma_{i}}
\end{array}\right\}=\left\{\begin{array}{c}
\boldsymbol{e}_{\Gamma_{u}}^{1} \\
-Q_{\Omega}^{1} \\
-Q_{\Gamma_{t}}^{1} \\
\boldsymbol{e}_{\Gamma_{u}}^{2} \\
-Q_{\Omega}^{2} \\
-Q_{\Gamma_{t}}^{2} \\
0
\end{array}\right\}
$$

Nota-se, mais uma vez, que de acordo com a hipótese adotada, a fronteira $\boldsymbol{\Gamma}_{\boldsymbol{i}}$ em cada elemento passa a ser entendida como um contorno de Dirichlet (cinemático) ou de Neumann (estático). Na primeira hipótese considerada, ao se admitir 'a priori' a reciprocidade de forças entre elementos, $\boldsymbol{\Gamma}_{\boldsymbol{i}}$ passou a ser interpretado como um contorno cinemático. Porque a equação adicional foi de continuidade em deslocamento, a formulação resultante é dita Híbrido-Mista de Deslocamento.

Continuidade em forma fraca

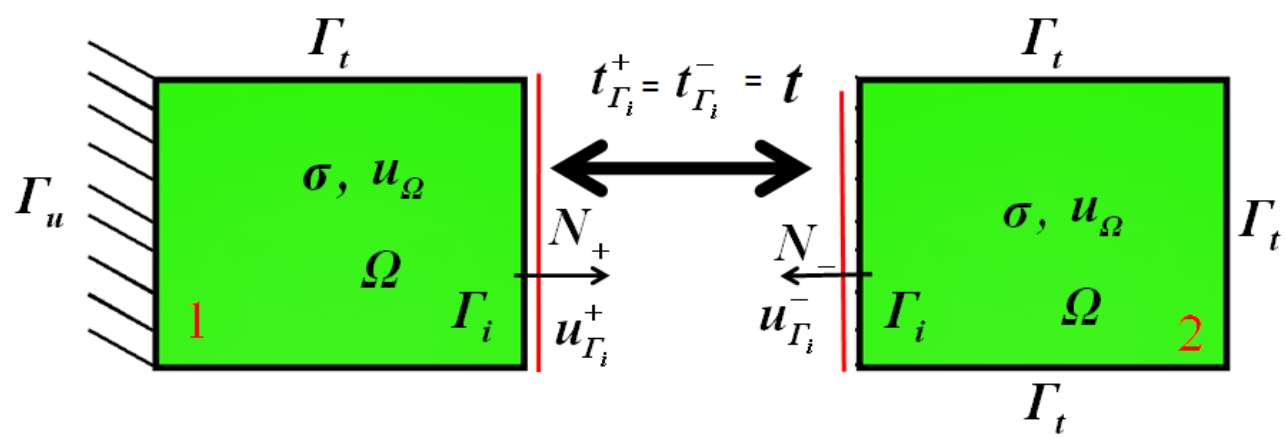

Reciprocidade em forma fraca

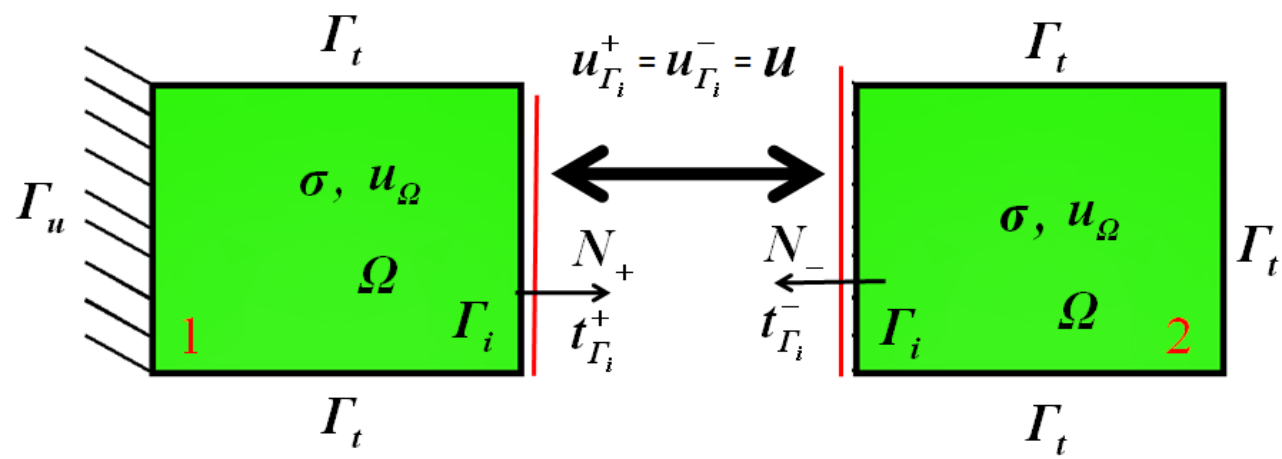

Figura 2.4 - Dois elementos de volume $\boldsymbol{\Omega}$ e contornos $\boldsymbol{\Gamma}_{t}, \boldsymbol{\Gamma}_{u}$ e $\boldsymbol{\Gamma}_{\boldsymbol{i}}$ - continuidade e reciprocidade em forma fraca. 
$\mathrm{Na}$ segunda hipótese, onde se admitiu a continuidade de deslocamentos entre elementos, a interpretação para $\boldsymbol{\Gamma}_{\boldsymbol{i}}$ passou a ser de um contorno estático. Porque a equação adicional foi de reciprocidade de forças, a formulação resultante é dita Híbrido-Mista de Tensão (FHMT), uma das formulações estudas neste trabalho.

\subsection{Formulações Híbridas para a Elasticidade Plana}

Nas formulações híbridas as aproximações para os campos de tensão são autoequilibradas no domínio ou volume $\boldsymbol{\Omega}$, de modo que a equação de equilíbrio correspondente é identicamente satisfeita na hipótese de forças volúmicas nulas:

$$
L S_{\Omega}=0
$$

Nessa condição, a matriz $\boldsymbol{A}_{\boldsymbol{\Omega}}$ para todos os elementos se anula e os sistemas exemplificados para as formulações híbrido-mistas de deslocamento (eq.(2.59)) e híbridomistas de tensão (eq.(2.68)) se simplificam apresentando respectivamente as seguintes formas:

$$
\begin{aligned}
& {\left[\begin{array}{ccccccc}
F^{1} & -A_{\Gamma_{t}}^{1} & -A_{\Gamma_{i}}^{+} & 0 & 0 & 0 & 0 \\
-A_{\Gamma_{t}}^{I^{T}} & 0 & 0 & 0 & 0 & 0 & 0 \\
-A_{\Gamma_{i}}^{+^{T}} & 0 & 0 & 0 & 0 & 0 & C_{\Gamma_{i}}^{+^{T}} \\
0 & 0 & 0 & F^{2} & -A_{\Gamma_{t}}^{2} & -A_{\Gamma_{i}}^{-} & 0 \\
0 & 0 & 0 & -A_{\Gamma_{t}}^{2^{T}} & 0 & 0 & 0 \\
0 & 0 & 0 & -A_{\Gamma_{i}}^{-T} & 0 & 0 & -C_{\Gamma_{i}}^{-^{T}} \\
0 & 0 & 0 & 0 & 0 & -C_{\Gamma_{i}}^{-} & 0
\end{array}\right]\left\{\begin{array}{c}
s_{\Omega}^{1} \\
\boldsymbol{q}_{\Gamma_{t}}^{1} \\
\boldsymbol{q}_{\Gamma_{i}}^{+} \\
\boldsymbol{s}_{\Omega}^{2} \\
\boldsymbol{q}_{\Gamma_{t}}^{2} \\
\boldsymbol{q}_{\Gamma_{i}}^{-} \\
\boldsymbol{o}_{\Gamma_{i}}
\end{array}\right\}=\left\{\begin{array}{c}
\boldsymbol{e}_{\Gamma_{u}}^{1} \\
-Q_{\Gamma_{t}}^{1} \\
0 \\
\boldsymbol{e}_{\Gamma_{u}}^{2} \\
-Q_{\Gamma_{t}}^{2} \\
0 \\
0
\end{array}\right\}} \\
& {\left[\begin{array}{ccccc}
\boldsymbol{F}^{1} & -A_{\Gamma_{t}}^{1} & 0 & 0 & -A_{\Gamma_{i}}^{+} \\
-A_{\Gamma_{t}}^{I^{T}} & 0 & 0 & 0 & 0 \\
0 & 0 & F^{2} & -A_{\Gamma_{t}}^{2} & -A_{\Gamma_{i}}^{-} \\
0 & 0 & -A_{\Gamma_{t}}^{2^{T}} & 0 & 0 \\
-A_{\Gamma_{i}}^{+^{T}} & 0 & -A_{\Gamma_{i}}^{-T} & 0 & 0
\end{array}\right]\left\{\begin{array}{c}
s_{\Omega}^{1} \\
q_{\Gamma_{t}}^{1} \\
s_{\Omega}^{2} \\
q_{\Gamma_{t}}^{2} \\
\boldsymbol{q}_{\Gamma_{i}}
\end{array}\right\}=\left\{\begin{array}{c}
\boldsymbol{e}_{\Gamma_{u}}^{1} \\
-Q_{\Gamma_{t}}^{1} \\
\boldsymbol{e}_{\Gamma_{u}}^{2} \\
-Q_{\Gamma_{t}}^{2} \\
0
\end{array}\right\}}
\end{aligned}
$$


Definem-se, assim, formulações ditas: híbrida de deslocamento e híbrida de tensão (FHT). Como se observa em particular na forma híbrida de tensão (FHT- adotada neste trabalho) restam como incógnitas: tensões no volume e deslocamentos no contorno $\boldsymbol{\Gamma}_{\boldsymbol{t}}$. 


\section{FHT e FHMT com Enriquecimento Nodal - Método dos Elementos Finitos Generalizados (MEFG)}

\subsection{O Método dos Elementos Finitos Generalizados (MEFG)}

Não é objetivo deste trabalho apresentar por completo a fundamentação teórica dos métodos sem malha e do Método dos Elementos Finitos Generalizados (MEFG). Foca-se essencialmente a aplicação da técnica de enriquecimento nodal, originária do Método das Nuvens “hp” (Duarte (1996), Duarte e Oden (1995, 1996)), na FHT e FHMT. Dessa forma, recomenda-se a leitura de trabalhos como: Duarte, Babuška e Oden (2000), Barros (2002), Torres (2003) e Góis (2004) para um melhor entendimento sobre essas formas nãoconvencionais de aproximação numérica do PVC.

O MEFG pode ser entendido como uma combinação da forma clássica do Método dos Elementos Finitos (MEF) com técnicas dos métodos sem malha, especificamente a estratégia de enriquecimento da aproximação adotada no Método das Nuvens "hp”. O MEFG surgiu na tentativa de superar alguns entraves dos métodos sem malha, tais como: dificuldades na imposição das condições de contorno na forma fraca do problema e no controle do erro da integração numérica.

Segundo o trabalho de Duarte, Babuška e Oden (2000), o MEFG foi proposto independentemente por:

- Babuška, Caloz e Osborn (1994) sob a denominação de Método dos Elementos Finitos Especiais;

- Melenk e Babuška (1996) e Babuška e Melenk (1997) como Método dos Elementos Finitos Partição Unidade (MEFPU); 
- Duarte (1996), Duarte e Oden $(1995,1996)$ com os trabalhos correspondentes à formulação do Método das Nuvens;

- Oden, Duarte e Zienkiewicz (1998) como uma formulação híbrida entre o Método das Nuvens “hp” e a forma clássica do MEF.

A fundamentação do MEFG vem com os seguintes trabalhos:

- Oden, Duarte e Zienkiewicz (1998): onde foi apresentada a possibilidade de empregar a rede de elementos finitos para a definição dos nós e das nuvens, e realizar o enriquecimento, à maneira do Método das Nuvens “hp”, sobre as funções de forma Lagrangianas usadas no MEF clássico (que constituem uma partição da unidade (PU), se relaxadas algumas das condições que definem uma PU).

- Strouboulis, Babuška e Cops (2000): que apresentam o MEFG como combinação do MEF clássico com o Método dos Elementos Finitos Partição da Unidade, Melenk e Babuška (1996) e Melenk (1992, 1995). A principal característica desse trabalho é a adição de funções solução do problema de valor de contorno estudado ao espaço de funções de enriquecimento da formulação clássica do MEF.

A partir desses trabalhos, as características principais do MEFG ficaram bem definidas, ou seja: utilização da malha de cobertura como domínio de integração, uso das funções de forma Lagrangianas do MEF convencional como PU e seu enriquecimento (utilizando as estratégias do Método das Nuvens “hp”) com funções polinomiais ou não.

Em relação à partição da unidade, classicamente, num ponto $\boldsymbol{x}$ o conjunto de funções de forma $\boldsymbol{\varphi}_{j}(\boldsymbol{x})$, definidas em subdomínios $\boldsymbol{\omega}_{\boldsymbol{j}}$ que contém o ponto, constitui uma PU caso satisfaçam as seguintes condições:

a) $\varphi_{j}(x) \in C_{0}^{\infty}\left(\omega_{j}\right)$;

As funções de forma pertencem ao conjunto de funções para os quais $\omega_{j}$ é um suporte compacto (incluindo-se suas derivadas até a ordem infinita). Isto significa que $\boldsymbol{\varphi}_{j}(\boldsymbol{x})$ e suas derivadas passam a ter valores não-nulos apenas no interior da região $\boldsymbol{\omega}_{j}$. 
b) $\sum_{j=1}^{N} \varphi_{j}(x)=1$;

Esta condição é essencial para assegurar que as funções de forma sejam capazes de reproduzir de modo exato uma função constante e assim garantir a convergência da aproximação à medida que novos pontos nodais sejam acrescentados ao domínio. Assim, admita-se que a função aproximada seja $\boldsymbol{u}(\boldsymbol{x})=\boldsymbol{k}$, de modo que todos os valores nodais sejam $\tilde{\boldsymbol{u}}_{\boldsymbol{j}}=\boldsymbol{k}, \forall \boldsymbol{j}=\mathbf{1}, 2, \ldots, \boldsymbol{N}$. Dessa forma a condição abordada neste item é necessária, pois:

$$
u(x) \approx \hat{u}(x)=\sum_{j=1}^{N} \varphi_{j} k=k \sum_{j=1}^{N} \varphi_{j}, \text { como } \sum_{j=1}^{N} \varphi_{j}=1
$$

então :

$$
u(x) \approx \hat{u}(x)=k
$$

c) $\varphi_{j}(x) \geq 0$ em $\boldsymbol{\Omega}$;

d) Todo sub-conjunto compacto de $\boldsymbol{\Omega}$ intercepta apenas um número finito de suportes $\varphi_{j}(x)$.

As quatro condições acima são bastante restritivas e para fins práticos elas podem ser “relaxadas”. Dessa forma, a primeira condição que exige suporte compacto até a ordem infinita nas derivadas, pode ser relaxada de tal forma que as funções de suporte compacto até uma ordem finita de derivada são ainda consideradas uma PU. Por isso, as funções Lagrangianas amplamente empregadas como funções de forma do MEF clássico podem ser consideradas uma PU.

A figura 3.1 apresenta um esquema de enriquecimento de uma PU bilinear Lagrangiana, atrelada a um nó $\boldsymbol{x}_{\boldsymbol{j}}$. A região de influência do nó $\boldsymbol{x}_{\boldsymbol{j}}$, denominada nuvem ou suporte $\omega_{j}$, é definida pelo conjunto dos 4 elementos finitos quadrilaterais que têm por vértice comum o nó $\boldsymbol{x}_{\boldsymbol{j}}$ em questão.

Assim, a Função Produto ou enriquecida da figura 3.1 é obtida da multiplicação da PU por uma aproximação local (função enriquecedora polinomial ou não). Nota-se que a função 
enriquecida preserva o suporte compacto da PU, juntamente com características herdadas da função enriquecedora.

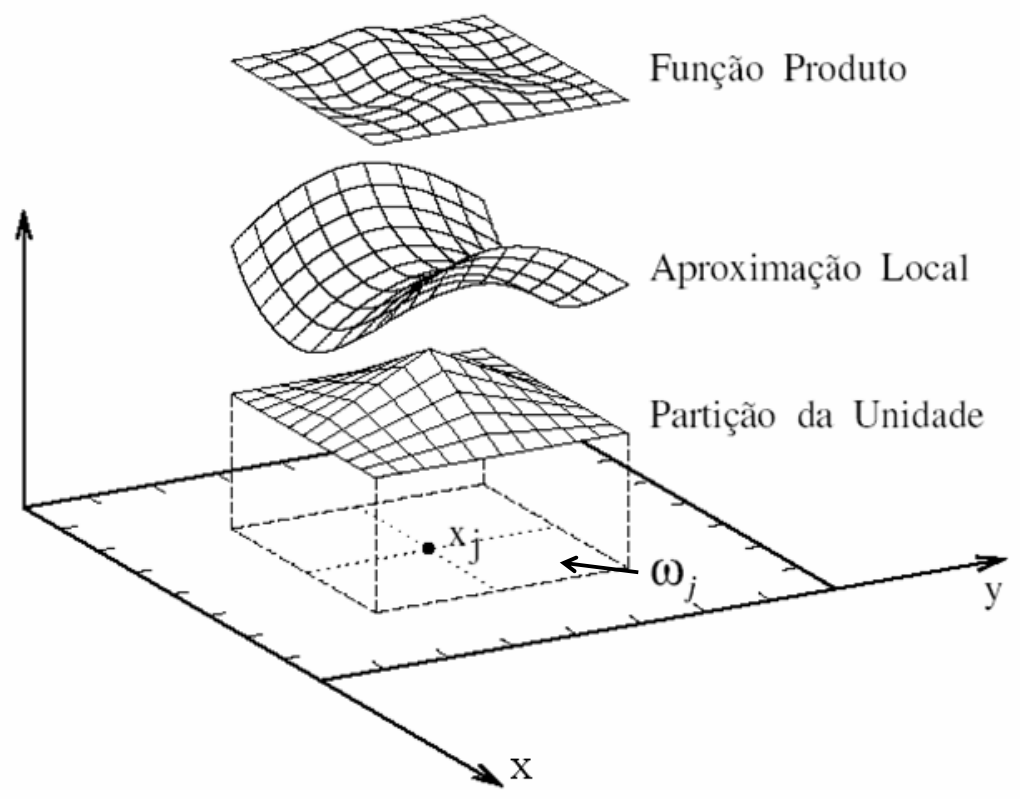

Figura 3.1 - Enriquecimento da Partição da Unidade num domínio global $\boldsymbol{x y}$.

No MEFG o enriquecimento da PU deve ser feito no domínio global, como mostra a figura 3.1. Portanto, ao se empregar domínios parametrizados, como no caso dos elementos isoparamétricos, pode-se recorrer a uma transformação de coordenadas para realizar o enriquecimento, que será apresentada com detalhes no próximo capítulo.

Por fim, como principais vantagens do MEFG, têm-se: aproveitamento da estrutura do MEF; facilidade na implementação de refinamentos $\boldsymbol{p}$; diminuição do efeito negativo da distorção dos elementos; possibilidade de utilizar funções especiais como funções enriquecedoras e inexistência de dificuldades quanto à imposição das condições de contorno do problema.

O MEFG pode ter sua estrutura estendida diretamente para a formulação híbrido-mista de tensão, no entanto para a formulação híbrida de tensão a extensão em princípio se limita ao campo de deslocamentos de contorno. Neste trabalho, aplica-se o MEFG para a FHMT e propõe-se uma alternativa que permite o enriquecimento nodal também para os campos de tensão da FHT. 


\subsection{FHMT e FHT com Enriquecimento Nodal}

\subsubsection{FHMT com Enriquecimento Nodal}

Todas as aproximações envolvidas na FHMT são atreladas a nós e, dessa forma, a metodologia clássica de enriquecimento do Método das Nuvens "hp” pode ser aplicada às tensões e deslocamentos no domínio e deslocamentos no contorno discretizados via rede de cobertura.

Assim, na FHMT com enriquecimento, aproveita-se, como no MEFG clássico, a rede de cobertura para definição das nuvens de domínio $\boldsymbol{\omega}_{j}$ e de contorno $\boldsymbol{\omega}_{\boldsymbol{j}_{\Gamma}}$ atreladas, respectivamente, a um determinado nó $\boldsymbol{x}_{\boldsymbol{j}}$ e $\boldsymbol{x}_{\boldsymbol{j}_{\boldsymbol{T}}}$. Para as redes de domínio e contorno a nuvem em torno de um nó é formada pelo conjunto de elementos que têm aquele nó como vértice, como indica a figura 3.2.

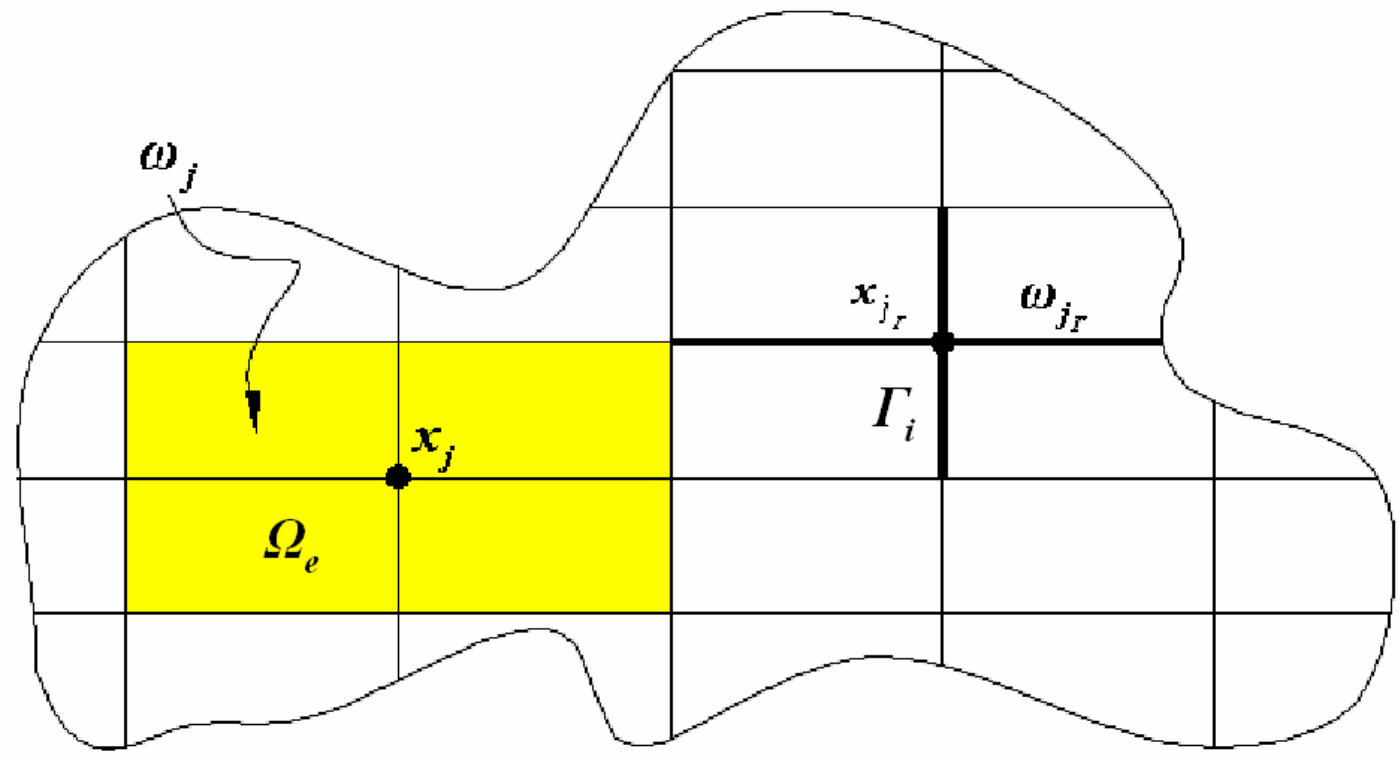

Figura 3.2 - FHMT e FHT - nuvens de influência para as redes de cobertura: domínio (bidimensional) e contorno (unidimensional).

Em cada nuvem, a aproximação nodal fica determinada aproveitando-se as funções de forma das redes de elementos finitos que compõe a nuvem e associadas ao nó base. Dessa forma, a continuidade entre elementos será da mesma ordem das funções adotadas para aproximação. Empregando-se o mesmo procedimento proposto no Método das Nuvens "hp”, o enriquecimento sobre as funções de forma nodais (para cada um dos campos aproximados 
na FHMT), em cada nuvem, é realizado pela sua multiplicação, por polinômios ou outras funções especiais. Assim, pode-se representar:

- A família de funções para o campo de tensões no domínio $\boldsymbol{\Omega}$ :

$$
\mathfrak{I}_{N}^{p}=\left\{\left\{S_{\Omega_{j}}\right\}_{j=1}^{N} \cup\left\{S_{\Omega_{j}} L_{j n_{e}}\right\}_{j=1}^{N}: j=1, \ldots, N ; n_{e}=1, \ldots, I(j)\right\}
$$

- A família de funções para o campo de deslocamentos no domínio $\boldsymbol{\Omega}$ :

$$
\Theta_{N}^{p}=\left\{\left\{U_{\Omega_{j}}\right\}_{j=1}^{N} \cup\left\{U_{\Omega_{j}} M_{j n_{e}}\right\}_{j=1}^{N}: j=1, \ldots, N ; n_{e}=1, \ldots, I(j)\right\}
$$

- A família de funções para o campo de deslocamentos nos contornos $\Gamma_{i}$ ou $\Gamma_{t}$ :

$$
\Xi_{N_{\Gamma}}^{p}=\left\{\left\{U_{\Gamma_{j}}\right\}_{j=1}^{N_{\Gamma}} \cup\left\{U_{\Gamma_{j}} O_{j n_{e_{\Gamma}}}\right\}_{j=1}^{N_{\Gamma}}: j=1, \ldots, N_{\Gamma} ; n_{e_{\Gamma}}=1, \ldots, I(j)\right\}
$$

onde, para as representações anteriores, têm-se que:

- $\boldsymbol{N}$ é o número total de nós no domínio $\boldsymbol{\Omega}$;

- $N_{\Gamma}$ é o número total de nós no contorno $\Gamma\left(\Gamma_{i}+\Gamma_{t}+\Gamma_{u}\right)$;

- $\boldsymbol{p}$ é o grau máximo da aproximação Lagrangiana resultante;

- $\boldsymbol{S}_{\boldsymbol{\Omega}_{j}}$ e $\boldsymbol{U}_{\boldsymbol{\Omega}_{j}}$ são as funções de forma (PU) para os campos de tensão e deslocamento respectivamente, referentes aos nós $\boldsymbol{j}=\mathbf{1}, \ldots ., \boldsymbol{N}$ do domínio $\boldsymbol{\Omega}$;

- $U_{\Gamma_{j}}$ é a função de forma (Partição da Unidade) para o campo de deslocamento referente aos nós do contorno $\Gamma\left(\Gamma_{i}+\Gamma_{t}+\Gamma_{u}\right)\left(j=1, \ldots ., N_{\Gamma}\right)$;

- $\boldsymbol{L}_{\boldsymbol{j} \boldsymbol{n}_{e}}, \boldsymbol{M}_{\boldsymbol{j} \boldsymbol{n}_{e}}$ são as $\boldsymbol{n}_{\boldsymbol{e}}$ funções que multiplicam ou enriquecem a função de forma de domínio definida em cada nó de índice $\boldsymbol{j}$;

- $\boldsymbol{O}_{j \boldsymbol{n}_{e \Gamma}}$ são as $\boldsymbol{n}_{\boldsymbol{e}_{\Gamma}}$ funções que multiplicam (enriquecem) a função de forma de contorno definida em cada nó de índice $\boldsymbol{j}$;

- $\boldsymbol{I}(\boldsymbol{j})$ é o contador para o número de funções adicionadas a cada nó de índice $\boldsymbol{j}$. 


\subsubsection{FHT com Enriquecimento Nodal}

Na FHT há aproximações das tensões e deslocamentos no domínio e contorno do elemento, respectivamente. Tendo-se em vista, inicialmente, a extensão do MEFG, define-se suportes ou nuvens associadas tanto ao domínio quanto ao contorno do elemento para fins de enriquecimento dos campos de aproximação.

Considere-se uma rede de elementos finitos híbridos de tensão, como apresenta a figura 3.2.

A nuvem de contorno $\omega_{j_{\Gamma}}$ está em destaque naquela figura. No elemento de contorno em $\boldsymbol{\Gamma}_{i}$, ou $\boldsymbol{\Gamma}_{\boldsymbol{t}}$, tem-se que a aproximação dos deslocamentos, que constitui uma PU, pode ser obtida da interpolação nodal de funções Lagrangianas clássicas. Assim, uma destas funções conectada ao nó $\boldsymbol{x}_{\boldsymbol{j}_{\boldsymbol{T}}}$ pode ser enriquecida por polinômios ou outras funções especiais.

Como o enriquecimento sobre as funções aproximativas dos deslocamentos no contorno do elemento, em cada nuvem de contorno $\omega_{j_{\Gamma}}$, é realizado empregando-se o mesmo procedimento proposto no Método das Nuvens “hp”, podendo-se representar a seguinte família de funções:

- A família de funções para o campo de deslocamentos no contorno $\boldsymbol{\Gamma}_{i}$ ou $\boldsymbol{\Gamma}_{t}$ :

$$
\mathfrak{R}_{N_{\Gamma}}^{p}=\left\{\left\{U_{\Gamma_{j}}\right\}_{j=1}^{N_{\Gamma}} \cup\left\{U_{\Gamma_{j}} O_{j n_{e}}\right\}_{j=1}^{N_{\Gamma}}: j=1, \ldots, N_{\Gamma} ; n_{e_{\Gamma}}=1, \ldots, I(j)\right\}
$$

onde:

- $N_{\Gamma}$ é o número total de nós no contorno $\Gamma\left(\Gamma_{i}+\Gamma_{t}+\Gamma_{u}\right)$;

- $\boldsymbol{p}$ é o grau máximo da aproximação Lagrangiana resultante;

- $\boldsymbol{U}_{\Gamma_{j}}$ é a função de forma (PU) para o campo de deslocamento referente aos nós do contorno $\Gamma\left(\Gamma_{i}+\Gamma_{t}+\Gamma_{u}\right)\left(j=1, \ldots, N_{\Gamma}\right)$;

- $\boldsymbol{O}_{\boldsymbol{j} \boldsymbol{n}_{\boldsymbol{e}}}$ são as $\boldsymbol{n}_{\boldsymbol{e}_{\Gamma}}$ funções que multiplicam (enriquecem) a função de forma de contorno definidas em cada nó de índice $\boldsymbol{j}$;

- $\boldsymbol{I}(\boldsymbol{j})$ é o contador para o número de funções adicionadas a cada nó de índice $\boldsymbol{j}$.

Não se pode escrever a família de funções para o campo de tensões no domínio $\boldsymbol{\Omega}$ à maneira apresentada pelo Método das Nuvens “hp”, pois as funções aproximativas das 
tensões utilizadas na FHT não são uma PU. Mesmo assim, propõe-se uma alternativa que permite o enriquecimento nodal.

Na rede representada na figura 3.2, as funções aproximativas do campo de tensões no domínio de um determinado elemento $\boldsymbol{\Omega}_{\boldsymbol{e}}$ são enriquecidas da seguinte forma: ao nó $\boldsymbol{x}_{\boldsymbol{j}}$, em destaque na rede, atrela-se uma função de suporte compacto $\boldsymbol{g}$ que será enriquecida por uma função $\boldsymbol{h}$. A nuvem $\boldsymbol{\omega}_{j}$ (em amarelo), associada ao $\boldsymbol{x}_{\boldsymbol{j}}$, mostra os elementos de domínio $\boldsymbol{\Omega}_{\boldsymbol{e}}$ onde as aproximações das tensões são enriquecidas. Pode-se, então, construir a seguinte representação:

- A família de funções para o campo de tensões no domínio $\boldsymbol{\Omega}$

$$
\mathfrak{I}_{e}^{p}=\left\{\left\{S_{\Omega_{e}}\right\}_{e=1}^{n_{\Omega}} \cup\left\{g_{j} L_{j}\right\}_{j=1}^{N}: e=1, \ldots, n_{\Omega ;} j=1, \ldots, N\right\}
$$

onde:

- $\boldsymbol{N}$ é o número total de nós no domínio $\boldsymbol{\Omega}$;

- p é o grau máximo da aproximação resultante;

- $\boldsymbol{S}_{\Omega_{e}}$ é função aproximativa para o campo de tensão, referentes aos elementos $\boldsymbol{e}=1, \ldots, \boldsymbol{n}_{\Omega}$ do domínio $\boldsymbol{\Omega}$;

- $\boldsymbol{g}_{\boldsymbol{j}}$ são funções com suporte compacto sobre nuvens definidas em cada nó de índice $\boldsymbol{j}$ com auxílio da rede de elementos finitos de cobertura;

- $\boldsymbol{L}_{\boldsymbol{j}}$ são funções que multiplicam ou enriquecem as funções $\boldsymbol{g}_{\boldsymbol{j}}$. 


\section{Modelo Discreto da FHMT com Enriquecimento Nodal}

\subsection{Introdução}

Nesta parte do trabalho destaca-se a formalização da FHMT com enriquecimento nodal polinomial para redes de cobertura compostas por elementos finitos quadrangulares convencionais de quatro nós, triangulares de três nós e elementos lineares de contorno. Também serão abordadas questões relacionadas à transformação de coordenadas de cada um dos elementos planos tratados, de interesse para a sua formulação.

\subsection{Modelo Discreto da FHMT com Enriquecimento Nodal}

No capítulo 2, para um maior entendimento das variáveis envolvidas nas formulações híbridas estudadas nesta pesquisa, abordam-se a FHT e a FHMT com uma discretização contendo um e dois elementos, mas sem considerações relativas à vinculação das aproximações da FHT/FHMT atreladas, ou não, a nós dispersos no domínio $\boldsymbol{\Omega}$ e contorno $\Gamma\left(\Gamma_{t}, \Gamma_{i}\right.$ е $\left.\Gamma_{u}\right)$.

Para generalização dos modelos e focando inicialmente o modelo híbrido-misto de tensão, considere-se um domínio $\boldsymbol{\Omega}$ coberto por uma rede de $\boldsymbol{n}_{\boldsymbol{\Omega}}$ elementos finitos ditos de

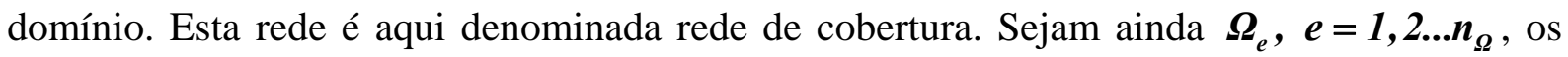
domínios de cada um dos elementos finitos. Definem-se também $\boldsymbol{n}_{\boldsymbol{\Gamma}_{t}}$ elementos no contorno de Neumann $\boldsymbol{\Gamma}_{\boldsymbol{t}_{\boldsymbol{T}_{t}}}, \boldsymbol{e}_{\boldsymbol{\Gamma}_{t}}=1, \ldots \boldsymbol{n}_{\Gamma_{t}}$, e $\boldsymbol{n}_{\Gamma_{i}}$ elementos do contorno entre os elementos de domínio $\Gamma_{i_{e_{i}}}, e_{\Gamma_{i}}=1, \ldots n_{\Gamma_{i}}$.

Agora, sejam $\boldsymbol{s}_{\Omega_{e}}, \boldsymbol{q}_{\Omega_{e}}, \quad \boldsymbol{q}_{\Gamma_{e_{\Gamma_{t}}}}$ e $\boldsymbol{q}_{\Gamma_{e_{\Gamma_{i}}}}$ os vetores que guardam os parâmetros generalizados correspondentes às aproximações locais para as tensões e deslocamentos dos 
elementos de domínio $\boldsymbol{e}$ e deslocamentos dos elementos dos contornos $\boldsymbol{e}_{\Gamma_{t}}$ e $\boldsymbol{e}_{\Gamma_{i}}$ nas fronteiras de Neumann e internas, respectivamente. Sejam também $\boldsymbol{s}_{\Omega}, \boldsymbol{q}_{\Omega}, \boldsymbol{q}_{\Gamma_{t}}$ e $\boldsymbol{q}_{\Gamma_{i}}$ os parâmetros globais para as tensões e deslocamentos no domínio, deslocamentos nas fronteiras de Neumann e internas, respectivamente.

O parâmetro generalizado $\boldsymbol{s}_{\Omega_{e}}$ pode ser colocado em correspondência com o parâmetro generalizado global $\boldsymbol{s}_{\boldsymbol{\Omega}}$, da mesma forma que o $\boldsymbol{q}_{\boldsymbol{\Omega}_{e}}$ pode ser colocado em correspondência com o parâmetro generalizado global $\boldsymbol{q}_{\Omega}$. Igualmente, os parâmetros de deslocamentos $\boldsymbol{q}_{\boldsymbol{T}_{\boldsymbol{r}_{t}}}$ do elemento no contorno $\boldsymbol{e}_{\Gamma_{t}}$ e $\boldsymbol{q}_{\Gamma_{e_{T_{i}}}}$ do elemento no contorno $\boldsymbol{e}_{\Gamma_{i}}$ podem ser colocados em correspondência com os parâmetros ou graus de liberdade globais $\boldsymbol{q}_{\boldsymbol{\Gamma}_{t}}$ e $\boldsymbol{q}_{\boldsymbol{\Gamma}_{i}}$, respectivamente. Nesse sentido, valem as seguintes relações:

$$
\begin{aligned}
& s_{\Omega_{e}}=\boldsymbol{F}_{\boldsymbol{e}} s_{\Omega}, e=1, \ldots n_{\Omega} \\
& q_{\Omega_{e}}=\boldsymbol{A}_{\boldsymbol{e}} q_{\Omega}, e=1, \ldots n_{\Omega} \\
& q_{\Gamma_{e_{T_{t}}}}=\boldsymbol{A}_{\boldsymbol{r}_{\boldsymbol{e}_{T_{t}}}} q_{\Gamma_{t}}, \boldsymbol{e}_{\Gamma_{t}}=1, \ldots n_{\Gamma_{t}} \\
& \boldsymbol{q}_{\Gamma_{e_{\Gamma_{i}}}}=\boldsymbol{A}_{\boldsymbol{r}_{\boldsymbol{e}_{\Gamma_{i}}}} q_{\Gamma_{i}}, \boldsymbol{e}_{\Gamma_{i}}=1, \ldots n_{\Gamma_{i}}
\end{aligned}
$$

onde $\boldsymbol{F}_{\boldsymbol{e}}$ e $\boldsymbol{A}_{\boldsymbol{e}}$ são matrizes Booleanas que extraem os parâmetros generalizados do elemento finito no domínio $\boldsymbol{\Omega}_{\boldsymbol{e}}$ e $\boldsymbol{A}_{\boldsymbol{r}_{\boldsymbol{e}_{t}}}$ e $\boldsymbol{A}_{\boldsymbol{r}_{\boldsymbol{e}_{i}}}$ são as matrizes Booleanas que extraem, respectivamente, os graus de liberdade dos elementos no contorno $\boldsymbol{\Gamma}_{t_{e_{T_{t}}}}$ e $\boldsymbol{\Gamma}_{\boldsymbol{i}_{e_{T_{i}}}}$.

Sejam as aproximações locais dos campos de tensões, eq.(2.30), dos deslocamentos no domínio, eq.(2.31), dos deslocamentos no contorno de Neumann, eq.(2.32), e dos deslocamentos no contorno entre elementos, eq.(2.34), respectivamente, dadas por interpolação dos graus de liberdade nas formas:

$$
\begin{gathered}
\tilde{\sigma}_{e}=S_{\Omega_{e}} s_{\Omega_{e}} \\
\tilde{u}_{e}=U_{\Omega_{e}} q_{\Omega_{e}}
\end{gathered}
$$




$$
\begin{aligned}
& \tilde{\boldsymbol{u}}_{\Gamma_{t}}=\boldsymbol{U}_{\Gamma_{\boldsymbol{t}_{e_{T_{t}}}}} \boldsymbol{q}_{\Gamma_{e_{\Gamma_{t}}}} \\
& \boldsymbol{u}_{\Gamma_{i}}=\boldsymbol{U}_{\Gamma_{\Gamma_{e_{\Gamma_{i}}}}} \boldsymbol{q}_{\Gamma_{e_{\Gamma_{i}}}}
\end{aligned}
$$

onde $\boldsymbol{S}_{\Omega_{e}}, \boldsymbol{U}_{\Omega_{e}}, \boldsymbol{U}_{\Gamma_{t_{e_{T_{t}}}}}$ e $\boldsymbol{U}_{{\boldsymbol{T _ { i }}}_{i_{T_{i}}}}$ são as matrizes que coletam as funções de interpolação dos campos de tensões no domínio, deslocamentos no domínio, deslocamentos no contorno de Neumann e deslocamentos no contorno interno, respectivamente. Considere também que nos contornos de Dirichlet $\Gamma_{u_{e_{\Gamma_{u}}}}$ existem $\boldsymbol{n} \boldsymbol{e}_{\Gamma_{u}}, \boldsymbol{n} \boldsymbol{e}_{\Gamma_{u}}=1, \ldots \boldsymbol{n}_{\Gamma_{u}}$, elementos com deslocamentos prescritos.

A partir da contribuição de cada um dos elementos no domínio e contorno as matrizes das eq.(2.24) a eq.(2.29) e eq.(2.37) podem ser calculadas por meio de

$$
\begin{aligned}
& \boldsymbol{F}=\sum_{e=1}^{n_{\Omega}} \boldsymbol{F}_{\boldsymbol{e}}^{\boldsymbol{T}} \boldsymbol{F}_{e} \boldsymbol{F}_{\boldsymbol{e}} \\
& A_{\Omega}=\sum_{e=1}^{n_{\Omega}} \boldsymbol{F}_{\boldsymbol{e}}^{\boldsymbol{T}} A_{\Omega_{e}} \boldsymbol{A}_{e} \\
& A_{\Gamma_{t}}=\sum_{e_{\Gamma_{t}}=1}^{n_{\Gamma_{t}}} \boldsymbol{F}_{\boldsymbol{e}}^{\boldsymbol{T}} A_{\Gamma_{t_{e_{T_{t}}}}} \boldsymbol{A}_{\boldsymbol{r}_{\boldsymbol{e}_{T_{t}}}} \\
& A_{\Gamma_{i}}=\sum_{e_{\Gamma_{i}}=1}^{n_{\Gamma_{i}}} \boldsymbol{F}_{\boldsymbol{e}}^{\boldsymbol{T}} A_{\Gamma_{i_{e_{i}}}} \boldsymbol{A}_{\boldsymbol{r}_{\boldsymbol{o}_{\Gamma_{i}}}} \\
& \boldsymbol{e}_{\Gamma_{u}}=\sum_{n e_{\Gamma_{u}}=1}^{n_{\Gamma_{u}}} \boldsymbol{F}_{\boldsymbol{e}}^{\boldsymbol{T}} \boldsymbol{e}_{\Gamma_{u_{n e_{T_{u}}}}} \\
& \boldsymbol{Q}_{\Omega}=\sum_{e=1}^{n_{\Omega}} \boldsymbol{A}_{e}^{\boldsymbol{T}} \boldsymbol{Q}_{\Omega_{e}} \\
& \boldsymbol{Q}_{\Gamma_{t}}=\sum_{e_{\Gamma_{t}}=1}^{n_{\Gamma_{t}}} \boldsymbol{A}_{\boldsymbol{r}_{\boldsymbol{I}_{t}}}^{\boldsymbol{T}} \boldsymbol{Q}_{\Gamma_{e_{\Gamma_{t}}}}
\end{aligned}
$$

onde:

$$
F_{e}=\int_{\Omega} S_{\Omega_{e}}^{T} f S_{\Omega_{e}} d \Omega
$$




$$
\begin{aligned}
& A_{\Omega_{e}}=\int_{\Omega}\left(L S_{\Omega_{e}}\right)^{T} U_{\Omega_{e}} d \Omega \\
& A_{\Gamma_{\bar{t}_{e_{T_{t}}}}}=\int_{\Gamma_{t}}\left(N S_{\Omega_{e}}\right)^{T} U_{\Gamma_{t_{t_{T_{t}}}}} d \Gamma \\
& A_{\Gamma_{i_{e_{T_{i}}}}}=\int_{\Gamma_{i}}\left(N S_{\Omega_{e}}\right)^{T} U_{\Gamma_{i_{e_{\Gamma_{i}}}}} d \Gamma
\end{aligned}
$$

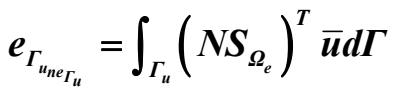

$$
\begin{aligned}
& Q_{\Omega_{e}}=\int_{\Omega} U_{\Omega_{e}}^{T} \bar{b} d \Omega \\
& Q_{\Gamma_{e_{\Gamma_{t}}}}=\int_{\Gamma_{t}} U_{\Gamma_{t_{e_{\Gamma_{t}}}}}^{T}(\bar{t}) d \Gamma
\end{aligned}
$$

As matrizes que guardam as aproximações (enriquecidas ou não) dos campos de tensões e deslocamentos no domínio e deslocamentos no contorno, apresentadas nas eq.(4.5) a eq.(4.8), são constituídas por sub-matrizes cujas dimensões estão associadas ao número de nós dos elementos, ao número de graus de liberdade definido em cada nó, ao número de nós enriquecidos e aos graus de enriquecimento nodal adotados.

\subsection{Elementos Finitos Híbrido-Mistos de Tensão com Enriquecimento Nodal Polinomial}

\subsubsection{Elemento Quadrilateral de Quatro Nós}

\subsubsection{Características Geométricas e Transformação de Coordenadas}

Adotam-se, inicialmente, os elementos finitos quadrilaterais de quatro nós, como elementos da rede de cobertura.

A figura 4.1 apresenta a numeração dos nós do elemento (sempre no sentido antihorário), utilizada no programa computacional desenvolvido. Além disso, a mesma figura destaca os elementos lineares no contorno $\left(\boldsymbol{\Gamma}_{\boldsymbol{u}_{e_{\Gamma_{u}}}}, \boldsymbol{\Gamma}_{\boldsymbol{t}_{e_{\boldsymbol{T}_{t}}}}\right.$ ou $\left.\boldsymbol{\Gamma}_{\boldsymbol{i}_{e_{\Gamma_{i}}}}\right)$ e indica o mapeamento do elemento do domínio regular mestre, de coordenadas normalizadas $\boldsymbol{\xi} \boldsymbol{\eta}$ ortogonais, para um elemento quadrilateral no domínio físico, de coordenadas cartesianas $\boldsymbol{x y}$. 


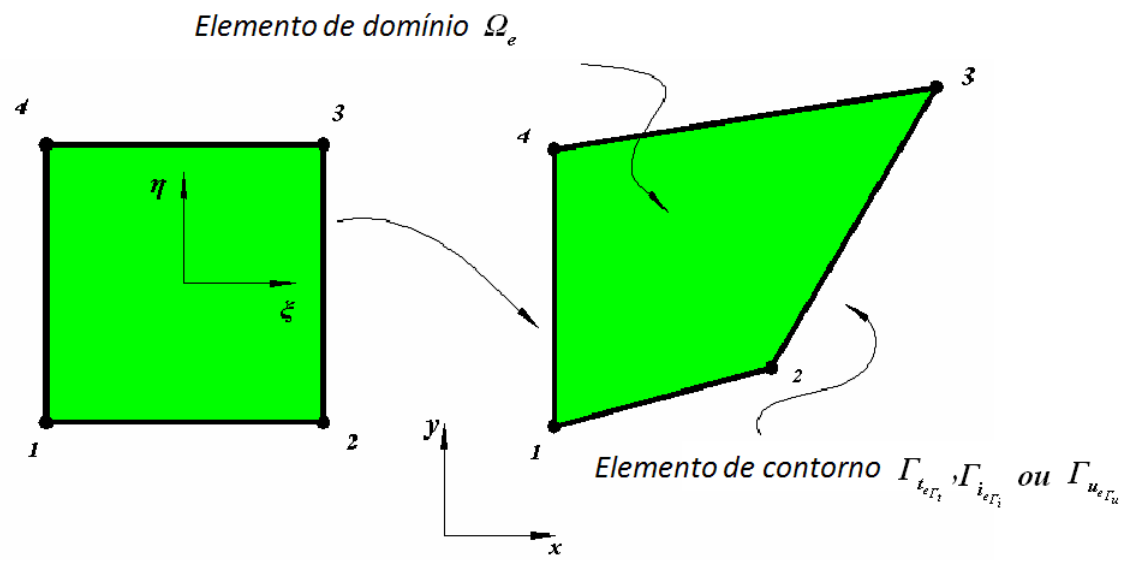

Figura 4.1 - Elemento quadrilateral de quatro nós.

Para a transformação de coordenadas do domínio paramétrico para o domínio físico, utilizam-se as funções Lagrangianas bilineares clássicas, ver figura 4.1, tendo a seguinte apresentação em relação a um sistema de referência cartesiano localizado no centro do elemento:

$$
\begin{aligned}
& \varphi_{1}=\frac{1}{4}(\xi-1)(\eta-1) \\
& \varphi_{2}=-\frac{1}{4}(\xi+1)(\eta-1) \\
& \varphi_{3}=\frac{1}{4}(\xi+1)(\eta+1) \\
& \varphi_{4}=-\frac{1}{4}(\xi-1)(\eta+1)
\end{aligned}
$$

onde $\boldsymbol{\xi}$ e $\boldsymbol{\eta}$ são coordenadas adimensionais variando entre -1 e 1 .

Define-se ainda a matriz $\boldsymbol{M}$ composta pelas funções de interpolação das eq.(4.23) a eq.(4.26). Assim:

$$
M=\left[\begin{array}{cccccccc}
\varphi_{1} & 0 & \varphi_{2} & 0 & \varphi_{3} & 0 & \varphi_{4} & 0 \\
0 & \varphi_{1} & 0 & \varphi_{2} & 0 & \varphi_{3} & 0 & \varphi_{4}
\end{array}\right]
$$

Seja também o vetor $c$ que guarda as coordenadas nodais do elemento finito, dado por:

$$
c^{T}=\left[\begin{array}{llllllll}
x_{1} & y_{1} & x_{2} & y_{2} & x_{3} & y_{3} & x_{4} & y_{4}
\end{array}\right]
$$


Então as coordenadas $\boldsymbol{x}, \boldsymbol{y}$ globais de um ponto do elemento finito podem ser mapeadas a partir do elemento parametrizado por meio da seguinte transformação:

$$
\left\{\begin{array}{l}
x \\
y
\end{array}\right\}=M c^{T}
$$

Com a transformação de coordenadas, podem-se escrever os operadores diferenciais no referencial paramétrico $\boldsymbol{\xi} \boldsymbol{\eta}$ em função dos definidos no referencial global $\boldsymbol{x y}$, da seguinte forma:

$$
\left\{\begin{array}{l}
\frac{\partial}{\partial \xi} \\
\frac{\partial}{\partial \eta}
\end{array}\right\}=J\left\{\begin{array}{l}
\frac{\partial}{\partial x} \\
\frac{\partial}{\partial y}
\end{array}\right\}
$$

sendo

$$
J=\left[\begin{array}{ll}
\frac{\partial x}{\partial \xi} & \frac{\partial y}{\partial \xi} \\
\frac{\partial x}{\partial \eta} & \frac{\partial y}{\partial \eta}
\end{array}\right]
$$

a matriz jacobiana.

Considere-se agora um elemento no contorno $\Gamma\left(\boldsymbol{\Gamma}_{u_{e_{\tau_{u}}}}, \boldsymbol{\Gamma}_{\boldsymbol{t}_{\boldsymbol{e}_{\tau_{t}}}}\right.$ ou $\left.\boldsymbol{\Gamma}_{\boldsymbol{i}_{e_{\Gamma_{i}}}}\right)$ coincidente com um dos lados no elemento quadrilateral $\boldsymbol{\Omega}_{e}$, ver figura 4.2. Ainda na figura o elemento linear é apresentado nos contornos paramétrico ( $\xi$ é a coordenada local adimensional com origem no centro do elemento) e físico, de coordenadas $\boldsymbol{x y}$. 


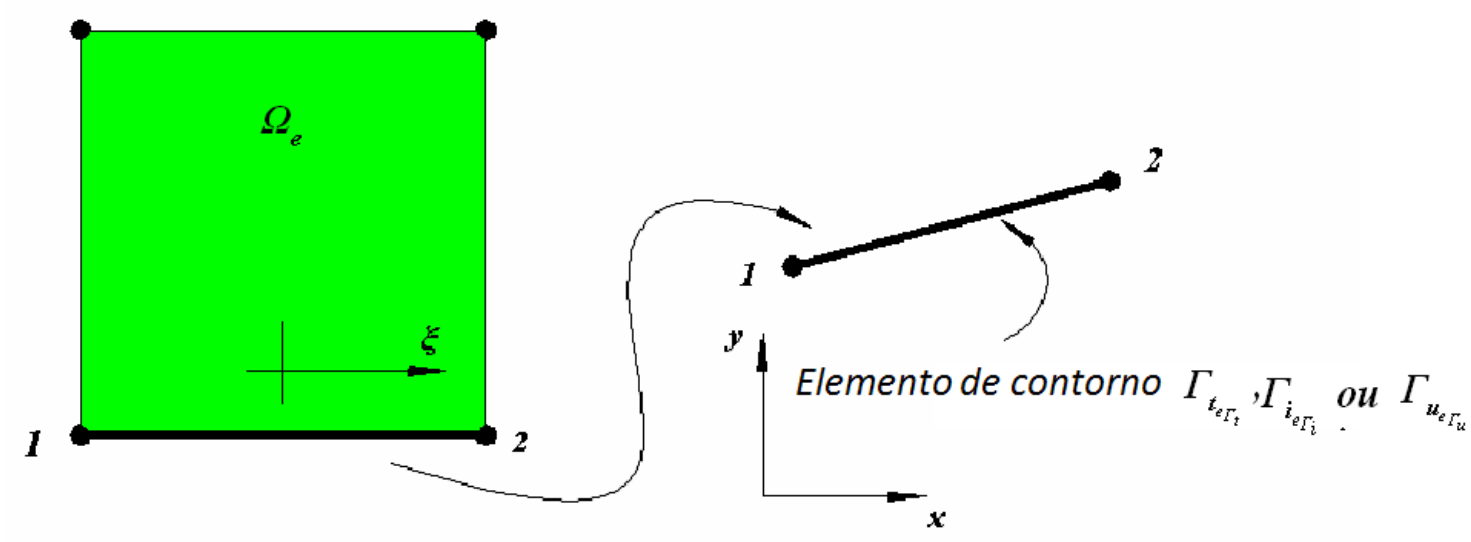

Figura 4.2 - Elemento finito linear com sistema local de referência com origem no seu centro.

No mapeamento de coordenadas do elemento linear no contorno paramétrico para o contorno físico, utilizam-se as funções lineares Lagrangianas clássicas. Para o elemento em $\boldsymbol{\eta}=\boldsymbol{- 1}$, tais funções têm a seguinte apresentação:

$$
\begin{gathered}
\psi_{1}=-\frac{1}{2}(\xi-1) \\
\psi_{2}=\frac{1}{2}(\xi+1)
\end{gathered}
$$

onde $\xi$ é a coordenada adimensional com origem no centro do elemento e variando entre -1 e 1.

As equações aqui propostas para o elemento no contorno em destaque na figura 4.2 também são válidas para elementos que compõem os outros lados do elemento no domínio, desde que se façam as seguintes mudanças na definição da coordenada adimensional:

- Se o elemento no contorno é o vertical esquerdo, a coordenada adimensional passa ser $\boldsymbol{\eta}$ (variando entre -1 e 1 ) com $\boldsymbol{\xi}=-\boldsymbol{1}$.

- Se o elemento no contorno é o vertical direito, a coordenada adimensional passa ser $\boldsymbol{\eta}$ (variando entre -1 e 1) com $\xi=1$.

- Se o elemento no contorno é o horizontal superior, a coordenada adimensional passa ser $\boldsymbol{\xi}$ (variando entre -1 e 1 ) com $\boldsymbol{\eta}=\boldsymbol{1}$.

Agora, define-se ainda a matriz $\boldsymbol{M}_{\boldsymbol{\Gamma}}$ composta pelas funções de interpolação das eq.(4.32) e eq.(4.33). Dessa forma: 


$$
M_{\Gamma}=\left[\begin{array}{cccc}
\psi_{1} & 0 & \psi_{2} & 0 \\
0 & \psi_{1} & 0 & \psi_{2}
\end{array}\right]
$$

Seja também o vetor $\boldsymbol{c}_{\boldsymbol{\Gamma}}$ que guarda as coordenadas nodais no domínio físico do elemento finito no contorno, dado por:

$$
c_{\Gamma}^{T}=\left[\begin{array}{llll}
x_{1} & y_{1} & x_{2} & y_{2}
\end{array}\right]
$$

Então as coordenadas $\boldsymbol{x y}$ globais de um ponto da rede dos elementos finitos lineares podem ser mapeadas a partir do elemento parametrizado por meio da seguinte transformação:

$$
\left\{\begin{array}{l}
x \\
y
\end{array}\right\}=M_{\Gamma} c_{\Gamma}^{T}
$$

Em geral, os elementos no contorno representam, no espaço parametrizado, uma reta $\boldsymbol{\xi}$ constante ou $\boldsymbol{\eta}$ constante. Dessa forma, se um dos lados do elemento quadrilateral corresponde, por exemplo, a $\boldsymbol{\eta}=\mathbf{1}$ ou $\boldsymbol{\eta}=-\mathbf{1}$ ( $\boldsymbol{\eta}$ constante), o diferencial de comprimento no domínio global $\boldsymbol{x y}$ em relação ao diferencial de comprimento no domínio paramétrico é calculado da seguinte forma:

$$
d s=\sqrt{\left(\frac{\partial x}{\partial \xi}\right)^{2}+\left(\frac{\partial y}{\partial \xi}\right)^{2}} d \xi
$$

Para $\xi$ constante, de forma análoga ao caso anterior, tem-se:

$$
d s=\sqrt{\left(\frac{\partial x}{\partial \eta}\right)^{2}+\left(\frac{\partial y}{\partial \eta}\right)^{2}} d \eta
$$

\subsubsection{Funções Aproximativas do Elemento Quadrilateral de Quatro Nós da FHMT}

Para aproximação das variáveis de tensão e deslocamento no domínio serão adotadas as mesmas funções utilizadas para aproximação da geometria do elemento quadrilateral de quatro nós. Assim, as matrizes $S_{\Omega_{e}}$ (eq.(4.5)) e $\boldsymbol{U}_{\boldsymbol{\Omega}_{e}}$ (eq.(4.6)) sem enriquecimento são apresentadas da seguinte forma: 


$$
\begin{aligned}
& S_{\Omega_{e}}=\left[\begin{array}{cccccccccccc}
\varphi_{1} & 0 & 0 & \varphi_{2} & 0 & 0 & \varphi_{3} & 0 & 0 & \varphi_{4} & 0 & 0 \\
0 & \varphi_{1} & 0 & 0 & \varphi_{2} & 0 & 0 & \varphi_{3} & 0 & 0 & \varphi_{4} & 0 \\
0 & 0 & \varphi_{1} & 0 & 0 & \varphi_{2} & 0 & 0 & \varphi_{3} & 0 & 0 & \varphi_{4}
\end{array}\right] \\
& U_{\Omega_{e}}=\left[\begin{array}{cccccccc}
\varphi_{1} & 0 & \varphi_{2} & 0 & \varphi_{3} & 0 & \varphi_{4} & 0 \\
0 & \varphi_{1} & 0 & \varphi_{2} & 0 & \varphi_{3} & 0 & \varphi_{4}
\end{array}\right] \\
& \text { Com } s_{\Omega_{e}}\left(\text { eq.(4.5)) e } \boldsymbol{q}_{\Omega_{e}}(\text { eq.(4.6)) definidos por: }\right. \\
& s_{\Omega_{e}}^{T}=\left[\begin{array}{llllllllllll}
\sigma_{x_{1}} & \sigma_{y_{1}} & \tau_{x y_{1}} & \sigma_{x_{2}} & \sigma_{y_{2}} & \tau_{x y_{2}} & \sigma_{x_{3}} & \sigma_{y_{3}} & \tau_{x y_{3}} & \sigma_{x_{4}} & \sigma_{y_{4}} & \tau_{x y_{4}}
\end{array}\right] \\
& \boldsymbol{q}_{\Omega_{e}}^{T}=\left[\begin{array}{llllllll}
u_{x_{1}} & u_{y_{1}} & u_{x_{2}} & u_{y_{2}} & u_{x_{3}} & u_{y_{3}} & u_{x_{4}} & u_{y_{4}}
\end{array}\right]
\end{aligned}
$$

As funções aproximativas para os deslocamentos no contorno $\Gamma\left(\Gamma_{u_{e_{\Gamma_{u}}}}, \Gamma_{\boldsymbol{t}_{e_{\Gamma_{t}}}}\right.$ ou $\left.\Gamma_{\boldsymbol{i}_{e_{\Gamma_{i}}}}\right)$ também são as mesmas definidas para aproximação da geometria do elemento linear de dois nós. Então as matrizes $\boldsymbol{U}_{\Gamma_{t_{e_{T_{t}}}}}$ (eq.(4.7)) e $\boldsymbol{U}_{\boldsymbol{\Gamma}_{i_{e_{\Gamma_{i}}}}}$ (eq.(4.8)) sem enriquecimento são escritas:

$$
\left[\begin{array}{cccc}
\psi_{1} & 0 & \psi_{2} & 0 \\
0 & \psi_{1} & 0 & \psi_{2}
\end{array}\right]
$$

Os vetores $\boldsymbol{q}_{\Gamma_{e_{\Gamma_{t}}}}$ (eq.(4.7)) e $\boldsymbol{q}_{\Gamma_{{ }_{e_{i}}}}$ (eq.(4.8)) dados por:

$$
\boldsymbol{q}_{\Gamma_{e_{T_{t}}}}^{T} \text { ou } \boldsymbol{q}_{\Gamma_{e_{\Gamma_{i}}}}^{T}=\left[\begin{array}{llll}
u_{x_{1}} & u_{y_{1}} & u_{x_{2}} & u_{y_{2}}
\end{array}\right]
$$

Vale lembrar que todas as funções aproximativas de domínio e contorno envolvidas na FHMT são atreladas a nós.

\subsubsection{Enriquecimento das Funções Aproximativas do Elemento Quadrilateral de Quatro Nós da FHMT}

Utilizando-se a metodologia de enriquecimento nodal apresentada no capítulo 3, ampliam-se as bases de aproximação da FHMT (eq.(4.39), eq.(4.40) e eq.(4.43)), por exemplo, com funções polinomiais. Dessa forma as eq.(3.6), eq.(3.7) e eq.(3.8) podem ser reescritas da seguinte forma: 
- A família de funções para o campo de tensões no domínio $\boldsymbol{\Omega}$ :

$$
\mathfrak{I}_{N}^{2}=\left\{\left\{S_{\Omega_{j}}\right\}_{j=1}^{N} \cup\left\{S_{\Omega_{j}} h_{j n_{e}}\right\}_{j=1}^{N}: j=1, \ldots, N ; n_{e}=1, \ldots, I(j)\right\}
$$

utilizada para construir a seguinte aproximação:

$$
\hat{\sigma}=\sum_{j=1}^{N} S_{\Omega_{j}}\left\{s_{\Omega_{j}}+\sum_{i=1}^{n_{e}} h_{j i} b_{j i}\right\}
$$

onde $\boldsymbol{s}_{\boldsymbol{\Omega}_{j}}$ são os graus de liberdade de tensões associadas às funções de forma originais e $\boldsymbol{b}_{\boldsymbol{j} \boldsymbol{i}}$ são os novos parâmetros nodais correspondentes a cada uma das parcelas de enriquecimento.

- A família de funções para o campo de deslocamentos no domínio $\boldsymbol{\Omega}$ :

$$
\Theta_{N}^{2}=\left\{\left\{U_{\Omega_{j}}\right\}_{j=1}^{N} \cup\left\{U_{\Omega_{j}} h_{j n_{e}}\right\}_{j=1}^{N}: j=1, \ldots, N ; n_{e}=1, \ldots, I(j)\right\}
$$

utilizada para construir a seguinte aproximação:

$$
\hat{\boldsymbol{u}}=\sum_{j=1}^{N} U_{\Omega_{j}}\left\{u_{\Omega_{j}}+\sum_{i=1}^{n_{e}} h_{j i} c_{j i}\right\}
$$

onde $\boldsymbol{u}_{\boldsymbol{\Omega}_{j}}$ são graus de liberdade em deslocamento associados às funções de forma originais e $\boldsymbol{c}_{j i}$ são os novos parâmetros nodais correspondentes a cada uma das parcelas de enriquecimento.

- A família de funções para o campo de deslocamentos no contorno $\Gamma\left(\Gamma_{t}, \Gamma_{i}\right.$ e $\left.\Gamma_{u}\right):$

$$
\Xi_{N \Gamma}^{1}=\left\{\left\{U_{\Gamma_{j}}\right\}_{j=1}^{N_{\Gamma}} \cup\left\{U_{\Gamma_{j}} h_{j n_{e_{\Gamma}}}\right\}_{j=1}^{N_{\Gamma}}: j=1, \ldots, N_{\Gamma} ; n_{e_{\Gamma}}=1, \ldots, I(j)\right\}
$$

utilizada para construir a seguinte aproximação: 


$$
\hat{\boldsymbol{u}}_{\Gamma}=\sum_{j=1}^{N_{\Gamma}} \boldsymbol{U}_{\Gamma_{j}}\left\{\boldsymbol{u}_{\Gamma_{j}}+\sum_{i=1}^{n_{e_{\Gamma}}} \boldsymbol{h}_{j i} \boldsymbol{d}_{j i}\right\}
$$

onde $\boldsymbol{u}_{\Gamma_{j}}$ são graus de liberdade em deslocamento associados às funções de forma originais e $\boldsymbol{d}_{j i}$ são os novos parâmetros nodais correspondentes a cada uma das parcelas de enriquecimento.

Nas eq.(4.45) a eq.(4.48) as $\boldsymbol{h}_{\boldsymbol{j} \boldsymbol{n}_{\boldsymbol{e}}}$ são as $\boldsymbol{n}_{\boldsymbol{e}}$ funções polinomiais que multiplicam ou enriquecem as tensões e deslocamentos de domínio definida em cada nó de índice $\boldsymbol{j}$. Já nas eq.(4.49) e eq.(4.50) as $\boldsymbol{h}_{\boldsymbol{j} \boldsymbol{n}_{\boldsymbol{T}}}$ são as $\boldsymbol{n}_{\boldsymbol{e}_{\Gamma}}$ funções polinomiais que multiplicam ou enriquecem os deslocamentos no contorno definida em cada nó de índice $\boldsymbol{j}$.

Foi salientado no capítulo 3 que o enriquecimento das bases aproximativas do MEFG deve ser realizado no domínio global.

Por meio de um desenvolvimento simples, apresentar-se-á a alternativa de enriquecimento das aproximações da FHMT desenvolvida neste trabalho.

Então, por exemplo, considere-se que a transformação de um elemento mestre para um elemento do domínio global seja dada pelas seguintes relações:

$$
\left\{\begin{array}{l}
x \\
y
\end{array}\right\}=\left\{\begin{array}{c}
\frac{1}{2}+\frac{3}{2} \xi \\
\frac{3}{2} \eta+\frac{1}{2} \xi \eta
\end{array}\right\}
$$

Considere também a função de forma bilinear (eq.(4.23)) atrelada ao nó 1 do elemento mestre, representado na figura 4.1, escrita da seguinte forma:

$$
\varphi_{1}=\frac{1}{4}(1-\xi-\eta+\xi \eta)
$$

O que se deseja é enriquecê-la no domínio global pela função $\boldsymbol{x}$ e determinar o valor da função enriquecida no seguinte ponto do domínio global:

$$
\left\{\begin{array}{l}
x \\
y
\end{array}\right\}=\left\{\begin{array}{c}
\frac{1}{2} \\
0
\end{array}\right\}
$$


Da transformação inversa da eq.(4.51), tem-se que o ponto definido na eq.(4.53) tem a seguinte correspondência no domínio paramétrico:

$$
\left\{\begin{array}{l}
\xi \\
\eta
\end{array}\right\}=\left\{\begin{array}{l}
0 \\
0
\end{array}\right\}
$$

Para que o enriquecimento seja conduzido de forma correta, faz-se necessário escrever a função $\boldsymbol{x}$ em coordenadas naturais. Assim, com auxílio da transformação dada pela eq.(4.51), tem-se:

$$
x=\frac{1}{2}+\frac{3}{2} \xi
$$

Segue que o enriquecimento é dado pela multiplicação de $x(\xi)$ por $\varphi_{1}(\xi, \eta)$ :

$$
x(\xi) \cdot \varphi_{1}(\xi, \eta)=\frac{1}{8}(1-\xi-\eta+\xi \eta)+\frac{3}{8}\left(\xi-\xi^{2}-\xi \eta+\xi^{2} \eta\right)
$$

Substituindo-se $\boldsymbol{\xi}=\boldsymbol{\eta}=\boldsymbol{0}$ na eq.(4.56), resulta:

$$
x(\xi) \cdot \varphi_{1}(\xi, \eta)=\frac{1}{8}
$$

Observa-se que, operando simplesmente o enriquecimento da função de forma $\varphi_{1}(\xi, \eta)$ pela variável $\xi$ do domínio paramétrico e substituindo-se as coordenadas da origem do sistema natural, eq.(4.54), não se obtém o mesmo resultado. De fato:

$$
\left.\xi \cdot \varphi_{1}(\xi, \eta)\right|_{\xi=0}=\left.\frac{1}{4}\left(\xi-\xi^{2}-\xi \eta+\xi^{2} \eta\right)\right|_{\xi=0}=0
$$

O procedimento descrito pode ser aplicado para a determinação do valor da função de forma enriquecida no domínio global para qualquer outro ponto do domínio. Nota-se que a transformação inversa desempenha um papel fundamental para relacionar o ponto desejado no domínio global com sua posição no domínio natural. Resta finalmente observar que são normalmente os pontos de integração numérica aqueles de interesse para a determinação de valores da função enriquecida. 
Outra recomendação para as funções $\boldsymbol{h}_{\boldsymbol{j} \boldsymbol{n}_{e}}$ e $\boldsymbol{h}_{\boldsymbol{j} \boldsymbol{n}_{\boldsymbol{e}}}$ escolhidas para o enriquecimento, é que sejam tais que:

$$
\begin{aligned}
& \boldsymbol{h}_{j n_{e}}\left(\xi_{j}, \eta_{j}\right)=0 \\
& \boldsymbol{h}_{j n_{e_{\Gamma}}}\left(\xi_{j}\right)=0
\end{aligned}
$$

onde $\boldsymbol{\xi}_{j}, \boldsymbol{\eta}_{j}$ são as coordenadas adimensionais dos nós $\boldsymbol{j}$ dos elementos de domínio e contorno. Como se observa nas eq.(4.59) e eq.(4.60) essas funções são nulas no nó enriquecido ('funções bolhas'). A vantagem deste procedimento é que se preserva o significado físico de graus de liberdade nodais originais $\boldsymbol{s}_{\boldsymbol{\Omega}_{j}}, \boldsymbol{u}_{\boldsymbol{\Omega}_{j}}$ e $\boldsymbol{u}_{\boldsymbol{\Gamma}_{j}}$.

Finalmente, as bases de aproximação da FHMT originais (eq.(4.39), eq.(4.40) e eq.(4.43)), podem ser escritas da seguinte forma:

$$
\begin{aligned}
& S_{\Omega_{e}}=\left[\begin{array}{llll}
\varphi_{1} \Delta_{1} & \varphi_{2} \Delta_{2} & \varphi_{3} \Delta_{3} & \varphi_{4} \Delta_{4}
\end{array}\right] \\
& U_{\Omega_{e}}=\left[\begin{array}{lllll}
\varphi_{1} \Delta_{1} & \varphi_{2} \Delta_{2} & \varphi_{3} \Delta_{3} & \varphi_{4} \Delta_{4}
\end{array}\right]
\end{aligned}
$$

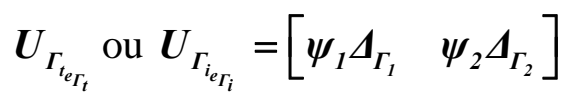

onde $\boldsymbol{\Delta}_{j}, \boldsymbol{j}=1, \ldots, 4$ e $\boldsymbol{\Delta}_{\boldsymbol{\Gamma}_{j}}, \boldsymbol{j}=\mathbf{1 , 2}$ são, respectivamente, as matrizes de enriquecimento polinomial do nó $\boldsymbol{j}$ de domínio ou contorno.

Considere-se agora que as matrizes de enriquecimento polinomial sejam dadas por:

$$
\Delta_{j}=\left[\begin{array}{llllll}
I_{3} & h_{11} I_{3} & \ldots & h_{j k} I_{3} & \ldots & h_{j n_{e}} I_{3}
\end{array}\right]
$$

quando do enriquecimento do campo de tensões no domínio;

$$
\Delta_{j}=\left[\begin{array}{llllll}
I_{2} & h_{11} I_{2} & \ldots & h_{j k} I_{2} & \ldots & h_{j n_{e}} I_{2}
\end{array}\right]
$$

quando do enriquecimento do campo de deslocamentos no domínio; e

$$
\Delta_{\Gamma_{j}}=\left[\begin{array}{lllllll}
I_{2} & h_{11} I_{2} & \ldots & h_{j k} I_{2} & \ldots & h_{j n_{e_{\Gamma}}} I_{2}
\end{array}\right]
$$

quando do enriquecimento do campo de deslocamentos no contorno. 
Nas eq.(4.64) a eq.(4.66) $\boldsymbol{I}_{2}$ e $\boldsymbol{I}_{3}$ são as matrizes identidades de terceira e segunda ordem respectivamente, uma vez que em cada nó são definidos três graus de liberdade de tensão no domínio e dois de deslocamentos no domínio e contorno.

Claramente, se as funções $\boldsymbol{h}_{\boldsymbol{j} \boldsymbol{n}_{\boldsymbol{e}}}$ e $\boldsymbol{h}_{\boldsymbol{j} \boldsymbol{n}_{\boldsymbol{e}}}$ são nulas, preserva-se, com as matrizes $\boldsymbol{I}_{2} \mathrm{e}$ $\boldsymbol{I}_{3}$, a estrutura convencional do MEF.

Formas habituais para as funções bolhas $\boldsymbol{h}_{\boldsymbol{j} \boldsymbol{n}_{\boldsymbol{e}}}$ e $\boldsymbol{h}_{\boldsymbol{j} \boldsymbol{n}_{\boldsymbol{e}}}$ (expressas em coordenadas naturais) são:

$$
\begin{aligned}
& \text { - } x \\
& h_{j n_{e}}(\xi, \eta)=\left(\varphi_{1} x_{1}+\varphi_{2} x_{2}+\varphi_{3} x_{3}+\varphi_{4} x_{4}\right)-x_{j} \\
& \text { - } y \\
& h_{j n_{e}}(\xi, \eta)=\left(\varphi_{1} y_{1}+\varphi_{2} y_{2}+\varphi_{3} y_{3}+\varphi_{4} y_{4}\right)-y_{j} \\
& \text { - } x y \\
& h_{j n_{e}}(\xi, \eta)=\left[\left(\varphi_{1} x_{1}+\varphi_{2} x_{2}+\varphi_{3} x_{3}+\varphi_{4} x_{4}\right)-x_{j}\right]\left[\left(\varphi_{1} y_{1}+\varphi_{2} y_{2}+\varphi_{3} y_{3}+\varphi_{4} y_{4}\right)-y_{j}\right] \\
& \text { - } x^{2} \\
& h_{j n_{e}}(\xi, \eta)=\left[\left(\varphi_{1} x_{1}+\varphi_{2} x_{2}+\varphi_{3} x_{3}+\varphi_{4} x_{4}\right)-x_{j}\right]^{2} \\
& \text { - } y^{2} \\
& h_{j n_{e}}(\xi, \eta)=\left[\left(\varphi_{1} y_{1}+\varphi_{2} y_{2}+\varphi_{3} y_{3}+\varphi_{4} y_{4}\right)-y_{j}\right]^{2} \\
& \text { - } x \\
& h_{j n_{e_{\Gamma}}}(\xi, \eta)=\left(\psi_{1} x_{1}+\psi_{2} x_{2}\right)-x_{j} \\
& \text { - } y \\
& h_{j n_{e_{\Gamma}}}(\xi, \eta)=\left(\psi_{1} y_{1}+\psi_{2} y_{2}\right)-y_{j}
\end{aligned}
$$

Assim para qualquer nó $\boldsymbol{j},(\boldsymbol{j}=\mathbf{1}, \ldots, 4)$ do elemento finito retangular $\boldsymbol{e}$, escolhido para ser enriquecido, pode-se escrever: 
- Para a matriz representada pela eq.(4.16) e com a eq.(4.61), tem-se:

$$
\begin{aligned}
& S_{\Omega_{e}}^{T} f S_{\Omega_{e}}=\left[\varphi_{\alpha} \varphi_{\beta} \Delta_{\alpha}^{T} f \Delta_{\beta}\right], \alpha=\beta=1, \ldots 4 \\
& F_{e}=\left[F_{\alpha \beta}\right], \alpha=\beta=1, \ldots 4
\end{aligned}
$$

onde:

$$
F_{\alpha \beta}=\left(\int_{\Omega_{e}} \varphi_{\alpha} \varphi_{\beta} \Delta_{\alpha}^{T} f \Delta_{\beta} d \Omega\right), \alpha, \beta=1, \ldots 4
$$

Nota-se que:

$$
\Delta_{a}^{T} f \Delta_{\beta}=\left[\begin{array}{ccccc}
f & \ldots & h_{j \beta} f & \ldots & h_{n_{e} \beta} f \\
\vdots & \ddots & \vdots & \ddots & \vdots \\
h_{j a} f & \cdots & h_{j \alpha} h_{j \beta} f & \cdots & h_{j \alpha} h_{n_{e} \beta} f \\
\vdots & \ddots & \vdots & \ddots & \vdots \\
h_{n_{e} a} f & \cdots & h_{n_{e} \alpha} f & \cdots & h_{n_{e} \alpha} h_{n_{e} \beta} f
\end{array}\right]
$$

Da mesma forma:

- Para a matriz representada pela eq.(4.17) e com as eq.(4.61) e eq.(4.62), escreve-se:

$$
\begin{aligned}
& L S_{\Omega_{e}}=L\left[\begin{array}{llll}
\varphi_{1} \Delta_{1} & \varphi_{2} \Delta_{2} & \varphi_{3} \Delta_{3} & \varphi_{4} \Delta_{4}
\end{array}\right]= \\
& =\left[\begin{array}{lll}
\left(L \varphi_{1}\right) \Delta_{1}+\varphi_{1}\left(L \Delta_{1}\right) & \ldots & \left(L \varphi_{4}\right) \Delta_{4}+\varphi_{4}\left(L \Delta_{4}\right)
\end{array}\right] \\
& \left(L S_{\Omega_{e}}\right)^{T}=\left[\begin{array}{c}
\Delta_{1}^{T}\left(L \varphi_{1}\right)^{T}+\varphi_{1}\left(L \Delta_{1}\right)^{T} \\
\vdots \\
\Delta_{4}^{T}\left(L \varphi_{4}\right)^{T}+\varphi_{4}\left(L \Delta_{4}\right)^{T}
\end{array}\right]
\end{aligned}
$$

$$
\begin{aligned}
& \left(L S_{\Omega_{e}}\right)^{T} U_{\Omega_{e}}=\left(\Delta_{\alpha}^{T}\left(L \varphi_{\alpha}\right)^{T}+\varphi_{\alpha}\left(L \Delta_{\alpha}\right)\right)\left(\varphi_{\beta} \Delta_{\beta}\right), \alpha=\beta=1, \ldots, 4 \\
& A_{\Omega_{e}}=\left[A_{\Omega_{\alpha \beta}}\right], \alpha=\beta=1, \ldots 4
\end{aligned}
$$

onde:

$$
A_{\Omega \alpha \beta}=\left(\int_{\Omega_{e}}\left(\Delta_{a}^{T}\left(L \varphi_{a}\right)^{T}+\varphi_{a}\left(L \Delta_{a}\right)\right)\left(\varphi_{\beta} \Delta_{\beta}\right) d \Omega\right), \alpha, \beta=1, \ldots 4,
$$


- Para a matriz representada pela eq.(4.21) e com a eq.(4.62), escreve-se:

$$
\begin{gathered}
U_{\Omega_{e}}^{T}=\left[\begin{array}{c}
\varphi_{1} \Delta_{1}^{T} \\
\vdots \\
\varphi_{4} \Delta_{4}^{T}
\end{array}\right] \\
Q_{\Omega_{e}}=\left[Q_{\Omega_{\alpha}}\right], \alpha=1 \ldots 4
\end{gathered}
$$

onde:

$$
Q_{\Omega_{\alpha}}=\left[\int_{\Omega_{e}} \varphi_{\alpha} \Delta_{\alpha}^{T} \bar{b} d \Omega\right], \alpha=1 \ldots 4
$$

Para qualquer nó $\boldsymbol{j}(\boldsymbol{j}=\mathbf{1 , 2})$, de cada lado do elemento finito quadrilateral que pertence ao contorno $\Gamma\left(\Gamma_{t}, \Gamma_{i}\right.$ e $\left.\Gamma_{u}\right)$, que é escolhido para ser enriquecido, pode-se escrever:

- Para as matrizes representadas pelas eq.(4.18) e eq.(4.19) e com as eq.(4.61) eq.(4.63), tem-se:

$$
\begin{aligned}
& N S_{\Omega_{e}}=\left[\begin{array}{llll}
\left(N \varphi_{1}\right) \Delta_{1} & \left(N \varphi_{2}\right) \Delta_{2} & \left(N \varphi_{3}\right) \Delta_{3} & \left(N \varphi_{4}\right) \Delta_{4}
\end{array}\right] \\
& \left(N S_{\Omega_{e}}\right)^{T}=\left[\begin{array}{c}
\Delta_{1}^{T}\left(N \varphi_{1}\right)^{T} \\
\vdots \\
\Delta_{4}^{T}\left(N \varphi_{4}\right)^{T}
\end{array}\right] \\
& \left(N S_{\Omega_{e}}\right)^{T}\left\{U_{\Gamma_{T_{e_{T_{t}}}}} \text { ou } U_{\Gamma_{i_{e_{e_{i}}}}}\right\}=\left(\Delta_{a}^{T}\left(N \varphi_{a}\right)^{T}\right)\left(\psi_{\gamma} \Delta_{\Gamma_{\gamma}}\right), \alpha=1, \ldots, 4, \gamma=1,2
\end{aligned}
$$

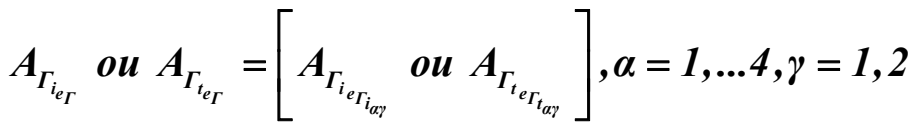

onde:

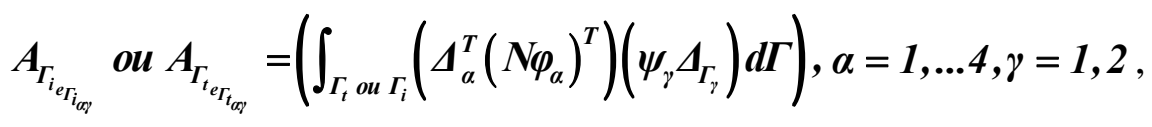

- $\quad$ Para a matriz representada pela eq.(4.20) e com as eq.(4.87), tem-se:

$$
e_{\Gamma_{u_{\text {ue } e_{u}}}}=\left[e_{\Gamma_{u_{n e e_{u_{\alpha}}}}}\right], \alpha=1 . .4
$$


onde:

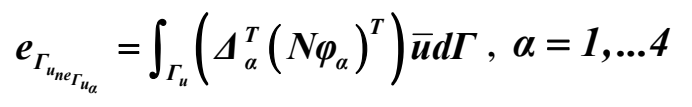

- $\quad$ Para a matriz representada pela eq.(4.22) e com eq.(4.63), escreve-se:

$$
\begin{aligned}
U_{\Gamma_{t}}^{T} & =\left[\begin{array}{l}
\psi_{1} \Delta_{\Gamma_{1}}^{T} \\
\psi_{2} \Delta_{\Gamma_{2}}^{T}
\end{array}\right] \\
Q_{\Gamma_{e_{T_{t}}}} & =\left[Q_{\Gamma_{e_{\Gamma_{t_{y}}}}}\right], \gamma=1,2
\end{aligned}
$$

onde:

$$
Q_{\Gamma_{e_{T_{t_{\gamma}}}}}=\left[\int_{\Gamma_{t}} \psi_{\gamma} \Delta_{\Gamma_{\gamma}}^{T} \bar{t} d \Gamma\right], \gamma=1,2
$$

\subsubsection{Integrais Intervenientes}

Neste item, ilustram-se as integrais intervenientes das eq.(4.75), eq.(4.81), eq.(4.84), eq.(4.89), eq.(4.91) e eq.(4.94) e o procedimento numérico para resolução destas integrais nos domínios paramétricos dos elementos quadrilateral (ver figura 4.1) e linear (ver figura 4.2).

Considere inicialmente a integral envolvida na eq.(4.75). Observa-se que cada integrando $\varphi_{a} \varphi_{\beta} \Delta_{\alpha}^{T} f \Delta_{\beta}, \alpha, \beta=1, \ldots 4$ é constituído basicamente de funções do tipo:

$$
p(\xi, \eta)
$$

assim cada integrando da eq.(4.75) pode ser expressa por:

$$
\int_{\Omega_{e}} p(\xi, \eta) d \Omega
$$

Além disso, tem-se:

$$
d \Omega=d x d y=|J| d \xi d \eta
$$

onde $|\boldsymbol{J}|$ é o determinante da matriz jacobiana ou jacobiano. Para se obter o jacobiano sempre positivo, a conectividade dos nós do elemento finito é feita sempre no sentido de giro de $\boldsymbol{\xi}$ para $\boldsymbol{\eta}$. 
Com todas as variáveis no domínio paramétrico do elemento finito quadrilateral, a eq.(4.97) é escrita da seguinte forma:

$$
\int_{\Omega_{e}} p(\xi, \eta) d \Omega=\int_{-1}^{1} \int_{-1}^{1} p(\xi, \eta)|J| d \xi d \eta
$$

O determinante da matriz jacobiana é dado por:

$$
\begin{gathered}
|J|=\frac{1}{8}\left(-x_{3} y_{2}+x_{3} y_{4}-x_{2} y_{1}-x_{1} y_{4}+x_{2} y_{3}+x_{1} y_{2}-x_{4} y_{3} \eta+\ldots\right. \\
\cdots+x_{4} y_{1}-x_{4} y_{3}+x_{4} y_{2} \eta+x_{2} y_{1} \eta+x_{3} y_{4} \eta+x_{1} y_{4} \xi-x_{1} y_{2} \eta-x_{4} y_{1} \xi-\cdots \\
\left.\cdots-x_{3} y_{1} \eta-x_{2} y_{4} \eta-x_{2} y_{4} \xi+x_{1} y_{3} \eta+x_{3} y_{1} \xi+x_{4} y_{2} \xi-x_{1} y_{3} \xi+x_{2} y_{3} \xi-x_{3} y_{2} \xi\right)
\end{gathered}
$$

Utilizando-se o método de integração numérica de Gauss-Legendre, pode-se calcular a eq.(4.99) da seguinte forma:

$$
\int_{\Omega_{e}} p(\xi, \eta) d x d y=\int_{-1}^{1} \int_{-1}^{1} p(\xi, \eta)|J| d \xi d \eta=\sum_{i=1}^{n} \sum_{j=1}^{m} w_{i} w_{j} p(\xi, \eta)|J|
$$

onde $\boldsymbol{n}$ e $\boldsymbol{m}$ são os números de pontos de Gauss utilizados em cada direção $\boldsymbol{\xi}$ e $\boldsymbol{\eta}$ e $\boldsymbol{w}_{\boldsymbol{i}}$ e $\boldsymbol{w}_{\boldsymbol{j}}$ são os pesos associados aos pontos de Gauss $\boldsymbol{i}$ e $\boldsymbol{j}$, respectivamente. Agora na eq.(4.101), tem-se que a função $p(\xi, \eta)$ e o $|\boldsymbol{J}|$ são calculadas para as coordenadas adimensionais $\boldsymbol{\xi}_{\boldsymbol{i}}$ e $\boldsymbol{\eta}_{\boldsymbol{j}}$ associadas também aos pontos de Gauss $\boldsymbol{i}$ e $\boldsymbol{j}$.

Para a eq.(4.81) o integrando $\left(\Delta_{\alpha}^{T}\left(L \varphi_{\alpha}\right)^{T}+\varphi_{\alpha}\left(L \Delta_{\alpha}\right)\right)\left(\varphi_{\beta} \Delta_{\beta}\right), \alpha, \beta=1, \ldots 4$ envolve funções do tipo:

$$
\frac{\partial p(\xi, \eta)}{\partial x} \cdot s(\xi, \eta) \text { e } \frac{\partial p(\xi, \eta)}{\partial y} \cdot s(\xi, \eta)
$$

dessa forma, com auxílio da eq.(4.98) , eq.(4.30) e eq.(4.31), pode-se escrever:

$$
\left\{\begin{array}{l}
\frac{\partial}{\partial x} \\
\frac{\partial}{\partial y}
\end{array}\right\}=J^{-1}\left\{\begin{array}{l}
\frac{\partial}{\partial \xi} \\
\frac{\partial}{\partial \eta}
\end{array}\right\}
$$




$$
\begin{aligned}
& \operatorname{com} J^{-1}=\frac{1}{|J|}\left[\begin{array}{cc}
\frac{\partial y}{\partial \eta} & -\frac{\partial y}{\partial \xi} \\
-\frac{\partial x}{\partial \eta} & -\frac{\partial x}{\partial \xi}
\end{array}\right] \Rightarrow J^{-1}=\left[\begin{array}{ll}
J_{11}^{-1} & J_{12}^{-1} \\
J_{21}^{-1} & J_{22}^{-1}
\end{array}\right] \text { e }|J|=\frac{\partial x}{\partial \xi} \frac{\partial y}{\partial \eta}-\frac{\partial y}{\partial \xi} \frac{\partial x}{\partial \eta} \\
& J_{11}^{-1}=\left[-2\left(-y_{1} \xi+y_{1}+y_{2} \xi+y_{2}-y_{3} \xi-y_{3}+y_{4} \xi-y_{4}\right)\right] /\left(x_{2} y_{3} \xi-x_{1} y_{3} \xi-x_{4} y_{1} \xi \cdots\right. \\
& \cdots-x_{4} \eta y_{3}+x_{2} y_{3}-x_{2} \eta y_{4}+x_{2} \eta y_{1}-x_{2} y_{4} \xi-x_{3} \eta y_{1}+x_{1} y_{4} \xi-x_{3} y_{2} \xi+x_{3} y_{1} \xi+\cdots \\
& \cdots+x_{4} \xi y_{2}+x_{3} \eta y_{4}+x_{4} y_{2} \eta-x_{2} y_{1}+x_{1} y_{2}-x_{1} y_{4}-\cdots \\
& \left.\cdots-x_{1} \eta y_{2}+x_{1} \eta y_{3}-x_{4} y_{3}-x_{3} y_{2}+x_{3} y_{4}+x_{4} y_{1}\right) \\
& J_{12}^{-1}=\left[-2\left(-y_{1} \eta+y_{1}+y_{2} \eta-y_{2}-y_{3} \eta-y_{3}+y_{4} \eta+y_{4}\right)\right] /\left(x_{2} y_{3} \xi-x_{1} y_{3} \xi-x_{4} y_{1} \xi \cdots\right. \\
& \cdots-x_{4} \eta y_{3}+x_{2} y_{3}-x_{2} \eta y_{4}+x_{2} \eta y_{1}-x_{2} y_{4} \xi-x_{3} \eta y_{1}+x_{1} y_{4} \xi-x_{3} y_{2} \xi+x_{3} y_{1} \xi+\cdots \\
& \cdots+x_{4} \xi y_{2}+x_{3} \eta y_{4}+x_{4} y_{2} \xi-x_{2} y_{1}+x_{1} y_{2}-x_{1} y_{4}-\cdots \\
& \left.\cdots-x_{1} \eta y_{2}+x_{1} \eta y_{3}-x_{4} y_{3}-x_{3} y_{2}+x_{3} y_{4}+x_{4} y_{1}\right) \\
& J_{21}^{-1}=\left[-2\left(-x_{1} \xi-x_{1}-x_{2} \xi-x_{2}+x_{3} \xi+x_{3}-x_{4} \xi+x_{4}\right)\right] /\left(x_{2} y_{3} \xi-x_{1} y_{3} \xi-x_{4} y_{1} \xi \ldots\right. \\
& \cdots-x_{4} \eta y_{3}+x_{2} y_{3}-x_{2} \eta y_{4}+x_{2} \eta y_{1}-x_{2} y_{4} \xi-x_{3} \eta y_{1}+x_{1} y_{4} \xi-x_{3} y_{2} \xi+x_{3} y_{1} \xi+\cdots \\
& \cdots+x_{4} \xi y_{2}+x_{3} \eta y_{4}+x_{4} y_{2} \xi-x_{2} y_{1}+x_{1} y_{2}-x_{1} y_{4}-\cdots \\
& \left.\cdots-x_{1} \eta y_{2}+x_{1} \eta y_{3}-x_{4} y_{3}-x_{3} y_{2}+x_{3} y_{4}+x_{4} y_{1}\right) \\
& J_{22}^{-1}=\left[2\left(-x_{1} \eta-x_{1}+x_{2} \eta+x_{2}+x_{3} \eta+x_{3}-x_{4} \eta-x_{4}\right)\right] /\left(x_{2} y_{3} \xi-x_{1} y_{3} \xi-x_{4} y_{1} \xi \cdots\right. \\
& \cdots-x_{4} \eta y_{3}+x_{2} y_{3}-x_{2} \eta y_{4}+x_{2} \eta y_{1}-x_{2} y_{4} \xi-x_{3} \eta y_{1}+x_{1} y_{4} \xi-x_{3} y_{2} \xi+x_{3} y_{1} \xi+\cdots \\
& \cdots+x_{4} \xi y_{2}+x_{3} \eta y_{4}+x_{4} y_{2} \eta-x_{2} y_{1}+x_{1} y_{2}-x_{1} y_{4}-\cdots \\
& \left.\cdots-x_{1} \eta y_{2}+x_{1} \eta y_{3}-x_{4} y_{3}-x_{3} y_{2}+x_{3} y_{4}+x_{4} y_{1}\right)
\end{aligned}
$$

Assim, expressa-se todo o integrando $\left(\Delta_{\alpha}^{T}\left(L \varphi_{\alpha}\right)^{T}+\varphi_{\alpha}\left(L \Delta_{\alpha}\right)\right)\left(\varphi_{\beta} \Delta_{\beta}\right), \alpha, \beta=1, \ldots 4$, numa única função $q(\xi, \eta)$ nas variáveis do domínio paramétrico $(\xi, \eta)$. Dessa forma:

$$
\int_{\Omega_{e}} q(\xi, \eta) d \Omega=\int_{-1}^{1} \int_{-1}^{1} q(\xi, \eta)|J| d \xi d \eta
$$


Aplicando-se o método de integração numérica de Gauss-Legendre, calcula-se a eq.(4.108) da seguinte forma:

$$
\int_{\Omega_{e}} q(\xi, \eta) d x d y=\int_{-1}^{1} \int_{-1}^{1} q(\xi, \eta)|J| d \xi d \eta=\sum_{i=1}^{n} \sum_{j=1}^{m} w_{i} w_{j} q(\xi, \eta)|J|
$$

onde $\boldsymbol{n}$ e $\boldsymbol{m}$ são os números de pontos de Gauss utilizados em cada direção $\boldsymbol{\xi}$ e $\boldsymbol{\eta}$ e $\boldsymbol{w}_{\boldsymbol{i}}$ e $\boldsymbol{w}_{\boldsymbol{j}}$ são os pesos associados aos pontos de Gauss $\boldsymbol{i}$ e $\boldsymbol{j}$, respectivamente. Agora na eq.(4.109), tem-se que a função $q(\xi, \eta), J_{11}^{-1}, J_{12}^{-1}, J_{21}^{-1}, J_{22}^{-1}$ e o $|\boldsymbol{J}|$ são calculadas para as coordenadas adimensionais $\boldsymbol{\xi}_{\boldsymbol{i}}$ e $\boldsymbol{\eta}_{\boldsymbol{j}}$ associadas também aos pontos de Gauss $\boldsymbol{i}$ e $\boldsymbol{j}$.

$\mathrm{Na}$ eq. (4.84) o integrando possui funções que se assemelham às da eq.(4.97) e por isso a eq.(4.101) pode ser aplicada para a solução numérica de cada integrando da eq.(4.84).

Já para cada integrando da eq.(4.89), $\left(\Delta_{a}^{T}\left(N \varphi_{\alpha}\right)^{T}\right)\left(\psi_{\gamma} \Delta_{\Gamma_{\gamma}}\right), \alpha=1, \ldots 4, \gamma=1,2$, duas das funções $\varphi_{a}, \alpha=1, \ldots 4$, são nulas e as outras tem $\boldsymbol{\xi}$ ou $\boldsymbol{\eta}$ constante, pois a integral é desenvolvida no contorno $\Gamma\left(\Gamma_{t}, \Gamma_{i}\right.$ e $\left.\Gamma_{u}\right)$. Dessa forma, compactam-se os integrandos da eq.(4.89) na seguinte função:

$$
r\left(\xi_{\text {constante }}, \eta\right) \text { ou } r\left(\xi, \eta_{\text {constante }}\right)
$$

Para as integrais de contorno, definem-se:

$$
d \Gamma=J d \xi
$$

ou

$$
d \Gamma=J d \eta
$$

dependendo se o contorno do elemento quadrilateral paramétrico coincide com as retas $\boldsymbol{\eta}$ constante ou $\boldsymbol{\xi}$ constante, respectivamente. O fator Jacobiano da transformação $\boldsymbol{J}$ é calculado com auxílio da eq.(4.37):

$$
J=\frac{\left[\sqrt{\left(x_{2}-x_{1}\right)^{2}+\left(y_{2}-y_{1}\right)^{2}}\right]}{2}
$$


Então, para o elemento finito da figura 4.2, considerando-se $\boldsymbol{\eta}$ constante, por exemplo, a integral da eq.(4.89) pode ser expressa resumidamente da seguinte forma:

$$
\int_{\Gamma_{t} \text { ou } \Gamma_{i}} r\left(\xi, \eta_{\text {constante }}\right) d \Gamma=\int_{-1}^{1} r\left(\xi, \eta_{\text {constante }}\right) J d \xi
$$

Utilizando-se o método de integração numérica de Gauss-Legendre, pode-se calcular eq.(4.114) da seguinte forma:

$$
\int_{\Gamma_{t} \text { ou } \Gamma} r(x, y) d \Gamma=\int_{-1}^{1} r\left(\xi, \eta_{\text {constante }}\right) J d \xi=\sum_{i=1}^{n} w_{i} r\left(\xi, \eta_{\text {constante }}\right) J
$$

onde $\boldsymbol{n}$ são os números de pontos de Gauss utilizados na direção $\boldsymbol{\xi}$ e $\boldsymbol{w}_{\boldsymbol{i}}$ são os pesos associados aos pontos de Gauss $\boldsymbol{i}$. Na eq.(4.115), tem-se que a função $\boldsymbol{r}\left(\boldsymbol{\xi}, \boldsymbol{\eta}_{\text {constante }}\right)$ e $\boldsymbol{J}$ são calculadas para os $\boldsymbol{\xi}_{i}$, também associados a cada ponto de Gauss $\boldsymbol{i}$.

Finalmente, a eq.(4.115) também pode ser utilizada na solução numérica das integrais das eq.(4.91) e eq.(4.94).

Para tornar o cálculo da integração numérica eficiente, foi utilizado o menor número de pontos de Gauss que integre sem erros as equações do tipo eq.(4.101), eq.(4.109) e eq.(4.115). Este número de pontos foi validado por meio de testes numéricos.

\subsubsection{Elemento Triangular de Três Nós}

\subsubsection{Características Geométricas e Transformação de Coordenadas}

Para os elementos triangulares é comum a utilização das coordenadas triangulares normalizadas, Savassi (1996), Assan (2003) e Soriano (2003). Para defini-las, considere-se inicialmente o triângulo de área $\boldsymbol{A}_{\boldsymbol{e}}$, ver figura 4.3. Seja ainda nesta figura um ponto $\boldsymbol{P}$ interno ao elemento de domínio triangular $\boldsymbol{\Omega}_{e}$, com coordenadas $\boldsymbol{x}$ e $\boldsymbol{y}$ num domínio global $\boldsymbol{x y}$. Definem-se também as áreas dos triângulos $\boldsymbol{P 1 2}, \boldsymbol{P} 23$ e $\boldsymbol{P 3 1}$, respectivamente, $\boldsymbol{A}_{1}, \boldsymbol{A}_{2}$ e $\boldsymbol{A}_{3}$, ver figura 4.3 . 


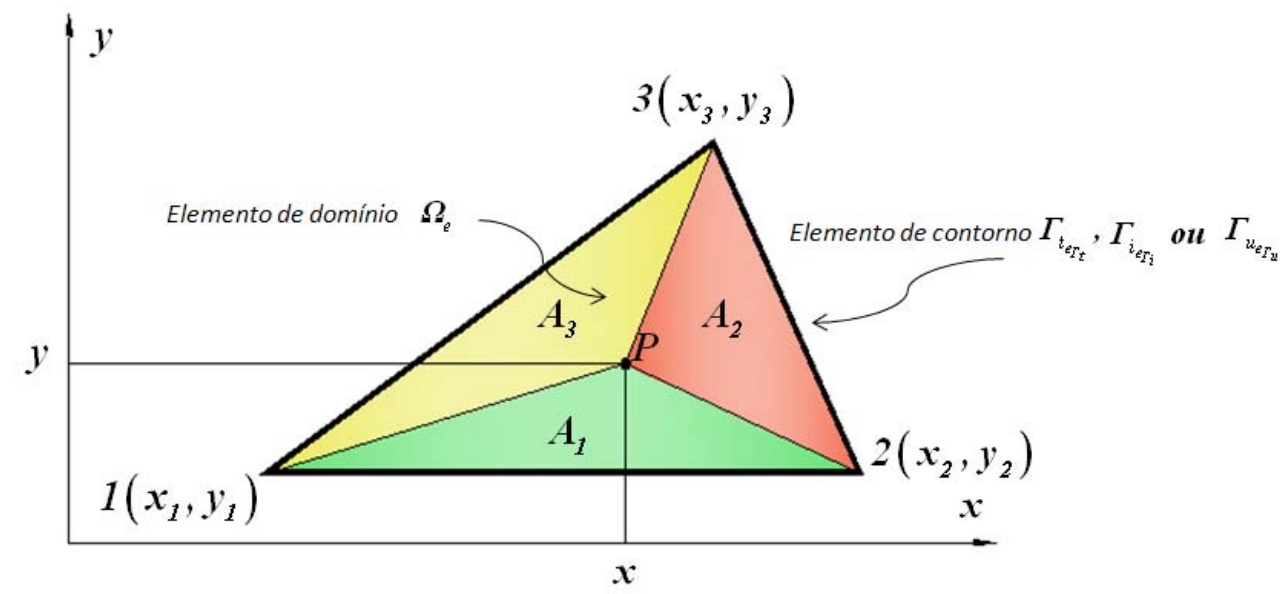

Figura 4.3 - Elemento triangular de três nós.

Assim, as coordenadas triangulares normalizadas podem ser interpretadas como relações entre as áreas dos triângulos $\boldsymbol{A}_{1}, \boldsymbol{A}_{2}$ e $\boldsymbol{A}_{3}$ e a área total, conforme as equações abaixo:

$$
\begin{aligned}
& \xi_{1}=\frac{A_{1}}{A_{e}} \\
& \xi_{2}=\frac{A_{2}}{A_{e}} \\
& \xi_{3}=\frac{A_{3}}{A_{e}}
\end{aligned}
$$

É evidente que o ponto $\boldsymbol{P}$ fica definido por $\xi_{1}, \xi_{2}$ e $\xi_{3}$. Como $\boldsymbol{A}_{1}+\boldsymbol{A}_{2}+\boldsymbol{A}_{3}=\boldsymbol{A}_{e}$ e considerando-se as coordenadas dos nós do elemento triangular definido na figura 4.3, têm-se as seguintes relações:

$$
\begin{aligned}
& x=\xi_{1} x_{1}+\xi_{2} x_{2}+\xi_{3} x_{3} \\
& y=\xi_{1} y_{1}+\xi_{2} y_{2}+\xi_{3} y_{3} \\
& \xi_{1}+\xi_{2}+\xi_{3}=1
\end{aligned}
$$

Da eq. (4.121), pode-se concluir que as coordenadas triangulares normalizadas não são independentes, pois $\xi_{3}$ pode ser obtida por meio das outras duas coordenadas. 
As eq.(4.119) e eq.(4.120) são as relações entre as coordenadas cartesianas $\boldsymbol{x y}$ (domínio global) e as coordenadas triangulares normalizadas (domínio paramétrico). Assim, defini-se a matriz $\boldsymbol{M}_{T}$ composta pelas coordenadas triangulares normalizadas:

$$
M_{T}=\left[\begin{array}{cccccc}
\xi_{1} & 0 & \xi_{2} & 0 & \xi_{3} & 0 \\
0 & \xi_{1} & 0 & \xi_{2} & 0 & \xi_{3}
\end{array}\right]
$$

Propõe-se também o vetor $\boldsymbol{c}_{\boldsymbol{T}}$ que guarda as coordenadas nodais do elemento finito triangular, dado por:

$$
c_{T}^{T}=\left[\begin{array}{llllll}
x_{1} & y_{1} & x_{2} & y_{2} & x_{3} & y_{3}
\end{array}\right]
$$

Então as coordenadas $\boldsymbol{x y}$ globais de um ponto $\boldsymbol{P}$ do elemento finito triangular podem ser mapeadas a partir do elemento parametrizado (via coordenadas normalizadas do elemento triangular) por meio da seguinte transformação:

$$
\left\{\begin{array}{c}
x \\
y
\end{array}\right\}=M_{T} c_{T}^{T}
$$

Para melhor compreender a utilização das coordenadas triangulares normalizadas como forma de parametrização do elemento finito triangular, mapeia-se o elemento da figura 4.3 no espaço $\xi_{1} \xi_{2}$, ver figura 4.4 .

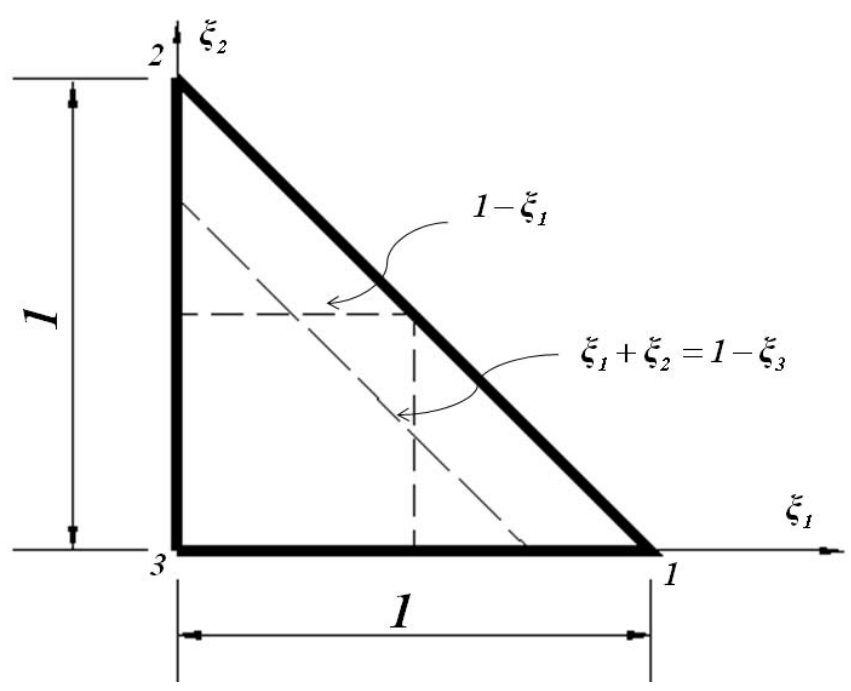

Figura 4.4 - Elemento triangular de três nós no espaço $\xi_{1} \xi_{2}$. 
Na figura 4.4, a equação da reta paralela ao lado é $\xi_{1}+\xi_{2}=1-\xi_{3}$ (eq.(4.121)). O domínio triangular na figura 4.4 pode ser idealizado parametrizado em termos do sistema de coordenadas $(\xi, \eta)$, com $\xi_{1}=\xi, \xi_{2}=\boldsymbol{\eta}, \xi_{3}=1-\xi-\eta$, origem no nó $3, \boldsymbol{\xi}$ variando de 0 a 1 e $\boldsymbol{\eta}$ variando de 0 a $(\boldsymbol{1}-\boldsymbol{\xi})$. Destaca-se ainda na figura 4.4 a numeração de nós (sempre no sentido anti-horário) do elemento utilizada na ferramenta computacional desenvolvida neste trabalho.

Com auxílio das eq.(4.116) a eq.(4.118) e lembrando que as áreas $\boldsymbol{A}_{1}, \boldsymbol{A}_{2}, \boldsymbol{A}_{3}$ e $\boldsymbol{A}_{\boldsymbol{e}}$ podem ser obtidas de:

$$
\begin{aligned}
& A=\frac{1}{2}\left[\begin{array}{lll}
1 & x_{1} & y_{1} \\
1 & x_{2} & y_{2} \\
1 & x_{3} & y_{3}
\end{array}\right] \\
& A_{1}=\frac{1}{2}\left[\begin{array}{lll}
1 & x & y \\
1 & x_{2} & y_{2} \\
1 & x_{3} & y_{3}
\end{array}\right] \\
& A_{2}=\frac{1}{2}\left[\begin{array}{lll}
1 & x_{1} & y_{1} \\
1 & x & y \\
1 & x_{3} & y_{3}
\end{array}\right] \\
& A_{3}=\frac{1}{2}\left[\begin{array}{lll}
1 & x_{1} & y_{1} \\
1 & x_{2} & y_{2} \\
1 & x & y
\end{array}\right]
\end{aligned}
$$

é possível estabelecer relações entre as coordenadas triangulares parametrizadas e as coordenadas cartesianas $\boldsymbol{x y}$. Logo, tem-se:

$$
\begin{aligned}
& \xi_{1}=\frac{1}{2 A_{e}}\left[x_{2} y_{3}-x_{3} y_{2}+x\left(y_{2}-y_{3}\right)+y\left(x_{3}-x_{2}\right)\right] \\
& \xi_{2}=\frac{1}{2 A_{e}}\left[x_{3} y_{1}-x_{1} y_{3}+x\left(y_{3}-y_{1}\right)+y\left(x_{1}-x_{3}\right)\right] \\
& \xi_{3}=\frac{1}{2 A_{e}}\left[x_{1} y_{2}-x_{2} y_{1}+x\left(y_{1}-y_{2}\right)+y\left(x_{2}-x_{1}\right)\right]
\end{aligned}
$$


Para o elemento triangular de três nós são válidas também as eq.(4.30) e eq.(4.31) que versam sobre a apresentação de operadores diferenciais no referencial paramétrico $\boldsymbol{\xi} \boldsymbol{\eta}$ em função dos definidos no referencial global $\boldsymbol{x y}$.

Para o elemento no contorno $\Gamma\left(\boldsymbol{\Gamma}_{u_{e_{T_{u}}}}, \boldsymbol{\Gamma}_{\boldsymbol{t}_{e_{\Gamma_{t}}}}\right.$ ou $\left.\boldsymbol{\Gamma}_{\boldsymbol{i}_{e_{\Gamma_{i}}}}\right)$ pertencente a um dos lados do elemento triangular $\boldsymbol{\Omega}_{\boldsymbol{e}}$ definidos nas coordenadas $\boldsymbol{x y}$ globais mapeado para um domínio paramétrico, ver figura 4.5. Neste domínio paramétrico, define-se $\boldsymbol{\xi}$ como coordenada adimensional com sistema de referência sempre no nó 1 do elemento linear.

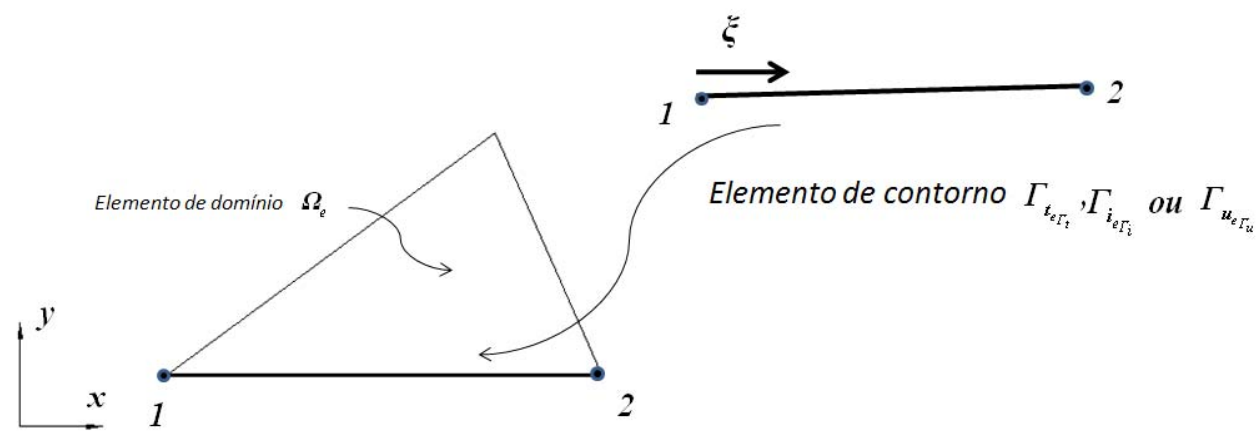

Figura 4.5 - Elemento finito linear com sistema de referência $\boldsymbol{\xi}$.

Na transformação de coordenadas do elemento linear no contorno físico para o contorno paramétrico, utilizam-se as seguintes funções:

$$
\begin{gathered}
\psi_{1}=1-\xi \\
\psi_{2}=\xi
\end{gathered}
$$

Seja a matriz $\boldsymbol{M}_{\boldsymbol{I}_{T}}$ composta pelas funções de interpolação das eq.(4.132) e eq.(4.133), dada por:

$$
M_{\Gamma_{T}}=\left[\begin{array}{cccc}
\psi_{1} & 0 & \psi_{2} & 0 \\
0 & \psi_{1} & 0 & \psi_{2}
\end{array}\right]
$$

Considere-se também o vetor $\boldsymbol{c}_{\boldsymbol{\Gamma}_{T}}$ que guarda as coordenadas nodais no domínio físico do elemento finito no contorno, dado por:

$$
c_{\Gamma_{T}}^{T}=\left[\begin{array}{llll}
x_{1} & y_{1} & x_{2} & y_{2}
\end{array}\right]
$$


Então as coordenadas $\boldsymbol{x y}$ globais de um ponto da rede dos elementos finitos lineares podem ser mapeadas a partir do elemento parametrizado por meio da seguinte transformação:

$$
\left\{\begin{array}{l}
x \\
y
\end{array}\right\}=M_{\Gamma_{T}} c_{\Gamma_{T}}^{T}
$$

Por fim, o diferencial de comprimento no domínio global $\boldsymbol{x y}$ em relação ao diferencial de comprimento no domínio paramétrico é calculado igualmente à forma apresentada nas eq.(4.37).

\subsubsection{Funções Aproximativas do Elemento Triangular de Três Nós da FHMT}

Igualmente ao elemento quadrilateral, para as funções aproximativas das tensões e deslocamentos no domínio, serão adotadas as mesmas funções utilizadas na aproximação da geometria do elemento triangular de três nós. Dessa forma, as matrizes $\boldsymbol{S}_{\Omega_{e}}$ (eq.(4.5)) e $\boldsymbol{U}_{\boldsymbol{\Omega}_{e}}$ (eq.(4.6)) sem enriquecimento são escritas da seguinte forma:

$$
\begin{aligned}
S_{\Omega_{e}} & =\left[\begin{array}{ccccccccc}
\xi_{1} & 0 & 0 & \xi_{2} & 0 & 0 & \xi_{3} & 0 & 0 \\
0 & \xi_{1} & 0 & 0 & \xi_{2} & 0 & 0 & \xi_{3} & 0 \\
0 & 0 & \xi_{1} & 0 & 0 & \xi_{2} & 0 & 0 & \xi_{3}
\end{array}\right] \\
U_{\Omega_{e}} & =\left[\begin{array}{cccccc}
\xi_{1} & 0 & \xi_{2} & 0 & \xi_{3} & 0 \\
0 & \xi_{1} & 0 & \xi_{2} & 0 & \xi_{3}
\end{array}\right]
\end{aligned}
$$

Com $s_{\Omega_{e}}$ (eq.(4.5)) e $\boldsymbol{q}_{\Omega_{e}}$ (eq.(4.6)) definidos por:

$$
\begin{aligned}
s_{\Omega_{e}}^{T} & =\left[\begin{array}{lllllllll}
\sigma_{x_{1}} & \sigma_{y_{1}} & \tau_{x y_{1}} & \sigma_{x_{2}} & \sigma_{y_{2}} & \tau_{x y_{2}} & \sigma_{x_{3}} & \sigma_{y_{3}} & \tau_{x y_{3}}
\end{array}\right] \\
\boldsymbol{q}_{\Omega_{e}}^{T} & =\left[\begin{array}{lllllll}
u_{x_{1}} & u_{y_{1}} & u_{x_{2}} & u_{y_{2}} & u_{x_{3}} & u_{y_{3}}
\end{array}\right]
\end{aligned}
$$

Já as funções de forma dos deslocamentos no contorno $\Gamma\left(\Gamma_{u_{e_{\Gamma_{u}}}}, \boldsymbol{\Gamma}_{\boldsymbol{t}_{\boldsymbol{e}_{\Gamma_{t}}}}\right.$ ou $\left.\boldsymbol{\Gamma}_{\boldsymbol{i}_{e_{\Gamma_{i}}}}\right)$ também são as mesmas definidas para aproximação da geometria do elemento linear de dois nós. Então as matrizes $\boldsymbol{U}_{\Gamma_{{ }_{e_{e_{t}}}}}$ (eq.(4.7)) e $\boldsymbol{U}_{\Gamma_{{ }_{i_{e_{i}}}}}$ (eq.(4.8)) sem enriquecimento são escritas: 
$\left[\begin{array}{cccc}\psi_{1} & 0 & \psi_{2} & 0 \\ 0 & \psi_{1} & 0 & \psi_{2}\end{array}\right]$

Os vetores $\boldsymbol{q}_{\Gamma_{e_{\Gamma_{t}}}}$ (eq.(4.7)) e $\boldsymbol{q}_{\Gamma_{{ }_{\Gamma_{i}}}}$ (eq.(4.8)) dados por:

$$
\boldsymbol{q}_{\Gamma_{e_{T_{t}}}}^{T} \text { ou } \boldsymbol{q}_{\Gamma_{e_{T_{i}}}}^{T}=\left[\begin{array}{llll}
u_{x_{1}} & u_{y_{1}} & u_{x_{2}} & u_{y_{2}}
\end{array}\right]
$$

\subsubsection{Enriquecimento das Funções Aproximativas do Elemento Triangular de Três Nós da FHMT}

As famílias de funções das tensões e deslocamentos no domínio e deslocamentos no contorno e suas aproximações, construídas para o elemento quadrilateral (eq.(4.45) a eq.(4.50)) também podem ser aplicadas ao elemento triangular, observando-se, é claro, as mudanças no tocante às bases de aproximação tensões (eq.(4.137)) e deslocamentos (eq.(4.138)) no domínio, deslocamentos no contorno (eq.(4.141)) e das funções polinomiais enriquecedoras das variáveis de domínio e contorno $\boldsymbol{h}_{\boldsymbol{j} \boldsymbol{n}_{\boldsymbol{e}}}$ e $\boldsymbol{h}_{\boldsymbol{j} \boldsymbol{n}_{\boldsymbol{e}}}$, respectivamente.

Todas as recomendações relacionadas ao enriquecimento das bases de aproximação do elemento quadrilateral da FHMT são válidas para o elemento triangular da FHMT, ou seja, o enriquecimento deve ser desenvolvido sempre no domínio global.

Assim, as bases de aproximação da FHMT originais (eq.(4.137), eq.(4.138) e eq.(4.141)), são expressas da seguinte forma:

$$
\begin{aligned}
& S_{\Omega_{e}}=\left[\begin{array}{llll}
\xi_{1} \Delta_{1} & \xi_{2} \Delta_{2} & \xi_{3} \Delta_{3}
\end{array}\right] \\
& U_{\Omega_{e}}=\left[\begin{array}{llll}
\xi_{1} \Delta_{1} & \xi_{2} \Delta_{2} & \xi_{3} \Delta_{3}
\end{array}\right] \\
& U_{\Gamma_{T_{e_{T_{t}}}}} \text { ou } U_{\Gamma_{i_{e_{T_{i}}}}}=\left[\begin{array}{ll}
\psi_{1} \Delta_{\Gamma_{1}} & \psi_{2} \Delta_{\Gamma_{2}}
\end{array}\right]
\end{aligned}
$$

onde $\boldsymbol{\Delta}_{j}, j=1, \ldots, 3$ e $\boldsymbol{\Delta}_{\Gamma_{j}}, \boldsymbol{j}=1,2$ são, respectivamente, as matrizes de enriquecimento polinomial do nó $\boldsymbol{j}$ de domínio ou contorno.

As matrizes de enriquecimento polinomial são dadas por:

$$
\Delta_{j}=\left[\begin{array}{llllll}
I_{3} & h_{11} I_{3} & \ldots & h_{j k} I_{3} & \ldots & h_{j n_{e}} I_{3}
\end{array}\right]
$$

quando do enriquecimento do campo de tensões no domínio; 


$$
\Delta_{j}=\left[\begin{array}{llllll}
I_{2} & h_{11} I_{2} & \ldots & h_{j k} I_{2} & \ldots & h_{j n_{e}} I_{2}
\end{array}\right]
$$

quando do enriquecimento do campo de deslocamentos no domínio; e

$$
\Delta_{\Gamma_{j}}=\left[\begin{array}{lllllll}
I_{2} & h_{11} I_{2} & \ldots & h_{j k} I_{2} & \ldots & h_{j n_{e_{\Gamma}}} I_{2}
\end{array}\right]
$$

quando do enriquecimento do campo de deslocamentos no contorno.

$\boldsymbol{I}_{2}$ e $\boldsymbol{I}_{3}$ pontuadas nas eq.(4.146) a eq.(4.148) são as matrizes identidades de terceira e segunda ordem respectivamente, uma vez que em cada nó são definidos três graus de liberdade de tensão no domínio e dois de deslocamentos no domínio e contorno.

Como já salientado, se as funções de enriquecimento $\boldsymbol{h}_{\boldsymbol{j} \boldsymbol{n}_{\boldsymbol{e}}}$ e $\boldsymbol{h}_{\boldsymbol{\boldsymbol { j } _ { \boldsymbol { e } }} \boldsymbol{}}$ são nulas, preservase, com as matrizes $\boldsymbol{I}_{2}$ e $\boldsymbol{I}_{3}$, a estrutura convencional do MEF.

Formas habituais para as funções bolhas enriquecedoras $\boldsymbol{h}_{\boldsymbol{j} \boldsymbol{n}_{\boldsymbol{e}}}$ e $\boldsymbol{h}_{\boldsymbol{j} \boldsymbol{n}_{\boldsymbol{e}_{\Gamma}}}$ (expressas em coordenadas naturais) são:

- $\boldsymbol{x}$

$$
\begin{gathered}
h_{j n_{e}}\left(\xi_{1}, \xi_{2}\right)=\left(\xi_{1} x_{1}+\xi_{2} x_{2}+\xi_{3} x_{3}\right)-x_{j} \\
\bullet \quad y \\
h_{j n_{e}}\left(\xi_{1}, \xi_{2}\right)=\left(\xi_{1} y_{1}+\xi_{2} y_{2}+\xi_{3} y_{3}\right)-y_{j}
\end{gathered}
$$

- $x y$

$$
\begin{aligned}
& h_{j n_{e}}\left(\xi_{1}, \xi_{2}\right)=\left[\left(\xi_{1} x_{1}+\xi_{2} x_{2}+\xi_{3} x_{3}\right)-x_{j}\right]\left[\left(\xi_{1} y_{1}+\xi_{2} y_{2}+\xi_{3} y_{3}\right)-y_{j}\right] \\
& \qquad x^{2} \\
& h_{j n_{e}}\left(\xi_{1}, \xi_{2}\right)=\left[\left(\xi_{1} x_{1}+\xi_{2} x_{2}+\xi_{3} x_{3}\right)-x_{j}\right]^{2} \\
& \bullet y^{2} \\
& h_{j n_{e}}\left(\xi_{1}, \xi_{2}\right)=\left[\left(\xi_{1} y_{1}+\xi_{2} y_{2}+\xi_{3} y_{3}\right)-y_{j}\right]^{2}
\end{aligned}
$$




$$
\begin{gathered}
h_{j n_{e_{\Gamma}}}\left(\psi_{1}, \psi_{2}\right)=\left(\psi_{1} x_{1}+\psi_{2} x_{2}\right)-x_{j} \\
\bullet \quad y \\
h_{j n_{e_{\Gamma}}}(\xi, \eta)=\left(\psi_{1} y_{1}+\psi_{2} y_{2}\right)-y_{j}
\end{gathered}
$$

Dessa forma para qualquer nó $j,(j=1, \ldots, 3)$ do elemento finito triangular $\boldsymbol{e}$, escolhido para ser enriquecido, pode-se escrever:

- $\quad$ Para a matriz representada pela eq.(4.16) e com a eq.(4.143), tem-se:

$$
\begin{aligned}
& S_{\Omega_{e}}^{T} f S_{\Omega_{e}}=\left[\varphi_{\alpha} \varphi_{\beta} \Delta_{\alpha}^{T} f \Delta_{\beta}\right], \alpha=\beta=1, \ldots 3 \\
& F_{e}=\left[F_{\alpha \beta}\right], \alpha=\beta=1, \ldots 3
\end{aligned}
$$

onde:

$$
F_{\alpha \beta}=\left(\int_{\Omega_{e}} \varphi_{\alpha} \varphi_{\beta} \Delta_{\alpha}^{T} f \Delta_{\beta} d \Omega\right), \alpha, \beta=1, \ldots 3,
$$

Nota-se que:

$$
\Delta_{\alpha}^{T} f \Delta_{\beta}=\left[\begin{array}{ccccc}
f & \ldots & h_{j \beta} f & \ldots & h_{n_{e} \beta} f \\
\vdots & \ddots & \vdots & \ddots & \vdots \\
h_{j \alpha} f & \cdots & h_{j \alpha} h_{j \beta} f & \cdots & h_{j \alpha} h_{n_{e} \beta} f \\
\vdots & \ddots & \vdots & \ddots & \vdots \\
h_{n_{e} a} f & \cdots & h_{n_{e} a} f & \cdots & h_{n_{e} \alpha} h_{n_{e} \beta} f
\end{array}\right]
$$

Da mesma forma:

- Para a matriz representada pela eq.(4.17) e com as eq.(4.143) e eq.(4.144), escreve-se:

$$
\begin{aligned}
& L S_{\Omega_{e}}=L\left[\begin{array}{lll}
\xi_{1} \Delta_{1} & \xi_{2} \Delta_{2} & \xi_{3} \Delta_{3}
\end{array}\right]= \\
& =\left[\begin{array}{lll}
\left(L \xi_{1}\right) \Delta_{1}+\xi_{1}\left(L \Delta_{1}\right) & \ldots & \left(L \xi_{3}\right) \Delta_{3}+\xi_{3}\left(L \Delta_{3}\right)
\end{array}\right] \\
& \left(L S_{\Omega_{e}}\right)^{T}=\left[\begin{array}{c}
\Delta_{1}^{T}\left(L \xi_{1}\right)^{T}+\xi_{1}\left(L \Delta_{1}\right)^{T} \\
\vdots \\
\Delta_{3}^{T}\left(L \xi_{3}\right)^{T}+\xi_{3}\left(L \Delta_{3}\right)^{T}
\end{array}\right]
\end{aligned}
$$




$$
\begin{aligned}
& \left(L S_{\Omega_{e}}\right)^{T} U_{\Omega_{e}}=\left(\Delta_{\alpha}^{T}\left(L \xi_{\alpha}\right)^{T}+\xi_{\alpha}\left(L \Delta_{\alpha}\right)\right)\left(\xi_{\beta} \Delta_{\beta}\right), \alpha=\beta=1, \ldots, 3 \\
& A_{\Omega_{e}}=\left[A_{\Omega_{\alpha \beta}}\right], \alpha=\beta=1, \ldots 3
\end{aligned}
$$

onde:

$$
A_{\Omega \alpha \beta}=\left(\int_{\Omega_{e}}\left(\Delta_{a}^{T}\left(L \varphi_{a}\right)^{T}+\varphi_{a}\left(L \mathcal{A}_{a}\right)\right)\left(\varphi_{\beta} \Delta_{\beta}\right) d \Omega\right), \alpha, \beta=1, \ldots .3
$$

- Para a matriz representada pela eq.(4.21) e com a eq.(4.144), escreve-se:

$$
\begin{aligned}
U_{\Omega_{e}}^{T} & =\left[\begin{array}{c}
\xi_{1} \Delta_{1}^{T} \\
\vdots \\
\xi_{3} \Delta_{3}^{T}
\end{array}\right] \\
Q_{\Omega_{e}} & =\left[Q_{\Omega_{\alpha}}\right], \alpha=1 \ldots 3
\end{aligned}
$$

onde:

$$
Q_{\Omega_{\alpha}}=\left[\int_{\Omega_{e}} \xi_{\alpha} \Delta_{\alpha}^{T} \bar{b} d \Omega\right], \alpha=1 \ldots 3
$$

Para qualquer nó $\boldsymbol{j}(\boldsymbol{j}=\mathbf{1 , 2})$, de cada lado do elemento finito quadrilateral que pertence ao contorno $\Gamma\left(\Gamma_{t}, \Gamma_{i}\right.$ e $\left.\Gamma_{u}\right)$, que é escolhido para ser enriquecido, pode-se escrever:

- Para as matrizes representadas pelas eq.(4.18) e eq.(4.19) e com as eq.(4.143) eq.(4.145), tem-se:

$$
\begin{aligned}
& N S_{\Omega_{e}}=\left[\begin{array}{lll}
\left(N \xi_{1}\right) \Delta_{1} & \left(N \xi_{2}\right) \Delta_{2} & \left(N \xi_{3}\right) \Delta_{3}
\end{array}\right] \\
& \left(N S_{\Omega_{e}}\right)^{T}=\left[\begin{array}{c}
\Delta_{1}^{T}\left(N \xi_{1}\right)^{T} \\
\vdots \\
\Delta_{3}^{T}\left(N \xi_{3}\right)^{T}
\end{array}\right] \\
& \left(N S_{\Omega_{e}}\right)^{T}\left\{U_{\Gamma_{i_{e_{T_{t}}}}} \text { oи } U_{\Gamma_{i_{e_{T_{i}}}}}\right\}=\left(\Delta_{\alpha}^{T}\left(N \xi_{\alpha}\right)^{T}\right)\left(\psi_{\gamma} \Delta_{\Gamma_{\gamma}}\right), \alpha=1, \ldots, 3, \gamma=1,2
\end{aligned}
$$

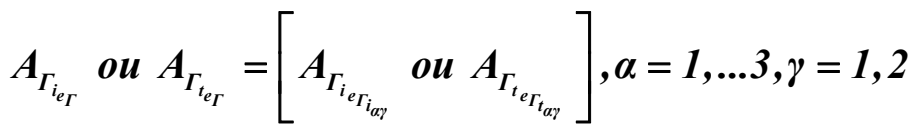


onde:

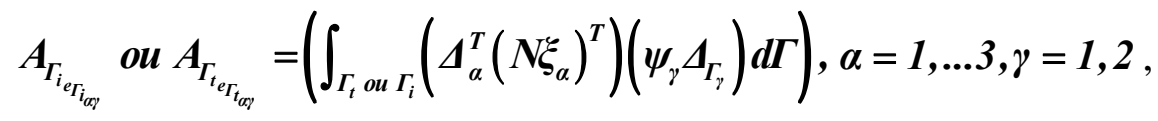

- Para a matriz representada pela eq.(4.20) e com as eq.(4.169), tem-se:

$$
e_{\Gamma_{u_{u_{e} T_{u}}}}=\left[e_{\Gamma_{u_{n e_{u_{\alpha}}}}}\right], \alpha=1 \ldots 3
$$

onde:

$$
e_{\Gamma_{u_{n e_{T_{u}}}}}=\int_{\Gamma_{u}}\left(\Delta_{a}^{T}\left(N \xi_{\alpha}\right)^{T}\right) \bar{u} d \Gamma, \alpha=1, \ldots 3
$$

- $\quad$ Para a matriz representada pela eq.(4.22) e com eq.(4.145), escreve-se:

$$
\begin{aligned}
& U_{\Gamma_{t}}^{T}=\left[\begin{array}{ll}
\psi_{1} & \Delta_{\Gamma_{1}}^{T} \\
\psi_{2} \Delta_{\Gamma_{2}}^{T}
\end{array}\right] \\
& Q_{\Gamma_{e_{T_{t}}}}=\left[Q_{\Gamma_{e_{\Gamma_{t_{\gamma}}}}}\right], \gamma=1,2
\end{aligned}
$$

onde:

$$
Q_{\Gamma_{e_{T_{y}}}}=\left[\int_{\Gamma_{t}} \psi_{\gamma} \Delta_{\Gamma_{\gamma}}^{T} \bar{t} d \Gamma\right], \gamma=1,2
$$

\subsubsection{Integrais Intervenientes}

Agora, ilustram-se as integrais intervenientes das eq.(4.157), eq.(4.163), eq.(4.166), eq.(4.171), eq.(4.173) e eq.(4.176) e o procedimento numérico para resolução destas integrais nos domínios paramétricos dos elementos triangular (ver figura 4.4) e linear (ver figura 4.5).

Considere inicialmente a integral envolvida na eq.(4.157). Observa-se que cada integrando $\xi_{\alpha} \xi_{\beta} \Delta_{\alpha}^{T} f \Delta_{\beta}, \alpha, \beta=1, \ldots 3$ é constituído basicamente de funções do tipo:

$$
p\left(\xi_{1}, \xi_{2}\right)
$$

assim a eq.(4.178) pode ser expressa por: 


$$
\int_{\Omega_{e}} p\left(\xi_{1}, \xi_{2}\right) d \Omega
$$

Além disso, tem-se:

$$
d \Omega=d x d y=|J| d \xi_{1} d \xi_{2}
$$

onde $|\boldsymbol{J}|$ é o determinante da matriz jacobiana.

Com todas as variáveis no domínio paramétrico do elemento finito triangular, a eq.(4.179) é escrita da seguinte forma:

$$
\int_{\Omega_{e}} p\left(\xi_{1}, \xi_{2}\right) d \Omega=\int_{0}^{1} \int_{0}^{1-\xi_{2}} p\left(\xi_{1}, \xi_{2}\right)|J| d \xi_{1} d \xi_{2}
$$

O determinante da matriz jacobiana para o elemento triangular é dado por:

$$
|J|=x_{1}\left(y_{2}-y_{3}\right)+x_{2}\left(y_{3}-y_{1}\right)+x_{3}\left(y_{1}-y_{2}\right)
$$

Utilizando-se pontos e pesos de Hammer, pode-se calcular a eq.(4.181) da seguinte forma:

$$
\int_{\Omega_{e}} p(\xi, \eta) d x d y=\int_{0}^{1} \int_{0}^{1-\xi_{2}} p\left(\xi_{1}, \xi_{2}\right)|J| d \xi_{1} d \xi_{2}=\sum_{i=1}^{n} w_{i} p\left(\xi_{1}, \xi_{2}\right)|J|
$$

onde $\boldsymbol{n}$ é o número de pontos de Hammer utilizado e $\boldsymbol{w}_{\boldsymbol{i}}$ é o peso associado a cada ponto de Hammer $\boldsymbol{i}$. Agora na eq.(4.183), tem-se que a função $p\left(\xi_{1}, \xi_{2}\right)$ é calculada para as coordenadas adimensionais $\boldsymbol{\xi}_{1_{i}}$ e $\boldsymbol{\xi}_{2_{i}}$ associadas também aos pontos de Hammer $\boldsymbol{i}$.

Para a eq.(4.163) o integrando $\left(\boldsymbol{\Delta}_{a}^{T}\left(\boldsymbol{L} \boldsymbol{\xi}_{\alpha}\right)^{T}+\boldsymbol{\xi}_{\alpha}\left(\boldsymbol{L} \boldsymbol{\Delta}_{\alpha}\right)\right)\left(\xi_{\beta} \Delta_{\beta}\right), \alpha, \boldsymbol{\beta}=1, \ldots 3$ envolve funções do tipo:

$$
\frac{\partial p\left(\xi_{1}, \xi_{2}\right)}{\partial x} \cdot s\left(\xi_{1}, \xi_{2}\right) \text { e } \frac{\partial p\left(\xi_{1}, \xi_{2}\right)}{\partial y} \cdot s\left(\xi_{1}, \xi_{2}\right)
$$

dessa forma, com auxílio da eq.(4.180) e da transformação 


$$
\left\{\begin{array}{c}
\frac{\partial}{\partial x} \\
\frac{\partial}{\partial y}
\end{array}\right\}=J^{-1}\left\{\begin{array}{c}
\frac{\partial}{\partial \xi} \\
\frac{\partial}{\partial \eta}
\end{array}\right\}
$$

com

$$
\begin{aligned}
& J_{11}^{-1}=\left[\left(-y_{2}+y_{3}\right)\right] /\left(-x_{1} y_{2}+x_{1} y_{3}+x_{3} y_{2}+x_{2} y_{1}-y_{1} x_{3}-x_{2} y_{3}\right) \\
& J_{12}^{-1}=\left[\left(-y_{3}+y_{1}\right)\right] /\left(-x_{1} y_{2}+x_{1} y_{3}+x_{3} y_{2}+x_{2} y_{1}-y_{1} x_{3}-x_{2} y_{3}\right) \\
& J_{21}^{-1}=\left[\left(-x_{3}+x_{2}\right)\right] /\left(-x_{1} y_{2}+x_{1} y_{3}+x_{3} y_{2}+x_{2} y_{1}-y_{1} x_{3}-x_{2} y_{3}\right) \\
& J_{22}^{-1}=\left[\left(-x_{1}+x_{3}\right)\right] /\left(-x_{1} y_{2}+x_{1} y_{3}+x_{3} y_{2}+x_{2} y_{1}-y_{1} x_{3}-x_{2} y_{3}\right)
\end{aligned}
$$

expressa-se todo o integrando $\left(\Delta_{\alpha}^{T}\left(\boldsymbol{L} \boldsymbol{\xi}_{\alpha}\right)^{T}+\boldsymbol{\xi}_{\alpha}\left(\boldsymbol{L} \boldsymbol{\Delta}_{\alpha}\right)\right)\left(\boldsymbol{\xi}_{\beta} \boldsymbol{\Delta}_{\beta}\right), \alpha, \boldsymbol{\beta}=1, \ldots 3$, numa única função $q\left(\xi_{1}, \xi_{2}\right)$ nas variáveis do domínio paramétrico $\left(\xi_{1}, \xi_{2}\right)$. Assim:

$$
\int_{\Omega_{e}} q\left(\xi_{1}, \xi_{2}\right) d \Omega=\int_{0}^{1} \int_{0}^{1-\xi_{2}} q\left(\xi_{1}, \xi_{2}\right)|J| d \xi_{1} d \xi_{2}
$$

Aplicando-se pontos e pesos de Hammer, calcula-se a eq.(4.190) da seguinte forma:

$$
\int_{\Omega_{e}} q\left(\xi_{1}, \xi_{2}\right) d x d y=\int_{\Omega_{e}} q\left(\xi_{1}, \xi_{2}\right) d \Omega=\int_{0}^{1} \int_{0}^{1-\xi_{2}} q\left(\xi_{1}, \xi_{2}\right)|J| d \xi_{1} d \xi_{2}=\sum_{i=1}^{n} w_{i} q\left(\xi_{1}, \xi_{2}\right)|J|
$$

onde $\boldsymbol{n}$ é o número de pontos de Hammer utilizado e $\boldsymbol{w}_{\boldsymbol{i}}$ é o peso associado a cada ponto de Hammer $\boldsymbol{i}$. Agora na eq.(4.191), tem-se que a função $q\left(\xi_{1}, \xi_{2}\right)$ é calculada para as coordenadas adimensionais $\boldsymbol{\xi}_{\boldsymbol{1}_{i}}$ e $\boldsymbol{\xi}_{2_{i}}$ associadas também aos pontos de Hammer $\boldsymbol{i}$.

Na eq. (4.166) o integrando possui funções que se assemelham as da eq.(4.179) e por isso a eq.(4.183) pode ser aplicada para solução numérica de cada integrando da eq.(4.166).

Já no integrando da eq.(4.171), $\left(\Delta_{\alpha}^{T}\left(N \xi_{\alpha}\right)^{T}\right)\left(\psi_{\gamma} \Delta_{\Gamma_{\gamma}}\right), \alpha=1, \ldots 3, \gamma=1,2$, uma das funções $\xi_{\alpha}, \alpha=1, \ldots 3$ não pertence ao contorno paratrizado onde é desenvolvida a integração numérica e por isso é nula. Dessa forma, podem-se compactar os integrandos da eq.(4.171) na seguinte função: 
$r(\xi)$

Para as integrais de contorno, definem-se:

$$
d \Gamma=J d \xi
$$

O fator Jacobiano da transformação $\boldsymbol{J}$ é calculado com auxílio da eq.(4.37):

$$
J=\left[\sqrt{\left(x_{2}-x_{1}\right)^{2}+\left(y_{2}-y_{1}\right)^{2}}\right]
$$

Então, para o elemento finito da figura 4.5, cada integral da eq.(4.171) pode ser expressas resumidamente da seguinte forma:

$$
\int_{\Gamma_{t} \text { ou } \Gamma_{i}} r(\xi) d \Gamma=\int_{0}^{1} r(\xi) J d \xi
$$

Utilizando-se o método de integração numérica de Gauss-Legendre, pode-se calcular eq.(4.195) da seguinte forma:

$$
\int_{\Gamma_{t} \text { ou } \Gamma_{i}} r(x, y) d \Gamma=\int_{0}^{1} r(\xi) J d \xi=\sum_{i=1}^{n} w_{i} r(\xi) J
$$

onde $\boldsymbol{n}$ são os números de pontos de Gauss utilizados na direção $\boldsymbol{\xi}$ e $\boldsymbol{w}_{\boldsymbol{i}}$ são os pesos associados aos pontos de Gauss $\boldsymbol{i}$. Na eq.(4.195), tem-se que a função $\boldsymbol{r}(\xi)$ e $\boldsymbol{J}$ são calculadas para os $\boldsymbol{\xi}_{\boldsymbol{i}}$, também associados a cada ponto de Gauss $\boldsymbol{i}$.

Enfim, a eq.(4.196) também pode ser utilizada na solução numérica das integrais das eq.(4.173) e eq.(4.176).

Para maior eficiência do cálculo da integração numérica, foi utilizado o menor número de pontos de Hammer que integre sem erros nas equações do tipo eq.(4.183) e eq.(4.191) e pontos de Gauss na eq.(4.196). Alguns testes numéricos foram aplicados para determinação do número ótimo de pontos de integração.

Vale lembrar que no apêndice A são tabulados alguns valores de pontos e pesos de Hammer utilizado no trabalho. 


\subsubsection{Solução do Sistema de Equações da FHMT com Enriquecimento Nodal - Método de Babuška}

Ressalta-se que o enriquecimento das bases aproximativas dos elementos quadrilaterais, triangulares e lineares empregando-se bases também polinomiais pode gerar famílias de funções linearmente dependentes. Neste trabalho, adaptou-se o procedimento numérico de Babuška, sugerido por Strouboulis, Babuška e Copps (2000), para contornar esse problema.

Para sua apresentação, considere-se a eq.(2.68) aplicada a um domínio $\boldsymbol{\Omega}$ coberto por uma rede de $\boldsymbol{n}_{\Omega}$ elementos finitos ditos de domínio com $\boldsymbol{\Omega}_{\boldsymbol{e}}, \boldsymbol{e}=\mathbf{1}, \mathbf{2} \ldots \boldsymbol{n}_{\Omega}$, os domínios de cada um destes elementos finitos. Definem-se também $\boldsymbol{n}_{\boldsymbol{\Gamma}_{t}}$ elementos no contorno de Neumann $\boldsymbol{\Gamma}_{\boldsymbol{t}_{\boldsymbol{e}_{t}}}, \boldsymbol{e}_{\Gamma_{t}}=1, \ldots \boldsymbol{n}_{\Gamma_{t}}$, e $\boldsymbol{n}_{\Gamma_{i}}$ elementos do contorno entre os elementos de domínio $\Gamma_{i_{e_{\Gamma_{i}}}}, \boldsymbol{e}_{\Gamma_{i}}=1, \ldots \boldsymbol{n}_{\Gamma_{i}}$. Assim, a eq.(2.68) é escrita da seguinte forma:

$$
A \boldsymbol{x}=\boldsymbol{b}
$$

onde

$$
A=\left[\begin{array}{cccc}
\boldsymbol{F} & A_{\Omega} & -A_{\Gamma_{t}} & -A_{\Gamma_{i}} \\
A_{\Omega}^{T} & 0 & 0 & 0 \\
-A_{\Gamma_{t}}^{T} & 0 & 0 & 0 \\
-A_{\Gamma_{i}}^{T} & 0 & 0 & 0
\end{array}\right] ; \boldsymbol{x}=\left\{\begin{array}{c}
s_{\Omega} \\
\boldsymbol{q}_{\Omega} \\
\boldsymbol{q}_{\Gamma_{t}} \\
\boldsymbol{q}_{\Gamma_{i}}
\end{array}\right\} \text { e } \boldsymbol{b}=\left\{\begin{array}{c}
\boldsymbol{e}_{\Gamma_{u}} \\
-Q_{\Omega} \\
-Q_{\Gamma_{t}} \\
0
\end{array}\right\}
$$

com os elementos da matriz $\boldsymbol{A}$ e do vetor $\boldsymbol{b}$ dados pelas eq.(4.9) a eq.(4.12) e eq.(4.13) a eq.(4.15), respectivamente. Já os elementos do vetor $\boldsymbol{x}$ guardam os parâmetros generalizados correspondentes às aproximações locais para as tensões e deslocamentos dos elementos de domínio $\boldsymbol{e}$ e deslocamentos dos elementos dos contornos $\boldsymbol{e}_{\boldsymbol{\Gamma}_{t}}$ e $\boldsymbol{e}_{\boldsymbol{\Gamma}_{\boldsymbol{i}}}$ nas fronteiras de Neumann e internas, respectivamente.

Com a ajuda da matriz diagonal dada por:

$$
B=[\operatorname{diag}(F)]^{-\frac{1}{2}}
$$

o sistema representado pela eq.(4.197) pode ser balanceado conforme: 


$$
\overline{\boldsymbol{A}} \overline{\boldsymbol{x}}=\overline{\boldsymbol{b}}
$$

onde

$$
\bar{A}=\left[\begin{array}{cccc}
\boldsymbol{B} F \boldsymbol{B} & \boldsymbol{B} A_{\Omega} & -\boldsymbol{B} \boldsymbol{A}_{\Gamma_{t}} & -\boldsymbol{B} \boldsymbol{A}_{\Gamma_{i}} \\
\boldsymbol{A}_{\Omega}^{T} \boldsymbol{B} & 0 & 0 & 0 \\
-A_{\Gamma_{t}}^{T} \boldsymbol{B} & 0 & 0 & 0 \\
-A_{\Gamma_{i}}^{T} \boldsymbol{B} & 0 & 0 & 0
\end{array}\right] ; \overline{\boldsymbol{x}}=\left\{\begin{array}{l}
\overline{\boldsymbol{s}}_{\Omega} \\
\boldsymbol{q}_{\Omega} \\
\boldsymbol{q}_{\Gamma_{t}} \\
\boldsymbol{q}_{\Gamma_{i}}
\end{array}\right\} \text { e } \bar{b}=\left\{\begin{array}{c}
\boldsymbol{e}_{\Gamma_{u}} \\
-Q_{\Omega} \\
-Q_{\Gamma_{t}} \\
0
\end{array}\right\}
$$

Nota-se que:

$$
\operatorname{diag}(B F B)=I
$$

e

$$
s_{\Omega}=B \bar{s}_{\Omega}
$$

A eq.(4.201) deve ser utilizada após se obter a solução da eq.(4.199). Seja então a seguinte aproximação de $\overline{\boldsymbol{A}}$ :

$$
\tilde{A}=\bar{A}+\varepsilon \boldsymbol{I}
$$

onde $\boldsymbol{\varepsilon}$ é uma número positivo pequeno. A eq.(4.202) garante que $\widetilde{\boldsymbol{A}}$ seja positiva definida. O método de Babuška pode ser resumido no seguinte algoritmo a seguir:

1. $\boldsymbol{j}=\mathbf{0} ; \overline{\boldsymbol{x}}_{\boldsymbol{0}}=\mathbf{0} ; \bar{r}_{0}=\bar{b} ;$

2. resolva $\widetilde{A} a_{j}=\bar{r}_{j}$;

3. $\overline{\boldsymbol{x}}_{j+1}=\overline{\boldsymbol{x}}_{j}+\boldsymbol{a}_{j}$;

4. $\overline{\boldsymbol{r}}_{j+1}=\overline{\boldsymbol{r}}_{j}-\bar{A}_{j} a_{j}$;

5. $\boldsymbol{j} \leftarrow \boldsymbol{j}+\mathbf{1}$;

6. se $\sqrt{\frac{\overline{\boldsymbol{r}}_{j}^{T} \overline{\boldsymbol{r}}_{j}}{\boldsymbol{x}_{j}^{T} \overline{\boldsymbol{A}} \overline{\boldsymbol{x}}_{j}}}>\boldsymbol{t o l}$ volte para o passo 2 .

onde $\boldsymbol{a}_{\boldsymbol{j}}$ é o erro na iteração $\boldsymbol{j}$ e $\overline{\boldsymbol{r}}_{\boldsymbol{j}}$ é o resíduo no iteração $\boldsymbol{j}$.

No passo 2 do algoritmo anterior o sistema deve ser resolvido por um método numérico que leve em conta a estrutura e a esparsidade da matriz $\widetilde{\boldsymbol{A}}$. Neste trabalho foram experimentados tanto os métodos diretos, como o de Choleski com armazenamento esparso, como iterativos como, o método dos Gradientes Conjugados pré-condicionado pela decomposição incompleta de Choleski de $\tilde{\boldsymbol{A}}$. 


\section{Modelo Discreto da FHT com Enriquecimento Nodal}

\subsection{Introdução}

Agora serão abordadas as características gerais da FHT com enriquecimento polinomial discretizada com rede de cobertura. Após uma apresentação geral, particularizarse-á a discretização para redes de elementos finitos quadrilaterais de quatro nós e triangulares de três nós. Vale ressaltar que alguns aspectos da rede de cobertura utilizada na análise da FHMT com enriquecimento nodal serão aplicados à rede de cobertura da FHT enriquecida nodalmente.

\subsection{Modelo Discreto da FHT com Enriquecimento}

Toda estrutura desenvolvida no item 4.2 para a rede de cobertura da FHMT é utilizada aqui com a seguinte consideração:

$$
L S_{\Omega_{e}}=0 \Rightarrow A_{\Omega_{e}}=0
$$

Então são válidas para este capítulo as eq.(4.1), eq.(4.3) a eq.(4.5), eq.(4.7) a eq.(4.9), eq.(4.11) a eq.(4.13), eq.(4.15), eq.(4.16), eq.(4.18) a eq.(4.20) e eq.(4.22).

Vale lembrar que na FHT com enriquecimento nodal as aproximações adotadas para $\boldsymbol{S}_{\Omega_{e}}$ (eq.(4.5)) não estão atreladas a nós.

Em relação à dimensão das matrizes envolvidas na FHT com enriquecimento nodal, a matriz $\boldsymbol{F}_{\boldsymbol{e}}$ é quadrada, com dimensão determinada em função do número total de parâmetros generalizados de tensão do elemento e do número de nós enriquecidos. A matriz $\boldsymbol{F}$ também é quadrada e esparsa por blocos, sendo montada segundo a sua diagonal principal pela justaposição das matrizes (sem sobreposição) correspondentes a cada elemento. Ainda, as 
matrizes $\boldsymbol{F}, \boldsymbol{A}_{\boldsymbol{\Gamma}_{t}}$ e $\boldsymbol{A}_{\boldsymbol{\Gamma}_{i}}$ dependem da geometria do elemento finito adotado na rede de cobertura.

A matriz $\boldsymbol{Q}_{\Gamma_{t}}$ tem dimensão determinada em função do número de parâmetros adotados nas aproximações dos campos de deslocamento dos elementos de contorno, do número dos nós enriquecidos e do grau de enriquecimento adotado.

\subsection{Elementos Finitos Híbrido de Tensão com Enriquecimento Nodal Polinomial}

\subsubsection{Elemento Quadrilateral de Quatro Nós}

\subsubsection{Características Geométricas e Transformação de Coordenadas}

As características geométricas e de transformação de coordenadas do elemento quadrilateral da FHT são iguais à apresentada para o elemento quadrilateral da FHMT.

\subsubsection{Funções Aproximativas do Elemento Quadrilateral de Quatro Nós da FHT}

As aproximações do campo de tensões, para a FHT, devem ser auto-equilibradas, ou seja, satisfazem localmente a equação de equilíbrio, eq.(2.1), a menos de forças volúmicas. A eq.(2.69) apresenta a condição para que a matriz $S_{\Omega}$, que guarda as funções de aproximação dos campos de tensão, satisfaça essa restrição.

Especificamente no domínio $\boldsymbol{\Omega}_{\boldsymbol{e}}$ do elemento quadrilateral híbrido de tensão, a matriz $\boldsymbol{S}_{\Omega_{e}}$ (eq.(4.5)) também deve satisfazer a eq.(5.1).

Funções polinomiais algébricas são utilizadas para aproximações do campo de tensões no domínio do elemento quadrilateral apresentado. Para garantir que o conjunto de funções polinomiais empregadas respeite a eq.(5.1), pode-se adotar a metodologia seguinte:

Considere-se a função de Airy:

$$
A(x, y)
$$

$\log 0$

$$
\begin{gathered}
\sigma_{x}=\frac{\partial^{2} A(x, y)}{\partial y^{2}} \\
\sigma_{y}=\frac{\partial^{2} A(x, y)}{\partial x^{2}}
\end{gathered}
$$


$\tau_{x y}=-\frac{\partial^{2} A(x, y)}{\partial x \partial y}$

Com $A(x, y)$ dado por:

$$
A(x, y)=\frac{1}{2} b x^{2}-c x y+\frac{1}{2} a y^{2}
$$

então

$$
\sigma=\left[\begin{array}{lll}
1 & 0 & 0 \\
0 & 1 & 0 \\
0 & 0 & 1
\end{array}\right]\left\{\begin{array}{l}
a \\
b \\
c
\end{array}\right\}
$$

com $A(x, y)$ dado por:

$$
A(x, y)=\frac{1}{6} d x^{3}-\frac{1}{2} c x^{2} y-\frac{1}{2} b x y^{2}+\frac{1}{6} a y^{3}
$$

então

$$
\sigma=\left[\begin{array}{cccc}
y & -x & 0 & 0 \\
0 & 0 & -y & x \\
0 & y & x & 0
\end{array}\right]\left\{\begin{array}{l}
a \\
b \\
c \\
d
\end{array}\right\}
$$

Com $A(x, y)$ dado por:

$$
A(x, y)=\frac{1}{12} e x^{4}-\frac{1}{3} d x^{3} y+\frac{1}{2} c x^{2} y^{2}-\frac{1}{3} b x y^{3}+\frac{1}{12} a y^{4}
$$

então

$$
\sigma=\left[\begin{array}{ccccc}
y^{2} & -2 x y & x^{2} & 0 & 0 \\
0 & 0 & y^{2} & -2 x y & x^{2} \\
0 & y^{2} & -2 x y & x^{2} & 0
\end{array}\right]\left\{\begin{array}{l}
a \\
b \\
c \\
d \\
e
\end{array}\right\}
$$

Nas equações anteriores $\boldsymbol{a}, \boldsymbol{b}, \boldsymbol{c}, \boldsymbol{d}$ e $\boldsymbol{e}$ são parâmetros generalizados das tensões. Podese agora definir $\boldsymbol{S}_{\boldsymbol{\Omega}_{e}}$ para os seguintes casos de aproximação do campo de tensões: 
- Aproximação constante no elemento

$$
S_{\Omega_{e}}=\left[\begin{array}{lll}
1 & 0 & 0 \\
0 & 1 & 0 \\
0 & 0 & 1
\end{array}\right]
$$

- Aproximação linear no elemento

$$
S_{\Omega_{e}}=\left[\begin{array}{cccccccc}
1 & 0 & 0 & \vdots & y & -x & 0 & 0 \\
0 & 1 & 0 & \vdots & 0 & 0 & -y & x \\
0 & 0 & 1 & \vdots & 0 & y & x & 0
\end{array}\right]
$$

- Aproximação quadrática no elemento

$$
S_{\Omega_{e}}=\left[\begin{array}{cccccccccccccc}
1 & 0 & 0 & \vdots & y & -x & 0 & 0 & \vdots & y^{2} & -2 x y & x^{2} & 0 & 0 \\
0 & 1 & 0 & \vdots & 0 & 0 & -y & x & \vdots & 0 & 0 & y^{2} & -2 x y & x^{2} \\
0 & 0 & 1 & \vdots & 0 & y & x & 0 & \vdots & 0 & y^{2} & -2 x y & x^{2} & 0
\end{array}\right]
$$

Nota-se que para as aproximações constante, linear e quadrática, o número de parâmetros generalizados de tensões no domínio $\boldsymbol{s}_{\boldsymbol{\Omega}_{e}}$ (eq.(4.5)) do elemento vale: três (3), sete (7) e doze (12), respectivamente. No capítulo que abordará as condições necessárias e suficientes para convergência da solução obtida com a FHT/FHMT, será visto que a escolha do número de parâmetros generalizados de tensões no domínio está intimamente ligada ao grau de aproximação do campo de deslocamentos no contorno do elemento.

Para a interpolação do campo de deslocamentos no contorno $\boldsymbol{\Gamma}_{\boldsymbol{t}_{e_{\Gamma_{t}}}}$ ou $\boldsymbol{\Gamma}_{\boldsymbol{i}_{\boldsymbol{e}_{\boldsymbol{T}_{i}}}}$ do elemento de domínio $\boldsymbol{\Omega}_{\boldsymbol{e}}$, funções lineares Lagrangianas clássicas são aplicadas. Essas funções também foram aplicadas ao elemento de contorno da FHMT. Assim, para o elemento de contorno da FHT derivado do elemento quadrilateral valem as eq.(4.43) e eq.(4.44). 


\subsubsection{Enriquecimento das Funções Aproximativas do Elemento Quadrilateral de Quatro Nós da FHT}

Na FHT são aproximados dois campos independentes e, como já salientado, o enriquecimento também pode ser realizado de forma independente para os dois campos. Aqui, a estratégia de enriquecimento é desenvolvida com ajuda de funções polinomiais.

Supondo que num domínio plano tenha sido adotada uma rede de cobertura com elementos quadrilaterais de quatro nós. Admitindo-se que se deseja enriquecer a aproximação correspondente às tensões nos domínios dos elementos finitos com ajuda de funções de suporte compacto $\boldsymbol{g}$ e polinomiais $\boldsymbol{h}$, a eq.(3.10) pode ser reescrita da seguinte forma:

- A família de funções para o campo de tensões no domínio $\boldsymbol{\Omega}$ :

$$
\mathfrak{J}_{e}^{p}=\left\{\left\{S_{\Omega_{e}}\right\}_{e=1}^{n_{\Omega}} \cup\left\{g_{j} h_{j}\right\}_{j=1}^{N}: e=1, \ldots, n_{\Omega ;} j=1, \ldots, N\right\}
$$

utilizada para construir a seguinte aproximação:

$$
\hat{\sigma}=\sum_{e=1}^{n_{\Omega}}\left\{S_{\Omega_{e}} s_{\Omega_{e}}\right\}+\sum_{j=1}^{N}\left\{g_{j} h_{j} b_{j}\right\}
$$

onde $\boldsymbol{s}_{\boldsymbol{\Omega}_{e}}$ são parâmetros das tensões associadas aos elementos de domínio $\boldsymbol{\Omega}_{\boldsymbol{e}}$ e $\boldsymbol{b}_{\boldsymbol{j}}$ são os novos parâmetros das tensões correspondentes ao enriquecimento.

Como a nova composição das aproximações para o campo das tensões no domínio dado pela eq.(5.15) deve garantir o equilíbrio local, eq.(5.1), desenvolveu-se a seguinte metodologia para o enriquecimento desse campo:

Seja então a função de Airy dada por:

$$
A=g^{3} h
$$

onde $\boldsymbol{g}(\boldsymbol{x}, \boldsymbol{y})$ tem suporte compacto dado pela rede de elementos finitos da rede de cobertura e $\boldsymbol{h}(\boldsymbol{x}, \boldsymbol{y})$ é polinomial com $\boldsymbol{x}, \boldsymbol{y}$ centrados nos nós. Assim:

$$
\sigma_{x}=\frac{\partial^{2} A}{\partial y^{2}}=3 g h\left[2\left(\frac{\partial g}{\partial y}\right)^{2}+g \frac{\partial^{2} g}{\partial y^{2}}\right]+6 g^{2} \frac{\partial g}{\partial y} \frac{\partial h}{\partial y}+g^{3} \frac{\partial^{2} h}{\partial y^{2}}
$$




$$
\begin{aligned}
& \sigma_{y}=\frac{\partial^{2} A}{\partial x^{2}}=3 g h\left[2\left(\frac{\partial g}{\partial x}\right)^{2}+g \frac{\partial^{2} g}{\partial x^{2}}\right]+6 g^{2} \frac{\partial g}{\partial x} \frac{\partial h}{\partial x}+g^{3} \frac{\partial^{2} h}{\partial x^{2}} \\
& \tau_{x y}=-\frac{\partial^{2} A}{\partial x \partial y}=-3 g h\left[2\left(\frac{\partial g}{\partial x} \frac{\partial g}{\partial y}\right)+g \frac{\partial^{2} g}{\partial x \partial y}\right]-3 g^{2}\left(\frac{\partial g}{\partial x} \frac{\partial h}{\partial y}+\frac{\partial g}{\partial y} \frac{\partial h}{\partial x}\right)-g^{3} \frac{\partial^{2} h}{\partial x \partial y}
\end{aligned}
$$

logo

$$
L\left\{\begin{array}{c}
\sigma_{x} \\
\sigma_{y} \\
\tau_{x y}
\end{array}\right\}=\left[\begin{array}{c}
\frac{\partial \sigma_{x}}{\partial x}+\frac{\partial \tau_{x y}}{\partial y} \\
\frac{\partial \tau_{x y}}{\partial x}+\frac{\partial \sigma_{y}}{\partial y}
\end{array}\right]=\left[\begin{array}{c}
\frac{\partial^{3} A}{\partial x \partial y^{2}}-\frac{\partial^{3} A}{\partial x \partial y^{2}} \\
-\frac{\partial^{3} A}{\partial x^{2} \partial y}+\frac{\partial^{3} A}{\partial x^{2} \partial y}
\end{array}\right]=\left[\begin{array}{l}
0 \\
0
\end{array}\right]
$$

Assim as aproximações do campo de tensões $\boldsymbol{S}_{\boldsymbol{\Omega}_{e}}$ dadas pelas eq.(5.12), eq.(5.13) e eq.(5.14), podem ser ampliadas da seguinte forma:

$$
S_{\Omega_{e}}^{*}=\left[\begin{array}{lllll}
S_{\Omega_{e}} & \Sigma_{1} & \Sigma_{2} & \Sigma_{3} & \Sigma_{4}
\end{array}\right]
$$

onde $\boldsymbol{S}_{\Omega_{e}}^{*}$ é a matriz de interpolação das tensões enriquecidas e

$$
\underset{(j=1, \ldots, 4)}{\Sigma_{j}}=\left[\begin{array}{c}
3 g_{j} h_{j}\left[2\left(\frac{\partial g_{j}}{\partial y}\right)^{2}+g_{j} \frac{\partial^{2} g_{j}}{\partial y^{2}}\right]+6 g_{j}{ }^{2} \frac{\partial g_{j}}{\partial y} \frac{\partial h_{j}}{\partial y}+g_{j}{ }^{3} \frac{\partial^{2} h_{j}}{\partial y^{2}} \\
3 g_{j} h_{j}\left[2\left(\frac{\partial g_{j}}{\partial x}\right)^{2}+g_{j} \frac{\partial^{2} g_{j}}{\partial x^{2}}\right]+6 g_{j}{ }^{2} \frac{\partial g_{j}}{\partial x} \frac{\partial h_{j}}{\partial x}+g_{j}{ }^{3} \frac{\partial^{2} h_{j}}{\partial x^{2}} \\
-3 g_{j} h_{j}\left[2\left(\frac{\partial g_{j}}{\partial x} \frac{\partial g_{j}}{\partial y}\right)+g_{j} \frac{\partial^{2} g_{j}}{\partial x \partial y}\right]-3 g_{j}{ }^{2}\left(\frac{\partial g_{j}}{\partial x} \frac{\partial h_{j}}{\partial y}+\frac{\partial g_{j}}{\partial y} \frac{\partial h_{j}}{\partial x}\right)-g_{j}{ }^{3} \frac{\partial^{2} h_{j}}{\partial x \partial y}
\end{array}\right]
$$

reúne as interpolações de suporte compacto com $\boldsymbol{x}, \boldsymbol{y}$ centrados nos nós $\boldsymbol{j}$ da rede de elementos finitos. Nota-se que $\boldsymbol{S}_{\Omega_{e}}^{*}$ também satisfaz a eq.(5.1).

Podem-se adotar como funções de suporte compacto, $\boldsymbol{g}_{\boldsymbol{j}}, \boldsymbol{j}=\mathbf{1}, \ldots, 4$, as funções Lagrangianas bilineares, utilizadas para aproximação da geometria do elemento quadrilateral híbrido de tensão. Assim: 


$$
g_{j}=\frac{1}{4} \xi_{j} \eta_{j}\left(\xi+\xi_{j}\right)\left(\eta+\eta_{j}\right), j=1, \ldots, 4
$$

onde $\boldsymbol{\xi}$ e $\boldsymbol{\eta}$ são coordenadas normalizadas ortogonais no domínio paramétrico e $\boldsymbol{\xi}_{\boldsymbol{j}}, \boldsymbol{\eta}_{\boldsymbol{j}}$ são coordenadas adimensionais dos nós.

Uma opção conveniente para as funções $\boldsymbol{h}_{\boldsymbol{j}}$ é que sejam do tipo bolha, isto é:

$$
h_{j}\left(\xi_{j}, \eta_{j}\right)=0
$$

Observa-se que formas habituais para as funções bolha podem ser iguais às dadas nas eq.(4.67) a eq.(4.71).

Com a transformação apresentada na eq.(4.103), podem-se expressar as derivadas $\frac{\partial \boldsymbol{g}_{j}}{\partial x}, \frac{\partial \boldsymbol{h}_{j}}{\partial x}, \frac{\partial \boldsymbol{g}_{j}}{\partial \boldsymbol{y}}, \frac{\partial \boldsymbol{h}_{j}}{\partial y}, \frac{\partial^{2} \boldsymbol{g}_{j}}{\partial x^{2}}, \frac{\partial^{2} \boldsymbol{h}_{j}}{\partial x^{2}}, \frac{\partial^{2} \boldsymbol{g}_{j}}{\partial \boldsymbol{y}^{2}}, \frac{\partial^{2} \boldsymbol{h}_{j}}{\partial \boldsymbol{y}^{2}}, \frac{\partial^{2} \boldsymbol{g}_{j}}{\partial x \partial y} \mathrm{e} \frac{\partial^{2} \boldsymbol{h}_{j}}{\partial x \partial y}$ com $\boldsymbol{g}_{j}$ e $\boldsymbol{h}_{j}$ escritas em função das coordenadas $\boldsymbol{\xi} \boldsymbol{\eta}$.

Nota-se que, para esse tipo de enriquecimento, somente uma função de enriquecimento $\boldsymbol{h}_{\boldsymbol{j}}$ é acrescida a um nó $\boldsymbol{j}$ da rede de cobertura de domínio.

Para a rede de contorno são empregados elementos retos de dois nós com funções de forma Lagrangianas lineares. Supondo que se deseja enriquecer a aproximação correspondente a um nó $\boldsymbol{j}$ da rede de cobertura de contorno com ajuda de funções polinomiais $\boldsymbol{h}_{\boldsymbol{j} \boldsymbol{n}_{e_{T}}}, \boldsymbol{j} \boldsymbol{n}_{\boldsymbol{e}_{\Gamma}}=\mathbf{1}, \ldots, \boldsymbol{n}_{\boldsymbol{e}_{\Gamma}}, \boldsymbol{n}_{\boldsymbol{e}_{\Gamma}}$ é o número de funções de enriquecimento adotadas, a eq.(3.9) pode ser re-escrita da seguinte forma:

- A família de funções para o campo de deslocamentos no contorno $\boldsymbol{\Gamma}$ :

$$
\mathfrak{R}_{N_{\Gamma}}^{p}=\left\{\left\{U_{\Gamma_{i_{j}}}\right\}_{j=1}^{N_{\Gamma}} \cup\left\{U_{\Gamma_{i_{j}}} h_{j n_{e_{\Gamma}}}\right\}_{j=1}^{N_{\Gamma}}: j=1, \ldots, N_{\Gamma} ; n_{e_{\Gamma}}=1, \ldots, I(j)\right\}
$$

utilizada para construir a seguinte aproximação:

$$
\hat{\boldsymbol{u}}_{\Gamma}=\sum_{j=1}^{N_{\Gamma}} \boldsymbol{U}_{\Gamma_{j}}\left\{\boldsymbol{q}_{\Gamma_{j}}+\sum_{l=1}^{n_{e_{\Gamma}}} \boldsymbol{h}_{j l} \boldsymbol{d}_{j l}\right\}
$$


onde $\boldsymbol{q}_{\Gamma_{j}}$ são graus de liberdade em deslocamento associados às funções de forma originais e $\boldsymbol{d}_{\boldsymbol{j} \boldsymbol{l}}$ são os novos parâmetros nodais correspondentes a cada uma das parcelas de enriquecimento.

Uma opção conveniente para as funções $\boldsymbol{h}_{\boldsymbol{j}_{\boldsymbol{n}_{\boldsymbol{T}}}}$ escolhidas para o enriquecimento, é que sejam tais que:

$$
\boldsymbol{h}_{j n_{e_{\Gamma}}}\left(\xi_{j}\right)=0
$$

onde $\xi_{j}$ é a coordenada adimensional para o elemento da rede de cobertura de contorno. Nesse caso, essas funções são nulas no nó enriquecido ('funções bolhas') e a vantagem desse procedimento, como já comentado, é que se preserva o significado físico de graus de liberdade nodais $\boldsymbol{q}_{\Gamma}$.

Formas habituais para as funções bolhas $\boldsymbol{h}_{\boldsymbol{j} \boldsymbol{n}_{\boldsymbol{e}}}$ são apresentadas nas eq.(4.72) e eq.(4.73).

Assim sendo, a matriz de interpolação do campo de deslocamento no contorno, eq.(4.43), pode ser escrita igualmente a forma apresentada na eq.(4.63).

Dessa forma para qualquer nó $\boldsymbol{j},(\boldsymbol{j}=\mathbf{1}, \ldots, 4)$ do elemento finito retangular $\boldsymbol{\Omega}_{e}$, escolhido para ser enriquecido, pode-se escrever:

- Para a matriz representada pela eq.(4.16) e com a eq.(5.22), tem-se

$$
\int_{\Omega_{e}}\left(S_{\Omega_{e}}^{*}\right)^{T} f\left(S_{\Omega_{e}}^{*}\right) d \boldsymbol{\Omega}=\left[\begin{array}{cccccc}
\int_{\Omega_{e}}\left(S_{\Omega_{e}}\right)^{T} f\left(S_{\Omega_{e}}\right) d \boldsymbol{\Omega} & \cdots & \int_{\Omega_{e}}\left(S_{\Omega_{e}}\right)^{T} f\left(\Sigma_{k}\right) d \boldsymbol{\Omega} & \cdots & \int_{\Omega_{e}}\left(S_{\Omega_{e}}\right)^{T} f\left(\Sigma_{n}\right) d \boldsymbol{\Omega} \\
\vdots & \ddots & \vdots & \ddots & \vdots \\
\int_{\Omega_{e}}\left(\Sigma_{l}\right) f\left(S_{\Omega_{e}}\right) d \boldsymbol{\Omega} & \cdots & \int_{\Omega_{e}}\left(\Sigma_{l}\right)^{T} f\left(\Sigma_{k}\right) d \boldsymbol{\Omega} & \cdots & \int_{\Omega_{e}}\left(\Sigma_{l}\right)^{T} f\left(\Sigma_{n}\right) d \boldsymbol{\Omega} \\
\vdots & \ddots & \vdots & \ddots & \vdots \\
\int_{\Omega_{e}}\left(\Sigma_{n}\right)^{T} f\left(S_{\Omega_{e}}\right) d \boldsymbol{\Omega} & \cdots & \int_{\Omega_{e}}\left(\Sigma_{n}\right)^{T} f\left(\Sigma_{k}\right) d \boldsymbol{\Omega} & \cdots & \int_{\Omega_{e}}\left(\Sigma_{n}\right)^{T} f\left(\Sigma_{n}\right) d \boldsymbol{\Omega}
\end{array}\right]
$$

onde $\boldsymbol{l}, \boldsymbol{k}$ são os nós enriquecidos do elemento quadrilateral e $\boldsymbol{n}$ é o número de nós enriquecidos no mesmo elemento. Observa-se que são acrescentadas $\boldsymbol{n}$ linhas e colunas à estrutura inicial da eq.(4.16).

Para qualquer nó $\boldsymbol{j}(\boldsymbol{j}=\mathbf{1 , 2})$, de cada lado do elemento finito quadrilateral que é escolhido para ser enriquecido, pode-se escrever: 
- Para a matriz representada pelas eq.(4.18) e eq.(4.19), juntamente com as eq.(5.22) e eq.(4.63), escreve-se:

$$
\int_{\Gamma_{t} \text { ou } \Gamma_{i}}\left(N S_{\Omega_{e}}^{*}\right)^{T} U_{\Gamma_{\text {er }}} d \Gamma=\left[\begin{array}{c}
\int_{\Gamma_{\text {t ou } \Gamma_{i}}}\left(N S_{\Omega_{e}}\right)^{T}\left(\Psi_{j} \Delta_{\Gamma_{j}}\right) d \Gamma \\
\vdots \\
\int_{\Gamma_{t} \text { ou } \Gamma_{i}}\left(N \Sigma_{l}\right)^{T}\left(\Psi_{j} \Delta_{\Gamma_{j}}\right) d \Gamma \\
\vdots \\
\int_{\Gamma_{t} \text { ou } \Gamma_{i}}\left(N \Sigma_{n}\right)^{T}\left(\Psi_{j} \Delta_{\Gamma_{j}}\right) d \Gamma
\end{array}\right]
$$

onde $\boldsymbol{l}$ é o nó enriquecido do elemento quadrilateral de domínio e $\boldsymbol{n}$ é o número de nós enriquecidos no mesmo elemento. Observa-se que são acrescentadas $\boldsymbol{n}$ linhas à estrutura inicial da eq.(4.18) e eq.(4.19). O número de colunas adicionais à eq.(4.18) e eq.(4.19) depende das $\boldsymbol{n}_{\boldsymbol{e}_{\boldsymbol{T}}}$ funções de enriquecimento adotadas para o campo de deslocamento no contorno.

- Para a matriz representada pela eq.(4.22), juntamente com a eq.(4.63), tem-se:

$$
\begin{gathered}
U_{\Gamma_{e_{\Gamma}}}^{T}=\left[\begin{array}{c}
\psi_{1} \Delta_{\Gamma_{1}}^{T} \\
\psi_{2} \Delta_{\Gamma_{2}}^{T}
\end{array}\right] \\
Q_{\Gamma_{e_{\Gamma}}}=\left[Q_{\Gamma_{\gamma}}\right], \gamma=1,2
\end{gathered}
$$

onde:

$$
Q_{\Gamma_{\gamma}}=\left[\int_{\Gamma_{t}} \psi_{\gamma} \Delta_{\Gamma_{\gamma}}^{T} \bar{t} d \Gamma\right], \gamma=1,2
$$

\subsubsection{Integrais Intervenientes}

Agora, ilustram-se as integrais intervenientes das eq.(5.29), eq.(5.30) e eq.(5.32) e o procedimento numérico para resolução destas integrais nos domínios paramétricos dos elementos quadrilateral (ver figura 4.1) e linear (ver figura 4.2).

Para as integrais envolvidas na eq.(5.29), observa-se que cada integrando é constituído basicamente de funções do tipo:

$$
\frac{\partial g}{\partial x}, \frac{\partial g}{\partial y}, \frac{\partial h_{j}}{\partial y}, \frac{\partial^{2} g}{\partial x^{2}}, \frac{\partial^{2} h}{\partial x^{2}}, \frac{\partial^{2} g}{\partial y^{2}}, \frac{\partial^{2} h}{\partial y^{2}}, \frac{\partial^{2} g}{\partial x \partial y}, \frac{\partial^{2} h}{\partial x \partial y}, g, h, x \text { е } y
$$


Os monômios das funções aproximativas das tensões no domínio $\left(\boldsymbol{x}, \boldsymbol{y}, \boldsymbol{x}^{2}, \boldsymbol{y}^{2}, \boldsymbol{x y}\right)$ escritas no domínio global $\boldsymbol{x} \boldsymbol{y}$ podem ser escritos no domínio paramétrico $\boldsymbol{\xi} \boldsymbol{\eta}$ via transformação dada pela eq.(4.29).

Os operadores diferenciais $\frac{\partial \boldsymbol{g}}{\partial \boldsymbol{x}}, \frac{\partial \boldsymbol{g}}{\partial \boldsymbol{y}}, \frac{\partial \boldsymbol{h}}{\partial \boldsymbol{y}}$ podem ser referenciadas no domínio paramétrico $\xi \boldsymbol{\eta}$ com auxílio da transformação dada na eq.(4.103).

Já os operadores diferenciais $\frac{\partial^{2} g}{\partial x^{2}}, \frac{\partial^{2} \boldsymbol{h}}{\partial x^{2}}, \frac{\partial^{2} g}{\partial y^{2}}, \frac{\partial^{2} \boldsymbol{h}}{\partial y^{2}}, \frac{\partial^{2} g}{\partial x \partial y}$ e $\frac{\partial^{2} \boldsymbol{h}}{\partial x \partial y}$ também podem ser expressados no domínio paramétrico $\xi \eta$ com auxílio da transformação apresentada na eq.(4.103), mas para uma melhor compreensão, seguem-se alguns desenvolvimentos.

Da transformação pontuada na eq.(4.103), pode-se escrever:

$$
\begin{aligned}
& \frac{\partial}{\partial x}=J_{11}^{-1} \frac{\partial}{\partial \xi}+J_{12}^{-1} \frac{\partial}{\partial \eta} \\
& \frac{\partial}{\partial y}=J_{21}^{-1} \frac{\partial}{\partial \xi}+J_{22}^{-1} \frac{\partial}{\partial \eta}
\end{aligned}
$$

O operador $\frac{\partial^{2}}{\partial x^{2}}$ é então calculado da seguinte forma:

$$
\frac{\partial^{2}}{\partial x^{2}}=J_{11}^{-1} \frac{\partial}{\partial \xi}\left(J_{11}^{-1} \frac{\partial}{\partial \xi}+J_{12}^{-1} \frac{\partial}{\partial \eta}\right)+J_{12}^{-1} \frac{\partial}{\partial \eta}\left(J_{11}^{-1} \frac{\partial}{\partial \xi}+J_{12}^{-1} \frac{\partial}{\partial \eta}\right)
$$

O operador $\frac{\partial^{2}}{\partial y^{2}}$ tem a seguinte apresentação:

$$
\frac{\partial^{2}}{\partial y^{2}}=J_{21}^{-1} \frac{\partial}{\partial \xi}\left(J_{21}^{-1} \frac{\partial}{\partial \xi}+J_{22}^{-1} \frac{\partial}{\partial \eta}\right)+J_{22}^{-1} \frac{\partial}{\partial \eta}\left(J_{21}^{-1} \frac{\partial}{\partial \xi}+J_{22}^{-1} \frac{\partial}{\partial \eta}\right)
$$

Já o operador $\frac{\partial^{2}}{\partial x \partial y}$ é expresso por:

$$
\frac{\partial^{2}}{\partial x y}=J_{21}^{-1} \frac{\partial}{\partial \xi}\left(J_{11}^{-1} \frac{\partial}{\partial \xi}+J_{12}^{-1} \frac{\partial}{\partial \eta}\right)+J_{22}^{-1} \frac{\partial}{\partial \eta}\left(J_{11}^{-1} \frac{\partial}{\partial \xi}+J_{12}^{-1} \frac{\partial}{\partial \eta}\right)
$$

Das eq.(5.37) a eq.(5.39), verifica-se a necessidade de calcular: 
$J_{11}^{-1}, J_{12}^{-1}, J_{21}^{-1}, J_{22}^{-1}, \frac{\partial J_{11}^{-1}}{\partial \xi}, \frac{\partial J_{12}^{-1}}{\partial \xi}, \frac{\partial J_{11}^{-1}}{\partial \eta}, \frac{\partial J_{12}^{-1}}{\partial \eta}, \frac{\partial J_{21}^{-1}}{\partial \xi}, \frac{\partial J_{22}^{-1}}{\partial \xi}, \frac{\partial J_{11}^{-1}}{\partial \eta}$ e $\frac{\partial J_{12}^{-1}}{\partial \eta}$

O cálculo de $\boldsymbol{J}_{11}^{-1}, \boldsymbol{J}_{12}^{-1}, \boldsymbol{J}_{21}^{-1}, \boldsymbol{J}_{22}^{-1}$ para um elemento paramétrico quadrilateral (ver figura 4.1) foi apresentado nas eq.(4.104) a eq.(4.107), respectivamente.

Agora, expande-se o cálculo dos outros operadores citados na eq.(5.40):

$$
\begin{aligned}
& \frac{\partial J_{11}^{-1}}{\partial \xi}= 2\left(x_{1} \eta y_{3}^{2}-y_{1} x_{3} y_{2}+y_{1} x_{4} y_{3}-y_{3} x_{1} y_{4}+y_{1} x_{1} y_{2}-x_{3} \eta y_{4}^{2}-y_{2} x_{4} y_{1}+x_{2} \eta y_{1}^{2}+\cdots\right. \\
& \cdots+x_{1} \eta y_{2}^{2}-x_{4} \eta y_{3}^{2}+y_{2} x_{1} y_{4}+x_{2} \eta y_{4}^{2}+y_{1} x_{2} y_{3}-\cdots \\
& \cdots-x_{3} \eta y_{1}^{2}-x_{4} \eta y_{2}^{2}-x_{2} y_{1}^{2}-x_{1} y_{2}^{2}-y_{3} x_{3} y_{1}-\cdots \\
& \cdots-y_{4} x_{4} y_{2}+y_{2} x_{2} y_{1}+y_{3} x_{3} y_{4}-y_{1} x_{1} y_{3}-y_{2} x_{2} y_{4}+\cdots \\
& \cdots+y_{4} x_{4} y_{3}-y_{4} x_{2} y_{3}+y_{2} x_{3} y_{4}-x_{4} y_{3}^{2}-x_{3} y_{4}^{2}+x_{3} y_{1}^{2}+\cdots \\
& \cdots+x_{4} y_{2}^{2}+x_{1} y_{3}^{2}+x_{2} y_{4}^{2}+y_{1} x_{1} \eta y_{3}-2 y_{1} x_{2} \eta y_{4}-\cdots \\
& \cdots-y_{1} x_{1} \eta y_{2}+2 y_{1} x_{3} \eta y_{4}-y_{1} x_{4} \eta y_{3}+y_{1} x_{4} \eta y_{2}-\cdots \\
& \cdots-2 y_{2} x_{1} \eta y_{3}+y_{2} x_{2} \eta y_{4}-y_{2} x_{3} \eta y_{4}-y_{2} x_{2} \eta y_{1}+\cdots \\
& \cdots+y_{2} x_{3} \eta y_{1}+2 y_{2} x_{4} \eta y_{3}-y_{3} x_{2} \eta y_{4}+y_{3} x_{3} \eta y_{4}+\cdots \\
&\left.\cdots+y_{3} x_{2} \eta y_{1}-y_{3} x_{3} \eta y_{1}-y_{4} x_{1} \eta y_{3}+y_{4} x_{1} \eta y_{2}+y_{4} x_{4} \eta y_{3}-y_{4} x_{4} \eta y_{2}\right) / \Phi \\
&\left.*\left(x_{3} y_{2}+x_{2} y_{4}-x_{3} y_{1}-x_{2} y_{3}-x_{4} y_{2}+x_{4} y_{1}+x_{1} y_{3}-x_{1} y_{4}\right)\right] / \Phi \\
&\left.\frac{\partial J_{12}^{-1}}{\partial \xi}\left[-x_{1} y_{3}+x_{2} y_{4}+x_{1} y_{2}-x_{3} y_{4}-x_{2} y_{1}+x_{3} y_{1}+x_{4} y_{3}-x_{4} y_{2}\right)\right] / \Phi \\
& \frac{\partial J_{11}^{-1}}{\partial \eta}\left[-2\left(-y_{1} \xi+y_{1}+y_{2} \xi+y_{2}-y_{3} \xi-y_{3}+y_{4} \xi-y_{4}\right) *\right. \\
& \cdots \\
& \cdots
\end{aligned}
$$




$$
\begin{aligned}
& \frac{\partial J_{12}^{-1}}{\partial \eta}=-2\left(y_{1} x_{3} y_{4}-y_{1} x_{4} y_{3}-y_{1} x_{1} y_{4}+y_{2} x_{4} y_{3}-x_{4} y_{1}^{2} \xi+y_{3} x_{1} y_{2}-y_{2} x_{2} y_{3}-x_{4} \xi y_{2}^{2}+\cdots\right. \\
& \cdots+x_{2} \xi y_{4}^{2}+x_{3} \xi y_{1}^{2}+x_{3} y_{2}^{2} \xi-x_{1} \xi y_{3}^{2}-\cdots \\
& \cdots-x_{1} \xi y_{4}^{2}+x_{2} \xi y_{3}^{2}-y_{3} x_{3} y_{2}+y_{4} x_{2} y_{1}-y_{4} x_{4} y_{1}+\cdots \\
& \cdots+x_{4} y_{1}^{2}-y_{2} x_{1} y_{4}-y_{1} x_{2} y_{3}+x_{3} y_{2}^{2}+x_{1} y_{4}^{2}+x_{2} y_{3}^{2}-\cdots \\
& \cdots-y_{3} x_{4} y_{1} \xi+2 y_{3} x_{1} y_{4} \xi+y_{4} x_{3} y_{2} \xi+y_{2} x_{1} y_{3} \xi-\cdots \\
& \cdots-y_{2} x_{1} y_{4} \xi-y_{3} x_{3} y_{2} \xi-2 y_{3} x_{2} y_{4} \xi+y_{3} x_{3} y_{1} \xi+\cdots \\
& \cdots+y_{3} x_{4} y_{2} \xi-y_{4} x_{3} y_{1} \xi-y_{4} x_{4} y_{2} \xi+y_{4} x_{4} y_{1} \xi-\cdots \\
& \cdots-y_{1} x_{1} y_{3} \xi+y_{1} x_{1} \xi y_{4}+y_{2} x_{2} \xi y_{4}-y_{2} x_{2} \xi y_{3}-\cdots \\
& \cdots-2 y_{1} x_{3} y_{2} \xi-y_{1} x_{2} y_{4} \xi+y_{1} x_{2} y_{3} \xi+2 y_{1} x_{4} \xi y_{2}+\cdots \\
& \cdots+y_{3} x_{3} y_{1}+y_{4} x_{4} y_{2}+y_{1} x_{1} y_{3}+y_{2} x_{2} y_{4}-\cdots \\
& \left.\cdots-y_{2} x_{3} y_{4}-x_{3} y_{1}^{2}-x_{4} y_{2}^{2}-x_{1} y_{3}^{2}-x_{2} y_{4}^{2}\right] / \Phi \\
& \frac{\partial J_{21}^{-1}}{\partial \xi}=-2\left(y_{1} x_{3} x_{4}-y_{2} x_{1} x_{3}-x_{1}^{2} \eta y_{2}+x_{1} x_{2} y_{3}+x_{4} x_{3} y_{2}+x_{1}^{2} \eta y_{3}-x_{3} x_{4} y_{3}-\cdots\right. \\
& \cdots-x_{3}^{2} \eta y_{1}-x_{2}^{2} \eta y_{1}-x_{2} x_{4} y_{1}-x_{2} x_{4} y_{3}-x_{4}^{2} \eta y_{2}+\cdots \\
& \cdots+x_{1} x_{3} y_{1}+x_{4}^{2} \eta y_{3}-x_{1} x_{2} y_{1}+x_{3}^{2} \eta y_{4}-x_{1} x_{3} y_{4}+\cdots \\
& \cdots+x_{2}^{2} \eta y_{4}-x_{4} x_{3} y_{4}+x_{2} x_{4} y_{2}+x_{3} x_{1} y_{3}+x_{4} x_{2} y_{4}-\cdots \\
& \cdots-x_{2} x_{1} y_{2}+x_{2} x_{1} y_{4}+x_{1}^{2} y_{2}+x_{2}^{2} y_{1}+x_{3}^{2} y_{4}+x_{4}^{2} y_{3}-\cdots \\
& \cdots-x_{1}^{2} y_{3}-x_{2}^{2} y_{4}-x_{3}^{2} y_{1}-x_{4}^{2} y_{2}-x_{1} x_{2} \eta y_{4}+x_{1} x_{3} \eta y_{4}+\cdots \\
& \cdots+x_{1} x_{2} y_{1} \eta-x_{1} x_{3} y_{1} \eta-2 x_{1} x_{4} y_{3} \eta+2 x_{1} x_{4} y_{2} \eta-\cdots \\
& \cdots-x_{2} x_{1} y_{3} \eta+x_{2} x_{1} \eta y_{2}-2 x_{2} x_{3} \eta y_{4}+\cdots \\
& \cdots+2 x_{2} x_{3} y_{1} \eta+x_{2} x_{4} y_{3} \eta-x_{2} x_{4} y_{2} \eta+x_{3} x_{1} \eta y_{3}-\cdots \\
& \cdots-x_{3} x_{1} \eta y_{2}-x_{3} x_{4} \eta y_{3}+x_{3} x_{4} y_{2} \eta+x_{4} x_{2} y_{4} \eta-\cdots \\
& \left.\cdots-x_{4} x_{3} \eta y_{4}-x_{4} x_{2} y_{1} \eta+x_{4} x_{3} \eta y_{1}\right] / \Phi \\
& \frac{\partial J_{21}^{-1}}{\partial \eta}=\left[2\left(-x_{1} \xi+x_{1}+x_{2} \xi+x_{2}-x_{3} \xi-x_{3}+x_{4} \xi-x_{4}\right) *\right. \\
& \left.*\left(-x_{1} y_{3}+x_{2} y_{4}+x_{1} y_{2}-x_{3} y_{4}-x_{2} y_{1}+x_{3} y_{1}+x_{4} y_{3}-x_{4} y_{2}\right)\right] / \Phi
\end{aligned}
$$




$$
\begin{gathered}
\frac{\partial J_{22}^{-1}}{\partial \xi}=\left[-2\left(-x_{1} \eta+x_{1}+x_{2} \eta-x_{2}-x_{3} \eta-x_{3}+x_{4} \eta+x_{4}\right) *\right. \\
\left.*\left(x_{3} y_{2}+x_{2} y_{4}-x_{3} y_{1}-x_{2} y_{3}-x_{4} y_{2}+x_{4} y_{1}+x_{1} y_{3}-x_{1} y_{4}\right)\right] / \Phi \\
\frac{\partial J_{12}^{-1}}{\partial \eta}=2\left(x_{1} x_{3} y_{2}+x_{1} x_{4} y_{1}-x_{4}^{2} y_{1} \xi+x_{2}^{2} y_{4} \xi+x_{2} x_{4} y_{1}-x_{3}^{2} y_{2} \xi+x_{2} x_{4} y_{3}-x_{1} x_{3} y_{1}+\cdots\right. \\
\cdots+x_{1} x_{3} y_{4}-x_{2}^{2} \xi y_{3}+x_{1}^{2} y_{4} \xi-x_{2} x_{4} y_{2}-x_{3} x_{2} y_{1}+\cdots \\
\cdots+x_{2} x_{3} y_{2}+x_{4} x_{1} y_{2}-x_{2} x_{3} y_{4}-x_{1} x_{4} y_{3}-x_{3} x_{1} y_{3}-\cdots \\
\cdots-x_{4} x_{2} y_{4}+x_{3}^{2} y_{1} \xi-x_{1}^{2} y_{3} \xi-x_{4}^{2} y_{2} \xi+x_{3} x_{2} y_{3}+\cdots \\
\cdots+x_{1}^{2} y_{3}+x_{2}^{2} y_{4}+x_{3}^{2} y_{1}+x_{4}^{2} y_{2}-x_{1}^{2} y_{4}-x_{2}^{2} y_{3}-\cdots \\
\cdots-x_{3}^{2} y_{2}-x_{4}^{2} y_{1}-x_{1} x_{3} y_{2} \xi-2 x_{1} x_{2} y_{4} \xi+x_{1} x_{3} y_{1} \xi+\cdots \\
\cdots+2 x_{1} x_{2} y_{3} \xi+x_{1} x_{4} y_{2} \xi-x_{1} x_{4} y_{1} \xi+x_{2} x_{3} y_{2} \xi-\cdots \\
\cdots-x_{2} x_{3} y_{1} \xi-x_{2} x_{4} \xi y_{2}+x_{2} x_{4} \xi y_{1}-x_{3} x_{2} \xi y_{4}+\cdots \\
\cdots+x_{3} x_{2} y_{3} \xi+2 x_{3} x_{4} y_{2} \xi-2 x_{3} x_{4} y_{1} \xi-x_{3} x_{1} \xi y_{3}+\cdots \\
\left.\cdots+x_{3} x_{1} y_{4} \xi+x_{4} x_{2} y_{4} \xi-x_{4} x_{2} y_{3} \xi+x_{4} x_{1} y_{3} \xi-x_{4} x_{1} y_{4} \xi\right] / \Phi \\
\left.y_{2} x_{3} y_{4}-x_{3} y_{1}^{2}-x_{4} y_{2}^{2}-x_{1} y_{3}^{2}-x_{2} y_{4}^{2}\right] / \Phi
\end{gathered}
$$

com

$$
\begin{array}{r}
\Phi=-x_{1} \eta y_{3}+x_{2} \eta y_{4}+x_{3} \xi y_{2}+x_{1} \eta y_{2}-x_{3} \eta y_{4}+\cdots \\
\cdots+x_{2} \xi y_{4}-x_{2} \eta y_{1}+x_{3} \eta y_{1}-x_{3} \xi y_{1}-x_{2} \xi y_{3}-x_{4} \xi y_{2}+\cdots \\
\cdots+x_{4} \xi y_{1}+x_{4} \eta y_{3}-x_{4} \eta y_{2}-x_{1} y_{2}+x_{1} y_{4}+x_{1} \xi y_{3}-x_{4} y_{1}+\cdots \\
\left.\cdots+x_{4} y_{3}-x_{3} y_{4}+x_{3} y_{2}+x_{2} y_{1}-x_{2} y_{3}-x_{1} \xi y_{4}\right)^{2}
\end{array}
$$

Das eq.(5.41) a eq.(5.49), tem-se que todos os possíveis parâmetros dos integrandos da eq.(5.29) podem ser expressos no domínio paramétrico $\boldsymbol{\xi} \boldsymbol{\eta}$. Então, cada elemento da matriz da eq.(5.29) é escrito da seguinte forma:

$$
\int_{\Omega_{e}} f(\xi, \eta) d \Omega
$$

A solução numérica da integral representada na eq.(5.50) pode ser obtida da eq.(4.101) com auxílio das eq.(4.98) e eq.(4.100). 
Para o integrando da eq.(5.30), além dos parâmetros da eq.(5.34), existem funções do tipo $\left(\boldsymbol{\Psi}_{j} \boldsymbol{\Delta}_{\Gamma_{j}}\right), j(j=1,2)$. Assim, todo este integrando pode ser desenvolvido em termos de $\xi$ ou $\boldsymbol{\eta}$ constante, pois a integral é calculada no contorno $\boldsymbol{\Gamma}\left(\boldsymbol{\Gamma}_{t}, \boldsymbol{\Gamma}_{\boldsymbol{i}}\right.$ e $\left.\boldsymbol{\Gamma}_{u}\right)$. Com o auxílio das eq.(4.110) a eq.(4.114), pode-se aplicar a eq.(4.115) para a integração numérica das integrais presentes na eq.(5.30).

Por fim, a eq.(4.115) também pode ser aplicada na solução numérica da integral da eq.(5.32).

\subsubsection{Elemento Triangular de Três Nós}

\subsubsection{Características Geométricas e Transformação de Coordenadas}

As características geométricas e de transformação de coordenadas do elemento triangular da FHT são iguais à apresentada para o elemento triangular da FHMT.

\subsubsection{Funções Aproximativas do Elemento Triangular de Três Nós da FHT}

As aproximações base das tensões no domínio do elemento triangular de três nós da FHT são iguais às do elemento quadrilateral da FHT. Portanto as eq.(5.12) a eq.(5.14) são também utilizadas no elemento triangular de três nós da FHT.

Para a interpolação do campo de deslocamentos no contorno $\boldsymbol{\Gamma}_{\boldsymbol{t}_{\boldsymbol{e}_{\boldsymbol{T}_{t}}}}$ ou $\boldsymbol{\Gamma}_{\boldsymbol{i}_{\boldsymbol{e}_{\boldsymbol{i}}}}$ do elemento de domínio $\boldsymbol{\Omega}_{e}$, funções lineares Lagrangianas clássicas são aplicadas. Essas funções também foram empregadas no elemento de contorno do elemento triangular da FHMT. Assim, para o elemento de contorno da FHT derivado do elemento triangular valem as eq.(4.141) e eq.(4.142).

\subsubsection{Enriquecimento das Funções Aproximativas do Elemento Triangular de Três Nós da FHT}

As famílias de funções das tensões e deslocamentos no contorno e suas respectivas aproximações, construídas para o elemento quadrilateral da FHT (eq.(5.15), eq.(5.16), eq.(5.26) e eq.(5.27)) podem ser aproveitadas para a definição das famílias de funções e aproximações do elemento triangular, verificando-se as mudanças em relação às funções de suporte compacto $\boldsymbol{g}_{\boldsymbol{j}}$, de enriquecimento polinomial $\boldsymbol{h}_{\boldsymbol{j}}$ e das funções polinomiais enriquecedoras das variáveis de contorno $\boldsymbol{h}_{\boldsymbol{j} \boldsymbol{e}_{\boldsymbol{e}_{T}}}$. 
Então, para este elemento finito, adotam-se como funções de suporte compacto, $\boldsymbol{g}_{\boldsymbol{j}}, \boldsymbol{j}=\mathbf{1}, \ldots, \mathbf{3}$, as funções utilizadas para aproximação da geometria do elemento triangular híbrido misto de tensão, ver eq.(4.116) a eq.(4.118).

Para as $\boldsymbol{h}_{\boldsymbol{j}}$ (tipo bolha) são selecionadas funções iguais às apresentadas nas eq.(4.149) a eq.(4.153).

A matriz de interpolação das tensões enriquecidas $S_{\Omega_{e}}^{*}$ agora é dada por:

$$
S_{\Omega_{e}}^{*}=\left[\begin{array}{llll}
S_{\Omega_{e}} & \Sigma_{1} & \Sigma_{2} & \Sigma_{3}
\end{array}\right]
$$

valendo-se ainda as eq.(5.23), com $j=1, \ldots, 3$.

Como funções bolhas $\boldsymbol{h}_{\boldsymbol{j} \boldsymbol{n}_{\boldsymbol{e}}}$ podem ser utilizadas as ilustradas nas eq.(4.154) e eq.(4.155).

Com a transformação apresentada na eq.(4.103), podem-se expressar as derivadas $\frac{\partial \boldsymbol{g}_{j}}{\partial \boldsymbol{x}}, \frac{\partial \boldsymbol{h}_{j}}{\partial \boldsymbol{x}}, \frac{\partial \boldsymbol{g}_{j}}{\partial \boldsymbol{y}}, \frac{\partial \boldsymbol{h}_{j}}{\partial \boldsymbol{y}}, \frac{\partial^{2} \boldsymbol{g}_{j}}{\partial \boldsymbol{x}^{2}}, \frac{\partial^{2} \boldsymbol{h}_{j}}{\partial \boldsymbol{x}^{2}}, \frac{\partial^{2} \boldsymbol{g}_{j}}{\partial \boldsymbol{y}^{2}}, \frac{\partial^{2} \boldsymbol{h}_{j}}{\partial \boldsymbol{y}^{2}}, \frac{\partial^{2} \boldsymbol{g}_{j}}{\partial x \partial y} \mathrm{e} \frac{\partial^{2} \boldsymbol{h}_{j}}{\partial x \partial y}$ com $\boldsymbol{g}_{j}$ e $\boldsymbol{h}_{j}$ escritas em função das coordenadas $\xi_{1} \xi_{2}$.

Como já comentado, observa-se que, para esse tipo de enriquecimento, somente uma função de enriquecimento $\boldsymbol{h}_{\boldsymbol{j}}$ é acrescida a um nó $\boldsymbol{j}$ da rede de cobertura de domínio.

Assim para qualquer nó $\boldsymbol{j},(\boldsymbol{j}=\mathbf{1}, \ldots, 3)$ do elemento finito triangular $\boldsymbol{\Omega}_{e}$, escolhido para ser enriquecido, pode-se escrever:

- Para a matriz representada pela eq.(4.16) e com a eq.(5.51), tem-se

$$
\int_{\Omega_{e}}\left(S_{\Omega_{e}}^{*}\right)^{T} f\left(S_{\Omega_{e}}^{*}\right) d \boldsymbol{\Omega}=\left[\begin{array}{cccccc}
\int_{\Omega_{e}}\left(S_{\Omega_{e}}\right)^{T} f\left(S_{\Omega_{e}}\right) d \boldsymbol{\Omega} & \cdots & \int_{\Omega_{e}}\left(S_{\Omega_{e}}\right)^{T} f\left(\Sigma_{k}\right) d \boldsymbol{\Omega} & \cdots & \int_{\Omega_{e}}\left(S_{\Omega_{e}}\right)^{T} f\left(\Sigma_{n}\right) d \boldsymbol{\Omega} \\
\vdots & \ddots & \vdots & \ddots & \vdots \\
\int_{\Omega_{e}}\left(\Sigma_{l}\right) f\left(S_{\Omega_{e}}\right) d \boldsymbol{\Omega} & \cdots & \int_{\Omega_{e}}\left(\Sigma_{l}\right)^{T} f\left(\Sigma_{k}\right) d \boldsymbol{\Omega} & \cdots & \int_{\Omega_{e}}\left(\Sigma_{l}\right)^{T} f\left(\Sigma_{n}\right) d \boldsymbol{\Omega} \\
\vdots & \ddots & \vdots & \ddots & \vdots \\
\int_{\Omega_{e}}\left(\Sigma_{n}\right)^{T} f\left(S_{\Omega_{e}}\right) d \boldsymbol{\Omega} & \cdots & \int_{\Omega_{e}}\left(\Sigma_{n}\right)^{T} f\left(\Sigma_{k}\right) d \boldsymbol{\Omega} & \cdots & \int_{\Omega_{e}}\left(\Sigma_{n}\right)^{T} f\left(\Sigma_{n}\right) d \boldsymbol{\Omega}
\end{array}\right]
$$

onde $\boldsymbol{l}, \boldsymbol{k}$ são os nós enriquecidos do elemento quadrilateral e $\boldsymbol{n}$ é o número de nós enriquecidos no mesmo elemento. Observa-se que são acrescentadas $\boldsymbol{n}$ linhas e colunas à estrutura inicial da eq.(4.16). 
Para qualquer nó $\boldsymbol{j}(\boldsymbol{j}=\mathbf{1 , 2})$, de cada lado do elemento finito triangular que é escolhido para ser enriquecido, pode-se escrever:

- Para a matriz representada pelas eq.(4.18) e eq.(4.19), juntamente com as eq.(5.51) e eq.(4.145), escreve-se:

$$
\int_{\Gamma_{t} \text { ou } \Gamma_{i}}\left(N S_{\Omega_{e}}^{*}\right)^{T} U_{\Gamma_{e_{\Gamma}}} d \Gamma=\left[\begin{array}{c}
\int_{\Gamma_{t} \text { ou } \Gamma_{i}}\left(N S_{\Omega_{e}}\right)^{T}\left(\Psi_{j} \Delta_{\Gamma_{j}}\right) d \Gamma \\
\vdots \\
\int_{\Gamma_{\text {o }} \text { ou } \Gamma_{i}}\left(N \Sigma_{l}\right)^{T}\left(\Psi_{j} \Delta_{\Gamma_{j}}\right) d \Gamma \\
\vdots \\
\int_{\Gamma_{t} \text { ou } \Gamma_{i}}\left(N \Sigma_{n}\right)^{T}\left(\Psi_{j} \Delta_{\Gamma_{j}}\right) d \Gamma
\end{array}\right]
$$

onde $\boldsymbol{l}$ é o nó enriquecido do elemento quadrilateral de domínio e $\boldsymbol{n}$ é o número de nós enriquecidos no mesmo elemento. Observa-se que são acrescentadas $\boldsymbol{n}$ linhas à estrutura inicial da eq.(4.18) e eq.(4.19). O número de colunas adicionais à eq.(4.18) e eq.(4.19) depende das $\boldsymbol{n}_{\boldsymbol{e}_{\Gamma}}$ funções de enriquecimento adotadas para o campo de deslocamento no contorno.

- Para a matriz representada pela eq.(4.22), juntamente com a eq.(4.145), tem-se:

$$
\begin{gathered}
U_{\Gamma_{e_{\Gamma}}}^{T}=\left[\begin{array}{c}
\psi_{1} \Delta_{\Gamma_{1}}^{T} \\
\psi_{2} \Delta_{\Gamma_{2}}^{T}
\end{array}\right] \\
Q_{\Gamma_{e_{\Gamma}}}=\left[Q_{\Gamma_{y}}\right], \gamma=1,2
\end{gathered}
$$

onde:

$$
Q_{\Gamma_{y}}=\left[\int_{\Gamma_{t}} \psi_{y} \Delta_{\Gamma_{y}}^{T} \bar{t} d \Gamma\right], \gamma=1,2
$$




\subsubsection{Integrais Intervenientes}

Nesta etapa, ilustra-se a resolução numérica das integrais representadas nas eq.(5.52), eq.(5.53) e eq.(5.55) para os domínios paramétricos dos elementos triangular (ver figura 4.3) e linear (ver figura 4.5).

Nas integrais da eq.(5.52), valem as eq.(5.34) a eq.(5.40) além da transformação dada pela eq.(4.124).

Especificamente, o desenvolvimento de $\boldsymbol{J}_{11}^{-1}, \boldsymbol{J}_{12}^{-1}, \boldsymbol{J}_{21}^{-1}, \boldsymbol{J}_{22}^{-1}$ para um elemento paramétrico triangular (ver figura 4.3) foi apresentado nas eq.(4.186) a eq.(4.189), respectivamente.

Para os outros operadores destacados na eq.(5.40), tem-se:

$$
\frac{\partial J_{11}^{-1}}{\partial \xi}=\frac{\partial J_{11}^{-1}}{\partial \eta}=\frac{\partial J_{12}^{-1}}{\partial \eta}=\frac{\partial J_{21}^{-1}}{\partial \xi}=\frac{\partial J_{21}^{-1}}{\partial \eta}=\frac{\partial J_{22}^{-1}}{\partial \xi}=\frac{\partial J_{12}^{-1}}{\partial \eta}=0
$$

Dessa forma, tem-se que todos os possíveis parâmetros dos integrandos da eq.(5.52) podem ser expressos no domínio paramétrico $\xi_{1} \xi_{2}$. Então, cada elemento da matriz da eq.(5.52) é escrito da seguinte forma:

$$
\int_{\Omega_{e}} f(\xi, \eta) d \Omega
$$

A solução numérica da integral representada na eq.(5.58) pode ser obtida da eq.(4.183) com auxílio das eq.(4.180) e eq.(4.182).

No integrando da eq.(5.53), além dos parâmetros da eq.(5.34), existem funções do tipo

$\left(\boldsymbol{\Psi}_{j} \boldsymbol{L}_{\Gamma_{j}}\right), j \quad(j=1,2)$. Então, todo este integrando pode ser desenvolvido em termos de $\boldsymbol{\xi}_{1}$ ou $\xi_{2}$ constante, pois a integral é calculada no contorno $\Gamma\left(\Gamma_{t}, \Gamma_{i}\right.$ e $\left.\Gamma_{u}\right)$. Com o auxílio das eq.(4.192) a eq.(4.195), pode-se aplicar a eq.(4.196) para a integração numérica das integrais presentes na eq.(5.53).

Finalmente, a eq.(4.196) também pode ser aplicada na solução numérica da integral da eq.(5.55).

Vale lembrar que o desenvolvimento apresentado nas eq.(4.197) a eq.(4.203) também é aplicado ao sistema de equações da FHT com enriquecimento nodal. 


\section{Estudo das Condições de Convergência da FHT e FHMT com Enriquecimento Nodal}

\subsection{Introdução}

O objetivo do capítulo é contribuir com o estudo sobre questões relativas às condições necessárias e suficientes para convergência das soluções obtidas da FHT e FHMT com enriquecimento nodal, Góis (2004) e Góis e Proença (2005, 2006a, 2006b, 2007a, 2007b, 2007c).

De forma geral, para que se garanta com o refinamento da rede, a convergência da solução aproximativa de um problema linear obtida por qualquer formulação do MEF (envolvendo um campo ou múltiplos campos), o espaço de aproximação escolhido deve satisfazer basicamente duas condições: consistência e estabilidade.

Uma das garantias oferecidas pela condição de consistência é que com o tamanho dos elementos finitos tendendo a zero, a aproximação adotada passa a representar exatamente, no limite, a solução da equação diferencial e as condições de contorno do problema. Um aspecto, em particular inserido na condição de consistência, é a compatibilidade. A compatibilidade exige que as funções de forma utilizadas na aproximação dos campos devam ser tais que garantam a sua continuidade entre elementos. No MEF e no MEFG a consistência é garantida pelo conceito da Partição da Unidade (PU).

Já se a condição de estabilidade é satisfeita, a solução do sistema de equações discretas existe e converge, com o refinamento, para uma solução única. Tendo-se em vista um elemento ou uma rede de elementos finitos, a condição de estabilidade pressupõe o atendimento a duas outras condições: a primeira é representada pela elipsidade e a segunda pela condição de Babuška-Brezzi (inf-sup), proposta independentemente por Babuška (1971, 1973) e Brezzi (1974). 
Bathe (1996) afirma que as condições de consistência e elipsidade são relativamente fáceis de atender, ao contrário da condição de Babuška-Brezzi (inf-sup). O mesmo autor ainda pontua que a prova analítica dessa última condição é freqüentemente de difícil obtenção e, por isso, procedimentos numéricos foram desenvolvidos para sua análise, Chapelle e Bathe (1993), Bathe (1996) e Bathe et al. (2000).

Neste trabalho são propostos dois testes numéricos para verificar condições necessárias e suficientes de convergência da solução da FHT e FHMT com enriquecimento nodal. O primeiro (denominado 'Teste por Inspeção'), decorre da análise das condições para solvabilidade do sistema e está baseado em Zienkiewicz et al. (1986). O segundo (denominado teste inf-sup) é fundamentado nos trabalhos de Babuška (1996), Chapelle e Bathe (1993), caracterizando-se como um critério de estabilidade. Esse teste foi usado para verificar se a FHT e a FHMT com enriquecimento nodal pode proporcionar soluções que convergem para a solução exata.

\subsection{O 'Teste por Inspeção'}

\subsubsection{Considerações Iniciais}

Neste item a ênfase é dada à solvabilidade do sistema de equações lineares que governam a FHT e FHMT com enriquecimento nodal, como primeiro passo no estudo da estabilidade de soluções obtidas destas duas formulações. Essencialmente, adapta-se a técnica definida no trabalho de Zienkiewicz et al. (1986) que se constitui condição algébrica simples para garantir a estabilidade de formulações mistas.

Verifica-se que esta condição está ligada à solvibilidade do sistema de equações lineares discretas da formulação Mista, e constitui-se numa condição algébrica necessária para a não singularidade desse sistema linear.

\subsubsection{O 'Teste por Inspeção'}

No 'Teste por Inspeção' para Formulações Mistas, apresentado no trabalho de Zienkiewicz et al. (1986), analisa-se um sistema típico resultante da aproximação mista para dois campos, dado por:

$$
\left[\begin{array}{cc}
A & B \\
B^{T} & 0
\end{array}\right]\left\{\begin{array}{l}
x \\
y
\end{array}\right\}=\left\{\begin{array}{l}
f_{1} \\
f_{2}
\end{array}\right\}
$$


onde $\boldsymbol{A}$ e $\boldsymbol{B}$ são matrizes e $\boldsymbol{x}$ e $\boldsymbol{y}$ são vetores com dimensões $\left(\boldsymbol{n}_{\boldsymbol{x}} \times \boldsymbol{n}_{\boldsymbol{x}}\right),\left(\boldsymbol{n}_{\boldsymbol{x}} \times \boldsymbol{n}_{\boldsymbol{y}}\right),\left(\boldsymbol{n}_{\boldsymbol{x}}\right)$ e $\left(\boldsymbol{n}_{\boldsymbol{y}}\right)$ respectivamente. $\left(\boldsymbol{n}_{\boldsymbol{x}}\right)$ e $\left(\boldsymbol{n}_{\boldsymbol{y}}\right)$ são os números de componentes dos vetores $\boldsymbol{x}$ e $\boldsymbol{y}$, respectivamente.

Em particular os vetores $\boldsymbol{x}$ e $\boldsymbol{y}$ podem representar os campos de tensão e deslocamento $(\boldsymbol{\sigma}$ e $\boldsymbol{u}$ ) e a eq. (6.1) pode resultar da aproximação do funcional clássico de Reissner-Helinger.

A condição algébrica necessária para que o sistema de equações dado pela eq.(6.1) seja não-singular é:

$$
\boldsymbol{n}_{\boldsymbol{x}} \geq \boldsymbol{n}_{\boldsymbol{y}}
$$

ou seja, a dimensão do vetor $\boldsymbol{x}$ deve ser maior ou igual a dimensão do vetor $\boldsymbol{y}$.

A condição representada pela eq.(6.2) fica evidente com o seguinte desenvolvimento:

Assume-se que na eq.(6.1) a matriz $\boldsymbol{A}$ seja não-singular. Assim, pode-se eliminar o vetor $\boldsymbol{x}$ da primeira linha e substituí-lo na segunda para obter:

$$
\left(B^{T} A^{-1} B\right) y=-f_{2}+B^{T} A^{-1} f_{1}
$$

Para a determinação do vetor $\boldsymbol{y}$ é necessário garantir que o produto matricial $\left(\boldsymbol{B}^{T} \boldsymbol{A}^{-1} \boldsymbol{B}\right)$ seja inversível. Então:

$$
\underbrace{B_{\left(n_{y} \times n_{x}\right)}^{T} A_{\left(n_{x} \times n_{x}\right)}^{-I}}_{(I)} \underbrace{B_{\left(n_{x} \times n_{y}\right)}}_{(I I)}
$$

Da primeira multiplicação matricial da eq.(6.4), representada por (I), resultará a seguinte matriz:

$$
C_{\left(n_{y} \times n_{x}\right)}
$$

Agora, multiplicando-se a eq.(6.5) por (II), tem-se:

$$
C_{\left(n_{y} \times n_{x}\right)} B_{\left(n_{x} \times n_{y}\right)}=D_{\left(n_{y} \times n_{y}\right)}
$$


Da Álgebra Matricial, tem-se a seguinte condição: dadas uma matriz $\boldsymbol{C}$ de dimensão $\left(\boldsymbol{n}_{\boldsymbol{y}} \times \boldsymbol{n}_{x}\right)$ e uma matriz $\boldsymbol{B}$ de dimensão $\left(\boldsymbol{n}_{x} \times \boldsymbol{n}_{\boldsymbol{y}}\right)$, a matriz $\boldsymbol{D}$ resultante da multiplicação de $\boldsymbol{C} \boldsymbol{B}$ só será inversível se $\boldsymbol{n}_{\boldsymbol{x}} \geq \boldsymbol{n}_{\boldsymbol{y}}$.

A condição dada pela eq.(6.2), ao evitar múltiplas soluções para a eq.(6.1), garante ainda que o vetor nulo não seja uma solução para o vetor $\boldsymbol{x}$. Vale salientar que a eq.(6.2) é uma condição necessária, mas não suficiente para garantir a não singularidade da matriz $\boldsymbol{D}$. Para que exista solução única da eq.(6.1), deve-se ainda ter:

$$
B y \neq 0 \quad \forall \quad y \neq 0
$$

Os autores ainda afirmam que todas as condições apresentadas anteriormente, de certa forma, estão presentes na condição de Babuška-Brezzi.

Em termos práticos, o 'Teste por Inspeção' para Formulações Mistas consiste basicamente em verificar se o vetor incógnito do sistema de equações lineares, obtido da discretização utilizada na análise, satisfaz a condição dada pela eq.(6.2). Para aplicar os conceitos fundamentais do trabalho de Zienkiewicz et al. (1986) à FHT e FHMT sem enriquecimento nodal, considere-se o sistema de equações dado pela eq.(2.36). Assim, definem-se as seguintes condições algébricas necessárias para existência de uma solução numérica:

$$
\begin{aligned}
& \boldsymbol{s}_{\Omega} \geq \boldsymbol{q}_{\Omega} \\
& \boldsymbol{s}_{\Omega} \geq \boldsymbol{q}_{\Gamma_{t}} \text { ou } \boldsymbol{s}_{\Omega} \geq \boldsymbol{q}_{\Gamma_{i}}
\end{aligned}
$$

As eq.(6.8) e eq.(6.9) são aplicadas a FHMT e para a FHT só a eq.(6.9) faz sentido quando se aplica a eq.(2.69) a eq.(2.36).

Quando se considera o enriquecimento dos campos de tensão e deslocamento no domínio e contorno do elemento, respectivamente, o ‘Teste por Inspeção’ é ampliado pela inclusão dos novos parâmetros de tensões no domínio $\boldsymbol{b}_{j i}$ (eq.(4.46) - FHMT) e $\boldsymbol{b}_{\boldsymbol{j}}$ (eq.(5.16) - FHT), deslocamentos no domínio $\boldsymbol{c}_{j i}$ (eq.(4.48) - FHMT) e deslocamento no contorno $\boldsymbol{d}_{j i}$ (eq.(4.50) - FHMT) e $\boldsymbol{d}_{j l}$ (eq.(5.27) - FHT).

Assim, as eq.(6.8) e eq.(6.9) passam a ser escritas da seguinte forma:

$$
\boldsymbol{s}_{\Omega}+\boldsymbol{b}_{i j} \geq \boldsymbol{q}_{\Omega}+\boldsymbol{c}_{j i} \text { para a FHMT }
$$




$$
\begin{aligned}
& \boldsymbol{s}_{\Omega}+\boldsymbol{b}_{i j} \geq \boldsymbol{q}_{\Gamma_{t}}+\boldsymbol{d}_{j i} \text { ou } \boldsymbol{s}_{\Omega}+\boldsymbol{b}_{i j} \geq \boldsymbol{q}_{\Gamma_{i}}+\boldsymbol{d}_{j i} \text { para a FHMT } \\
& \boldsymbol{s}_{\Omega}+\boldsymbol{b}_{j} \geq \boldsymbol{q}_{\Gamma_{t}}+\boldsymbol{d}_{j l} \text { ou } \boldsymbol{s}_{\Omega}+\boldsymbol{b}_{j} \geq \boldsymbol{q}_{\Gamma_{i}}+\boldsymbol{d}_{j l} \text { para a FHT }
\end{aligned}
$$

Deste modo, o 'Teste por Inspeção' para FHT e FHMT com enriquecimento nodal pode ser aplicado da mesma forma que o 'Teste por Inspeção' para Formulações Mistas. Escolhidas as discretizações do problema, investigam-se as condições eq.(6.10) a eq.(6.12) para essas redes de elementos finitos. Vale ressaltar, novamente, que esse teste é uma condição necessária, mas não suficiente para garantia da solvibilidade da eq.(2.36), incluindo-se ou não a restrição dada pela eq.(2.69). A condição suficiente para solvibilidade do sistema discreto da FHT e FHMT com enriquecimento é observada pela análise do significado físico dos autovalores da matriz dos coeficientes da eq.(2.36) (especificamente para a FHT é mandatório ainda considerar a eq.(2.69) aplicada à eq.(2.36)).

\subsection{Estudo da Condição de Babuška-Brezzi (inf-sup) Aplicada à FHT e FHMT}

\subsubsection{Introdução}

No item anterior, tratou-se do 'Teste por Inspeção’ para a FHT e FHMT, que constitui condição necessária para que uma seqüência de soluções aproximadas possa convergir para a solução de certo problema. Neste item, aborda-se a condição de Babuška-Brezzi (ou condição inf-sup), Babuška $(1971,1973)$ e Brezzi (1974), que apresenta um caráter mais amplo: trata-se de condição suficiente para a convergência de aproximações numéricas, obtida com o MEF, de toda uma classe de problemas caracterizada por certo operador linear.

Inicialmente faz-se uma apresentação da condição, em sua forma originalmente proposta, considerando um operador, ou forma bilinear, representativo de uma classe genérica de problemas de valor de contorno, destacando-se a associação direta da condição de Babuška-Brezzi com aspectos de estabilidade do operador e, portanto, de convergência.

Por outro lado, a extensão dessa condição para as formas não-convencionais FHT e FHMT não tem proposição formal na literatura. Por essa razão, propõe-se uma alternativa numérica, baseada em Babuška (1996) e no trabalho de Chapelle e Bathe (1993). Tal alternativa é então empregada para verificar se aproximações geradas por elementos quadrilaterais de quatro nós e triangulares de três nós da FHT e FHMT com enriquecimento nodal satisfazem ou não essa condição. 


\subsubsection{A Condição de Babuška-Brezzi (inf-sup)}

Seja um problema variacional geral caracterizado por uma forma bilinear $\boldsymbol{B}(\boldsymbol{\varphi}, \boldsymbol{\psi})$ definida em $\boldsymbol{W} \times \boldsymbol{W}$, onde $\boldsymbol{W}$ é um espaço de Hilbert (um espaço vetorial, munido de um produto interno, e completo em relação à norma definida com esse produto interno, Bathe (1996), Schwab (1998) e Brezzi e Fortin (1991)).

O primeiro argumento da forma bilinear $\boldsymbol{B}(\cdot, \cdot)$ é denominado função admissível ou solução e o segundo função peso ou teste.

Além disso, admita-se que para a classe de problemas a ser considerada, defina-se o espaço de Hilbert como:

$$
W=\left\{u \mid u \in L^{2}(\Omega) ; \frac{\partial u}{\partial x_{j}} \in L^{2}(\Omega), j=1,2,3 ; u=0 \text { em } \Gamma_{u}\right\}
$$

onde $\boldsymbol{L}^{2}(\boldsymbol{\Omega})$ é o espaço das funções quadrado integráveis no domínio $\boldsymbol{\Omega}$ do corpo considerado e se admite que a condição de contorno de Dirichlet é zero em $\boldsymbol{\Gamma}_{u}$. Formalmente, representa-se o espaço $\boldsymbol{L}^{2}(\boldsymbol{\Omega})$ como:

$$
L^{2}(\Omega)=\left\{w \mid w \text { é definido em } \Omega \text { e } \int_{\Omega} w^{2} d \Omega=\|w\|_{L^{2}(\Omega)}^{2}<+\infty\right\}
$$

Isto posto, dado um funcional linear contínuo $\boldsymbol{F}(\boldsymbol{\psi}): \boldsymbol{W} \rightarrow \mathbb{R}$, um problema de valor de contorno pode ser representado na seguinte forma:

Determinar $\boldsymbol{\varphi} \in \boldsymbol{W}$ tal que

$$
B(\varphi, \psi)=F(\psi) \forall \psi \in W
$$

Admitindo-se que a abordagem adotada para gerar uma aproximação para a forma bilinear seja tipo Galerkin, então o espaço das funções admissíveis é igual ao espaço das funções peso. Além disso, o emprego do método dos elementos finitos para a geração das aproximações acaba por atribuir uma dimensão finita ao espaço solução.

Assim sendo, pode-se introduzir o subespaço de $\boldsymbol{W}$, de dimensão finita, como:

$$
W_{n}=\left\{u_{n} \mid u_{n} \in L^{2}(\Omega) ; \frac{\partial u_{n}}{\partial x_{j}} \in L^{2}(\Omega), j=1,2,3 ; u_{n} \in Q_{n}\left(\Omega^{(m)}\right) ; u_{n}=0 \text { em } \Gamma_{u}\right\}
$$


onde $\boldsymbol{Q}_{n}\left(\boldsymbol{\Omega}^{(\boldsymbol{m})}\right)$ indica o caráter polinomial de grau “ $\boldsymbol{n}$ ” da aproximação global $\boldsymbol{u}_{n}$ quando restrita ao elemento $\boldsymbol{m}$ do domínio discretizado. Nessas condições, tem-se que a solução por Elementos Finitos da eq.(6.15) é obtida resolvendo-se o seguinte problema:

Encontrar $\varphi_{n} \in \boldsymbol{W}_{n}$ tal que

$$
B\left(\varphi_{n}, \psi_{n}\right)=F\left(\psi_{n}\right) \forall \psi_{n} \in W_{n}
$$

Para medir a qualidade da aproximação por Elementos Finitos, no sentido de erro em relação à solução exata, é necessário introduzir normas, representadas por: $\|\cdot\|_{S}$ e $\|\cdot\|_{T}$ nos espaços das funções admissíveis e teste, respectivamente. Assim, no espaço das funções admissíveis, podem ser definidas:

$$
\left(\left\|\varphi-\varphi_{n}\right\|_{S}\right)
$$

e

$$
\underset{\eta_{n} \in W_{n}}{\inf }\left(\left\|\varphi-\eta_{n}\right\|_{S}\right), \operatorname{com} \eta_{n} \in W_{n}
$$

A norma representada pela eq.(6.18) mede o erro entre a solução exata $\varphi$ e uma solução aproximada $\varphi_{n}$. Tal norma pode ser empregada como uma medida de convergência de uma seqüência de soluções se: $\left\|\boldsymbol{\varphi}-\boldsymbol{\varphi}_{n}\right\|_{S} \rightarrow \boldsymbol{0} \boldsymbol{p} / \boldsymbol{n} \rightarrow \infty$. Um aspecto importante que se pode demonstrar é que a solução por elementos finitos é aquela que apresenta o menor erro de aproximação. Assim sendo, uma vez adotado o MEF, a convergência passa a depender da ordem do espaço de aproximação adotado e da própria solução exata, isto é:

$$
\inf _{\eta_{h} \in W_{h}}\left\|\varphi-\eta_{h}\right\|_{S}=\Phi^{n}(\varphi) \rightarrow 0 \text { para } n \rightarrow \infty
$$

Já a norma da eq.(6.19) mede o menor erro possível entre todas as possíveis aproximações $\boldsymbol{\eta}_{\boldsymbol{n}}$ do espaço discretizado $\boldsymbol{W}_{\boldsymbol{n}}$; este resultado pode ser tomado como uma medida da 'aproximabilidade’ obtida com uma seqüência de soluções.

Ao se aplicar uma alternativa numérica como o método dos elementos finitos a expectativa é que a metodologia de geração de aproximações produza soluções convergentes não somente em relação à certa solução exata, mas para todo conjunto de soluções exatas de uma classe de problemas. Assim sendo, a análise de convergência deve assumir um sentido 
mais amplo, passando a depender da capacidade de aproximação do espaço de funções escolhido e, também, da estabilidade forma bilinear que rege a classe de problemas. Nesse sentido, Bathe (1996), Brezzi e Bathe (1990) e Brezzi e Fortin (1991), demonstram a seguinte expressão que relaciona as medidas de convergência e 'aproximabilidade' mencionadas, envolvendo uma constante relacionada à classe de problemas:

$$
\left\|\varphi-\varphi_{n}\right\|_{S} \leq\left(1+\frac{k_{m}}{\lambda}\right) \inf _{\eta_{n} \in W_{n}}\left\|\varphi-\eta_{n}\right\|_{S}
$$

onde $\boldsymbol{k}_{\boldsymbol{m}}$ vem da condição de continuidade da forma bilinear, que pode ser expressa por:

$$
B(\eta, \psi) \leq k_{m}\|\eta\|_{S}\|\psi\|_{T} \forall \eta, \psi \in W
$$

Aliás, um requisito mínimo sobre $\boldsymbol{B}(\boldsymbol{\eta}, \boldsymbol{\psi})$ e $\boldsymbol{F}(\boldsymbol{\psi})$, para que a eq.(6.17) tenha sentido, é a sua continuidade.

Ainda na eq.(6.21), a constante $\lambda$ é obtida da condição de Babuška-Brezzi (inf-sup), e se apresentar um valor positivo aponta para a estabilidade da forma bilinear. Para espaços de dimensão finita a condição inf-sup passa a ser definida por:

$$
\underset{\eta_{h} \in W_{h} \psi_{h} \in W_{h}}{\sup } \frac{B\left(\eta_{h}, \psi_{h}\right)}{\left\|\eta_{h}\right\|_{S}\left\|\psi_{h}\right\|_{T}}=\lambda>0
$$

Claramente o valor de $\lambda$ está associado à forma bilinear que rege a classe de problemas.

Pode-se mostrar, ainda, que as eq.(6.22) (continuidade) e eq.(6.23) (condição inf-sup) implicam na eq.(6.21). Assim, se a eq.(6.23) é atendida, conclui-se pela eq.(6.21) que as soluções por elementos finitos apresentam-se convergentes para toda solução exata da classe de problemas governada pela forma bilinear.

Ao contrário, se $\boldsymbol{\lambda} \rightarrow \boldsymbol{0}^{+}$a análise da eq.(6.21) mostra que haverá convergência somente para soluções em relação às quais $\Phi^{n}(\varphi)$ tenda à zero mais rapidamente do que o multiplicador $\left(\boldsymbol{1}+\frac{\boldsymbol{k}_{\boldsymbol{m}}}{\boldsymbol{\lambda}}\right)$ tender ao infinito. Isto não é sempre garantido, podendo haver ao menos uma solução $\boldsymbol{\varphi}$ da classe de problemas para a qual essa situação se inverta e a solução por elementos finitos não apresente convergência. 
Em termos do emprego prático das condições anteriores, numa primeira situação se a condição de Babuška-Brezzi indicar $(\boldsymbol{\lambda}>\boldsymbol{0})$ para certa solução aproximada de um dado problema então a eq.(6.21) fornece uma medida do erro de aproximação. Numa segunda etapa a constatação de $\boldsymbol{\lambda} \rightarrow \boldsymbol{0}$ para sucessivas aproximações mais refinadas indica que pode haver problemas de convergência em relação à solução exata daquele problema.

Numa situação mais geral, pode-se, em última análise, comprovar, ou não, a robustez de determinado elemento finito, avaliando pela eq.(6.23) a evolução das constantes de estabilidade para as seqüências de soluções, de todos os problemas de uma classe, obtidas por discretizações realizadas com aquele elemento. Esta situação é, porém, impraticável, podendo ser conduzida parcialmente em termos operacionais, mas ainda assim em grau suficiente para oferecer um indicativo de robustez, conforme se mostra no capítulo de aplicações.

\subsubsection{Determinação Numérica de $\lambda$}

Como salientado anteriormente, a condição dada pela eq.(6.21) pode servir ao controle das soluções obtidas com o MEF e por esta razão é de interesse estimar o valor de $\lambda$. O valor analítico de $\lambda$, dependendo da formulação do MEF estudada, não é facilmente determinado, seguindo daí a necessidade de sua avaliação numérica. O desenvolvimento a seguir reproduz, em parte, o conteúdo apresentado em Babuška (1996), o qual será estendido nos próximos itens para a FHMT e FHT.

Assim, considere-se a eq.(6.17) escrita na forma matricial:

Determinar $\mathbf{x} \in \mathbb{R}^{n}$ tal que:

$$
\mathbf{B x}=\mathbf{f}
$$

onde $\mathbf{B}$ é uma matriz simétrica com dimensão $\boldsymbol{n} \times \boldsymbol{n}$ e $\mathbf{f} \in \mathbb{R}^{\boldsymbol{n}}$. Agora a eq.(6.23), para uma determinada rede de cobertura, que define certo grau $\boldsymbol{n}$ de aproximação, pode ser apresentada da seguinte forma:

$$
\underset{\eta}{\inf \sup } \frac{\psi^{\mathrm{T}} \mathrm{B} \eta}{\left(\eta^{\mathrm{T}} \mathrm{S} \eta\right)^{\frac{1}{2}}\left(\psi^{\mathrm{T}} \mathrm{T} \psi\right)^{\frac{1}{2}}}=\lambda_{n} \geq \lambda>0
$$

onde $\mathbf{S}$ e $\mathbf{T}$ são matrizes simétricas positivo-definidas obtidas das normas $\left\|\boldsymbol{\eta}_{n}\right\|_{S}^{2}=\left(\boldsymbol{\eta}^{\mathrm{T}} \mathbf{S} \boldsymbol{\eta}\right)$ e $\left\|\psi_{n}\right\|_{T}^{2}=\left(\psi^{\mathrm{T}} \mathbf{T} \psi\right) ; \boldsymbol{\eta}$ e $\psi$ são vetores que reúnem os valores nodais de $\eta_{n}$ e $\psi_{n}$, respectivamente e $\boldsymbol{B}\left(\boldsymbol{\eta}_{n}, \boldsymbol{\psi}_{n}\right)$ foi escrito na forma $\boldsymbol{\psi}^{\mathrm{T}} \mathbf{B} \boldsymbol{\eta}$. 
Introduzindo:

$$
\mathbf{R}(\eta, \psi)=\underset{\psi}{\sup } \frac{\psi^{\mathrm{T}} \mathrm{B} \eta}{\left(\psi^{\mathrm{T}} \mathrm{T} \psi\right)^{\frac{1}{2}}}
$$

a condição dada pela eq.(6.23), pode ser escrita como:

$$
\inf _{\eta} \frac{\mathrm{R}(\eta, \psi)}{\left(\eta^{\mathrm{T}} \mathrm{S} \eta\right)^{\frac{1}{2}}}=\lambda_{n}>0
$$

Por um lado, como $\mathbf{T}$ é simétrica e positivo-definida existe uma matriz simétrica positivo-definida $\mathbf{T}^{\frac{1}{2}}$ tal que $\mathbf{T}^{\frac{1}{2}} \mathbf{T}^{\frac{1}{2}}=\mathbf{T}$, Babuška (1996). Então, independente do resultado que implica na igualdade para a eq.(6.26), pode-se escrever que:

$$
\mathbf{R}(\boldsymbol{\eta}, \psi) \geq \frac{\psi^{\mathrm{T}} \mathbf{B} \boldsymbol{\eta}}{\left(\psi^{\mathrm{T}} \mathbf{T}^{\frac{1}{2}} \mathbf{T}^{\frac{1}{2}} \psi\right)^{\frac{1}{2}}}
$$

Por outro lado, a (6.26) deve valer para qualquer $\boldsymbol{\psi}$, assim sendo, seja, em particular:

$$
\psi=\mathbf{T}^{-1} \mathbf{B} \boldsymbol{\eta}
$$

Assim sendo, a eq.(6.28) passa a ser dada por:

$$
\mathbf{R}(\boldsymbol{\eta}, \psi) \geq \frac{\overbrace{\boldsymbol{\eta}^{\mathrm{T}} \mathbf{B}^{\mathrm{T}} \mathbf{T}^{\frac{-1}{2}}}^{\mathrm{C}^{\mathrm{T}}} \overbrace{\mathbf{T}^{\frac{-1}{2}} \mathbf{B} \boldsymbol{\eta}}^{\mathrm{C}}}{(\underbrace{\boldsymbol{\eta}^{\mathrm{T}} \mathbf{B}^{\mathrm{T}} \mathbf{T}^{\frac{-1}{2}}}_{\mathrm{C}^{\mathrm{T}}} \underbrace{\mathbf{T}^{\frac{-1}{2}} \mathbf{B} \boldsymbol{\eta}}_{\mathrm{C}})^{\frac{1}{2}}}
$$

Da desigualdade de Schwartz $\left(\frac{\left|\mathbf{C}^{\mathbf{T}} \mathbf{D}\right|}{|\mathbf{D}|} \leq|\mathbf{D}|\right)$ pode-se concluir que: 


$$
\frac{\left|\boldsymbol{\eta}^{\mathrm{T}} \mathbf{B}^{\mathrm{T}} \mathbf{T}^{\frac{-1}{2}} \mathbf{T}^{-1} \mathbf{B} \boldsymbol{\eta}\right|}{\left(\boldsymbol{\eta}^{\mathrm{T}} \mathbf{B}^{\mathrm{T}} \mathbf{T}^{\frac{-1}{2}} \mathbf{T}^{\frac{-1}{2}} \mathbf{B} \boldsymbol{\eta}\right)^{\frac{1}{2}}} \leq\left(\boldsymbol{\eta}^{\mathrm{T}} \mathbf{B}^{\mathrm{T}} \mathbf{T}^{-1} \mathbf{B} \boldsymbol{\eta}\right)^{\frac{1}{2}}
$$

Assim:

$$
\mathbf{R}(\boldsymbol{\eta}, \Psi)=\left(\boldsymbol{\eta}^{\mathrm{T}} \mathbf{B}^{\mathrm{T}} \mathbf{T}^{-1} \mathbf{B} \boldsymbol{\eta}\right)^{\frac{1}{2}}
$$

Das eq.(6.27) e eq.(6.32), tem-se:

$$
\lambda_{n}^{2}=\underset{\eta}{\eta} \frac{\eta^{\mathrm{T}} \mathbf{B}^{\mathrm{T}} \mathbf{T}^{-1} \mathbf{B} \boldsymbol{\eta}}{\eta^{\mathrm{T}} \mathrm{S} \eta}
$$

Na expressão à direita da eq.(6.33) reconhece-se o quociente de Rayleigh generalizado, Bathe (1996). Uma propriedade deste quociente é que valores estacionários para ele são obtidos por um problema de autovalor, no caso, dado por:

$$
\mathbf{B}^{\mathrm{T}} \mathbf{T}^{-1} \mathbf{B} \boldsymbol{\eta}=\mu \mathbf{S} \eta
$$

onde $\boldsymbol{\eta}$ e $\boldsymbol{\mu}$ são respectivamente os auto-vetores e os autovalores da eq.(6.34). Dessa forma, para certa formulação, constantes físicas e espaços dimensionais finitos, a eq.(6.33) fica satisfeita por um $\lambda_{n}^{2}$ que corresponde ao menor autovalor do problema descrito pela eq.(6.34); naturalmente, o valor de $\lambda_{n}$ é igual $\sqrt{\boldsymbol{\mu}_{\min }}$.

\subsubsection{O Teste Numérico da Condição de Babuška-Brezzi (inf-sup) aplicado à FHMT com Enriquecimento Nodal.}

Sejam as equações que governam a FHMT escritas da seguinte forma:

$$
\begin{aligned}
& \int_{\Omega} \delta \sigma^{T} f \sigma d \Omega+\int_{\Omega} u^{T}(L \delta \sigma) d \Omega-\int_{\Gamma_{t}} u_{\Gamma_{t}}^{T}(N \delta \sigma) d \Gamma-\int_{\Gamma_{i}} u_{\Gamma_{i}}^{T}\left(N_{+} \delta \sigma\right) d \Gamma=\int_{\Gamma_{u}} \bar{u}^{T}(N \delta \sigma) d \Gamma \\
& \int_{\Omega} \delta u^{T}(L \sigma) d \Omega=-\int_{\Omega} \delta u^{T} b d \Omega \\
& \int_{\Gamma_{t}} \delta u_{\Gamma_{t}}^{T}(N \sigma) d \Gamma=\int_{\Gamma_{t}} \delta u_{\Gamma_{t}}^{T} \bar{t} d \Gamma \\
& \int_{\Gamma_{i}} \delta u_{\Gamma_{i}}^{T}\left(N_{+} \sigma\right) d \Gamma+\int_{\Gamma_{i}} \delta u_{\Gamma_{i}}^{T}\left(N_{-} \sigma\right) d \Gamma=0
\end{aligned}
$$


Baseado em Schwab (1998), para a formulação híbrido-mista de tensão, pode-se definir para as eq.(6.35) a eq.(6.38) uma forma bilinear $\boldsymbol{B}(.,$.$) e um funcional linear \boldsymbol{F}($.$) ,$ como segue abaixo:

$$
\begin{gathered}
B(\eta, \psi)=\int_{\Omega} \delta \sigma^{T} f \sigma d \Omega+\int_{\Omega} u^{T}(L \delta \sigma) d \Omega-\int_{\Gamma_{t}} u_{\Gamma_{t}}^{T}(N \delta \sigma) d \Gamma+\cdots \\
\cdots+\int_{\Omega} \delta u^{T}(L \sigma) d \Omega+\int_{\Gamma_{t}} \delta u_{\Gamma_{t}}^{T}(N \sigma) d \Gamma-\int_{\Gamma_{i}} u_{\Gamma_{i}}^{T}\left(N_{+} \delta \sigma\right) d \Gamma+\cdots \\
\cdots+\int_{\Gamma_{i}} \delta u_{\Gamma_{i}}^{T}\left(N_{+} \sigma\right) d \Gamma+\int_{\Gamma_{i}} \delta u_{\Gamma_{i}}^{T}\left(N_{-} \sigma\right) d \Gamma \\
F(\psi)=\int_{\Gamma_{u}} \bar{u}^{T}(N \delta \sigma) d \Gamma-\int_{\Omega} \delta u^{T} b d \Omega+\int_{\Gamma_{t}} \delta u_{\Gamma_{t}}^{T} \bar{t} d \Gamma
\end{gathered}
$$

onde $\boldsymbol{\eta}=\left(\boldsymbol{\sigma}, \boldsymbol{u}, \boldsymbol{u}_{\Gamma_{t}}, \boldsymbol{u}_{\Gamma_{i}}\right)$ e $\boldsymbol{\psi}=\left(\boldsymbol{\delta} \boldsymbol{\sigma}, \boldsymbol{\delta} \boldsymbol{u}, \boldsymbol{\delta} \boldsymbol{u}_{\Gamma_{t}}, \boldsymbol{\delta} \boldsymbol{u}_{\Gamma_{i}}\right)$ são definidos em $\boldsymbol{S} \times \boldsymbol{T}$ com:

$$
\begin{aligned}
& S=\left\{\left(\sigma, u, u_{\Gamma_{t}}, u_{\Gamma_{i}}\right): \sigma, u \in L^{2}(\Omega) ; u_{\Gamma_{t}}, u_{\Gamma_{i}} \in L^{2}(\Gamma)\right\} \\
& T=\left\{\left(\delta \sigma, \delta u, \delta u_{\Gamma_{t}}, \delta u_{\Gamma_{i}}\right): \delta \sigma, \delta u \in L^{2}(\Omega) ; \delta u_{\Gamma_{t}}, \delta u_{\Gamma_{i}} \in L^{2}(\Gamma)\right\}
\end{aligned}
$$

Pode-se, ainda, definir as seguintes normas:

$$
\begin{aligned}
& \|\eta\|_{S}^{2}=\left\|\left(\sigma, u, u_{\Gamma_{t}}, u_{\Gamma_{i}}\right)\right\|_{S}^{2}=\int_{\Omega} \sigma^{2} d \Omega+\int_{\Omega} u^{2} d \Omega+\int_{\Gamma_{t}} u_{\Gamma_{t}}^{2} d \Gamma+\int_{\Gamma_{i}} u_{\Gamma_{i}}^{2} d \Gamma \\
& \|\psi\|_{T}^{2}=\left\|\left(\delta \sigma, \delta u, \delta u_{\Gamma_{t}}, \delta u_{\Gamma_{i}}\right)\right\|_{T}^{2}=\int_{\Omega} \delta \sigma^{2} d \Omega+\int_{\Omega} \delta u^{2} d \Omega+\int_{\Gamma_{t}} \delta u_{\Gamma_{t}}^{2} d \Gamma+\int_{\Gamma_{i}} \delta u_{\Gamma_{i}}^{2} d \Gamma
\end{aligned}
$$

Agora, para a aplicação do "teste inf-sup" a certa rede de cobertura com elementos finitos quadrilaterais de quatro nós e triangular de três nós da FHMT com enriquecimento, é preciso determinar todas as matrizes envolvidas na eq.(6.34). Para isso, consideram-se as mesmas bases aproximativas para os campos de tensão e deslocamento no domínio e deslocamento no contorno, apresentadas no capítulo 4.

Então, para o cálculo das matrizes $\mathbf{S}$ e $\mathbf{T}$ considere as eq.(6.43) e eq.(6.44). Como as aproximações para os campos virtuais de tensão e deslocamento de contorno no espaço $\boldsymbol{T}$ 
(eq.(6.42)) são representadas pelo mesmo conjunto de função interpoladoras apresentadas para o espaço $\boldsymbol{S}$ (eq.(6.41)), tem-se que: $\mathbf{S}=\mathbf{T}$.

Da eq.(6.43) e as aproximações definidas nas eq.(2.30) a eq.(2.32) e eq.(2.34), para certa rede de cobertura, escreve-se:

$$
\left\|\eta_{h}\right\|_{S}^{2}=s_{\Omega}^{T} \int_{\Omega} S_{\Omega}^{T} S_{\Omega} d \Omega s_{\Omega}+q_{\Omega}^{T} \int_{\Gamma_{t}} U_{\Omega}^{T} U_{\Omega} d \Omega q_{\Omega}+q_{\Gamma_{t}}^{T} \int_{\Gamma_{t}} U_{\Gamma_{t}}^{T} U_{\Gamma_{t}} d \Gamma q_{\Gamma_{t}}+q_{\Gamma_{i}}^{T} \int_{\Gamma_{i}} U_{\Gamma_{i}}^{T} U_{\Gamma_{i}} d \Gamma q_{\Gamma_{i}}
$$

Como já definido, a norma $\left\|\boldsymbol{\eta}_{\boldsymbol{h}}\right\|_{S}^{2}$ da eq.(6.45) pode ser escrita da seguinte forma:

$$
\left\|\boldsymbol{\eta}_{h}\right\|_{S}^{2}=\left(\boldsymbol{\eta}^{\mathrm{T}} \mathbf{S} \boldsymbol{\eta}\right)
$$

onde

$$
\eta=\left\{\begin{array}{l}
s_{\Omega} \\
q_{\Omega} \\
q_{\Gamma_{t}} \\
q_{\Gamma_{i}}
\end{array}\right\}
$$

e

$$
S=\left[\begin{array}{cccc}
\int_{\Omega} S_{\Omega}^{T} S_{\Omega} d \Omega & O & O & O \\
O & \int_{\Omega} U_{\Omega}^{T} U_{\Omega} d \Omega & O & O \\
O & O & \int_{\Gamma_{t}} U_{\Gamma_{t}}^{T} U_{\Gamma_{t}} d \Gamma & O \\
O & O & O & \int_{\Gamma_{i}} U_{\Gamma_{i}}^{T} U_{\Gamma_{i}} d \Gamma
\end{array}\right]
$$

Na eq.(6.48) $\boldsymbol{O}$ representa a submatriz nula.

A forma bilinear definida na eq.(6.34) é apresentada da seguinte forma:

$$
B\left(\eta_{h}, \psi_{h}\right)=\psi^{\mathrm{T}} \mathrm{B} \eta
$$

onde

$$
\psi^{\mathrm{T}}=\left\{\begin{array}{llll}
\delta s_{\Omega}^{T} & \delta q_{\Omega}^{T} & \delta q_{\Gamma_{t}}^{T} & \delta q_{\Gamma_{i}}^{T}
\end{array}\right\}
$$




$$
\mathbf{B}=\left[\begin{array}{cccc}
\boldsymbol{F} & A_{\Omega} & -A_{\Gamma_{t}} & -A_{\Gamma_{i}} \\
A_{\Omega}^{T} & 0 & 0 & 0 \\
-A_{\Gamma_{t}}^{T} & 0 & 0 & 0 \\
-A_{\Gamma_{i}}^{T} & 0 & 0 & 0
\end{array}\right]
$$

onde foram introduzidas as seguintes matrizes:

$$
\begin{aligned}
& F=\int_{\Omega} S_{\Omega}^{T} f S_{\Omega} d \Omega \\
& A_{\Omega}=\int_{\Omega}\left(L S_{\Omega}\right)^{T} U_{\Omega} d \Omega \\
& A_{\Gamma_{t}}=\int_{\Gamma_{t}}\left(N S_{\Omega}\right)^{T} U_{\Gamma_{t}} d \Gamma \\
& A_{\Gamma_{i}}=\int_{\Gamma_{i}}\left(N S_{\Omega}\right)^{T} U_{\Gamma_{i}} d \Gamma
\end{aligned}
$$

Assim, estão definidos todos os parâmetros da eq.(6.34) necessários para o cálculo de $\lambda_{n}$. Agora, para a determinação de $\mathbf{S}=\mathbf{T}$ e $\mathbf{B}$ dentro das várias condições de enriquecimento é só ampliar da forma desejada as bases aproximativas iniciais dos campos de tensão e deslocamento no domínio e deslocamento no contorno.

Como a aplicação da condição inf-sup para avaliar a robustez dos elementos é impraticável, opta-se por verificar a estabilidade das soluções obtidas em cada um dos diferentes problemas, de uma mesma classe, abordados no capítulo de exemplos; nesse sentido, realiza-se uma espécie de "teste inf-sup”. Para tanto, a metodologia adotada é similar à sugerida no trabalho de Chapelle e Bathe (1993), isto é: em problema da FHMT com enriquecimento nodal será considerada uma seqüência de redes com elementos quadrilaterais e triangulares. Para cada rede o valor de $\lambda_{n}=\sqrt{\boldsymbol{\mu}_{\min }}$ será calculado. Se os $\sqrt{\boldsymbol{\mu}_{\min }}$ não tenderem a zero, o elemento quadrilateral e triangular da FHMT com enriquecimento nodal será considerado estável.

A estabilidade em todos os problemas testados, se constatada, pode ser interpretada como um bom indicativo da robustez dos elementos. 


\subsubsection{O Teste Numérico da Condição de Babuška-Brezzi (inf-sup) aplicado à FHT com Enriquecimento Nodal.}

Com a consideração da condição imposta pela eq.(2.69) e a hipótese de forças volúmicas nulas, as eq.(6.35) a eq.(6.38) são escritas agora da seguinte forma:

$$
\begin{aligned}
& \int_{\Omega} \delta \sigma^{T} f \sigma d \Omega-\int_{\Gamma_{t}} u_{\Gamma_{t}}^{T}(N \delta \sigma) d \Gamma-\int_{\Gamma_{i}} u_{\Gamma_{i}}^{T}\left(N_{+} \delta \sigma\right) d \Gamma=\int_{\Gamma_{u}} \bar{u}^{T}(N \delta \sigma) d \Gamma \\
& \int_{\Gamma_{t}} \delta u_{\Gamma_{t}}^{T}(N \sigma) d \Gamma=\int_{\Gamma_{t}} \delta u_{\Gamma_{t}}^{T} \bar{t} d \Gamma \\
& \int_{\Gamma_{i}} \delta u_{\Gamma_{i}}^{T}\left(N_{+} \sigma\right) d \Gamma+\int_{\Gamma_{i}} \delta u_{\Gamma_{i}}^{T}\left(N_{-} \sigma\right) d \Gamma=0
\end{aligned}
$$

Ainda baseado em Schwab (1998), para a formulação híbrida de tensão, pode-se definir para as eq.(6.56) a eq.(6.58) uma forma bilinear $\boldsymbol{B}(.,$.$) e um funcional linear \boldsymbol{F}($.$) ,$ como segue abaixo:

$$
\begin{gathered}
B(\eta, \psi)=\int_{\Omega} \delta \sigma^{T} f \sigma d \Omega-\int_{\Gamma_{t}} u_{\Gamma_{t}}^{T}(N \delta \sigma) d \Gamma+\cdots \\
\cdots+\int_{\Gamma_{t}} \delta u_{\Gamma_{t}}^{T}(N \sigma) d \Gamma-\int_{\Gamma_{i}} u_{\Gamma_{i}}^{T}\left(N_{+} \delta \sigma\right) d \Gamma+\cdots \\
\cdots+\int_{\Gamma_{i}} \delta u_{\Gamma_{i}}^{T}\left(N_{+} \sigma\right) d \Gamma+\int_{\Gamma_{i}} \delta u_{\Gamma_{i}}^{T}\left(N_{-} \sigma\right) d \Gamma \\
F(\psi)=\int_{\Gamma_{u}} \bar{u}^{T}(N \delta \sigma) d \Gamma+\int_{\Gamma_{t}} \delta u_{\Gamma_{t}}^{T} \bar{t} d \Gamma
\end{gathered}
$$

onde $\boldsymbol{\eta}=\left(\boldsymbol{\sigma}, \boldsymbol{u}_{\Gamma_{t}}, \boldsymbol{u}_{\Gamma_{i}}\right)$ e $\boldsymbol{\psi}=\left(\boldsymbol{\delta} \boldsymbol{\sigma}, \boldsymbol{\delta} \boldsymbol{u}_{\Gamma_{t}}, \boldsymbol{\delta} \boldsymbol{u}_{\Gamma_{i}}\right)$ são definidos em $\boldsymbol{S} \times \boldsymbol{T}$ com:

$$
\begin{aligned}
& S=\left\{\left(\sigma, u_{\Gamma_{t}}, u_{\Gamma_{i}}\right): \sigma \in L^{2}(\Omega) ; u_{\Gamma_{t}}, u_{\Gamma_{i}} \in L^{2}(\Gamma)\right\} \\
& T=\left\{\left(\delta \sigma, \delta u_{\Gamma_{t}}, \delta u_{\Gamma_{i}}\right): \delta \sigma \in L^{2}(\Omega) ; \delta u_{\Gamma_{t}}, \delta u_{\Gamma_{i}} \in L^{2}(\Gamma)\right\}
\end{aligned}
$$

Da eq.(6.59), definem-se as seguintes normas:

$$
\|\eta\|_{S}^{2}=\left\|\left(\sigma, u_{\Gamma_{t}}, u_{\Gamma_{i}}\right)\right\|_{S}^{2}=\int_{\Omega} \sigma^{2} d \Omega+\int_{\Gamma_{t}} u_{\Gamma_{t}}^{2} d \Gamma+\int_{\Gamma_{i}} u_{\Gamma_{i}}^{2} d \Gamma
$$




$$
\|\psi\|_{T}^{2}=\left\|\left(\delta \sigma, \delta u_{\Gamma_{t}}, \delta u_{\Gamma_{i}}\right)\right\|_{T}^{2}=\int_{\Omega} \delta \sigma^{2} d \Omega+\int_{\Gamma_{t}} \delta u_{T_{t}}^{2} d \Gamma+\int_{\Gamma_{i}} \delta u_{\Gamma_{i}}^{2} d \Gamma
$$

Igualmente a FHMT, para a aplicação do "teste inf-sup" a certa rede de cobertura com elementos finitos quadrilaterais de quatro nós e triangular de três nós da FHT com enriquecimento, é preciso determinar todas as matrizes envolvidas na eq.(6.34). Assim, consideram-se as mesmas bases aproximativas para os campos de tensão e deslocamento no contorno, apresentadas no capítulo 5.

Dessa forma, para o desenvolvimento das matrizes $\mathbf{S}$ e $\mathbf{T}$ considere as eq.(6.63) e eq.(6.64). Como as aproximações para os campos virtuais de tensão e deslocamento de contorno no espaço $\boldsymbol{T}$ (eq.(6.62)) são representadas pelo mesmo conjunto de função interpoladoras apresentadas para o espaço $\boldsymbol{S}$ (eq.(6.61)), tem-se que $\mathbf{S}=\mathbf{T}$.

Da eq.(6.43) e as aproximações definidas nas eq.(2.30), eq.(2.32) e eq.(2.34), para certa rede de cobertura, escreve-se:

$$
\left\|\boldsymbol{\eta}_{h}\right\|_{S}^{2}=s_{\Omega}^{T} \int_{\Omega} S_{\Omega}^{T} S_{\Omega} d \Omega s_{\Omega}+q_{\Gamma_{t}}^{T} \int_{\Gamma_{t}} U_{\Gamma_{t}}^{T} U_{\Gamma_{t}} d \Gamma q_{\Gamma_{t}}+q_{\Gamma_{i}}^{T} \int_{\Gamma_{i}} U_{\Gamma_{i}}^{T} U_{\Gamma_{i}} d \Gamma q_{\Gamma_{i}}
$$

Como já definido, a norma $\left\|\boldsymbol{\eta}_{h}\right\|_{S}^{2}$ da eq.(6.65) pode ser escrita da seguinte forma:

$$
\left\|\boldsymbol{\eta}_{\boldsymbol{h}}\right\|_{S}^{2}=\left(\boldsymbol{\eta}^{\mathrm{T}} \mathbf{S} \boldsymbol{\eta}\right)
$$

onde

$$
\boldsymbol{\eta}=\left\{\begin{array}{l}
s_{\Omega} \\
\boldsymbol{q}_{\Gamma_{t}} \\
\boldsymbol{q}_{I_{i}}
\end{array}\right\}
$$

e

$$
\mathbf{S}=\left[\begin{array}{ccc}
\int_{\Omega} S_{\Omega}^{T} S_{\Omega} d \Omega & o & O \\
o & \int_{\Gamma_{t}} U_{\Gamma_{t}}^{T} U_{\Gamma_{t}} d \Gamma & O \\
o & O & \int_{\Gamma_{i}} U_{\Gamma_{i}}^{T} U_{\Gamma_{i}} d \Gamma
\end{array}\right]
$$

Na eq.(6.68) $\boldsymbol{O}$ é a sub-matriz nula. 
A forma bilinear definida na eq.(6.34) é apresentada da seguinte forma:

$$
B\left(\eta_{h}, \psi_{h}\right)=\psi^{\mathrm{T}} \mathrm{B} \eta
$$

onde

$$
\psi^{\mathrm{T}}=\left\{\begin{array}{lll}
\delta s_{\Omega}^{T} & \delta q_{\Gamma_{t}}^{T} & \delta q_{\Gamma_{i}}^{T}
\end{array}\right\}
$$

e

$$
\mathbf{B}=\left[\begin{array}{ccc}
\boldsymbol{F} & -A_{\Gamma_{t}} & -A_{\Gamma_{i}} \\
-A_{\Gamma_{t}}^{T} & 0 & 0 \\
-A_{\Gamma_{i}}^{T} & 0 & 0
\end{array}\right]
$$

onde foram introduzidas as seguintes matrizes:

$$
\begin{aligned}
& F=\int_{\Omega} S_{\Omega}^{T} f S_{\Omega} d \Omega \\
& A_{\Gamma_{t}}=\int_{\Gamma_{t}}\left(N S_{\Omega}\right)^{T} U_{\Gamma_{t}} d \Gamma \\
& A_{\Gamma_{i}}=\int_{\Gamma_{i}}\left(N S_{\Omega}\right)^{T} U_{\Gamma_{i}} d \Gamma
\end{aligned}
$$

Assim, estão determinados todos os parâmetros da eq.(6.34) necessários para o cálculo de $\lambda_{n}$. Para aplicar o "teste inf-sup" à FHT segue a mesma metodologia descrita anteriormente para a FHMT. 

Nodal 


\section{Análise Numérica da FHT e FHMT com Enriquecimento Nodal}

\subsection{Introdução}

Neste capítulo o desempenho dos elementos triangulares e quadrilaterais da FHT e FHMT com enriquecimento nodal é avaliado pela representação dos campos de tensão e deslocamento e também pela convergência dos resultados em relação à energia de deformação.

As soluções obtidas da FHT e FHMT com enriquecimento nodal, em todos os exemplos, são confrontadas com resultados exatos (quando possível) ou com os de uma análise pelo MEF convencional, gerados com o software $\operatorname{ANSYS}^{\circledR}$, a partir de uma discretização bastante refinada. Por simplificação não foram adotadas unidades para comprimento e força nos exemplos analisados.

O conjunto de exemplos testados inclui casos de distorção da rede e duas situações limite em termos de regularidade esperada para as respostas: chapa tracionada simetricamente e chapa tracionada com fenda central.

Particularmente, em relação à sensibilidade à distorção da rede três exemplos são explorados: viga engastada submetida a momento concentrado, viga engastada submetida à carga de cisalhamento e o problema clássico - Painel de Cook. Os dois primeiros também foram pontuados em Punch \& Atluri (1984) e por isso os resultados dessa referência serão confrontados com os obtidos dos elementos finitos da FHT e FHMT com enriquecimento nodal tratados nesta pesquisa.

Todos os resultados servem também para subsidiar a análise das condições de convergência da FHT e FHMT com enriquecimento nodal que será desenvolvida mais adiante, a partir do item 7.3. 


\subsection{Distorção da Rede}

\subsubsection{Exemplo 1 - Viga Engastada - Momento Concentrado}

Na figura 7.1 apresenta-se uma chapa retangular de espessura unitária, $(\mathbf{1 0} \times 2)$ unidades de comprimento, com borda vertical esquerda fixa $\left(\boldsymbol{u}_{\boldsymbol{x}}=\boldsymbol{0} ; \boldsymbol{u}_{\boldsymbol{y}}=\boldsymbol{0}\right)$ e submetida a um momento concentrado no lado vertical direito de $\boldsymbol{M}=\mathbf{1 0 0}$ unidades de momento.

Este exemplo tem resultados exatos (teoria clássica de vigas) para as tensões, deslocamentos e energia de deformação.

Assim, adotando-se para o material da chapa um módulo de Young $\boldsymbol{E}=\boldsymbol{1}$ e um coeficiente de Poisson $\boldsymbol{v}=\boldsymbol{0 , 2 5}$, têm-se os seguintes valores:

a) Tensão: $\sigma_{x}=150$ (lado horizontal superior), $\boldsymbol{\sigma}_{x}=\mathbf{- 1 5 0}$ (lado horizontal inferior);

b) Deslocamento: $\boldsymbol{u}_{\boldsymbol{y}}=\mathbf{- 7 . 5 0 0}$ no lado vertical direito;

c) Energia de Deformação: $\boldsymbol{U}_{\text {exemplo } 1}=\mathbf{7 5 . 0 0 0}$.

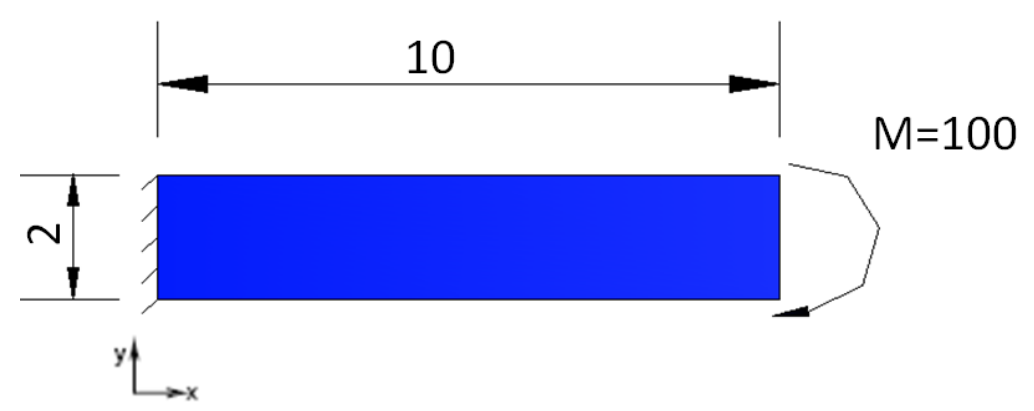

Figura 7.1 - Chapa com 100 unidades de momento concentrado.

Com objetivo de verificar a sensibilidade à distorção da rede dos elementos quadrilateral e triangular da FHT e FHMT com enriquecimento nodal, este exemplo será analisado por um conjunto de cinco (5) redes distorcidas, como ilustra a figura 7.2.

Cada situação de distorção é definida por um fator denominado parâmetro de distorção $\boldsymbol{\mu}$. Este parâmetro é calculado pela razão entre a diferença das coordenadas “ $\boldsymbol{x}$ ” dos dois nós centrais, pertencentes a cada lado horizontal das chapas, e o comprimento da viga (10), conforme a figura 7.2. Assim, para as redes a), b), c), d) e e), respectivamente, computam-se: $\mu=0, \mu=0,2, \mu=0,4, \mu=0,6$ е $\mu=0,8$. 


\section{Redes Quadrilaterais}

a)

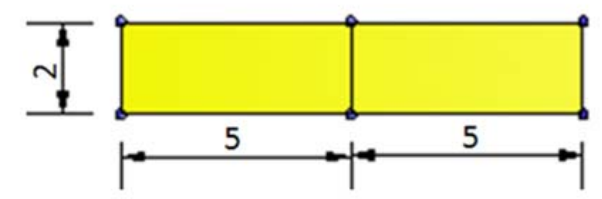

b)

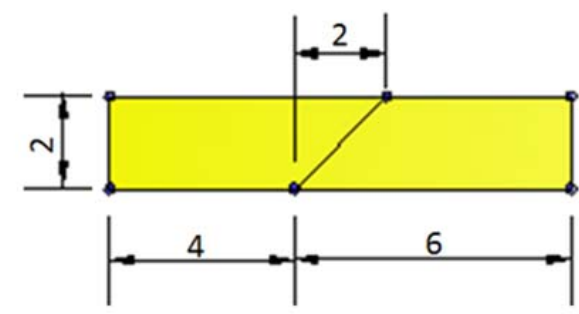

c)

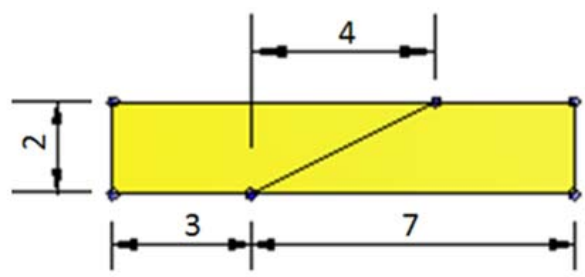

d)

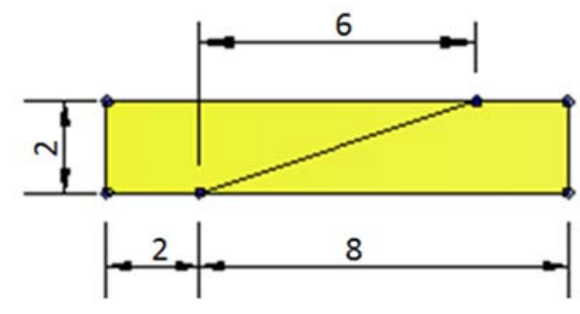

e)

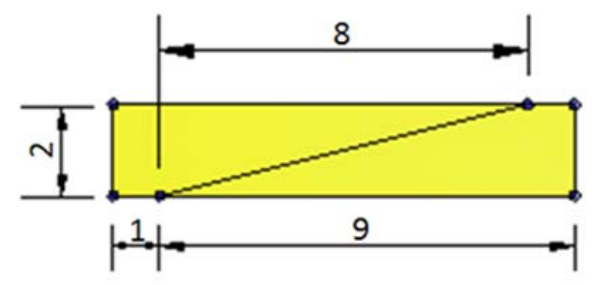

Redes Triangulares

a)
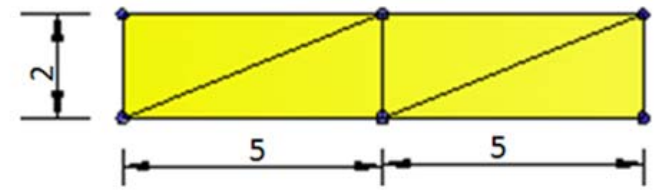

b)

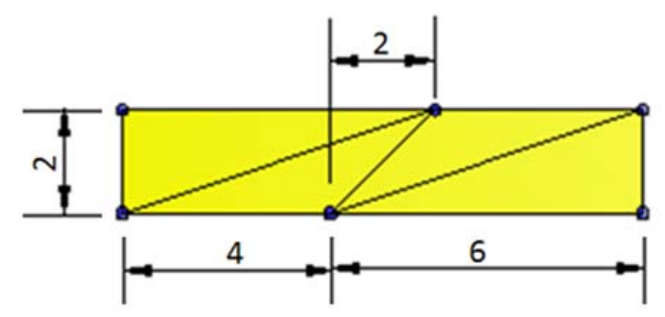

c)

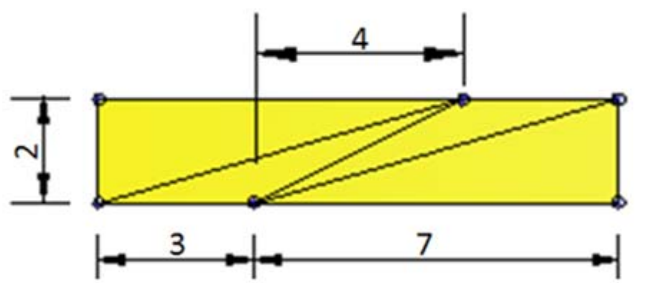

d)

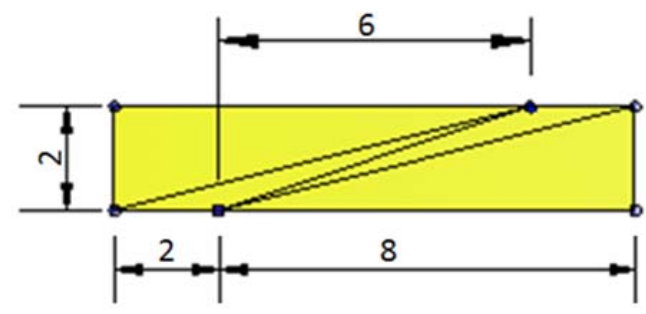

e)

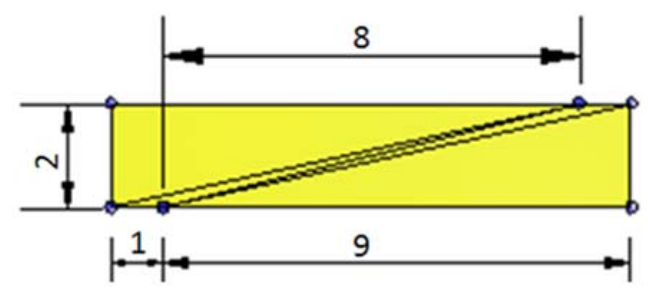

Figura 7.2 - Conjunto de Redes Quadrilaterais e Triangulares: Exemplo 1. 
Em Punch and Atluri (1984), este Exemplo foi utilizado para avaliar a sensibilidade à distorção da rede dos seguintes elementos quadrilaterais de quatro nós:

- APR: Elemento Quadrilateral Híbrido de Tensão de 4 nós - Equilíbrio a priori - Funcional Híbrido de Tensão;

- APO: Elemento Quadrilateral Híbrido de Tensão de 4 nós - Equilíbrio a posteriori - Funcional de Hellinger-Reissner;

- APC: Elemento Quadrilateral Híbrido de Tensão de 4 nós - Equilíbrio a posteriori - Funcional de Hellinger-Reissner;

- APS: Elemento Quadrilateral Híbrido de Tensão de 4 nós - Equilíbrio a posteriori - Funcional de Hellinger-Reissner;

Confrontam-se as respostas apresentadas por estes elementos com os resultados dos elementos quadrilaterais da FHT e FHMT com enriquecimento nodal. O nó do canto superior direito das redes da figura 7.2 será utilizado como nó de referência para o deslocamento.

Para a situação $\boldsymbol{\mu}=\boldsymbol{0}$, obtiveram-se os valores postados nas tabelas 7.1 a 7.3.

Tabela 7.1 - Exemplo 1 - FHMT sem enriquecimento nodal- $\boldsymbol{\mu}=\boldsymbol{0}$ - resultados para deslocamento e energia de deformação.

\begin{tabular}{cccc}
\hline FHMT sem Enriquecimento & \multicolumn{2}{c}{ FHMT sem Enriquecimento } \\
\multicolumn{2}{c}{ Elemento Triangular } & \multicolumn{2}{c}{ Elemento Quadrilateral } \\
\hline Deslocamento no & Energia de & Deslocamento no & Energia de \\
Contorno & Deformação & Contorno & Deformação \\
\hline$-1.915,90$ & $68.578,56$ & -7.500 & 75.000 \\
\hline
\end{tabular}

Tabela 7.2 - Exemplo 1 - FHT sem enriquecimento nodal $-\boldsymbol{\mu}=\boldsymbol{0}$ - resultados para deslocamento e energia de deformação.

FHT sem Enriquecimento $\quad$ FHT sem Enriquecimento

Elemento Triangular Elemento Quadrilateral

\begin{tabular}{ccccc}
\hline $\begin{array}{c}\text { Base de } \\
\text { Aproximação } \\
\text { das Tensões }\end{array}$ & $\begin{array}{c}\text { Deslocamento no } \\
\text { Contorno }\end{array}$ & $\begin{array}{c}\text { Energia de } \\
\text { Deformação }\end{array}$ & $\begin{array}{c}\text { Deslocamento no } \\
\text { Contorno }\end{array}$ & $\begin{array}{c}\text { Energia de } \\
\text { Deformação }\end{array}$ \\
\hline Quadrática & $-702,53$ & $7.227,05$ & $-2.363,30$ & $23.632,58$ \\
\hline Linear & $-702,53$ & $7.227,05$ & $-2.363,30$ & $23.632,58$ \\
\hline Constante & $-702,53$ & $7.227,05$ & - & - \\
\hline
\end{tabular}


Tabela 7.3 - Exemplo 1 - MEF Clássico $-\boldsymbol{\mu}=\boldsymbol{0}$ - resultados para deslocamento e energia de deformação.

\begin{tabular}{cccc}
\hline \multicolumn{2}{c}{ MEF Clássico } & \multicolumn{2}{c}{ MEF Clássico } \\
\multicolumn{2}{c}{ Elemento Triangular } & \multicolumn{2}{c}{ Elemento Quadrilateral } \\
\hline Deslocamento no & Energia de & Deslocamento no & Energia de \\
Contorno & Deformação & Contorno & Deformação \\
\hline$-702,53$ & $7.227,05$ & -7.500 & 75.000 \\
\hline
\end{tabular}

Dos resultados apresentados nas tabelas 7.1 a 7.3, observa-se que tanto o elemento quadrilateral sem enriquecimento nodal da FHMT como o do MEF Clássico recuperaram a resposta da teoria clássica de vigas. Ainda deste conjunto de resultados, pode-se concluir que o elemento triangular sem enriquecimento nodal da FHT converge, no mínimo, para a resposta obtida com o elemento triangular do MEF Clássico.

A figura 7.3 mostra as distribuições de tensão na direção $\boldsymbol{x}$ para os elementos quadrilateral e triangular sem enriquecimento da FHMT $-\boldsymbol{\mu}=\boldsymbol{0}$.

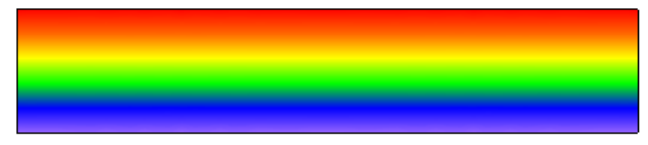

$\sigma_{x}$ Elem ento Quadrilateral

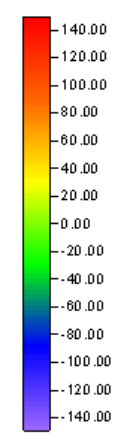

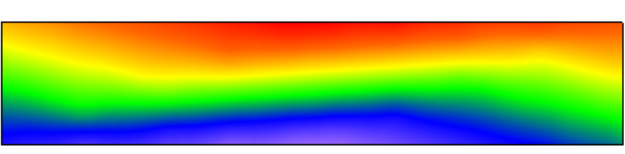

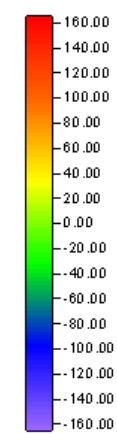

$\sigma_{x}$ Elemento Triangular

Figura 7.3 - Tensão plana $-\boldsymbol{\sigma}_{\boldsymbol{x}}$ - elemento quadrilateral e triangular sem enriquecimento $-(\boldsymbol{\%} \boldsymbol{\mu}=\boldsymbol{0})$ - FHMT.

Ainda da figura 7.3, verifica-se que o elemento triangular não consegue reproduzir a distribuição de tensão $\left(\sigma_{x}\right)$ exata da formulação clássica de viga.

As tensões na direção $\boldsymbol{x}$ para os elementos triangular (considerando-se: valores e distribuição) e quadrilateral (considerando-se: valores) sem enriquecimento da FHT (base quadrática da aproximação do campo de tensões no domínio) não são representativas, quando comparadas com a distribuição e valores de tensão $\left(\sigma_{x}\right)$ exata obtida com a formulação clássica de vigas, ver figura 7.4. 


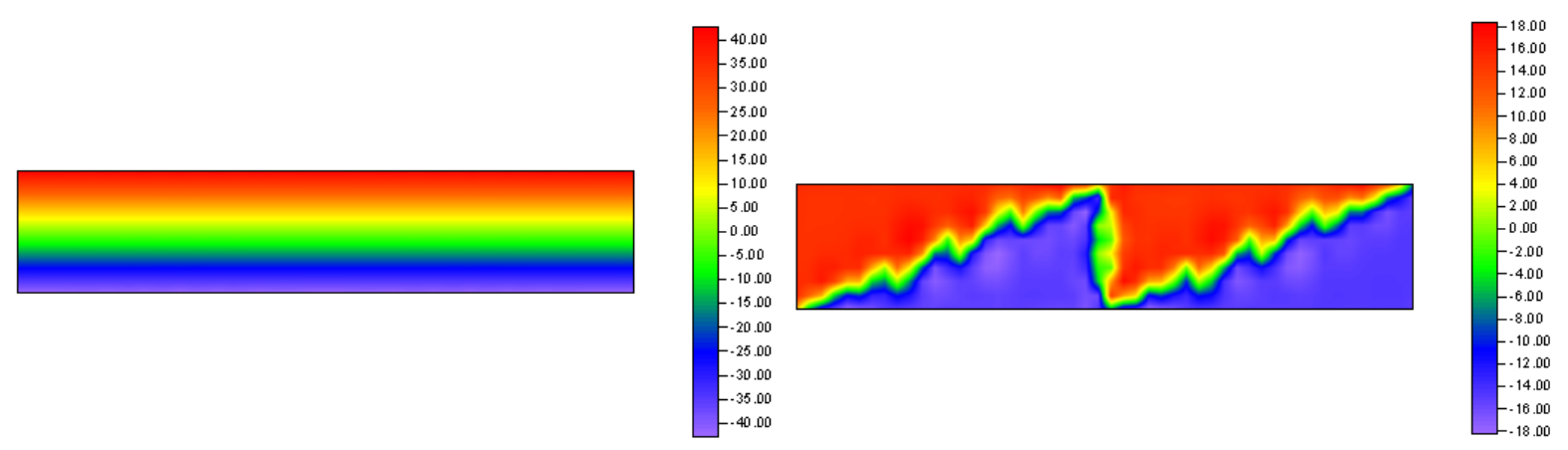

$\sigma_{x}$ Elem ento Quadrilateral

$\sigma_{x}$ Elemento Triangular

Figura 7.4 - Tensão plana $-\boldsymbol{\sigma}_{\boldsymbol{x}}$ - elemento quadrilateral e triangular sem enriquecimento $-(\boldsymbol{\%} \boldsymbol{\mu}=\boldsymbol{0})$ - Aproximação Quadrática das Tensões - FHT.

Agora, passa-se à análise das alterações de valores para o deslocamento, energia de deformação e na representação do campo de tensão na direção $\boldsymbol{x}$ gerados quando se aumenta gradativamente o parâmetro de distorção $\boldsymbol{\mu}$.

Assim, considera-se o conjunto de redes da figura 7.2, aplicando-se, ainda, os enriquecimentos (com funções polinomiais) dos campos envolvidos na FHT e FHMT propostos neste trabalho. Foram definidas especificamente as seguintes possibilidades:

a) Elementos quadrilateral e triangular da FHT, com aproximações constante, linear ou quadrática para tensões no domínio e linear para deslocamentos no contorno, sem enriquecimento;

b) Elementos quadrilateral e triangular da FHT, com aproximações constante, linear ou quadrática para tensões no domínio, enriquecimento nodal das tensões (todos os nós) e sem enriquecimento sobre a aproximação linear para deslocamentos no contorno;

c) Elementos quadrilateral e triangular da FHT, com aproximações constante, linear ou quadrática para tensões no domínio, enriquecimento nodal das tensões (todos os nós) e enriquecimento linear para deslocamentos no contorno (excluindo-se os nós com deslocamentos prescritos);

d) Elementos quadrilateral e triangular da FHT, com aproximação quadrática para tensões no domínio e somente com enriquecimento linear para deslocamentos no contorno (excluindo-se os nós com deslocamentos prescritos);

e) Elementos quadrilateral e triangular da FHMT, sem enriquecimento sobre qualquer dos campos de aproximação; 
f) Elementos quadrilateral e triangular da FHMT, com enriquecimento sobre os campos de tensões no domínio (todos os nós) e sem enriquecimento dos deslocamentos no domínio e deslocamentos no contorno;

g) Elementos quadrilateral e triangular da FHMT, com enriquecimento sobre os campos de tensões e deslocamentos no domínio (todos os nós) e deslocamentos no contorno (excluindo-se os nós com deslocamentos prescritos).

Destacam-se na figura 7.5 os nós enriquecidos para as combinações de enriquecimento das grandezas de domínio e contorno envolvidas na FHMT/FHT apresentadas anteriormente. Embora a figura 7.5 apresente somente o elemento quadrilateral, esta metodologia de enriquecimento também é aplicada ao elemento triangular.

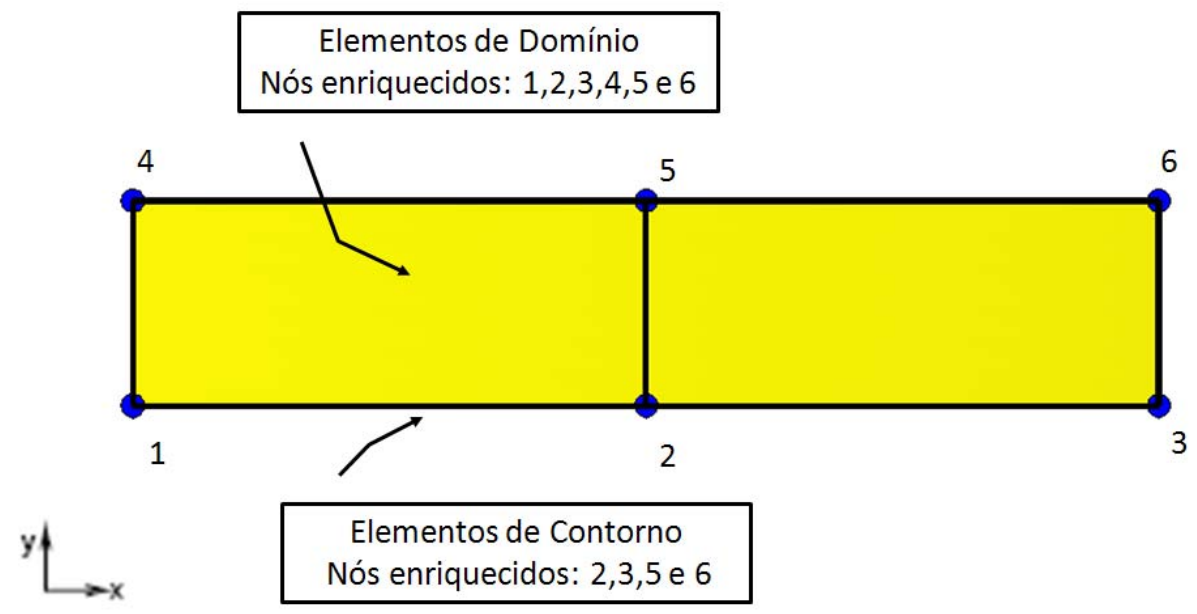

Figura 7.5 - Nós enriquecidos no Exemplo 1- FHT e FHMT.

Nas figuras a seguir, encontram-se os resultados obtidos nos vários testes de sensibilidade à distorção de redes propostos. Cada curva foi construída com o parâmetro de distorção $\boldsymbol{\mu}(\boldsymbol{0} \leq \boldsymbol{\mu} \leq \boldsymbol{0}, \boldsymbol{8})$ definido nas abscissas e o valor normalizado da energia de deformação, ou de deslocamentos, nas ordenadas. Cada valor normalizado foi calculado dividindo-se a grandeza analisada (num certo nível de distorção $\boldsymbol{\mu}$ ) pelo valor exato dessa grandeza na teoria clássica de vigas.

A figura 7.6 mostra que o elemento quadrilateral da FHMT/FHT sem enriquecimento, igualmente ao elemento quadrilateral do MEF clássico, não consegue manter o nível de deslocamento exato com o aumento do parâmetro $\boldsymbol{\mu}$. 


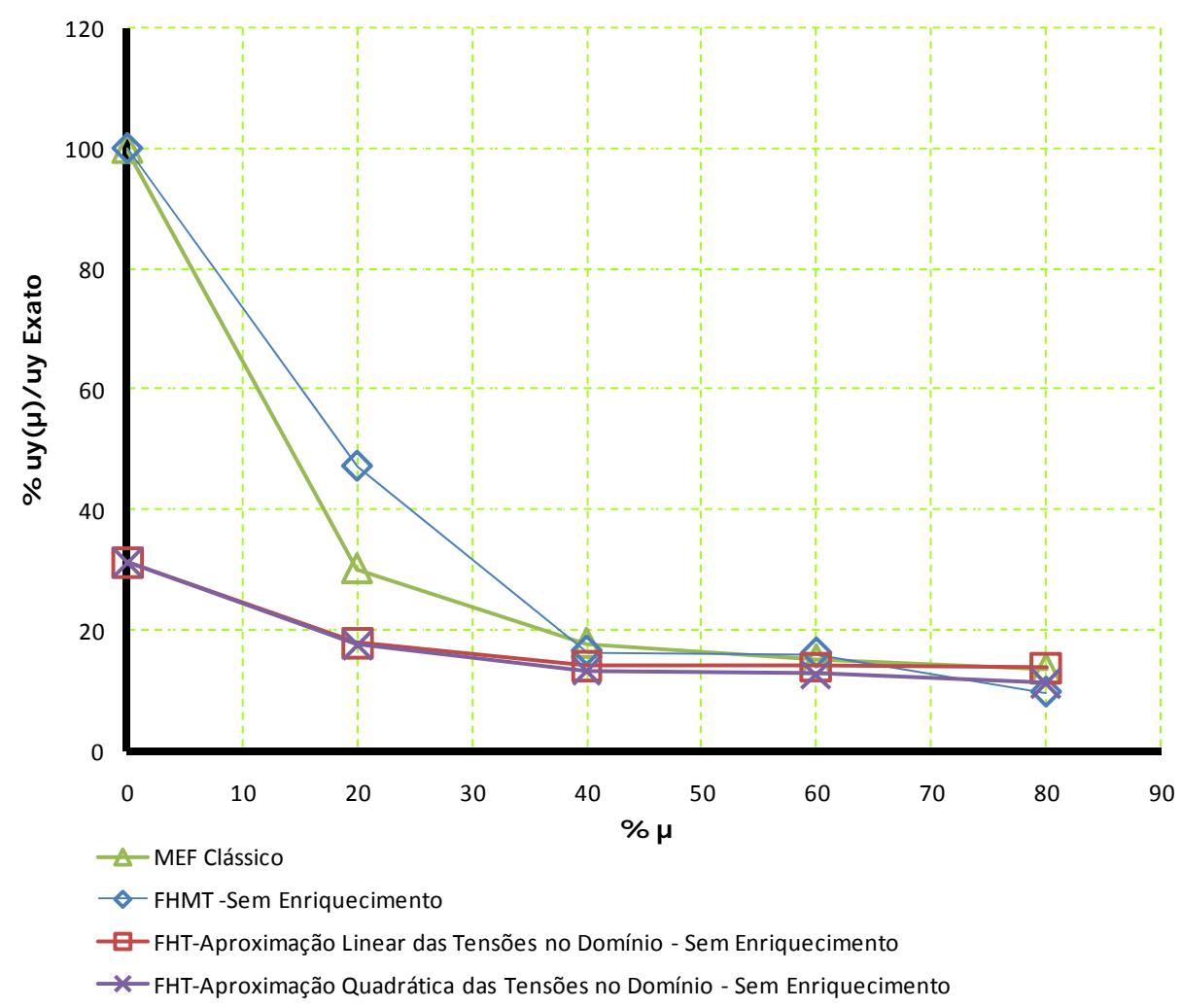

Figura 7.6 - Sensibilidade do deslocamento à distorção da rede - Elemento Quadrilateral - FHT, FHMT e MEF Clássico.

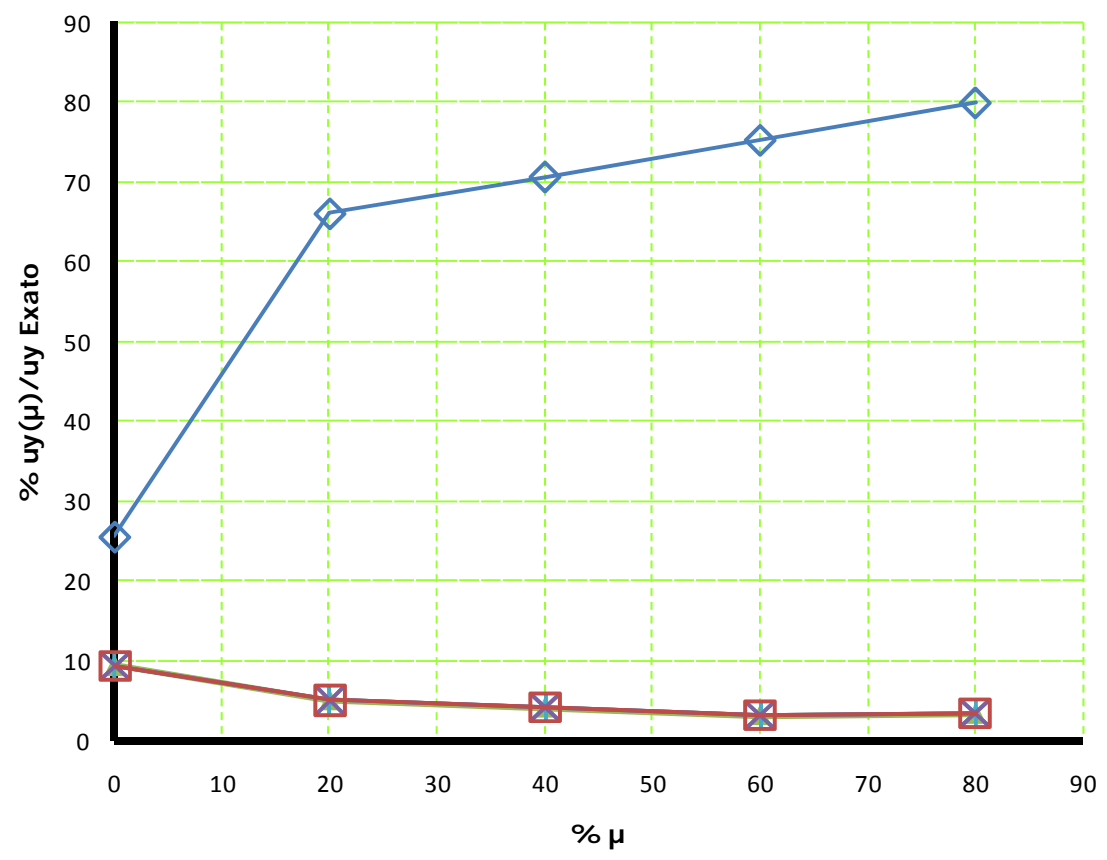

\footnotetext{
- MEF Clássico

$\diamond$ FHMT -Sem Enriquecimento

- FHT-Aproximação Constante das Tensões no Domínio - Sem Enriquecimento

* FHT-Aproximação Linear das Tensões no Domínio - Sem Enriquecimento

—FHT-Aproximação Quadrática das Tensões no Domínio - Sem Enriquecimento
}

Figura 7.7 - Sensibilidade do deslocamento à distorção da rede - Elemento Triangular - FHT, FHMT e MEF Clássico. 
O elemento triangular da FHT sem enriquecimento recuperou a mesma resposta do MEF Clássico, para todos os níveis de distorção das redes analisadas. Com o elemento triangular da FHMT sem enriquecimento, mesmo para a condição nula de distorção $(\boldsymbol{\mu}=\boldsymbol{0})$, não se obteve o valor exato do deslocamento, ver figura 7.7.

O enriquecimento simultâneo das tensões no domínio e deslocamentos no contorno, bem como o enriquecimento exclusivo dos deslocamentos no contorno, mantém a resposta do elemento quadrilateral da FHT - Aproximação Quadrática das Tensões - bem próximo ao valor exato do deslocamento, mesmo numa condição extrema de distorção $(\boldsymbol{\mu}=\boldsymbol{0 , 8})$, como apresenta a figura 7.8 .

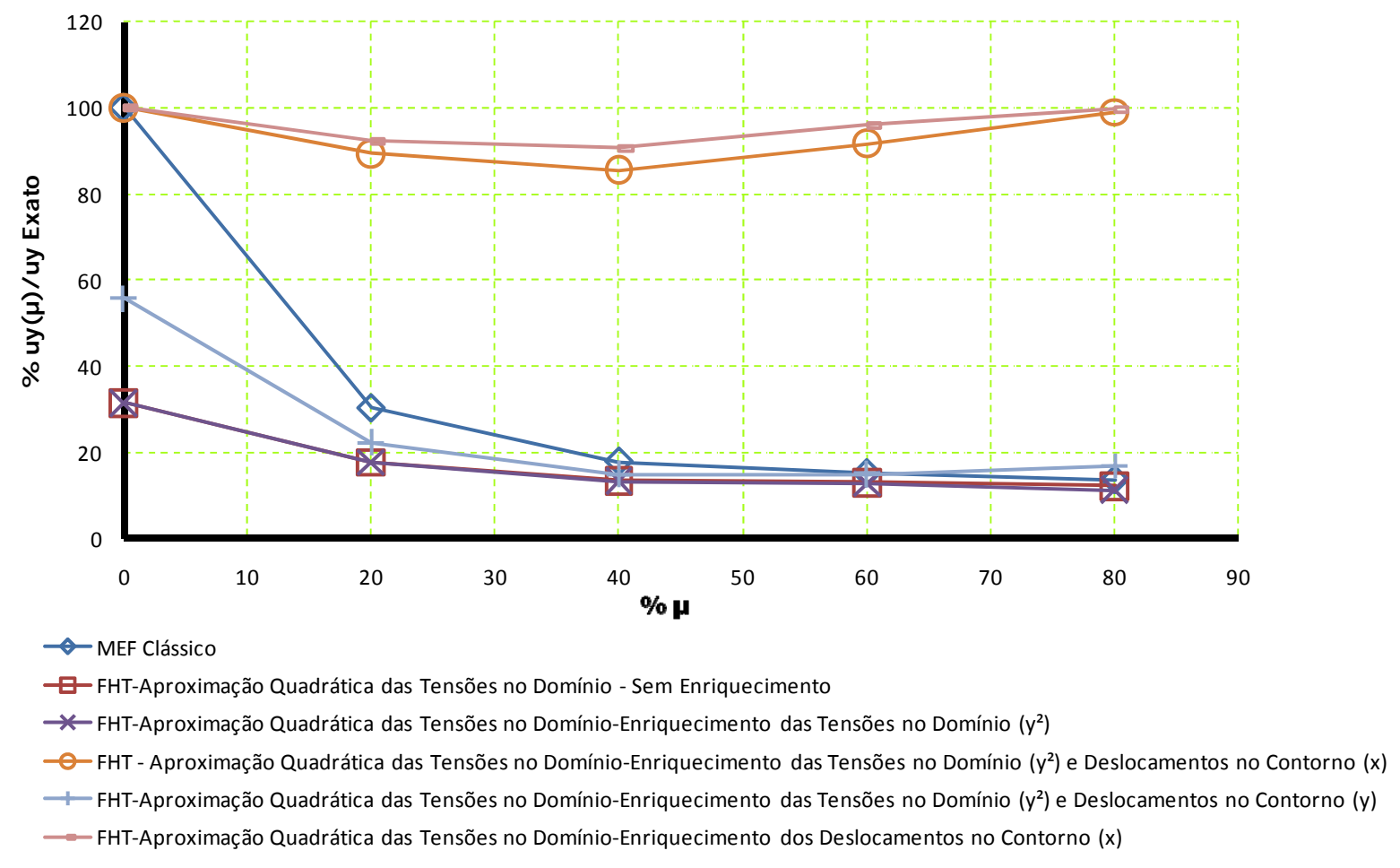

Figura 7.8 - Sensibilidade do deslocamento à distorção da rede - Elemento Quadrilateral - FHT e MEF Clássico.

A figura 7.9 destaca que o elemento triangular da FHT enriquecido com algumas combinações sobre as tensões no domínio e deslocamentos no contorno é sensível à distorção da rede, mas apresenta respostas melhores que as obtidas com o MEF clássico. 


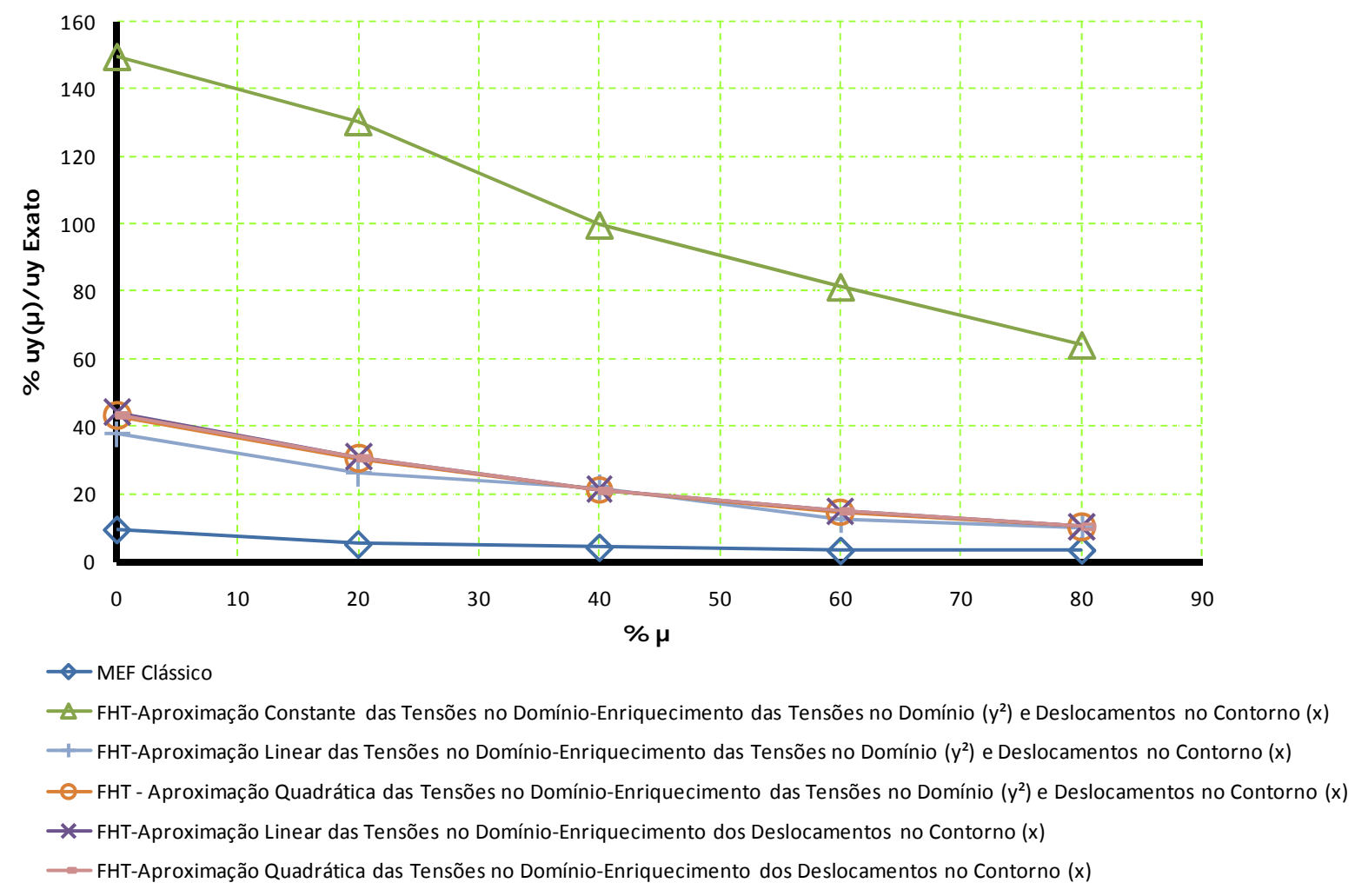

Figura 7.9 - Sensibilidade do deslocamento à distorção da rede - Elemento Triangular - FHT e MEF clássico.

Os elementos quadrilaterais do trabalho de Punch and Atluri (1984) são mais sensíveis à distorção da rede, quando comparados ao elemento quadrilateral da FHT com enriquecimento exclusivo dos deslocamentos no contorno $(\boldsymbol{x})$ ou simultâneo das tensões no domínio $\left(y^{2}\right)$ e deslocamentos no contorno $(x)$ - Aproximação Quadrática das Tensões no Domínio, figura 7.10. 


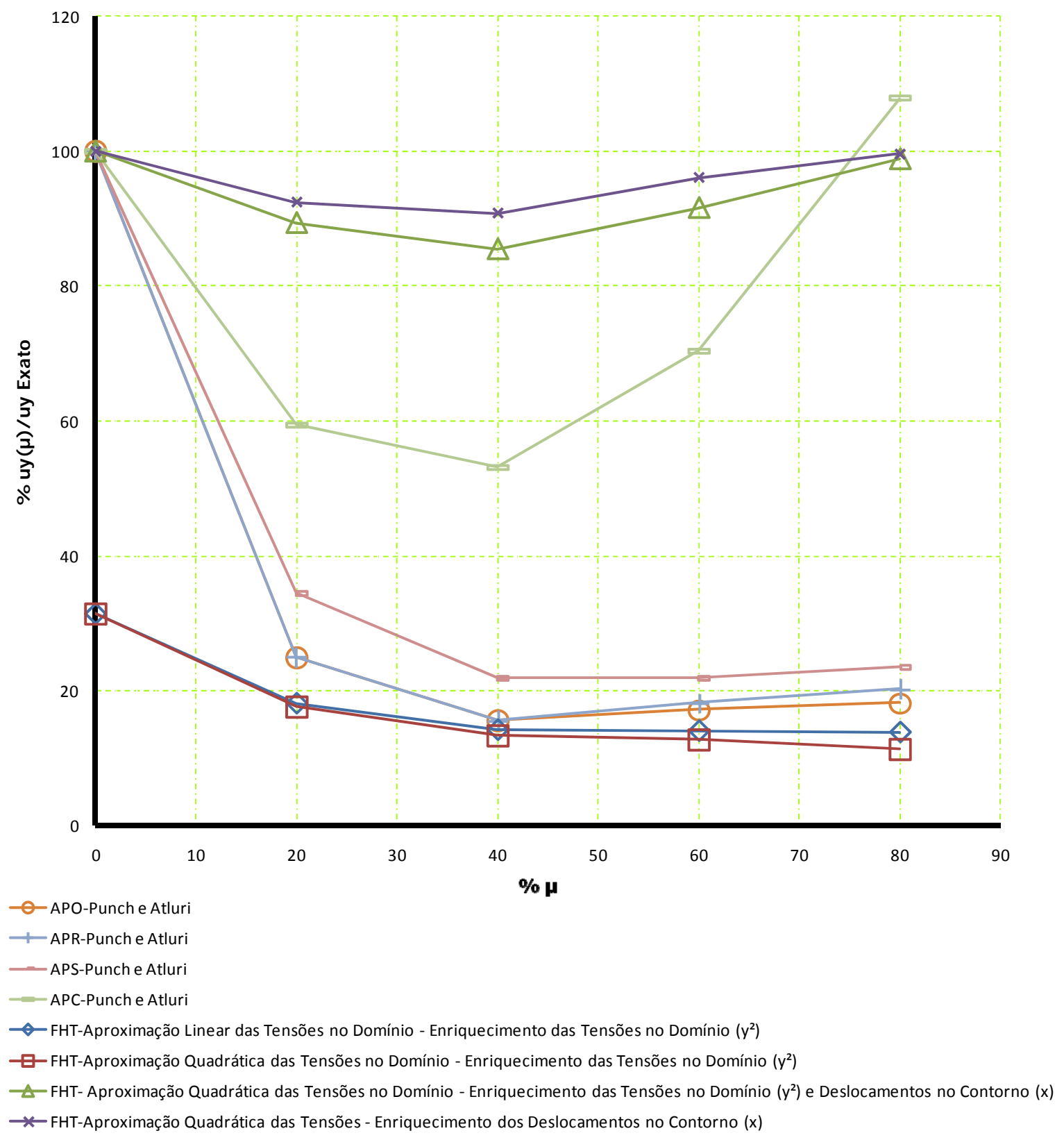

Figura 7.10 - Sensibilidade do deslocamento à distorção da rede - Elemento Quadrilateral - FHT e Punch e Atluri (1984). 


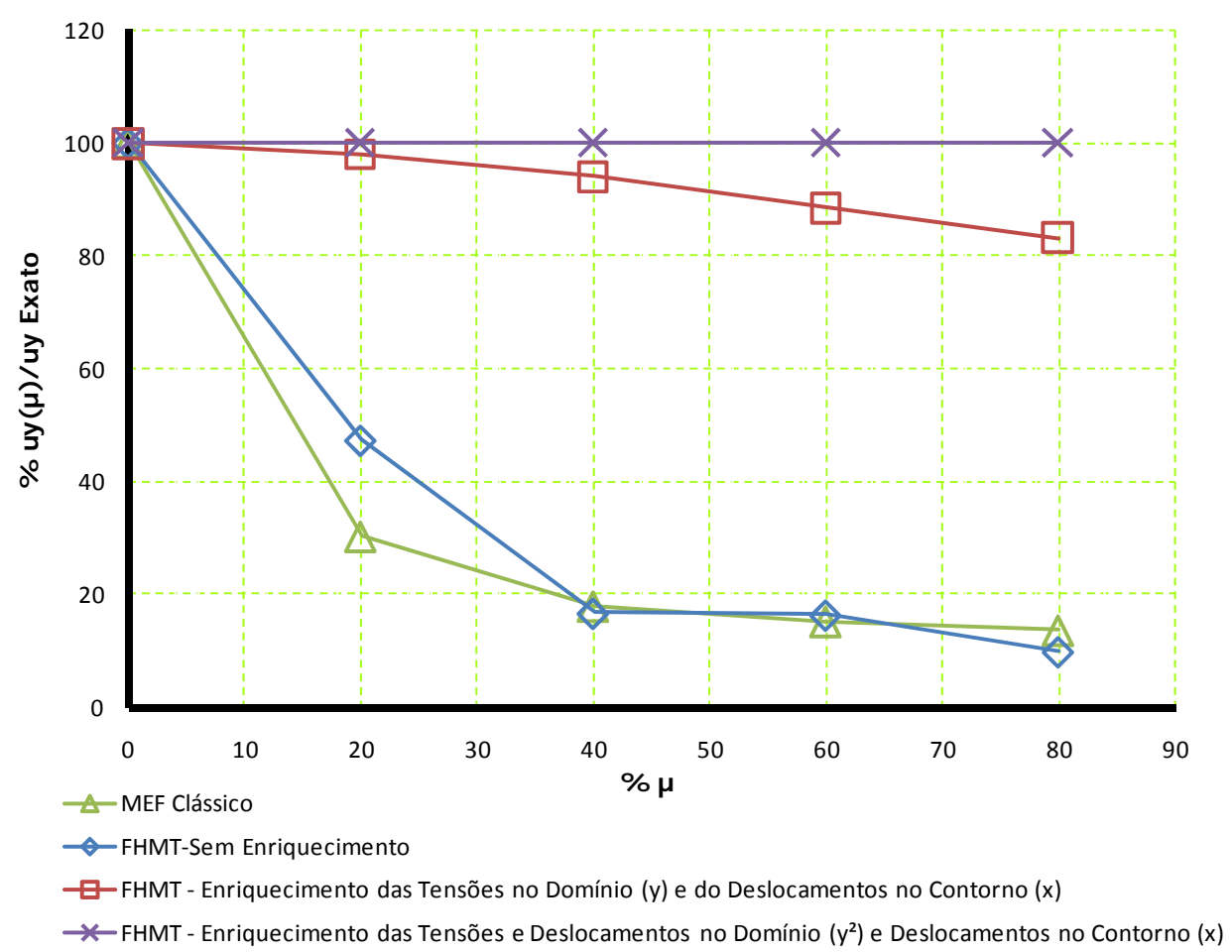

Figura 7.11 - Sensibilidade do deslocamento à distorção da rede - Elemento Quadrilateral - FHMT e MEF clássico.

O elemento quadrilateral enriquecido - FHMT - tensões e deslocamentos no domínio $\left(y^{2}\right)$ e deslocamentos no contorno $(x)$ - mostrou-se extremamente robusto, pois, para todos os níveis de distorção $(\boldsymbol{\mu})$ estudados, o deslocamento de referência obtido foi sempre igual ao exato, ver figura 7.11. Este elemento, nas condições de enriquecimento anteriores, quando confrontado com os elementos quadrilaterais do trabalho de Punch and Atluri (1984) é o menos sensível à distorção da rede, como destaca a figura 7.13.

Mesmo não tendo a mesma robustez do elemento quadrilateral enriquecido (figura 7.11), o elemento Triangular da FHMT enriquecido - tensões e deslocamentos no domínio $\left(y^{2}\right)$ e deslocamentos no contorno $(x)$ - figura 7.12, apresentou uma baixa sensibilidade à distorção da rede. 


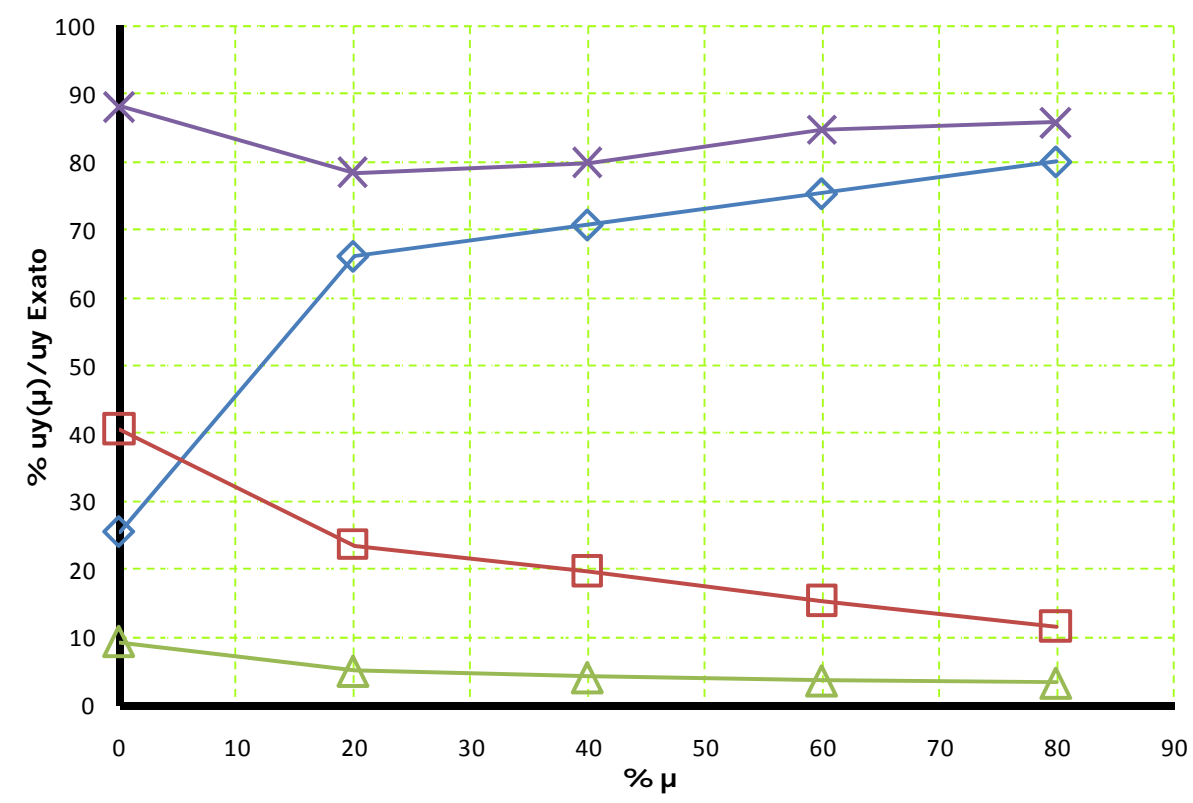

$\triangle$ MEF Clássico

$\leadsto$ FHMT-Sem Enriquecimento

—FHMT - Enriquecimento das Tensões no Domínio (y) e do Deslocamentos no Contorno (x)

$\rightarrow$ FHMT - Enriquecimento das Tensões e Deslocamentos no Domínio $\left(\mathrm{y}^{2}\right)$ e Deslocamentos no Contorno $(\mathrm{x})$

Figura 7.12 - Sensibilidade do deslocamento à distorção da rede - Elemento Triangular - FHMT e MEF clássico.

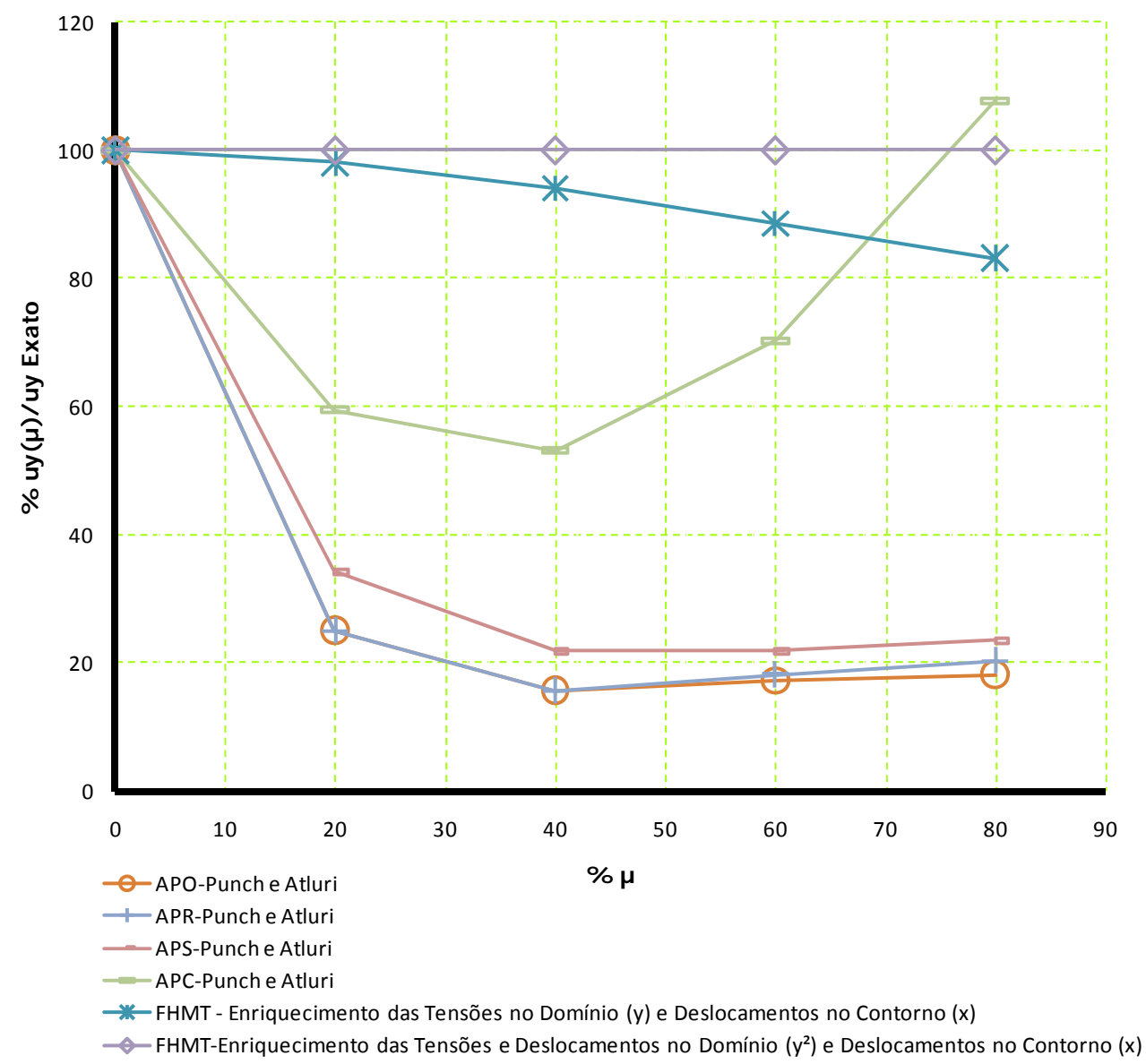

Figura 7.13 - Sensibilidade do deslocamento à distorção da rede - Elemento Quadrilateral - FHMT e Punch and Atluri (1984). 
A representação da tensão plana $\left(\sigma_{x}\right)$ do Exemplo 1, discretizado com elementos quadrilaterais e triangulares da FHT/FHMT - $(\boldsymbol{\mu}=\boldsymbol{0 , 8})$, perde precisão em relação à distribuição de tensão $\left(\sigma_{x}\right)$ exata, ver figuras 7.14 e 7.15.

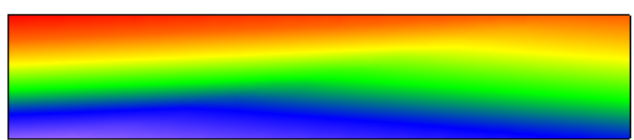

$\sigma_{x}$ Elem ento Quadrilateral $(\% \mu=80)$
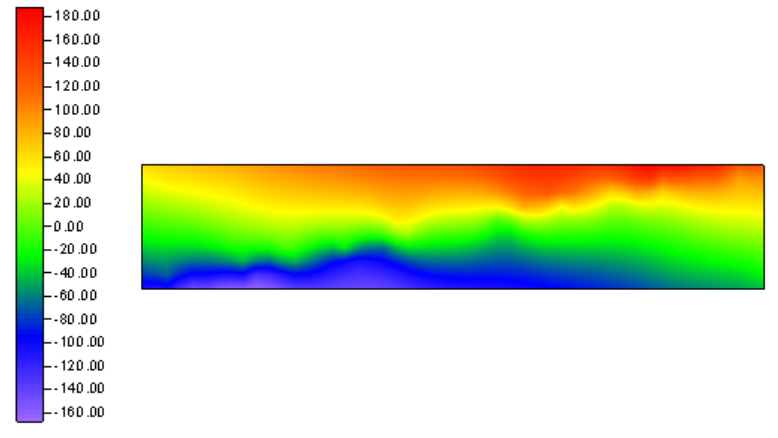

$\sigma_{x}$ Elemento Triangular $(\% \mu=80)$

Figura 7.14 - Tensão plana $-\boldsymbol{\sigma}_{\boldsymbol{x}}$ - elemento quadrilateral e triangular sem enriquecimento $(\% \boldsymbol{\mu}=80)-$ FHMT.

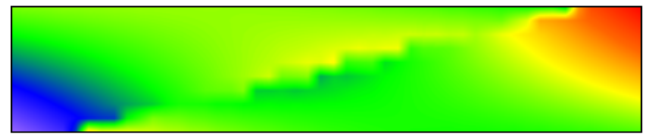

$\sigma_{x}$ Elemento Quadrilateral $(\% \mu=80)$
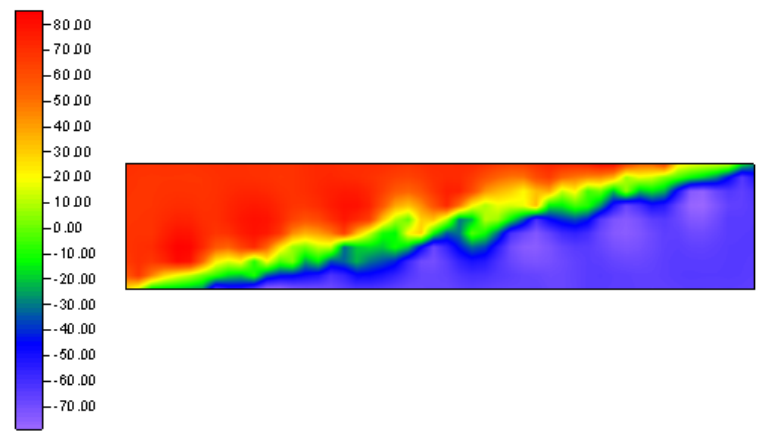

$\sigma_{x}$ Elemento Triangular $(\% \mu=80)$

Figura 7.15 - Tensão plana - $\boldsymbol{\sigma}_{\boldsymbol{x}}$ - elemento quadrilateral e triangular sem enriquecimento $(\boldsymbol{\%} \boldsymbol{\mu}=\boldsymbol{8 0})$ - Aproximação Quadrática das Tensões - FHT.

Na figura 7.16, destaca-se à distribuição de tensão $\left(\sigma_{x}\right)$ para o Exemplo 1 analisado com o elemento quadrilateral da FHT com $\boldsymbol{\mu}=\boldsymbol{0 , \boldsymbol { 8 }}$. Ressalta-se que o enriquecimento exclusivo sobre os deslocamentos no contorno possibilitou a representação fiel da distribuição exata de tensão na direção $\boldsymbol{x}$ do Exemplo 1, mesmo nesse nível de distorção. 


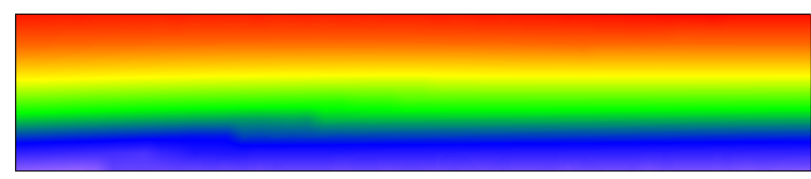

$\sigma_{x}$ Elemento Quadrilateral $(\% \mu=80) \Rightarrow$ Com Enriquecimento

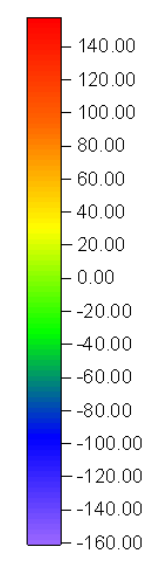

Figura 7.16 - Tensão plana - $\boldsymbol{\sigma}_{\boldsymbol{x}}$ - elemento quadrilateral com enriquecimento seletivo dos Deslocamentos no Contorno $(\boldsymbol{x})-(\boldsymbol{\%} \boldsymbol{\mu}=\boldsymbol{8 0})$ - Aproximação Quadrática das Tensões - FHT.

A figura 7.17 confirma a forte sensibilidade do elemento triangular da FHT à distorção da rede, pois observa-se que mesmo com enriquecimento seletivo dos deslocamentos no contorno não foi possível melhorar a estimativa e a representação do $\left(\sigma_{x}\right)$.

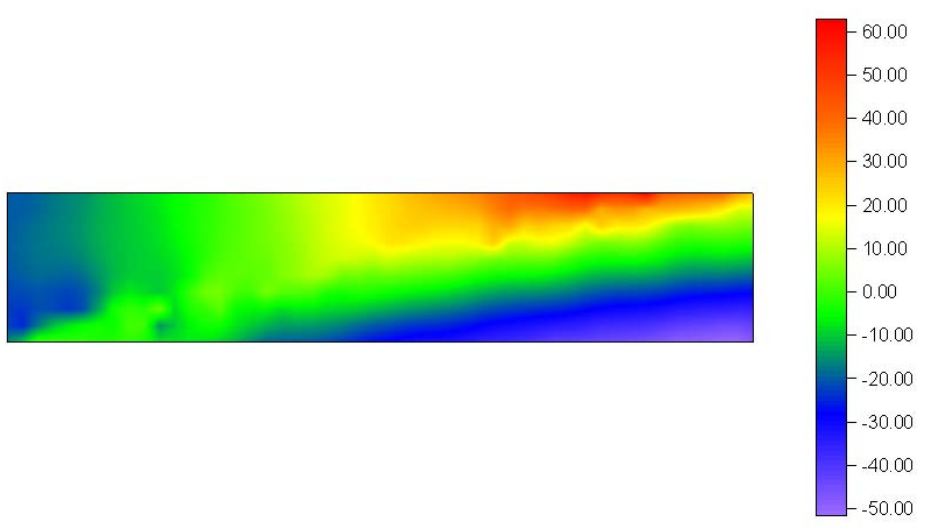

$\sigma_{x}$ Elemento Triangular $(\% \mu=80) \Rightarrow$ Com Enriquecimento

Figura 7.17 - Tensão plana - $\boldsymbol{\sigma}_{\boldsymbol{x}}$ - elemento triangular com enriquecimento seletivo dos Deslocamentos no Contorno $(\boldsymbol{x})-(\boldsymbol{\%} \boldsymbol{\mu}=\boldsymbol{8 0})$ - Aproximação Quadrática das Tensões - FHT.

A representação da tensão plana $\left(\sigma_{x}\right)$ obtida das análises do Exemplo 1 com os elementos da FHMT (quadrilateral e triangular) enriquecidos - tensões e deslocamentos no domínio $\left(y^{2}\right)$ e deslocamentos no contorno $(x)$ - concordam com o valor exato da teoria clássica de vigas e não sofrem qualquer influência com o aumento do parâmetro $(\boldsymbol{\mu})$, ver figuras 7.18 e 7.19 . 


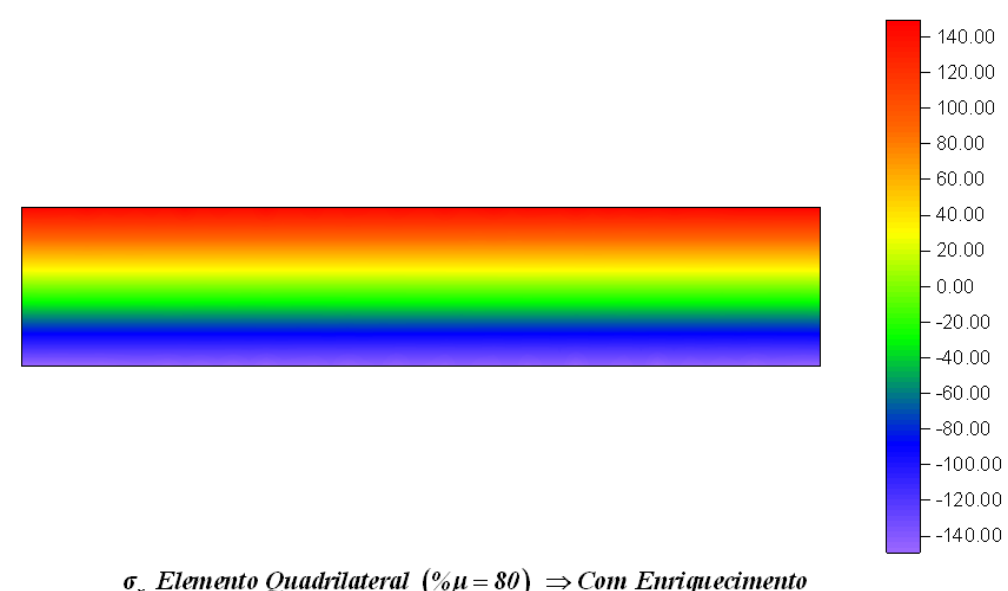

Figura 7.18 - Tensão plana - $\boldsymbol{\sigma}_{\boldsymbol{x}}$ - elemento quadrilateral com enriquecimento das Tensões e Deslocamentos no Domínio $\left(\boldsymbol{y}^{2}\right)$ e Deslocamentos no Contorno $(\boldsymbol{x})-(\boldsymbol{\%} \boldsymbol{\mu}=\boldsymbol{8 0})$ - FHMT.

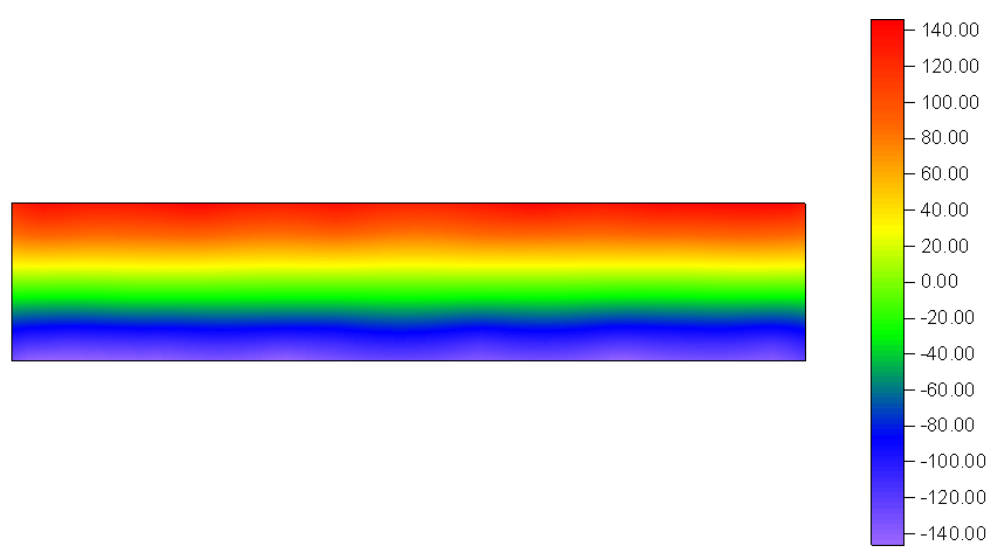

$\sigma_{x}$ Elem ento Triangular $(\% \mu=80) \Rightarrow$ Com Enriquecimento

Figura 7.19 - Tensão plana - $\boldsymbol{\sigma}_{\boldsymbol{x}}$ - elemento triangular com enriquecimento das Tensões e Deslocamentos no Domínio $\left(\boldsymbol{y}^{2}\right)$ e Deslocamentos no Contorno $(\boldsymbol{x})-(\boldsymbol{\%} \boldsymbol{\mu}=\boldsymbol{8 0})$ - FHMT.

O valor da energia de deformação do Exemplo 1, igualmente ao deslocamento, também destoa do valor exato à medida que se aumenta o parâmetro de distorção $(\boldsymbol{\mu})$ da rede de elementos quadrilaterais e triangulares da FHT/FHMT sem enriquecimento, como mostram, respectivamente, as figuras 7.20 e 7.21. 


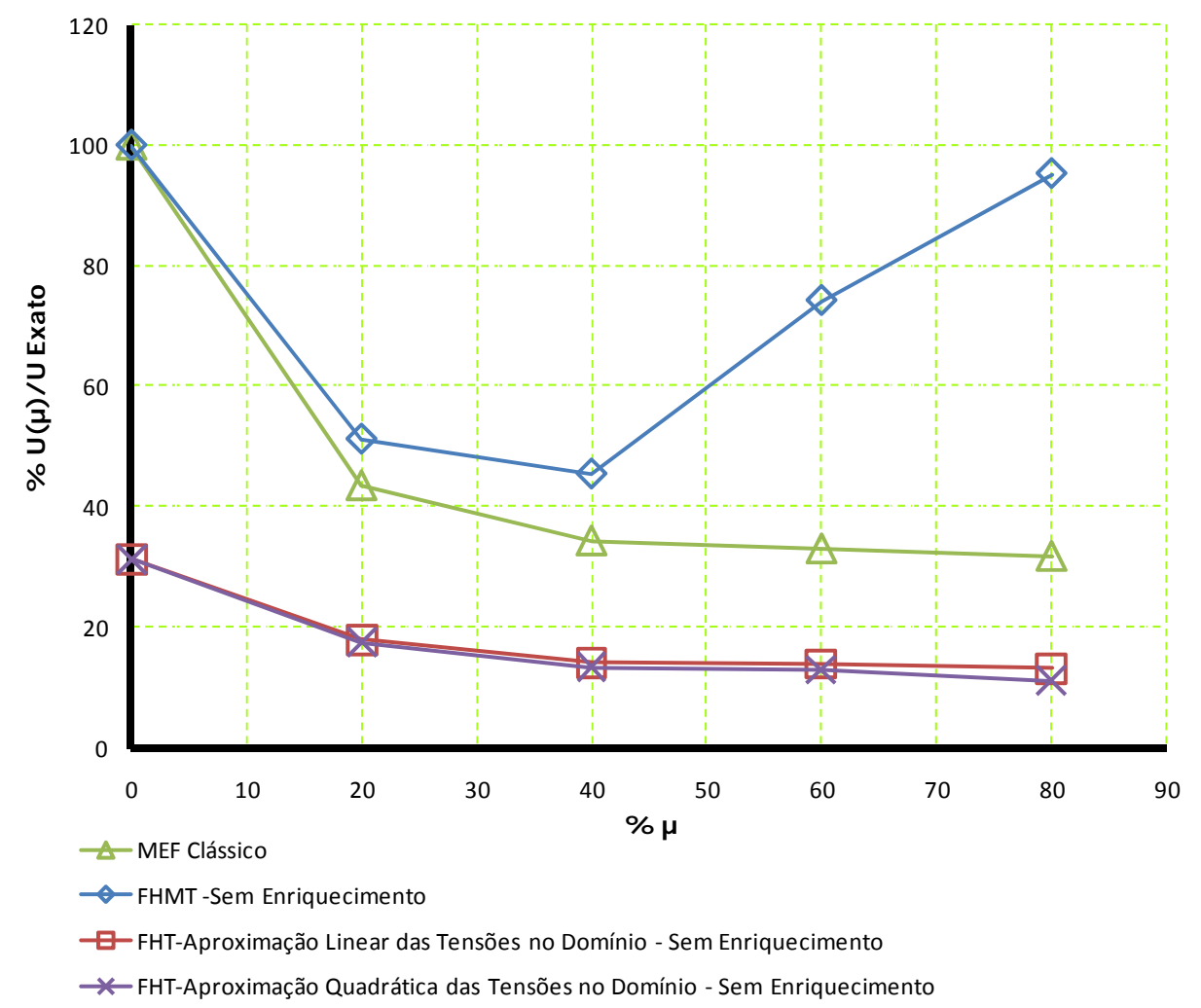

Figura 7.20 - Sensibilidade da energia de deformação à distorção da rede - Elemento Quadrilateral FHT, FHMT e MEF clássico.

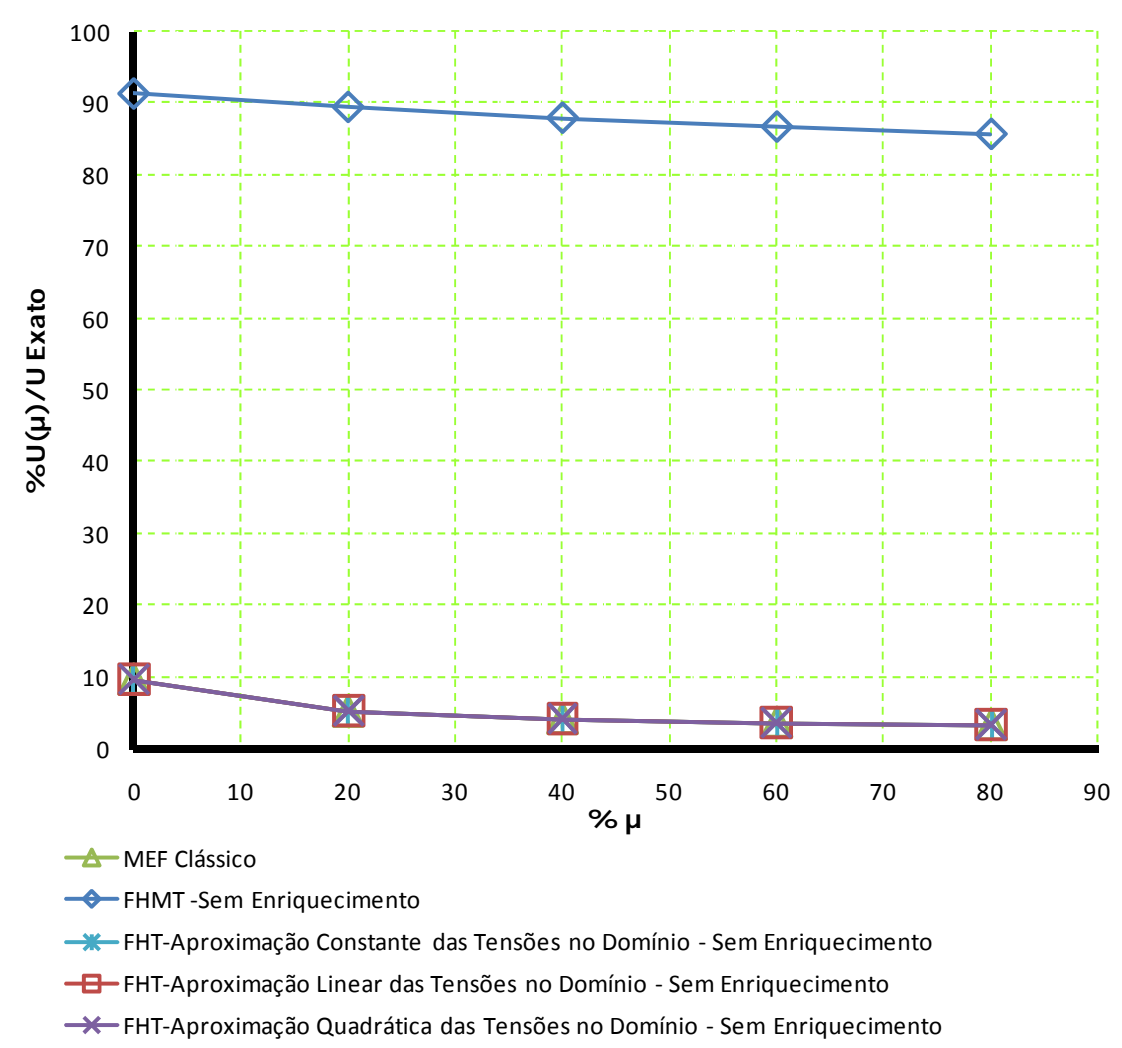

Figura 7.21 - Sensibilidade da energia de deformação à distorção da rede - Elemento Triangular FHT, FHMT e MEF clássico. 
Tomando-se como grandeza de referência a energia de deformação, o elemento quadrilateral da FHT (aproximação quadrática das tensões no domínio) sem enriquecimento é mais sensível a distorção da rede do que o elemento quadrilateral do MEF Clássico, como apresenta a figura 7.22. Vale salientar que essa situação é invertida quando, por exemplo, combinam-se enriquecimentos das tensões no domínio $\left(y^{2}\right)$ e deslocamentos no contorno $(x)$.

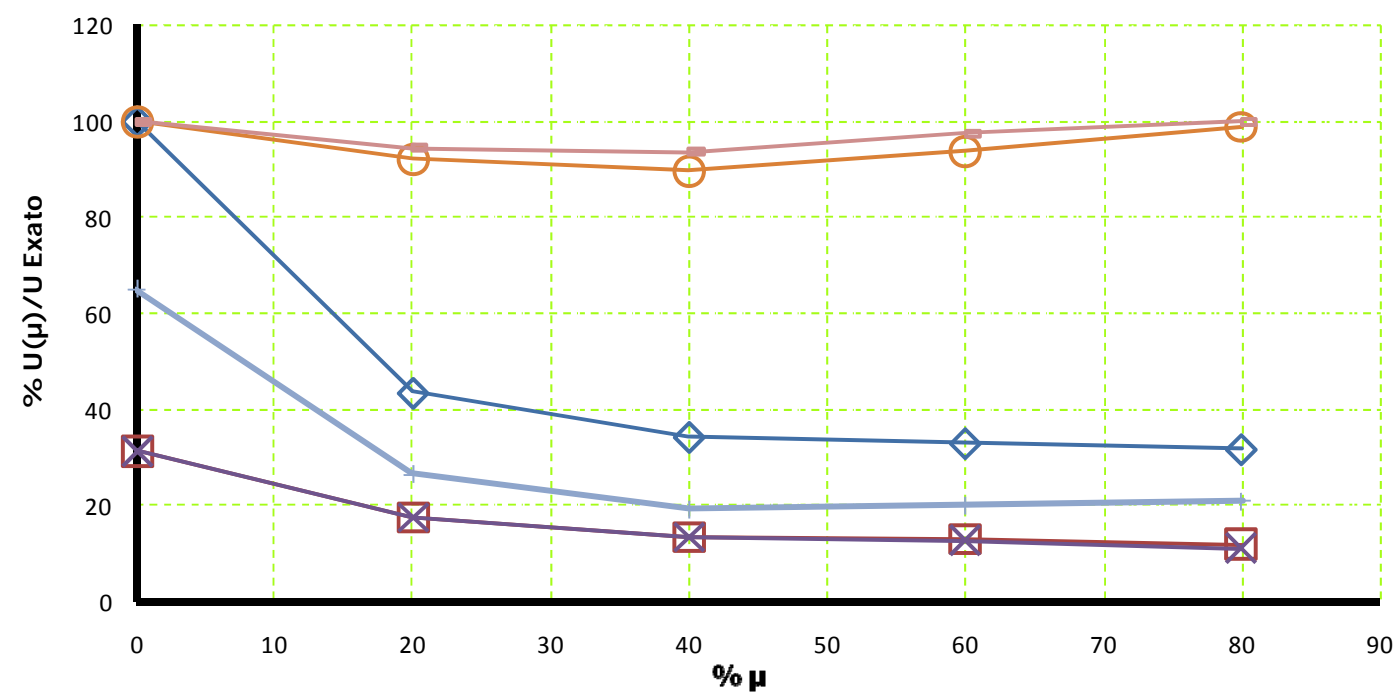

$\smile$ MEF Clássico

—-FHT-Aproximação Quadrática das Tensões no Domínio - Sem Enriquecimento

* FHT-Aproximação Quadrática das Tensões no Domínio-Enriquecimento das Tensões no Domínio $\left(\mathrm{y}^{2}\right)$

— FHT - Aproximação Quadrática das Tensões no Domínio-Enriquecimento das Tensões no Domínio ( $\mathrm{y}^{2}$ ) e Deslocamentos no Contorno (x)

- FHT-Aproximação Quadrática das Tensões no Domínio-Enriquecimento das Tensões no Domínio ( $\mathrm{y}^{2}$ ) e Deslocamentos no Contorno (y)

—- FHT-Aproximação Quadrática das Tensões no Domínio-Enriquecimento dos Deslocamentos no Contorno (x)

Figura 7.22 - Sensibilidade da energia de deformação à distorção da rede - Elemento Quadrilateral FHT e MEF clássico.

A figura 7.23 destaca a forte sensibilidade do elemento triangular da FHT à distorção da rede, mas com respostas no mínimo iguais ao elemento triangular do MEF clássico. 


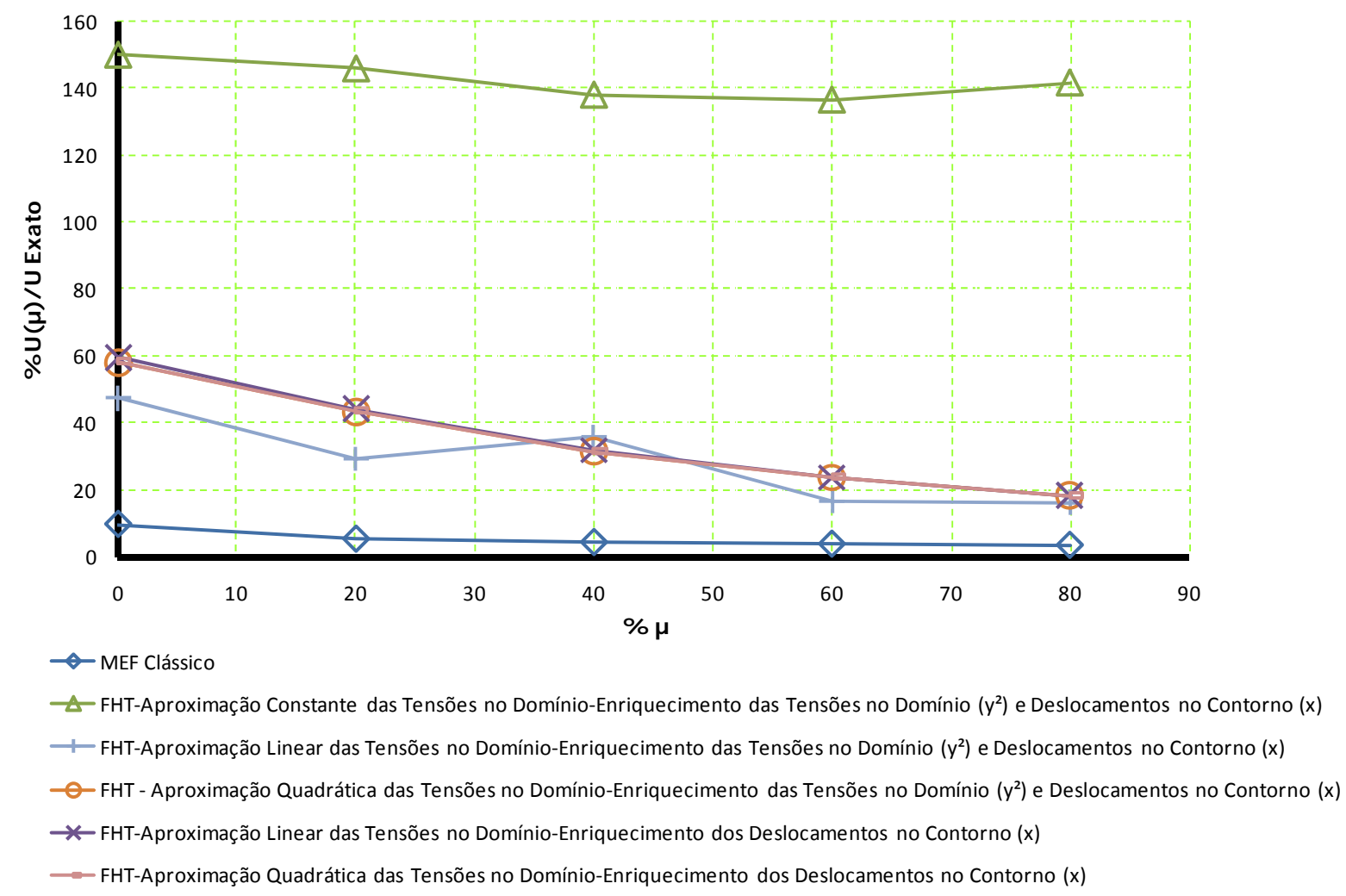

Figura 7.23 - Sensibilidade da energia de deformação à distorção da rede - Elemento TriangularFHT e MEF clássico.

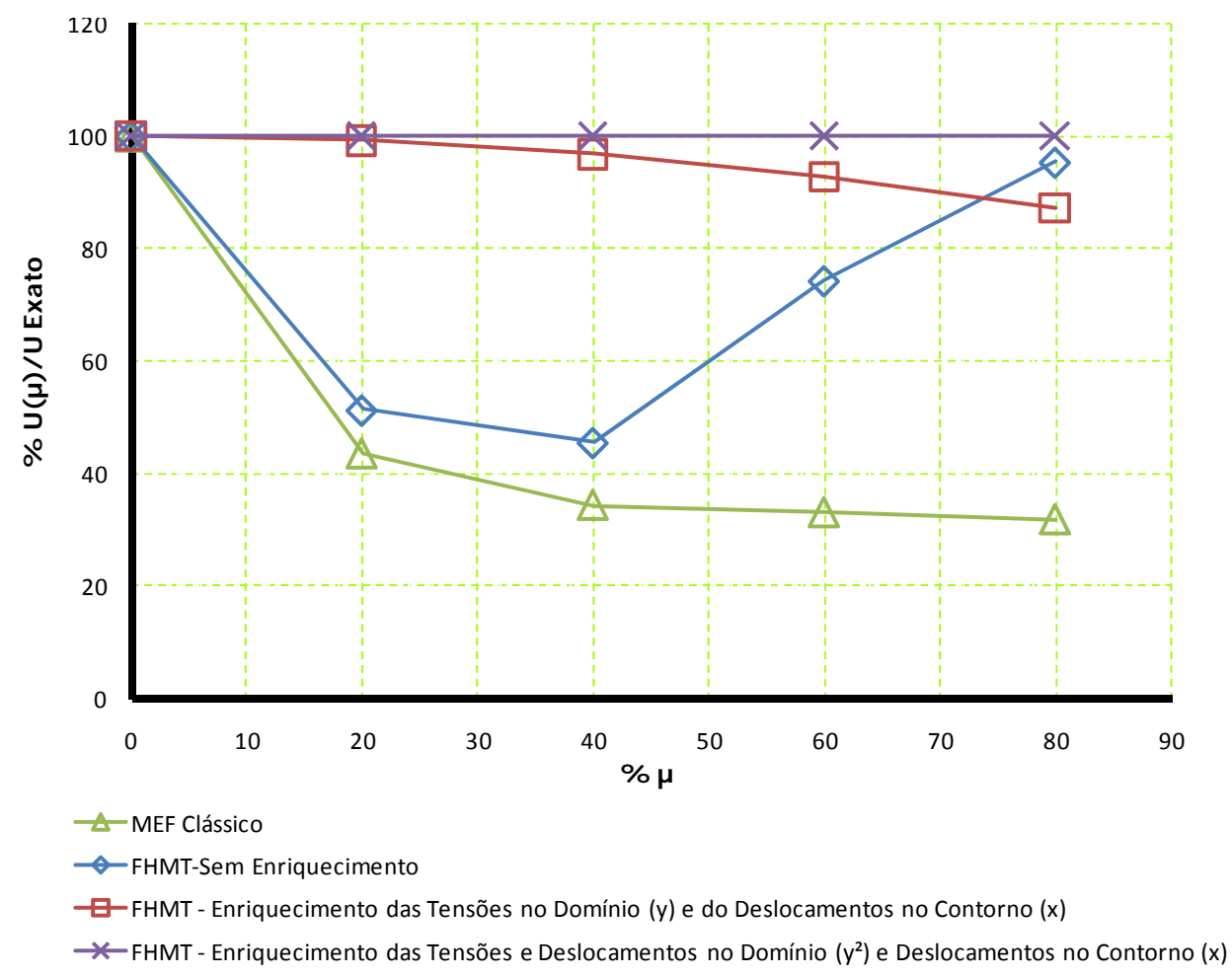

Figura 7.24 - Sensibilidade da energia de deformação à distorção da rede - Elemento Quadrilateral FHMT e MEF clássico. 
Os resultados obtidos das análises do Exemplo 1 (energia de deformação) com os elementos da FHMT (quadrilateral e triangular) enriquecidos - tensões e deslocamentos no domínio $\left(\boldsymbol{y}^{2}\right)$ e deslocamentos no contorno $(\boldsymbol{x})$ - concordam com o valor exato da teoria clássica de vigas e não sofrem qualquer influência com o aumento do parâmetro $(\boldsymbol{\mu})$. Este fato pode ser observado dos resultados ilustrados nas figuras 7.24 e 7.25.

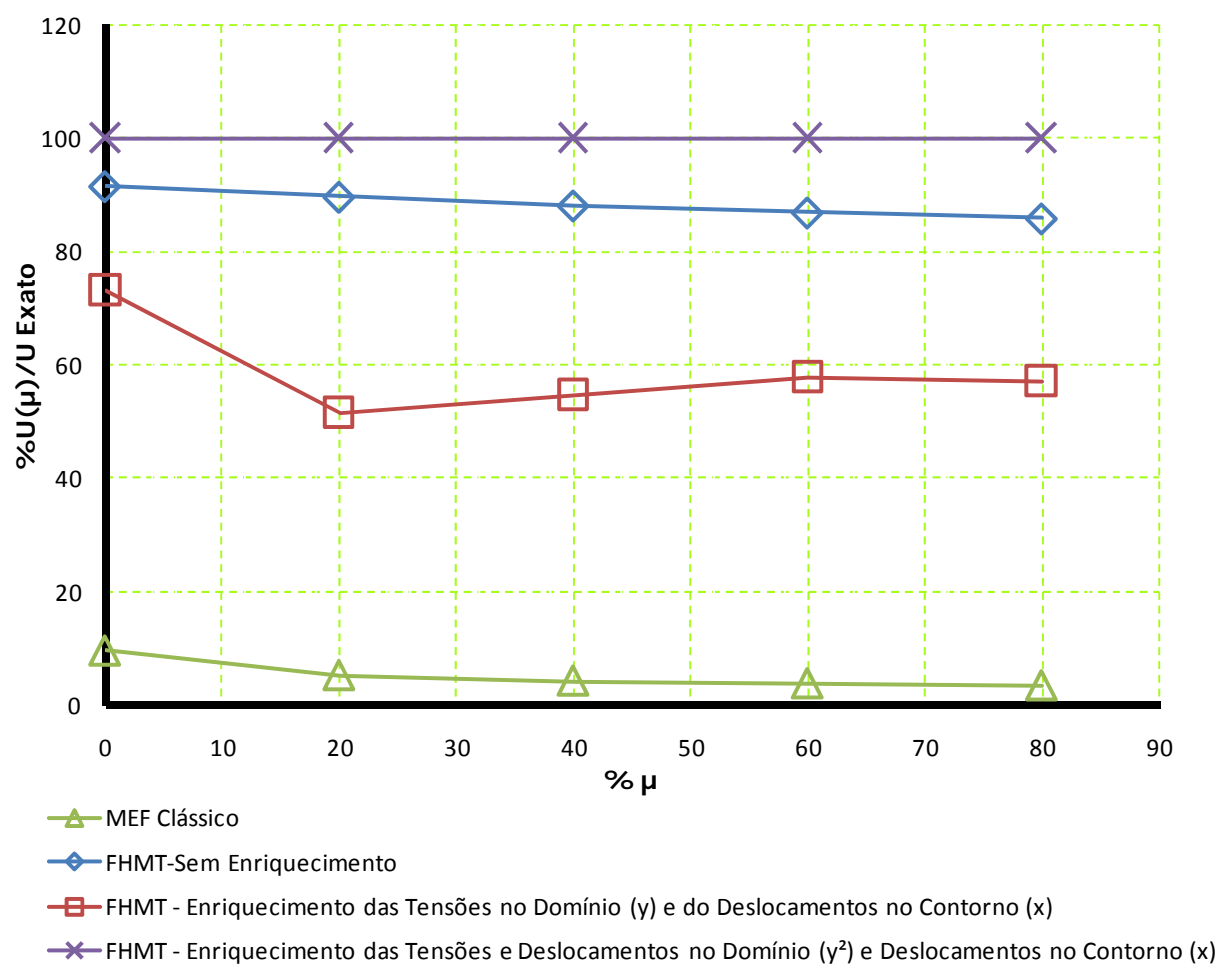

Figura 7.25 - Sensibilidade da energia de deformação à distorção da rede - Elemento TriangularFHMT e MEF clássico.

\subsubsection{Exemplo 2 - Viga Engastada - Cisalhamento}

A figura 7.26 ilustra uma chapa retangular de espessura unitária, $(\mathbf{1 0} \times \mathbf{2})$ unidades de comprimento, com borda vertical esquerda fixa $\left(\boldsymbol{u}_{\boldsymbol{x}}=\boldsymbol{0} ; \boldsymbol{u}_{\boldsymbol{y}}=\boldsymbol{0}\right)$ e submetida a uma força de cisalhamento no lado vertical direito de $\boldsymbol{q}=\mathbf{1 0 0}$ unidades de força por unidades de comprimento. 


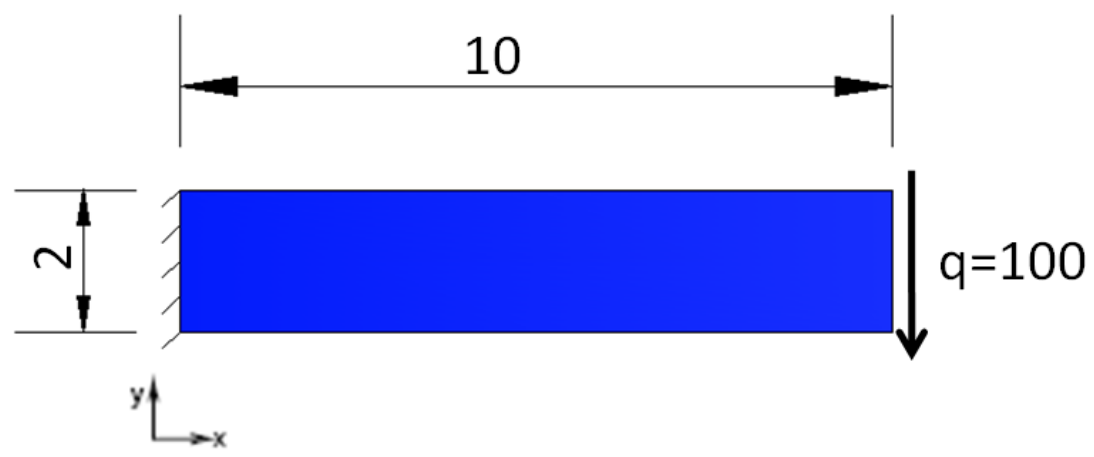

Figura 7.26 - Exemplo 2 - Chapa com 100 unidades de força por unidade de comprimento.

Para definir os resultados de referência, o Exemplo 2 foi analisado via ANSYS ${ }^{\circledR}$ com uma seqüência de redes regulares até 10.000 elementos quadrilaterais PLANE 42. Assim, admitindo-se que o módulo de Young é $\boldsymbol{E}=\mathbf{1}$ e o coeficiente de Poisson $\boldsymbol{v}=\mathbf{0 , 2 5}$, obteve-se para este problema os seguintes valores:

a) Energia de Deformação de referência: $U_{\text {exemplo } 2}=1,03 \cdot 10^{7}$;

b) Deslocamento de referência: $\boldsymbol{u}_{\boldsymbol{y}}=-\mathbf{1 , 0 3 \cdot 1 0 ^ { 5 }}$ no lado vertical esquerdo (ver figura 7.27);
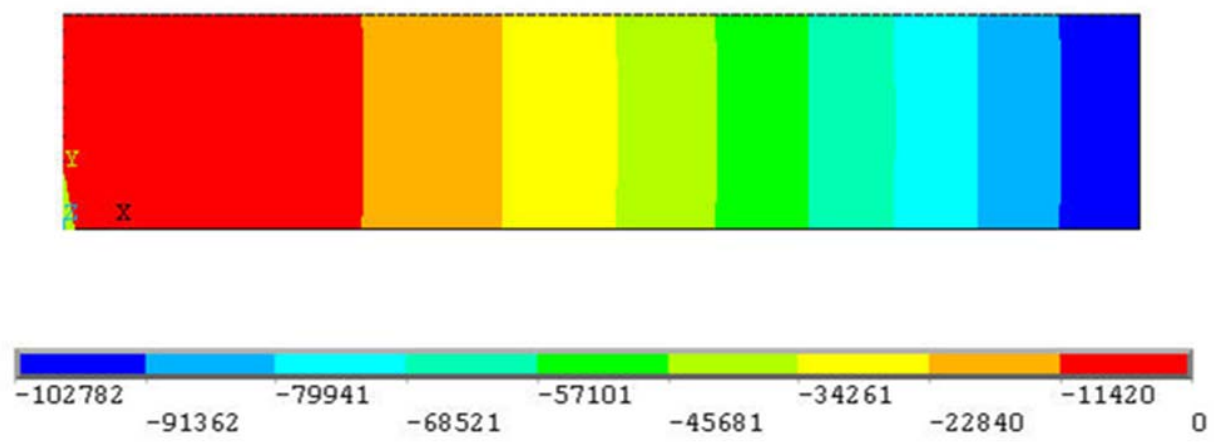

Figura 7.27 - Exemplo 2 - Deslocamento $\boldsymbol{u}_{\boldsymbol{y}}$ para a discretização adotada.

c) Distribuição de tensões planas como mostra a figura 7.28. 


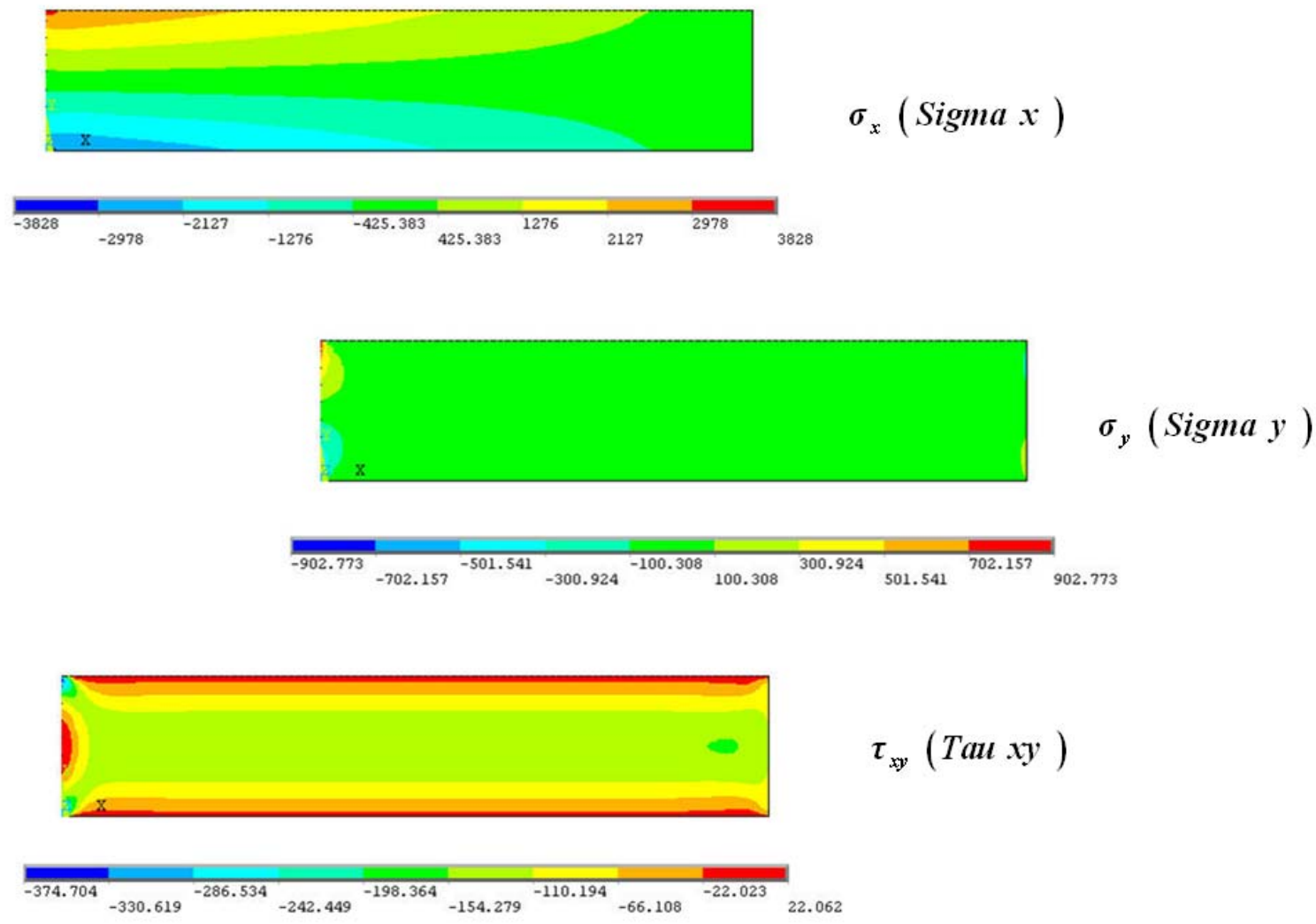

Figura 7.28 - Exemplo 2 - Tensões Planas para a discretização adotada.

As redes quadrilaterais e triangulares utilizadas no Exemplo 1 (em todos os níveis do parâmetro de distorção $\boldsymbol{\mu}$ - vide figura 7.2) também serão aplicadas ao Exemplo 2 para avaliar a sensibilidade à distorção da rede dos elementos quadrilateral e triangular da FHT e FHMT com enriquecimento nodal.

O Exemplo 2 também foi abordado no trabalho de Punch and Atluri (1984) e os seus resultados serão confrontados com os resultados dos elementos quadrilaterais da FHT e FHMT com enriquecimento nodal. Analogamente ao Exemplo 1, o nó do canto superior direito das redes da figura 7.2 será utilizado como nó de referência para comparar a grandeza deslocamento.

Os resultados para deslocamento e energia de deformação do Exemplo 2, discretizado com elementos sem distorção $(\boldsymbol{\mu}=\boldsymbol{0})$ da FHMT/FHT e MEF, são ilustrados nas tabelas 7.4, 7.5 e 7.6 . 
Tabela 7.4 - Exemplo 2 - FHMT sem enriquecimento $-\boldsymbol{\mu}=\boldsymbol{0}$ - resultados para deslocamento e energia de deformação.

\begin{tabular}{cccc}
\hline \multicolumn{2}{c}{ FHMT sem Enriquecimento } & \multicolumn{2}{c}{ FHMT sem Enriquecimento } \\
\multicolumn{2}{c}{ Elemento Triangular } & \multicolumn{2}{c}{ Elemento Quadrilateral } \\
\hline Deslocamento no & Energia de & Deslocamento no & Energia de \\
Contorno & Deformação & Contorno & Deformação \\
\hline $3,33.10^{11}$ & $5,71.10^{5}$ & $-8,54.10^{4}$ & $\mathbf{8 , 5 3 . 1 0}^{6}$ \\
\hline
\end{tabular}

Tabela 7.5 - Exemplo 2 - FHT sem enriquecimento- $\boldsymbol{\mu}=\boldsymbol{0}$ - resultados para deslocamento e energia de deformação.

\begin{tabular}{ccccc}
\hline & \multicolumn{2}{c}{ FHT sem Enriquecimento } & \multicolumn{2}{c}{ FHT sem Enriquecimento } \\
& \multicolumn{2}{c}{ Elemento Triangular } & \multicolumn{2}{c}{ Elemento Quadrilateral } \\
\hline $\begin{array}{c}\text { Base de } \\
\begin{array}{c}\text { Aproximação } \\
\text { das Tensões }\end{array}\end{array}$ & $\begin{array}{c}\text { Deslocamento no } \\
\text { Contorno }\end{array}$ & $\begin{array}{c}\text { Energia de } \\
\text { Deformação }\end{array}$ & $\begin{array}{c}\text { Deslocamento no } \\
\text { Contorno }\end{array}$ & $\begin{array}{c}\text { Energia de } \\
\text { Deformação }\end{array}$ \\
\hline Quadrática & $\mathbf{- 1 , 1 4 . 1 0 ^ { 4 }}$ & $\mathbf{1 , 1 4 . 1 0 ^ { 6 }}$ & $-3,20.10^{4}$ & $3,20.10^{6}$ \\
\hline Linear & $-\mathbf{1 , 1 4 . 1 0 ^ { 4 }}$ & $\mathbf{1 , 1 4 . 1 0 ^ { 6 }}$ & $-3,20.10^{4}$ & $3,20.10^{6}$ \\
\hline Constante & $-\mathbf{1 , 1 4 . 1 0 ^ { 4 }}$ & $\mathbf{1 , 1 4 . 1 0 ^ { 6 }}$ & - & - \\
\hline
\end{tabular}

Tabela 7.6 - Exemplo 1 - MEF Clássico - $\boldsymbol{\mu}=\boldsymbol{0}$ - resultados para deslocamento e energia de deformação.

\begin{tabular}{cccc}
\hline \multicolumn{2}{c}{ MEF Clássico } & \multicolumn{2}{c}{ MEF Clássico } \\
Elemento Triangular & \multicolumn{2}{c}{ Elemento Quadrilateral } \\
\hline $\begin{array}{c}\text { Deslocamento no } \\
\text { Contorno }\end{array}$ & Energia de & Deslocamento no & Energia de \\
\hline$-1,14.10^{4}$ & Deformação & Contorno & Deformação \\
\hline
\end{tabular}

Os resultados apresentados nas tabelas 7.4 a 7.6 mostram que ambos os elementos da FHT/FHMT sem enriquecimento nodal e os elementos do MEF Clássico não conseguiram reproduzir os resultados tomados como referência para o deslocamento e energia de deformação. Isto se deve ao fato do Exemplo 2 não ser trivial e a rede adotada ser muito grosseira. É importante ressaltar que as discretizações utilizadas nesta parte do trabalho são para analisar a sensibilidade dos elementos da FHT/FHMT à distorção da rede.

Vale salientar que o elemento triangular da FHMT apresentou convergência muito baixa na energia de deformação e consequentemente obteve-se um resultado não representativo para o deslocamento do nó de referência, ver tabela 7.4. 
Ainda destes resultados, garante-se que o elemento triangular sem enriquecimento nodal da FHT converge, no mínimo, para a resposta obtida com o elemento triangular do MEF Clássico.

A figura 7.29 apresenta a distribuição de tensões planas para o elemento quadrilateral sem enriquecimento da FHMT - $\boldsymbol{\mu}=\boldsymbol{0}$. Como já era de se esperar, com uma discretização tão simples, não foi possível recuperar a representação de referência das tensões planas do Exemplo 2.

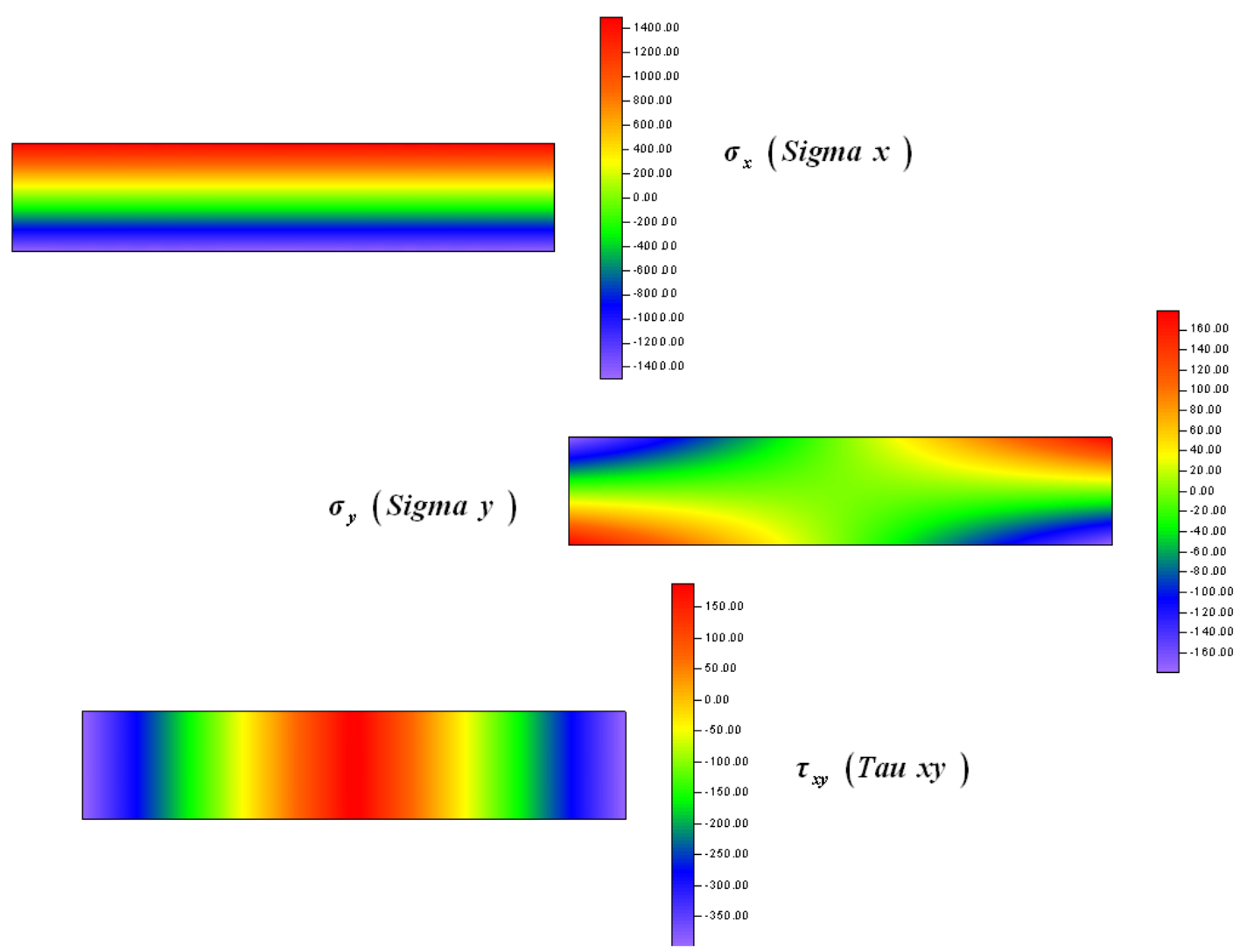

Figura 7.29 - Tensões planas - elemento quadrilateral sem enriquecimento - $(\boldsymbol{\%} \boldsymbol{\mu}=\boldsymbol{0})$ - FHMT.

As tensões planas para o elemento triangular e quadrilateral sem enriquecimento da FHT (base quadrática das tensões no domínio) também não é representativa, quando comparada com a distribuição de tensões de referência, ver figuras 7.30, 7.31 e 7.28. 


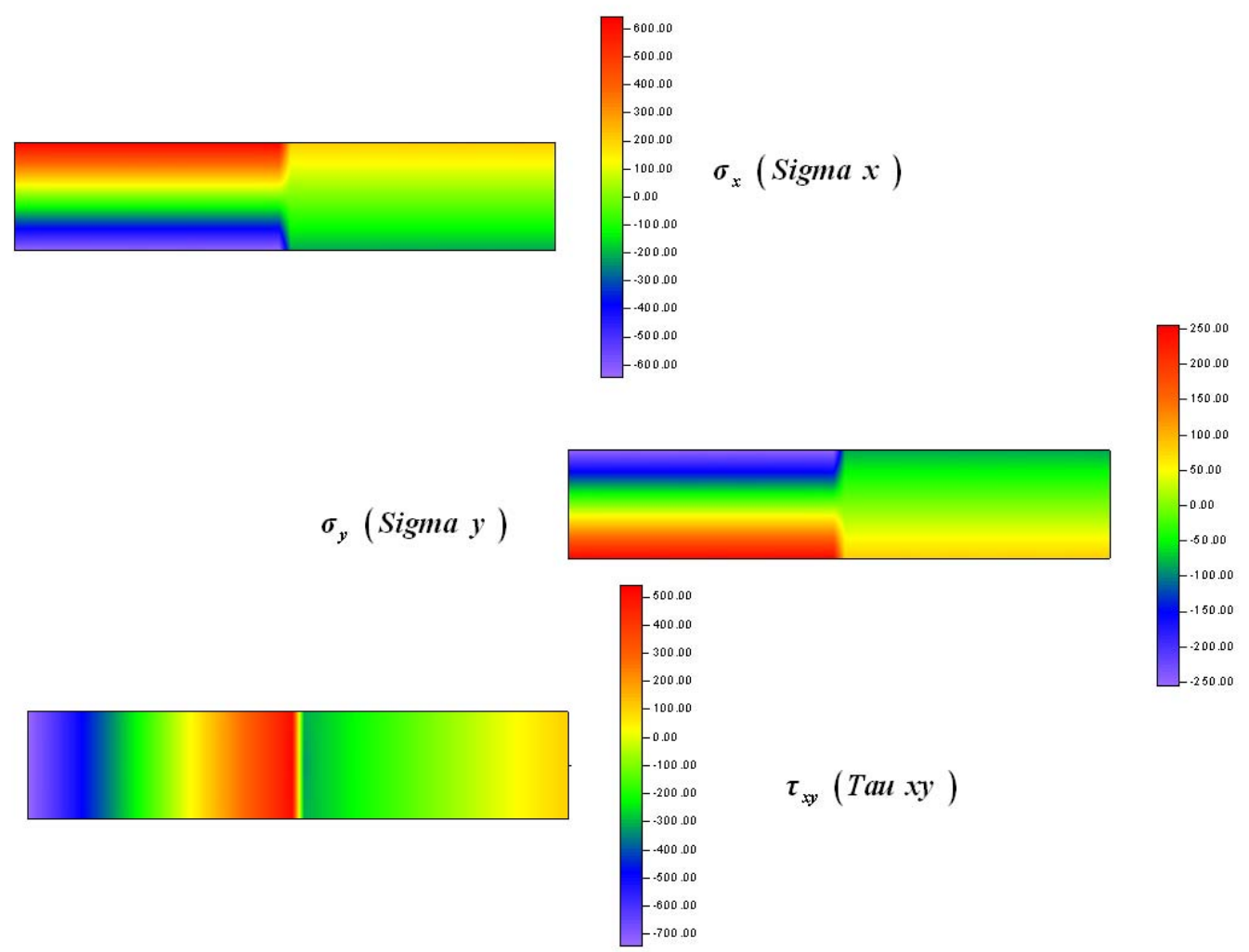

Figura 7.30 - Tensões planas - elemento quadrilateral sem enriquecimento - $(\boldsymbol{\%} \boldsymbol{\mu}=\boldsymbol{0})$ Aproximação Quadrática das Tensões - FHT.

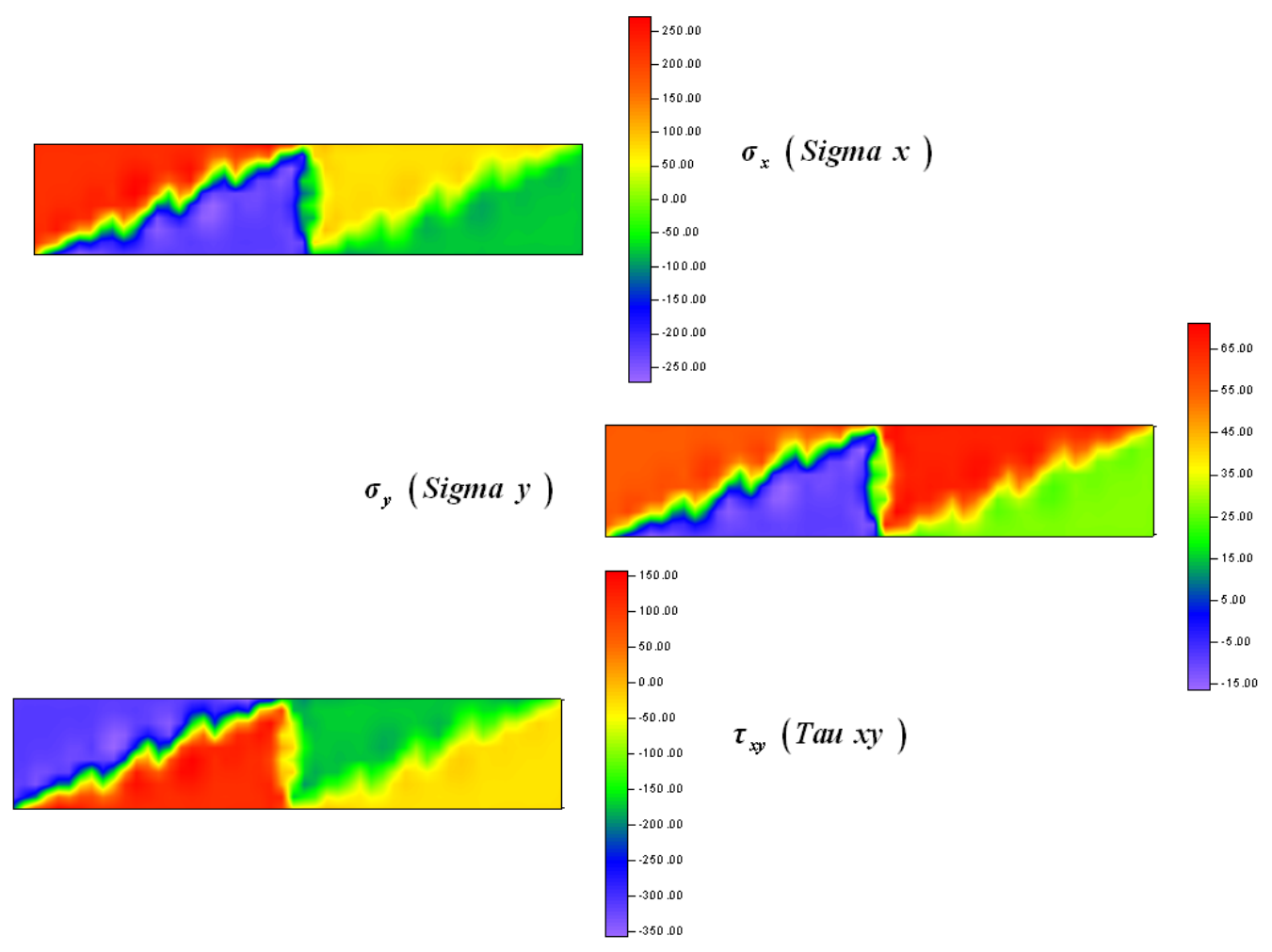

Figura 7.31 - Tensões planas - elemento triangular sem enriquecimento - $(\boldsymbol{\%} \boldsymbol{\mu}=\boldsymbol{0})$ Aproximação Quadrática das Tensões - FHT. 


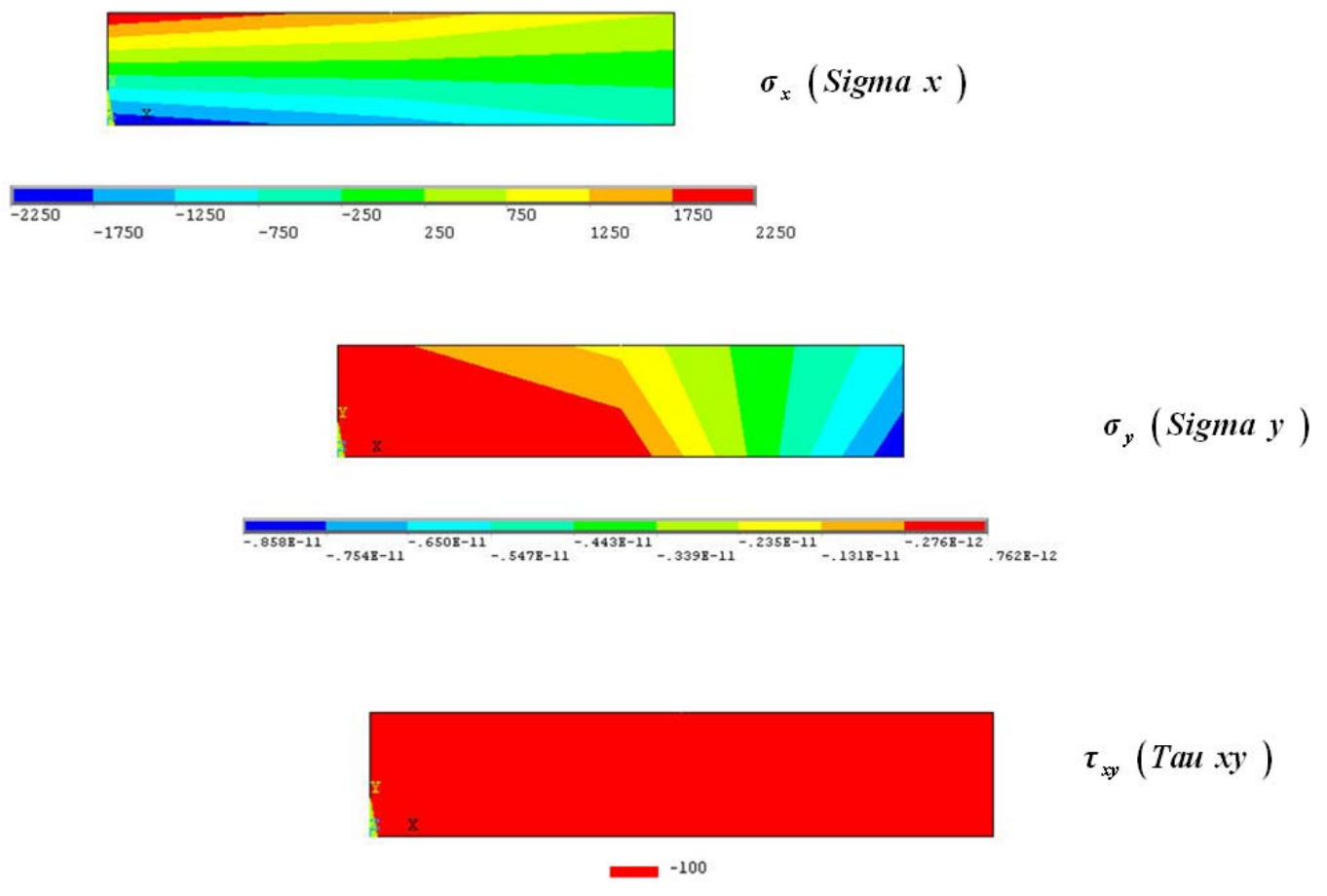

Figura 7.32 - Tensões planas - elemento quadrilateral - $(\boldsymbol{\%} \boldsymbol{\mu}=\boldsymbol{0})$ - MEF.

Focando os estudos relacionados à distorção da rede, o Exemplo 2 será discretizado com todas as redes apresentadas na figura 7.2 e, ainda, consideradas as combinações de enriquecimento nodal (ver figura 7.5) que foram aplicadas ao Exemplo 1. Assim, poderão ser avaliadas as alterações de valores para a energia de deformação e deslocamento, bem como na representação dos campos de tensões planas gerados com os elementos triangulares e quadrilaterais da FHT/FHMT com enriquecimento nodal, quando se aumenta gradativamente o parâmetro de distorção $\boldsymbol{\mu}$.

No caso específico das tensões planas será utilizada a componente de tensão na direção $(\boldsymbol{x})$ como parâmetro de comparação, pois para elemento quadrilateral do MEF clássico $\boldsymbol{\mu}=\boldsymbol{0}$ (ver figura 7.32) somente a representação do $\boldsymbol{\sigma}_{x}$ é similar ao resultado de referência (figura 7.28), ou seja, para o nível de discretização adotado só 0 representatividade.

A seguir, são ilustrados os resultados obtidos nos vários testes propostos de sensibilidade à distorção das redes. Cada curva foi construída com o parâmetro de distorção $\boldsymbol{\mu}(\boldsymbol{0} \leq \boldsymbol{\mu} \leq \mathbf{0 , 8})$ definida nas abscissas e o valor normalizado da energia de deformação, ou 
dos deslocamentos, como ordenadas. Cada valor normalizado foi calculado dividindo-se a grandeza analisada (num certo nível de distorção $\boldsymbol{\mu}$ ) pelo valor de referência dessa grandeza.

A figura 7.33 destaca que os elementos quadrilaterais da FHT/FHMT sem enriquecimento e o do MEF clássico perdem precisão na representação dos deslocamentos do nó de referência quando se aumenta o parâmetro $\boldsymbol{\mu}$.

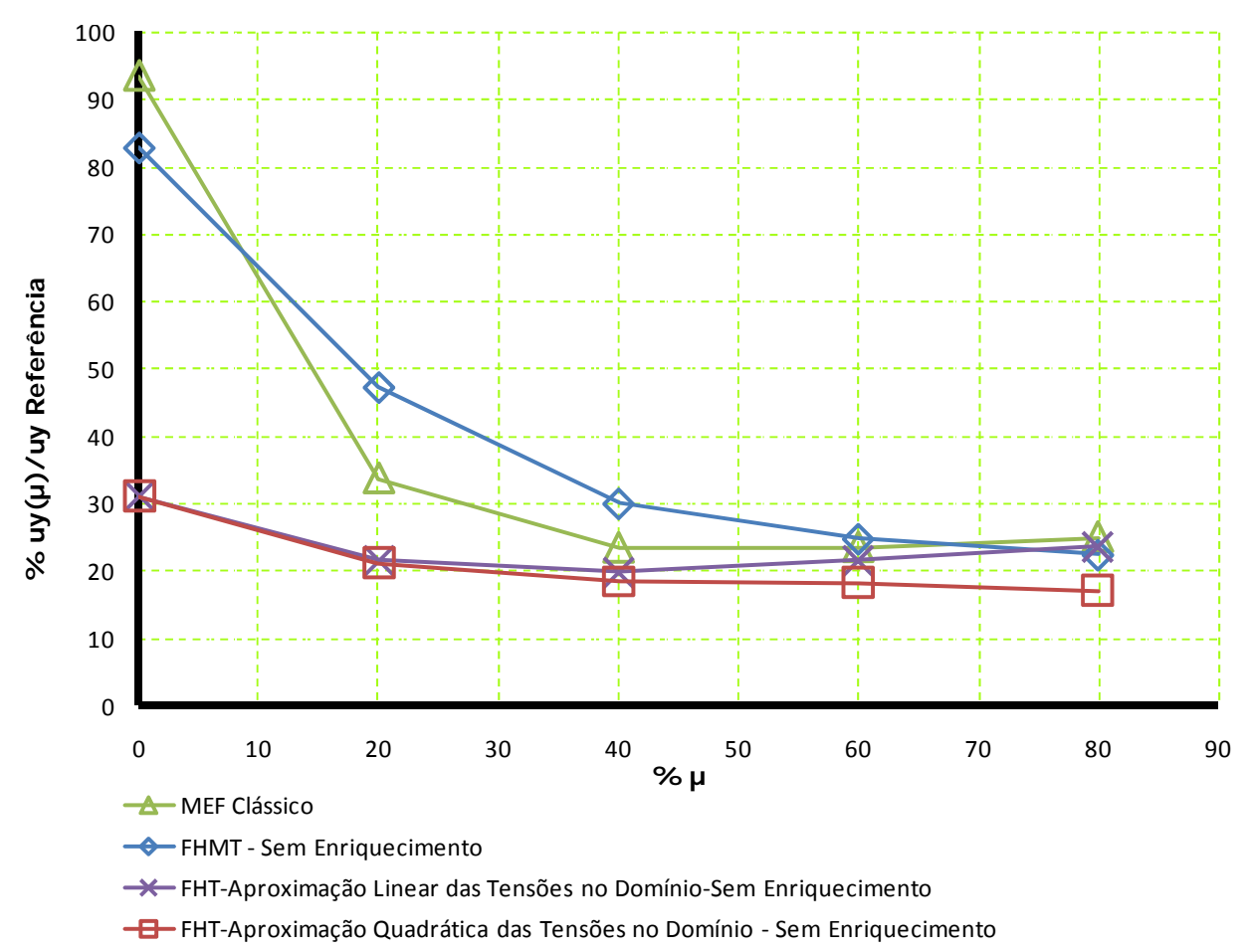

Figura 7.33 - Sensibilidade do deslocamento à distorção da rede - Elemento Quadrilateral - FHT, FHMT e MEF Clássico.

Como apresenta a figura 7.34, o elemento triangular da FHT sem enriquecimento recuperou a mesma resposta do MEF Clássico, para todos os níveis de distorção das redes analisadas. Ainda nesta figura, observa-se que o elemento triangular da FHMT sem enriquecimento, mesmo na condição nula de distorção $(\boldsymbol{\mu}=\boldsymbol{0})$, o deslocamento do nó padrão se distanciou bastante do deslocamento de referência, ver figura 7.34.

O enriquecimento simultâneo das tensões no domínio e deslocamentos no contorno, bem como o enriquecimento exclusivo dos deslocamentos no contorno, faz com que a resposta do elemento quadrilateral da FHT - Aproximação Quadrática das Tensões - supere os resultados obtidos com o elemento quadrilateral do MEF clássico para todos os níveis de distorção $(\boldsymbol{\mu})$, como mostra a figura 7.35. 


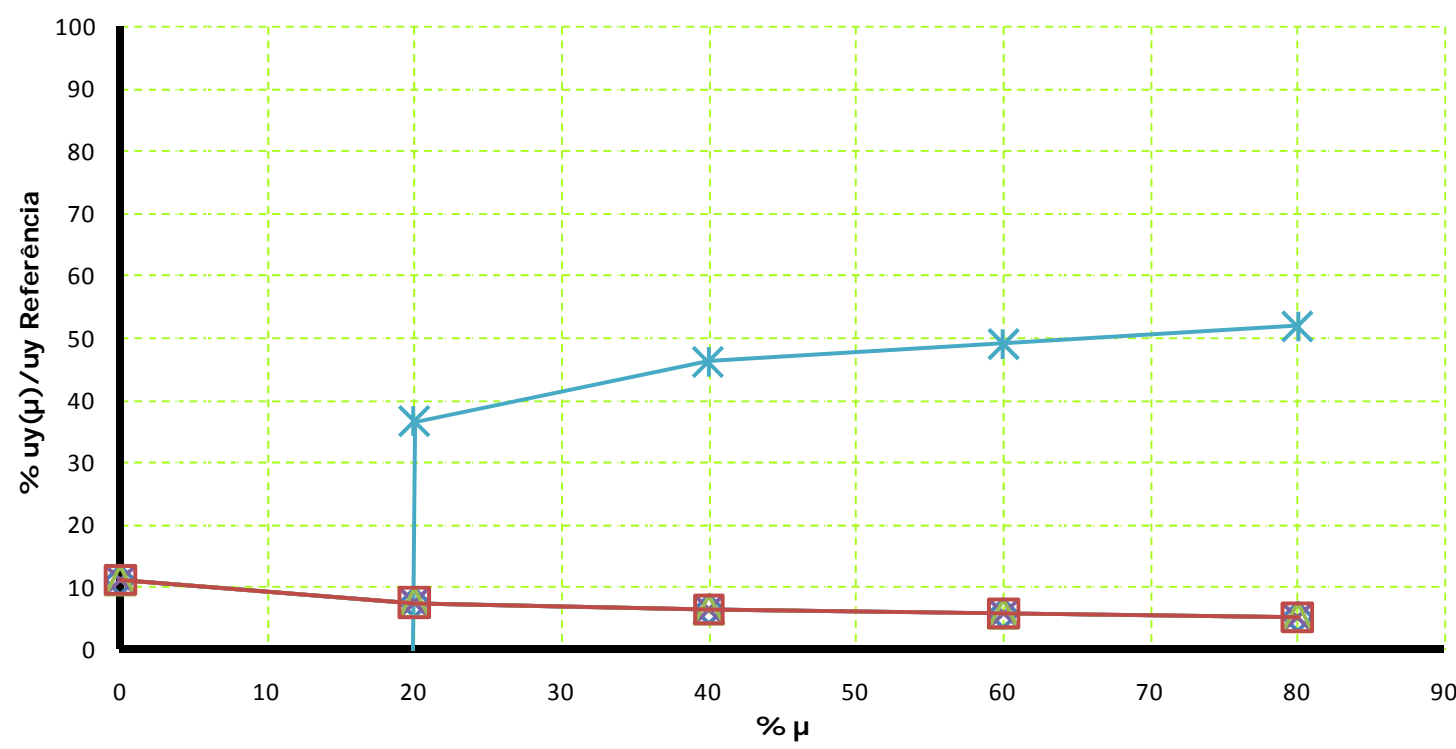

$\smile$ MEF Clássico

- FHMT - Sem Enriquecimento

* FHT-Aproximação Constante das Tensões no Domínio-Sem Enriquecimento

$\triangle$ FHT-Aproximação Linear das Tensões no Domínio-Sem Enriquecimento

—FHT-Aproximação Quadrática das Tensões no Domínio-Sem Enriquecimento

Figura 7.34 - Sensibilidade do deslocamento à distorção da rede - Elemento Triangular - FHT, FHMT e MEF Clássico.

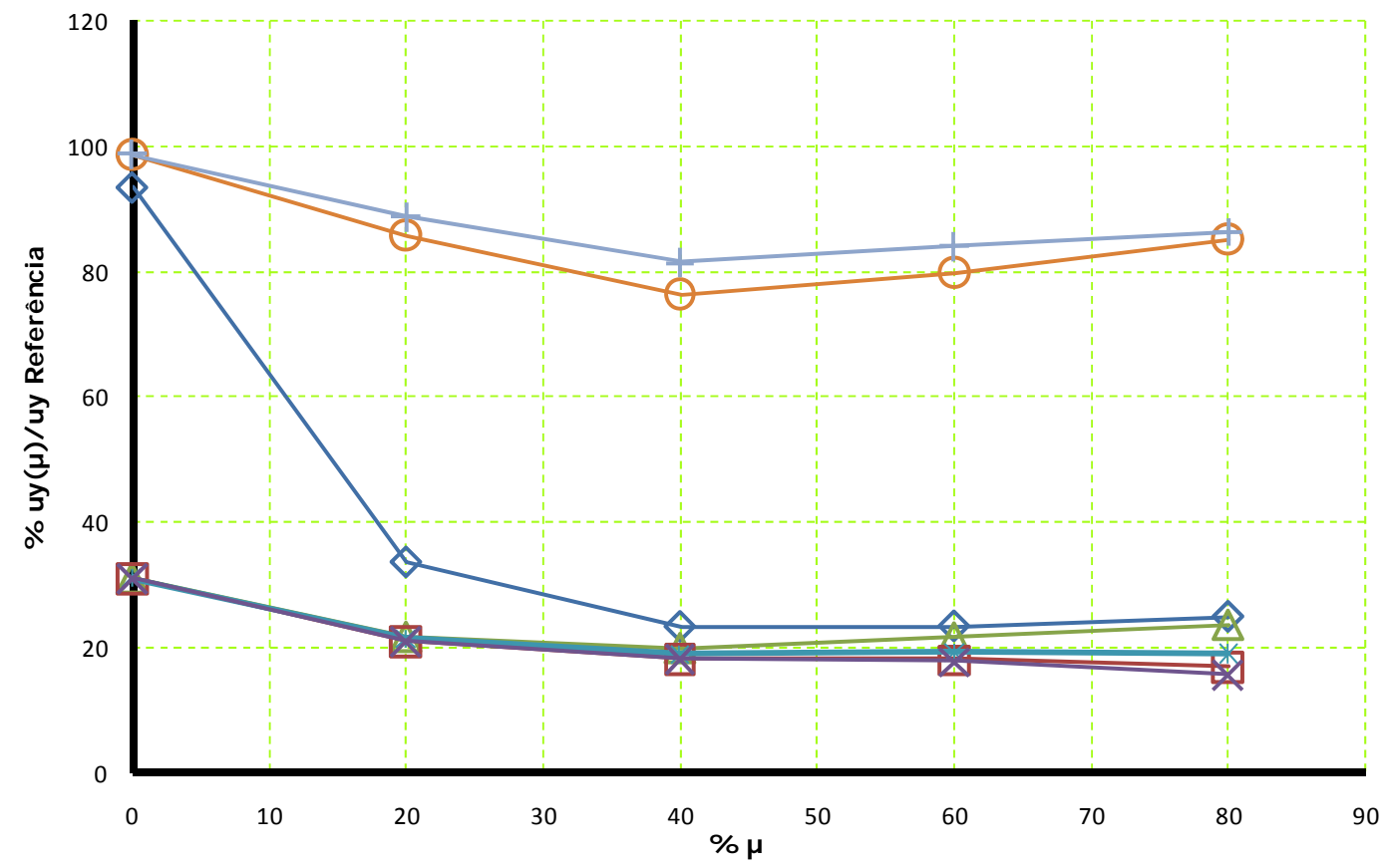

\footnotetext{
$\smile$ MEF Clássico

$\triangle$ FHT-Aproximação Linear das Tensões no Domínio - Sem Enriquecimento

—-FHT-Aproximação Quadrática das Tensões no Domínio-Sem Enriquecimento

* FHT-Aproximação Linear das Tensões no Domínio - Enriquecimento das Tensões no Domínio $\left(\mathrm{y}^{2}\right)$

* FHT-Aproximação Quadrática das Tensões no Domínio - Enriquecimento das Tensões no Domínio $\left(y^{2}\right)$

- - FHT -Aproximação Quadrática das Tensões no Domínio - Enriquecimento das Tensões no Domínio $\left(\mathrm{y}^{2}\right)$ e Deslocamentos no Contorno (x)

—-FHT-Aproximação Quadrática das Tensões no Domínio - Enriquecimento dos Deslocamentos no Contorno (x)
}

Figura 7.35 - Sensibilidade do deslocamento à distorção da rede - Elemento Quadrilateral - FHT e MEF Clássico. 


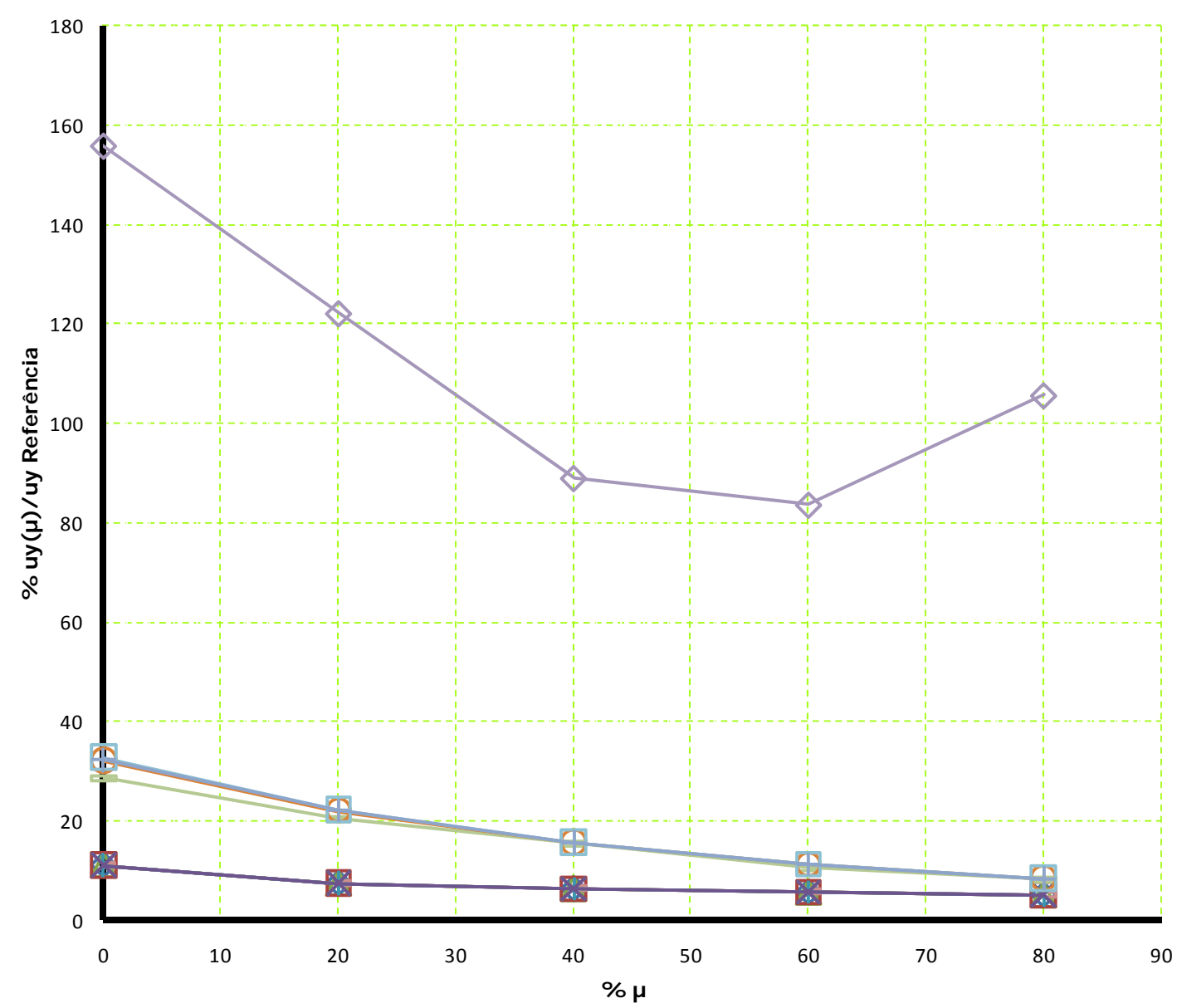

$\smile$ MEF Clássico

$\triangle$ _ FHT-Aproximação Linear das Tensões no Domínio - Sem Enriquecimento

—-FHT-Aproximação Quadrática das Tensões no Domínio-Sem Enriquecimento

- FHT-Aproximação Constante das Tensões no Domínio - Enriquecimento das Tensões no Domínio $\left(y^{2}\right)$

*-FHT-Aproximação Linear das Tensões no Domínio - Enriquecimento das Tensões no Domínio $\left(\mathrm{y}^{2}\right)$

* FHT-Aproximação Quadrática das Tensões no Domínio - Enriquecimento das Tensões no Domínio $\left(y^{2}\right)$

$\sim$ FHT -Aproximação Constante das Tensões no Domínio - Enriquecimento das Tensões no Domínio $\left(\mathrm{y}^{2}\right)$ e Deslocamentos no Contorno $(x)$

— FHT -Aproximação Linear das Tensões no Domínio - Enriquecimento das Tensões no Domínio ( $\mathrm{y}^{2}$ ) e Deslocamentos no Contorno ( $\mathrm{x}$ )

- FHT -Aproximação Quadrática das Tensões no Domínio - Enriquecimento das Tensões no Domínio $\left(\mathrm{y}^{2}\right)$ e Deslocamentos no Contorno (x)

- FHT-Aproximação Linear das Tensões no Domínio - Enriquecimento dos Deslocamentos no Contorno ( $x$ )

+ FHT-Aproximação Quadrática das Tensões no Domínio - Enriquecimento dos Deslocamentos no Contorno (x)

Figura 7.36 - Sensibilidade do deslocamento à distorção da rede - Elemento Triangular - FHT e MEF clássico.

O enriquecimento dos campos envolvidos na FHT não atenuou a influência da distorção da rede dos elementos triangulares nas respostas apresentadas na figura 7.36. Em todas as possibilidades de enriquecimento não se conseguiu manter o deslocamento do nó de referência no nível do deslocamento apresentado na figura 7.27. 


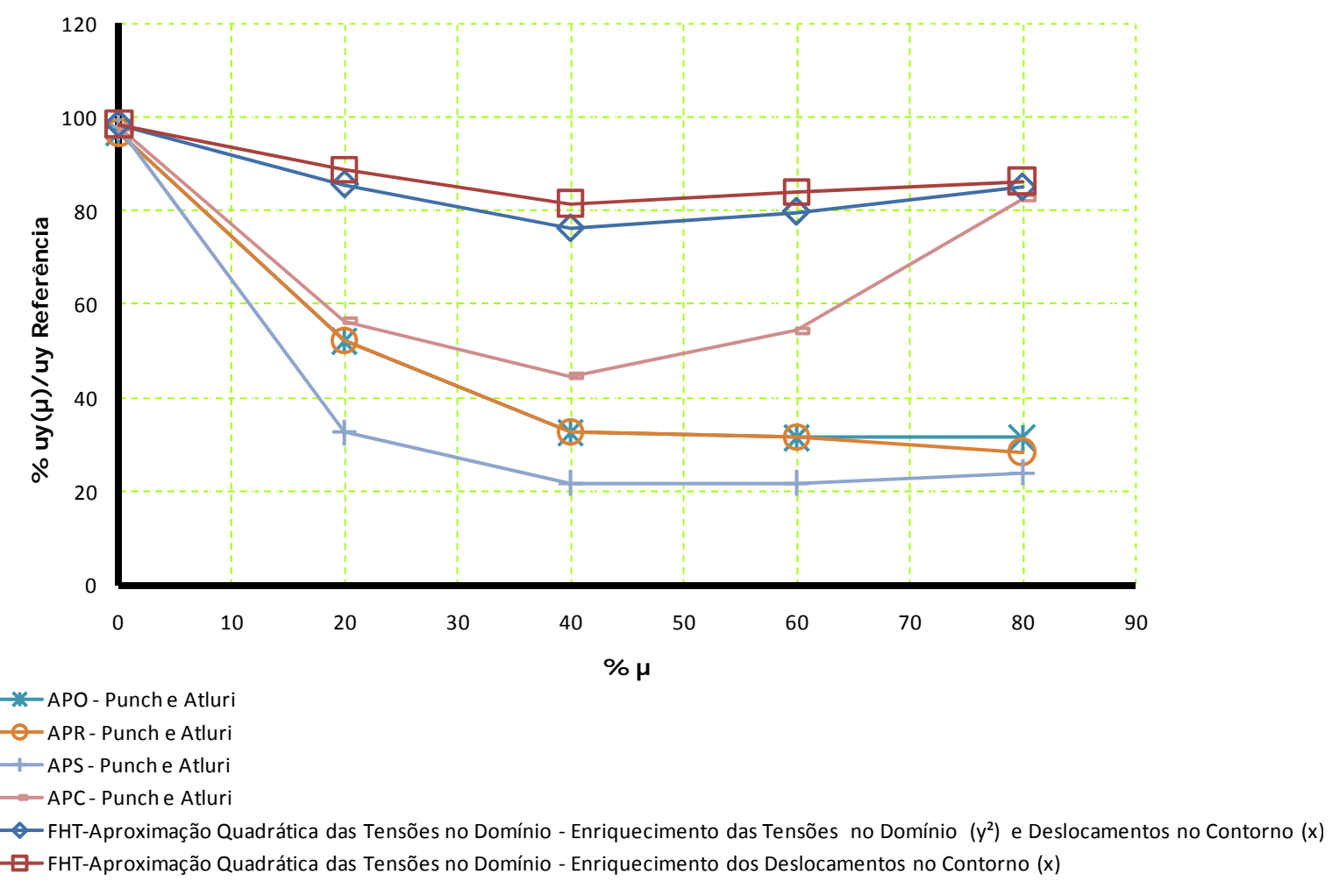

Figura 7.37 - Sensibilidade do deslocamento à distorção da rede - Elemento Quadrilateral - FHT e Punch and Atluri (1984).

O elemento quadrilateral da FHT com enriquecimento exclusivo dos deslocamentos no contorno $(x)$ ou simultâneo das tensões no domínio $\left(y^{2}\right)$ e deslocamentos no contorno $(x)$ - Aproximação Quadrática das Tensões no Domínio - possibilitou melhores resultados que os apresentados no trabalho de Punch and Atluri (1984) - ver figura 7.37. 
O elemento quadrilateral enriquecido da FHMT - tensões no domínio $\left(\boldsymbol{y}^{2}\right)$ e deslocamentos no contorno $(\boldsymbol{x})$ - é pouco sensível à distorção da rede, pois, em todos os níveis de distorção $(\boldsymbol{\mu})$ analisados, o deslocamento do nó do canto superior direito da chapa obtido foi sempre igual ao de referência, como destaca a figura 7.38. Este elemento, com as condições de enriquecimento aplicadas anteriormente, quando comparado com os elementos quadrilaterais do trabalho de Punch and Atluri (1984) é o menos sensível à distorção de rede, ver figura 7.40 .

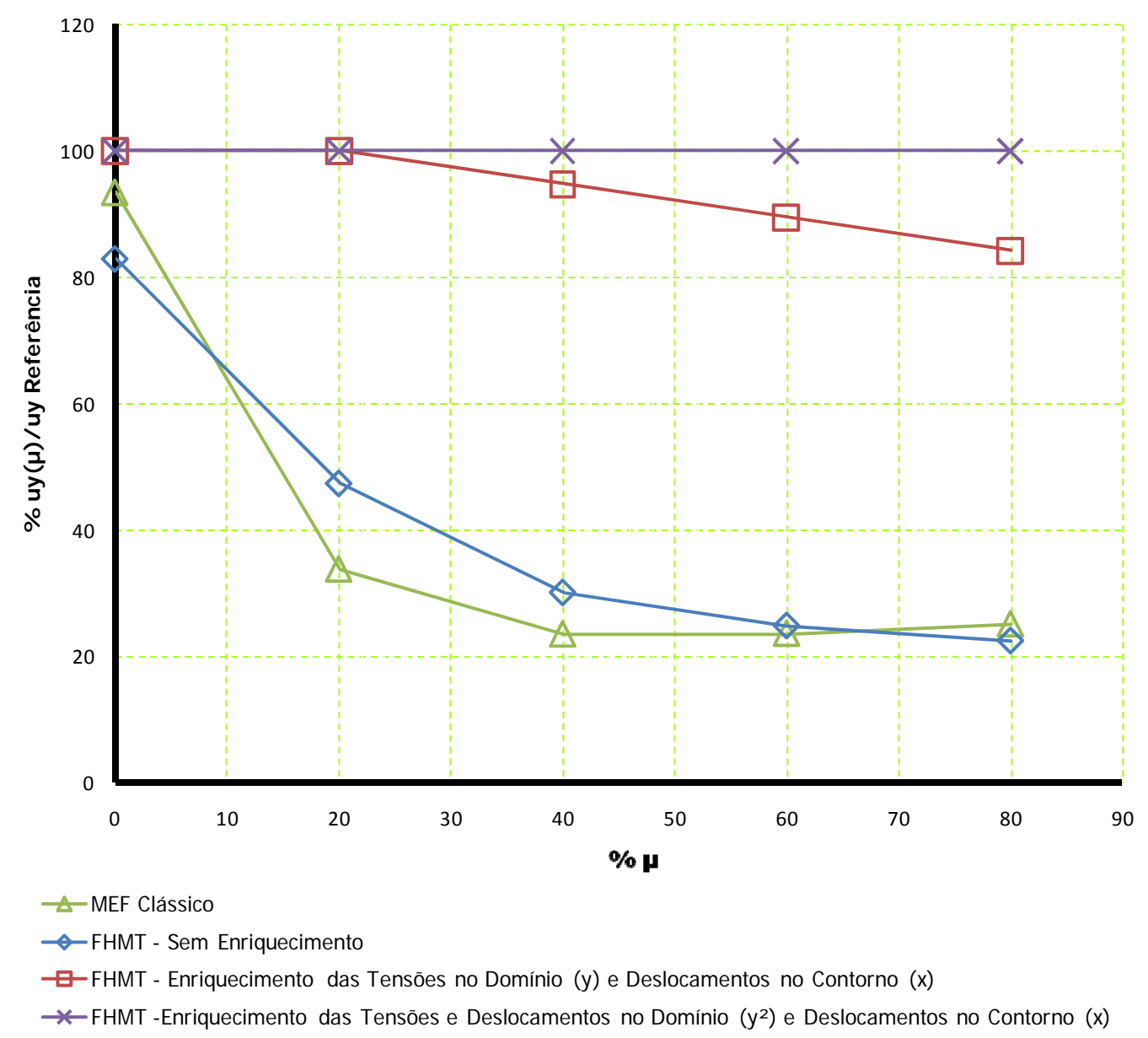

Figura 7.38 - Sensibilidade do deslocamento à distorção da rede - Elemento Quadrilateral - FHMT e MEF clássico.

Para o deslocamento do nó de referência se manter nó nível do deslocamento da figura 7.27, precisou-se enriquecer o elemento triangular da FHMT simultaneamente nas tensões no domínio $\left(y^{2}+y\right)$ ou $\left(y^{2}+x^{2}+x+y+x y\right)$ e deslocamentos no contorno $(x)$, ver figura 7.39. 


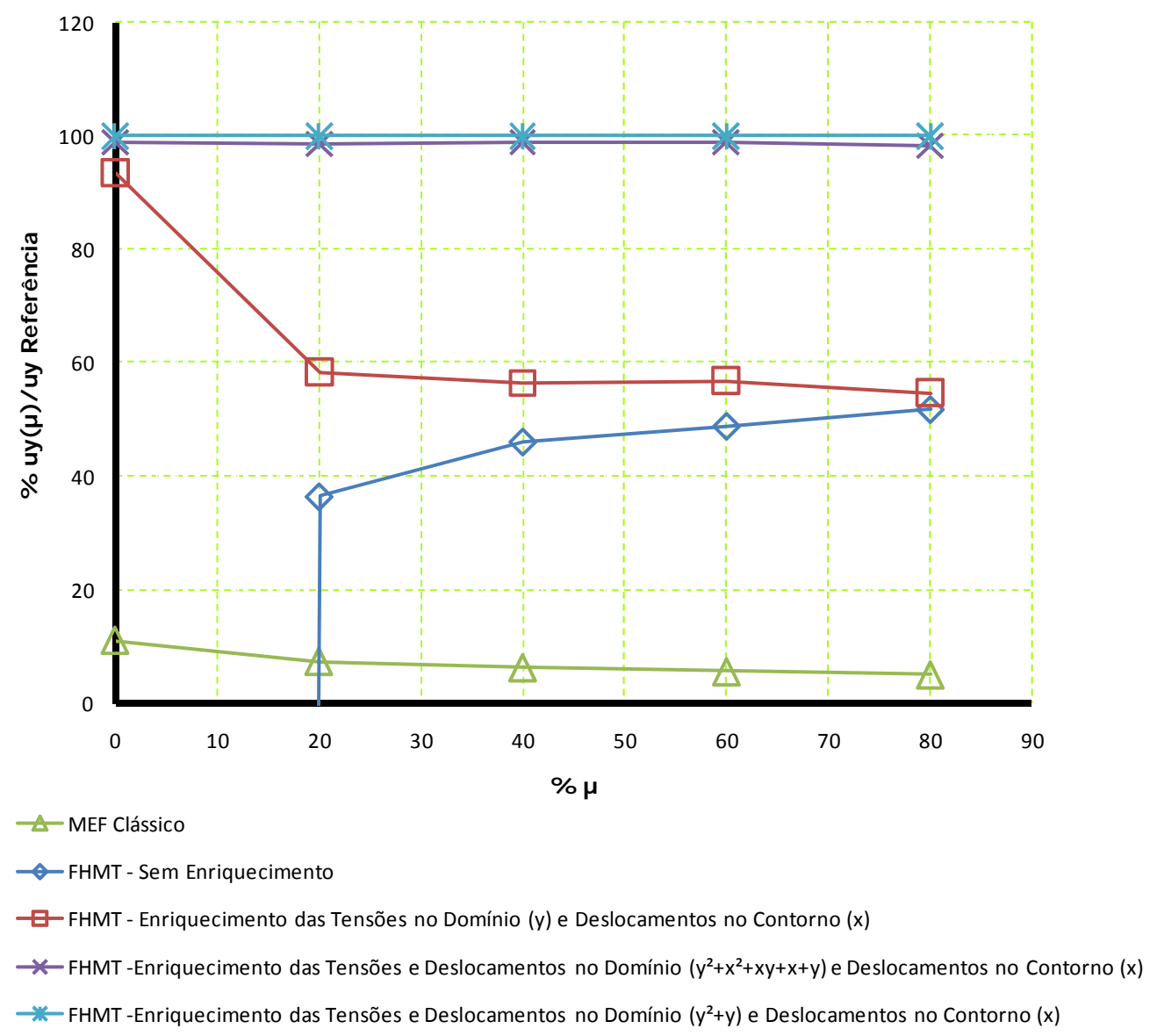

Figura 7.39 - Sensibilidade do deslocamento à distorção da rede - Elemento Triangular - FHMT e MEF clássico.

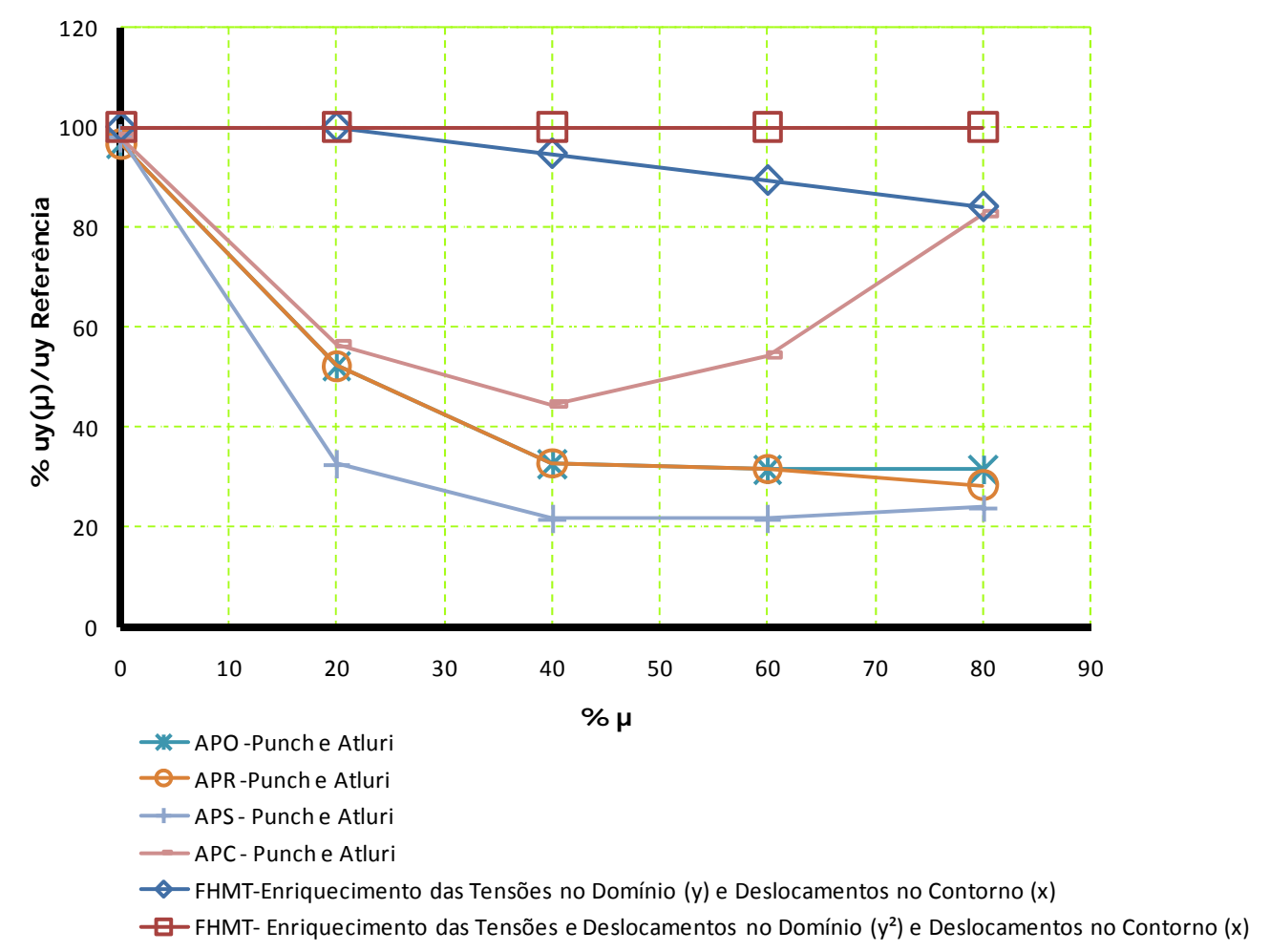

Figura 7.40 - Sensibilidade do deslocamento à distorção da rede - Elemento Quadrilateral - FHMT e Punch and Atluri (1984). 
Na figura 7.41, destaca-se à distribuição de tensão $\left(\sigma_{x}\right)$ para o Exemplo 2 analisado com o elemento quadrilateral da FHT com $\boldsymbol{\mu}=\boldsymbol{0}$ e $\boldsymbol{\mu}=\boldsymbol{0 , \boldsymbol { s }}$. Observa-se que o enriquecimento exclusivo sobre os deslocamentos no contorno possibilitou uma melhor representação da distribuição da tensão na direção $(\boldsymbol{x})$ para $\boldsymbol{\mu}=\boldsymbol{0}$. Quando este enriquecimento é aplicado para $\boldsymbol{\mu}=\boldsymbol{0 , \boldsymbol { 8 }}$, a distorção do elemento exerce ainda certa influência na distribuição do $\left(\sigma_{x}\right)$.

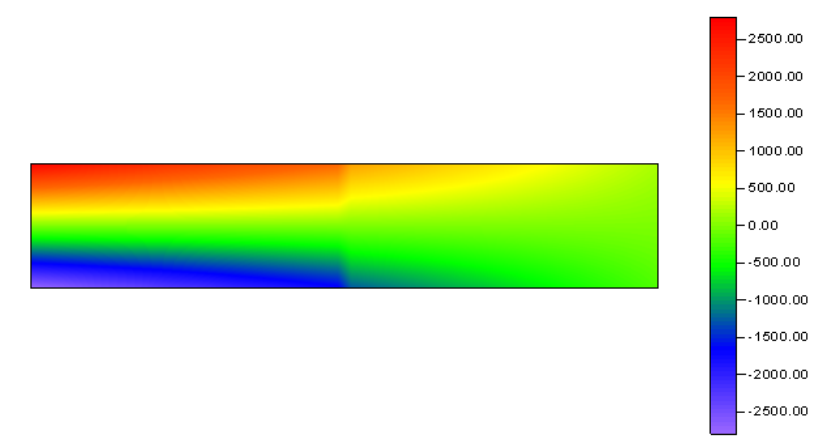

$\sigma_{x}$ Elemento Quadrilateral $(\% \mu=0) \Rightarrow$ Com Enriquecimento

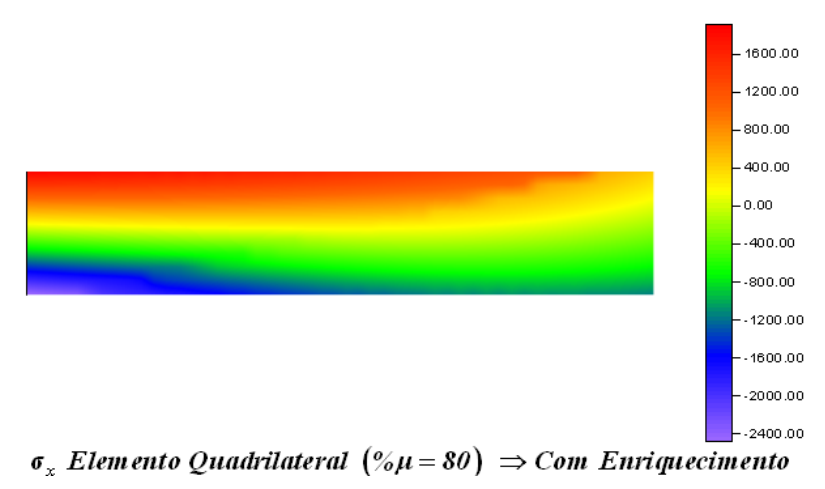

Figura 7.41 - Tensão plana - $\boldsymbol{\sigma}_{\boldsymbol{x}}$ - elemento quadrilateral com enriquecimento seletivo dos Deslocamentos no Contorno $(\boldsymbol{x})-(\boldsymbol{\%} \boldsymbol{\mu}=\boldsymbol{0})$ e $(\boldsymbol{\%} \boldsymbol{\mu}=\boldsymbol{8 0})$ - Aproximação Quadrática das Tensões - FHT.

A representação do $\left(\sigma_{x}\right)$, para o elemento triangular da FHT, mesmo com a condição de enriquecimento exclusivo dos deslocamentos no contorno $(\boldsymbol{x})$, não é satisfatória (ver figura 7.42). 


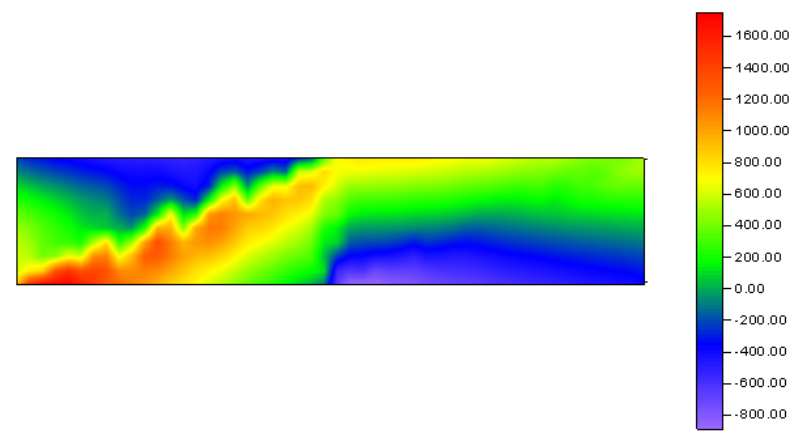

$\sigma_{x}$ Elem ento Triangular $(\% \mu=0) \Rightarrow$ Com Enriquecim ento

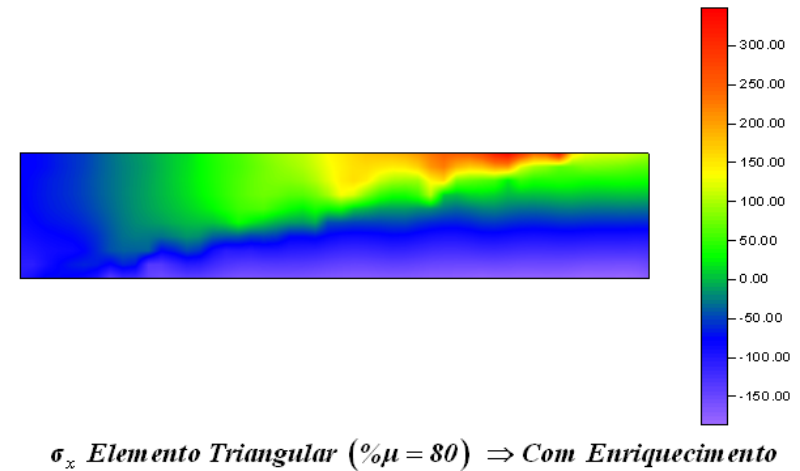

Figura 7.42 - Tensão plana - $\boldsymbol{\sigma}_{x}$ - elemento triangular com enriquecimento seletivo dos

Deslocamentos no Contorno $(\boldsymbol{x})-(\boldsymbol{\%} \boldsymbol{\mu}=\boldsymbol{8 0})$ - Aproximação Quadrática das Tensões - FHT.

Com o elemento quadrilateral da FHMT enriquecido - tensões e deslocamentos no domínio $\left(y^{2}\right)$ e deslocamentos no contorno $(x)$ - foi possível melhorar a representação da tensão plana $\left(\sigma_{x}\right)$ mesmo numa condição extrema de distorção $(\boldsymbol{\mu}=\boldsymbol{0 , 8})$, como ilustra a figura 7.43 .

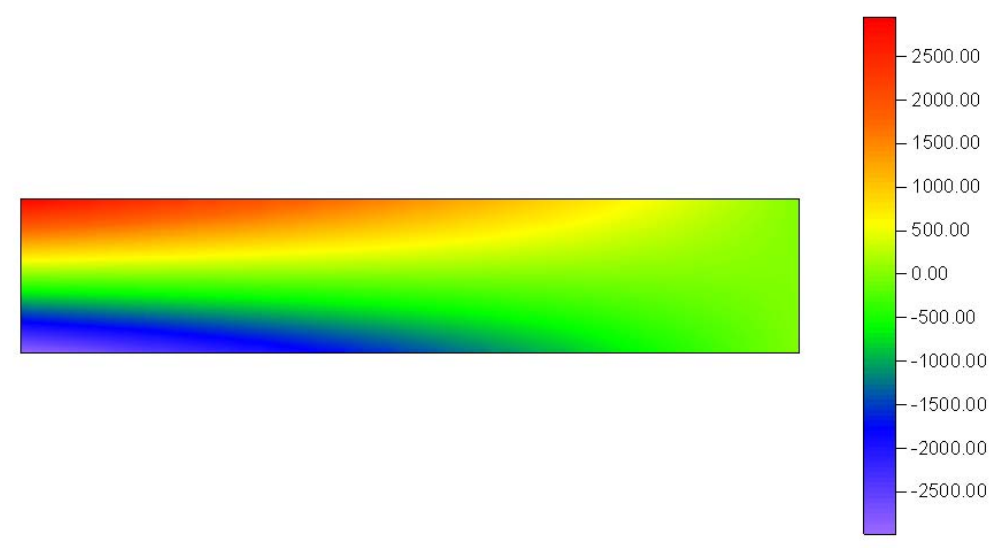

$\sigma_{x}$ Elemento Quadrilateral $(\% \mu=80) \Rightarrow$ Com Enriquecimento

Figura 7.43 - Tensão plana - $\sigma_{x}$ - elemento quadrilateral com enriquecimento das Tensões e Deslocamentos no Domínio $\left(\boldsymbol{y}^{2}\right)$ e Deslocamentos no Contorno $(\boldsymbol{x})$ $(\% \boldsymbol{\mu}=\mathbf{8 0})-$ FHMT. 
Já no elemento triangular da FHMT foi necessário aumentar o nível de enriquecimento das grandezas de domínio - tensões e deslocamentos no domínio $\left(\boldsymbol{y}+\boldsymbol{y}^{2}\right)$ e deslocamentos no contorno $(\boldsymbol{x})$ - para melhorar a representação da tensão plana $\left(\boldsymbol{\sigma}_{x}\right)$ na condição extrema de distorção $(\boldsymbol{\mu}=\boldsymbol{0}, \boldsymbol{8})$, ver figura 7.44 .

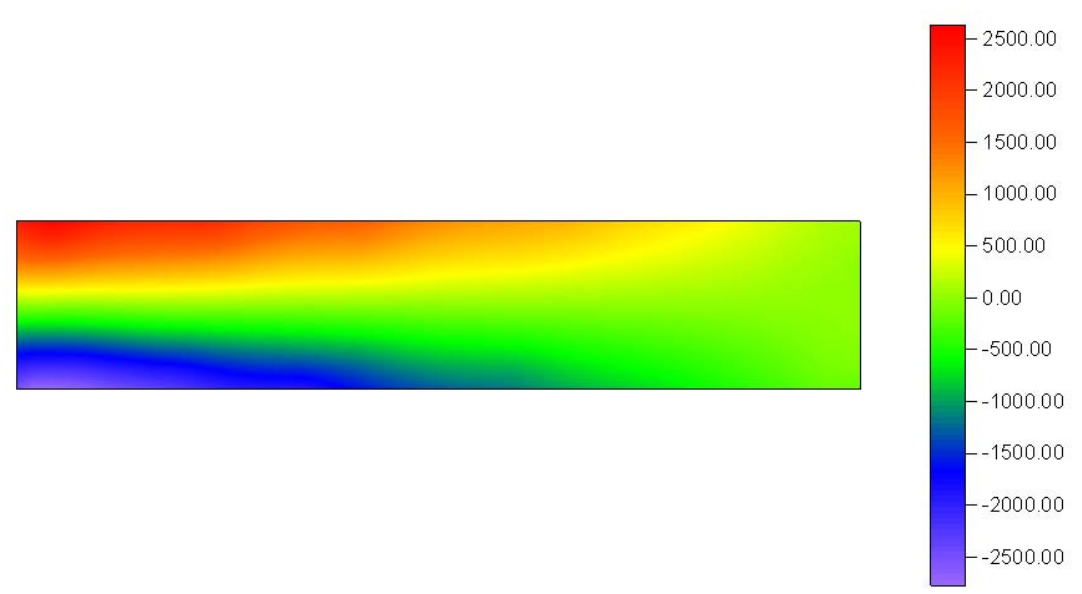

Figura 7.44 - Tensão plana - $\boldsymbol{\sigma}_{\boldsymbol{x}}$ - elemento triangular com enriquecimento das Tensões e Deslocamentos no Domínio $\left(\boldsymbol{y}+\boldsymbol{y}^{2}\right)$ e Deslocamentos no Contorno $(\boldsymbol{x})-$ $(\% \boldsymbol{\mu}=\mathbf{8 0})-$ FHMT.

As figuras 7.45 e 7.46 mostram que os elementos quadrilaterais e triangulares da FHT/FHMT são sensíveis à distorção da rede, pois à medida que se aumenta o parâmetro de distorção $(\boldsymbol{\mu})$ o valor da energia de deformação do Exemplo 2, igualmente ao deslocamento, também se distancia do valor de referência. Ainda na figura 7.46, observa-se o baixo nível de energia de deformação apresentado pelo elemento triangular da FHMT para $\boldsymbol{\mu}=\boldsymbol{0}$. 


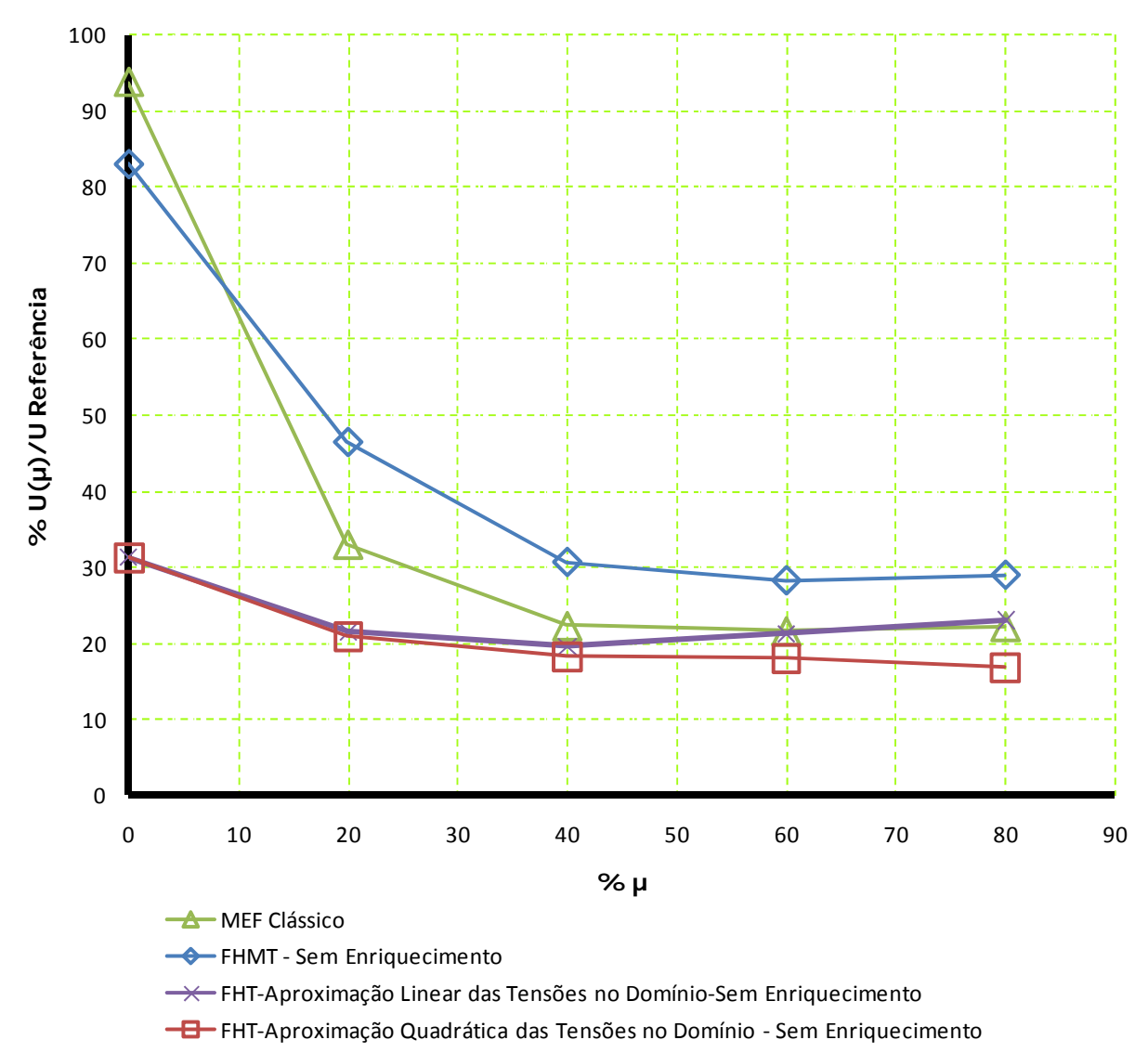

Figura 7.45 - Sensibilidade da energia de deformação à distorção da rede - Elemento Quadrilateral FHT, FHMT e MEF clássico.

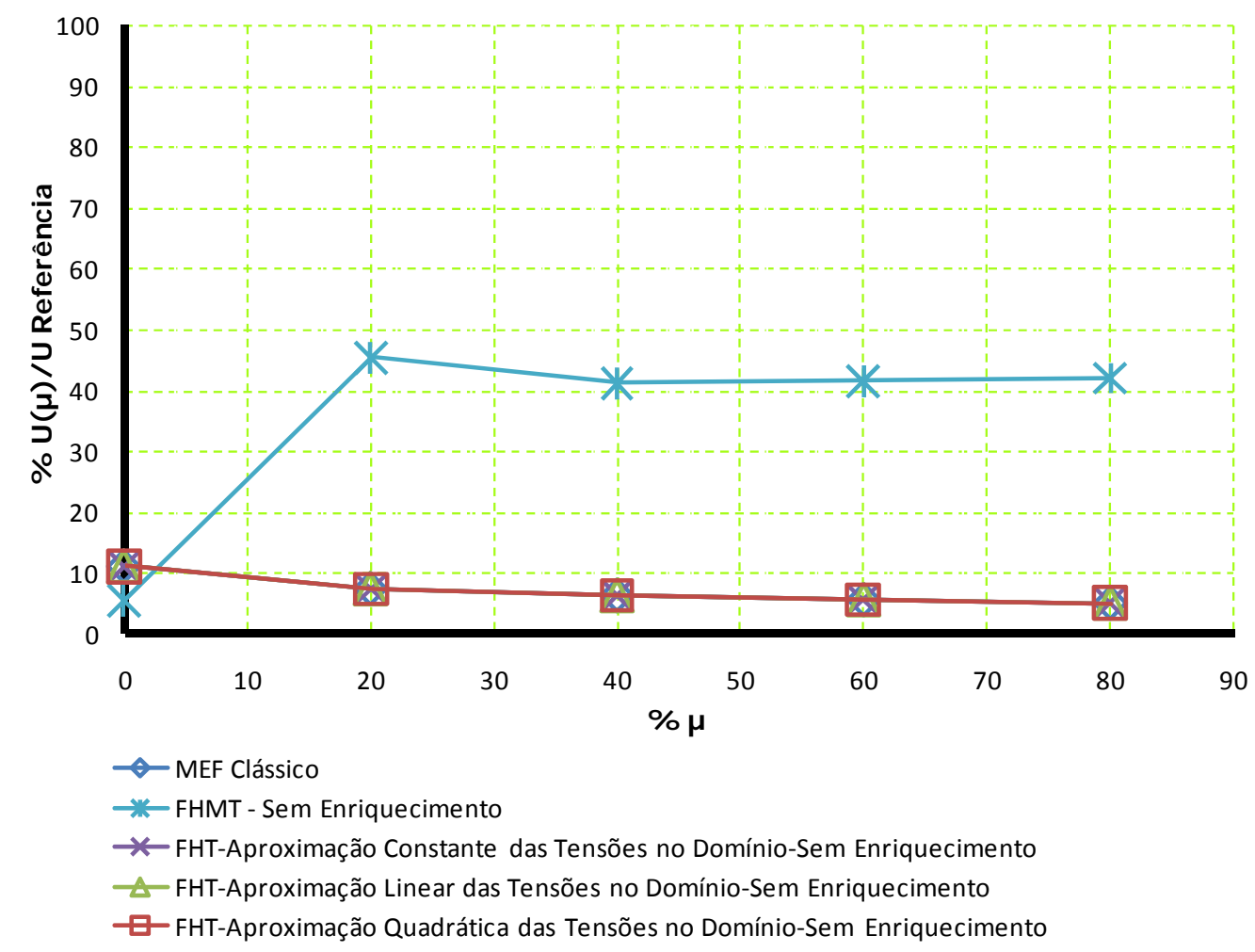

Figura 7.46 - Sensibilidade da energia de deformação à distorção da rede - Elemento Triangular FHT, FHMT e MEF clássico. 
Para a energia de deformação, o elemento quadrilateral da FHT sem enriquecimento é mais sensível à distorção da rede quando comparado com o elemento quadrilateral do MEF Clássico, como ilustra a figura 7.47. Porém, com o enriquecimento simultâneo das tensões no domínio $\left(\boldsymbol{y}^{2}\right)$ e deslocamentos no contorno $(\boldsymbol{x})$ ou exclusivamente os deslocamentos no contorno $(\boldsymbol{x})$ essa situação se inverte.

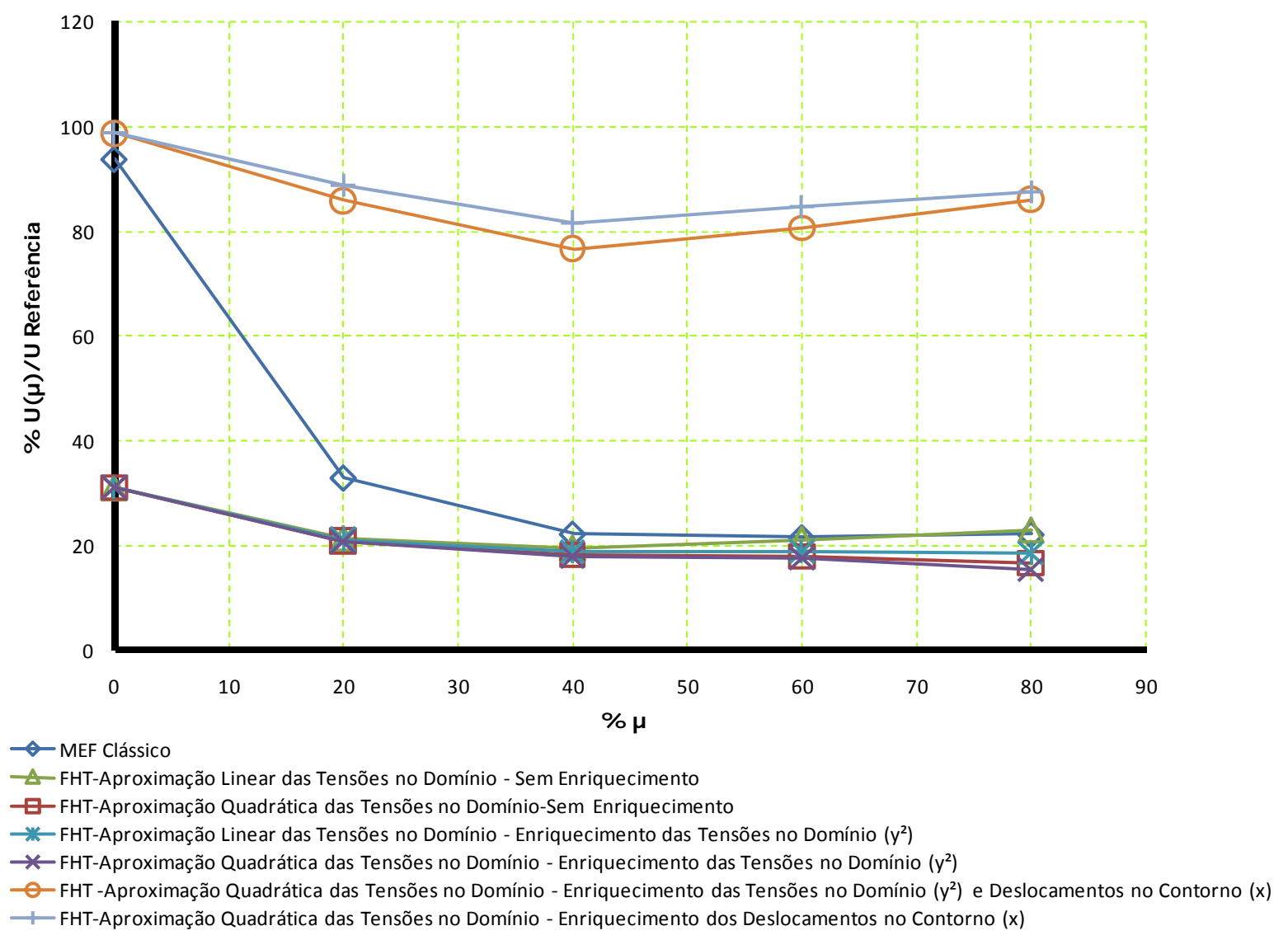

Figura 7.47 - Sensibilidade da energia de deformação à distorção da rede - Elemento Quadrilateral FHT e MEF clássico. 


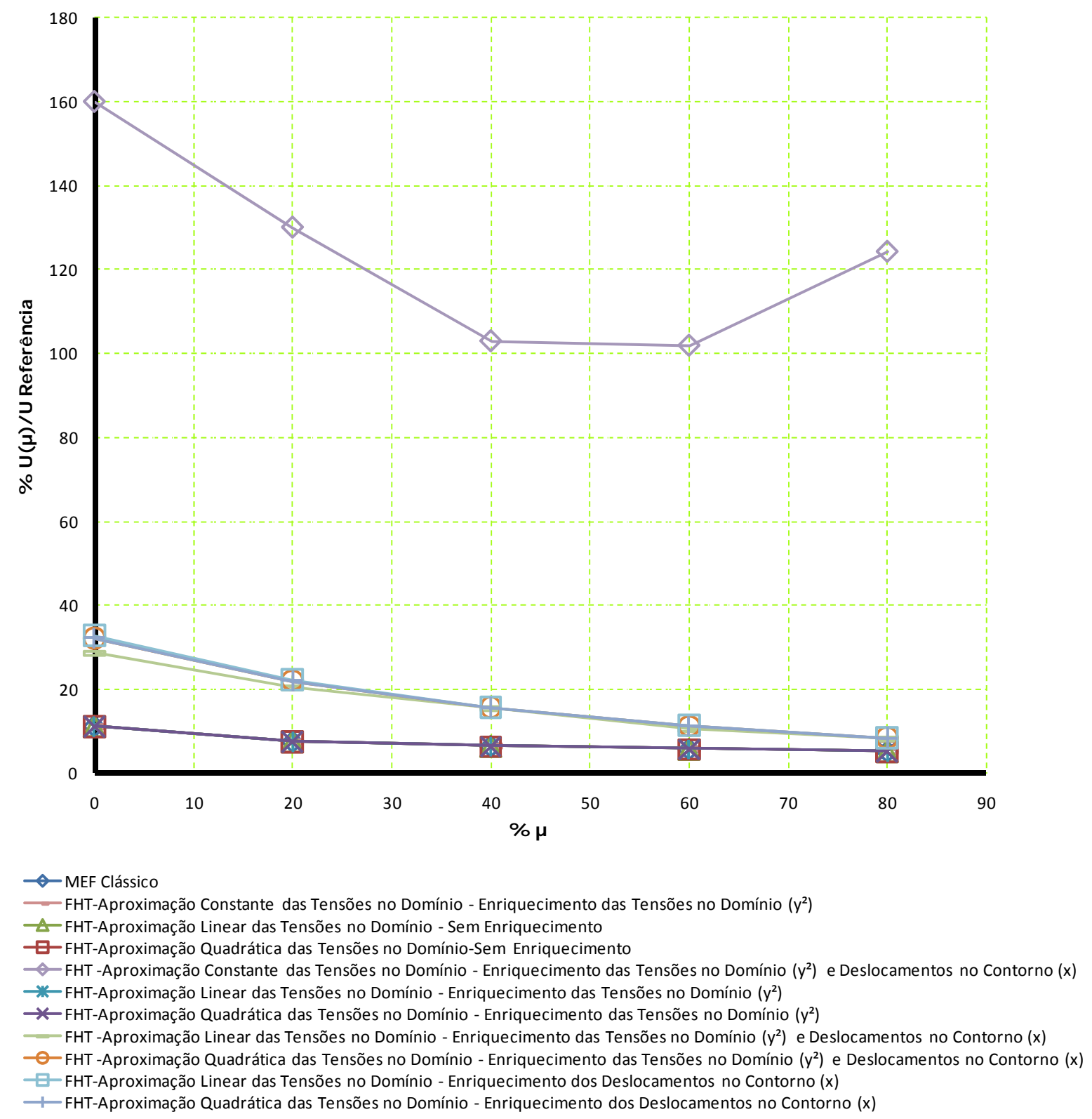

Figura 7.48 - Sensibilidade da energia de deformação à distorção da rede - Elemento TriangularFHT e MEF clássico.

A figura 7.48 confirma a forte sensibilidade do elemento triangular da FHT à distorção da rede, mas com respostas no mínimo iguais ao elemento triangular do MEF clássico. 


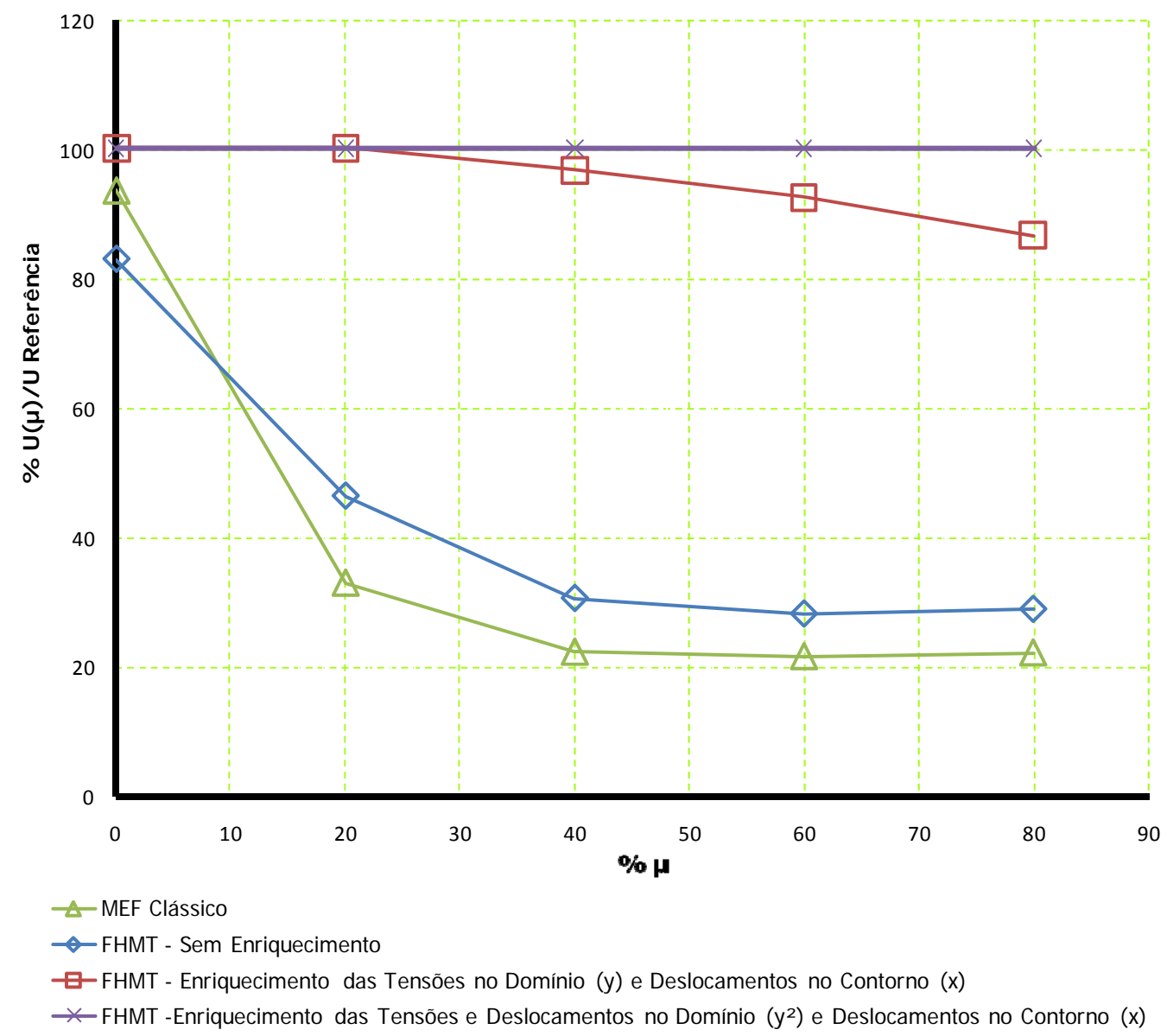

Figura 7.49 - Sensibilidade da energia de deformação à distorção da rede - Elemento Quadrilateral FHMT e MEF clássico.

Os resultados obtidos das análises do Exemplo 2 (energia de deformação) com o elemento quadrilateral da FHMT enriquecido - tensões e deslocamentos no domínio $\left(\boldsymbol{y}^{2}\right)$ e deslocamentos no contorno $(\boldsymbol{x})$ - concordam com o valor de referência e não sofrem qualquer influência com o aumento do parâmetro $(\boldsymbol{\mu})$, ver figura 7.49.

Para o elemento triangular da FHMT foi necessário aumentar o nível de enriquecimento das grandezas de domínio - tensões e deslocamentos no domínio $\left(\boldsymbol{y}+\boldsymbol{y}^{2}\right)$ e deslocamentos no contorno $(\boldsymbol{x})$ - para que o valor da energia de deformação não sofra qualquer influência com o aumento do parâmetro $(\boldsymbol{\mu})$, ver figura 7.50. 


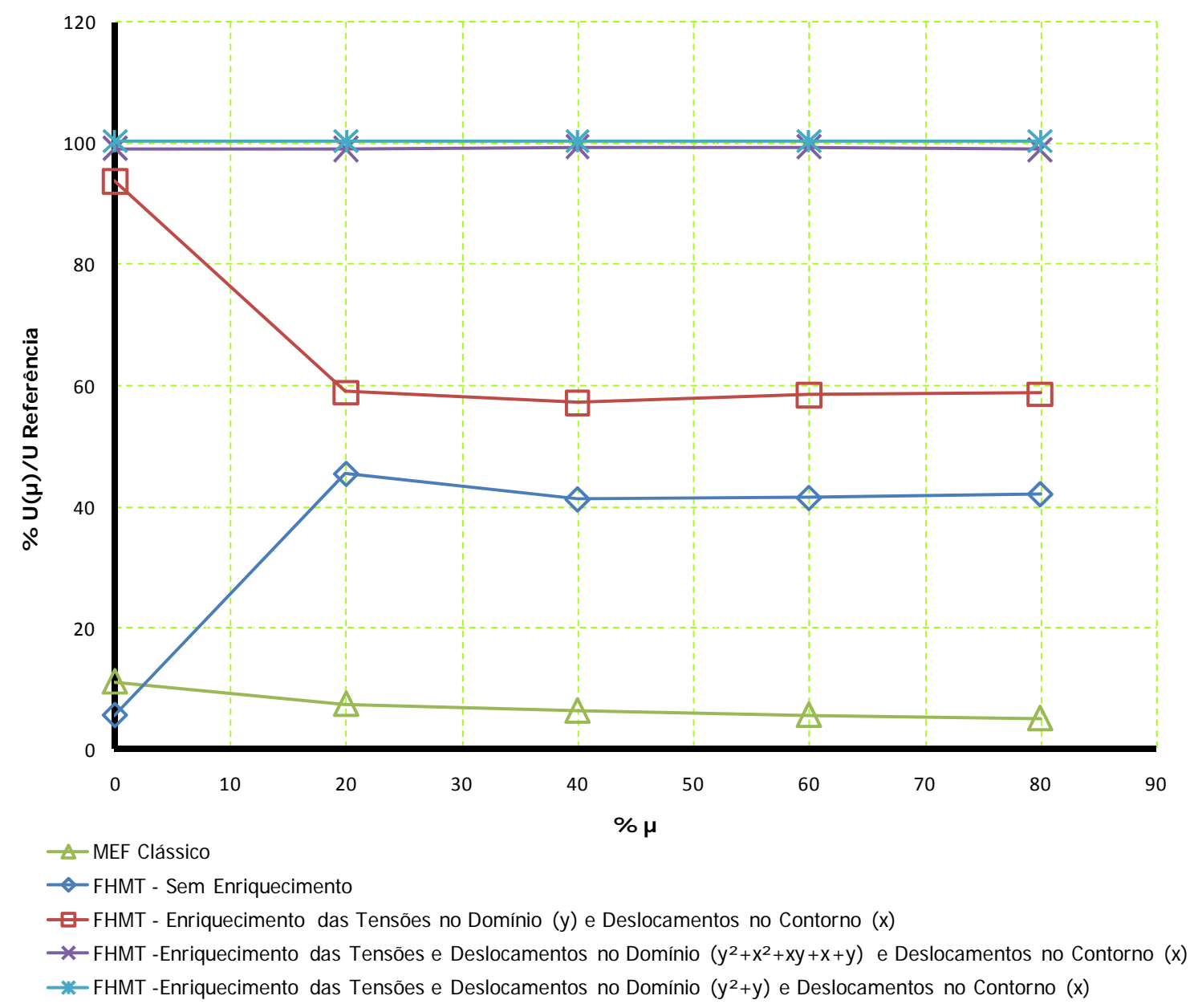

Figura 7.50 - Sensibilidade da energia de deformação à distorção da rede - Elemento Triangular FHMT e MEF clássico.

\subsubsection{Exemplo 3 - Painel de Cook}

Este exemplo, apresentado originalmente por Cook (1987), consiste em chapa de espessura unitária, fixa numa extremidade $\left(\boldsymbol{u}_{\boldsymbol{x}}=\boldsymbol{0} ; \boldsymbol{u}_{\boldsymbol{y}}=\boldsymbol{0}\right)$ e sujeita a uma carga uniformemente distribuída na outra $(\boldsymbol{q}=\mathbf{0 , 0 0 6 2 5}$ unidades de força por unidades de comprimento). As dimensões da chapa são apresentadas na figura 7.51. 


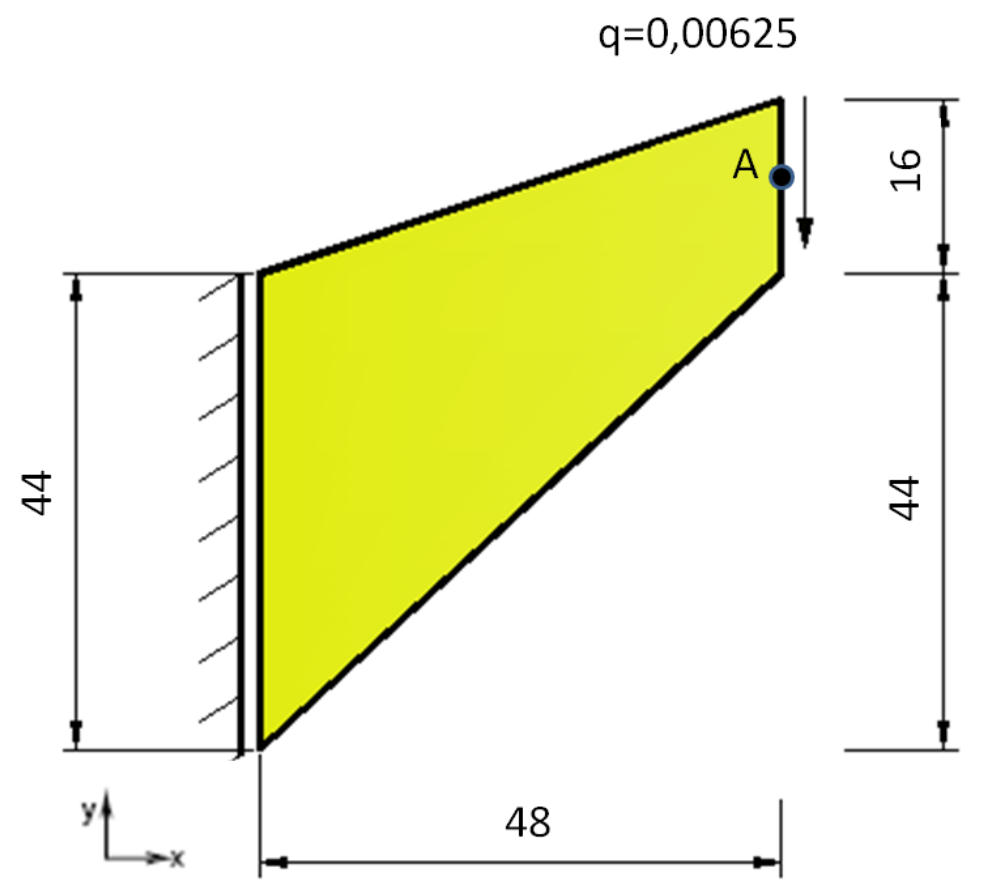

Figura 7.51 - Exemplo 3 - Painel de Cook.

Para estabelecer os resultados de referência, o Exemplo 3 foi analisado via ANSYS ${ }^{\circledR}$ com uma seqüência de redes regulares chegando a 10.000 elementos quadrilaterais PLANE 42 e totalizando 20.402,00 graus de liberdade. Assim, admitindo que o módulo de Young é $\boldsymbol{E}=10$ e o coeficiente de Poisson $v=\frac{1}{3}$, tem-se para este problema os seguintes valores de referência:

a) Energia de Deformação de referência: $U_{\text {exemplo } 3}=1,20 \cdot 10^{-2}$;

b) Deslocamento no ponto médio do lado vertical esquerdo (ponto A da figura 7.51): $u_{y}=-0,239$.

O objetivo deste problema é verificar a convergência na energia de deformação, considerando-se discretizações com elementos quadrilaterais e triangulares distorcidos da FHT e FHMT com enriquecimento nodal. Assim, propõe-se um conjunto de quatro (4) redes distorcidas, como ilustra a figura 7.52 . 


\section{Redes Quadrilaterais}
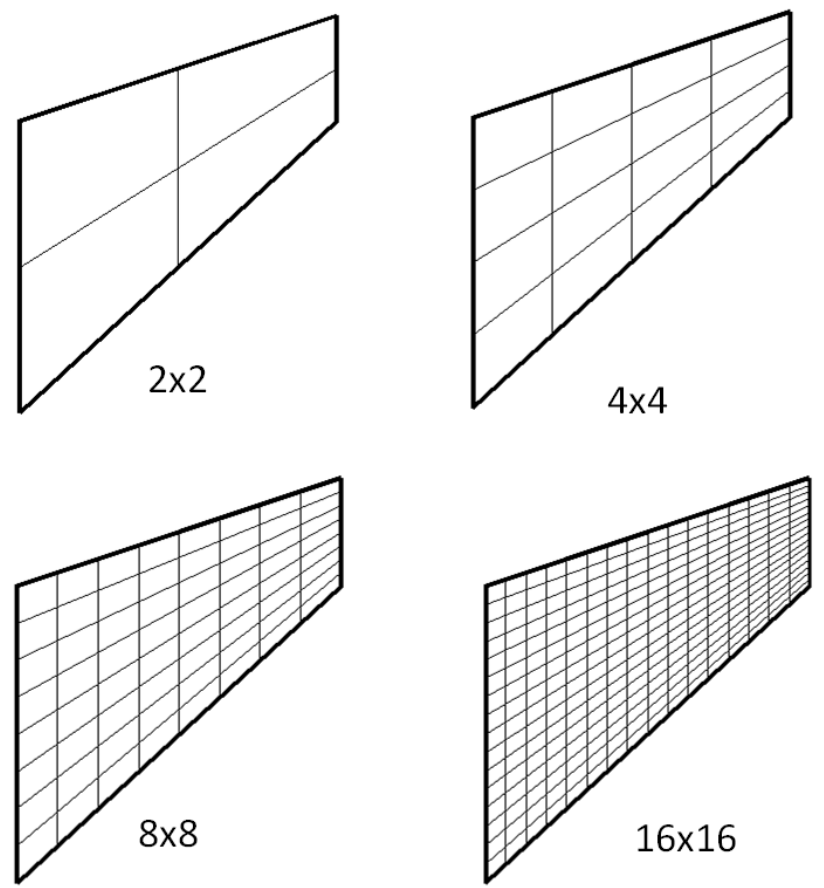

Redes Triangulares
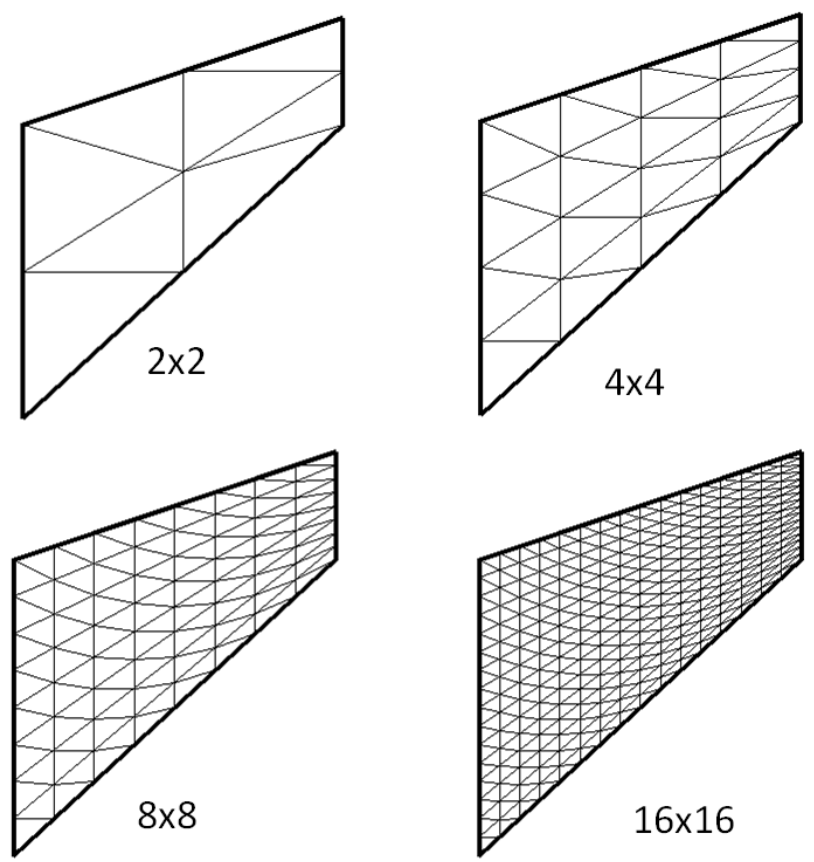

Figura 7.52 - Conjunto de redes distorcidas: Exemplo 3.

A figura 7.53 apresenta a convergência da energia de deformação para o elemento quadrilateral da FHT/FHMT sem enriquecimento e para o elemento quadrilateral do MEF clássico. Observa-se claramente que o elemento quadrilateral da FHT fornece respostas com 
tendência de convergência assintótica para o valor da energia de deformação de referência. Esta tendência, mesmo menos acentuada, existe ainda para a resposta obtida com o elemento quadrilateral da FHMT e não existe para o elemento do MEF clássico.

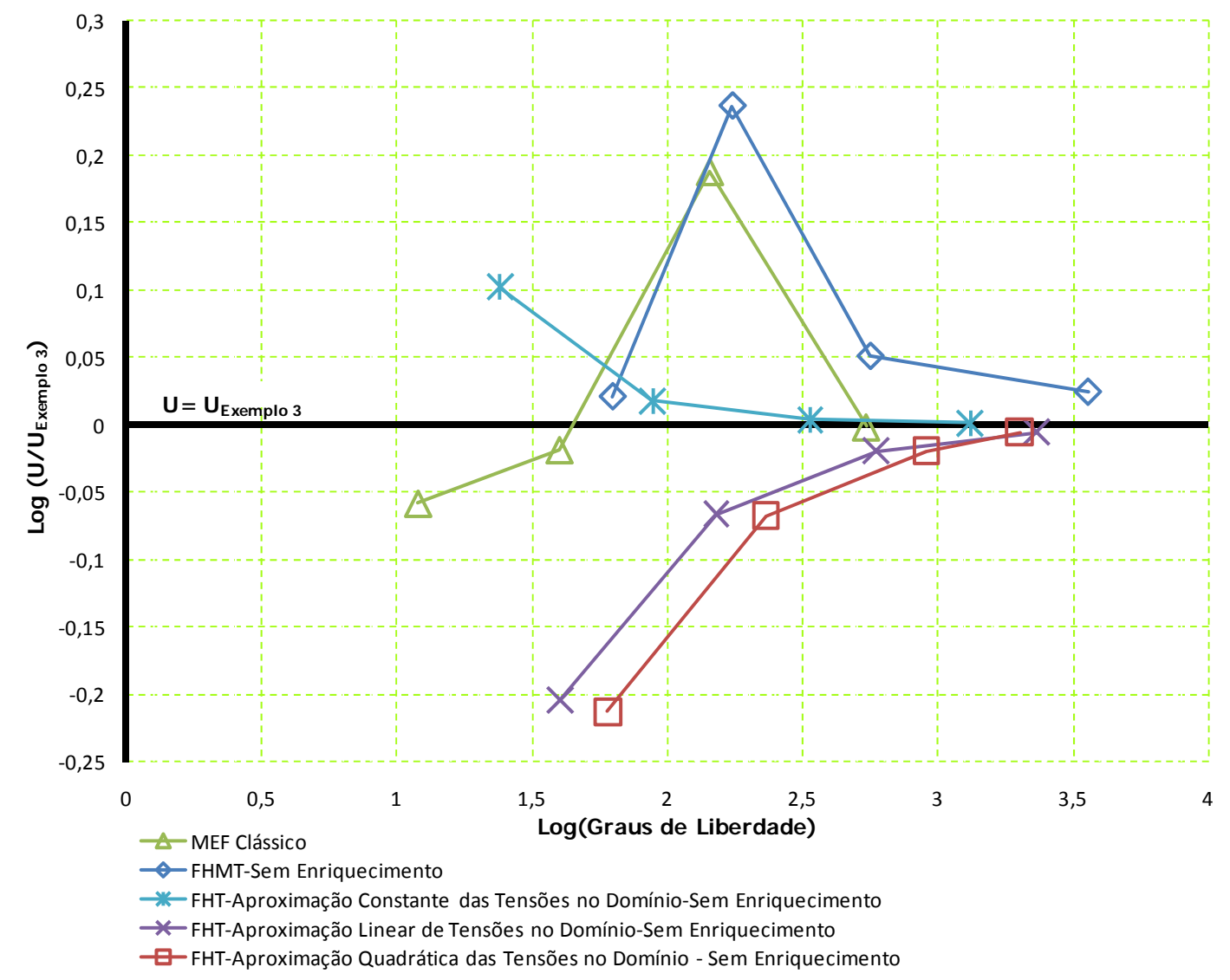

Figura 7.53 - Convergência da energia de deformação $U_{\text {exemplo } 3}$ - Elemento Quadrilateral FHT/FHMT e MEF clássico.

Nas tabelas 7.7, 7.8, 7.9 e 7.10, destacam-se o erro relativo entre o valor de referência do deslocamento no ponto A e os valores para cada uma das redes e condições de enriquecimento utilizadas na análise do Exemplo 3.

Os elementos quadrilaterais e triangulares da FHMT sem enriquecimento apresentam valores elevados para o erro relativo do deslocamento no ponto A, ver tabela 7.7. 
Tabela 7.7- Exemplo 3 - FHMT sem enriquecimento - resultados para o deslocamento no ponto A.

\begin{tabular}{cccccc}
\hline & \multicolumn{2}{c}{ Elemento Triangular } & \multicolumn{3}{c}{ Elemento Quadrilateral } \\
\hline Rede & $\begin{array}{c}\text { Deslocamento no } \\
\text { ponto A }\end{array}$ & $\begin{array}{c}\text { Erro } \\
\text { Relativo (\%) }\end{array}$ & Rede & $\begin{array}{c}\text { Deslocamento no } \\
\text { ponto A }\end{array}$ & $\begin{array}{c}\text { Erro } \\
\text { Relativo (\%) }\end{array}$ \\
\hline $2 \times 2$ & $-0,238$ & 0,42 & $2 \times 2$ & $-0,240$ & 0,42 \\
\hline $4 \times 4$ & $-0,100$ & 58,16 & $4 \times 4$ & 2,450 & $-1125,10$ \\
\hline $8 \times 8$ & $-0,090$ & 62,34 & $\mathbf{8 x 8}$ & 0,470 & $-296,65$ \\
\hline $16 \times 16$ & $-0,122$ & 48,95 & $16 \times 16$ & 0,152 & $-163,60$ \\
\hline
\end{tabular}

O elemento triangular da FHT sem enriquecimento, em todos os níveis de aproximação das tensões no domínio, recuperou a mesma resposta fornecida pelo elemento triangular do MEF clássico, como mostra a figura 7.54. O elemento triangular, em todas as formulações, apresenta tendência de convergência assintótica para o valor de referência da energia de deformação do Exemplo 3.

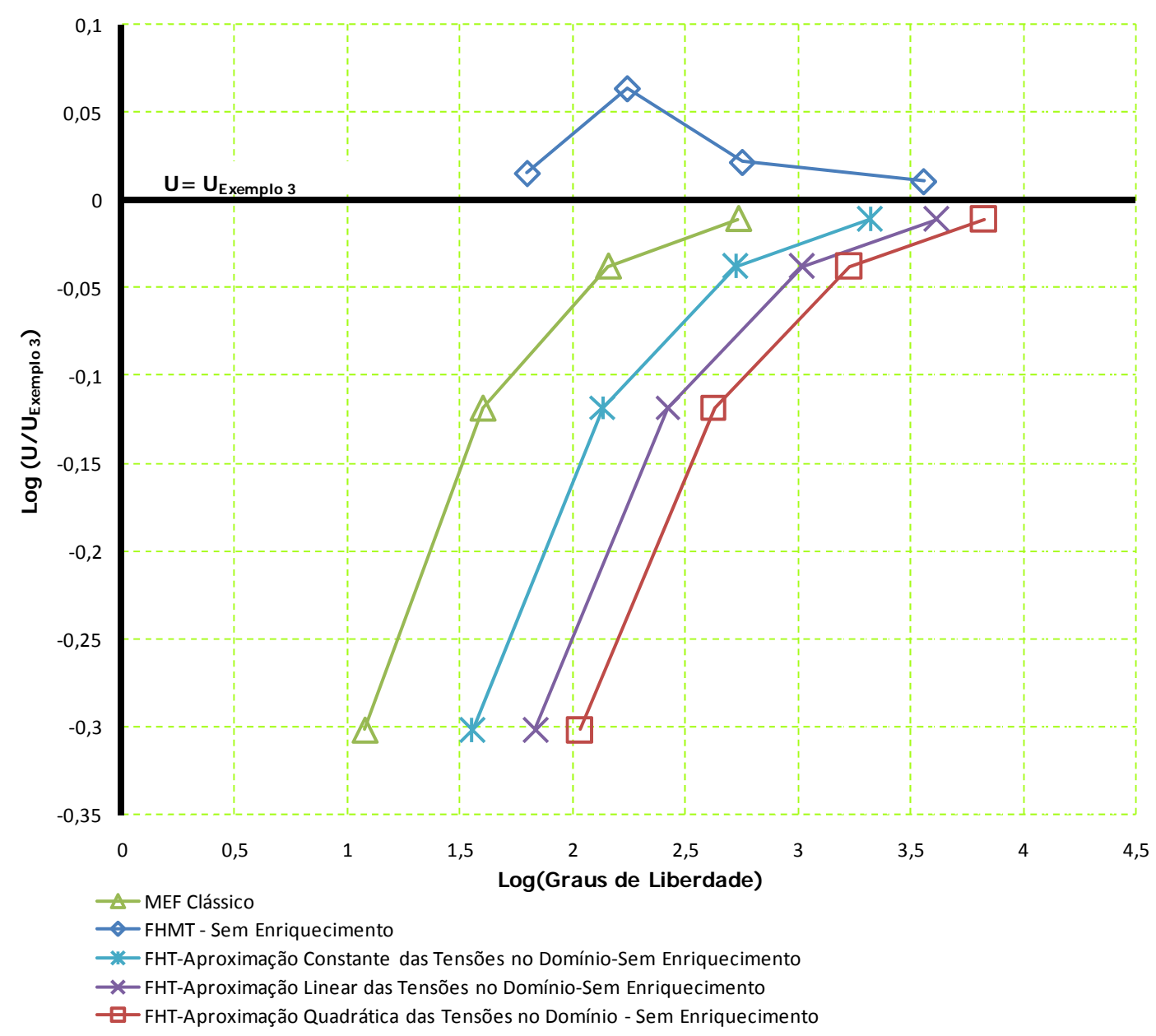

Figura 7.54 - Convergência da energia de deformação $\boldsymbol{U}_{\text {exemplo } 3}$ - Elemento Triangular - FHT/FHMT e MEF clássico.

A tabela 7.8 indica que o erro relativo do deslocamento do ponto A diminui à medida que se aumenta o grau de refinamento dos elementos da FHT sem enriquecimento. 
Tabela 7.8 - Exemplo 3 - FHT sem enriquecimento- resultados para o deslocamento no ponto A.

\section{Elemento Triangular}

Elemento Quadrilateral

\begin{tabular}{|c|c|c|c|c|c|c|}
\hline $\begin{array}{c}\text { Base de } \\
\text { Aproximação } \\
\text { das Tensões }\end{array}$ & Rede & $\begin{array}{c}\text { Deslocamento } \\
\text { no ponto A }\end{array}$ & $\begin{array}{c}\text { Erro } \\
\text { Relativo } \\
(\%)\end{array}$ & Rede & $\begin{array}{c}\text { Deslocamento no } \\
\text { ponto A }\end{array}$ & $\begin{array}{c}\text { Erro } \\
\text { Relativo } \\
(\%)\end{array}$ \\
\hline \multirow{4}{*}{ Quadrática } & $2 \times 2$ & $-0,120$ & 49,79 & $2 \times 2$ & $-0,148$ & 38,08 \\
\hline & $4 \times 4$ & $-0,183$ & 23,43 & $4 \times 4$ & $-0,206$ & 13,81 \\
\hline & $8 \times 8$ & $-0,220$ & 7,95 & $8 \times 8$ & $-0,229$ & 4,18 \\
\hline & $16 \times 16$ & $-0,234$ & 2,09 & $16 \times 16$ & $-0,237$ & 0,84 \\
\hline \multirow{4}{*}{ Linear } & $2 \times 2$ & $-0,120$ & 49,79 & $2 \times 2$ & $-0,150$ & 37,24 \\
\hline & $4 \times 4$ & $-0,183$ & 23,43 & $4 \times 4$ & $-0,206$ & 13,81 \\
\hline & $8 \times 8$ & $-0,220$ & 7,95 & $8 \times 8$ & $-0,229$ & 4,18 \\
\hline & $16 \times 16$ & $-0,234$ & 2,09 & $16 \times 16$ & $-0,237$ & 0,84 \\
\hline \multirow{4}{*}{ Constante } & $2 \times 2$ & $-0,120$ & 49,79 & $2 \times 2$ & $-0,303$ & $-26,78$ \\
\hline & $4 \times 4$ & $-0,183$ & 23,43 & $4 \times 4$ & $-0,248$ & $-3,77$ \\
\hline & $8 \times 8$ & $-0,220$ & 7,95 & $8 \times 8$ & $-0,242$ & $-1,26$ \\
\hline & $16 \times 16$ & $-0,234$ & 2,09 & $16 \times 16$ & $-0,240$ & $-0,42$ \\
\hline
\end{tabular}

O enriquecimento do elemento quadrilateral e triangular da FHMT - tensões no domínio $\left(y^{2}\right)$ e deslocamentos no contorno $(x)$ - possibilitou recuperar respostas muito próximas ao valor da energia de deformação e do deslocamento do ponto A de referência do problema, para todas as redes adotadas (ver figuras 7.55, 7.56 e tabela 7.9).

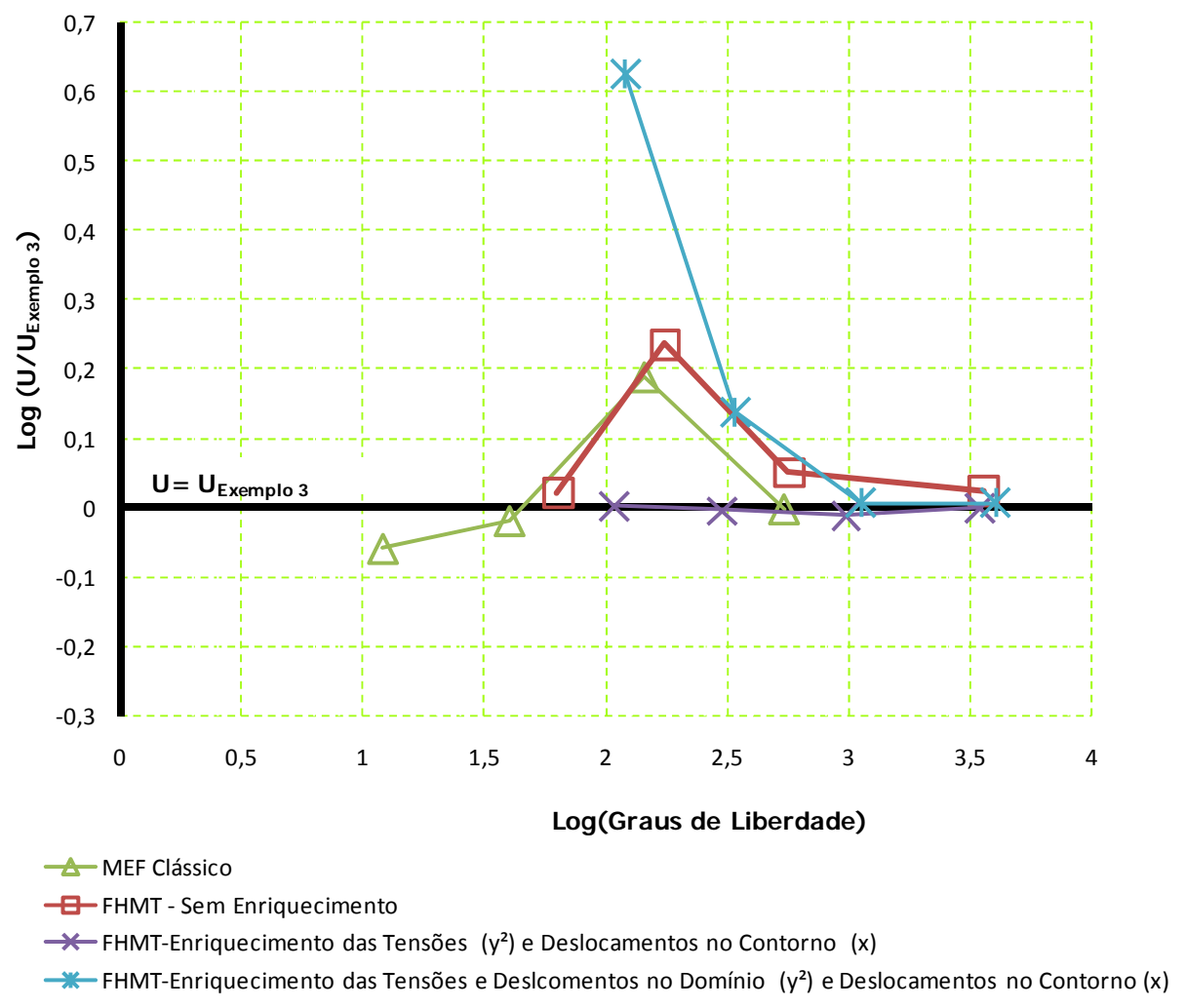

Figura 7.55 - Convergência da energia de deformação $\boldsymbol{U}_{\text {exemplo } 3}$ - Elemento Quadrilateral - FHMT e MEF clássico. 
Tabela 7.9- Exemplo 3 - FHMT com enriquecimento das tensões no domínio $\left(\boldsymbol{y}^{2}\right)$ e deslocamentos no contorno $(\boldsymbol{x})$ - resultados para o deslocamento no ponto A.

\begin{tabular}{cccccc}
\hline \multicolumn{3}{c}{ Elemento Triangular } & \multicolumn{3}{c}{ Elemento Quadrilateral } \\
\hline Rede & $\begin{array}{c}\text { Deslocamento no } \\
\text { ponto A }\end{array}$ & $\begin{array}{c}\text { Erro } \\
\text { Relativo (\%) }\end{array}$ & Rede & $\begin{array}{c}\text { Deslocamento no } \\
\text { ponto A }\end{array}$ & $\begin{array}{c}\text { Erro } \\
\text { Relativo (\%) }\end{array}$ \\
\hline $2 \times 2$ & $-0,210$ & 12,13 & $2 \times 2$ & $-0,250$ & $-4,60$ \\
\hline $4 \times 4$ & $-0,231$ & 3,35 & $4 \times 4$ & $-0,236$ & 1,26 \\
\hline $\mathbf{8 x 8}$ & $-0,238$ & 0,42 & $\mathbf{8 x 8}$ & $-0,232$ & 2,93 \\
\hline $16 \times 16$ & $-0,239$ & 0,01 & $16 \times 16$ & $-0,239$ & 0,01 \\
\hline
\end{tabular}

Vale ressaltar que o enriquecimento das grandezas de domínio em ambas as formulações é realizada na totalidade dos nós das redes da figura 7.52. Já o enriquecimento dos deslocamentos no contorno foi realizado em todos os nós, excluindo-se aqueles com condições de contorno essenciais aplicadas.

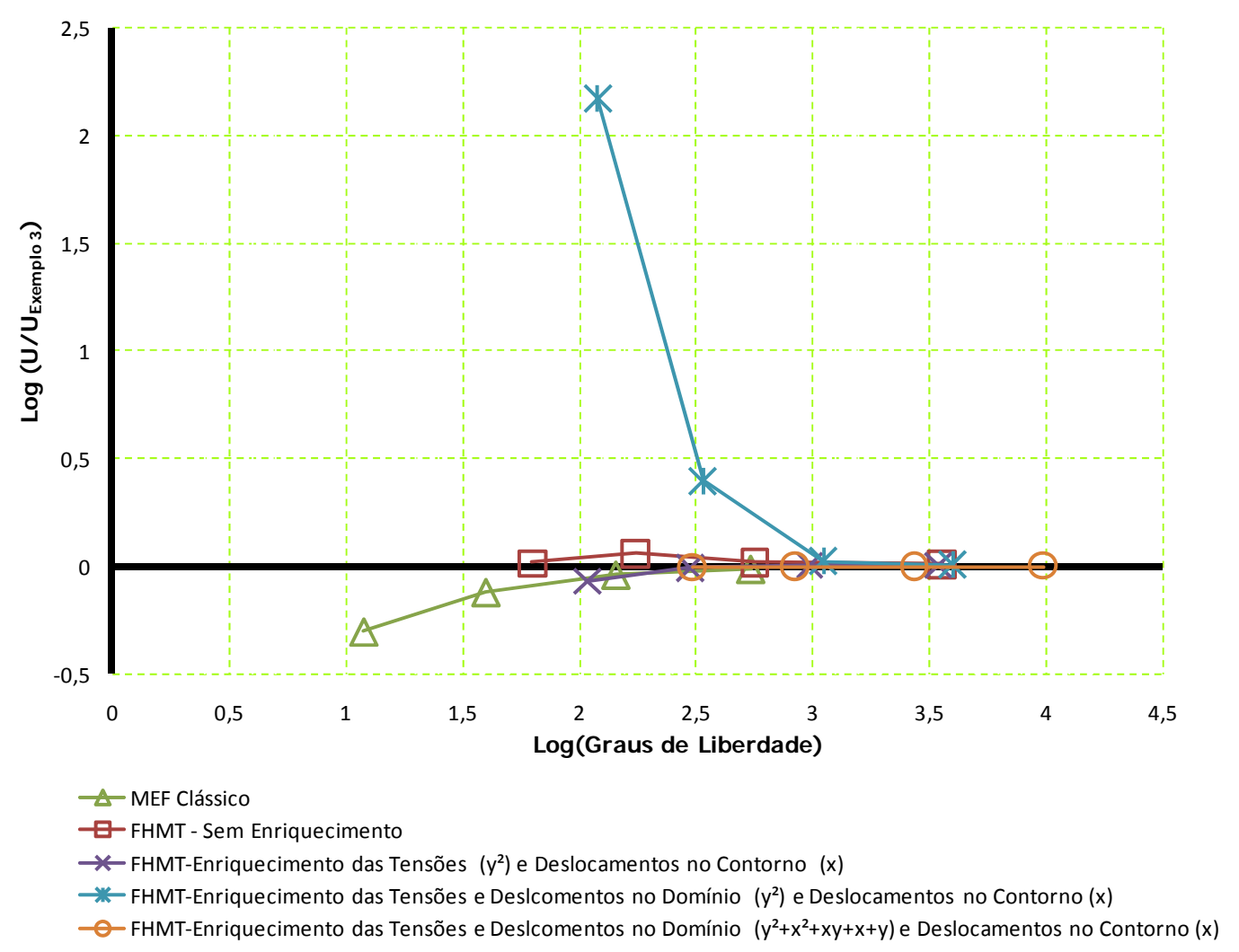

Figura 7.56 - Convergência da energia de deformação $\boldsymbol{U}_{\text {exemplo } 3}$ - Elemento Triangular - FHMT e MEF clássico.

A figura 7.56 mostra que o enriquecimento simultâneo das grandezas de domínio $\left(y^{2}+x^{2}+y+x+x y\right)$ e do deslocamento no contorno $(x)$ do elemento triangular da FHMT permite, mesmo com o menor grau de refinamento utilizado para análise do Exemplo 3, obter a energia de referência do problema. 


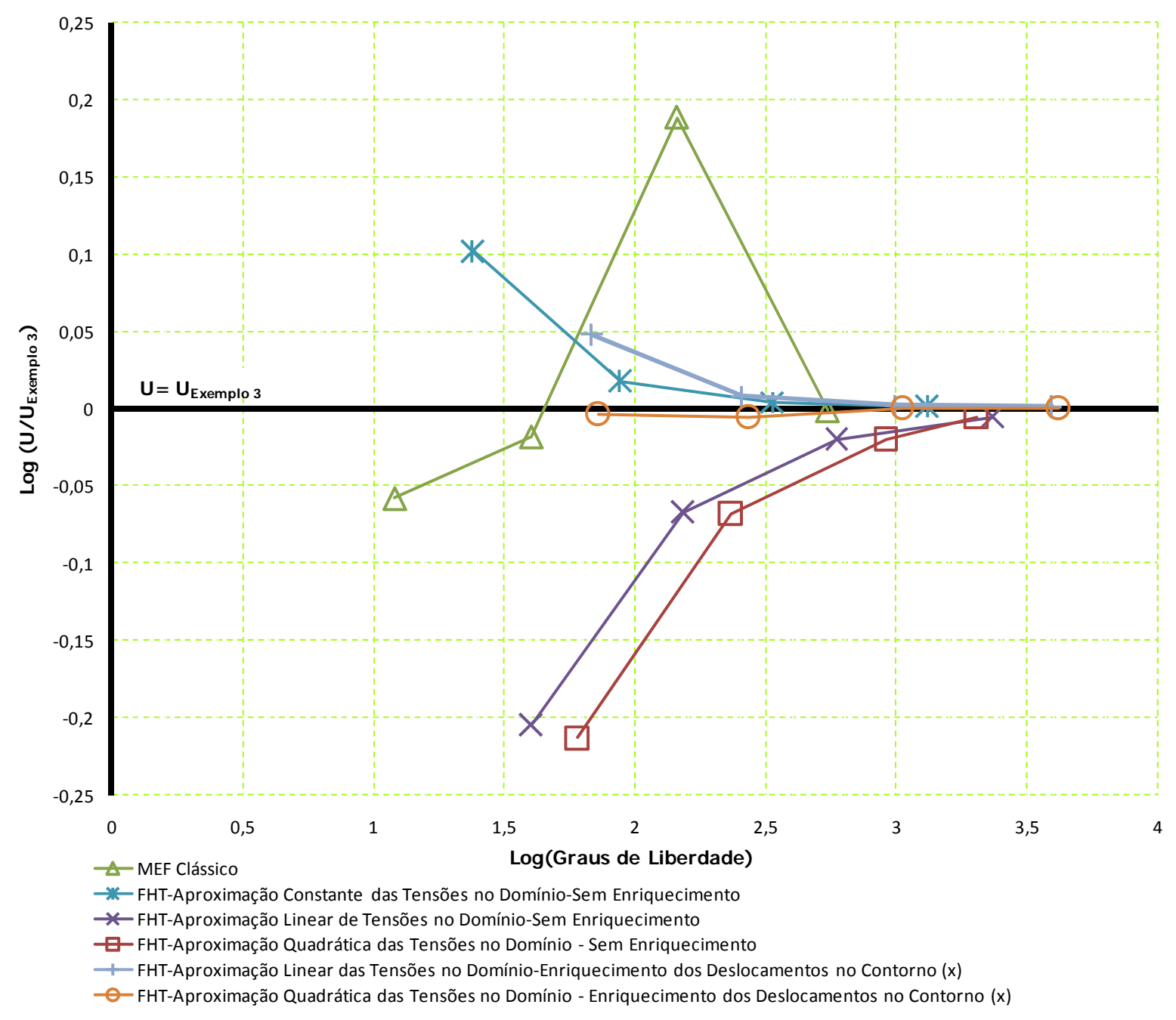

Figura 7.57 - Convergência da energia de deformação $\boldsymbol{U}_{\text {exemplo } 3}$ - Elemento Quadrilateral - FHT e MEF clássico.

Tabela 7.10 - Exemplo 3 - FHT com enriquecimento dos deslocamentos no contorno $(\boldsymbol{x})$ resultados para o deslocamento no ponto A.

\begin{tabular}{|c|c|c|c|c|c|c|}
\hline & \multicolumn{3}{|c|}{ Elemento Triangular } & \multicolumn{3}{|c|}{ Elemento Quadrilateral } \\
\hline $\begin{array}{c}\text { Base de } \\
\text { Aproximação } \\
\text { das Tensões }\end{array}$ & Rede & $\begin{array}{c}\text { Deslocamento } \\
\text { no ponto A }\end{array}$ & $\begin{array}{c}\text { Erro } \\
\text { Relativo } \\
(\%)\end{array}$ & Rede & $\begin{array}{c}\text { Deslocamento no } \\
\text { ponto A }\end{array}$ & $\begin{array}{c}\text { Erro } \\
\text { Relativo } \\
(\%)\end{array}$ \\
\hline \multirow{4}{*}{ Quadrática } & $2 \times 2$ & $-0,173$ & 27,61 & $2 \times 2$ & $-0,239$ & 0,00 \\
\hline & $4 \times 4$ & $-0,218$ & 8,79 & $4 \times 4$ & $-0,238$ & 0,42 \\
\hline & $8 \times 8$ & $-0,233$ & 2,51 & $8 \times 8$ & $-0,240$ & $-0,42$ \\
\hline & $16 \times 16$ & $-0,238$ & 0,42 & $16 \times 16$ & $-0,240$ & $-0,42$ \\
\hline \multirow{4}{*}{ Linear } & $2 \times 2$ & $-0,179$ & 25,10 & $2 \times 2$ & $-0,270$ & $-12,97$ \\
\hline & $4 \times 4$ & $-0,220$ & 7,95 & $4 \times 4$ & $-0,232$ & 2,93 \\
\hline & $8 \times 8$ & $-0,234$ & 2,09 & $8 \times 8$ & $-0,240$ & $-0,42$ \\
\hline & $16 \times 16$ & $-0,238$ & 0,42 & $16 \times 16$ & $-0,240$ & $-0,42$ \\
\hline
\end{tabular}

Com o enriquecimento dos deslocamentos no contorno $(\boldsymbol{x})$ para o elemento quadrilateral e triangular da FHT (aproximação quadrática e linear das tensões) é possível 
recuperar o valor do deslocamento de referência do ponto A, com o refinamento da rede, ver tabela 7.10. Entretanto, só o elemento quadrilateral juntamente com o enriquecimento dos deslocamentos no contorno $(\boldsymbol{x})$ foi possível obter o valor de referência da energia de deformação com a rede menos refinada usada na análise do Exemplo 3, como ilustram as figuras 7.57 e 7.58 .

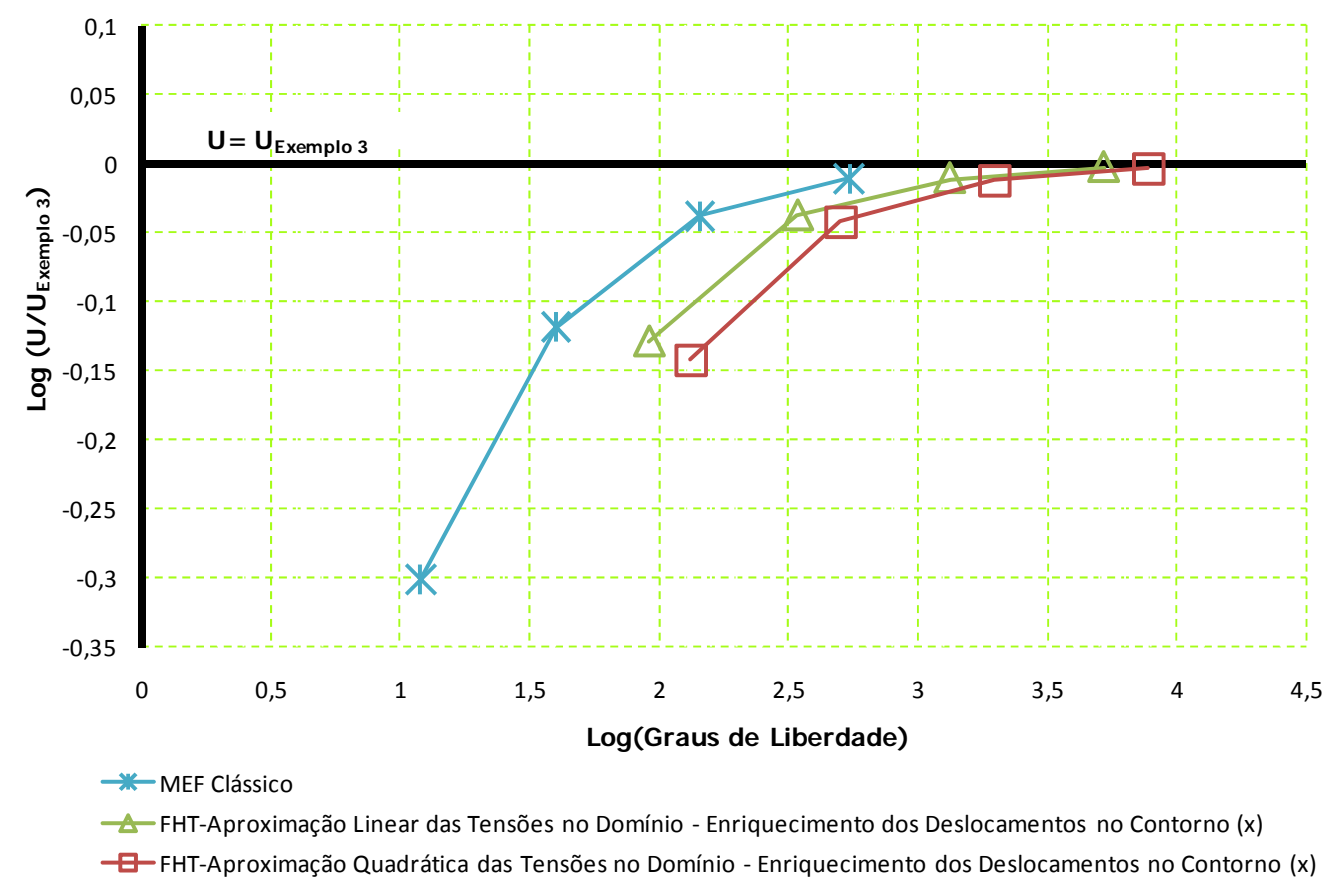

Figura 7.58 - Convergência da energia de deformação $\boldsymbol{U}_{\text {exemplo } 3}$ - Elemento Triangular - FHT e MEF clássico.

Das figuras 7.54 e 7.58, evidencia-se fortemente que o elemento triangular da FHT esteja sujeito ao princípio da limitação. Este princípio indica que os resultados obtidos para certa formulação não-convencional é idêntica a obtida com a formulação clássica do MEF, Zienkiewicz (2000). 


\subsection{O 'Teste por Inspeção’ Aplicado à FHT/FHMT com Enriquecimento Nodal}

\subsubsection{Considerações Iniciais}

Sabe-se que o 'Teste por Inspeção’ está ligado à estabilidade da FHT/FHMT com enriquecimento nodal e, como já citado, é somente condição necessária para garantia de solvabilidade do problema.

Basicamente o teste consiste em verificar se o vetor incógnita do sistema de equações lineares, resultante das discretizações dos problemas propostos, satisfaz as eq.(6.10) a eq.(6.12). Nos trabalhos de Góis e Proença (2005, 2006a, 2006b, 2007a, 2007b e 2007c) esse tema foi amplamente abordado.

É importante observar que o 'Teste por Inspeção’ foi aplicado em todos os exemplos anteriores associados à distorção da rede para selecionar as condições de enriquecimento que não violassem o mesmo. Agora, estende-se a aplicação do teste para dois problemas planos que essencialmente diferem entre si pela regularidade esperada da solução, conforme se explica mais adiante.

O primeiro é uma chapa quadrada com a borda vertical esquerda engastada $\left(\boldsymbol{u}_{\boldsymbol{x}}=\boldsymbol{0}, \boldsymbol{u}_{\boldsymbol{y}}=\boldsymbol{0}\right)$ e bordas horizontais com deslocamentos verticais nulos $\left(\boldsymbol{u}_{\boldsymbol{y}}=\boldsymbol{0}\right)$, ver figura 7.59 .

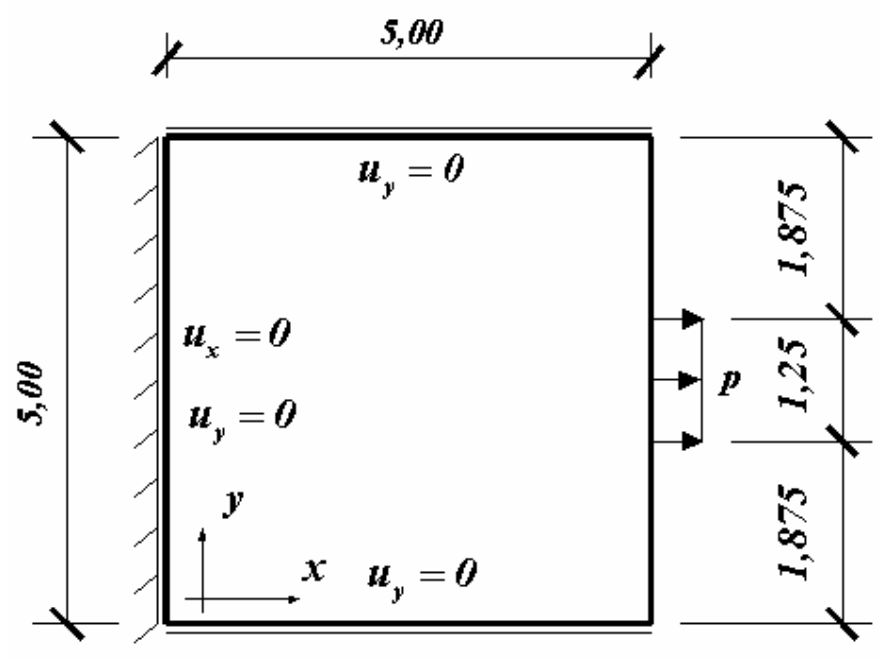

Figura 7.59 - Chapa tracionada.

O segundo problema é uma chapa retangular com fenda central, como mostra a figura 7.60. Devido à dupla simetria do problema, para efeitos do estudo do 'Teste por Inspeção', será analisado apenas $(1 / 4)$ desta chapa (figura 7.61). 


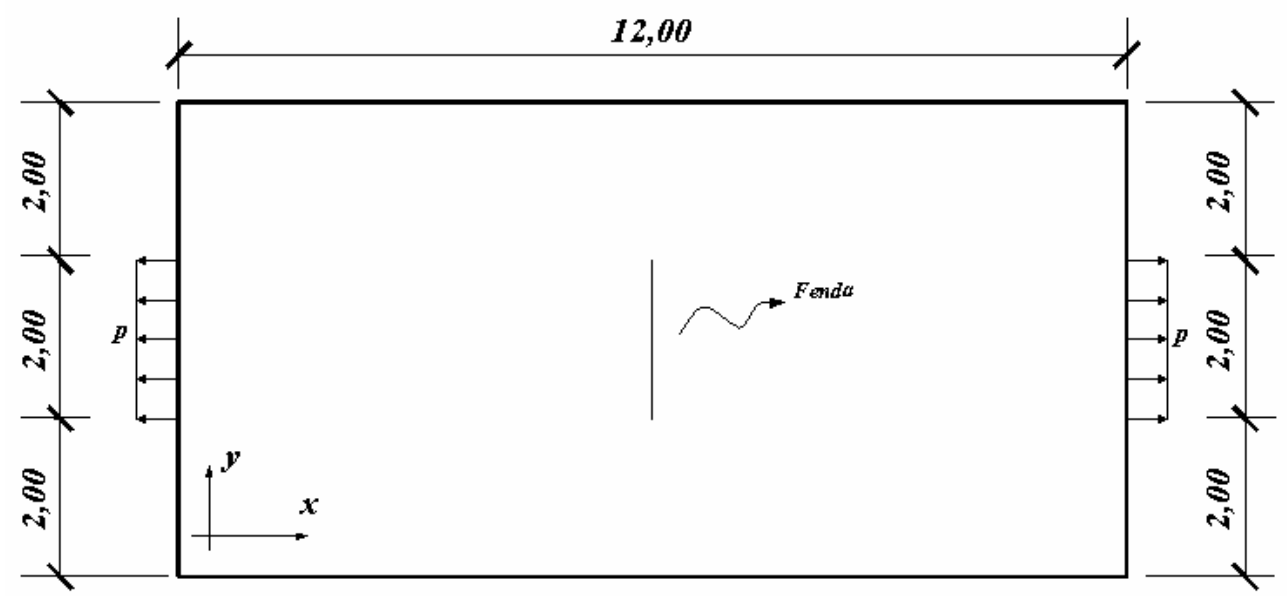

Figura 7.60 - Chapa tracionada com fenda central.

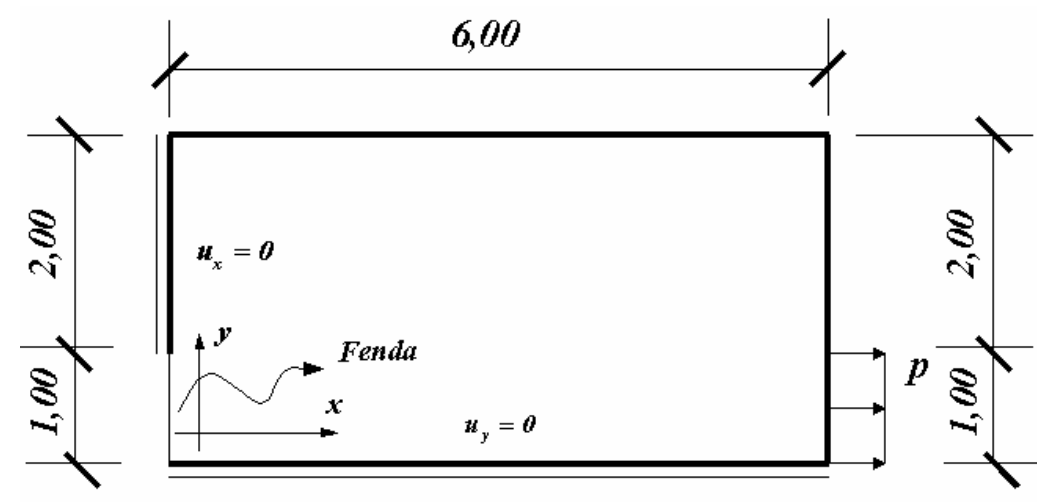

Figura 7.61 - Simetria da chapa tracionada com fenda central.

Nos dois exemplos propostos, adota-se $\boldsymbol{E}=\mathbf{1 0 0 0}$ para o módulo de Young, $\boldsymbol{v}=\mathbf{0 , 3}$ para o coeficiente de Poisson e ainda regime de comportamento elástico-linear. Ambas as chapas são tracionadas por $\boldsymbol{p}=\mathbf{1 0}$ unidades de força distribuída por unidade de comprimento.

As chapas planas das figuras 7.59 e 7.61 foram escolhidas para a aplicação do 'Teste por Inspeção’, por apresentarem, dentro da classe de problemas elástico-lineares planos, características bem distintas: a primeira com distribuição de tensões bastante regular, esperando-se, apenas, uma maior concentração de tensões próximo da aplicação do carregamento (simulação numérica simplificada); já a chapa da figura 7.61, além da concentração de tensões próxima à força aplicada, apresenta também um ponto de singularidade forte na extremidade da fenda (simulação numérica complexa).

Avalia-se o ‘Teste por Inspeção’ analisando-se a representação dos campos de tensões e deslocamentos dos problemas propostos, além da convergência da energia de deformação. 


\subsubsection{Chapa Tracionada}

Para a avaliação do 'Teste por Inspeção’ aplicado ao exemplo da figura 7.59, utilizouse o conjunto de redes regulares apresentado nas figuras 7.62 e 7.63.
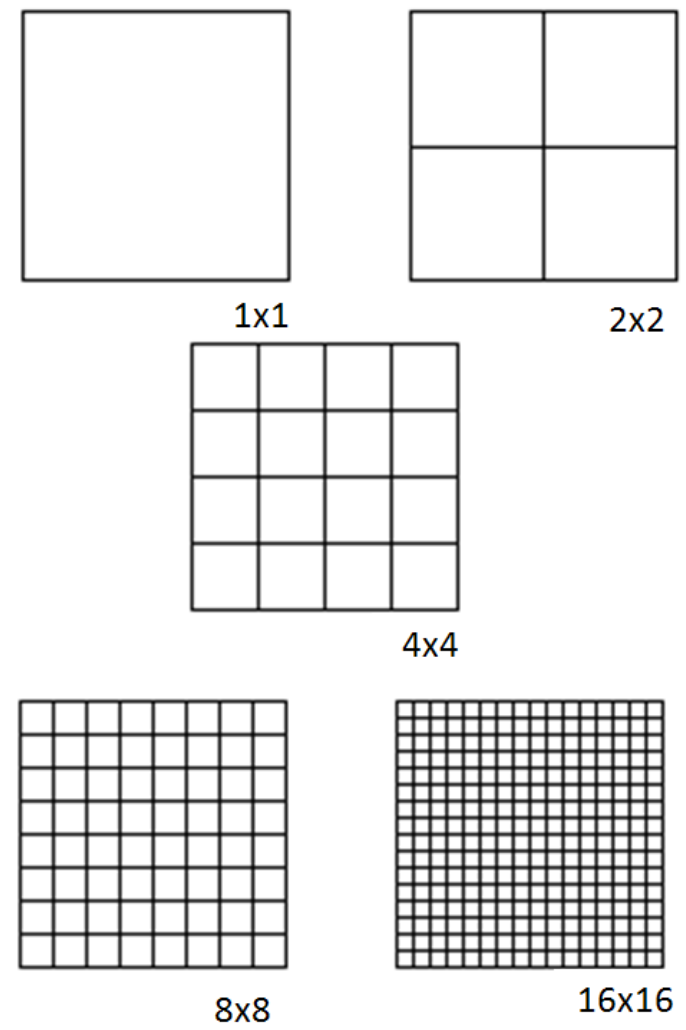

Figura 7.62 - Redes quadrilaterais regulares - chapa tracionada. 


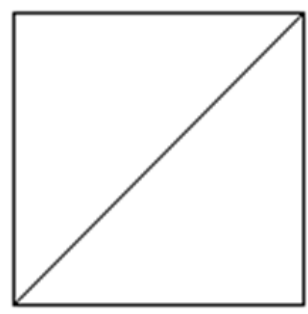

$1 \times 1$

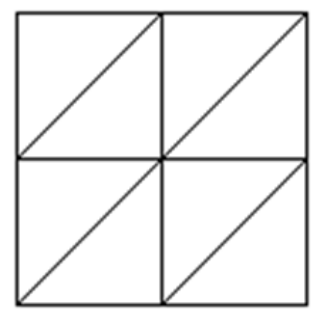

$2 \times 2$

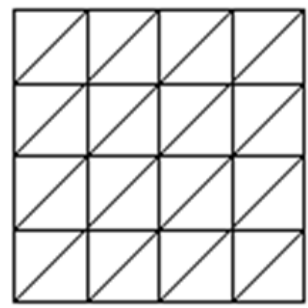

$4 \times 4$

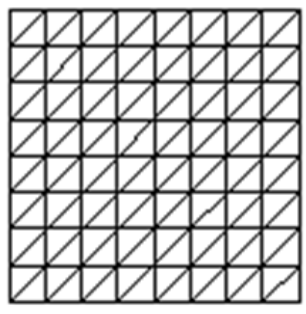

$8 \times 8$

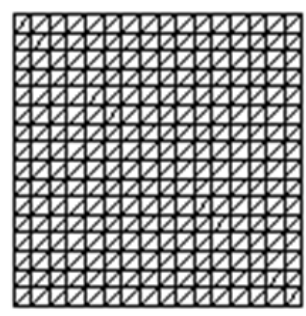

$16 \times 16$

Figura 7.63 - Redes triangulares regulares - chapa tracionada.

Nas tabelas a seguir estão indicados os números de graus de liberdade considerando-se cada uma das redes adotadas e as possibilidades de enriquecimento polinomial sobre os campos envolvidos na FHT/FHMT. No caso específico de enriquecimento dos campos da FHMT e do campo de deslocamento no contorno da FHT foi utilizado o primeiro nível de enriquecimento polinomial para construção das tabelas.

Para leitura dos resultados tabulados, considera-se a seguinte legenda:

- $\sigma$ : aproximação do campo de tensão no domínio do elemento da FHT ou FHMT;

- $\boldsymbol{u}_{\Omega}$ : aproximação do campo de deslocamento no domínio do elemento da FHMT;

- $\boldsymbol{u}_{\boldsymbol{\Gamma}}$ : aproximação do campo de deslocamento no contorno do elemento da FHT ou FHMT;

- $\boldsymbol{s}_{\boldsymbol{\Omega}}$ : vetor que guarda os parâmetros de tensão no domínio do elemento com ou sem enriquecimento - FHT ou FHMT;

- $\boldsymbol{q}_{\Omega}$ : vetor que guarda os graus de liberdade de deslocamento no domínio do elemento e ainda os possíveis parâmetros nodais enriquecidos - FHMT;

- $\boldsymbol{q}_{\Gamma}$ : vetor que guarda os graus de liberdade de deslocamento no contorno do elemento e ainda os possíveis parâmetros nodais enriquecidos - FHT ou FHMT; 
Tabela 7.11 - Graus de liberdade para a chapa tracionada - elemento quadrilateral - FHMT.

\begin{tabular}{|c|c|c|c|c|c|c|c|c|c|c|}
\hline \multirow{3}{*}{$\begin{array}{c}\text { Condições } \\
\text { de }\end{array}$} & \multicolumn{10}{|c|}{ Redes } \\
\hline & \multicolumn{2}{|c|}{$(1 \times 1)$} & \multicolumn{2}{|c|}{$(2 \times 2)$} & \multicolumn{2}{|c|}{$(4 \times 4)$} & \multicolumn{2}{|c|}{$(8 \times 8)$} & \multicolumn{2}{|c|}{$(16 \times 16)$} \\
\hline & $s_{\Omega}$ & $q_{\Omega}+q_{\Gamma}$ & $s_{\Omega}$ & $q_{\Omega}+q_{\Gamma}$ & $s_{\Omega}$ & $q_{\Omega}+q_{\Gamma}$ & $s_{\Omega}$ & $q_{\Omega}+q_{\Gamma}$ & $s_{\Omega}$ & $q_{\Omega}+q_{I}$ \\
\hline $\begin{array}{c}\text { Sem } \\
\text { enriquecimento }\end{array}$ & 12 & 10 & 27 & 26 & 75 & 82 & 243 & 290 & 867 & 1.090 \\
\hline $\begin{array}{c}\text { Todos os nós } \\
\text { enriquecidos } \sigma\end{array}$ & 24 & 10 & 54 & 26 & 150 & 82 & 486 & 290 & 1.734 & 1.090 \\
\hline $\begin{array}{c}\text { Todos os nós } \\
\text { enriquecidos } \\
\boldsymbol{\sigma} \text { e } \boldsymbol{u}_{\boldsymbol{\Omega}}\end{array}$ & 24 & 16 & 54 & 44 & 150 & 132 & 486 & 452 & 1.734 & 1.668 \\
\hline $\begin{array}{c}\text { Todos os nós } \\
\text { enriquecidos } \\
\boldsymbol{\sigma} \text { e } \boldsymbol{u}_{\boldsymbol{\Gamma}}\end{array}$ & 24 & 12 & 54 & 34 & 150 & 114 & 486 & 418 & 1.734 & 1.602 \\
\hline $\begin{array}{c}\text { Todos os nós } \\
\text { enriquecidos } \\
\boldsymbol{u}_{\boldsymbol{\Omega}} \text { e } \boldsymbol{u}_{\boldsymbol{\Gamma}}\end{array}$ & 12 & 20 & 27 & 52 & 75 & 164 & 243 & 580 & 867 & 2.180 \\
\hline $\begin{array}{c}\text { Todos os nós } \\
\text { enriquecidos } \\
\boldsymbol{u}_{\boldsymbol{\Gamma}} \\
\end{array}$ & 12 & 12 & 27 & 34 & 75 & 114 & 243 & 418 & 867 & 1.602 \\
\hline $\begin{array}{c}\text { Todos os nós } \\
\text { enriquecidos } \boldsymbol{\sigma} \\
\boldsymbol{u}_{\boldsymbol{\Omega}} \text { e } \boldsymbol{u}_{\boldsymbol{\Gamma}}\end{array}$ & 24 & 20 & 54 & 52 & 150 & 164 & 486 & 580 & 1.734 & 2.180 \\
\hline
\end{tabular}

Tabela 7.12 - Graus de liberdade para a chapa tracionada - elemento triangular - FHMT.

\begin{tabular}{|c|c|c|c|c|c|c|c|c|c|c|}
\hline \multirow{3}{*}{$\begin{array}{c}\text { Condições } \\
\text { de } \\
\text { Enriquecimento }\end{array}$} & \multicolumn{10}{|c|}{ Redes } \\
\hline & \multicolumn{2}{|c|}{$(1 \times 1)$} & \multicolumn{2}{|c|}{$(2 \times 2)$} & \multicolumn{2}{|c|}{$(4 \times 4)$} & \multicolumn{2}{|c|}{$(8 \times 8)$} & \multicolumn{2}{|c|}{$(16 \times 16)$} \\
\hline & $s_{\Omega}$ & $q_{\Omega}+q_{\Gamma}$ & $s_{\Omega}$ & $q_{\Omega}+q_{\Gamma}$ & $s_{\Omega}$ & $q_{\Omega}+q_{\Gamma}$ & $s_{\Omega}$ & $q_{\Omega}+q_{\Gamma}$ & $s_{\Omega}$ & $q_{\Omega}+q_{I}$ \\
\hline $\begin{array}{c}\text { Sem } \\
\text { enriquecimento }\end{array}$ & 12 & 10 & 27 & 26 & 75 & 82 & 243 & 290 & 867 & 1.090 \\
\hline $\begin{array}{c}\text { Todos os nós } \\
\text { enriquecidos } \boldsymbol{\sigma}\end{array}$ & 24 & 10 & 54 & 26 & 150 & 82 & 486 & 290 & 1.734 & 1.090 \\
\hline $\begin{array}{l}\text { Todos os nós } \\
\text { enriquecidos } \\
\boldsymbol{\sigma} \text { e } \boldsymbol{u}_{\boldsymbol{\Omega}}\end{array}$ & 24 & 16 & 54 & 44 & 150 & 132 & 486 & 452 & 1.734 & 1.668 \\
\hline $\begin{array}{c}\text { Todos os nós } \\
\text { enriquecidos } \\
\boldsymbol{\sigma} \text { e } \boldsymbol{u}_{\boldsymbol{\Gamma}} \\
\end{array}$ & 24 & 12 & 54 & 34 & 150 & 114 & 486 & 418 & 1.734 & 1.602 \\
\hline $\begin{array}{c}\text { Todos os nós } \\
\text { enriquecidos } \\
\boldsymbol{u}_{\boldsymbol{\Omega}} \text { e } \boldsymbol{u}_{\boldsymbol{\Gamma}}\end{array}$ & 12 & 20 & 27 & 52 & 75 & 164 & 243 & 580 & 867 & 2.180 \\
\hline $\begin{array}{c}\text { Todos os nós } \\
\text { enriquecidos } \\
\boldsymbol{u}_{\boldsymbol{\Gamma}} \\
\end{array}$ & 12 & 12 & 27 & 34 & 75 & 114 & 243 & 418 & 867 & 1.602 \\
\hline $\begin{array}{c}\text { Todos os nós } \\
\text { enriquecidos } \boldsymbol{\sigma} \\
\boldsymbol{u}_{\boldsymbol{\Omega}} \text { e } \boldsymbol{u}_{\boldsymbol{\Gamma}}\end{array}$ & 24 & 20 & 54 & 52 & 150 & 164 & 486 & 580 & 1.734 & 2.180 \\
\hline
\end{tabular}


As tabelas 7.11 e 7.12 indicam que somente com o enriquecimento das tensões no domínio é possível atender à condição $\boldsymbol{s}_{\Omega} \geq \boldsymbol{q}_{\Omega}+\boldsymbol{q}_{\Gamma}$ para todas as redes utilizadas na análise desse exemplo. As células em destaque nas tabelas 7.11 a 7.18 mostram sobre quais redes e combinações de enriquecimento as condições $\boldsymbol{s}_{\Omega} \geq \boldsymbol{q}_{\Omega}+\boldsymbol{q}_{\Gamma}$ (FHMT) ou $\boldsymbol{s}_{\Omega} \geq \boldsymbol{q}_{\Gamma}$ (FHT) não são satisfeitas.

Da tabela 7.13, mantida a aproximação constante para o campo de tensão no elemento quadrilateral da FHT, observa-se que o enriquecimento exclusivo do campo de deslocamento no contorno em todos os nós das redes não atende a condição $\boldsymbol{s}_{\Omega} \geq \boldsymbol{q}_{\Gamma}$. Já utilizando a aproximação linear ou quadrática para o campo de tensão (ver tabelas 7.15 e 7.17), garante-se a desigualdade $\boldsymbol{s}_{\Omega} \geq \boldsymbol{q}_{\Gamma}$, mesmo com enriquecimento exclusivo do campo de deslocamento.

Tabela 7. 13- Graus de liberdade para a chapa tracionada - elemento quadrilateral com aproximação constante para o campo de tensão - FHT.

\begin{tabular}{|c|c|c|c|c|c|c|c|c|c|c|}
\hline \multirow{3}{*}{$\begin{array}{c}\text { Condições } \\
\text { de }\end{array}$} & \multicolumn{10}{|c|}{ Redes } \\
\hline & \multicolumn{2}{|c|}{$(1 \times 1)$} & \multicolumn{2}{|c|}{$(2 \times 2)$} & \multicolumn{2}{|c|}{$(4 \times 4)$} & \multicolumn{2}{|c|}{$(8 \times 8)$} & \multicolumn{2}{|c|}{$(16 \times 16)$} \\
\hline & $s_{\Omega}$ & $\boldsymbol{q}_{\Gamma}$ & $s_{\Omega}$ & $q_{\Gamma}$ & $s_{\Omega}$ & $\boldsymbol{q}_{\Gamma}$ & $\boldsymbol{s}_{\boldsymbol{\Omega}}$ & $\boldsymbol{q}_{\Gamma}$ & $s_{\Omega}$ & $q_{\Gamma}$ \\
\hline $\begin{array}{c}\text { Sem } \\
\text { enriquecimento }\end{array}$ & 3 & 2 & 12 & 8 & 48 & 32 & 192 & 128 & 768 & 512 \\
\hline $\begin{array}{c}\text { Todos os nós } \\
\text { enriquecidos } \boldsymbol{\sigma}\end{array}$ & 7 & 2 & 28 & 8 & 112 & 32 & 448 & 128 & 1.792 & 512 \\
\hline $\begin{array}{c}\text { Todos os nós } \\
\text { enriquecidos } \\
\boldsymbol{u}_{\boldsymbol{\Gamma}}\end{array}$ & 3 & 4 & 12 & 16 & 48 & 64 & 192 & 256 & 768 & 1.024 \\
\hline $\begin{array}{c}\text { Todos os nós } \\
\text { enriquecidos } \\
\boldsymbol{\sigma} \text { e } \boldsymbol{u}_{\boldsymbol{\Gamma}}\end{array}$ & 7 & 4 & 28 & 16 & 112 & 64 & 448 & 256 & 1.792 & 1.024 \\
\hline
\end{tabular}

Tabela 7.14 - Graus de liberdade para a chapa tracionada - elemento triangular com aproximação constante para o campo de tensão - FHT.

\begin{tabular}{|c|c|c|c|c|c|c|c|c|c|c|}
\hline \multirow{3}{*}{$\begin{array}{c}\text { Condições } \\
\text { de }\end{array}$} & \multicolumn{10}{|c|}{ Redes } \\
\hline & \multicolumn{2}{|c|}{$(1 \times 1)$} & \multicolumn{2}{|c|}{$(2 \times 2)$} & \multicolumn{2}{|c|}{$(4 \times 4)$} & \multicolumn{2}{|c|}{$(8 \times 8)$} & \multicolumn{2}{|c|}{$(16 \times 16)$} \\
\hline & $\boldsymbol{s}_{\boldsymbol{\Omega}}$ & $q_{\Gamma}$ & $\boldsymbol{s}_{\boldsymbol{\Omega}}$ & $q_{\Gamma}$ & $\boldsymbol{S}_{\boldsymbol{\Omega}}$ & $q_{\Gamma}$ & $\boldsymbol{S}_{\boldsymbol{\Omega}}$ & $\boldsymbol{q}_{\Gamma}$ & $s_{\Omega}$ & $q_{\Gamma}$ \\
\hline $\begin{array}{c}\text { Sem } \\
\text { enriquecimento }\end{array}$ & 6 & 2 & 24 & 8 & 96 & 32 & 384 & 128 & 1.536 & 512 \\
\hline $\begin{array}{c}\text { Todos os nós } \\
\text { enriquecidos } \sigma\end{array}$ & 12 & 2 & 48 & 8 & 192 & 32 & 768 & 128 & 3.072 & 512 \\
\hline $\begin{array}{c}\text { Todos os nós } \\
\text { enriquecidos } \\
\boldsymbol{u}_{\boldsymbol{\Gamma}}\end{array}$ & 6 & 4 & 24 & 16 & 96 & 64 & 384 & 256 & 1.536 & 1.024 \\
\hline $\begin{array}{c}\text { Todos os nós } \\
\text { enriquecidos } \\
\boldsymbol{\sigma} \text { e } \boldsymbol{u}_{\boldsymbol{\Gamma}} \\
\end{array}$ & 12 & 4 & 48 & 16 & 192 & 64 & 768 & 256 & 3.072 & 1.024 \\
\hline
\end{tabular}


O elemento triangular da FHT, mesmo com aproximação constante para o campo de tensão no domínio, atende a condição $\boldsymbol{s}_{\Omega} \geq \boldsymbol{q}_{\Gamma}$ nas combinações de enriquecimento indicadas nas tabelas 7.14, 7.16 e 7.18.

Tabela 7.15 - Graus de liberdade para a chapa tracionada - elemento quadrilateral com aproximação linear para o campo de tensão - FHT.

\begin{tabular}{cccccccccccc}
\hline \multirow{2}{*}{$\begin{array}{c}\text { Condições } \\
\text { de }\end{array}$} & \multicolumn{10}{c}{$(1 \times 1)$} & \multicolumn{10}{c}{ Redes } \\
\cline { 2 - 12 } Enriquecimento & $s_{\Omega}$ & $q_{\Gamma}$ & $s_{\Omega}$ & $q_{\Gamma}$ & $s_{\Omega}$ & $q_{\Gamma}$ & $s_{\Omega}$ & $q_{\Gamma}$ & $s_{\Omega}$ & $q_{\Gamma}$ \\
\hline $\begin{array}{c}\text { Sem } \\
\text { enriquecimento }\end{array}$ & 7 & 2 & 28 & 8 & 112 & 32 & 448 & 128 & 1.792 & 512 \\
\hline $\begin{array}{c}\text { Todos os nós } \\
\text { enriquecidos } \sigma\end{array}$ & 11 & 2 & 44 & 8 & 176 & 32 & 704 & 128 & 2.816 & 512 \\
\hline $\begin{array}{c}\text { Todos os nós } \\
\text { enriquecidos } \\
\boldsymbol{u}_{\Gamma}\end{array}$ & 7 & 4 & 28 & 16 & 112 & 64 & 448 & 256 & 1.792 & 1.024 \\
\hline $\begin{array}{c}\text { Todos os nós } \\
\text { enriquecidos } \\
\sigma \text { e } \boldsymbol{u}_{\Gamma}\end{array}$ & 11 & 4 & 44 & 16 & 176 & 64 & 704 & 256 & 2.816 & 1.024 \\
\hline
\end{tabular}

Tabela 7.16 - Graus de liberdade para a chapa tracionada - elemento triangular com aproximação linear para o campo de tensão - FHT.

\begin{tabular}{cccccccccccc}
\hline \multirow{2}{*}{$\begin{array}{c}\text { Condições } \\
\text { de }\end{array}$} \\
\cline { 2 - 12 } Enriquecimento & \multicolumn{10}{c}{$(1 \times 1)$} & \multicolumn{10}{c}{ Redes } \\
\cline { 2 - 11 } & $s_{\Omega}$ & $q_{\Gamma}$ & $s_{\Omega}$ & $q_{\Gamma}$ & $s_{\Omega}$ & $q_{\Gamma}$ & $s_{\Omega}$ & $q_{\Gamma}$ & $s_{\Omega}$ & $q_{\Gamma}$ \\
\hline $\begin{array}{c}\text { Sem } \\
\text { enriquecimento }\end{array}$ & 14 & 2 & 56 & 8 & 224 & 32 & 896 & 128 & 3.584 & 512 \\
\hline $\begin{array}{c}\text { Todos os nós } \\
\text { enriquecidos } \sigma\end{array}$ & 20 & 2 & 80 & 8 & 320 & 32 & 1.280 & 128 & 5.120 & 512 \\
\hline $\begin{array}{c}\text { Todos os nós } \\
\text { enriquecidos } \\
\boldsymbol{u}_{\Gamma}\end{array}$ & 14 & 4 & 56 & 16 & 224 & 64 & 896 & 256 & 3.584 & 1.024 \\
\hline $\begin{array}{c}\text { Todos os nós } \\
\text { enriquecidos } \\
\sigma \text { e } \boldsymbol{u}_{\Gamma}\end{array}$ & 20 & 4 & 80 & 16 & 320 & 64 & 1.280 & 256 & 5.120 & 1.024 \\
\hline
\end{tabular}


Tabela 7.17 - Graus de liberdade para a chapa tracionada - elemento quadrilateral com aproximação quadrática para o campo de tensão - FHT.

\begin{tabular}{|c|c|c|c|c|c|c|c|c|c|c|}
\hline \multirow{3}{*}{$\begin{array}{c}\text { Condições } \\
\text { de }\end{array}$} & \multicolumn{10}{|c|}{ Redes } \\
\hline & \multicolumn{2}{|c|}{$(1 \times 1)$} & \multicolumn{2}{|c|}{$(2 \times 2)$} & \multicolumn{2}{|c|}{$(4 \times 4)$} & \multicolumn{2}{|c|}{$(8 \times 8)$} & \multicolumn{2}{|c|}{$(16 \times 16)$} \\
\hline & $s_{\Omega}$ & $q_{\Gamma}$ & $s_{\Omega}$ & $q_{\Gamma}$ & $\boldsymbol{s}_{\boldsymbol{\Omega}}$ & $\boldsymbol{q}_{\Gamma}$ & $s_{\Omega}$ & $\boldsymbol{q}_{\Gamma}$ & $s_{\Omega}$ & $q_{\Gamma}$ \\
\hline $\begin{array}{c}\text { Sem } \\
\text { enriquecimento }\end{array}$ & 12 & 2 & 48 & 8 & 192 & 32 & 768 & 128 & 3.072 & 512 \\
\hline $\begin{array}{c}\text { Todos os nós } \\
\text { enriquecidos } \sigma\end{array}$ & 16 & 2 & 64 & 8 & 256 & 32 & 1.024 & 128 & 4.096 & 512 \\
\hline $\begin{array}{c}\text { Todos os nós } \\
\text { enriquecidos } \\
\boldsymbol{u}_{\boldsymbol{\Gamma}}\end{array}$ & 12 & 4 & 48 & 16 & 192 & 64 & 768 & 256 & 3.072 & 1.024 \\
\hline $\begin{array}{c}\text { Todos os nós } \\
\text { enriquecidos } \\
\boldsymbol{\sigma} \text { е } \boldsymbol{u}_{\boldsymbol{\Gamma}} \\
\end{array}$ & 16 & 4 & 64 & 16 & 256 & 64 & 1.024 & 256 & 4.096 & 1.024 \\
\hline
\end{tabular}

Tabela 7.18 - Graus de liberdade para a chapa tracionada - elemento triangular com aproximação quadrática para o campo de tensão - FHT.

\begin{tabular}{|c|c|c|c|c|c|c|c|c|c|c|}
\hline \multirow{3}{*}{$\begin{array}{c}\text { Condições } \\
\text { de }\end{array}$} & \multicolumn{10}{|c|}{ Redes } \\
\hline & \multicolumn{2}{|c|}{$(1 \times 1)$} & \multicolumn{2}{|c|}{$(2 \times 2)$} & \multicolumn{2}{|c|}{$(4 \times 4)$} & \multicolumn{2}{|c|}{$(8 \times 8)$} & \multicolumn{2}{|c|}{$(16 \times 16)$} \\
\hline & $s_{\Omega}$ & $q_{\Gamma}$ & $s_{\Omega}$ & $q_{\Gamma}$ & $s_{\Omega}$ & $q_{\Gamma}$ & $s_{\Omega}$ & $q_{\Gamma}$ & $s_{\Omega}$ & $q_{\Gamma}$ \\
\hline $\begin{array}{c}\text { Sem } \\
\text { enriquecimento }\end{array}$ & 24 & 2 & 96 & 8 & 384 & 32 & 1.536 & 128 & 6.144 & 512 \\
\hline $\begin{array}{c}\text { Todos os nós } \\
\text { enriquecidos } \boldsymbol{\sigma}\end{array}$ & 30 & 2 & 120 & 8 & 480 & 32 & 1.920 & 128 & 7.680 & 512 \\
\hline $\begin{array}{c}\text { Todos os nós } \\
\text { enriquecidos } \\
\boldsymbol{u}_{\boldsymbol{\Gamma}}\end{array}$ & 24 & 4 & 96 & 16 & 384 & 64 & 1.536 & 256 & 6.144 & 1.024 \\
\hline $\begin{array}{c}\text { Todos os nós } \\
\text { enriquecidos } \\
\boldsymbol{\sigma} \text { e } \boldsymbol{u}_{\boldsymbol{\Gamma}}\end{array}$ & 30 & 4 & 120 & 16 & 480 & 64 & 1.920 & 256 & 7.680 & 1.024 \\
\hline
\end{tabular}

Com objetivo de complementar a análise do 'Teste por Inspeção’, foram determinados os autovalores da matriz de coeficientes (eq. (2.36), incluindo-se ou não a restrição dada pela eq.(2.69)), após aplicação das condições de contorno do problema proposto, para cada um dos casos apresentados nas tabelas 7.11 a 7.18.

Na FHT/FHMT com enriquecimento nodal existem autovalores positivos, negativos e, dependendo dos campos enriquecidos ou das funções utilizadas para o enriquecimento, podem aparecer autovalores nulos (modos espúrios).

Os autovalores positivos correspondem às tensões. Dessa forma, para que se garanta a inversibilidade da matriz $\boldsymbol{F}$ da FHT/FHMT (condição básica para o desenvolvimento do 'Teste por Inspeção') é necessário que tanto as aproximações, como as funções enriquecedoras das tensões no domínio e a quantidade de nós enriquecidos, não gerem 
dependências lineares e, consequentemente, autovalores nulos. Como as aproximações, funções enriquecedoras dos campos de tensões no domínio do elemento e números de nós enriquecidos adotados não resultam modos espúrios estáticos, o total de autovalores positivos é sempre igual à dimensão do vetor $\boldsymbol{s}_{\boldsymbol{\Omega}}$.

Os autovalores negativos correspondem aos deslocamentos no contorno do elemento no caso da FHT, e aos deslocamentos de domínio somados aos de contorno para a FHMT. O total de autovalores negativos e, quando existirem, os modos espúrios cinemáticos (autovalores nulos) são iguais à dimensão do vetor $\boldsymbol{q}_{\Gamma}$ e $\boldsymbol{q}_{\Omega}+\boldsymbol{q}_{\Gamma}$, respectivamente para a FHT e FHMT.

Tabela 7.19 - Autovalores para a chapa tracionada - elemento quadrilateral - sem enriquecimento FHMT.

\begin{tabular}{cccccc}
\hline \multirow{2}{*}{ Autovalores } & $(1 \times 1)$ & $(2 \times 2)$ & $(4 \times 4)$ & $(8 \times 8)$ & $(16 \times 16)$ \\
\cline { 2 - 6 } Positivos & 12 & 27 & 75 & 243 & 867 \\
\hline Negativos & 7 & 21 & 61 & 189 & 637 \\
\hline Nulos & 3 & 5 & 21 & 101 & 453 \\
\hline
\end{tabular}

No caso específico da chapa tracionada analisada com os elementos planos da FHMT sem enriquecimento, a avaliação dos autovalores da matriz de coeficientes revelou a existência de modos espúrios cinemáticos (autovalores nulos), mesmo com $\boldsymbol{s}_{\Omega} \geq \boldsymbol{q}_{\Omega}+\boldsymbol{q}_{\Gamma}$ para algumas redes, tabelas 7.19 e 7.20. Com essa condição, não existe garantia de solvabilidade do problema, a menos que se usem estratégias de resolução do sistema de equações lineares (eq. (2.36), incluindo-se ou não a restrição dada pela eq.(2.69)) como as utilizadas neste trabalho. Porém, caso $\boldsymbol{q}_{\Omega}+\boldsymbol{q}_{\Gamma}$ seja significativamente maior que $\boldsymbol{s}_{\Omega}$, não é possível obter convergência na estratégia de resolução do sistema de equações lineares.

Tabela 7.20 - Autovalores para a chapa tracionada - elemento triangular - sem enriquecimento FHMT.

\begin{tabular}{cccccc}
\hline \multirow{2}{*}{ Autovalores } & \multicolumn{5}{c}{ Redes } \\
\cline { 2 - 6 } & $(1 \times 1)$ & $(2 \times 2)$ & $(4 \times 4)$ & $(8 \times 8)$ & $(16 \times 16)$ \\
\hline Positivos & 12 & 27 & 75 & 243 & 867 \\
\hline Negativos & 6 & 19 & 59 & 188 & 636 \\
\hline Nulos & 4 & 7 & 23 & 102 & 454 \\
\hline
\end{tabular}


O 'Teste por Inspeção’ para o problema da chapa tracionada, discretizada com elementos quadrilaterais e triangulares sem enriquecimentos sobre os campos envolvidos na FHT, tem a condição $\boldsymbol{s}_{\boldsymbol{\Omega}} \geq \boldsymbol{q}_{\boldsymbol{\Gamma}}$ atendida para o conjunto de redes utilizadas e ainda a inexistência de autovalores nulos, como mostra a tabela 7.21 Neste caso, há garantia de solvabilidade do problema.

Tabela 7.21 - Autovalores para a chapa tracionada - sem enriquecimento - FHT.

\begin{tabular}{|c|c|c|c|c|c|c|c|c|}
\hline \multirow{3}{*}{$\begin{array}{c}\text { Base de } \\
\text { Aproximação } \\
\text { das Tensões }\end{array}$} & \multicolumn{4}{|c|}{ Elemento Triangular } & \multicolumn{4}{|c|}{ Elemento Quadrilateral } \\
\hline & \multirow{2}{*}{ Rede } & \multicolumn{3}{|c|}{ Autovalores } & \multirow{2}{*}{ Rede } & \multicolumn{3}{|c|}{ Autovalores } \\
\hline & & Positivo & Negativo & Nulo & & Positivo & Negativo & Nulo \\
\hline \multirow{5}{*}{ Quadrática } & $1 \times 1$ & 24 & 2 & 0 & 1x1 & 12 & 2 & 0 \\
\hline & $2 \times 2$ & 96 & 8 & 0 & $2 \times 2$ & 48 & 8 & 0 \\
\hline & $4 \times 4$ & 384 & 32 & 0 & $4 \times 4$ & 192 & 32 & 0 \\
\hline & $8 \times 8$ & 1.536 & 128 & 0 & $8 \times 8$ & 768 & 128 & 0 \\
\hline & $16 \times 16$ & 6.144 & 512 & 0 & $16 \times 16$ & 3.072 & 512 & 0 \\
\hline \multirow{5}{*}{ Linear } & $1 \times 1$ & 14 & 2 & 0 & $1 \times 1$ & 7 & 2 & 0 \\
\hline & $2 \times 2$ & 56 & 8 & 0 & $2 \times 2$ & 28 & 8 & 0 \\
\hline & $4 \times 4$ & 224 & 32 & 0 & $4 \times 4$ & 112 & 32 & 0 \\
\hline & $8 \times 8$ & 896 & 128 & 0 & $8 \times 8$ & 448 & 128 & 0 \\
\hline & $16 \times 16$ & 3.584 & 512 & 0 & $16 \times 16$ & 1.792 & 512 & 0 \\
\hline \multirow{5}{*}{ Constante } & $1 \times 1$ & 6 & 2 & 0 & $1 \times 1$ & 3 & 2 & 0 \\
\hline & $2 \times 2$ & 204 & 8 & 0 & $2 \times 2$ & 102 & 8 & 0 \\
\hline & $4 \times 4$ & 96 & 32 & 0 & $4 \times 4$ & 48 & 32 & 0 \\
\hline & $8 \times 8$ & 384 & 128 & 0 & $8 \times 8$ & 192 & 128 & 0 \\
\hline & $16 \times 16$ & 1.536 & 512 & 0 & $16 \times 16$ & 768 & 512 & 0 \\
\hline
\end{tabular}

Na aplicação da FHT para análise da chapa tracionada, a existência de modos espúrios cinemáticos (autovalores nulos) foi detectada quando se utilizou aproximação constante ou linear para o campo de tensões no domínio juntamente com o enriquecimento exclusivo sobre os deslocamentos no contorno do elemento. Assim, os resultados das tabelas 7.13 a 7.18 mostram que mesmo com a desigualdade $\boldsymbol{s}_{\Omega} \geq \boldsymbol{q}_{\Gamma}$ satisfeita, para a condição exclusiva de enriquecimento sobre o campo de deslocamento no contorno do elemento, os modos espúrios cinemáticos apareceram.

A existência desses modos caracteriza, claramente, o 'Teste por Inspeção' como condição necessária, mas não suficiente para a solvabilidade e estabilidade.

Passando à representação dos resultados, o valor de referência da energia de deformação do problema representado na figura $7.59\left(\boldsymbol{U}_{\text {chapa tracionada }}=\mathbf{0 , 1 2 4}\right)$ foi obtido por meio de uma análise (elástico-linear) com o ANSYS ${ }^{\circledR}$ utilizando uma rede regular de $\boldsymbol{8 0} \times \boldsymbol{8 0}$ elementos PLANE 42. 
As figuras 7.64 a 7.69 apresentam convergência da energia de deformação $\boldsymbol{U}_{\text {chapa tracionada }}$ em relação à energia de deformação de referência, para as discretizações das figuras 7.62 e 7.63 e algumas condições de enriquecimento. Vale salientar que todas as condições de enriquecimento aplicadas ao conjunto de discretizações utilizadas na análise deste exemplo tendem a recuperar o valor de referência da energia de deformação $\boldsymbol{U}_{\text {chapa tracionada }}$, como ilustram as figuras 7.64 a 7.69 .

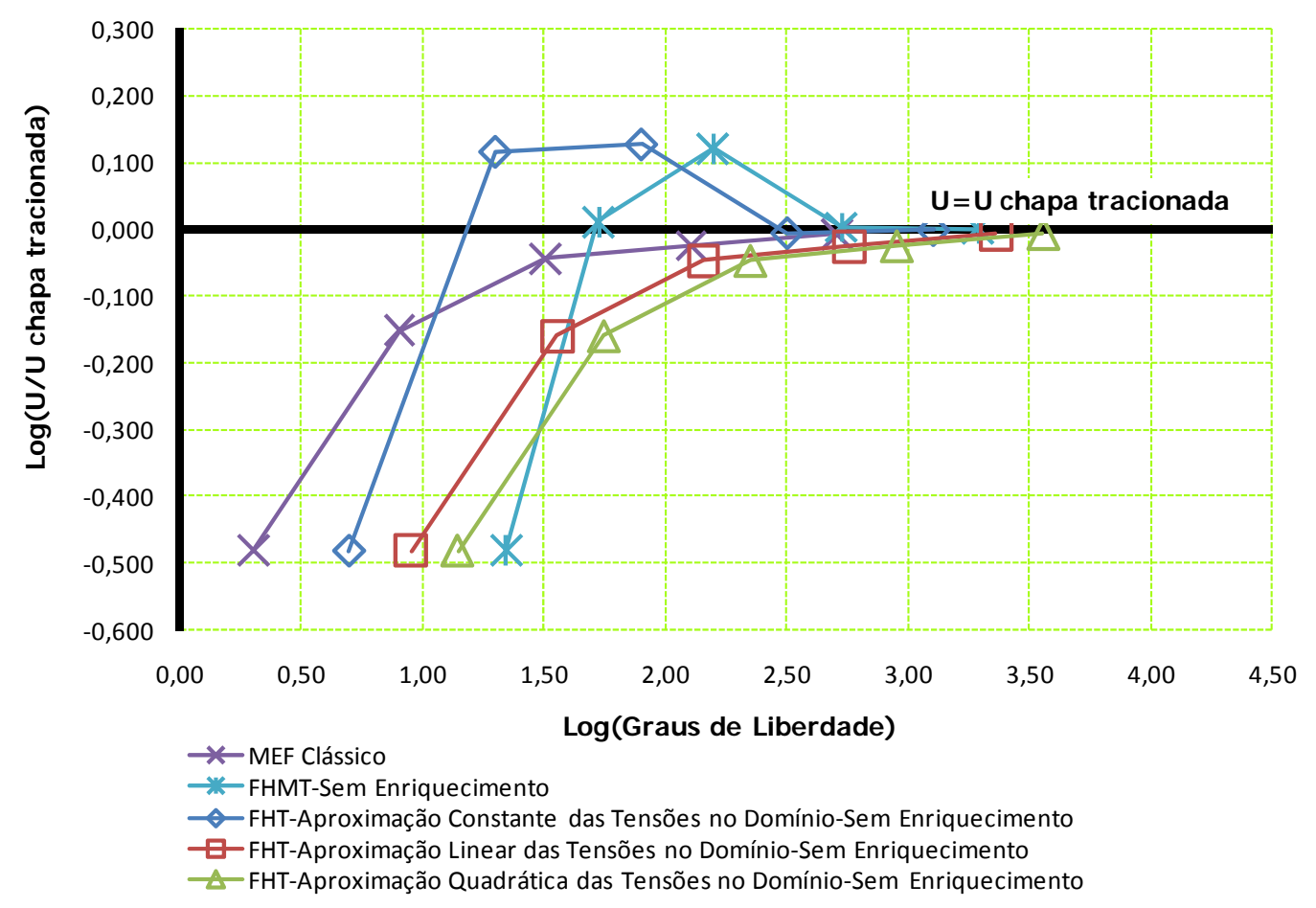

Figura 7.64 - Convergência da energia de deformação $\boldsymbol{U}_{\text {chapa tracionada }}$ - Elemento Quadrilateral - FHT, FHMT e MEF clássico.

Destaca-se que para a construção das figuras 7.64 a 7.69 foram adotadas as seguintes condições de enriquecimento: as grandezas de domínio da FHT/FHMT enriquecidos na totalidade dos nós. Já enriquecimento dos deslocamentos de contorno foi aplicado em todos os nós, excluindo-se aqueles onde existem condições de contorno essenciais.

Os elementos quadrilaterais regulares da FHT/FHMT sem enriquecimento, igualmente ao elemento quadrilateral do MEF Clássico, apresentam baixas estimativas para o valor da energia de deformação para redes menos refinadas, ver figura 7.64. 


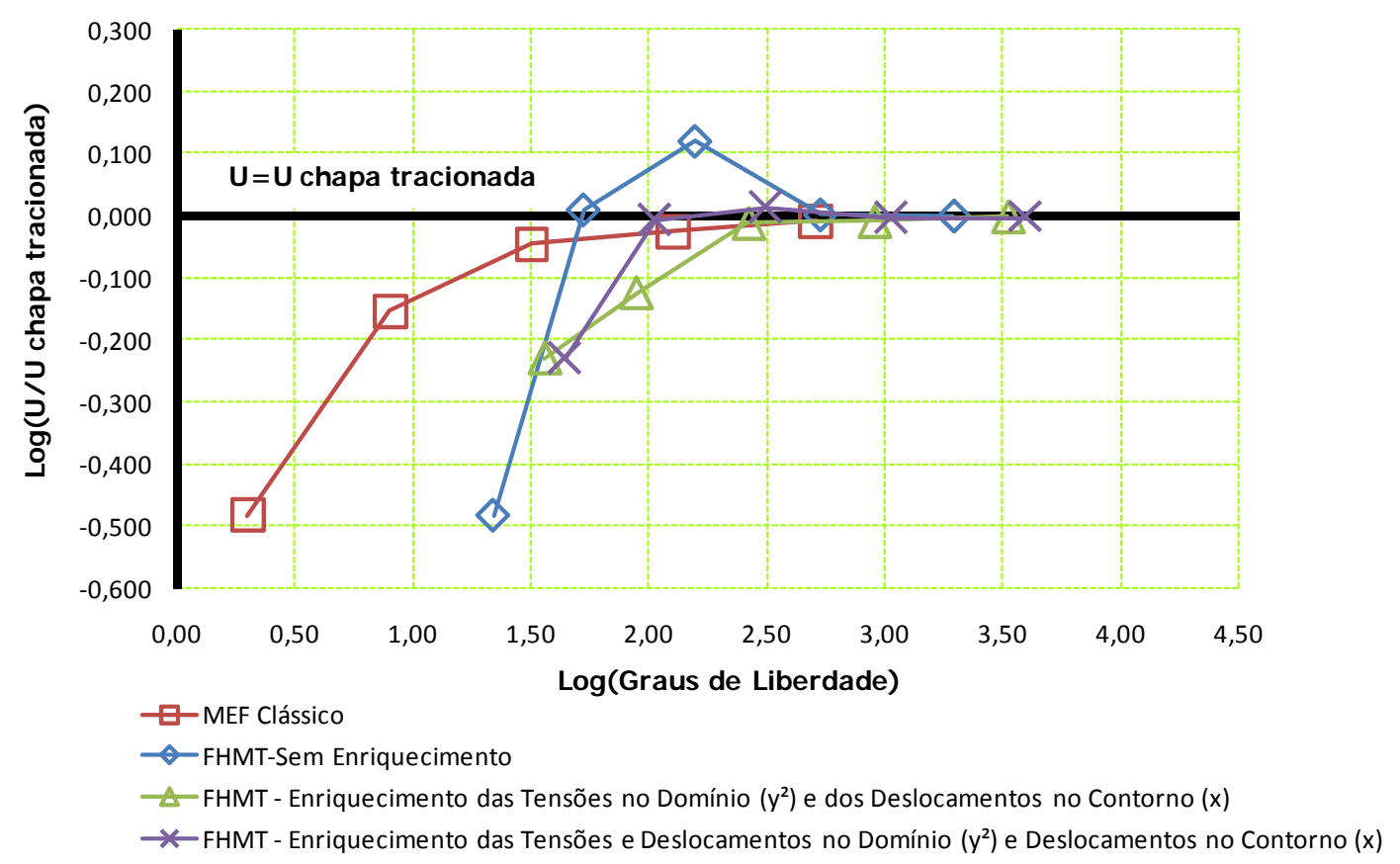

Figura 7.65 - Convergência da energia de deformação $\boldsymbol{U}_{\text {chapa tracionada }}$ - Elemento Quadrilateral FHMT e MEF clássico.

Na figura 7.65, percebe-se que o enriquecimento simultâneo das tensões e deslocamentos no domínio $\left(\boldsymbol{y}^{2}\right)$ e dos deslocamentos no contorno $(\boldsymbol{x})$ do elemento quadrilateral da FHMT trouxe melhorias nas estimativas da energia de deformação para redes regulares menos refinadas $(1 \times 1,2 \times 2$ e $4 \times 4)$.

Com aproximação quadrática para o campo de tensão no domínio do elemento, tem-se que o enriquecimento polinomial exclusivo sobre os deslocamentos resultou em melhor estimativa da energia de deformação, quando comparada com a situação sem enriquecimento ou com o resultado do MEF Clássico, ver figura 7.66. 


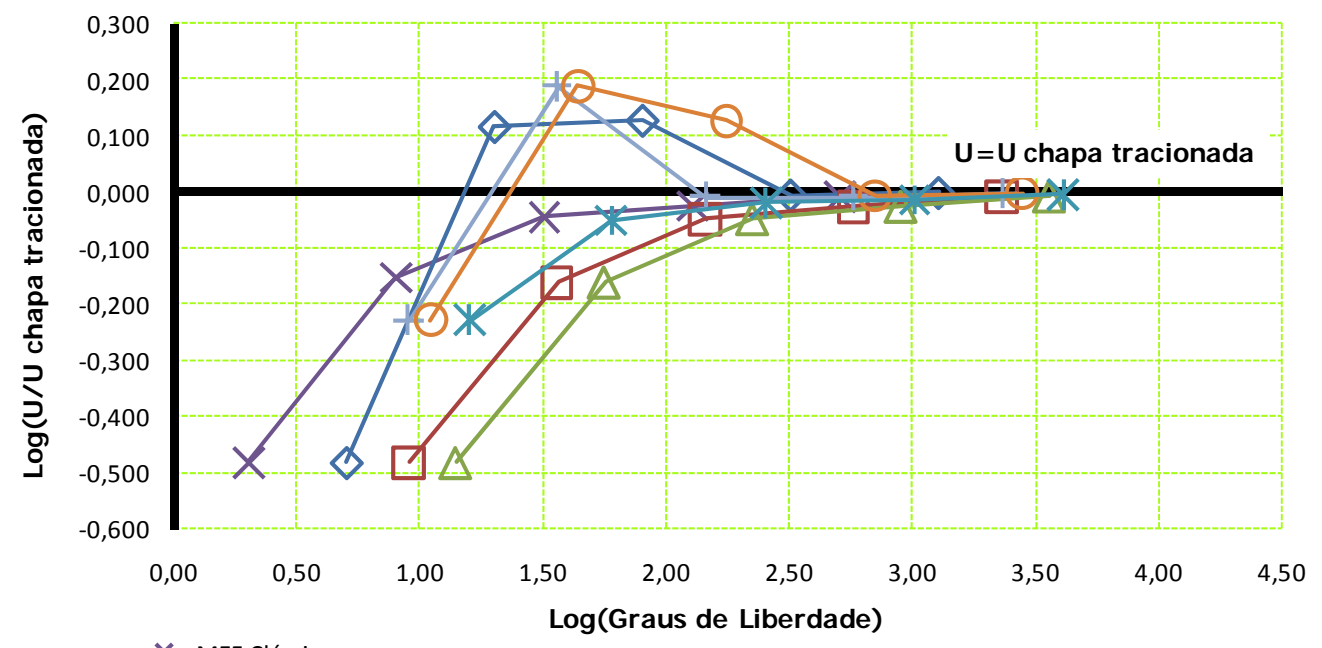

* MEF Clássico

— FHT-Aproximação Constante das Tensões no Domínio-Sem Enriquecimento

— FHT-Aproximação Linear das Tensões no Domínio-Sem Enriquecimento

$\triangle$ FHT-Aproximação Quadrática das Tensões no Domínio-Sem Enriquecimento

- FHT-Aproximação Constante das Tensões no Domínio-Enriquecimento das Tensões no Domínio $\left(y^{2}\right)$

- - FHT-Aproximação Linear das Tensões no Domínio-Enriquecimento dos Deslocamentos no Contorno $(x)$

*-FHT-Aproximação Quadrática das Tensões no Domínio-Enriquecimento dos Deslocamentos no Contorno (x)

Figura 7.66 - Convergência da energia de deformação $\boldsymbol{U}_{\text {chapa tracionada }}$ - Elemento Quadrilateral - FHT e MEF clássico.

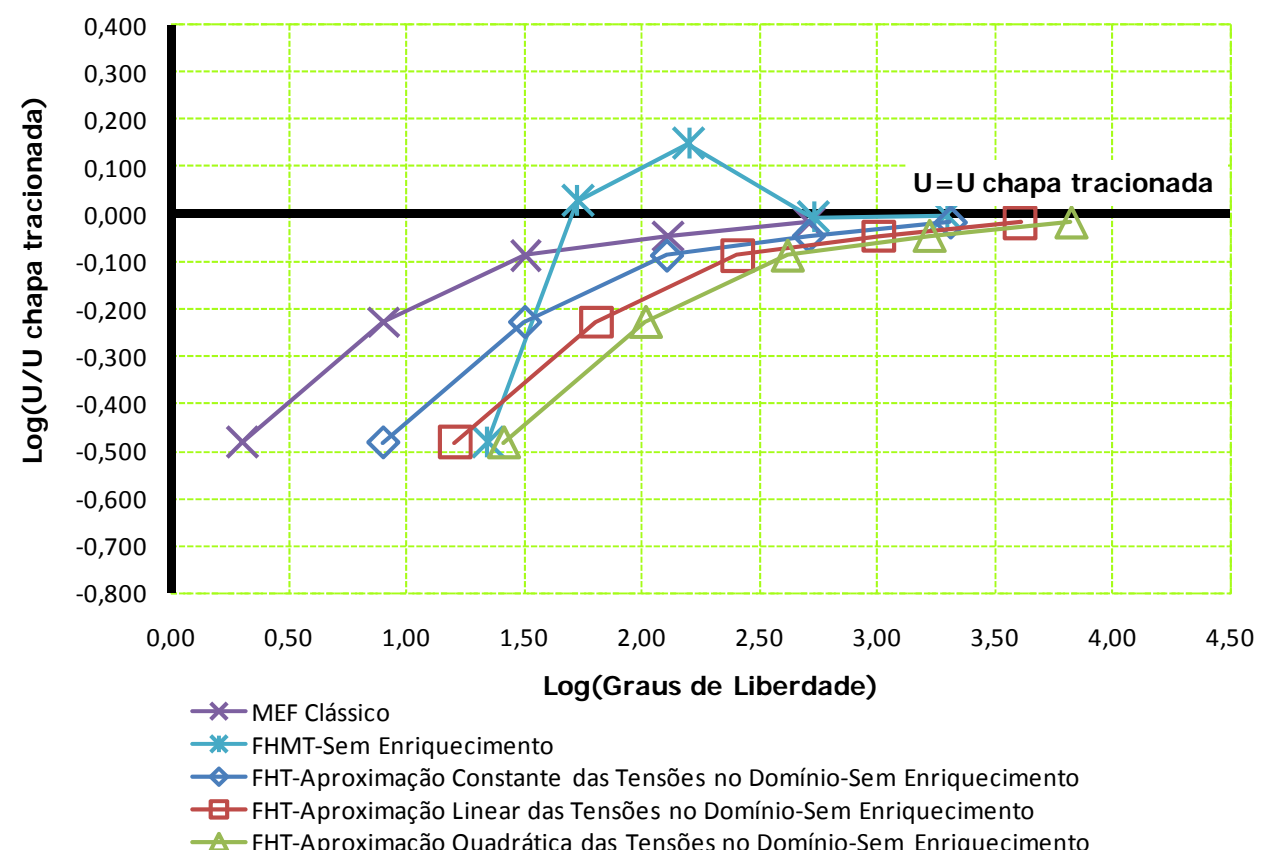

Figura 7.67 - Convergência da energia de deformação $\boldsymbol{U}_{\text {chapa tracionada }}$ - Elemento Triangular - FHT, FHMT e MEF clássico.

Já na figura 7.67, observa-se que o elemento triangular da FHT, para todas as bases aproximativas do campo de tensão no domínio, recupera a mesma estimativa da energia de deformação obtida com o elemento triangular do MEF Clássico. 


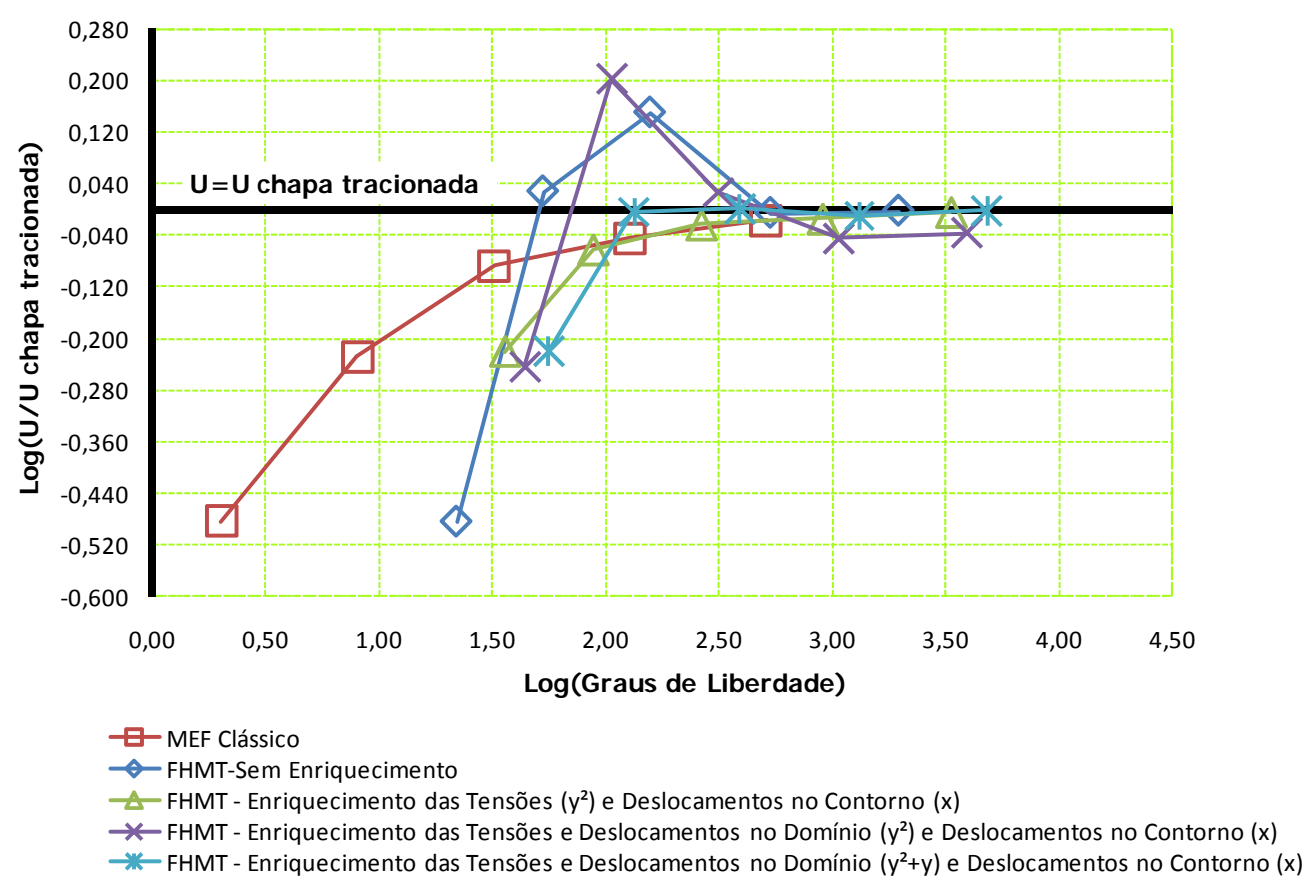

Figura 7.68 - Convergência da energia de deformação $\boldsymbol{U}_{\text {chapa tracionada }}$ - Elemento Triangular - FHMT e MEF clássico.

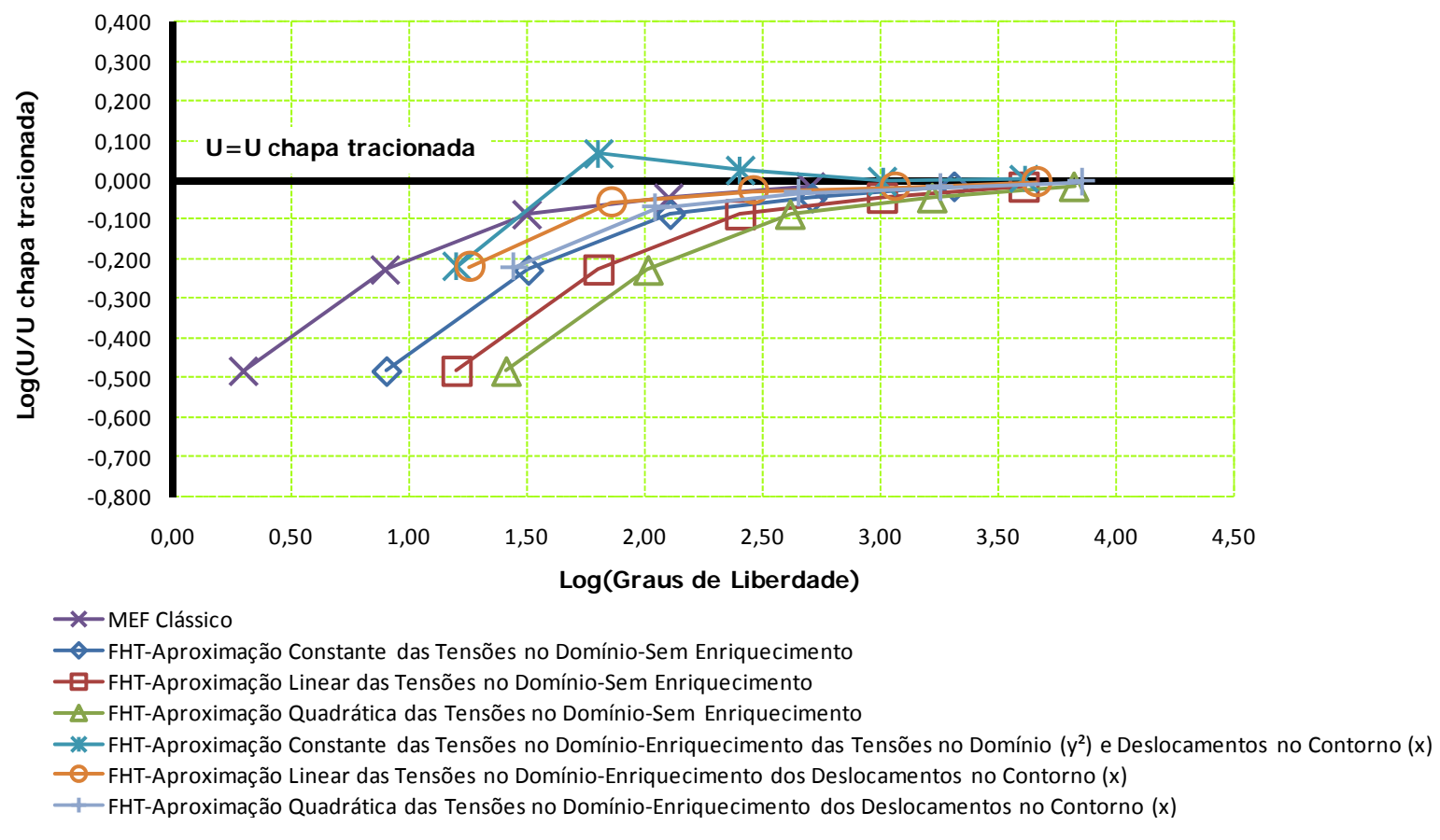

Figura 7.69 - Convergência da energia de deformação $\boldsymbol{U}_{\text {chapa tracionada }}$ - Elemento Triangular - FHT e MEF clássico.

A figura 7.68 mostra que para recuperar o mesmo nível do valor de referência da energia de deformação com redes menos refinadas $(2 \times 2$ e $4 \times 4)$, o elemento triangular da 
FHMT precisou ser enriquecido com um grau superior $\left(\boldsymbol{y}^{2}+\boldsymbol{y}\right)$ para as grandezas de domínio.

Nas figuras 7.66 e 7.69 não se apresentam resultados para o enriquecimento exclusivo sobre o campo de deslocamento no contorno dos elementos, pois para essa condição de enriquecimento ou não se atende o 'Teste por Inspeção' ou apresenta modos espúrios cinemáticos (autovalores nulos); consequentemente, não se obteve convergência no procedimento iterativo de Babuška, utilizado para resolver o sistema de equações lineares.

O último comentário aponta, novamente, para o fato que as condições $\boldsymbol{s}_{\Omega} \geq \boldsymbol{q}_{\Gamma}$ e $\boldsymbol{s}_{\Omega} \geq \boldsymbol{q}_{\Omega}+\boldsymbol{q}_{\Gamma}$ não são suficientes para garantia de solvabilidade, estabilidade e convergência do problema.

Como complemento, reproduz-se a seguir a visualização dos resultados de tensões e deslocamentos obtidos em algumas das análises realizadas.

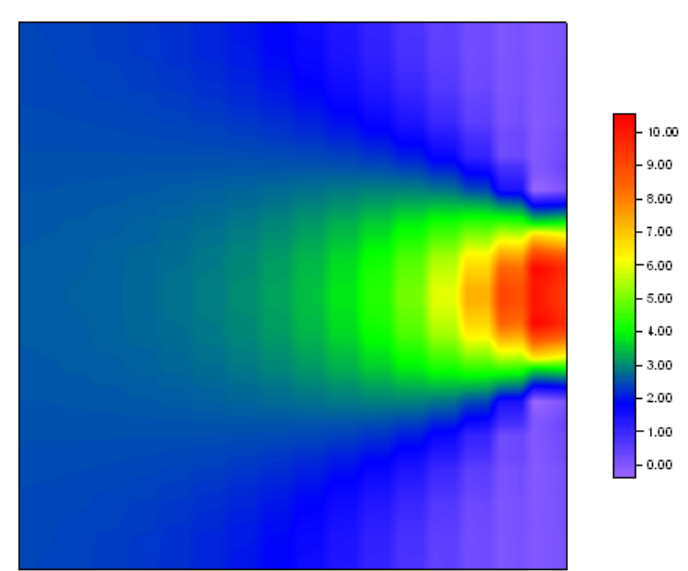

$\sigma_{x}(\operatorname{Sigma} x)$

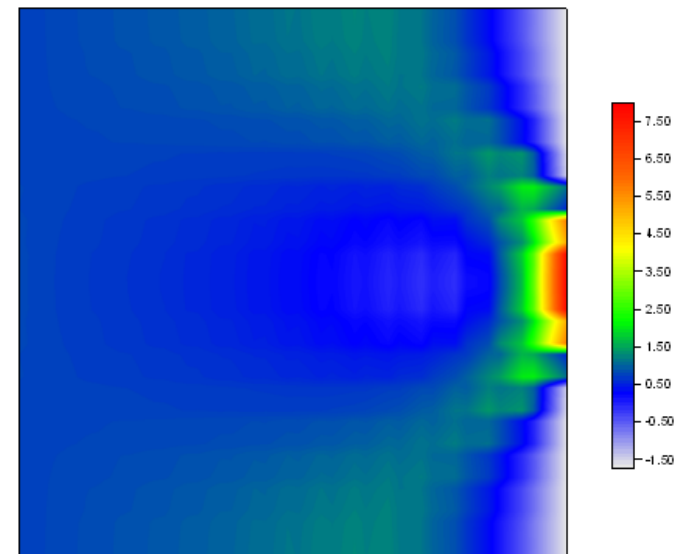

$\sigma_{y}(\operatorname{Sigma} y)$

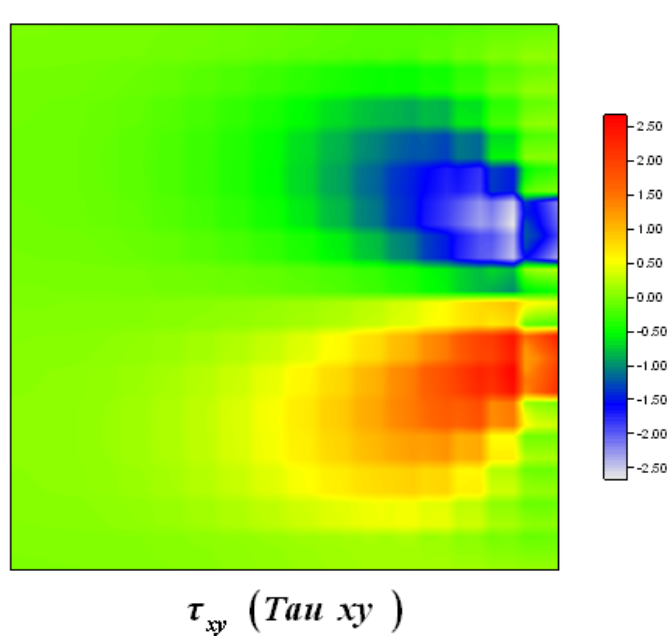

Figura 7.70 - Tensões planas para rede regular $\mathbf{1 6} \times \mathbf{1 6}$ - aproximação quadrática das tensões Elemento Quadrilateral - FHT - sem enriquecimento. 
Representação do campo de tensão para discretização com rede quadrilateral regular $16 \times 16$ - aproximação quadrática das tensões da FHT sem enriquecimento - é destacada na figura 7.70. O campo de tensões obtido via FHT sem enriquecimento apresenta descontinuidade (como as tensões ilustradas na figura 7.70), pois a FHT garante somente continuidade nos deslocamentos de contorno, enquanto o equilíbrio entre elementos é garantido de forma ponderada.

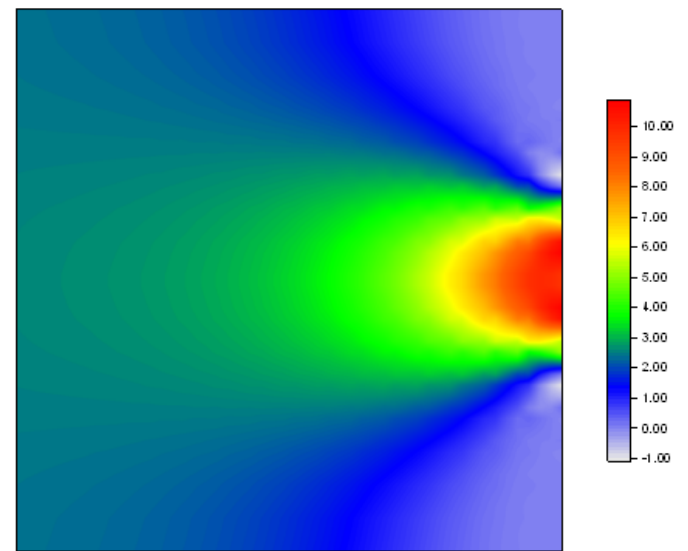

$\sigma_{x}(\operatorname{Sigma} x)$

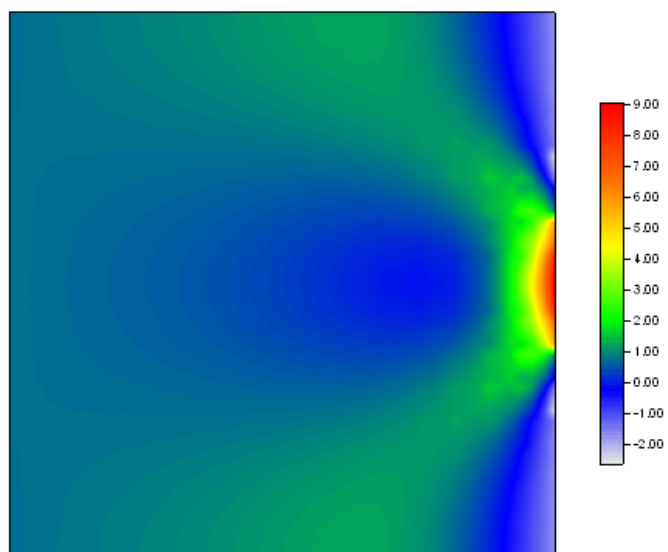

$\sigma_{y}($ Sigma $y)$

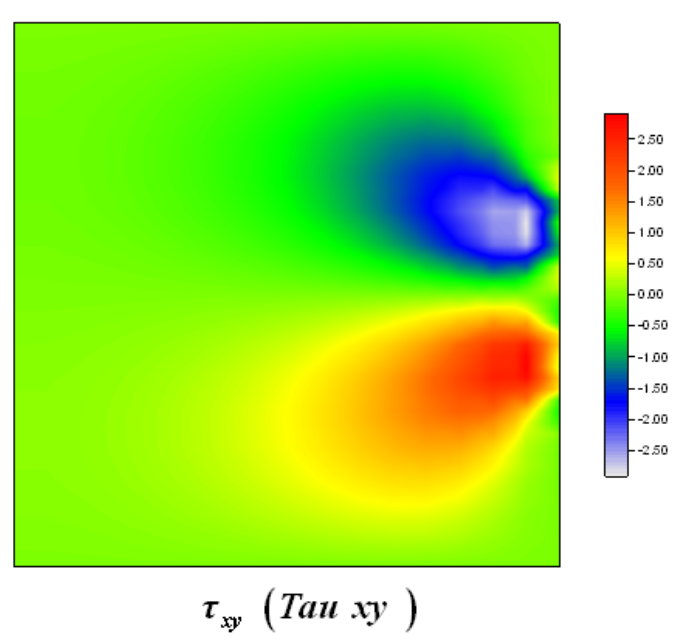

Figura 7.71 - Tensões planas para rede regular $16 \times 16$ - aproximação quadrática das tensões Elemento Quadrilateral - FHT - enriquecimento dos Deslocamentos no Contorno $(x)$.

A figura 7.71 mostra as tensões planas para a rede regular $\mathbf{1 6} \times \mathbf{1 6}$ - aproximação quadrática das tensões da FHT com enriquecimento dos deslocamentos no contorno $(\boldsymbol{x})$. Nesta figura, observa-se que o enriquecimento proposto melhorou a representação das tensões planas, pois o efeito de descontinuidade do campo de tensões presentes na figura 7.70 desapareceu. 


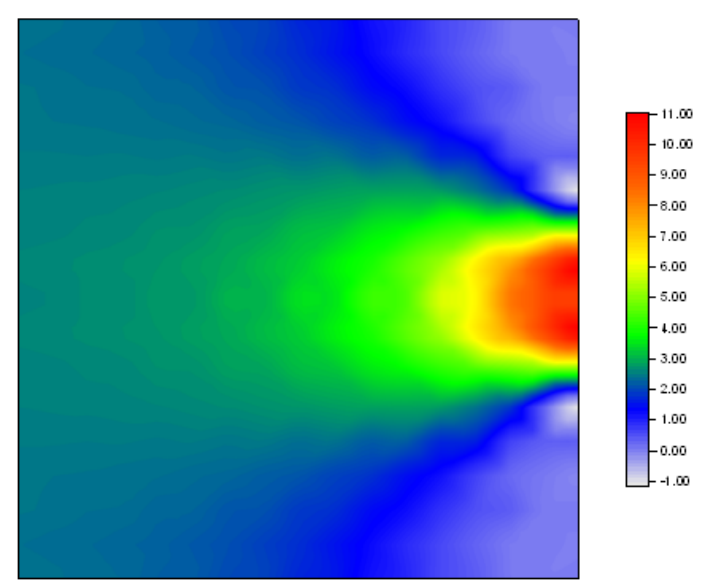

$\sigma_{x}(\operatorname{Sigma} x)$
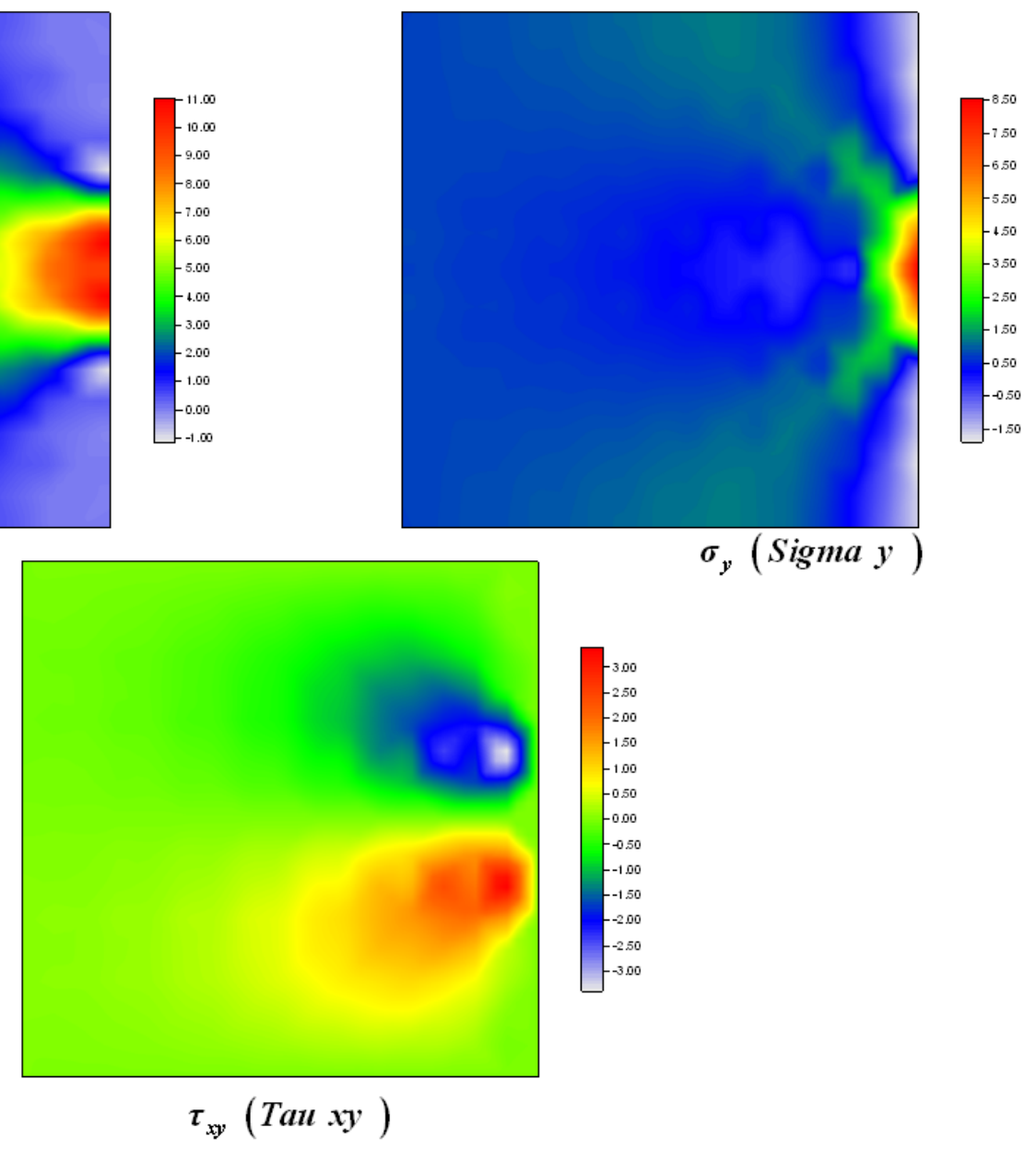

Figura 7.72 - Tensões planas para rede regular $\mathbf{1 6} \times \mathbf{1 6}$ com- Elemento Triangular - FHMT - sem enriquecimento.

As tensões planas obtidas com elementos triangulares da FHMT sem enriquecimento rede regular $\mathbf{1 6} \times 16$ - são apresentadas na figura 7.72. O enriquecimento das tensões e deslocamentos no domínio $\left(\boldsymbol{y}^{2}+\boldsymbol{y}\right)$ e deslocamentos no contorno $(\boldsymbol{x})$ proporcionou melhora na definição das tensões na região da aplicação do carregamento, figura 7.73.

Os resultados gerados para a chapa tracionada discretizada com elementos triangulares foram obtidos com 12 pontos de Gauss distribuídos no domínio e 12 pontos de Gauss no contorno, quando não existiu enriquecimento sobre os campos da FHT/FHMT. Se os campos da FHT/FHMT são enriquecidos, utilizam-se 16 pontos de Gauss no elemento de domínio e 16 pontos de Gauss no elemento de contorno. 

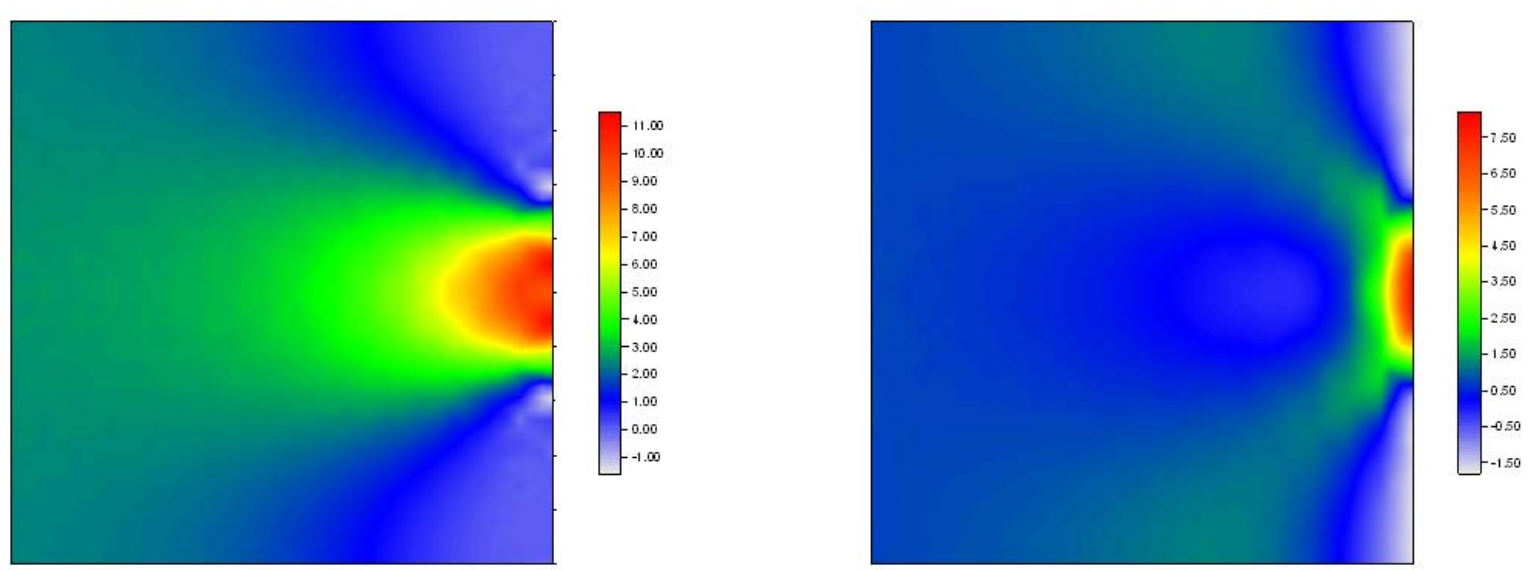

$\sigma_{x}(\operatorname{Sigma} x)$

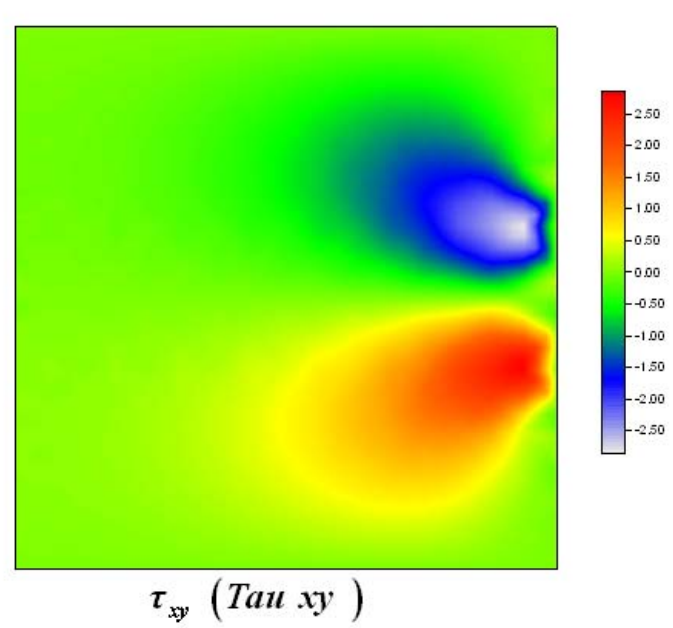

$\sigma_{y}(\operatorname{Sigma} y)$

Figura 7.73 - Tensões planas para rede regular $16 \times 16$ com - Elemento Triangular - FHMT enriquecimento das Tensões e Deslocamentos no Domínio $\left(\boldsymbol{y}^{2}+\boldsymbol{y}\right)$ dos Deslocamentos no Contorno $(\boldsymbol{x})$.

Quando os elementos quadrilaterais foram aplicados à análise da chapa tracionada, utilizaram-se 16 pontos de Gauss em cada direção do elemento quadrilateral de domínio e 16 pontos de Gauss no elemento de contorno, quando não existiu enriquecimento sobre os campos da FHT/FHMT. Se o campo de tensão e/ou deslocamentos no domínio ou o deslocamento no contorno do elemento quadrilateral for enriquecido, utilizam-se 24 pontos de Gauss em cada direção do elemento de domínio e 24 pontos de Gauss no elemento de contorno.

Os deslocamentos planos, para a discretização da chapa tracionada com elementos quadrilaterais da FHT- aproximação quadrática das tensões - com enriquecimento dos deslocamentos no contorno $(\boldsymbol{x})$ em todos os nós, estão representados na figura 7.74. 


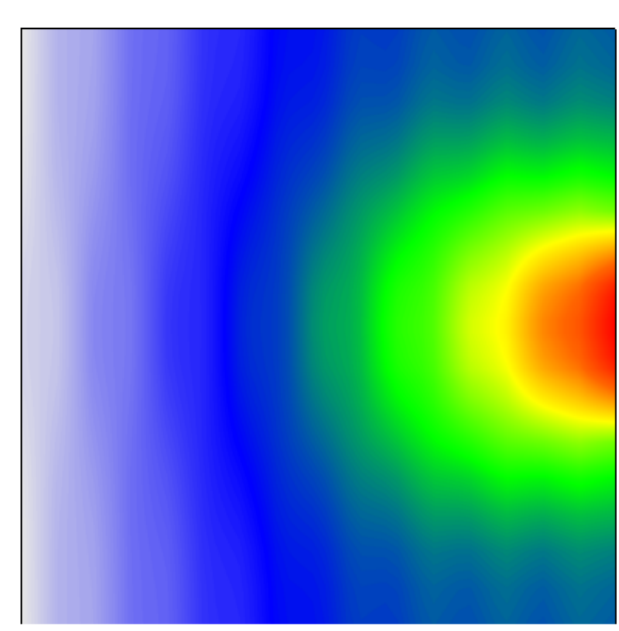

$u_{x}$

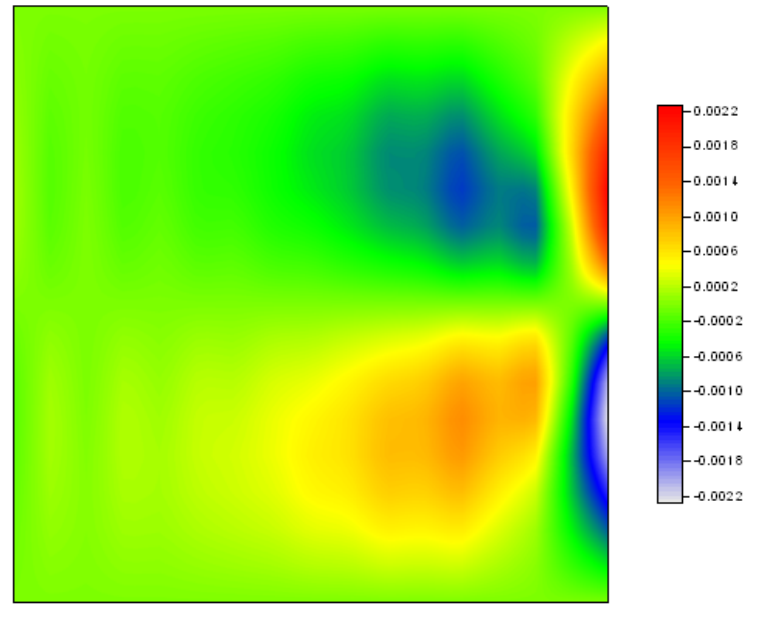

$u_{y}$

Figura 7.74 - Aproximação típica dos deslocamentos nas direções $\boldsymbol{x}\left(\boldsymbol{u}_{\boldsymbol{x}}\right)$ e $\boldsymbol{y}\left(\boldsymbol{u}_{\boldsymbol{y}}\right)$ para rede regular $16 \times 16$ - aproximação quadrática das tensões - Elemento Quadrilateral - FHT enriquecimento dos Deslocamentos no Contorno $(x)$.

Os deslocamentos e tensões planos de referência obtidos com o ANSYS $^{\circledR}$ utilizando uma rede regular de $\mathbf{8 0} \times \mathbf{8 0}$ elementos PLANE 42, estão mostrados nas figuras 7.75 e 7.76.
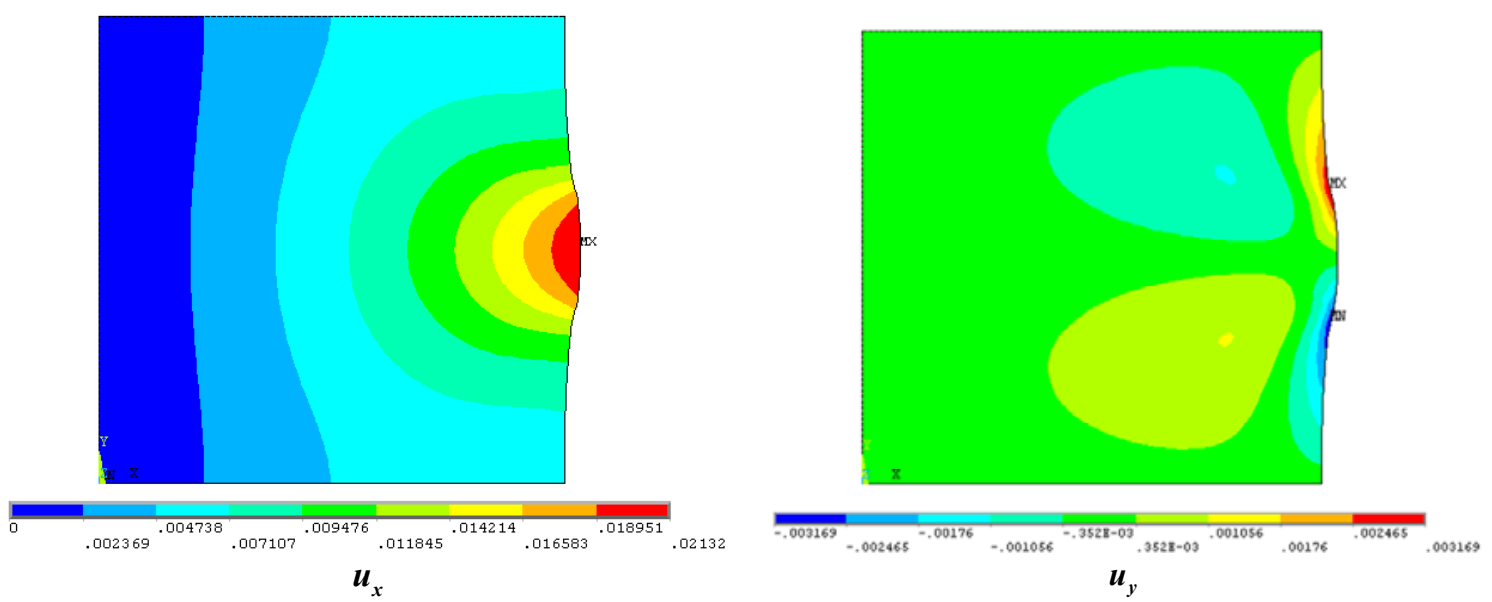

Figura 7.75 - Deslocamentos planos para uma rede regular $\mathbf{8 0} \times \mathbf{8 0}$ elementos PLANE 42 do ANSYS $^{\circledR}$ 

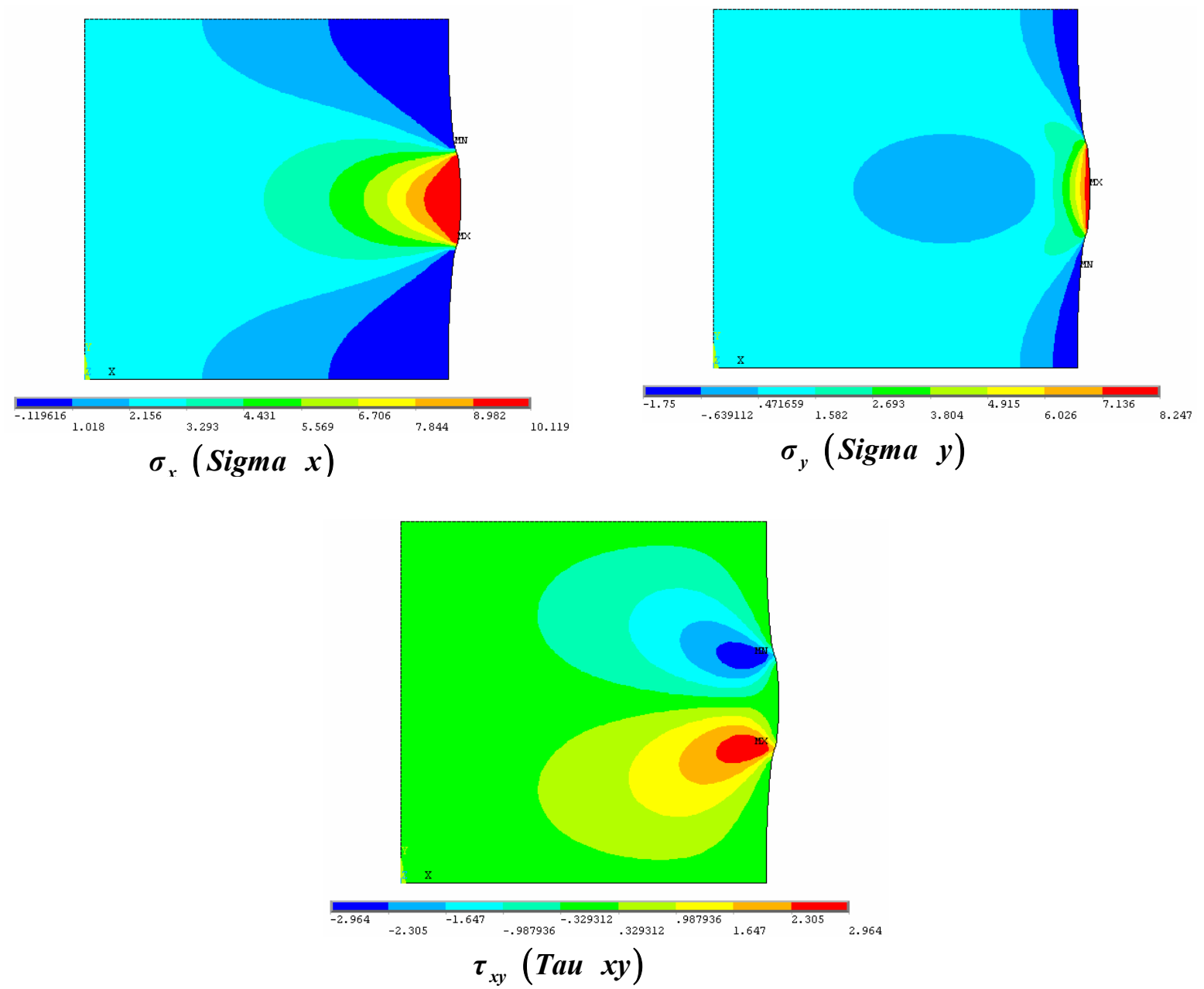

Figura 7.76 - Representação do campo de tensões para uma rede regular $\mathbf{8 0} \times \mathbf{8 0}$ elementos PLANE 42 do ANSYS $^{\circledR}$.

\subsubsection{Chapa Tracionada com Fenda Central}

O ‘Teste por Inspeção’ será aplicado ao problema da figura 7.61 com o auxílio das redes regulares apresentada nas figuras 7.77 e 7.78 . 


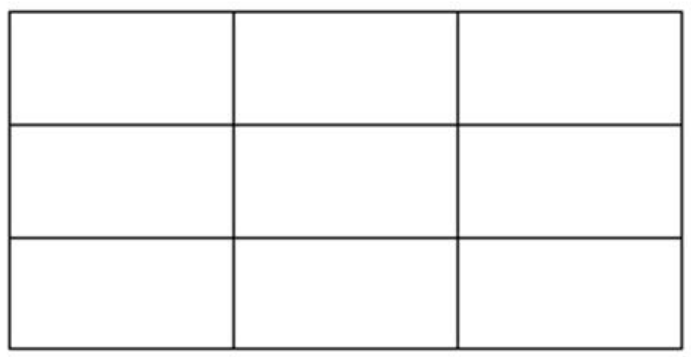

$3 \times 3$

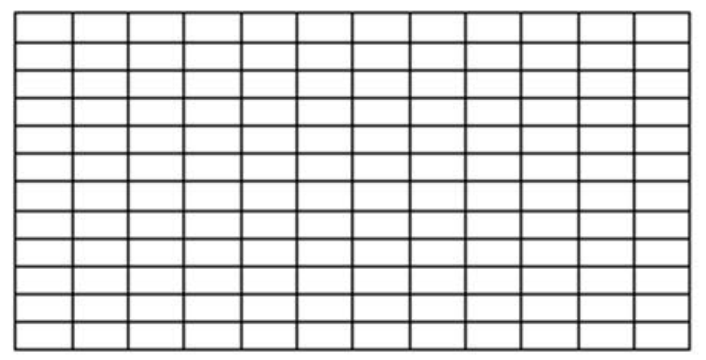

$12 \times 12$

\begin{tabular}{|l|l|l|l|l|l|}
\hline & & & & & \\
\hline & & & & & \\
\hline & & & & & \\
\hline & & & & & \\
\hline & & & & & \\
\hline & & & & & \\
\hline
\end{tabular}

$6 \times 6$

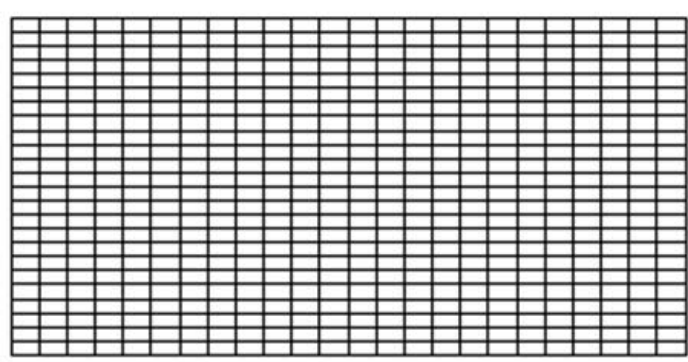

$24 \times 24$

Figura 7.77 - Redes quadrilaterais regulares - chapa tracionada com fenda central.

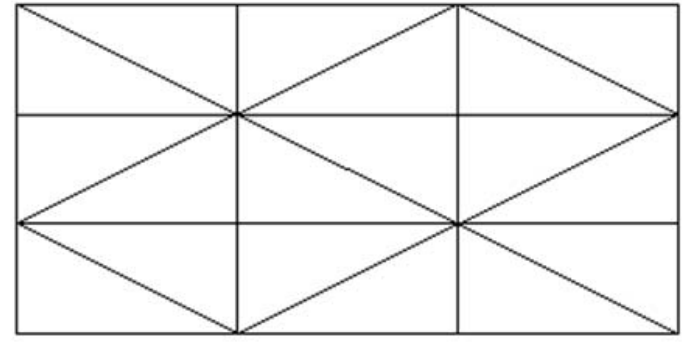

$3 \times 3$

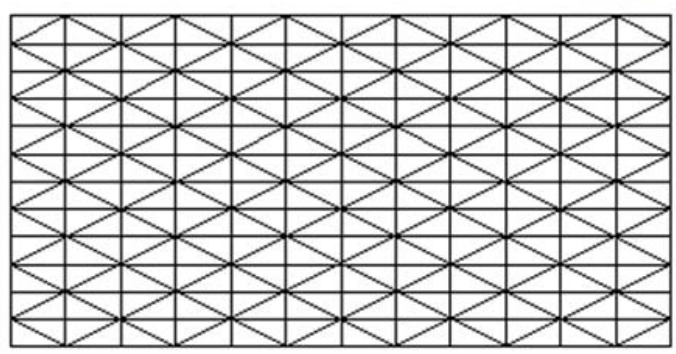

$12 \times 12$

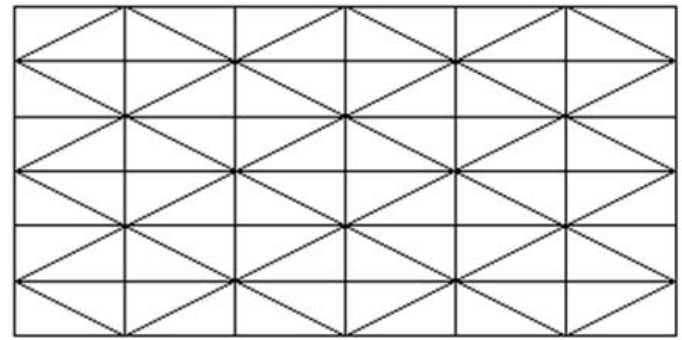

$6 \times 6$

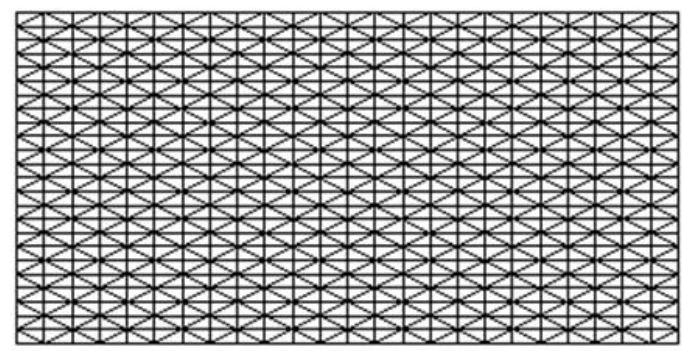

$24 \times 24$

Figura 7.78 - Redes triangulares regulares - chapa tracionada com fenda central.

Nas tabelas 7.22 a 7.29 apresentam-se os valores dos graus de liberdade envolvidos nas diferentes redes e condições de enriquecimento. Funções polinomiais foram utilizadas para enriquecer os campos dos elementos quadrilaterais e triangulares da FHT/FHMT; para o enriquecimento dos campos de domínio da FHMT e do campo de deslocamento no contorno da FHT foi utilizado o primeiro nível de enriquecimento polinomial. 
Para leitura dos resultados tabulados, considera-se a seguinte legenda:

- $\sigma$ : aproximação do campo de tensão no domínio do elemento da FHT ou FHMT;

- $\boldsymbol{u}_{\boldsymbol{\Omega}}$ : aproximação do campo de deslocamento no domínio do elemento da FHMT;

- $\boldsymbol{u}_{\boldsymbol{\Gamma}}$ : aproximação do campo de deslocamento no contorno do elemento da FHT ou FHMT;

- $\boldsymbol{s}_{\Omega}$ : vetor que guarda os parâmetros de tensão no domínio do elemento com ou sem enriquecimento - FHT ou FHMT ;

- $\boldsymbol{q}_{\Omega}$ : vetor que guarda os graus de liberdade de deslocamento no domínio do elemento e ainda os possíveis parâmetros nodais enriquecidos - FHMT;

- $\boldsymbol{q}_{\Gamma}$ : vetor que guarda os graus de liberdade de deslocamento no contorno do elemento e ainda os possíveis parâmetros nodais enriquecidos - FHT ou FHMT;

Como mostram as tabelas 7.22 e 7.23 , para este problema somente as condições de enriquecimento exclusivo sobre os campos de tensões ou enriquecimento simultâneo das tensões e deslocamentos no domínio, ou enriquecimento das tensões de domínio juntamente com os deslocamentos no contorno, atenderam à condição $s_{\Omega} \geq \boldsymbol{q}_{\Omega}+\boldsymbol{q}_{\Gamma}$.

Tabela 7.22 - Graus de liberdade para a chapa com fenda central - elemento quadrilateral - FHMT.

\begin{tabular}{|c|c|c|c|c|c|c|c|c|}
\hline \multirow{3}{*}{$\begin{array}{c}\text { Condições } \\
\text { de } \\
\text { Enriquecimento }\end{array}$} & \multicolumn{8}{|c|}{ Redes } \\
\hline & \multicolumn{2}{|c|}{$(3 \times 3)$} & \multicolumn{2}{|c|}{$(6 \times 6)$} & \multicolumn{2}{|c|}{$(12 \times 12)$} & \multicolumn{2}{|c|}{$(24 \times 24)$} \\
\hline & $\boldsymbol{s}_{\boldsymbol{\Omega}}$ & $q_{\Omega}+q_{\Gamma}$ & $\boldsymbol{s}_{\Omega}$ & $q_{\Omega}+q_{\Gamma}$ & $\boldsymbol{s}_{\boldsymbol{\Omega}}$ & $q_{\Omega}+q_{\Gamma}$ & $\boldsymbol{s}_{\boldsymbol{\Omega}}$ & $q_{\Omega}+q_{\Gamma}$ \\
\hline $\begin{array}{c}\text { Sem } \\
\text { enriquecimento }\end{array}$ & 48 & 57 & 147 & 184 & 507 & 654 & 1.875 & 2.458 \\
\hline $\begin{array}{c}\text { Todos os nós } \\
\text { enriquecidos } \boldsymbol{\sigma}\end{array}$ & 96 & 57 & 294 & 184 & 1.014 & 654 & 3.750 & 2.458 \\
\hline $\begin{array}{l}\text { Todos os nós } \\
\text { enriquecidos } \\
\boldsymbol{\sigma} \text { e } \boldsymbol{u}_{\boldsymbol{\Omega}}\end{array}$ & 96 & 89 & 294 & 282 & 1.014 & 992 & 3.750 & 3.708 \\
\hline $\begin{array}{l}\text { Todos os nós } \\
\text { enriquecidos } \\
\boldsymbol{\sigma} \text { e } \boldsymbol{u}_{\boldsymbol{\Gamma}}\end{array}$ & 96 & 75 & 294 & 258 & 1.014 & 948 & 3.750 & 3.624 \\
\hline $\begin{array}{c}\text { Todos os nós } \\
\text { enriquecidos } \\
\boldsymbol{u}_{\boldsymbol{\Omega}} \text { e } \boldsymbol{u}_{\boldsymbol{\Gamma}}\end{array}$ & 48 & 107 & 147 & 356 & 507 & 1.286 & 1.875 & 4.874 \\
\hline $\begin{array}{c}\text { Todos os nós } \\
\text { enriquecidos } \\
\boldsymbol{u}_{\boldsymbol{\Gamma}}\end{array}$ & 48 & 75 & 147 & 258 & 507 & 948 & 1.875 & 3.624 \\
\hline $\begin{array}{c}\text { Todos os nós } \\
\text { enriquecidos } \boldsymbol{\sigma} \\
\boldsymbol{u}_{\boldsymbol{\Omega}} \text { e } \boldsymbol{u}_{\boldsymbol{\Gamma}}\end{array}$ & 96 & 107 & 294 & 356 & 1.014 & 1.286 & 3.750 & 4.874 \\
\hline
\end{tabular}


As linhas em destaque nas tabelas 7.22 e 7.23 correspondem às redes e combinações de enriquecimento onde a condição $\boldsymbol{s}_{\Omega} \geq \boldsymbol{q}_{\boldsymbol{\Omega}}+\boldsymbol{q}_{\Gamma}$ (FHMT) não é satisfeita.

Tabela 7.23 - Graus de liberdade para a chapa com fenda central - elemento triangular - FHMT.

\begin{tabular}{|c|c|c|c|c|c|c|c|c|}
\hline \multirow{3}{*}{$\begin{array}{c}\text { Condições } \\
\text { de }\end{array}$} & \multicolumn{8}{|c|}{ Redes } \\
\hline & \multicolumn{2}{|c|}{$(3 \times 3)$} & \multicolumn{2}{|c|}{$(6 \times 6)$} & \multicolumn{2}{|c|}{$(12 \times 12)$} & \multicolumn{2}{|c|}{$(24 \times 24)$} \\
\hline & $s_{\Omega}$ & $q_{\Omega}+q_{\Gamma}$ & $s_{\Omega}$ & $q_{\Omega}+q_{\Gamma}$ & $s_{\Omega}$ & $q_{\Omega}+q_{\Gamma}$ & $s_{\Omega}$ & $q_{\Omega}+q_{I}$ \\
\hline $\begin{array}{c}\text { Sem } \\
\text { enriquecimento }\end{array}$ & 48 & 57 & 147 & 184 & 507 & 654 & 1.875 & 2.458 \\
\hline $\begin{array}{c}\text { Todos os nós } \\
\text { enriquecidos } \boldsymbol{\sigma}\end{array}$ & 96 & 57 & 294 & 184 & 1.014 & 654 & 3.750 & 2.458 \\
\hline $\begin{array}{c}\text { Todos os nós } \\
\text { enriquecidos } \\
\boldsymbol{\sigma} \text { e } \boldsymbol{u}_{\boldsymbol{\Omega}} \\
\end{array}$ & 96 & 89 & 294 & 282 & 1.014 & 992 & 3.750 & 3.708 \\
\hline $\begin{array}{c}\text { Todos os nós } \\
\text { enriquecidos } \\
\boldsymbol{\sigma} \text { e } \boldsymbol{u}_{\boldsymbol{\Gamma}}\end{array}$ & 96 & 82 & 294 & 270 & 1.014 & 970 & 3.750 & 3.666 \\
\hline $\begin{array}{c}\text { Todos os nós } \\
\text { enriquecidos } \\
\boldsymbol{u}_{\boldsymbol{\Omega}} \text { е } \boldsymbol{u}_{\boldsymbol{\Gamma}}\end{array}$ & 48 & 114 & 147 & 368 & 507 & 1.308 & 1.875 & 4.916 \\
\hline $\begin{array}{c}\text { Todos os nós } \\
\text { enriquecidos } \\
\boldsymbol{u}_{\boldsymbol{\Gamma}} \\
\end{array}$ & 48 & 82 & 147 & 270 & 507 & 970 & 1.875 & 3.666 \\
\hline $\begin{array}{c}\text { Todos os nós } \\
\text { enriquecidos } \boldsymbol{\sigma} \\
\boldsymbol{u}_{\boldsymbol{\Omega}} \text { е } \boldsymbol{u}_{\boldsymbol{\Gamma}}\end{array}$ & 96 & 114 & 294 & 368 & 1.014 & 1.308 & 3.750 & 4.916 \\
\hline
\end{tabular}

Tabela 7.24 - Graus de liberdade para a chapa tracionada com fenda central - elemento quadrilateral com aproximação constante para o campo de tensão - FHT.

\begin{tabular}{cccccccccc}
\hline \multirow{8}{*}{$\begin{array}{c}\text { Condições } \\
\text { de }\end{array}$} \\
\cline { 2 - 9 } Enriquecimento & \multicolumn{8}{c}{$(3 \times 3)$} & \multicolumn{7}{c}{$(6 \times 6)$} & \multicolumn{2}{c}{$(12 \times 12)$} & \multicolumn{2}{c}{$(24 \times 24)$} \\
\cline { 2 - 9 } & $s_{\Omega}$ & $q_{\Gamma}$ & $s_{\Omega}$ & $q_{\Gamma}$ & $s_{\Omega}$ & $q_{\Gamma}$ & $s_{\Omega}$ & $q_{\Gamma}$ \\
\hline $\begin{array}{c}\text { Sem } \\
\text { enriquecimento }\end{array}$ & 27 & 25 & 108 & 86 & 432 & 316 & 1.728 & 1.208 \\
\hline $\begin{array}{c}\text { Todos os nós } \\
\text { enriquecidos } \sigma\end{array}$ & 63 & 25 & 252 & 86 & 1.008 & 316 & 4.032 & 1.208 \\
\hline $\begin{array}{c}\text { Todos os nós } \\
\text { enriquecidos } \\
\boldsymbol{u}_{\Gamma}\end{array}$ & 27 & 50 & 108 & 172 & 432 & 632 & 1.728 & 2.416 \\
\hline $\begin{array}{c}\text { Todos os nós } \\
\text { enriquecidos } \\
\sigma \text { e } \boldsymbol{u}_{\Gamma}\end{array}$ & 63 & 50 & 252 & 172 & 1.008 & 632 & 4.032 & 2.416 \\
\hline
\end{tabular}

As tabelas 7.24 a 7.29 evidenciam que na chapa com fenda central, analogamente ao caso da chapa tracionada, somente a condição de enriquecimento exclusivo do campo de deslocamento no contorno do elemento quadrilateral (todos os nós das redes) sobre 
aproximação constante das tensões, não satisfaz à condição $\boldsymbol{s}_{\Omega_{2}} \geq \boldsymbol{q}_{\Gamma}$. A linha em destaque na tabela 7.24 apresenta justamente a situação que viola aquela condição.

Com a aproximação linear ou quadrática, para os campos de tensões no domínio dos elementos triangulares e quadrilaterais (ver tabelas 7.26 a 7.29), todas as condições de enriquecimento verificam a desigualdade $\boldsymbol{s}_{\boldsymbol{\Omega}} \geq \boldsymbol{q}_{\Gamma}$.

Tabela 7.25 - Graus de liberdade para a chapa tracionada com fenda central - elemento triangular com aproximação constante para o campo de tensão - FHT.

\begin{tabular}{|c|c|c|c|c|c|c|c|c|}
\hline \multirow{3}{*}{$\begin{array}{c}\text { Condições } \\
\text { de }\end{array}$} & \multicolumn{8}{|c|}{ Redes } \\
\hline & \multicolumn{2}{|c|}{$(3 \times 3)$} & \multicolumn{2}{|c|}{$(6 \times 6)$} & \multicolumn{2}{|c|}{$(12 \times 12)$} & \multicolumn{2}{|c|}{$(24 \times 24)$} \\
\hline & $s_{\Omega}$ & $q_{\Gamma}$ & $s_{\Omega}$ & $q_{\Gamma}$ & $s_{\Omega}$ & $q_{\Gamma}$ & $s_{\Omega}$ & $q_{\Gamma}$ \\
\hline $\begin{array}{c}\text { Sem } \\
\text { enriquecimento }\end{array}$ & 54 & 25 & 216 & 86 & 864 & 316 & 3.456 & 1.208 \\
\hline $\begin{array}{c}\text { Todos os nós } \\
\text { enriquecidos } \sigma\end{array}$ & 108 & 25 & 432 & 86 & 1.728 & 316 & 6.912 & 1.208 \\
\hline $\begin{array}{c}\text { Todos os nós } \\
\text { enriquecidos } \\
\boldsymbol{u}_{\boldsymbol{\Gamma}} \\
\end{array}$ & 54 & 50 & 216 & 172 & 864 & 632 & 3.456 & 2.416 \\
\hline $\begin{array}{c}\text { Todos os nós } \\
\text { enriquecidos } \\
\boldsymbol{\sigma} \text { e } \boldsymbol{u}_{\boldsymbol{\Gamma}}\end{array}$ & 108 & 50 & 432 & 172 & 1.728 & 632 & 6.912 & 2.416 \\
\hline
\end{tabular}

Tabela 7.26 - Graus de liberdade para a chapa tracionada com fenda central - elemento quadrilateral com aproximação linear para o campo de tensão - FHT.

\begin{tabular}{|c|c|c|c|c|c|c|c|c|}
\hline \multirow{3}{*}{$\begin{array}{c}\begin{array}{c}\text { Condições } \\
\text { de }\end{array} \\
\text { Enriquecimento }\end{array}$} & \multicolumn{8}{|c|}{ Redes } \\
\hline & \multicolumn{2}{|c|}{$(3 \times 3)$} & \multicolumn{2}{|c|}{$(6 \times 6)$} & \multicolumn{2}{|c|}{$(12 \times 12)$} & \multicolumn{2}{|c|}{$(24 \times 24)$} \\
\hline & $s_{\Omega}$ & $q_{\Gamma}$ & $s_{\Omega}$ & $q_{\Gamma}$ & $s_{\Omega}$ & $q_{\Gamma}$ & $s_{\Omega}$ & $q_{\Gamma}$ \\
\hline $\begin{array}{c}\text { Sem } \\
\text { enriquecimento }\end{array}$ & 63 & 25 & 252 & 86 & 1.008 & 316 & 4.032 & 1.208 \\
\hline $\begin{array}{c}\text { Todos os nós } \\
\text { enriquecidos } \boldsymbol{\sigma}\end{array}$ & 99 & 25 & 396 & 86 & 1.584 & 316 & 6.336 & 1.208 \\
\hline $\begin{array}{c}\text { Todos os nós } \\
\text { enriquecidos } \\
\boldsymbol{u}_{\boldsymbol{\Gamma}}\end{array}$ & 63 & 50 & 252 & 172 & 1.008 & 632 & 4.032 & 2.416 \\
\hline $\begin{array}{c}\text { Todos os nós } \\
\text { enriquecidos } \\
\qquad \boldsymbol{\sigma} \text { e } \boldsymbol{u}_{\boldsymbol{\Gamma}}\end{array}$ & 99 & 50 & 396 & 172 & 1.584 & 632 & 6.336 & 2.416 \\
\hline
\end{tabular}


Tabela 7.27 - Graus de liberdade para a chapa tracionada com fenda central - elemento triangular com aproximação linear para o campo de tensão - FHT.

\begin{tabular}{|c|c|c|c|c|c|c|c|c|}
\hline \multirow{3}{*}{$\begin{array}{c}\text { Condições } \\
\text { de } \\
\text { Enriquecimento }\end{array}$} & \multicolumn{8}{|c|}{ Redes } \\
\hline & \multicolumn{2}{|c|}{$(3 \times 3)$} & \multicolumn{2}{|c|}{$(6 \times 6)$} & \multicolumn{2}{|c|}{$(12 \times 12)$} & \multicolumn{2}{|c|}{$(24 \times 24)$} \\
\hline & $s_{\Omega}$ & $q_{\Gamma}$ & $s_{\Omega}$ & $q_{\Gamma}$ & $s_{\Omega}$ & $q_{\Gamma}$ & $s_{\Omega}$ & $q_{\Gamma}$ \\
\hline $\begin{array}{c}\text { Sem } \\
\text { enriquecimento }\end{array}$ & 126 & 25 & 504 & 86 & 2.016 & 316 & 8.064 & 1.208 \\
\hline $\begin{array}{c}\text { Todos os nós } \\
\text { enriquecidos } \boldsymbol{\sigma}\end{array}$ & 180 & 25 & 720 & 86 & 2.880 & 316 & 11.520 & 1.208 \\
\hline $\begin{array}{c}\text { Todos os nós } \\
\text { enriquecidos } \\
\boldsymbol{u}_{\boldsymbol{\Gamma}}\end{array}$ & 126 & 50 & 504 & 172 & 2.016 & 632 & 8.064 & 2.416 \\
\hline $\begin{array}{c}\text { Todos os nós } \\
\text { enriquecidos } \\
\boldsymbol{\sigma} \text { e } \boldsymbol{u}_{\boldsymbol{\Gamma}}\end{array}$ & 180 & 50 & 720 & 172 & 2.880 & 632 & 11.520 & 2.416 \\
\hline
\end{tabular}

Tabela 7.28 - Graus de liberdade para a chapa tracionada com fenda central - elemento quadrilateral com aproximação quadrática para o campo de tensão - FHT.

\begin{tabular}{|c|c|c|c|c|c|c|c|c|}
\hline \multirow{3}{*}{$\begin{array}{c}\text { Condições } \\
\text { de } \\
\text { Enriquecimento }\end{array}$} & \multicolumn{8}{|c|}{ Redes } \\
\hline & \multicolumn{2}{|c|}{$(3 \times 3)$} & \multicolumn{2}{|c|}{$(6 \times 6)$} & \multicolumn{2}{|c|}{$(12 \times 12)$} & \multicolumn{2}{|c|}{$(24 \times 24)$} \\
\hline & $s_{\Omega}$ & $q_{\Gamma}$ & $s_{\Omega}$ & $q_{\Gamma}$ & $s_{\Omega}$ & $q_{\Gamma}$ & $s_{\Omega}$ & $q_{\Gamma}$ \\
\hline $\begin{array}{c}\text { Sem } \\
\text { enriquecimento }\end{array}$ & 108 & 25 & 432 & 86 & 1.728 & 316 & 6.912 & 1.208 \\
\hline $\begin{array}{c}\text { Todos os nós } \\
\text { enriquecidos } \boldsymbol{\sigma}\end{array}$ & 144 & 25 & 576 & 86 & 2.304 & 316 & 9.216 & 1.208 \\
\hline $\begin{array}{c}\text { Todos os nós } \\
\text { enriquecidos } \\
\boldsymbol{u}_{\boldsymbol{\Gamma}} \\
\end{array}$ & 108 & 50 & 432 & 172 & 1.708 & 632 & 6.912 & 2.416 \\
\hline $\begin{array}{c}\text { Todos os nós } \\
\text { enriquecidos } \\
\boldsymbol{\sigma} \text { e } \boldsymbol{u}_{\boldsymbol{\Gamma}}\end{array}$ & 144 & 50 & 576 & 172 & 2.304 & 632 & 9.216 & 2.416 \\
\hline
\end{tabular}

Tabela 7.29 - Graus de liberdade para a chapa tracionada com fenda central - elemento triangular com aproximação quadrática para o campo de tensão - FHT.

\begin{tabular}{cccccccccc}
\hline \multirow{2}{*}{$\begin{array}{c}\text { Condições } \\
\text { de }\end{array}$} \\
\cline { 2 - 9 } Enriquecimento & \multicolumn{8}{c}{$(3 \times 3)$} & \multicolumn{8}{c}{$(6 \times 6)$} & \multicolumn{2}{c}{$(12 \times 12)$} & \multicolumn{2}{c}{$(24 \times 24)$} \\
\cline { 2 - 9 } & $s_{\Omega}$ & $q_{\Gamma}$ & $s_{\Omega}$ & $q_{\Gamma}$ & $s_{\Omega}$ & $q_{\Gamma}$ & $s_{\Omega}$ & $q_{\Gamma}$ \\
\hline $\begin{array}{c}\text { Sem } \\
\text { enriquecimento }\end{array}$ & 216 & 25 & 864 & 86 & 3.456 & 316 & 13.824 & 1.208 \\
\hline $\begin{array}{c}\text { Todos os nós } \\
\text { enriquecidos } \sigma\end{array}$ & 270 & 25 & 1.080 & 86 & 4.320 & 316 & 17.280 & 1.208 \\
\hline $\begin{array}{c}\text { Todos os nós } \\
\text { enriquecidos } \\
\boldsymbol{u}_{\Gamma}\end{array}$ & 216 & 50 & 864 & 172 & 3.456 & 632 & 13.824 & 2.416 \\
\hline $\begin{array}{c}\text { Todos os nós } \\
\text { enriquecidos } \\
\sigma \text { e } \boldsymbol{u}_{\Gamma}\end{array}$ & 270 & 50 & 1.080 & 172 & 4.320 & 632 & 17.280 & 2.416 \\
\hline
\end{tabular}


Neste problema, no tocante à análise de autovalores das matrizes de coeficientes dos sistemas obtidos para a FHMT sem enriquecimento (elementos quadrilateral e triangular), e após aplicação das condições de contorno, verifica-se a presença de modos espúrios cinemáticos (ver tabelas 7.30 e 7.31), como já era de se esperar observando-se os resultados das tabelas 7.22 e 7.23 .

Tabela 7.30 - Autovalores para a chapa com fenda central - elemento quadrilateral - sem enriquecimento - FHMT.

\begin{tabular}{ccccc}
\hline \multirow{2}{*}{ Autovalores } & \multicolumn{4}{c}{ Redes } \\
\cline { 2 - 5 } & $(3 \times 3)$ & $(6 \times 6)$ & $(12 \times 12)$ & $(24 \times 24)$ \\
\hline Positivos & 48 & 147 & 507 & 1.875 \\
\hline Negativos & 45 & 131 & 409 & 1.397 \\
\hline Nulos & 12 & 53 & 245 & 1.061 \\
\hline
\end{tabular}

Tabela 7.31 - Autovalores para a chapa com fenda central - elemento triangular - sem enriquecimento - FHMT.

\begin{tabular}{ccccc}
\hline \multirow{2}{*}{ Autovalores } & \multicolumn{4}{c}{ Redes } \\
\cline { 2 - 5 } & $(3 \times 3)$ & $(6 \times 6)$ & $(12 \times 12)$ & $(24 \times 24)$ \\
\hline Positivos & 48 & 147 & 507 & 1.875 \\
\hline Negativos & 44 & 130 & 408 & 1.396 \\
\hline Nulos & 13 & 54 & 246 & 1.062 \\
\hline
\end{tabular}

Como comentado anteriormente, não existe garantia de solvabilidade deste problema com a presença de autovalores nulos, a menos que se aplique uma estratégia de reconhecimento e eliminação de modos espúrios para solução do sistema de equações lineares da FHMT, Freitas, Almeida e Pereira (1996).

A análise de autovalores da matriz de coeficientes da FHT (elemento quadrilateral), com aproximação constante para o campo de tensão, sem qualquer enriquecimento, apontou, após aplicação das condições de contorno, modos espúrios cinemáticos (autovalores nulos), conforme destacado na tabela 7.32. Assim, neste problema, mesmo com a condição de enriquecimento anterior verificando $\boldsymbol{s}_{\boldsymbol{\Omega}} \geq \boldsymbol{q}_{\Gamma}$, detectou-se modos espúrios cinemáticos.

Já com o elemento triangular da FHT sem enriquecimento, a análise de autovalores não apresentou modos espúrios cinemáticos para a matriz de coeficientes, tabela 7.32. 
Tabela 7.32 - Autovalores para a chapa tracionada com fenda central - sem enriquecimento - FHT.

\begin{tabular}{|c|c|c|c|c|c|c|c|c|}
\hline \multirow{3}{*}{$\begin{array}{c}\text { Base de } \\
\text { Aproximação } \\
\text { das Tensões }\end{array}$} & \multirow{3}{*}{ Rede } & \multicolumn{3}{|c|}{ Ilemento Triangular } & \multicolumn{4}{|c|}{ Elemento Quadrilateral } \\
\hline & & \multicolumn{3}{|c|}{ Autovalores } & \multirow{2}{*}{ Rede } & \multicolumn{3}{|c|}{ Autovalores } \\
\hline & & Positivo & Negativo & Nulo & & Positivo & Negativo & Nulo \\
\hline \multirow{4}{*}{ Quadrática } & $3 \times 3$ & 216 & 25 & 0 & $3 \times 3$ & 108 & 25 & 0 \\
\hline & $6 \times 6$ & 864 & 86 & 0 & $6 \times 6$ & 432 & 86 & 0 \\
\hline & $12 \times 12$ & 3.456 & 316 & 0 & $12 \times 12$ & 1.728 & 316 & 0 \\
\hline & $24 \times 24$ & 13.824 & 1.208 & 0 & $24 \times 24$ & 6.912 & 1.208 & 0 \\
\hline \multirow{4}{*}{ Linear } & $3 \times 3$ & 126 & 25 & 0 & $3 \times 3$ & 63 & 25 & 0 \\
\hline & $6 \times 6$ & 504 & 86 & 0 & $6 \times 6$ & 252 & 86 & 0 \\
\hline & $12 \times 12$ & 2.016 & 316 & 0 & $12 \times 12$ & 1.008 & 316 & 0 \\
\hline & $24 \times 24$ & 8.064 & 1.208 & 0 & $24 \times 24$ & 4.032 & 1.208 & 0 \\
\hline \multirow{4}{*}{ Constante } & $3 \times 3$ & 54 & 25 & 0 & $3 \times 3$ & 27 & 24 & 1 \\
\hline & $6 \times 6$ & 216 & 86 & 0 & $6 \times 6$ & 108 & 85 & 1 \\
\hline & $12 \times 12$ & 864 & 316 & 0 & $12 \times 12$ & 432 & 315 & 1 \\
\hline & $24 \times 24$ & 3.456 & 1.208 & 0 & $24 \times 24$ & 1.728 & 1.207 & 1 \\
\hline
\end{tabular}

Os modos espúrios cinemáticos também aparecem quando do enriquecimento exclusivo do campo de deslocamentos no contorno dos elementos triangular e quadrilateral da FHT com aproximações constante $\left(s_{\Omega} \leq \boldsymbol{q}_{\Gamma}\right)$ e linear $\left(s_{\Omega} \geq \boldsymbol{q}_{\Gamma}\right)$ das tensões no domínio.

Portanto, mesmo com as desigualdades $\boldsymbol{s}_{\boldsymbol{\Omega}} \geq \boldsymbol{q}_{\Gamma}$ (FHT) e $\boldsymbol{s}_{\boldsymbol{\Omega}} \geq \boldsymbol{q}_{\boldsymbol{\Omega}}+\boldsymbol{q}_{\Gamma}$ (FHMT) satisfeitas para algumas condições de enriquecimento, não se garante solvabilidade do problema (a condição é necessária). Por outro lado, em todos os casos onde $\boldsymbol{s}_{\boldsymbol{\Omega}}<\boldsymbol{q}_{\Gamma} \mathrm{e}$ $\boldsymbol{s}_{\boldsymbol{\Omega}}<\boldsymbol{q}_{\Omega}+\boldsymbol{q}_{\Gamma}$ (mesmo para as condições seletivas de enriquecimento), foi confirmada a presença de modos espúrios cinemáticos (autovalores nulos) que comprometem a solvabilidade dos problemas.

Para a representação dos campos de tensões/deslocamentos planos e também das curvas de convergência da energia de deformação da chapa tracionada com fenda central, serão exploradas algumas condições de enriquecimento seletivo, isto é, restrito aos nós próximos à ponta da fenda. A figura 7.79 ilustra os nós que serão enriquecidos na FHT e FHMT para a rede quadrilateral 6x6. Para as outras redes das figuras 7.77 e 7.78 são selecionados para o enriquecimento os seis nós que circundam a ponta da fenda. Vale ressaltar que, se em algum dos nós selecionados existir uma condição de contorno essencial aplicada, o campo de deslocamento de contorno da FHT/FHMT vinculado a este nó não será enriquecido. 


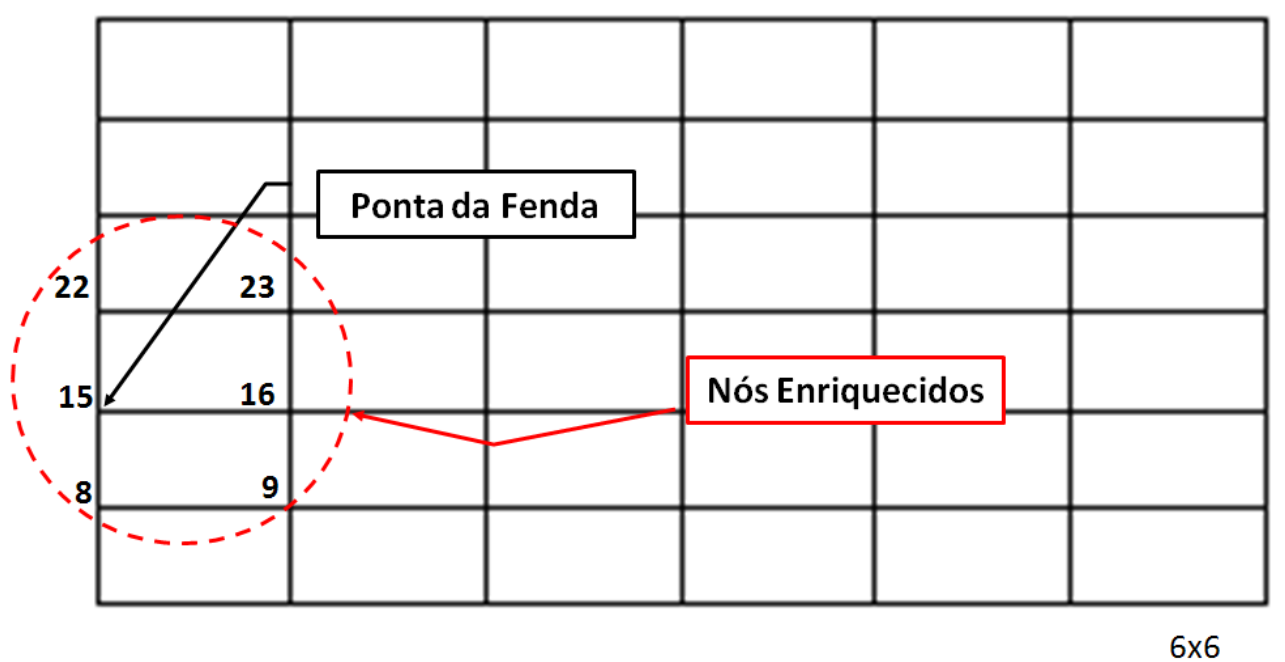

Figura 7.79 - Enriquecimento seletivo - chapa tracionado com fenda - rede quadrilateral regular 6x6.

O valor de referência da energia de deformação do problema representado na figura $7.61\left(\boldsymbol{U}_{\text {chapa com fenda }}=\boldsymbol{0 , 1 6 5}\right)$ foi obtido por meio de uma análise com o ANSYS ${ }^{\circledR}$ utilizando uma rede regular de $240 \times 240$ elementos PLANE 42 .

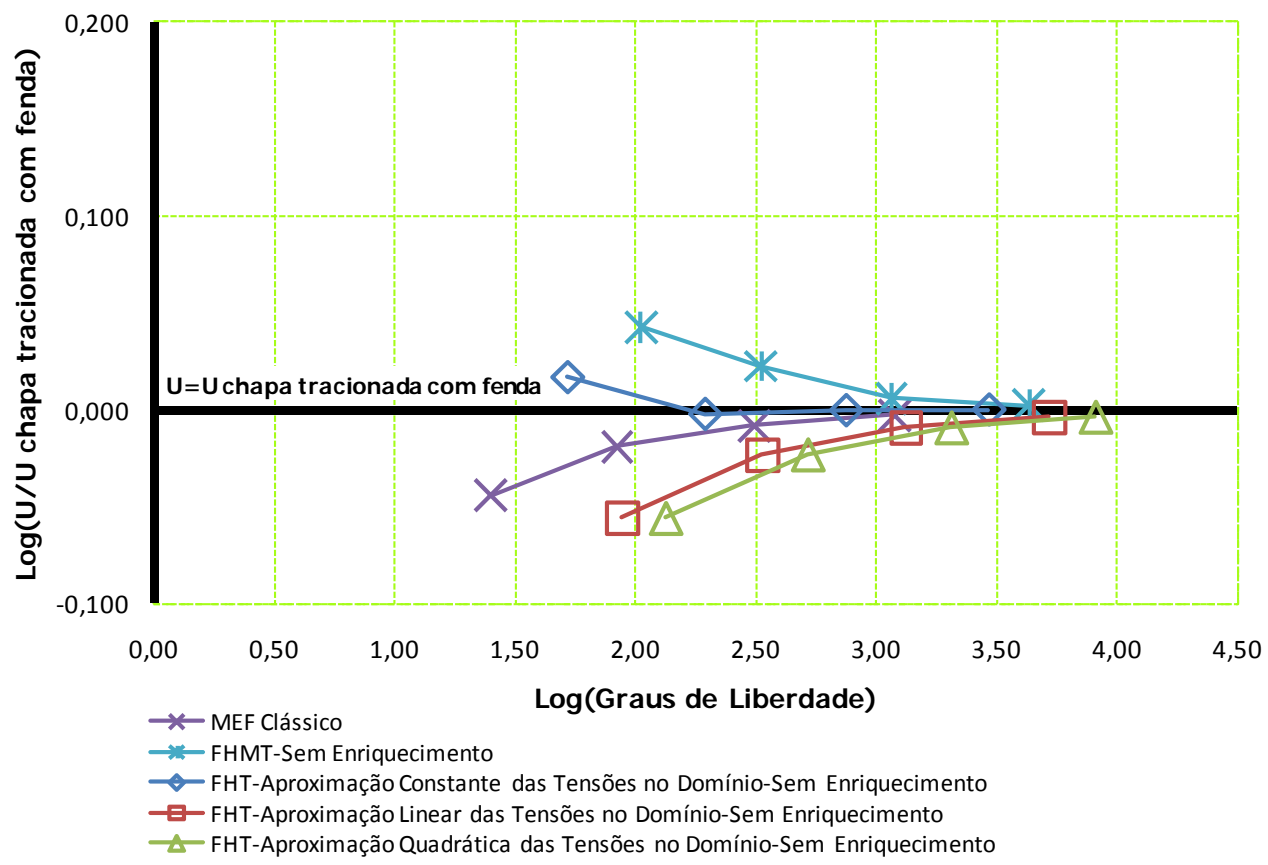

Figura 7.80 - Convergência da energia de deformação $\boldsymbol{U}_{\text {chapa tracionada com fenda }}$ - Elemento Quadrilateral - FHT, FHMT e MEF clássico.

A figura 7.80 indica que tanto o elemento quadrilateral sem enriquecimento da FHT como o da FHMT tendem a obter assintoticamente o valor da energia de deformação de referência. Pode-se observar ainda desta figura que o elemento quadrilateral sem 
enriquecimento da FHT com aproximação quadrática e linear recupera a mesma resposta obtida com o elemento quadrilateral do MEF Clássico.

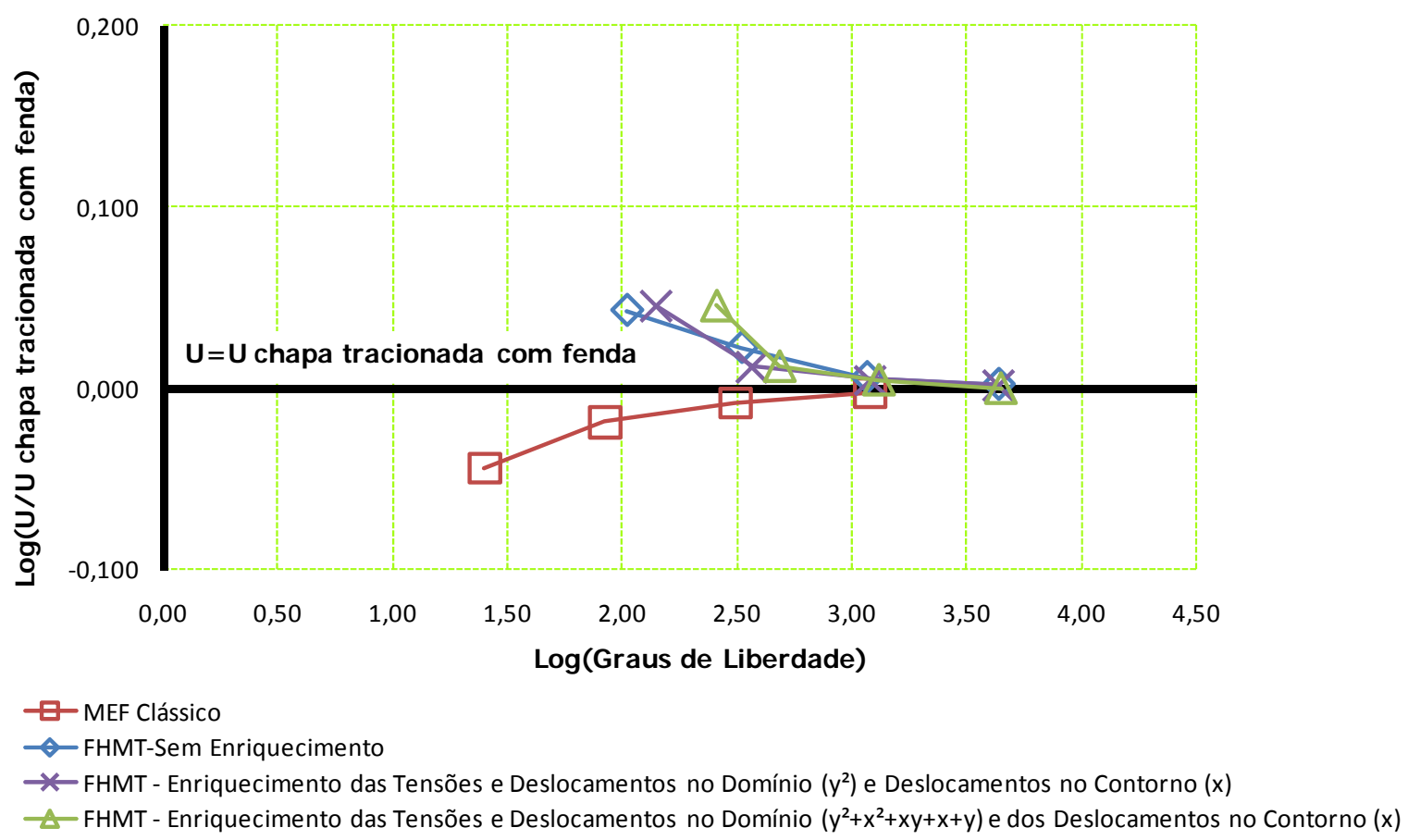

Figura 7.81 - Convergência da energia de deformação $\boldsymbol{U}_{\text {chapa tracionada com fenda }}$ - Elemento Quadrilateral - FHMT e MEF clássico.

O enriquecimento seletivo de alguns nós em torno da fenda proporcionaram uma melhor estimativa da energia de deformação para a rede quadrilateral $(\boldsymbol{6} \times \mathbf{6})$ da FHMT, ver figura 7.81. As condições de enriquecimento ilustradas na figura 7.81 indicam que o elemento quadrilateral enriquecido da FHMT também tende a recuperar o valor da energia de deformação de referência.

O elemento triangular da FHT, para todos os níveis de aproximação do campo de tensões no domínio, forneceu a mesma resposta obtida com o elemento triangular do MEF Clássico, figura 7.82 .

A figura 7.83 mostra que o enriquecimento das tensões no domínio $\left(y^{2}\right)$ e deslocamentos no contorno $(\boldsymbol{x})$ ou enriquecimento simultâneo das tensões e deslocamentos no domínio $\left(\boldsymbol{y}^{2}+\boldsymbol{x}^{2}+\boldsymbol{x}+\boldsymbol{y}+\boldsymbol{x y}\right)$ e deslocamentos no contorno $(\boldsymbol{x})$ do elemento triangular da FHMT conduziu a melhores estimativas da energia de deformação para redes menos refinadas $(6 \times 6$ e $6 \times 6)$. 


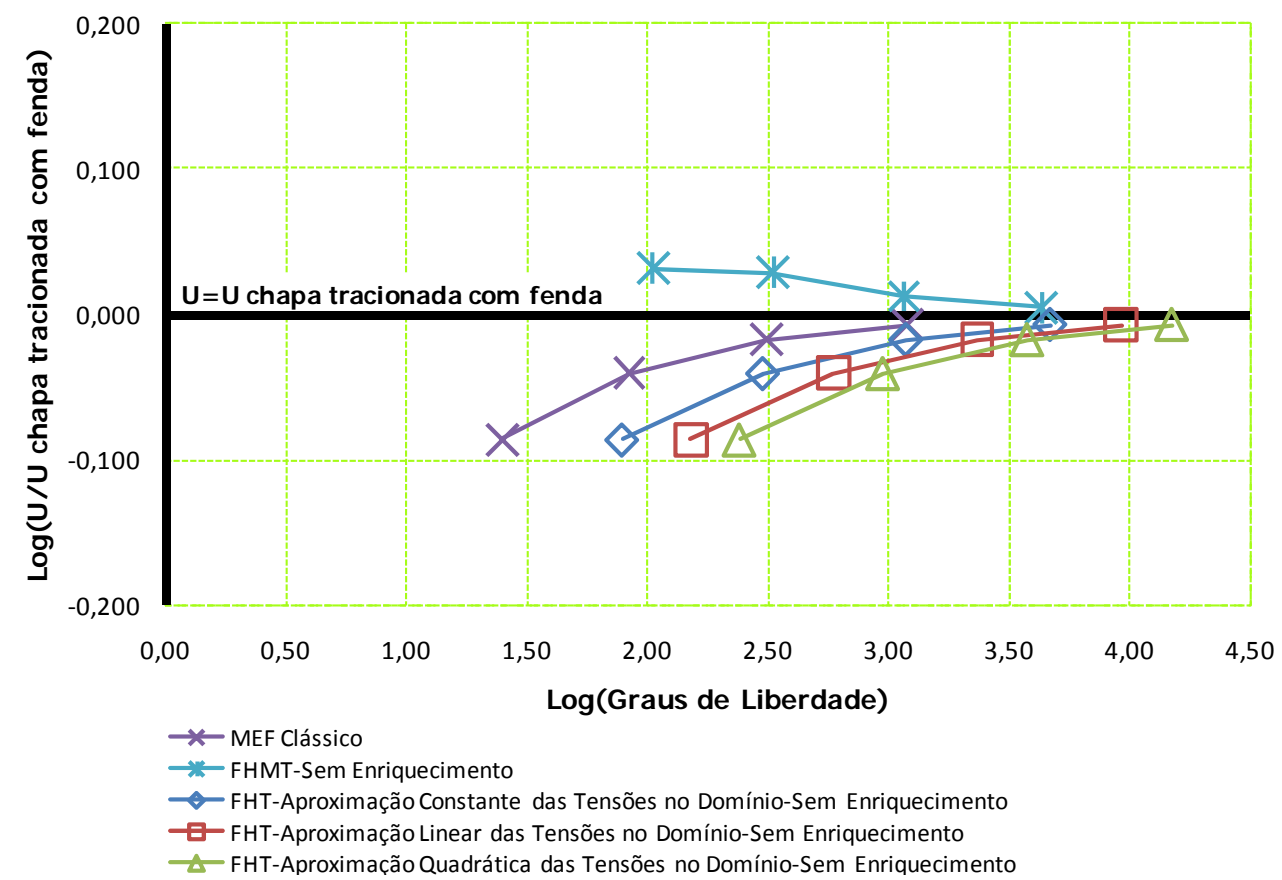

Figura 7.82 - Convergência da energia de deformação $\boldsymbol{U}_{\text {chapa tracionada com fenda }}$ - Elemento Triangular FHT, FHMT e MEF clássico.

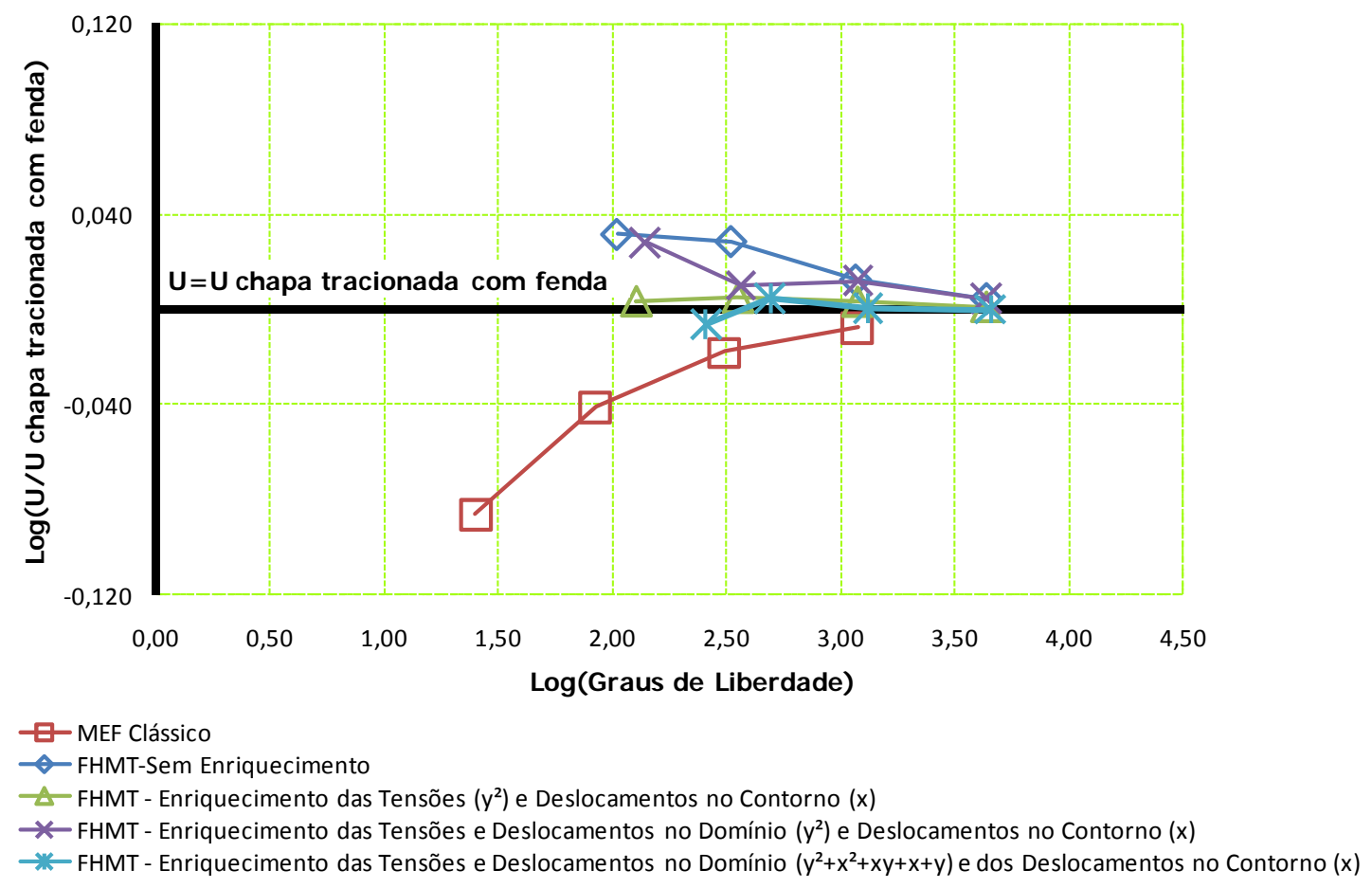

Figura 7.83 - Convergência da energia de deformação $\boldsymbol{U}_{\text {chapa tracionada com fenda }}$ - Elemento Triangular FHMT e MEF clássico.

As condições de enriquecimento seletivo aplicadas aos elementos quadrilaterais e triangulares da FHT apresentaram praticamente as mesmas respostas obtidas com estes elementos sem enriquecimento algum sobre os campos da FHT, ver figuras 7.84 e 7.85. 


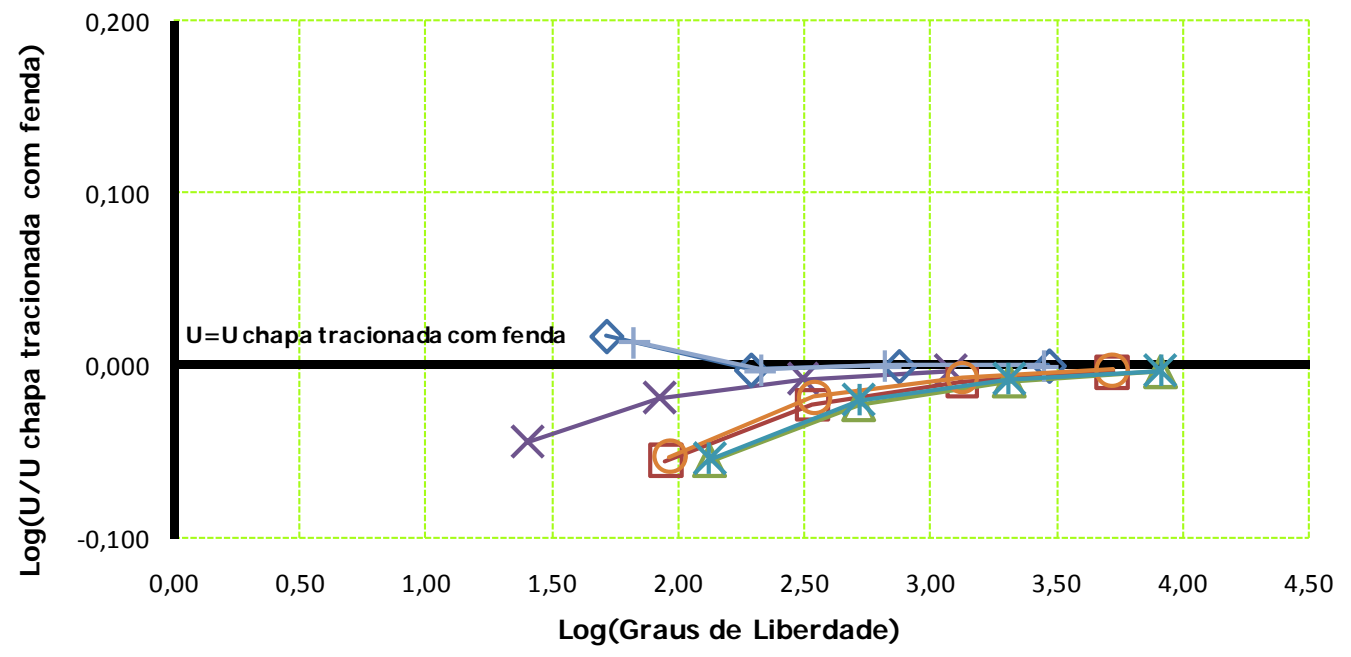

- MEF Clássico

—FHT-Aproximação Constante das Tensões no Domínio-Sem Enriquecimento

—FHT-Aproximação Linear das Tensões no Domínio-Sem Enriquecimento

_ FHT-Aproximação Quadrática das Tensões no Domínio-Sem Enriquecimento

- FHT-Aproximação Constante das Tensões no Domínio-Enriquecimento das Tensões no Domínio $\left(\mathrm{y}^{2}\right)$

- - FHT-Aproximação Linear das Tensões no Domínio-Enriquecimento dos Deslocamentos no Contorno (x)

-FHT-Aproximação Quadrática das Tensões no Domínio-Enriquecimento dos Deslocamentos no Contorno (x)

Figura 7.84 - Convergência da energia de deformação $\boldsymbol{U}_{\text {chapa tracionada com fenda }}$ - Elemento Quadrilateral - FHT e MEF clássico.

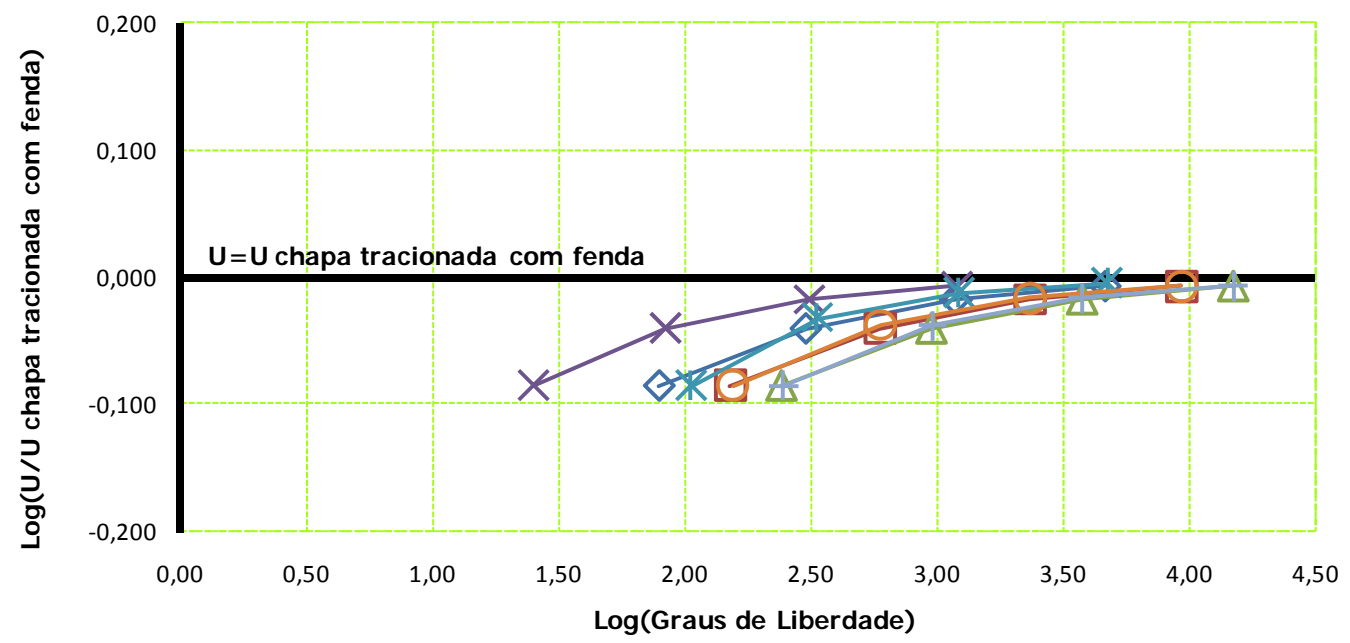

$\leftarrow$ MEF Clássico

—FHT-Aproximação Constante das Tensões no Domínio-Sem Enriquecimento

—FHT-Aproximação Linear das Tensões no Domínio-Sem Enriquecimento

$\triangle$ —FHT-Aproximação Quadrática das Tensões no Domínio-Sem Enriquecimento

- FHT-Aproximação Constante das Tensões no Domínio-Enriquecimento das Tensões no Domínio $\left(\mathrm{y}^{2}\right.$ ) e Deslocamentos no Contorno (x)

- FHT-Aproximação Linear das Tensões no Domínio-Enriquecimento dos Deslocamentos no Contorno $(x)$

+ FHT-Aproximação Quadrática das Tensões no Domínio-Enriquecimento dos Deslocamentos no Contorno $(x)$

Figura 7.85 - Convergência da energia de deformação $\boldsymbol{U}_{\text {chapa tracionada com fenda }}$ - Elemento Triangular FHT e MEF clássico.

Vale lembrar que para todos os resultados deste problema, utilizaram-se os mesmos números de pontos de Gauss/Hammer que foram aplicados ao problema da chapa tracionada. 
Representam-se, nas figuras a seguir, as tensões planas da chapa tracionada com fenda para a rede regular $24 \times 24$ (elementos quadrilaterais e triangulares) e algumas condições de enriquecimento seletivo dos campos da FHT/FHMT.
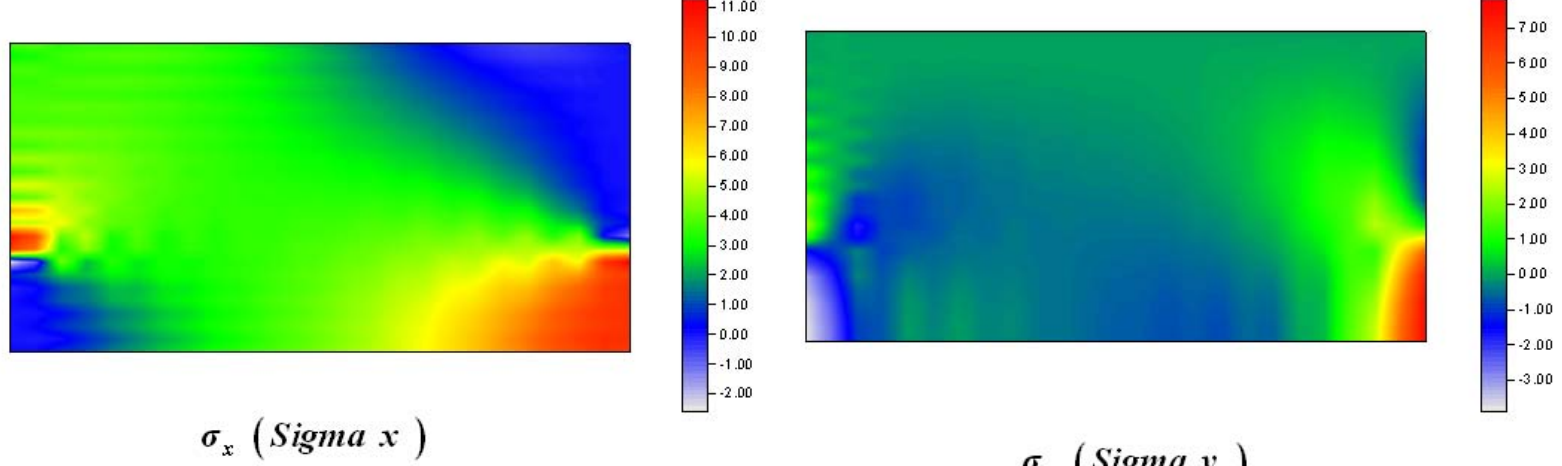
$\sigma_{y}($ Sigma $y)$

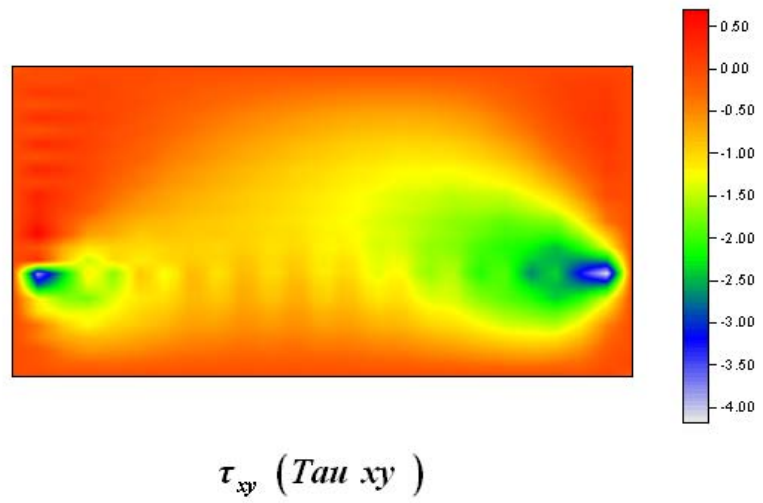

Figura 7.86 - Tensões planas para rede regular $24 \times 24$ - Elemento Quadrilateral - FHMT - sem enriquecimento.

Sabe-se, da teoria clássica da Mecânica da Fratura Elástica Linear, que a distribuição das tensões elásticas nas proximidades da extremidade de uma fenda é regida por funções singulares e tendem ao infinito. A figura 7.86 ilustra as tensões planas para o problema da chapa tracionada com fenda discretizadas com elementos quadrilaterais da FHMT. Esta figura apresenta uma região de concentração próxima à ponta da fenda, apesar da estimativa baixa dos valores das tensões planas nessa região.

O enriquecimento seletivo do elemento quadrilateral da FHMT (nós próximos à ponta da fenda) - tensões e deslocamento no domínio $\left(\boldsymbol{y}^{2}+\boldsymbol{x}^{2}+\boldsymbol{x}+\boldsymbol{y}+\boldsymbol{x y}\right)$ e deslocamentos no $\operatorname{contorno}(\boldsymbol{x})$ - melhorou a estimativa das tensões planas e definiu melhor o efeito da concentração na ponta da trinca, ver figura 7.87. 

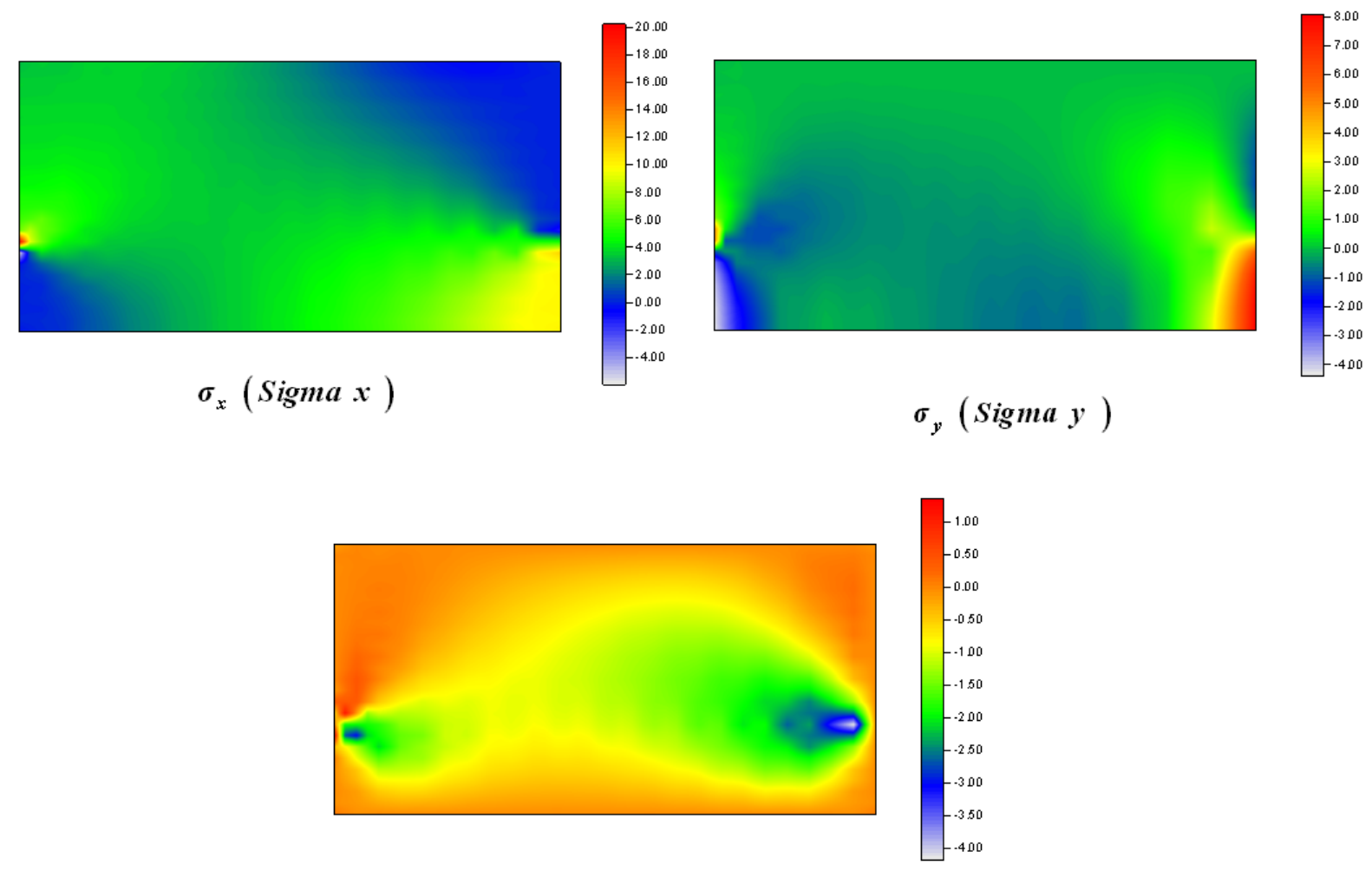

$$
\tau_{x y}(\text { Tau } x y)
$$

Figura 7.87 - Tensões planas para rede regular $24 \times 24$ - Elemento Quadrilateral - FHMT - com enriquecimento das Tensões e Deslocamentos no Domínio $\left(\boldsymbol{y}^{2}+\boldsymbol{x}^{2}+\boldsymbol{x}+\boldsymbol{y}+\boldsymbol{x y}\right)$ e dos Deslocamentos no Contorno $(\boldsymbol{x})$.

O elemento triangular sem enriquecimento da FHMT apresentou basicamente a mesma representação das tensões planas obtidas com o elemento quadrilateral da FHMT sem enriquecimento, ver figura 7.88. O elemento triangular da FHMT enriquecido seletivamente nas tensões e deslocamentos no domínio $\left(\boldsymbol{y}^{2}+\boldsymbol{x}^{2}+\boldsymbol{x}+\boldsymbol{y}+\boldsymbol{x y}\right)$ e deslocamentos no contorno $(\boldsymbol{x})$ também melhorou a definição do efeito de concentração na ponta da fenda como mostra a figura 7.89 . 


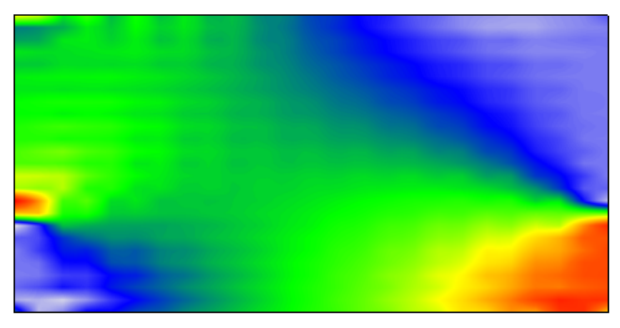

$\sigma_{x}(\operatorname{Sigma} x)$
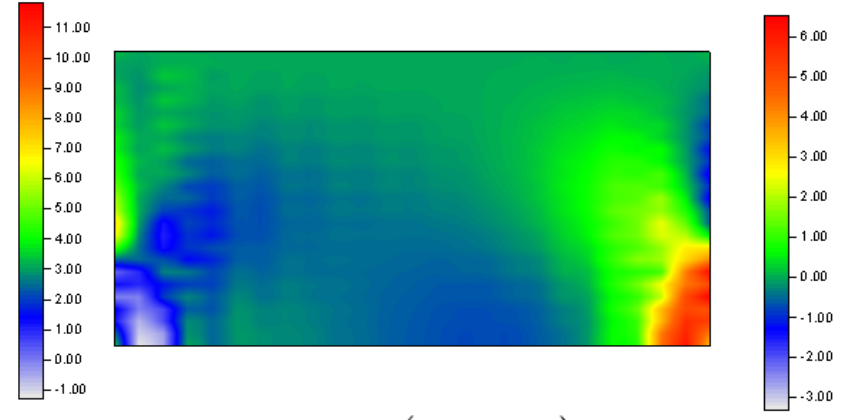

$\sigma_{y}(\operatorname{Sigma} y)$

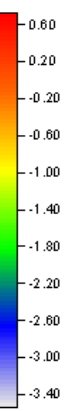

$\tau_{x y}($ Tau $x y)$

Figura 7.88 - Tensões planas para rede regular $24 \times 24$ - Elemento Triangular - FHMT - sem enriquecimento.

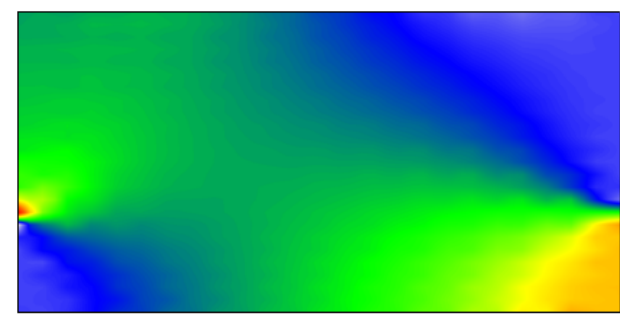

$\sigma_{x}(\operatorname{Sigma} x)$

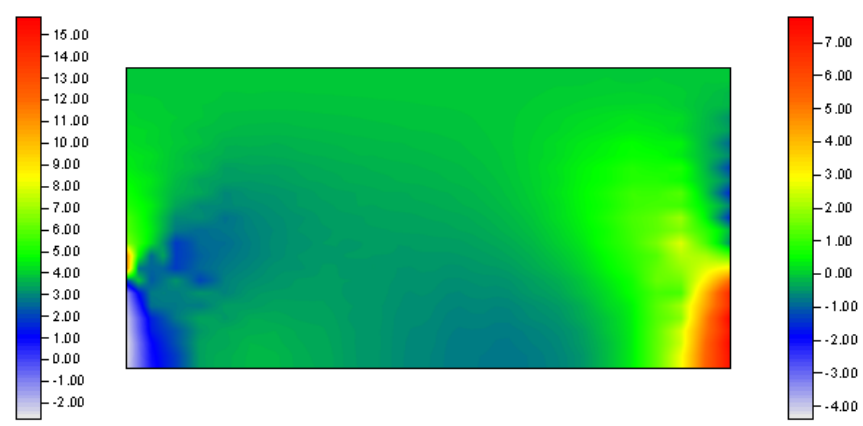

$\sigma_{y}(\operatorname{Sigma} y)$

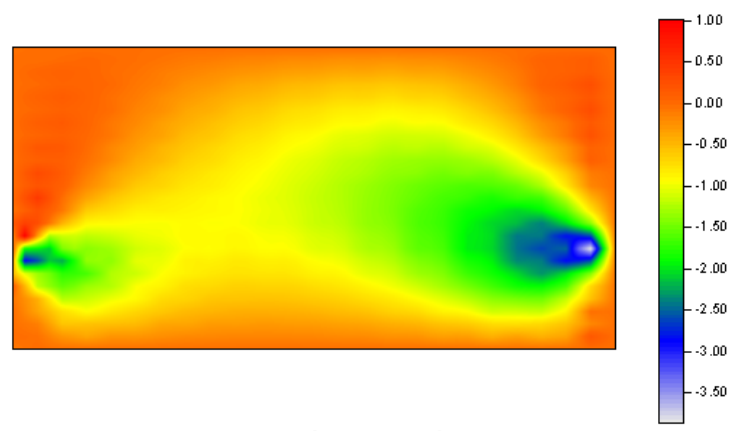

$\tau_{x y}($ Tau $x y)$

Figura 7.89 - Tensões planas para rede regular $24 \times 24$ - Elemento Triangular - FHMT - com enriquecimento das Tensões e Deslocamentos no Domínio $\left(\boldsymbol{y}^{2}+\boldsymbol{x}^{2}+\boldsymbol{x}+\boldsymbol{y}+\boldsymbol{x y}\right)$ e dos Deslocamentos no Contorno $(\boldsymbol{x})$. 
Com o elemento quadrilateral da FHT sem enriquecimento - aproximação constante das tensões - não foi possível definir precisamente o efeito da concentração de tensão na ponta da fenda, figura 7.90. Já se os campos do elemento quadrilateral da FHT - aproximação constante das tensões - forem enriquecidos seletivamente em nós próximos à ponta da trinca (tensões no domínio $\left(y^{2}\right)$ e deslocamento no contorno $(x)$ ), consegue-se uma melhor definição da concentração na ponta da fenda para o $\sigma_{x}$ (Sigma $\boldsymbol{x}$ ), como apresenta a figura 7.92.

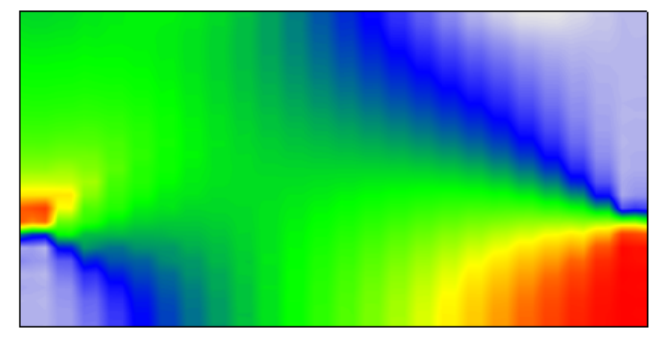

$\sigma_{x}(\operatorname{Sigma} x)$
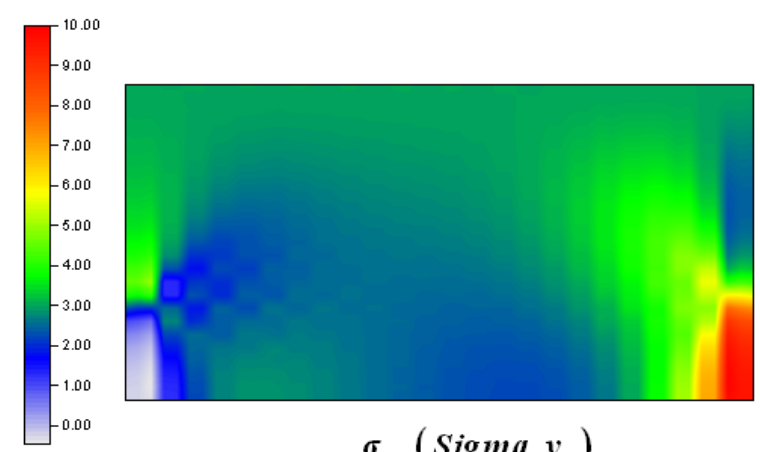

$\sigma_{y}(\operatorname{Sigma} y)$
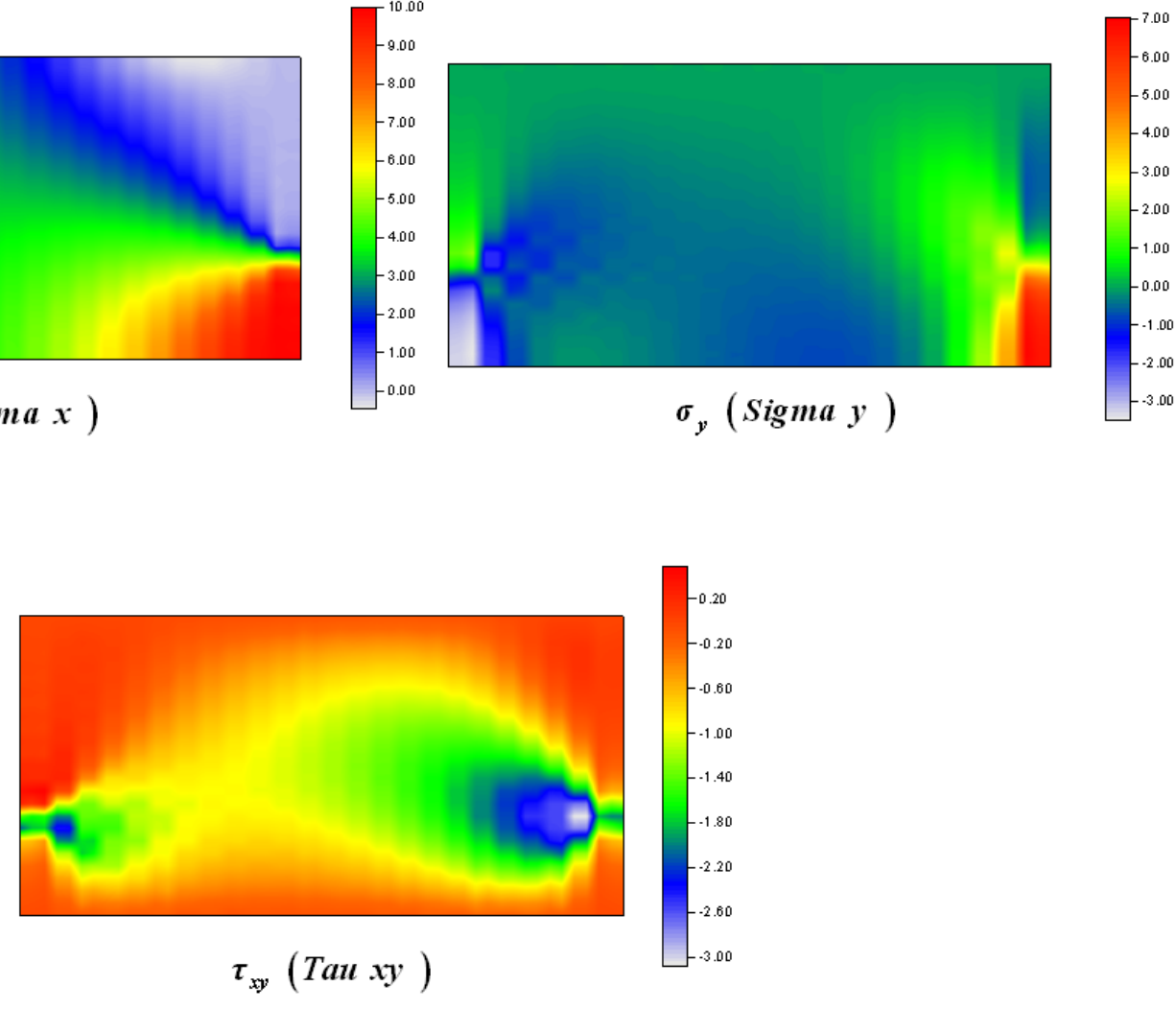

Figura 7.90 - Tensões planas para rede regular $24 \times 24$ - aproximação constante das tensões Elemento Quadrilateral - FHT - sem enriquecimento.

A figura 7.91 mostra os deslocamentos planos para o elemento quadrilateral da FHT sem enriquecimento - aproximação constante das tensões. 


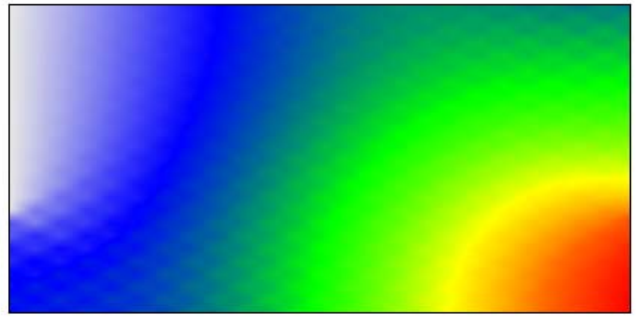

$\boldsymbol{u}_{\boldsymbol{x}}$

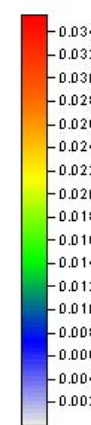

.0320
.0300
.0280
.0280
.0240
.0220
.0200
.0180
.0160
.0140
.0120
.0100
.0080
.0060
.0040
0020

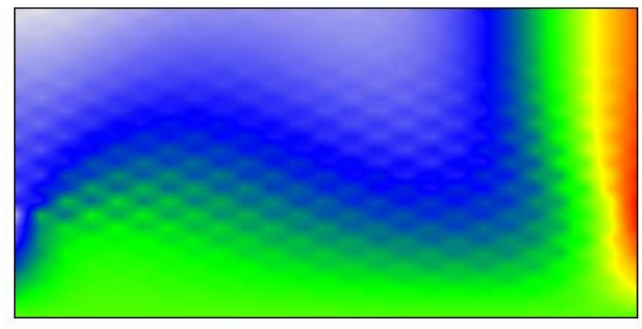

$\boldsymbol{u}_{\boldsymbol{y}}$

Figura 7.91- Aproximação típica dos deslocamentos nas direções $\boldsymbol{x}\left(\boldsymbol{u}_{\boldsymbol{x}}\right)$ e $\boldsymbol{y}\left(\boldsymbol{u}_{\boldsymbol{y}}\right)$ para rede regular $24 \times 24$ - aproximação constante das tensões - Elemento Quadrilateral - FHT sem enriquecimento.

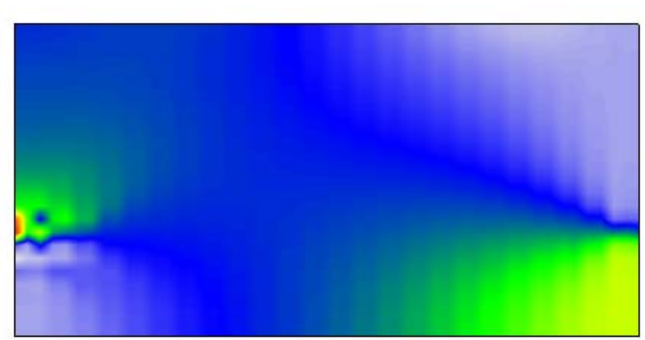

$\sigma_{x}(\operatorname{Sigma} x)$
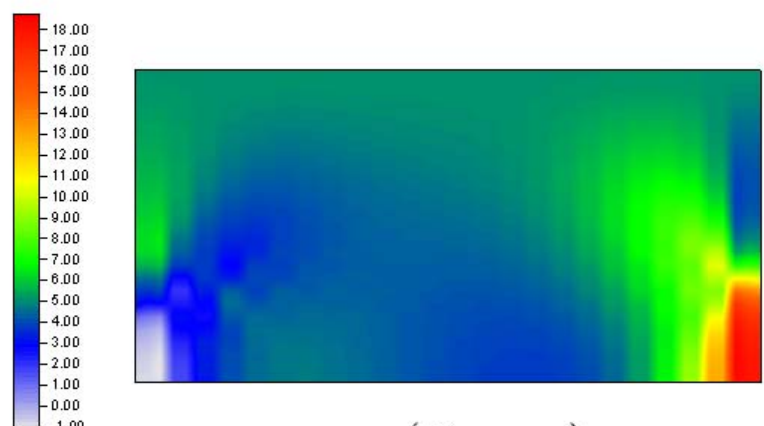

$\sigma_{y}(\operatorname{Sigma} y)$
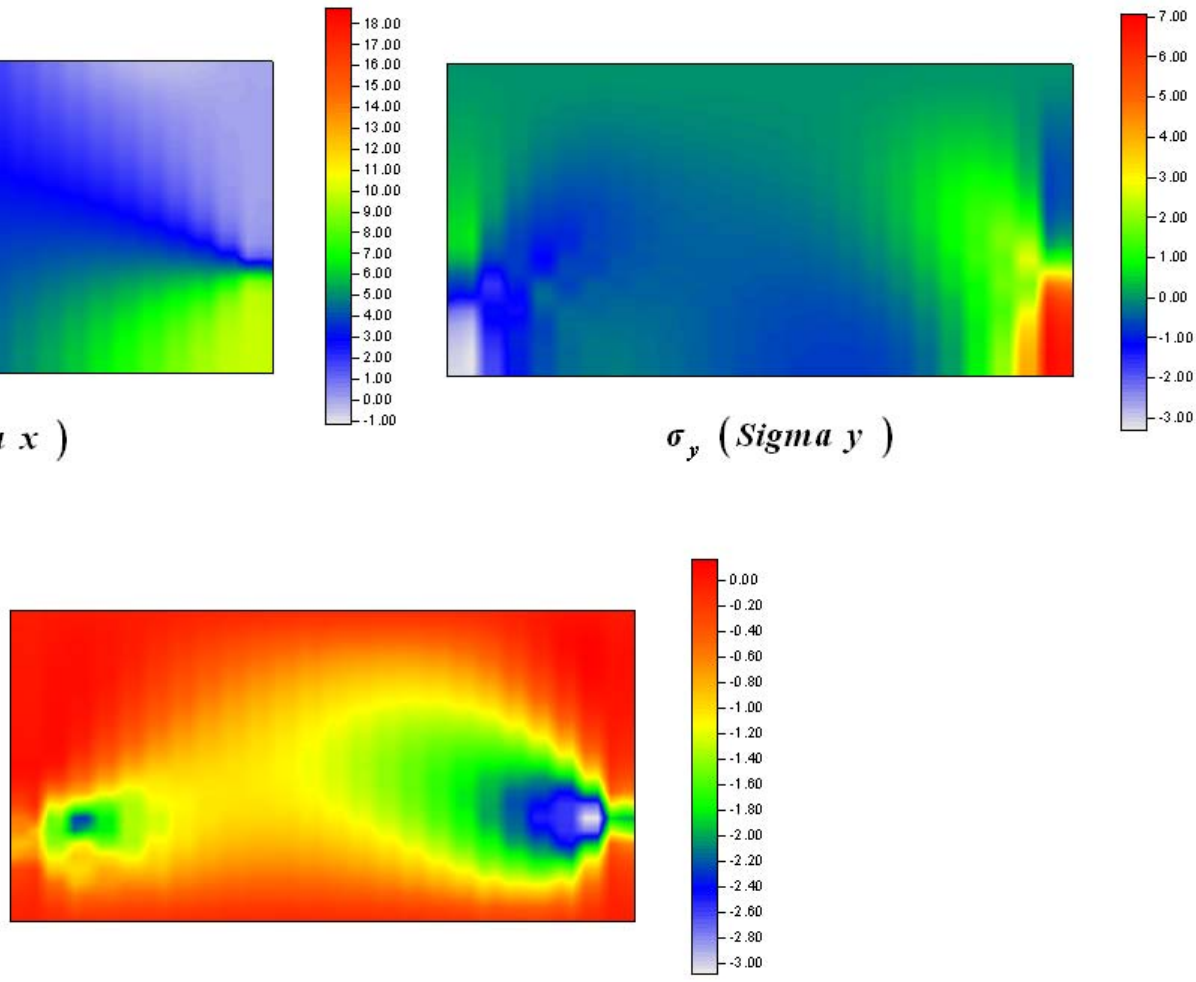

$\tau_{x y}($ Tau $x y)$

Figura 7.92 - Tensões planas para rede regular $24 \times 24$ - aproximação constante das tensões Elemento Quadrilateral - FHT - enriquecimento das Tensões no Domínio $\left(\boldsymbol{y}^{2}\right)$ e dos Deslocamentos no Contorno $(\boldsymbol{x})$.

O efeito de concentração de tensão próximo à ponta da fenda pode ser mais bem representado quando uma rede é gerada de tal forma que os seus elementos decresçam numa progressão geométrica para a ponta da região singular, (SZABÓ; BABUŠKA, 1991). 
Os elementos da rede irregular representada na figura 7.93 são dimensionados de tal forma que seus lados decrescem para a ponta da fenda e para a região da descontinuidade do carregamento, numa progressão geométrica de razão 0,15 .

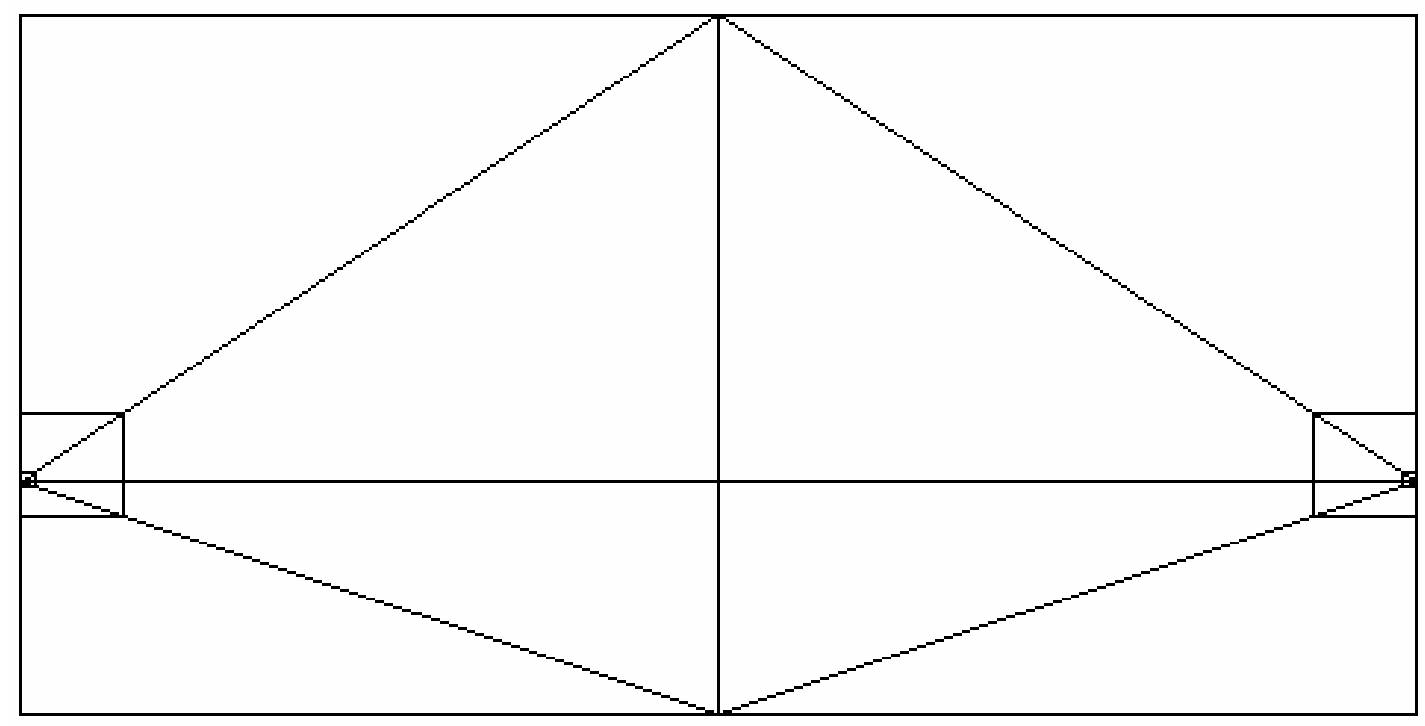

Figura 7.93 - Rede irregular com 28 elementos quadrilaterais.

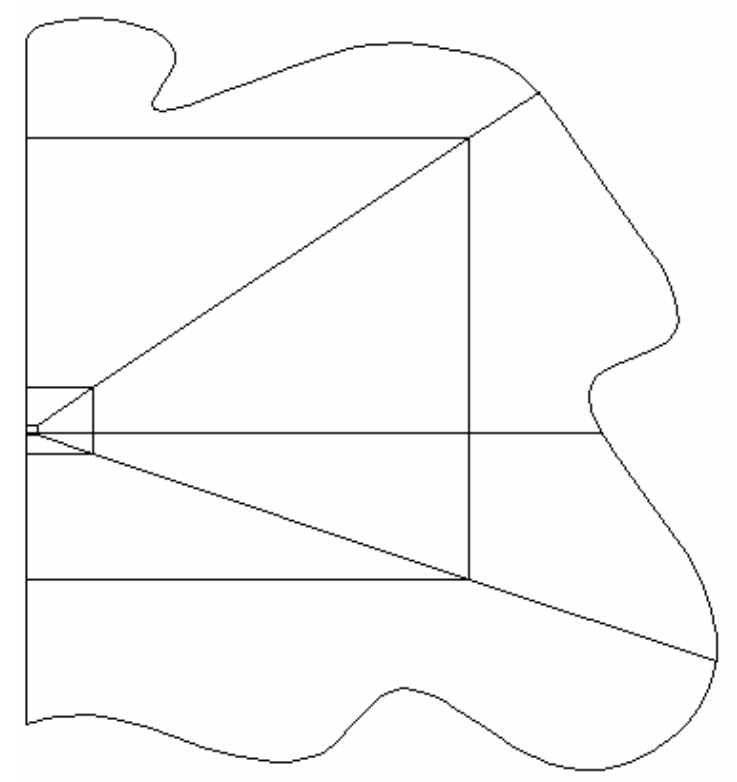

Figura 7.94 - Discretização próxima à ponta da fissura.

A figura 7.94 mostra uma ampliação da discretização próxima à ponta da fenda.

As tensões e deslocamentos planos (com aproximação quadrática das tensões) para a rede da figura 7.93 são representados, respectivamente, nas figuras 7.95 e 7.96. Da figura 
7.95, observa-se claramente a melhoria na estimativa das tensões em relação aos resultados anteriores.

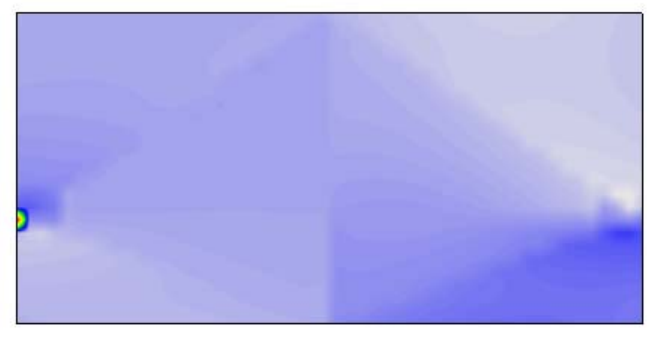

$\sigma_{x}(\operatorname{Sigma} x)$
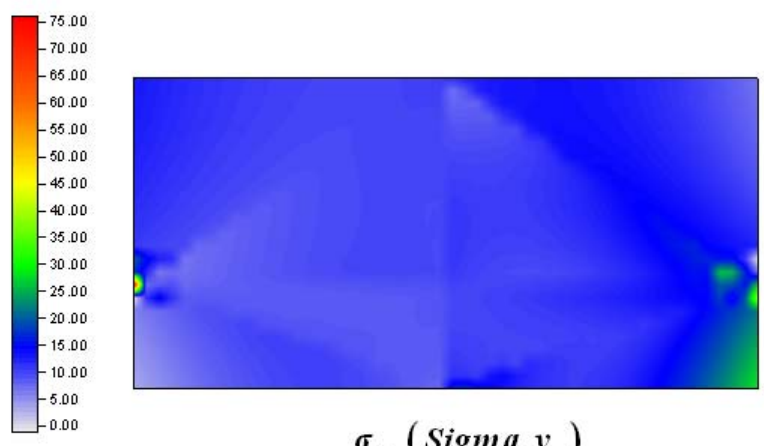

$\sigma_{y}(\operatorname{Sigma} y)$
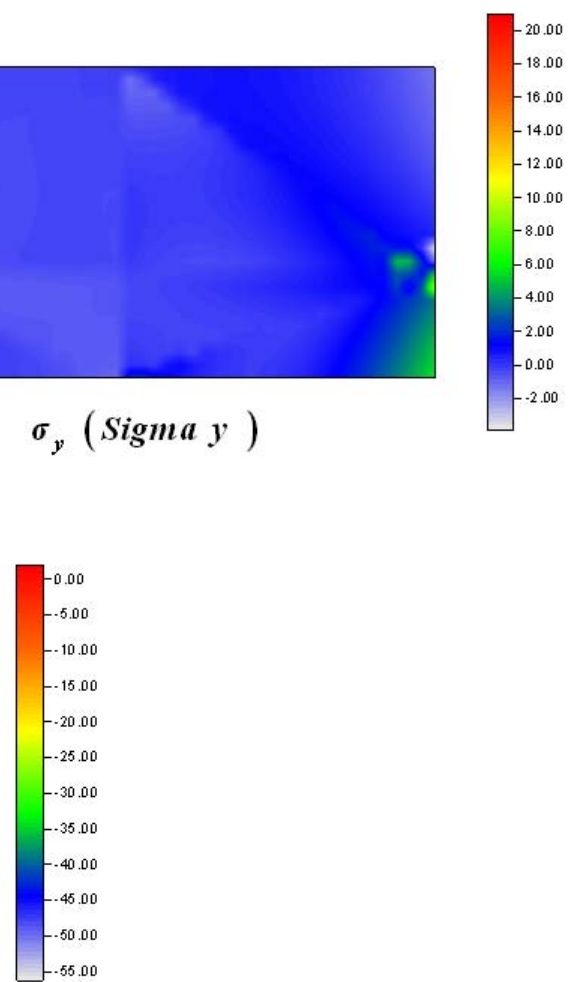

$\tau_{x y}($ Tau $x y)$

Figura 7.95 - Tensões planas para rede irregular - aproximação quadrática das tensões - Elemento Quadrilateral - FHT - sem enriquecimento.

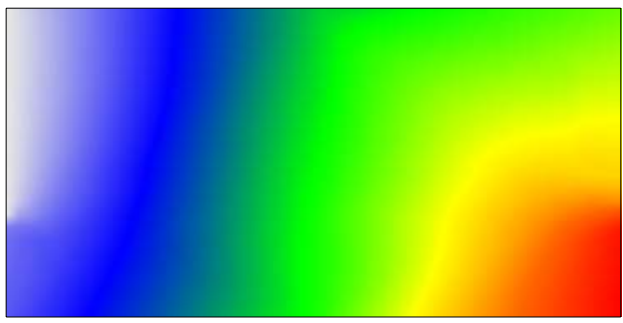

$u_{x}$

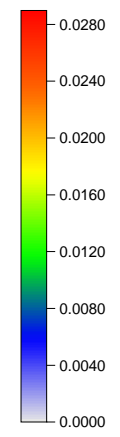

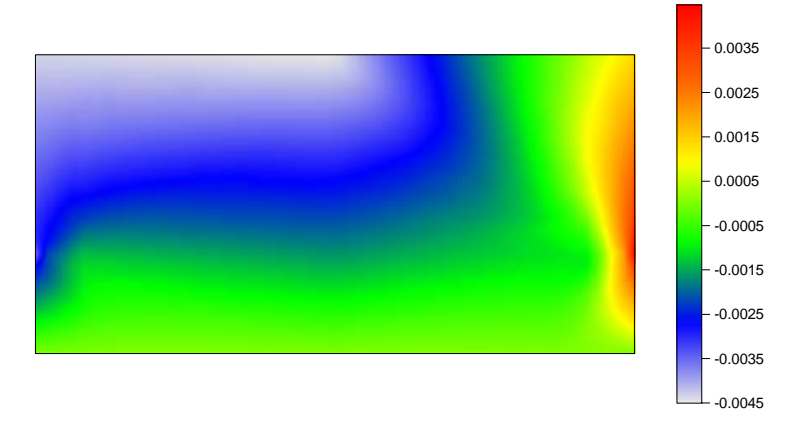

$\boldsymbol{u}_{\boldsymbol{y}}$

Figura 7.96 - Aproximação típica dos deslocamentos nas direções $\boldsymbol{x}\left(\boldsymbol{u}_{\boldsymbol{x}}\right)$ e $\boldsymbol{y}\left(\boldsymbol{u}_{\boldsymbol{y}}\right)$ para rede irregular - aproximação quadrática das tensões - Elemento Quadrilateral - FHT - sem enriquecimento.

Como complementação, representa-se as tensões e deslocamentos de referência obtidos com o ANSYS $^{\circledR}$ (utilizando uma rede regular de $240 \times 240$ elementos PLANE 42), ver figuras 7.97 e 7.98 . 

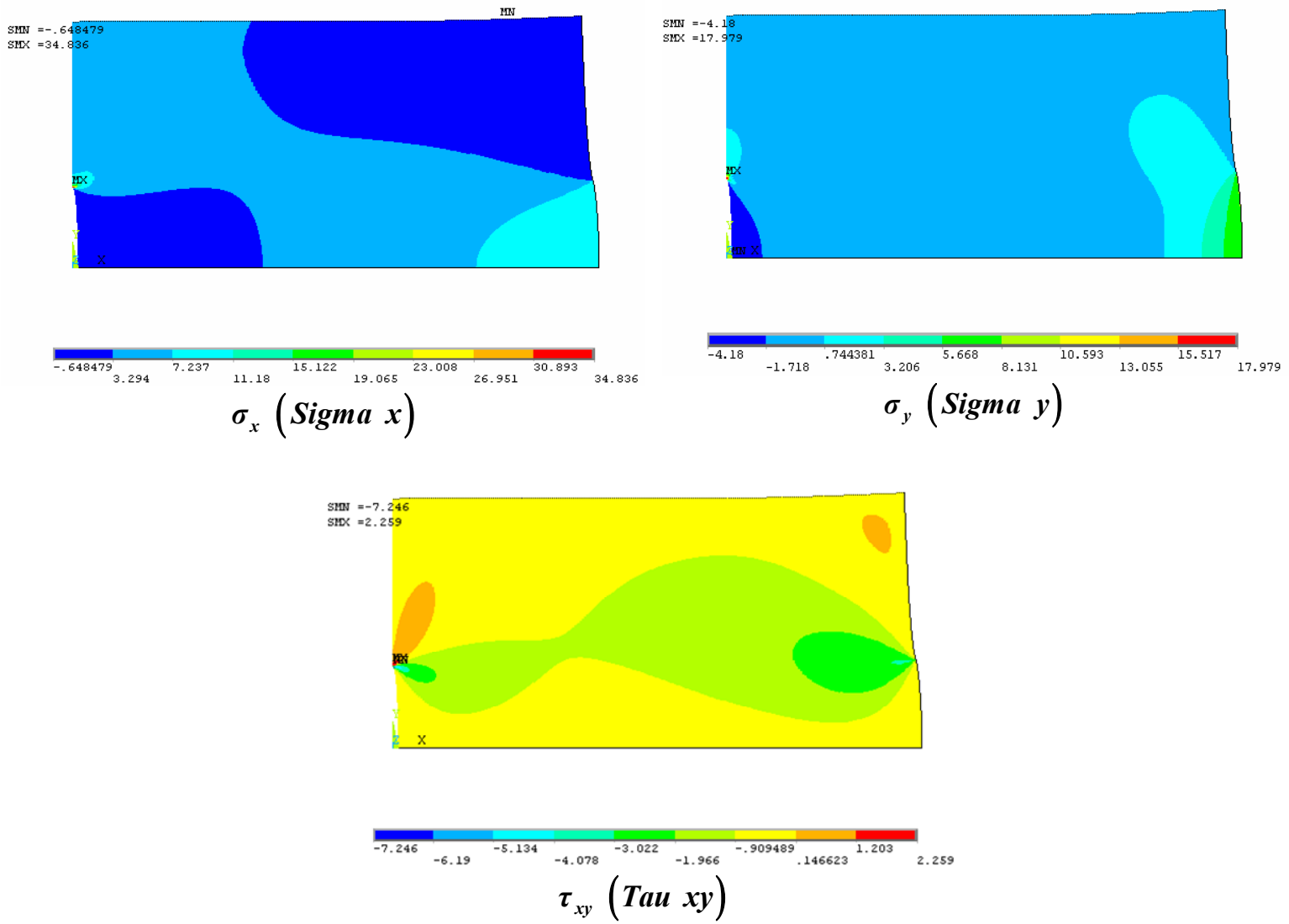

Figura 7.97 - Representação do campo de tensões para uma rede regular $240 \times 240$ elementos PLANE 42 do ANSYS ${ }^{\circledR}$.
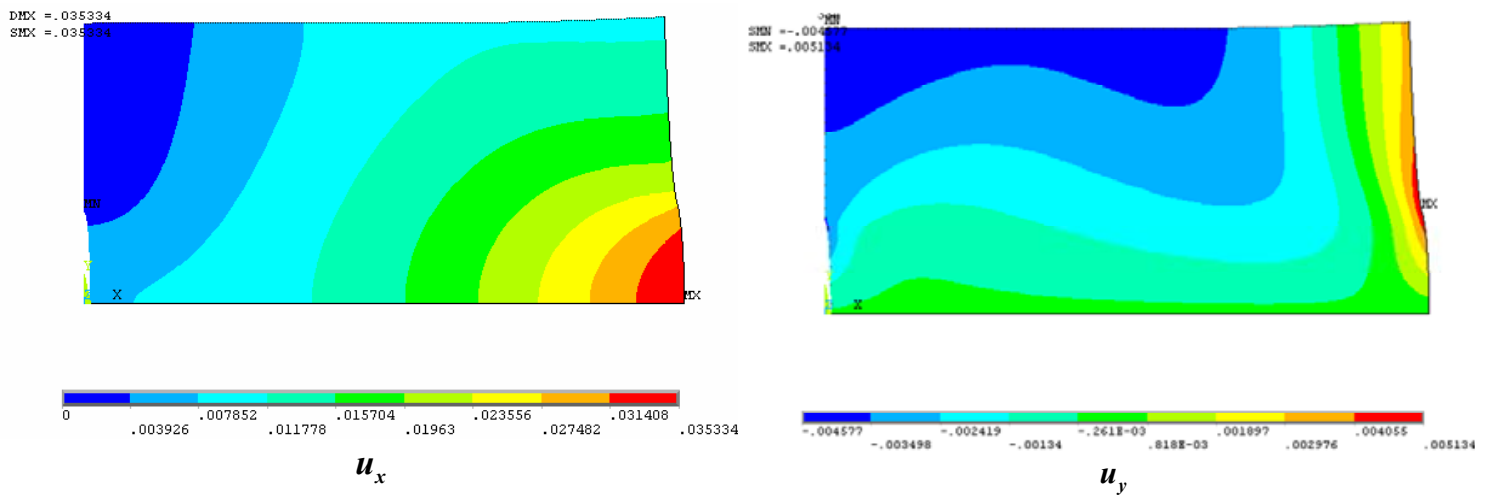

Figura 7.98 - Deslocamentos planos para uma rede regular $240 \times 240$ elementos PLANE 42 do ANSYS ${ }^{\circledR}$. 


\subsection{O Teste “inf-sup” Aplicado à FHT/FHMT com Enriquecimento Nodal: Resultados Numéricos}

\subsubsection{Introdução}

O teste “inf-sup” é de difícil aplicação pela exigência de inúmeras variações sobre as condições de contorno e seqüências de redes utilizadas nas discretizações de toda uma classe de problemas. No entanto, entende-se que sua aplicação considerando-se um número limitado de testes possa, ainda assim, fornecer indicativos de desempenho dos elementos da FHT/FHMT avaliados nesta pesquisa.

Entretanto, é importante ressaltar que se os elementos finitos quadrilaterais e triangulares da FHT/FHMT com enriquecimento nodal satisfazem o teste "inf-sup” para certo conjunto de problemas, não é possível garantir que esses mesmos elementos satisfaçam aquela condição para qualquer outro problema da classe.

Para a análise ‘indicativa’ da estabilidade dos elementos quadrilateral e triangular da FHT/FHMT, selecionaram-se dentro da classe dos problemas lineares planos três casos com comportamento mecânico e condições de contorno bem distintos: o painel de Cook (figura 7.51), a chapa tracionada (figura 7.59) e a chapa tracionada com fenda (figura 7.61).

Chapelle e Bathe (1993) sugerem que um elemento seja avaliado com o teste "inf-sup" utilizando-se redes com refinamentos que contemplam os nós da discretização anterior. Ao menos três refinamentos são recomendados para prever se $\lambda_{n}$ será limitado inferiormente por uma constante positiva, o que indica a verificação do teste. Seguindo as recomendações daquele trabalho, para aplicação do teste “inf-sup” na FHT/FHMT com enriquecimento nodal consideram-se seqüências de redes de elementos quadrilaterais e triangulares em cada um dos problemas.

As seqüências de redes quadrilaterais e triangulares $(2 \times 2,4 \times 4,8 \times 8$ e $16 \times 16)$, ilustradas na figura 7.52, são utilizadas na avaliação do painel de Cook. As redes apresentadas nas figuras 7.63 e $7.64(1 \times 1,2 \times 2,4 \times 4,8 \times 8$ e $16 \times 16)$ compõem as discretizações para a chapa tracionada. Já para o problema da chapa com fenda adotam-se as redes das figuras 7.77 e $7.78(3 \times 3,6 \times 6,12 \times 12$ e $24 \times 24)$.

Para cada uma das redes regulares apresentadas as matrizes $\mathbf{S}=\mathbf{T}$ e $\mathbf{B}$ foram montadas e o valor de $\lambda_{n}$ (inf-sup) calculado. Os resultados obtidos estão aqui representados na forma de gráficos $\log (1 / N) \times \log \left(\lambda_{n}\right)$ ( $N$ é a soma do número total de parâmetros de tensão e de graus de liberdade em deslocamentos). Se a curva $\log (1 / N) \times \log \left(\lambda_{n}\right)$ converge 
assimptoticamente para um determinado valor $\lambda_{n}>\boldsymbol{0}$, conclui-se que os elementos quadrilaterais e triangulares da FHT/FHMT satisfazem o teste “inf-sup”, indicando convergência da solução.

Primeiramente, os três problemas são analisados na situação sem enriquecimento. Posteriormente, as condições de enriquecimento sobre os campos envolvidos na FHT/FHMT com funções polinomiais são avaliadas.

\subsubsection{Painel de Cook}

Para as sequências de redes irregulares adotadas, os resultados do teste "inf-sup" para o elemento quadrilateral da FHT/FHMT na situação sem enriquecimento são ilustrados na figura 7.99.

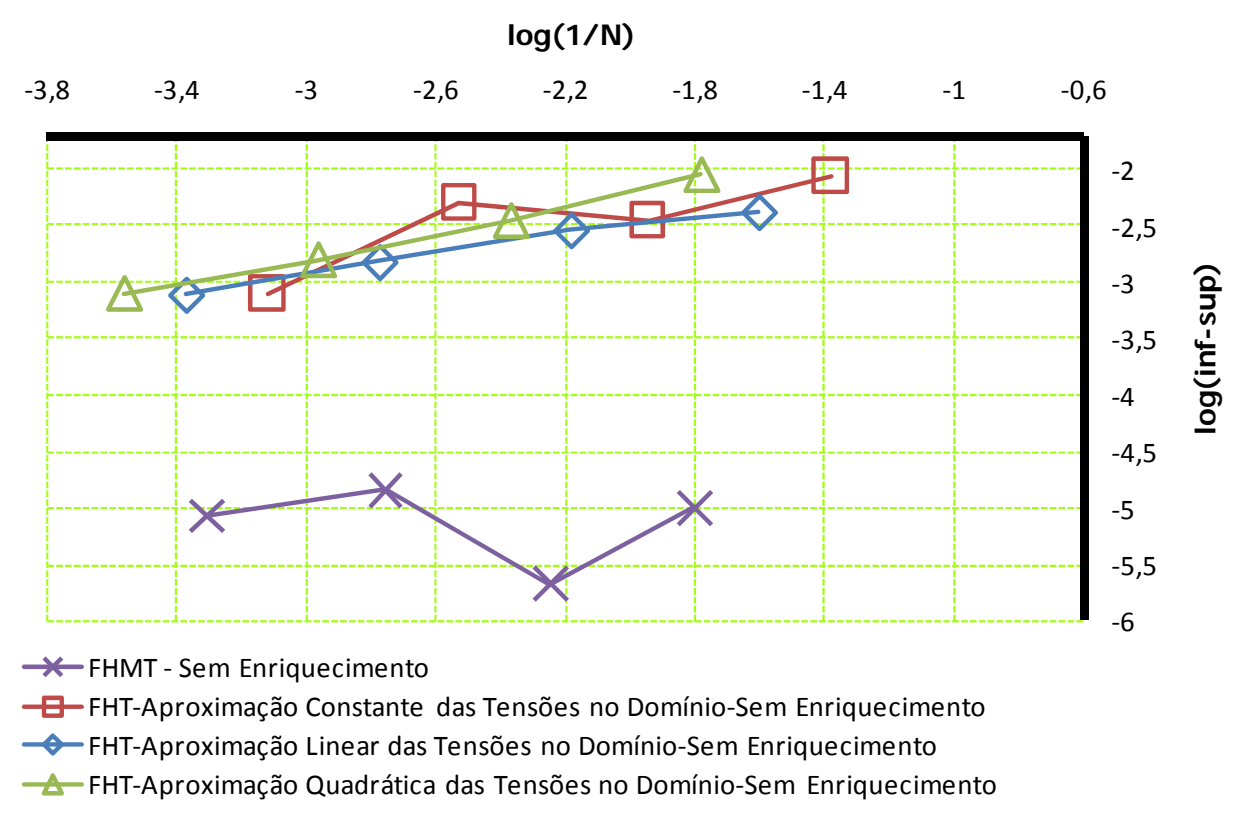

Figura 7.99 - Resultados do teste “inf-sup” - Elemento Quadrilateral - FHT/FHMT sem enriquecimento.

A figura 7.99 indica que o elemento quadrilateral da FHT/FHMT sem enriquecimento não satisfaz o teste "inf-sup", pois as curvas apontam para $\lambda_{n} \rightarrow \boldsymbol{0}$.

A seguir apresentam-se os resultados do teste para algumas combinações de enriquecimento dos campos envolvidos na FHT/FHMT. Vale lembrar que para o painel de Cook os enriquecimentos, quando aplicados, foram realizados na totalidade dos nós de domínio, bem como em todos os nós do contorno que não possuem condição de contorno essencial prescrita. 


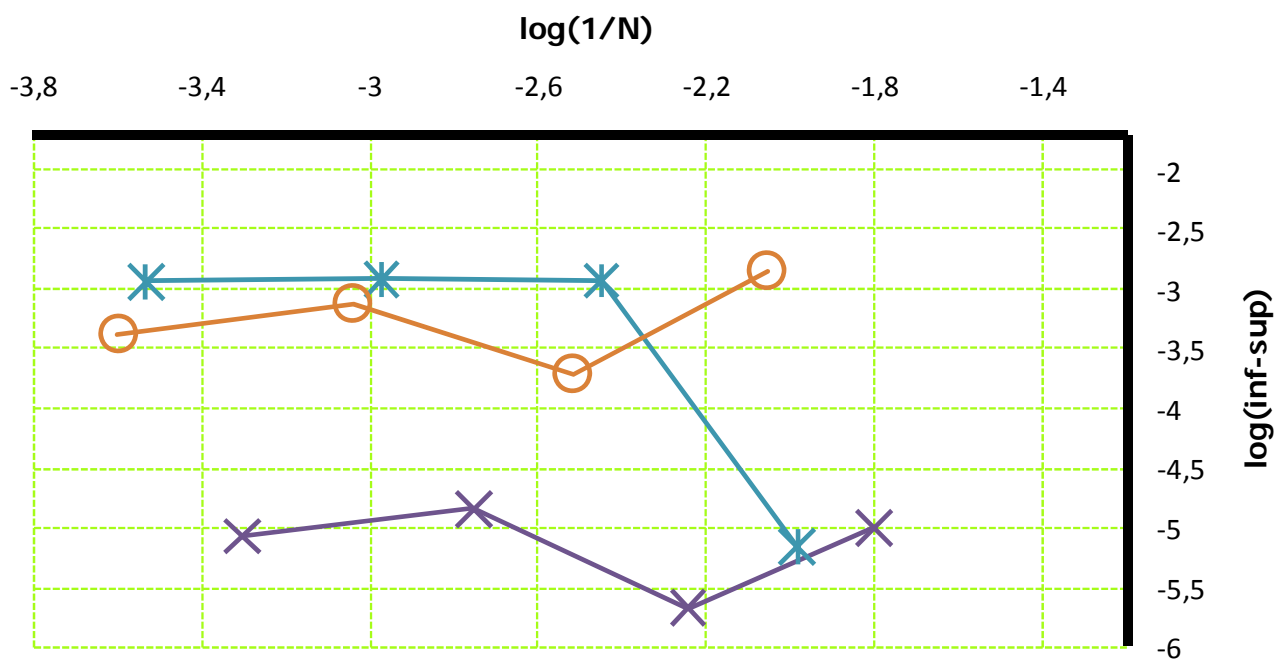

* FHMT - Sem Enriquecimento

- FHMT - Enriquecimento das Tensões no Domínio $\left(\mathrm{y}^{2}\right)$ e Deslocamentos no Contorno $(x)$

- FHMT-Enriquecimento das Tensões e Deslocamentos no Domínio $\left(\mathrm{y}^{2}\right)$ e Deslocamentos no Contorno $(\mathrm{x})$

Figura 7.100 - Resultados do teste “inf-sup” - Elemento Quadrilateral - FHMT com enriquecimento.

Especificamente para o elemento quadrilateral da FHMT enriquecido nas tensões no domínio $\left(\boldsymbol{y}^{2}\right)$ e deslocamentos no contorno $(\boldsymbol{x})$, conseguiu-se obter $\boldsymbol{\lambda}_{\boldsymbol{n}}>\boldsymbol{0}$, ver figura 7.100 .

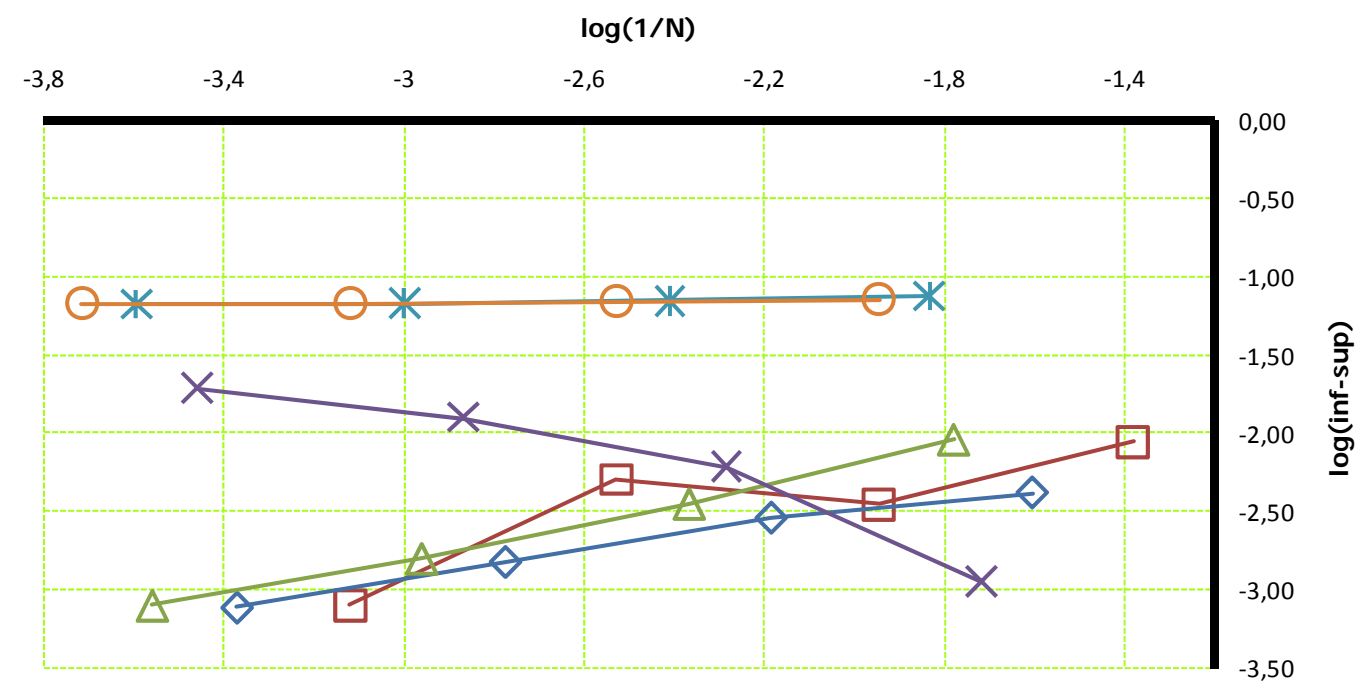

\footnotetext{
—FHT-Aproximação Constante das Tensões no Domínio-Sem Enriquecimento

$\smile$ FHT-Aproximação Linear das Tensões no Domínio-Sem Enriquecimento

_ FHT-Aproximação Quadrática das Tensões no Domínio-Sem Enriquecimento

* FHT-Aproximação Constante das Tensões no Domínio-Enriquecimento das Tensões no Domínio $\left(\mathrm{y}^{2}\right)$ e Deslocamentos no Contorno (x)

- FHT-Aproximação Linear das Tensões no Domínio-Enriquecimento das Tensões no Domínio $\left(\mathrm{y}^{2}\right.$ ) e Deslocamentos no Contorno (x)

- FHT-Aproximação Quadrática das Tensões no Domínio-Enriquecimento das Tensões no Domínio ( $\mathrm{y}^{2}$ ) e Deslocamentos no Contorno ( $\mathrm{x}$ )
}

Figura 7.101 - Resultados do teste “inf-sup” - Elemento Quadrilateral - FHT com enriquecimento. 
Todas as combinações de enriquecimentos sobre o elemento quadrilateral da FHT, postados na figura 7.101, apontam para a existência de $\boldsymbol{\lambda}_{\boldsymbol{n}}>\boldsymbol{0}$.

Entretanto, com o enriquecimento exclusivo dos deslocamentos no contorno $(\boldsymbol{x})$ para o elemento quadrilateral da FHT (considerando-se todas as bases aproximativas dos campos de tensão no domínio) não se obteve convergência para $\lambda_{n}>\boldsymbol{0}$, ver figura 7.102.

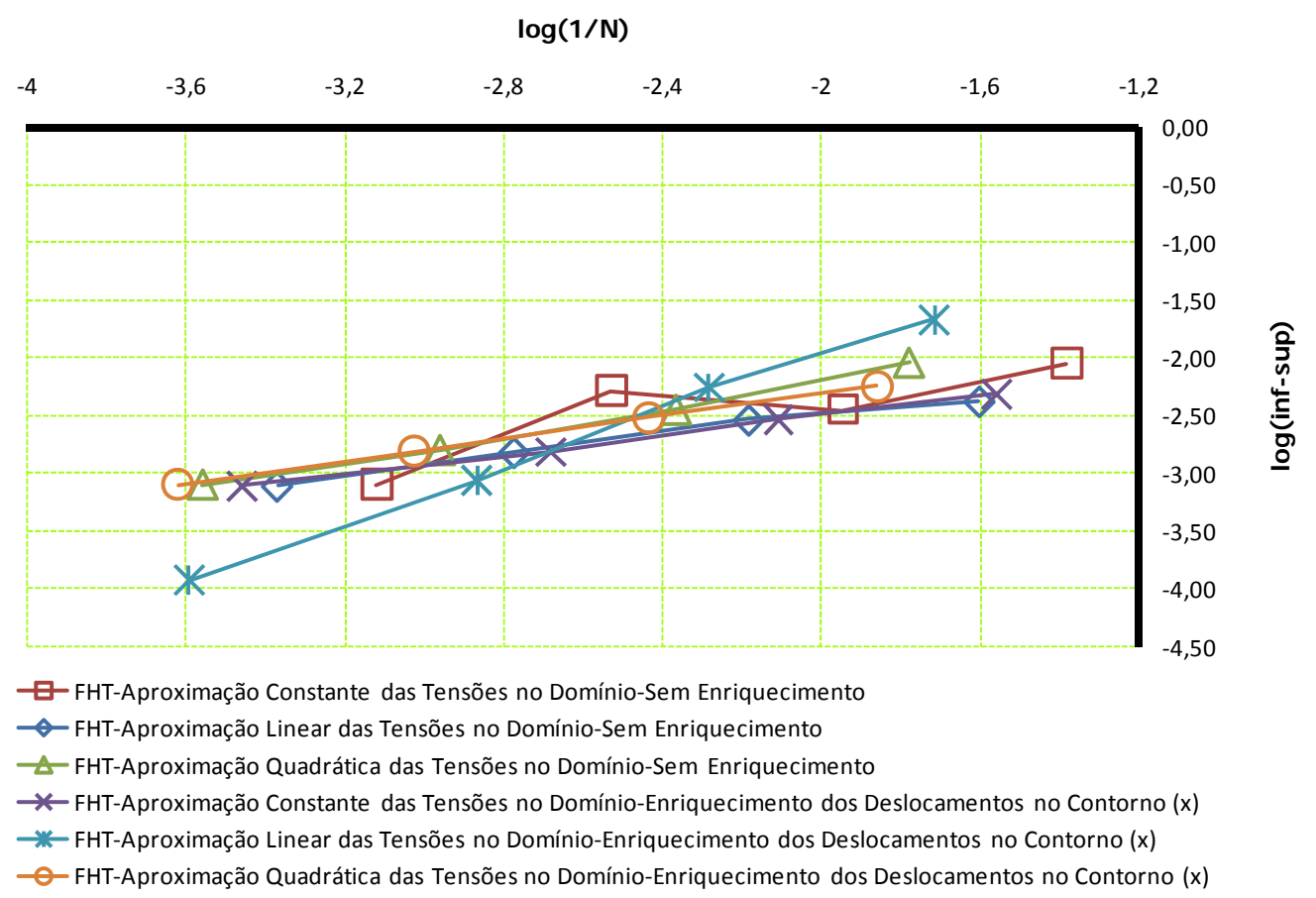

Figura 7.102 - Resultados do teste “inf-sup” - Elemento Quadrilateral - FHT com enriquecimento.

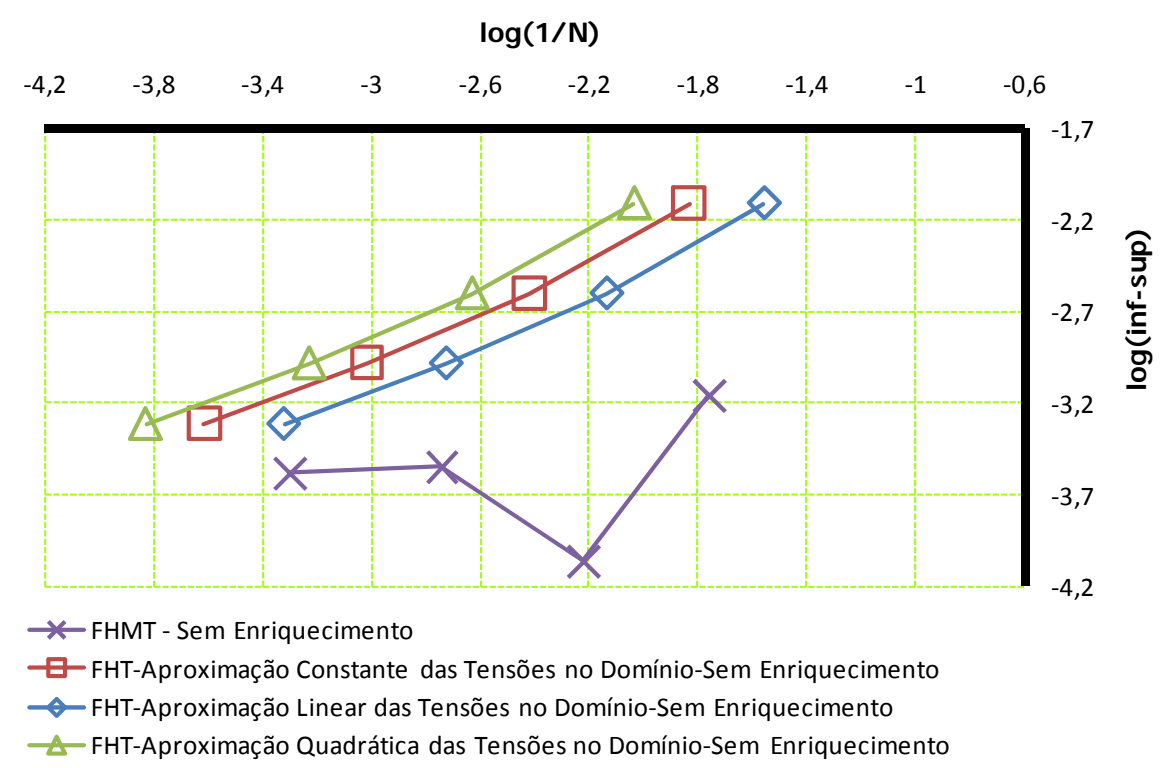

Figura 7.103 - Resultados do teste “inf-sup" - Elemento Triangular - FHT/FHMT sem enriquecimento. 
O elemento triangular da FHT/FHMT sem enriquecimento apresentou basicamente a mesma resposta do elemento quadrilateral sem enriquecimento, ou seja, não foi possível obter $\lambda_{n}>\mathbf{0}$, como mostra a figura 7.103.

$\log (1 / N)$

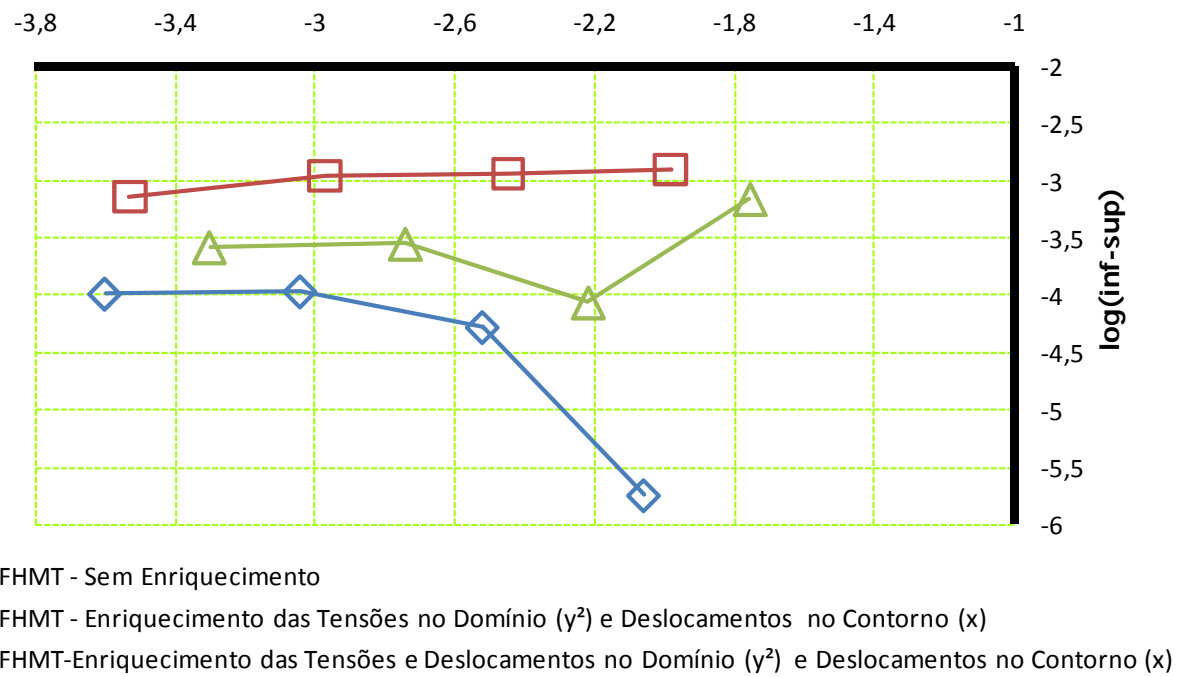

Figura 7.104 - Resultados do teste “inf-sup” - Elemento Triangular - FHMT com enriquecimento.

Os resultados da figura 7.104 mostram que com o enriquecimento do elemento triangular da FHMT nas tensões no domínio $\left(\boldsymbol{y}^{2}\right)$ e deslocamento no contorno $(\boldsymbol{x})$, conseguiu-se obter $\lambda_{n}>\boldsymbol{0}$.

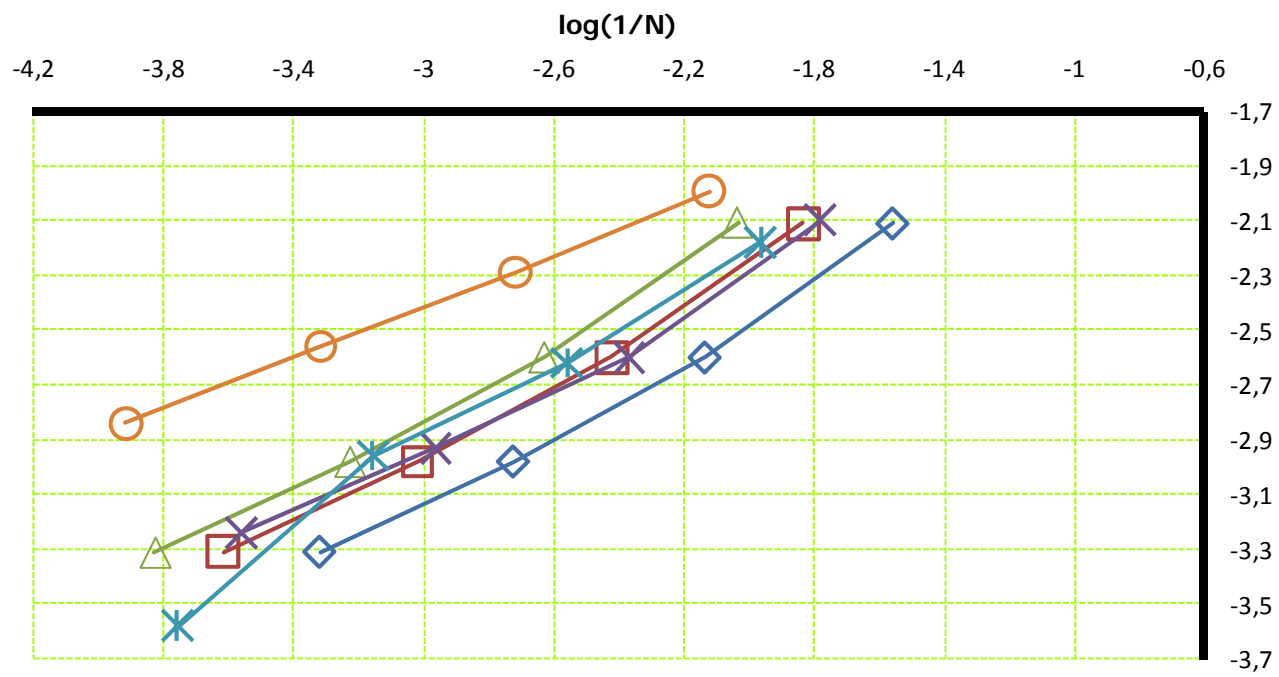

—-FHT-Aproximação Constante das Tensões no Domínio-Sem Enriquecimento

$\smile$ FHT-Aproximação Linear das Tensões no Domínio-Sem Enriquecimento

$\triangle$ FHT-Aproximação Quadrática das Tensões no Domínio-Sem Enriquecimento

* FHT-Aproximação Constante das Tensões no Domínio-Enriquecimento das Tensões no Domínio $\left(\mathrm{y}^{2}\right)$

*-FHT-Aproximação Linear das Tensões no Domínio-Enriquecimento das Tensões no Domínio $\left(\mathrm{y}^{2}\right)$

- FHT-Aproximação Quadrática das Tensões no Domínio-Enriquecimento das Tensões no Domínio $\left(\mathrm{y}^{2}\right)$

Figura 7.105 - Resultados do teste “inf-sup” - Elemento Triangular - FHT com enriquecimento. 
Na FHT é importante o enriquecimento simultâneo das tensões e deslocamentos. De fato, quando se enriquece somente tensões no domínio $\left(\boldsymbol{y}^{2}\right)$, figura 7.105, ou deslocamentos no contorno $(\boldsymbol{x})$, figura 7.106, não se consegue obter $\lambda_{n}>\mathbf{0}$ para o elemento triangular.

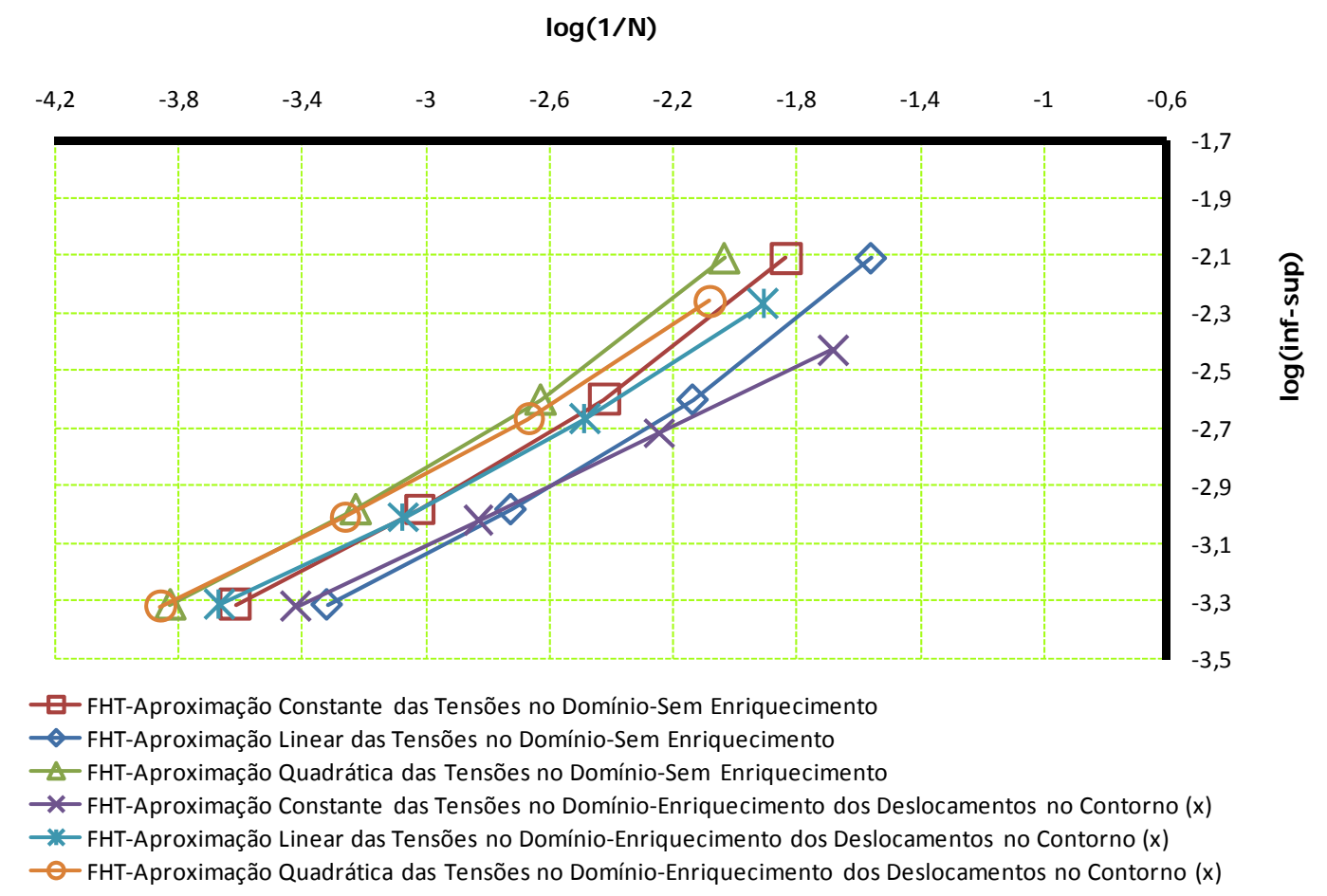

Figura 7.106 - Resultados do teste “inf-sup” - Elemento Triangular - FHT com enriquecimento.

\subsubsection{Chapa Tracionada}

Os resultados do teste "inf-sup” para o conjunto de redes regulares quadrilaterais da FHT/FHMT sem enriquecimento, utilizadas na discretização deste problema, são apresentados nas figuras 7.107 e 7.108.

Na figura 7.107, verifica-se que o elemento quadrático da FHT sem enriquecimento satisfaz o teste "inf-sup", com indicativo de $\boldsymbol{\lambda}_{\boldsymbol{n}}>\mathbf{0}$ em todos os níveis da aproximação das tensões no domínio. Com o elemento quadrilateral da FHMT, a condição de estabilidade $\left(\lambda_{n}>0\right)$ é clara, ver figura 7.108. 


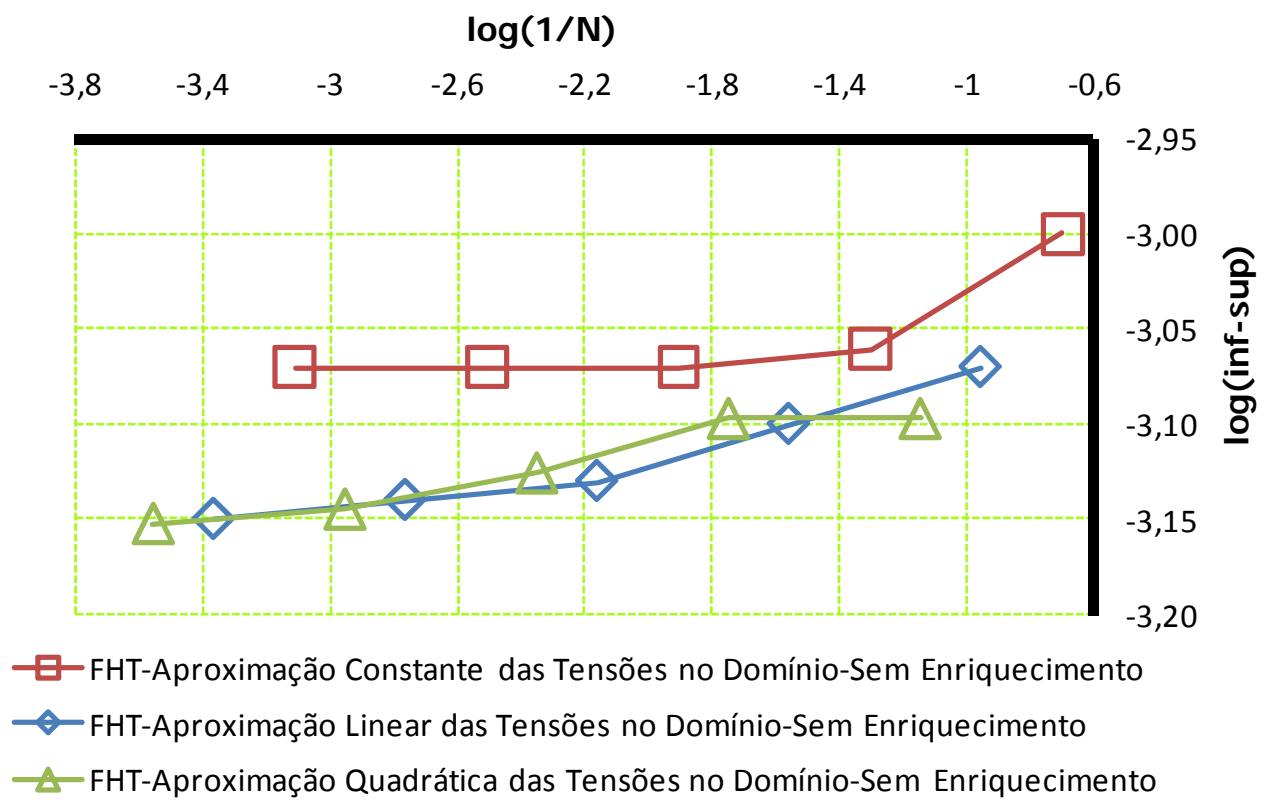

Figura 7.107 - Resultados do teste “inf-sup” - Elemento Quadrilateral - FHT sem enriquecimento.

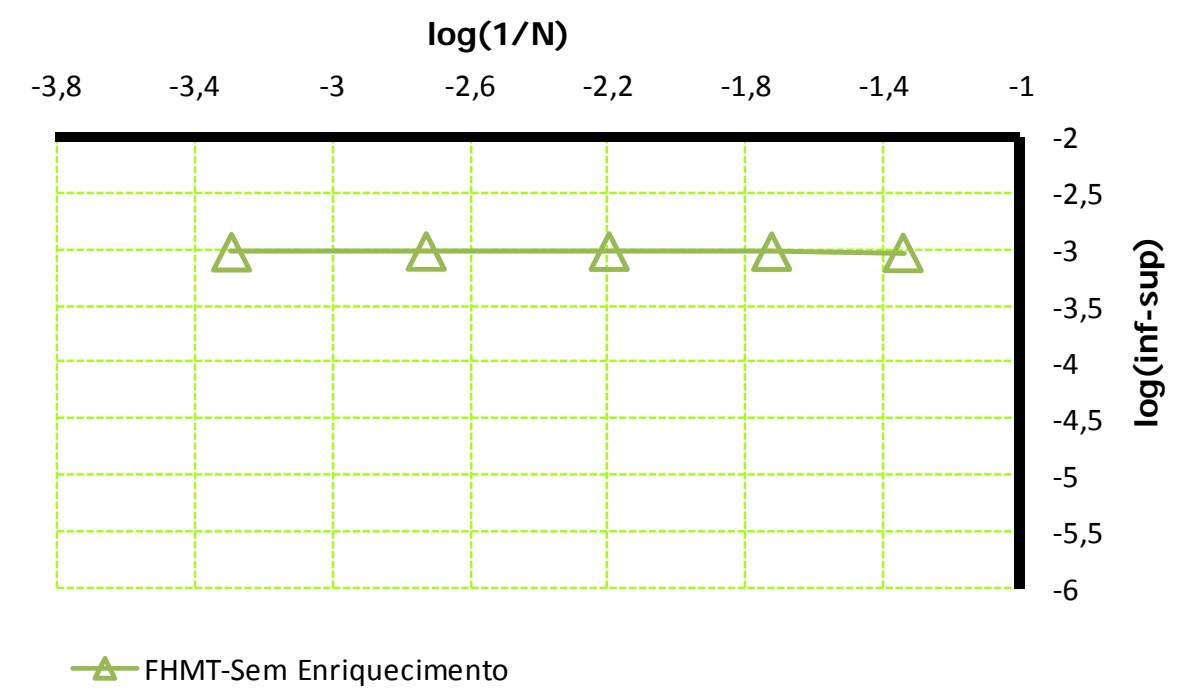

Figura 7.108 - Resultados do teste “inf-sup” - Elemento Quadrilateral - FHMT sem enriquecimento.

O elemento quadrilateral da FHT enriquecido, nos três níveis de aproximação do campo de tensão, satisfez o teste "inf-sup" para o problema da chapa tracionada, pois todas as curvas apresentam tendência de convergência para um $\lambda_{n}>\boldsymbol{0}$, ver figura 7.109. Ressalta-se que os enriquecimentos foram impostos na totalidade dos nós de domínio e em todos os nós de contorno que não possuem condição de contorno essencial prescrita. 


\section{$\log (1 / N)$}

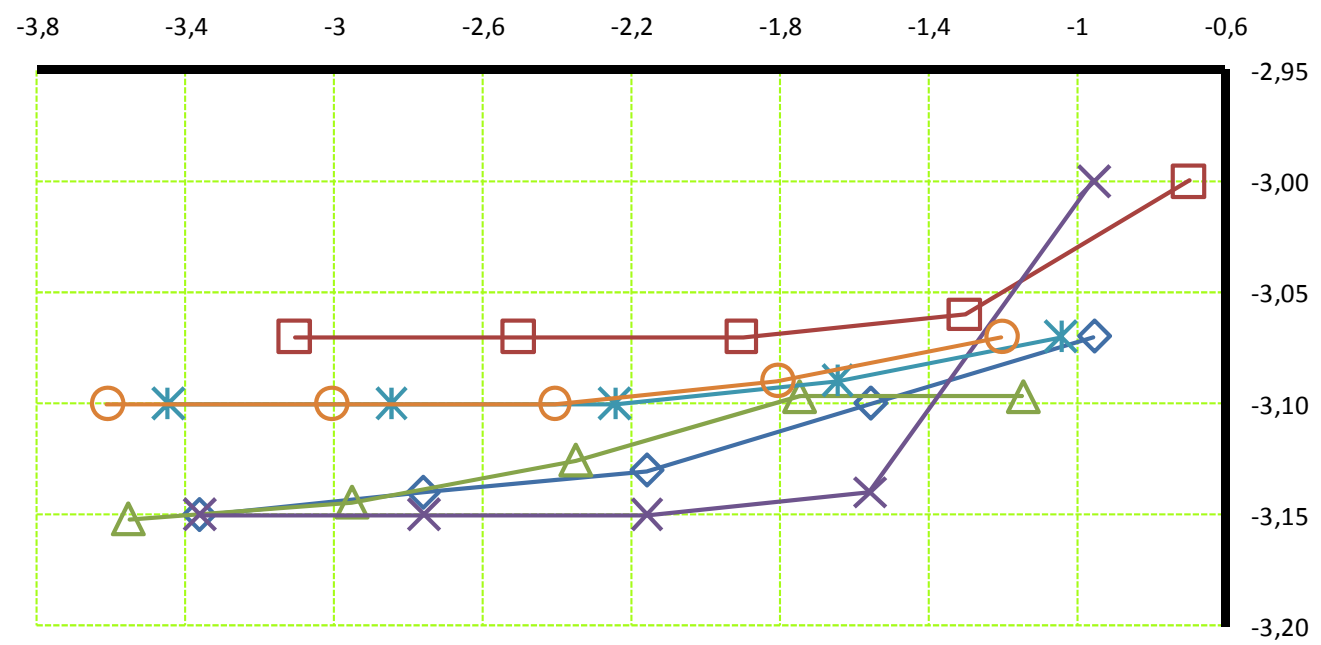

\footnotetext{
—-FHT-Aproximação Constante das Tensões no Domínio-Sem Enriquecimento $\multimap$ FHT-Aproximação Linear das Tensões no Domínio-Sem Enriquecimento — FHT-Aproximação Quadrática das Tensões no Domínio-Sem Enriquecimento * FHT-Aproximação Constante das Tensões no Domínio-Enriquecimento das Tensões no Domínio $\left(\mathrm{y}^{2}\right)$ e Deslocamentos no Contorno (x) - - FHT-Aproximação Linear das Tensões no Domínio-Enriquecimento dos Deslocamentos no Contorno (x)

- FHT-Aproximação Quadrática das Tensões no Domínio-Enriquecimento dos Deslocamentos no Contorno (x)
}

Figura 7.109 - Resultados do teste “inf-sup” - Elemento Quadrilateral - FHT com enriquecimento.

$\log (1 / N)$

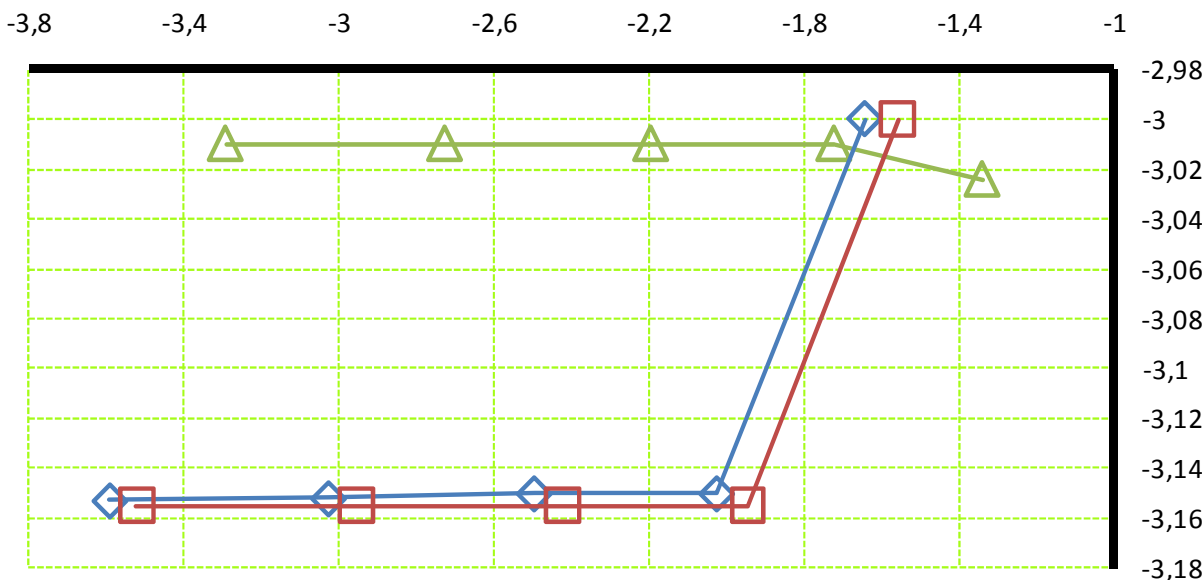

\footnotetext{
$\triangle$ FHMT-Sem Enriquecimento

$\multimap$ FHMT-Enriquecimento das Tensões e Deslocamentos no Domínio $\left(\mathrm{y}^{2}\right)$ e Deslocamentos no Contorno $(x)$

—-FHMT-Enriquecimento das Tensões no Domínio $\left(\mathrm{y}^{2}\right)$ e Deslocamentos no Contorno $(x)$
}

Figura 7.110 - Resultados do teste “inf-sup” - Elemento Quadrilateral - FHMT com enriquecimento.

Com a seleção de algumas combinações de enriquecimento aplicada ao elemento quadrilateral da FHMT, manteve-se a tendência de $\lambda_{n}>\boldsymbol{0}$ apresentada por este mesmo elemento sem enriquecimento, como mostra a figura 7.110. 
$\log (\mathbf{1} / \mathbf{N})$

$\begin{array}{llllllllllll}-4,4 & -4 & -3,6 & -3,2 & -2,8 & -2,4 & -2 & -1,6 & -1,2 & -0,8 & -0,4 & 0\end{array}$

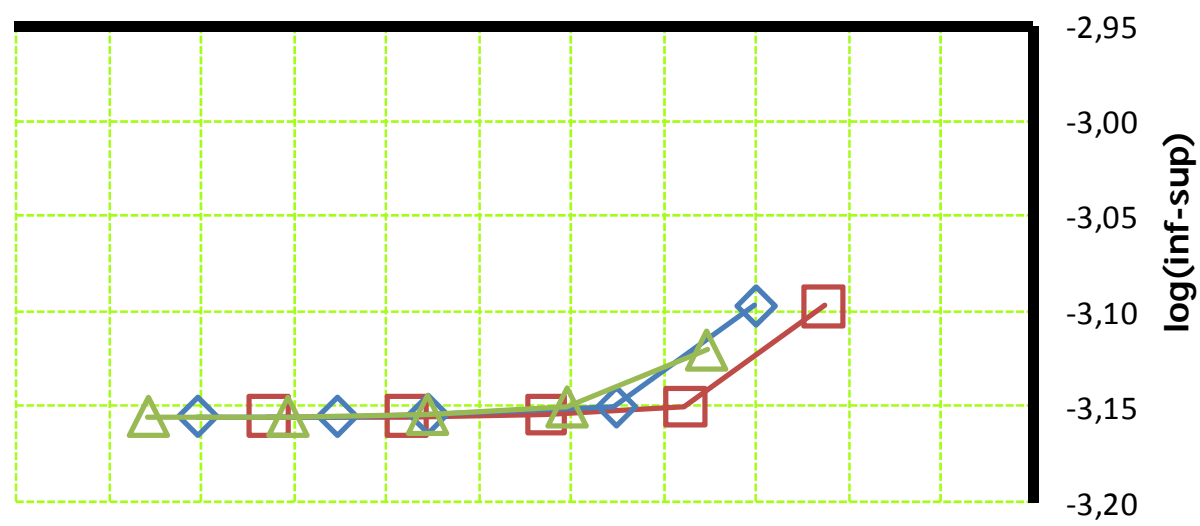

- FHT-Aproximação Constante das Tensões no Domínio-Sem Enriquecimento

—FHT-Aproximação Linear das Tensões no Domínio-Sem Enriquecimento

$\triangle$ FHT-Aproximação Quadrática das Tensões no Domínio-Sem Enriquecimento

Figura 7.111 - Resultados do teste “inf-sup” - Elemento Triangular - FHT sem enriquecimento.

A figura 7.111 mostra que, mesmo sem enriquecimento algum sobre as bases aproximativas do elemento triangular da FHT, é possível convergir para uma constante $\lambda_{n}$ positiva.

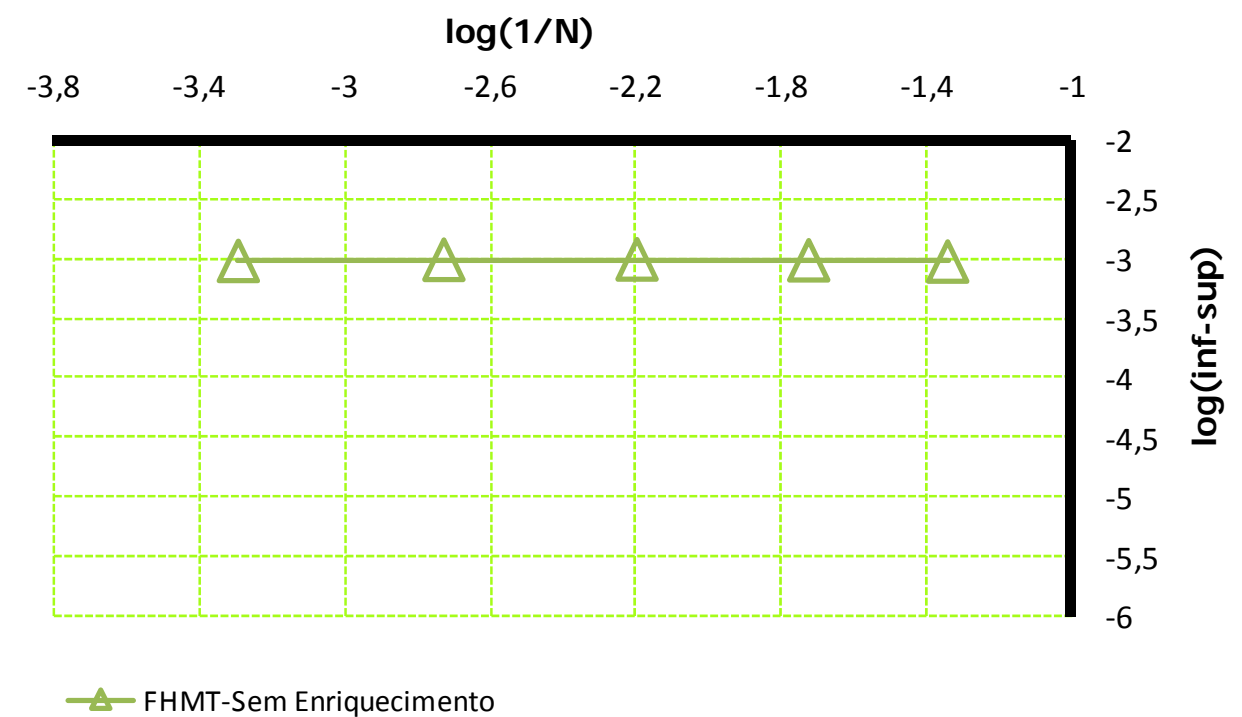

Figura 7.112 - Resultados do teste “inf-sup” - Elemento Triangular - FHMT sem enriquecimento.

Com o elemento triangular da FHMT sem enriquecimento também foi possível obter $\lambda_{n}>\boldsymbol{0}$, como destaca a figura 7.112.

$\mathrm{O}$ enriquecimento polinomial exclusivo sobre os deslocamentos no contorno ou simultâneo sobre as tensões no domínio e deslocamentos no contorno do elemento 
quadrilateral da FHT conduziu a resultados satisfatórios, $\lambda_{n}>\mathbf{0}$, nos casos da aproximação constante, linear e quadrática do campo de tensão, ver figura 7.113.

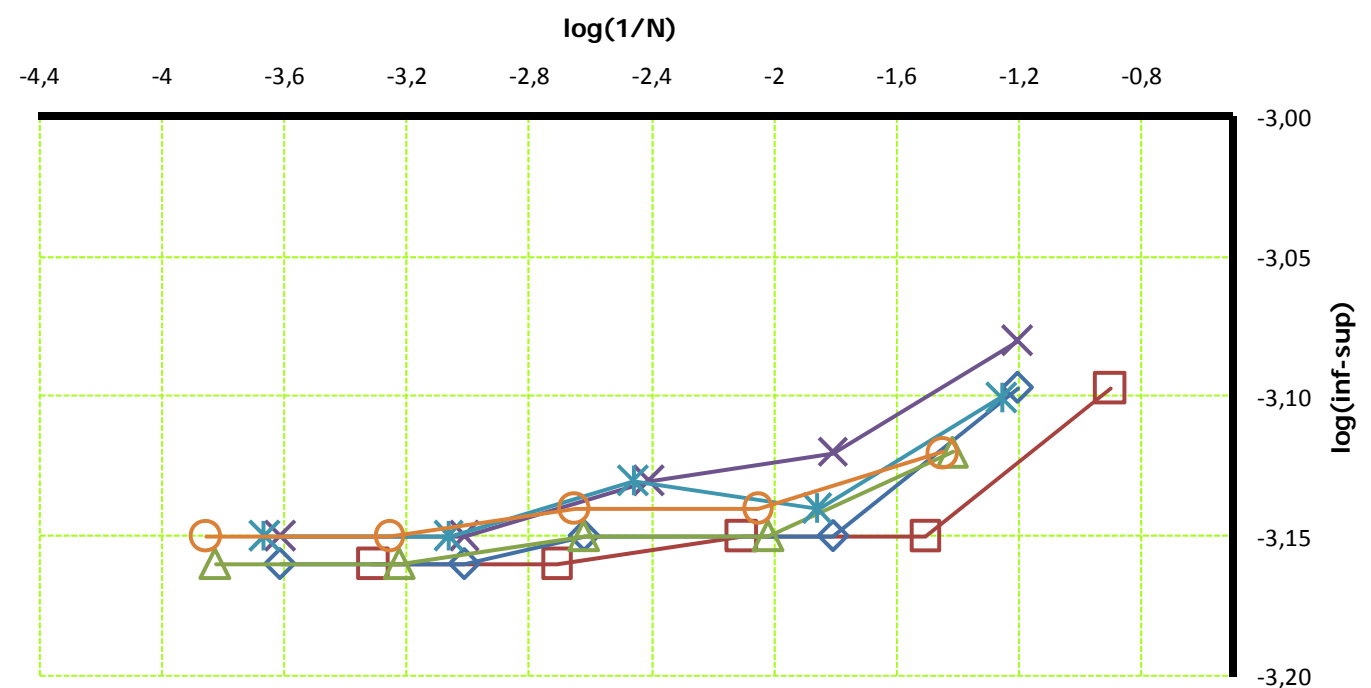

—FHT-Aproximação Constante das Tensões no Domínio-Sem Enriquecimento

$\sim$ FHT-Aproximação Linear das Tensões no Domínio-Sem Enriquecimento

- FHT-Aproximação Quadrática das Tensões no Domínio-Sem Enriquecimento

* FHT-Aproximação Constante das Tensões no Domínio-Enriquecimento das Tensões no Domínio $\left(\mathrm{y}^{2}\right)$ e Deslocamentos no Contorno ( $\mathrm{x}$ )

*-FHT-Aproximação Linear das Tensões no Domínio-Enriquecimento dos Deslocamentos no Contorno (x)

- FHT-Aproximação Quadrática das Tensões no Domínio-Enriquecimento dos Deslocamentos no Contorno (x)

Figura 7.113 - Resultados do teste "inf-sup” - Elemento Triangular - FHT com enriquecimento.

$\log (1 / N)$

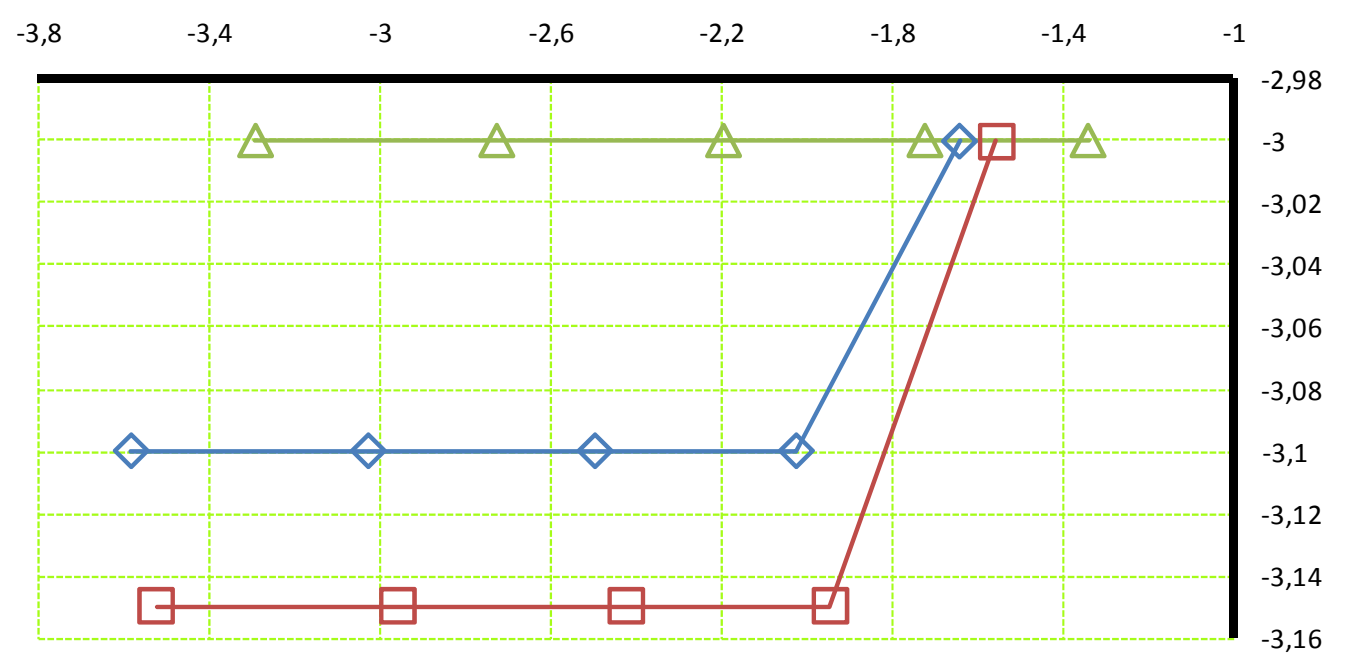

$\therefore$ FHMT-Sem Enriquecimento

- FHMT-Enriquecimento das Tensões no Domínio $\left(\mathrm{y}^{2}\right)$ e Deslocamentos no Contorno (x)

$\smile$ FHMT-Enriquecimento das Tensões e Deslocamentos no Domínio $\left(\mathrm{y}^{2}\right)$ e Deslocamentos no Contorno $(x)$

Figura 7.114 - Resultados do teste "inf-sup” - Elemento Triangular - FHMT com enriquecimento. 
A figura 7.114 destaca que as condições de enriquecimento testadas para ampliar os campos de tensão e deslocamento no domínio e deslocamento no contorno do elemento triangular da FHMT foram suficientes para alcançar $\boldsymbol{\lambda}_{\boldsymbol{n}}>\boldsymbol{0}$.

\subsubsection{Chapa Tracionada com Fenda Central}

Neste exemplo são aplicados enriquecimentos seletivos sobre os campos envolvidos na FHT/FHMT. Os nós escolhidos para o enriquecimento seletivo foram postados na figura 7.79 .

A aproximação constante das tensões no domínio sem enriquecimento gerou resposta $\lambda_{n} \rightarrow \boldsymbol{0}$. Nos demais níveis de aproximação do campo de tensão no domínio observaram-se convergência assintótica do $\lambda_{n}$ para um valor positivo, ver figura 7.115.

\section{$\log (\mathbf{1} / \mathbf{N})$}

$\begin{array}{llllllllll}-4,2 & -3,8 & -3,4 & -3 & -2,6 & -2,2 & -1,8 & -1,4 & -1 & -0,6\end{array}$

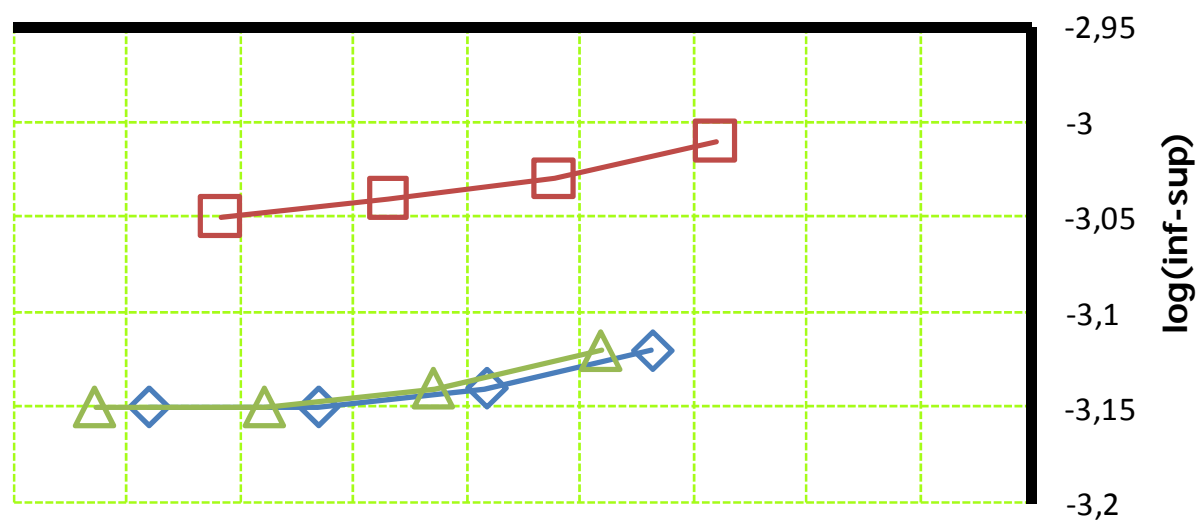

—-FHT-Aproximação Constante das Tensões no Domínio-Sem Enriquecimento

—FHT-Aproximação Linear das Tensões no Domínio-Sem Enriquecimento

$\triangle$ FHT-Aproximação Quadrática das Tensões no Domínio-Sem Enriquecimento

Figura 7.115 - Resultados do teste “inf-sup” - Elemento Quadrilateral - FHT sem enriquecimento. 
$\log (1 / N)$

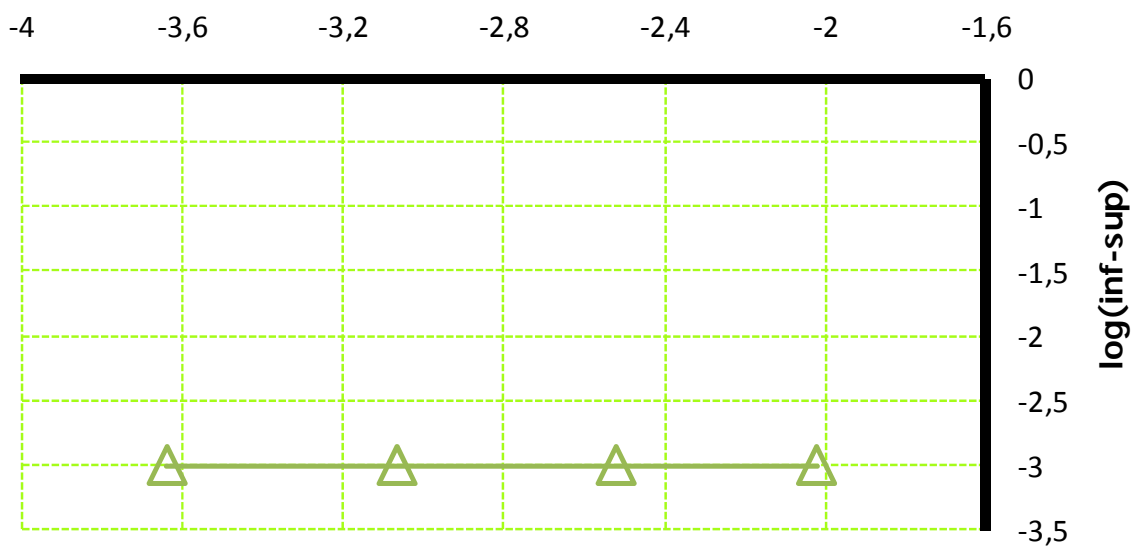

$\triangle$ FHMT-Sem Enriquecimento

Figura 7.116 - Resultados do teste “inf-sup” - Elemento Quadrilateral - FHMT sem enriquecimento.

A figura 7.116 indica que o elemento quadrilateral da FHMT sem enriquecimento satisfaz o teste "inf-sup”, pois $\lambda_{n}$ tende para um valor positivo.

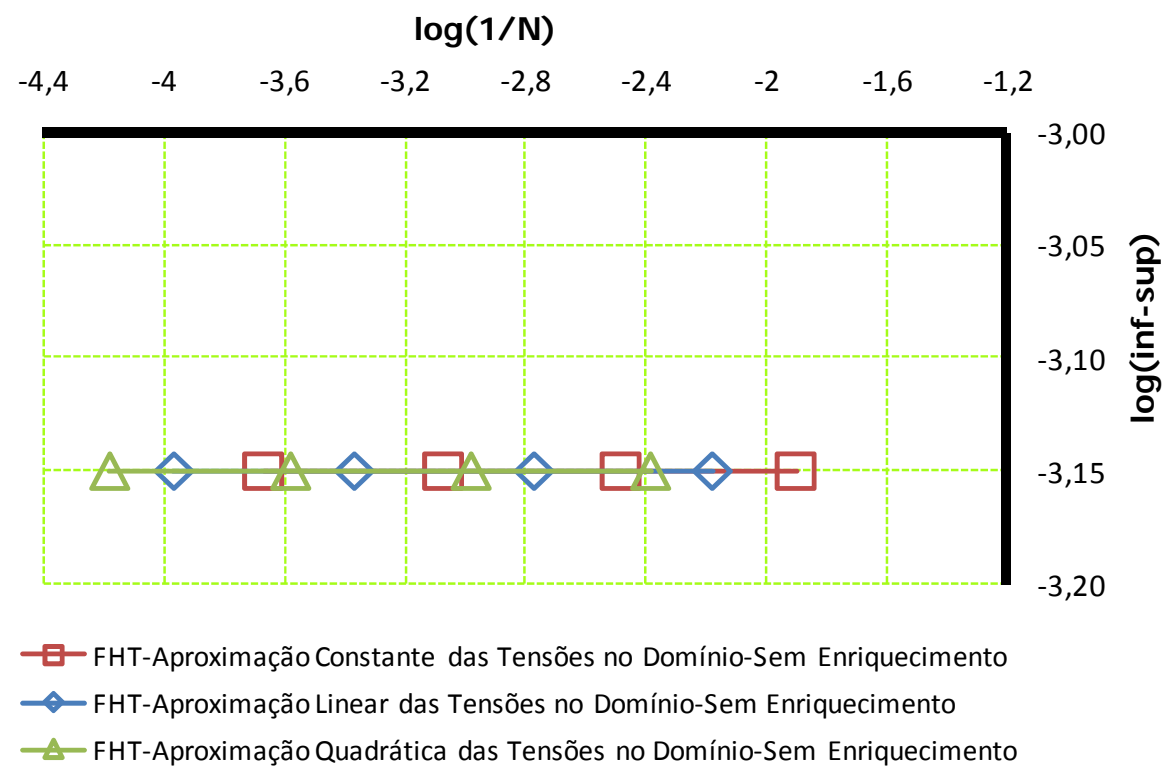

Figura 7.117 - Resultados do teste “inf-sup” - Elemento Triangular - FHT sem enriquecimento.

O elemento triangular da FHT (todas as bases aproximativas das tensões no domínio) sem enriquecimento satisfaz o teste "inf-sup" $\operatorname{com} \lambda_{n}$ tendendo para uma constante positiva, como mostra a figura 7.117. 


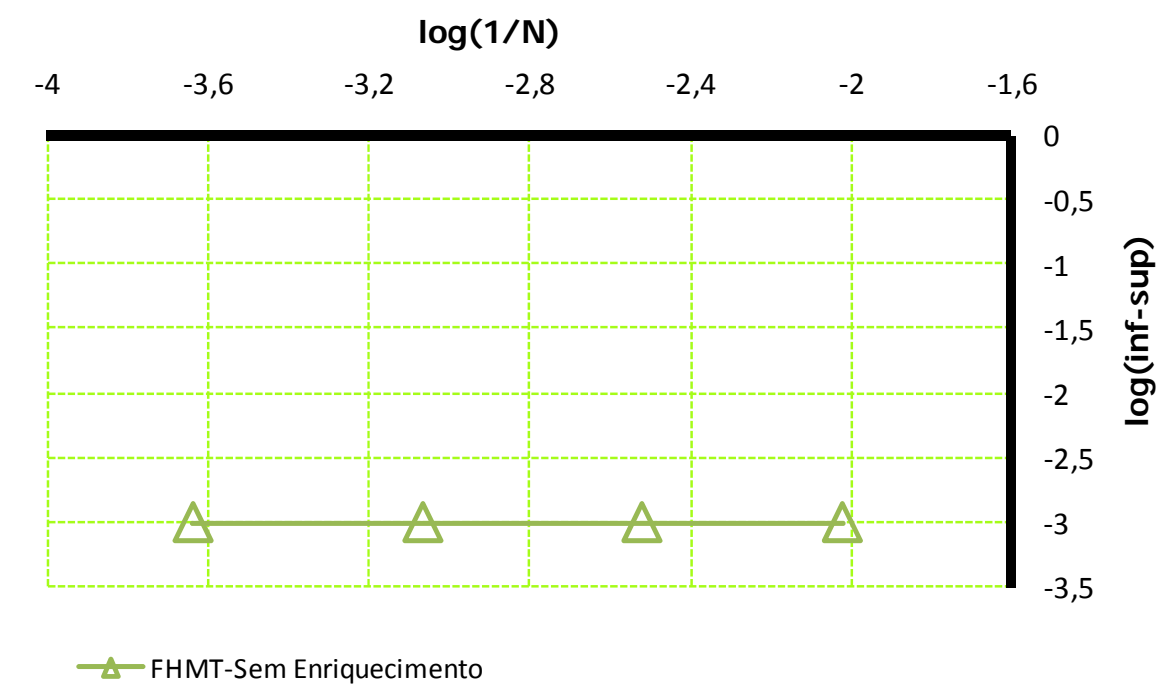

Figura 7.118 - Resultados do teste “inf-sup” - Elemento Triangular - FHMT sem enriquecimento.

Com o elemento triangular da FHMT sem enriquecimento claramente se alcança $\lambda_{n}>\mathbf{0}$, ver figura 7.118 .

Como apresenta a figura 7.119, para todas as combinações de enriquecimentos testados no elemento triangular da FHMT é possível à convergência para $\lambda_{n}>\boldsymbol{0}$.

$\log (1 / N)$

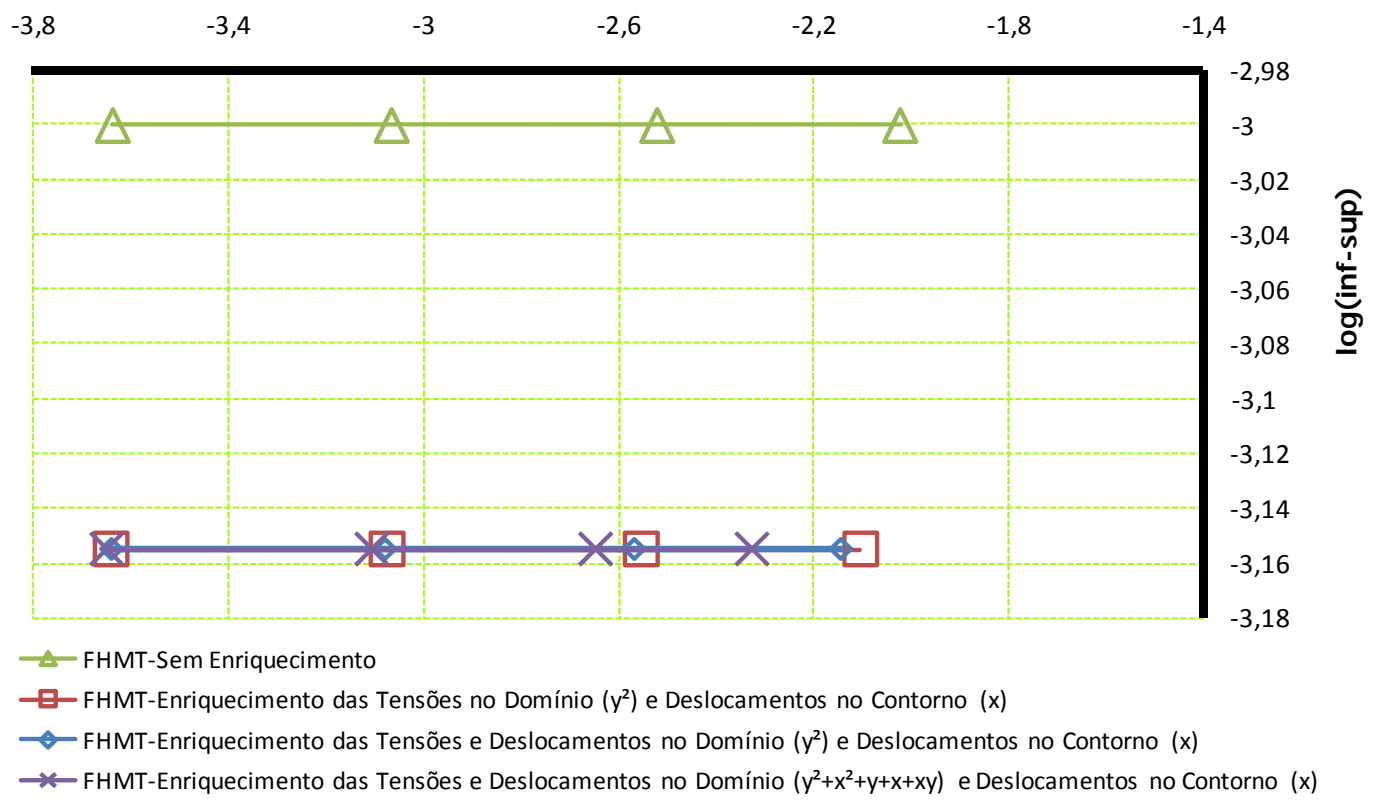

Figura 7.119 - Resultados do teste “inf-sup” - Elemento Triangular - FHMT com enriquecimento. 
$\log (1 / N)$

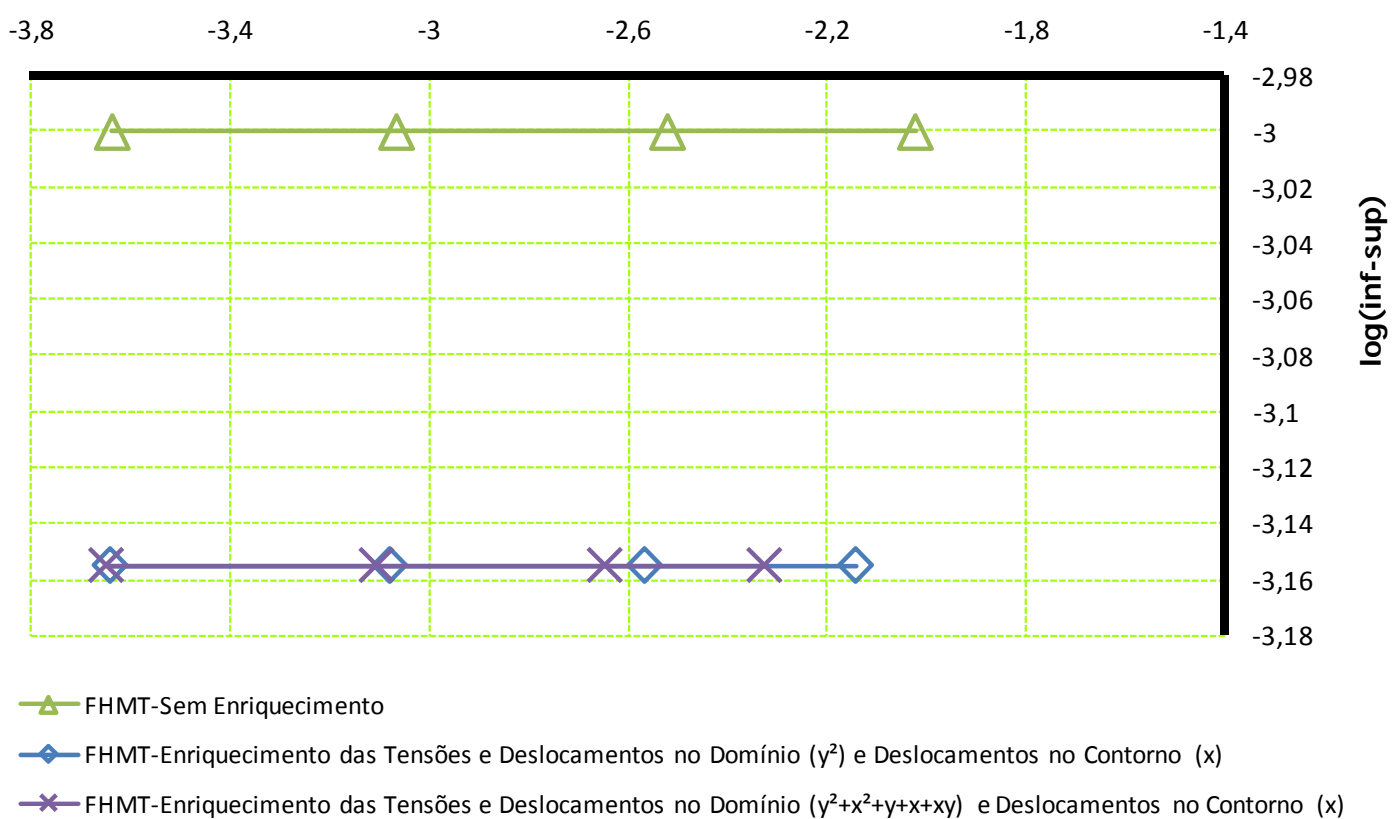

Figura 7.120 - Resultados do teste “inf-sup” - Elemento Quadrilateral - FHMT com enriquecimento.

O elemento quadrilateral enriquecido da FHMT apresentou o mesmo comportamento do elemento triangular enriquecido da mesma formulação (figura 7.119), ou seja, foi possível convergir para uma constante $\lambda_{n}$ positiva, ver figura 7.120.

\section{$\log (1 / N)$}

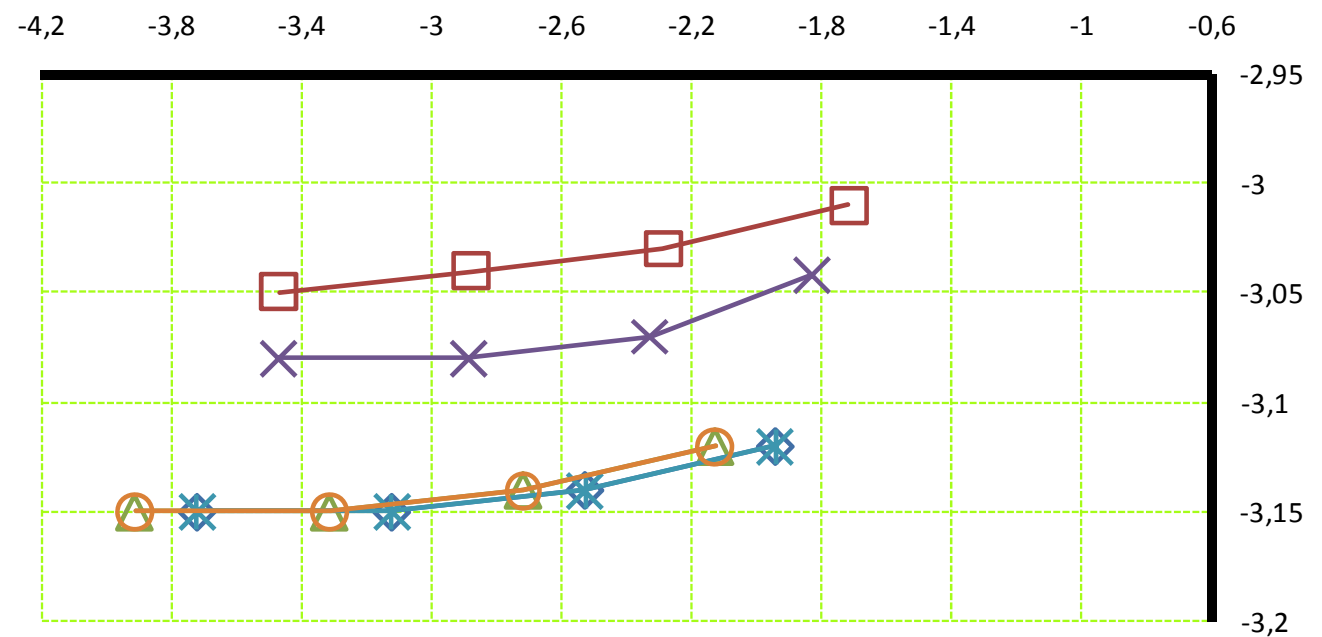

—FHT-Aproximação Constante das Tensões no Domínio-Sem Enriquecimento

—FHT-Aproximação Linear das Tensões no Domínio-Sem Enriquecimento

$\triangle$ FHT-Aproximação Quadrática das Tensões no Domínio-Sem Enriquecimento

* FHT-Aproximação Constante das Tensões no Domínio-Enriquecimento das Tensões no Domínio ( $\mathrm{y}^{2}$ )

*-FHT-Aproximação Linear das Tensões no Domínio-Enriquecimento dos Deslocamentos no Contorno (x)

- FHT-Aproximação Quadrática das Tensões no Domínio-Enriquecimento dos Deslocamentos no Contorno (x)

Figura 7.121 - Resultados do teste “inf-sup” - Elemento Quadrilateral - FHT com enriquecimento. 
As figuras 7.121 e 7.122 indicam que os elementos quadrilateral e triangular da FHT enriquecidos foram muito eficientes, pois, em todas as possibilidades de enriquecimento testadas, conseguiu-se convergência para $\lambda_{n}>\boldsymbol{0}$.

\section{$\log (1 / N)$}

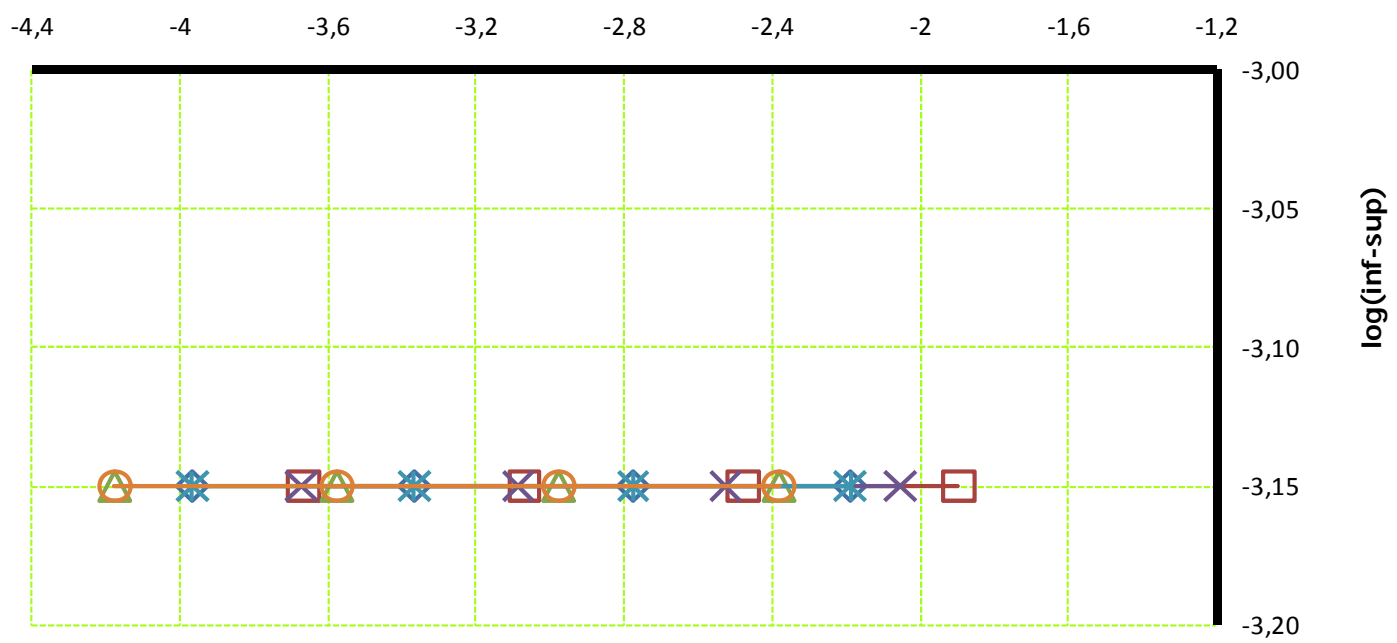

\footnotetext{
- FHT-Aproximação Constante das Tensões no Domínio-Sem Enriquecimento

—FHT-Aproximação Linear das Tensões no Domínio-Sem Enriquecimento

—- FHT-Aproximação Quadrática das Tensões no Domínio-Sem Enriquecimento

- FHT-Aproximação Constante das Tensões no Domínio-Enriquecimento das Tensões no Domínio $\left(\mathrm{y}^{2}\right)$ e Deslocmentos no Contorno ( $\mathrm{x}$ )

- FHT-Aproximação Linear das Tensões no Domínio-Enriquecimento dos Deslocamentos no Contorno (x)

- - FHT-Aproximação Quadrática das Tensões no Domínio-Enriquecimento dos Deslocamentos no Contorno (x)
}

Figura 7.122 - Resultados do teste “inf-sup” - Elemento Triangular - FHT com enriquecimento.

\subsection{Discussão dos Resultados}

Primeiramente, avaliou-se a sensibilidade dos elementos quadrilateral e triangular da FHT/FHMT com enriquecimento nodal à distorção de rede. Para isso, utilizaram-se três exemplos: os dois primeiros bem simples, de solução exata conhecida e fácil simulação numérica e o terceiro, o Painel de Cook, de simulação numérica mais complicada, pois existe um grau de distorção intrínseco na geometria e discretização adotada para análise deste problema.

Verificou-se que os elementos quadrilateral e triangular da FHMT e o quadrilateral da FHT, mesmo com forte distorção da rede, conseguiram representar corretamente as tensões (estimativa e distribuição), os deslocamentos e as estimativas da energia de deformação dos problemas propostos, para algumas possibilidades de enriquecimento nodal desenvolvidas no trabalho e que previamente verificavam o ‘Teste por Inspeção’. 
O elemento triangular da FHT com enriquecimento nodal foi o único que apresentou sensibilidade à distorção de rede, mas com respostas no mínimo iguais às apresentadas pelo elemento triangular do MEF clássico.

Como o emprego do MEFG possibilita, em princípio, explorar diferentes níveis de enriquecimento para os campos de tensão no domínio (FHT/FHMT), deslocamentos no domínio (FHMT) e deslocamento no contorno (FHT/FHMT), iniciaram-se análises para avaliar a eficiência das várias combinações de enriquecimento e identificar condições gerais para garantir a estabilidade da resposta numérica dos elementos estudados.

Naturalmente o tipo de problema (condições de contorno e geometria), redes usadas na discretização, grau de aproximação e condições de enriquecimento dos campos envolvidos na FHT/FHMT devem influenciar diretamente a estabilidade, e consequentemente a convergência das soluções aproximadas, dos elementos quadrilateral e triangular da FHT/FHMT com enriquecimento nodal.

O 'Teste por Inspeção' foi o primeiro passo para a identificação das combinações estáveis de enriquecimento. Esse teste, consistindo na verificação de desigualdades (eq.(6.10) a eq.(6.12)) relacionando os graus de liberdade envolvidos, foi aplicado a dois problemas planos com características de regularidade bem distintas.

Observou-se que o 'Teste por Inspeção’ é somente condição necessária para garantia da estabilidade, pois existiram situações onde as desigualdades que governam esse teste foram satisfeitas e não se obteve solução do procedimento numérico iterativo para solução do sistema de equações lineares.

A não-convergência no procedimento numérico deveu-se basicamente à presença de modos espúrios cinemáticos. Estes modos foram detectados em correspondência com autovalores nulos da matriz de coeficientes da FHT/FHMT com enriquecimento nodal (eq.(2.36), incluindo-se ou não a restrição dada pela eq.(2.69)).

A avaliação do 'Teste por Inspeção' foi complementada com a análise dos campos de tensão, deslocamento e gráficos de convergência da energia de deformação nos dois problemas estudados.

Ficou evidente que os elementos quadrilateral e triangular da FHT/FHMT, quando das estimativas da energia de deformação, estão sujeitos ao princípio da limitação, ou seja, apresentam respostas idênticas às obtidas via MEF Clássico, Zienkiewicz (2000). Vale ressaltar que mesmo com este entrave, justifica-se o estudo destas formas não-convencionais do MEF, pois, como demonstrado, os elementos da FHT/FHMT com enriquecimento nodal são menos sensíveis à distorção de rede. Além disso, quando se deseja maior precisão na 
representação dos campos de tensão, observa-se claramente a potencialidade dos elementos da FHT/FHMT com enriquecimento nodal em relação aos resultados apresentados pelos elementos do MEF Clássico.

Por outro lado, a condição de Babuška-Brezzi (ou teste “inf-sup”) é condição suficiente para estabilidade. Entretanto, trata-se de um teste de aplicação muito custosa, pois em tese, para que um elemento finito seja considerado estável seria necessário avaliá-lo sob diferentes condições de rede e vinculação em todos os problemas de uma classe.

Neste trabalho utilizaram-se três problemas, com graus distintos de complexidade e discretização (o primeiro discretizado com um conjunto de quatro redes, o segundo com cinco redes regulares e o terceiro com quatro redes também regulares), para analisar a estabilidade das soluções obtidas com os elementos quadrilateral e triangular da FHT/FHMT com enriquecimento nodal. Como o número de simulações realizadas foi limitado, considerou-se que a satisfação do teste "inf-sup" (convergência de $\lambda_{n}$ para um valor positivo), nos três problemas, forneceria um bom indicativo para estabilidade.

É importante observar que um dos entraves no desenvolvimento do teste "inf-sup" foi a limitação do número de redes que geram pontos para as curvas $\log (1 / N) \times \log \left(\lambda_{n}\right)$. Esse limitante foi de caráter exclusivamente computacional, pois para o problema de autovalor generalizado (eq.(6.34)), analisado com redes mais refinadas e enriquecimentos das aproximações, foram geradas matrizes com dimensões que ultrapassam 2 GB. Ocorre que a combinação entre processadores de 32 bits e o sistema operacional Windows, utilizados nos processamentos numéricos, impõe uma limitação de 2 GB de memória RAM por aplicativo. Apesar dessa limitação foi possível realizar os testes de forma conclusiva.

Com o teste "inf-sup” aplicado aos exemplos propostos (Painel de Cook, chapa tracionada e chapa com fenda central), obtiveram-se os seguintes indicativos de eficiência:

- Os elementos quadrilateral e triangular da FHMT sem enriquecimento não são estáveis;

- Os elementos quadrilateral e triangular da FHT sem enriquecimento não são estáveis;

- Os elementos quadrilateral e triangular da FHMT para algumas condições de enriquecimento são estáveis;

- O elemento quadrilateral da FHT com enriquecimento é estável;

- O elemento triangular da FHT com enriquecimento não é estável. 


\section{Considerações Finais e Conclusões}

No âmbito das formulações não-convencionais em elementos finitos este trabalho objetivou contribuir ao estudo das formulações Híbridas de tensão (FHT) e Híbrido-Mistas de Tensão (FHMT).

Elementos quadrilaterais de quatro nós e triangulares de três nós foram desenvolvidos para ambas as formulações, com funções de aproximação geradas por procedimento de enriquecimento baseado no método dos elementos finitos generalizados (MEFG).

Como características gerais, nos elementos finitos da FHT as tensões são aproximadas no domínio e os deslocamentos nos contornos dos elementos. Já nos elementos da FHMT dois campos são aproximados no domínio (tensões e deslocamentos) e um campo aproximado no contorno (deslocamento). Especificamente para os elementos da FHT, as aproximações dos campos de tensão no domínio são auto-equilibradas e não estão atreladas a nós. Já as aproximações para o deslocamento no contorno são obtidas por interpolação de valores nodais, tanto na FHT como na FHMT. Dada à independência, admitida previamente, do campo de deslocamentos de contorno em relação aos demais, em termos de discretização emprega-se uma rede de elementos quadrangulares ou triangulares de domínio sobreposta à outra de elementos unidimensionais de contorno.

Em razão de que as bases de aproximação inicial dos campos da FHMT constituem partição da unidade, o MEFG pôde ser estendido diretamente a esta formulação. Assim, podese afirmar que um resultado original obtido foi um método de elementos finitos generalizados para formulação híbrido-mista de tensão. Entretanto, em relação à FHT, a extensão não resulta direta, uma vez que os campos de tensão, admitidos a priori como equilibrados, não constituem partição da unidade. Nesse sentido, a semelhança com o MEFG decorre da técnica de enriquecimento nodal adotada e a formulação obtida pode ser simplesmente denominada: formulação híbrida de tensão com enriquecimento nodal. 
Justamente a metodologia de enriquecimento proposta para o enriquecimento das aproximações das tensões no domínio para elementos quadrilaterais de quatro nós e triangulares de três nós da FHT constitui outro aspecto de contribuição original do trabalho.

Em cada uma das formulações, FHT e FHMT, empregaram-se funções polinomiais para fins de enriquecimento nodal. Uma vez que as bases originais são também polinomiais, o enriquecimento pode produzir dependências entre as componentes da base expandida. A técnica de perturbação dos elementos da diagonal principal da matriz dos coeficientes do sistema (Strouboulis, Babuška e Copps (2000)) foi adaptada com sucesso na resolução dos sistemas das duas formulações estudadas, permitindo superar eventuais dificuldades decorrentes das dependências.

Por outro lado, em princípio, o enriquecimento dos campos envolvidos na FHT/FHMT pode ser arbitrário. No entanto, percebeu-se que algumas condições de enriquecimento não se mostraram eficientes, particularmente quando os enriquecimentos se concentraram nos campos de deslocamento, de tal modo a produzir variáveis generalizadas de deslocamento em número total superior às variáveis de tensão. As situações contrárias, nas quais o número de variáveis generalizadas de tensão permanecia sempre superior, produziram resultados estáveis. Por isso, iniciou-se um estudo sobre as condições necessárias e suficientes para convergência de soluções aproximadas obtidas com os métodos propostos.

Uma primeira condição decorreu da análise de existência de solução para os sistemas de equações lineares das formulações estudadas (enriquecidas ou não). Daí resultou o chamado 'Teste por Inspeção', que consiste basicamente em verificar se o vetor incógnito possui variáveis generalizadas de tensão em número superior às variáveis generalizadas de deslocamento.

Posteriormente, para melhor compreensão das condições de solvibilidade dos sistemas lineares discretos das FHT e FHMT com enriquecimento nodal, passou-se a analisar os autovalores das matrizes dos coeficientes desses sistemas. Notou-se que determinadas condições de enriquecimento, particularmente sobre os campos de deslocamento de domínio na FHMT, podem introduzir uma quantidade significativa de modos espúrios cinemáticos, afetando a convergência da rotina iterativa de solução adotada, mesmo que o teste por inspeção seja verificado. Concluiu-se, portanto, que o ‘Teste por Inspeção’ constitui condição necessária para convergência de soluções aproximadas obtidas via FHT/FHMT com enriquecimento nodal. Mesmo assim, o teste pode ser de grande valia para a definição preliminar de combinações de enriquecimento dos campos envolvidos na FHT/FHMT. 
Quanto a uma condição suficiente para estabilidade de solução dos sistemas enriquecidos, optou-se por partir da condição suficiente dada pelo teste 'inf-sup', ou condição de Babuška-Brezzi, existente na literatura para as formulações convencionais. Porém, não há uma demonstração formal dessa condição para as formulações não-convencionais aqui desenvolvidas. Além disso, trata-se de condição de difícil aplicação por exigir uma variação muito grande de discretizações e condições de contorno dentro de uma classe de problemas. Optou-se, então, por desenvolver um teste numérico, nos moldes do teste proposto no trabalho de Chappelle e Bathe (1993) para as formulações convencionais, para a obtenção de um indicativo de estabilidade dos elementos quadrilateral e triangular das FHT e FHMT com enriquecimento nodal; o procedimento resultante é outra contribuição original deste trabalho.

Nos testes realizados, sempre que o ‘Teste por Inspeção’ não era verificado, o teste 'inf-sup' indicava instabilidade. Ao final do conjunto de testes realizados, dos indicativos obtidos conclui-se, por um lado, que é possível detectar problemas de estabilidade em algumas situações aonde o 'Teste por Inspeção’ tenha sido previamente verificado. Por outro lado, em todos os casos testados aonde o teste 'inf-sup' foi verificado o 'Teste por Inspeção' também foi atendido.

Finalmente, o teste 'inf-sup' comprovou que a estabilidade dos elementos quadrilateral e triangular da FHMT com enriquecimento nodal pode ser garantida apenas com o 'Teste por Inspeção’. Já para a FHT, o ‘Teste por Inspeção’ garante estabilidade somente do elemento quadrilateral com enriquecimento nodal.

Como conclusão geral, com base nos problemas tratados neste trabalho (classe dos problemas lineares), pode-se afirmar que verificado preliminarmente o 'Teste por Inspeção’ o emprego de elementos quadrilaterais de quatro nós e triangulares de três nós para as FHT e FHMT com a técnica de enriquecimento nodal apresenta grande potencial para a solução, com precisão numérica e baixo custo computacional. Entre as vantagens oferecidas por esses elementos estão: a ótima representação dos campos de tensão (a depender do problema já com redes pouco refinadas) e a quase total independência da precisão das respostas em relação à distorção de rede. Neste sentido foram realizados testes de distorção que mostraram claramente o ótimo desempenho desses elementos no confronto com outros elementos similares, inclusive os derivados de formulações mistas convencionais.

Como propostas para desenvolvimentos futuros, propõem-se: 
- Avaliação das possibilidades de enriquecimento com funções especiais, como as de base trigonométrica que fundamentam as soluções dos problemas de fratura elástica. Esta opção pode reduzir bastante a necessidade de refinamento da rede nas vizinhanças da ponta da fissura, como é usual nas análises convencionais, e até com ganho de precisão. Além disso, nos problemas de fratura, a forma híbrida, caracterizada pelos campos de deslocamento de contorno pode vir a ser explorada com vantagens nas simulações que envolvam propagação de fissuras;

- Avaliação das possibilidades de extensão das formulações desenvolvidas para abranger classes de problemas não-lineares. Pode-se, em princípio, tomar por base as propostas para este tipo de análise já feita anteriormente para as formulações mistas convencionais (Simo e Rifai (1990)). 


\section{Referências Bibliográficas e Bibliografia Básica}

ANSYS RELEASE 5.5.1 (1998). Theory Manual. $3^{\text {rd }}$ edition. SAS IP, Inc.

ARNOLD, D. N. (1990). Mixed finite element methods for elliptic problems. Computer Methods in Applied Mechanics and Engineering, v.82, p. 281-300.

ASSAN, A.E. (2003). Método dos elementos finitos: primeiros passos. 2.ed. Campinas. Editora da Unicamp.

BABUŠKA, I. (1971). Error bounds for finite element methods. Numerische Mathematik, v.16, p. 322-333.

BABUŠKA, I. (1973). The finite element method with lagrange multipliers. Numerische Mathematik, v.20, p. 179-192.

BABUŠKA, I. (1996). On the inf-sup (babuška-brezzi) condition. The University of Texas at Austin. Technical Report \#5. TICAM

BABUŠKA, I.; CALOZ, G.; OSBORN, J. E. (1994). Special finite element method for a classe of second order elliptic problems with rough coefficients. SIAM Journal on Numerical Analysis, v.31, n.4, p. 727-981.

BABUŠKA, I.; MELENK, J. M. (1997). The partition of unity method. International Journal for Numerical Methods in Engineering, v. 40, p. 727-758.

BABUŠKA, I. et al. (1996). Finite element method for solving problems with singular solutions. Journal of Computational and Applied Mathematics, v.74, p. 51-70.

BARROS, F. B. (2002). Métodos sem malha e método dos elementos finitos generalizados em análise não-linear de estruturas. Tese (Doutorado). Escola de Engenharia de São Carlos, Universidade de São Paulo. 
BATHE, K. J. (1996). Finite element procedures. 2.ed. New Jersey: Prentice-Hall.

BATHE, K. J.; HENDRIANA, D.; BREZZI, F.; SANGALLI, G. (2000). Inf-sup test of upwind methods. International Journal for Numerical Methods in Engineering, v.48, p.745-760.

BATHE, K. J.; IOSILEVICH, A.; CHAPELLE, D. (2000). An inf-sup test for shell finite elements. Computer and Structures, v.75, p. 439-456.

BATHE, K. J. (2001). The inf-sup condition and its evaluation for mixed finite element method. Computer and Structures, v.79, p. 243-252.

BREZZI, F. (1974). On the existence, uniqueness and approximation of saddle point problems arising from lagrange multipliers. RAIRD, v.8 (r-2), p. 127-151.

BREZZI, F.; BATHE, K. J. (1990). A discourse on the stability conditions for mixed finite element formulation. Computer Methods in Applied Mechanics and Engineering, v.82, p. 27-57.

BREZZI, F.; FORTIN, M. (1991). Mixed and hybrid finite element methods. New York: Springer Verlag.

BUSSAMRA, F. L. S. (1999). Elementos finitos híbrido-trefftz: um modelo elastoplástico tridimensional. Tese (Doutorado). Escola Politécnica da Universidade de São Paulo.

CHAPELLE, D.; BATHE, K. J. (1993). The inf-sup test. Computers \& Structures, v. 47, n.4/5, p. 537-545.

COOK, R. (1987). A plane hybrid element with rotational d.o.f and adjustable stiffiness. International Journal for Numerical Methods in Engineering, v.24, p.1499-1508.

CUNHA, C. (2000). Métodos numéricos. 2.ed. Campinas: Editora da Unicamp.

DESAI, C. S.; ABEL, J.F. (1972). Introduction to the finite element: a numerical method for engineering analysis. New York: Van Nostrand Reinhold Company.

DUARTE, C. A. (1995). A review of some meshless methods to solve partial differential equations. The University of Texas at Austin. Technical Report. TICAM.

DUARTE, C. A. (1996). The hp-cloud method. Tese (Doutorado) - The University of Texas at Austin.

DUARTE, C. A.; BABUŠKA, I.; ODEN, J. T. (2000). Generalized finite element methods for three-dimensional structural mechanics problems. Computers \& Structures, v. 77, n. 2, p. 215-232.

DUARTE, C. A.; ODEN, J. T. (1995). Hp clouds - a meshless to solve boundary - value problem. The University of Texas at Austin. Technical Report. TICAM. 
DUARTE, C. A.; ODEN, J.T. (1996). Hp clouds - an hp meshless method. Numerical Methods for Partial Differential Equations. John Wiley \& Sons, p. 1 - 34.

FREITAS, J. A. T.; ALMEIDA, J. P. B. M.; PEREIRA, E. M. B. R. (1996). Non conventional formulations for the finite element method. Structural Engineering and Mechanics, v.4, p. 655-678.

GÓIS, W. (2004). Método dos elementos finitos generalizados em formulação variacional mista. Dissertação (Mestrado). Escola de Engenharia de São Carlos, Universidade de São Paulo.

GÓIS, W.; PROENÇA, S. P. B. (2005). Generalized finite element method in mixed variational formulation: a study of convergence and stability. In: THEMATIC CONFERENCE ON MESHLESS METHODS, 2005, Lisboa. Proceendings...Lisboa: ECCOMAS. p.B22.1-B22.8

GÓIS, W.; PROENÇA, S. P. B. (2006). On the stability of generalized finite element method solutions in mixed variational formulation. In: VII SIMPÓSIO DE MECÂNICA COMPUTACIONAL - SIMMEC, 7.,2006, Araxá. Resumo dos Trabalhos técnicos... Araxá: SIMMEC. p. 95

GÓIS, W.; PROENÇA, S. P. B. (2006). Elementos finitos híbridos com enriquecimento nodal. Cadernos de Engenharia de Estruturas v. 8, n.32, p. 165-168

GÓIS, W.; PROENÇA, S. P. B. (2007). Elementos finitos híbridos com enriquecimento nodal. In: CONGRESSO ÍBERO AMERICANO SOBRE MÉTODOS COMPUTACIONAIS PARA ENGENHARIA, 2007, Porto. Proceendings...Lisboa: Associação Portuguesa de Mecânica Teórica, Aplicada e Computacional. p.1-17

GÓIS, W.; PROENÇA, S. P. B. (2007). Generalized finite element method in mixed variational formulation: a study of convergence and stability. In: ADVANCES IN MESHFREE TECHNIQUES. v.5. Heilderberg: Springer Verlog.

GÓIS, W.; PROENÇA, S. P. B. (2007). Hybrid mixed and hybrid finite elements with nodal enrichment. In: NINTH US NATIONAL CONGRESS ON COMPUTATIONAL MECHANICS, 2007, San Francisco. Proceendings...San Francisco: USACM.

GOLUB, G. H.; LOAN, C. F. V. (1996). Matrix computation. 5.ed. Maryland: Johns Hopkins University Press.

HOFFMAN, K.; KUNZE, R. (1971). Algebra linear. São Paulo: Editora Polígono S. A.

IOSILEVICH, A.; BATHE, K. J.; BREZZI, F. (1997). On evaluating the inf-sup condition for plate bending elements. International Journal for Numerical Methods in Engineering, v.40, p. 3639-3663.

IRONS, B.M.; AHMAD, S. (1980). Techniques of finite elements. Chichester Ellis: Harwood. 
IRONS, B.M.; RAZZAQUE, A. (1972). Experience with the patch test for convergence of finite element method. Mathematical Foundations of Finite Element Method. Academic Press, p. 557-587.

LABORATORIO DE COMPUTAÇÃO CIENTÍFICA (1983). Curso de mecânica teórica e aplicada - módulo II - primeira parte. Rio de janeiro.

LANCASTER, P.; SALKAUSKAS, K. (1981). Surfaces generated by moving least squares methods. Mathematics of Computation, v. 37, n.155, p. 141-158.

LANCASTER, P.; SALKAUSKAS, K. (1990). Curve and surface fitting. Academic press.

MELENK, J. M. (1992). Finite element methods whit harmonic shape functions for solving laplace's equation. Dissertação (Mestrado) - University of Maryland, College Park, 1992.

MELENK, J. M. (1995). On generalized finite element methods. Tese (Doutorado) University of Maryland, College Park,1995.

MELENK, J. M.; BABUŠKA, I. (1996). The partition of unity finite element method: basic theory and applications. Computer Methods in Applied Mechanics and Engineering, v.139, p.289-314.

NETO, R. G. A. (2008). Elementos finitos não-convencionais em formulações variacionais mistas. Dissertação (Mestrado). Escola de Engenharia de São Carlos, Universidade de São Paulo.

ODEN, J. T.; DUARTE, C.A.; ZIENKIEWICZ, O. C. (1998). A new cloud - based hp finite element method. Computer Methods in Applied Mechanics and Engineering, v. 153, p. 117-126.

OÑATE, E. (1995). Calculo de estructuras por el metodo de elementos finitos: análisis estático lineal. 2.ed. Barcelona: Artes Gráficas Torres S.A.

OÑATE, E.; IDELSOHN, S.; ZIENKIEWICZ, O. C. (1995). Finite point methods in computational mechanics. Report 67.

OÑATE, E. et al. (1996). A finite point method in computational mechanics applications to convective transport and fluid flow. International Journal for Numerical Methods in Engineering, v.39, p. 3839-3866.

OÑATE, E. et al. (1996). A stabilized finite point method for analysis of fluid mechanics problems. Computer Methods in Applied Mechanics and Engineering, v. 139, p. 315-346.

PAVLIN, V.; PERRONE, N. (1975). Finite diference energy techniques for arbitrary meshes. Computer \& Structures, v. 5, p. 45-58.

PROENÇA, S. P. B. (2008). Introdução aos métodos numéricos. Notas de Aula. Escola de Engenharia de São Carlos, Universidade de São Paulo. 
PIMENTA, P. M.; PROENÇA, S. P. B.; FREITAS, J. A. T. (2002). Elementos finitos híbridos mistos com enriquecimento nodal. J. M. Gaicolea, C. Mota Soares, M. Pastor y G. Bugeda (Eds.), Métodos Numéricos em Ingeniería V, SEMNI.

PIAN, T.H H. (1964). Derivation of element stiffness matrices assumed stress distributions. AIAAJ, v. 2, p. 1333-1336.

PIAN, T.H H.; TONG, P. (1969). Basis of finite element methods for solid continua. International Journal for Numerical Methods in Engineering, v. 1, p. 3-29.

PUNCH, E.F.; ATLURI, S. N. (1984). Development and testing of stable, invariant, isoparametric curvilinear 2- and 3-d hybrid-stress elements. Computer Methods in Applied Mechanics and Engineering, v.47, p. 331-356.

SAVASSI, W. (1996). Introdução ao método dos elementos finitos em análise linear de estruturas. São Carlos. Escola de Engenharia de São Carlos. Universidade de São Paulo.

SCHWAB, CH. (1998). p- and hp- finite element methods: theory and applications in solid and fluid mechanics. Oxford: Oxford University Press Inc.

SHERPAD, D. (1968). A two-dimensional functions for irregularly spaced data. ACM National Conference. p. 517-524.

SIMO, J.C.; RIFAI, M.S. (1990). A class of mixed assumed strain method of incompatible modes. International for Numerical Methods in Engineering, v. 29, p.1595-1638.

SORIANO, H.L. (2003). Método de elementos finitos em análise de estruturas. 1.ed. São Paulo. EDUSP. Editora da Universidade de São Paulo.

SOUZA, C. O. (2008). Formulação híbrida-trefftz com enriquecimento seletivo: aplicação a problemas bidimensionais da elasticidade. Dissertação (Mestrado). Escola de Engenharia de São Carlos, Universidade de São Paulo.

STROUBOULIS, T.; BABUŠKA, I.; COPPS, K. (2000). The design and analysis of the generalized finite element method. Computer Methods in Applied Mechanics and Engineering, v. 181, n. 1-3 , p. 43-69.

SZABÓ, B.; BABUŠKA, I. (1991). Finite element analysis. John Wiley \& Sons.

TIMOSHENKO, S. P.; GOODIER, J. N. (1980). Teoria da Elasticidade. Guanabara Dois S. A.

TORRES, I. F. R. (2003). Desenvolvimento e aplicação do método dos elementos finitos generalizados em análise tridimensional não-linear de sólidos. Tese (Doutorado). Escola de Engenharia de São Carlos, Universidade de São Paulo.

VALLIAPAN, S. (1981). Continuum Mechanics: Fundamentals. A. A. Balkema.

WASHIZU, K. (1975). Variational methods in elasticity and plasticity. Pergamon Press. 
XU, Z. (1992). Applied Elasticity. John Wiley \& Sons.

ZIENKIEWICZ, O. C. (1980). El método de elementos finitos. Editorial Reverté S.A.

ZIENKIEWICZ, O. C. (2000). The finite element method-Volume 1: The Basis.5.ed. Butterworth Heinemann.

ZIENKIEWICZ, O. C.; LEFEBVRE, D. (1987). Three-field mixed approximation and the plate bending problem. Communications in Applied Numerical Methods, v.3, p. 301-309.

ZIENKIEWICZ, O. C. et al. (1986). The patch test for mixed formulation. International Journal for Numerical Methods in Engineering, v.23, p. 1873-1882. 


\section{APÊNDICE A - Pontos e Pesos de Hammer - Integração Numérica em Domínios Triangulares}

A tabela A.1 apresenta os pontos e pesos de Hammer implementados nos programas computacionais desenvolvidos nesta pesquisa:

Tabela A.1 - Pontos e Pesos de Hammer para $0 \leq \xi_{i} \leq 1, i=1,2,3$

\begin{tabular}{|c|c|c|c|c|c|}
\hline Grau & Pontos & $\xi_{1}$ & $\xi_{2}$ & $\xi_{3}$ & $w_{i}$ \\
\hline 1 & 1 & $1 / 3$ & $1 / 3$ & $1 / 3$ & $1 / 2$ \\
\hline \multirow{3}{*}{2} & \multirow{3}{*}{3} & $2 / 3$ & $1 / 6$ & $1 / 6$ & $1 / 6$ \\
\hline & & $1 / 6$ & $2 / 3$ & $1 / 6$ & $1 / 6$ \\
\hline & & $1 / 6$ & $1 / 6$ & $2 / 3$ & $1 / 6$ \\
\hline \multirow{4}{*}{3} & \multirow{4}{*}{4} & $1 / 3$ & $1 / 3$ & $1 / 3$ & $-0,28125000$ \\
\hline & & 0,60 & 0,20 & 0,20 & 0,26041666 \\
\hline & & 0,20 & 0,60 & 0,20 & 0,26041666 \\
\hline & & 0,20 & 0,20 & 0,60 & 0,26041666 \\
\hline \multirow{6}{*}{4} & \multirow{6}{*}{6} & 0,81684757 & 0,09157621 & 0,09157621 & 0,05497587 \\
\hline & & 0,09157621 & 0,81684757 & 0,09157621 & 0,05497587 \\
\hline & & 0,09157621 & 0,09157621 & 0,81684757 & 0,05497587 \\
\hline & & 0,10810301 & 0,44594849 & 0,44594849 & 0,11169079 \\
\hline & & 0,44594849 & 0,10810301 & 0,44594849 & 0,11169079 \\
\hline & & 0,44594849 & 0,44594849 & 0,10810301 & 0,11169079 \\
\hline
\end{tabular}


Tabela A.1 - Continuação

\begin{tabular}{|c|c|c|c|c|c|}
\hline Grau & Pontos & $\xi_{1}$ & $\xi_{2}$ & $\xi_{3}$ & $w_{i}$ \\
\hline \multirow{7}{*}{5} & \multirow{7}{*}{7} & $1 / 3$ & $1 / 3$ & $1 / 3$ & 0,11250000 \\
\hline & & 0,79742698 & 0,10128650 & 0,10128650 & 0,06296959 \\
\hline & & 0,10128650 & 0,79742698 & 0,10128650 & 0,06296959 \\
\hline & & 0,10128650 & 0,10128650 & 0,79742698 & 0,06296959 \\
\hline & & 0,05971587 & 0,47014206 & 0,47014206 & 0,06619708 \\
\hline & & 0,47014206 & 0,05971587 & 0,47014206 & 0,06619708 \\
\hline & & 0,47014206 & 0,47014206 & 0,05971587 & 0,06619708 \\
\hline \multirow{12}{*}{6} & \multirow{12}{*}{12} & 0,87382197 & 0,06308901 & 0,06308901 & 0,02542245 \\
\hline & & 0,06308901 & 0,87382197 & 0,06308901 & 0,02542245 \\
\hline & & 0,06308901 & 0,06308901 & 0,87382197 & 0,02542245 \\
\hline & & 0,50142650 & 0,24928674 & 0,24928674 & 0,05839314 \\
\hline & & 0,24928674 & 0,50142650 & 0,24928674 & 0,05839314 \\
\hline & & 0,24928674 & 0,24928674 & 0,50142650 & 0,05839314 \\
\hline & & 0,63650249 & 0,31035245 & 0,05314504 & 0,04142554 \\
\hline & & 0,31035245 & 0,63650249 & 0,05314504 & 0,04142554 \\
\hline & & 0,05314504 & 0,31035245 & 0,63650249 & 0,04142554 \\
\hline & & 0,63650249 & 0,05314504 & 0,31035245 & 0,04142554 \\
\hline & & 0,31035245 & 0,05314504 & 0,63650249 & 0,04142554 \\
\hline & & 0,05314504 & 0,63650249 & 0,31035245 & 0,04142554 \\
\hline
\end{tabular}


Tabela A.1 - Continuação

\begin{tabular}{|c|c|c|c|c|c|}
\hline Grau & Pontos & $\xi_{1}$ & $\xi_{2}$ & $\xi_{3}$ & $w_{i}$ \\
\hline \multirow{16}{*}{8} & \multirow{16}{*}{16} & $1 / 3$ & $1 / 3$ & $1 / 3$ & 0,07215780 \\
\hline & & 0,08141482 & 0,45929258 & 0,45929258 & 0,04754582 \\
\hline & & 0,45929258 & 0,08141482 & 0,45929258 & 0,04754582 \\
\hline & & 0,45929258 & 0,45929258 & 0,08141482 & 0,04754582 \\
\hline & & 0,65886138 & 0,17056930 & 0,17056930 & 0,05160869 \\
\hline & & 0,17056930 & 0,65886138 & 0,17056930 & 0,05160869 \\
\hline & & 0,17056930 & 0,17056930 & 0,65886138 & 0,05160869 \\
\hline & & 0,89890554 & 0,05054721 & 0,05054721 & 0,01622925 \\
\hline & & 0,05054721 & 0,89890554 & 0,05054721 & 0,01622925 \\
\hline & & 0,05054721 & 0,05054721 & 0,89890554 & 0,01622925 \\
\hline & & 0,00839477 & 0,26311282 & 0,72849239 & 0,01361516 \\
\hline & & 0,26311282 & 0,00839477 & 0,72849239 & 0,01361516 \\
\hline & & 0,72849239 & 0,26311282 & 0,00839477 & 0,01361516 \\
\hline & & 0,00839477 & 0,72849239 & 0,26311282 & 0,01361516 \\
\hline & & 0,26311282 & 0,72849239 & 0,00839477 & 0,01361516 \\
\hline & & 0,72849239 & 0,00839477 & 0,26311282 & 0,01361516 \\
\hline
\end{tabular}

